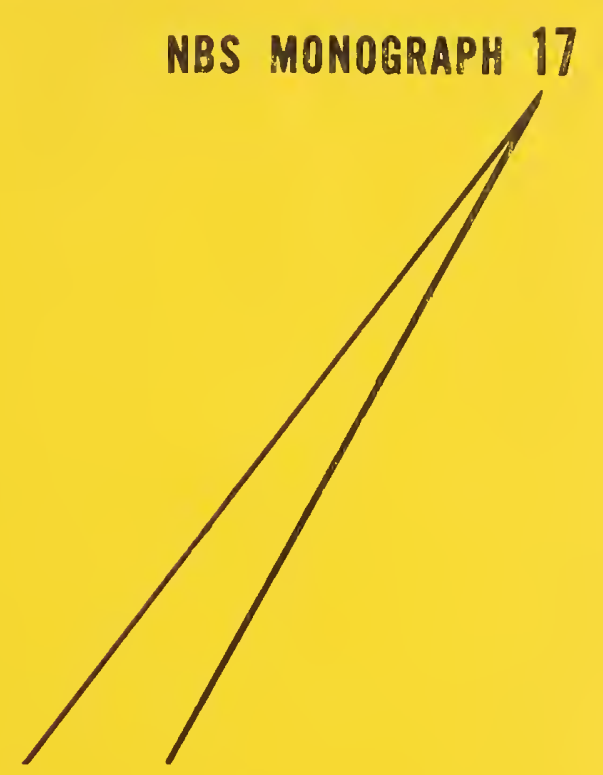

\title{
New Description of
}

\section{Thorium Spectra}

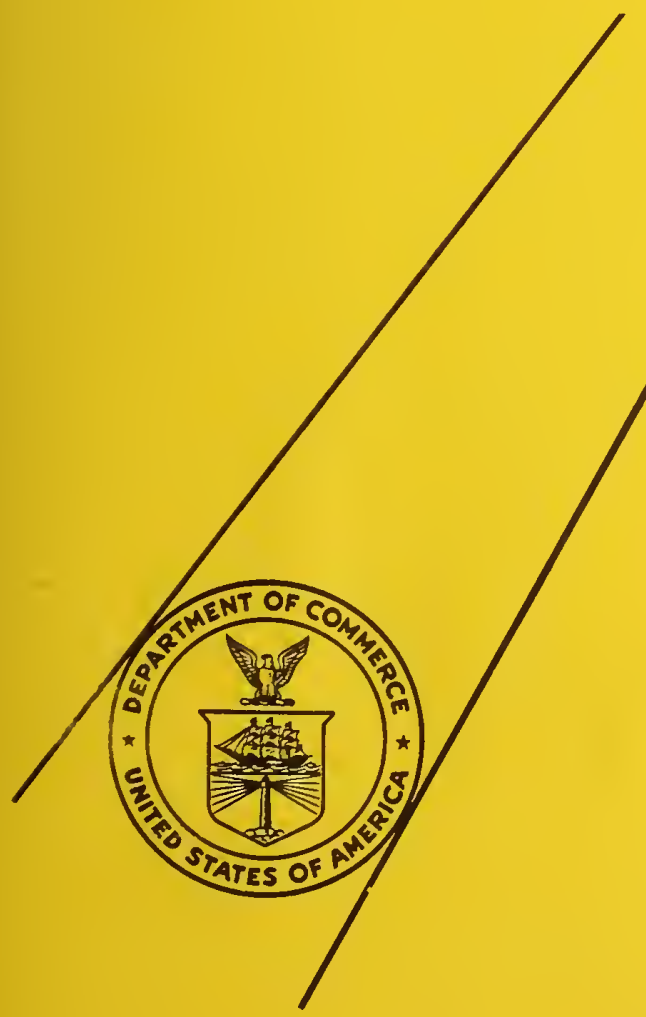

U.S. DEPARTMENT OF COMMERCE NATIONAL BUREAU OF STANDARDS 


\section{THE NATIONAL BUREAU OF STANDARDS}

\section{Functions and Activities}

The functions of the National Bureau of Standards are set forth in the Act of Congress, March 3, 1901, as amended by Congress in Public Law 619, 1950. These include the development and maintenance of the national standards of measurement and the provision of means and methods for making measurements consistent with these standards; the determination of physical constants and properties of materials; the development of methods and instruments for testing materials, devices, and structures; advisory services to government agencies on scientific and technical problems; invention and development of devices to serve special needs of the Government; and the development of standard practices, codes, and specifications. The work includes basic and applied research, development, engineering, instrumentation, testing, evaluation, calibration services, and various consultation and information services. Research projects are also performed for other government agencies when the work relates to and supplements the basic program of the Bureau or when the Bureau's unique competence is required. The scope of activities is suggested by the listing of divisions and sections on the inside of the back cover.

\section{Publications}

The results of the Bureau's work take the form of either actual equipment and devices or published papers. These papers appear either in the Bureau's own series of publications or in the journals of professional and scientific societies. The Bureau itself publishes three periodicals available from the Government Printing Office: The Journal of Research, published in four separate sections, presents complete scientific and technical papers; the Technical News Bulletin presents summary and preliminary reports on work in progress; and Basic Radio Propagation Predictions provides data for determining the best frequencies to use for radio communications throughout the world. There are also five series of nonperiodical publications: Monographs, Applied Mathematics Series, Handbooks, Miscellaneous Publications, and Technical Notes.

Information on the Bureau's publications can be found in NBS Circular 460, Publications of the National Bureau of Standards (\$1.25) and its Supplement (\$1.50), available from the Superintendent of Documents, Government Printing Office, Washington 25, D.C. 


\section{New Description of Thorium Spectra}

Romuald Zalubas

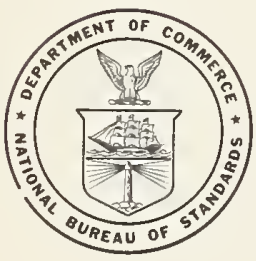

National Bureau of Standards Monograph 17

Issued June 21, 1960 


\section{Contents}

2. References

Table 1. Wavelengths of thorium spectra $\ldots \ldots \ldots \ldots \ldots$ 


\title{
New Description of Thorium Spectra
}

\author{
Romuald Zalubas
}

Wavelengths and estimated intensities in electrodeless lamp and spark sources are presented for 15121 lines of Th I, Th II, Th III, and. Th IV in the spectral range from 2000 to $11550 \mathrm{~A}$. Previously published interferometric values are in this table rounded off to three decimal places and followed by the letter $\mathrm{i}$.

\section{Introduction}

In 1868, forty years after Berzelius' discovery of thorium, Thalén measured the first spectral lines of this element [1]. ${ }^{1} \quad$ Later in 1909, Moore [2] was the first to observe the splitting of thorium lines in a magnetic field. Kayser in volume 6 of his Handbuch der Spectroskopie listed 23 papers on the thorium spectra which had appeared up to 1912 . His table contained about 1,600 arc and spark lines of thorium. This probably constituted about half of the total number of known lines, for weak lines were not included because at that time he did not see much use for them. Since then there have appeared about 50 more papers dealing with the thorium spectra, some being contributions to special regions of the spectra starting in the vacuum and ending in the infrared region; some dealing with its identification in the solar spectrum, in chemical compounds or in radioactivity decay products; some describing and analyzing Th 1 , Th II, Th IlI, and Th Iv; and some others being theoretical considerations of the thorium spectra among the other rare earth spectra. Since 1912, three new compilations have been published, namely; in 1939, the MIT Wavelength tables [3]; in 1946, the Specola Vaticana in Die Spektren der Seltenen Erden gave a list of the known Th 1, II, and III lines [4]; and in 1958, Oak Ridge National Laboratory published a Compilation of Data of some Spectra of Thorium [5]. In these four descriptions, the references to most of the literature on the thorium spectra can be found; therefore, there is little use in giving a complete bibliography here.

As early as 1926 [6], work on the thorium spectra at the National Bureau of Standards was initiated by Kiess, but never published. Kiess secured some spectrograms of arcs and sparks between thorium metal electrodes in air with a Hilger prism spectrograph having a dispersion of $0.6 \AA / \mathrm{mm}$ at $\lambda 2200 \AA$. He also measured some of these spectrograms, and these measurements are incorporated in the present publication.

In 1955 the thorium program was resumed at the Bureau under the supervision of Kiess, because halide electrodeless lamps excited by microwaves at 2,450 Mc became available. These lamps

\footnotetext{
1 Figures in brackets indicate the literature references on p. 2
}

emit nuch sharper and more numerable lines than conventional ares in air and do not excite the troublesome atmospheric bands that normally mask selected regions of the spectrum.

The first electrodeless thorium halide ( $\mathrm{Th} \mathrm{I}_{4}$ and $\mathrm{Th} \mathrm{Br}_{4}$ ) lamps were made of thorium metal by combining the metal and halogen. The metal, as later appeared, had impurities of zirconium. Later Bozman succeeded in making a thorium halide lamp, by using $\mathrm{Th} \mathrm{O}_{2}$. These spectrograms were free of zirconium impurities, thus enabling us to eliminate the zirconium lines from our list. The concave grating spectrographs of the Bureau with $7,500,15,000$, and 30,000 lines per inch were used. Spectrograms were taken with various sources, namely with an electrodeless lamp and with a spark having thorium metal electrodes. They also included iron electrodeless lamp exposures to furnish iron secondary wavelength standards.

Zeeman magnetic patterns of $\mathrm{Th}_{1}$ and $\mathrm{Th}$ II have been produced by using the Bureau electromagnet with fields of about 37,000 oersteds. About 1,000 of these patterns have been measured and the resulting $g$-values determined.

Th 1, Th I1, and Th 111 lines have been separated by using the following criteria: (a) Relative intensities in lamp and spark exposures; (b) relative intensities of $n$ and $p$ components of unresolved Zeeman patterns as compared with a no field exposure; (c) resolved Zeeman patterns; and (d) known classified lines of Th I, Th 1I, Th In, and Th $1 v$ from the literature [7].

Usually, lines relatively stronger in an electrodeless lamp than in a spark exposure are Th I lines; those relatively weak in lamp exposures and stronger in spark exposures are Th II lines; and lines either not present or very weak in lamp exposures but relatively strong in spark exposures are Th III lines.

At least three sets of plates in every region have been secured and measured by "hand and eye." Only near the end of the work some plates were measured by semiautomatic Bureau equipment, which in turn is designed for further processing by electronic computers. Most of the lines have been measured on three plates, and some of 
them on as many as eight plates. A few lines are included that have been measured only twice.

Simultaneously with this work, interferometric measurements of thorium secondary standards in the region $\lambda 3287 \mathrm{~A}-6989 \mathrm{~A}$ were made by Meggers and Stanley [8] at the Bureau. Their results have been used to improve the accuracy of our measurements of thorium lines. The relative intensities of the lines in lamp exposures were first estimated by eye, and later calibrated to a uniform scale in the region from 2000 to 9000 A by using Corliss' et al. [9] unpublished data of relative intensities for 2,000 lines of thorium. Spark intensities have not been calibrated to a uniform scale.

The wavelengths are tabulated in table 1 . The first column gives the wavelength in Angstrom units. The letter $i$ after the wavelength indicates the thorium standards, wavelengths of which are taken from published interferometric measurements [8] and rounded off to three decimal places. This was done in order to make the list more convenient for users of thorium standards. Our grating warelengths do not differ more than $\pm 0.005 \mathrm{~A}$ from the corresponding interferometric measurements. The second and the third columns contain intensities of lines in electrodeless lamps and sparks in air. The letter symbols are used to describe the character of the lines; $d$-double, $h$-hazy, $w$-wide, $l$-shaded to longer wavelength, and $s$-shaded to shorter wavelength.
The fourth column in the table gives the assignment of the individual line to successive spectra.

Table 1 contains more than twice as many lines as have heretofore been published. It should meet all the present requirements for spectrochemistry, and for an extension of the structural analysis of Th I and Th II between the wavelength limits 2000 and $12000 \mathrm{~A}$. The work on the analysis of Th I is continuing at this Bureau. A short account of the present state of analysis of Th I has been published separately [10].

This work could not have been carried to its present stage of completion without the cordial collaboration of several spectroscopists. The author is especially indebted to C. C. Kiess for suggesting the problem, for the use of his unpublished data and for taking a keen interest in the progress of the analysis. W. F. Meggers and R. W. Stanley generously furnished the thorium standard wavelengths used here. W. F. Meggers and $\mathrm{C} . \mathbf{H}$. Corliss provided the data for calibrating the intensities of Th I lines. C. H. Corliss and W. R. Bozman provided some of the lamps used for sources, and the latter assisted greatly with the coding, printing and other digital computer work. Mrs. Ruth Peterson has been an invaluable assistant with the computational and clerical work. To all of these it is a pleasure to record my gratitude.

\section{References}

[1] H. Kayser, Handbuch der Spektroskopie VI, 636, (1912).

[2] B. E. Moore, Ap. J. 33, 143 (1909).

[3] G. R. Harrison, MiT Wavelength Tables (1939).

[4] A. Gatterer, J. Junkes and V. Frodl, Die Spektren der Seltenen Erden p. 329 (1945).

[5] G. W. Charles, O.R.N.L. 2,319 pp (1958).

[6] (a) C. C. Kiess and R. Zalubas, Spectroscopy Symposium at Argonne National Laboratory, p. 16 (Feb. 1956).

(b) R. Zalubas, J. Opt. Soc. Am. 49, 512 (1959).

[7] (a) J. R. MeNally, Jr., C. R. Harrison and H. B. Park, J. Opt. Soc. Am. 32, 334 (1942).

(b) L. de Bruin, P. F. A. Klinkenberg, and $\mathrm{Ph}$. Schuurmans, Z. Physik 122, 23 (1944).

(c) J. R. MeNally, Jr., J. Opt. Soc. Am. 35, 390 (1945).

(d) R. J. Lang, Phys. Rev. 56, 272 (1939).

(e) L. de Bruin and P. F. A. Klinkenberg, Proc. Acad. Sci. Amsterdam 43, 581 (1940). (f) L. de Bruin, P. F. A. Klinkenberg and Ph. Schuurmans, Z. Physik. 118, 58 (1941).

(g) P. F. A. Klinkenberg, Physica 16, 618 (1950).

(h) R. J. Lang, Can. J. Res. A 14, 43 (1936).

(i) P. F. A. Klinkenberg and R. J. Lang, Physica 15, 774 (1949).

(k) P. F. A. Klinkenberg, Physica 16, 185 (1950).

(1) R. Zalubas, unpublished data (1959).

[8] W. F. Meggers and R. W. Stanley, J. Research NBS 61, 95-103 (1958).

[9] W. F. Meggers, C. H. Corliss, and B. F. Scribner, unpublished data on relative intensities of spectral lines (1959).

[10] R. Zalubas, J. Research NBS 63a, 275-278 (1959).

Washington, D. C., March 9, 1960. 
Table 1. Wavelengths of Thorium Spectra

\begin{tabular}{|c|c|c|c|c|c|c|c|c|c|c|c|}
\hline \multirow{2}{*}{$\begin{array}{l}\text { Wave- } \\
\text { length }\end{array}$} & \multicolumn{2}{|c|}{ Intensity } & \multirow{2}{*}{$\begin{array}{l}\text { Spec- } \\
\text { trum }\end{array}$} & \multirow{2}{*}{$\begin{array}{l}\text { Wave- } \\
\text { length }\end{array}$} & \multicolumn{2}{|c|}{ Intensity } & \multirow{2}{*}{$\begin{array}{l}\text { Spec- } \\
\text { trum }\end{array}$} & \multirow{2}{*}{$\begin{array}{l}\text { Wave- } \\
\text { length }\end{array}$} & \multicolumn{2}{|c|}{ Intensity } & \multirow{2}{*}{$\begin{array}{l}\text { Spec } \\
\text { trum }\end{array}$} \\
\hline & & Spark & & & Lamp & Spark & & & Lamp & Spark & \\
\hline $\begin{array}{l}2002.34 \\
2004.38 \\
2012.68 \\
2018.71 \\
2027.99\end{array}$ & 1 & $\begin{array}{r}125 \\
2 \\
20 \\
3 \\
2\end{array}$ & $\begin{array}{l}\text { I V } \\
\text { I I I } \\
\text { I I } \\
\text { II } \\
\text { II I }\end{array}$ & $\begin{array}{l}2134.77 \\
2135.08 \\
2135.45 \\
2136.16 \\
2137.77\end{array}$ & $\begin{array}{l}1 \\
2 \\
6\end{array}$ & 10 & $\begin{array}{l}\text { I I } \\
\text { I } \\
\text { I } \\
\text { I I I }\end{array}$ & $\begin{array}{l}2173.15 \\
2173.41 \\
2173.51 \\
2174.14 \\
2174.33\end{array}$ & $\begin{array}{l}l \\
l w \\
l w \\
1 \\
1\end{array}$ & & $\begin{array}{l}\text { I } \\
\text { I } \\
\text { I } \\
\text { I } \\
\text { I }\end{array}$ \\
\hline $\begin{array}{l}2028.93 \\
2029.28 \\
2032.93 \\
2033.46 \\
2035.72\end{array}$ & $\begin{array}{l}1 \\
1\end{array}$ & $\begin{array}{r}12 \\
2 \\
1\end{array}$ & $\begin{array}{l}\text { I } \\
\text { I I I } \\
\text { II } \\
\text { I I } \\
\text { I }\end{array}$ & $\begin{array}{l}2138.57 \\
2139.26 \\
2141.94 \\
2144.14 \\
2145.38\end{array}$ & $\begin{array}{c}10 \\
1 \\
2 \\
1 \mathrm{w}\end{array}$ & 10 & $\begin{array}{l}\text { I } \\
\text { I } \\
\text { I } \\
\text { I I I } \\
\text { I }\end{array}$ & $\begin{array}{l}2174.41 \\
2174.56 \\
2175.13 \\
2175.82 \\
2176.29\end{array}$ & $\begin{array}{l}1 \\
1 \\
2 \\
1\end{array}$ & $4 w$ & $\begin{array}{l}\text { I I I } \\
\text { I } \\
\text { I } \\
\text { I } \\
\text { I }\end{array}$ \\
\hline $\begin{array}{l}2036.18 \\
2037.26 \\
2039.58 \\
2040.33 \\
2059.79\end{array}$ & $\begin{array}{l}1 \\
1 \\
1\end{array}$ & $\begin{array}{r}10 \\
5 \\
2 \\
10 \\
2\end{array}$ & $\begin{array}{l}\text { I I I } \\
\text { II } \\
\text { II I } \\
\text { III } \\
\text { II }\end{array}$ & $\begin{array}{l}2146.93 \\
2147.14 \\
2148.26 \\
2149.21 \\
2151.31\end{array}$ & $\begin{array}{l}5 \\
2 \\
1 \\
1\end{array}$ & 50 & $\begin{array}{l}\text { IV } \\
\text { I } \\
\text { I } \\
\text { II I } \\
\text { I }\end{array}$ & $\begin{array}{l}2176.38 \\
2176.77 \\
2176.84 \\
2177.16 \\
2177.36\end{array}$ & $\begin{array}{l}1 \\
1 \\
2 \\
1 .\end{array}$ & $\begin{array}{l}2 \\
4 \\
2\end{array}$ & $\begin{array}{l}\text { I } \\
\text { I I I } \\
\text { I I } \\
\text { I I } \\
\text { I }\end{array}$ \\
\hline $\begin{array}{l}2062.29 \\
2062.41 \\
2064.80 \\
2065.73 \\
2066.74\end{array}$ & $\begin{array}{l}1 \\
1\end{array}$ & $\begin{array}{c}4 \\
3 \\
3 \\
50 w\end{array}$ & $\begin{array}{l}\text { I I I } \\
\text { I } \\
\text { II I } \\
\text { II } \\
\text { IV }\end{array}$ & $\begin{array}{l}2151.41 \\
2151.67 \\
2152.95 \\
2153.28 \\
2153.38\end{array}$ & $\begin{array}{l}1 \\
1 \\
1 \\
2 \\
3\end{array}$ & $\begin{array}{l}2 \\
1\end{array}$ & $\begin{array}{l}\text { II } \\
\text { I I } \\
\text { I } \\
\text { I } \\
\text { I }\end{array}$ & $\begin{array}{l}2177.76 \\
2177.87 \\
2177.96 \\
2178.35 \\
2178.47\end{array}$ & $\begin{array}{l}1 \\
1 \\
1 \\
1\end{array}$ & $\begin{array}{l}4 w \\
1\end{array}$ & $\begin{array}{l}\text { I } \\
\text { II I } \\
\text { I } \\
\text { I I } \\
\text { I }\end{array}$ \\
\hline $\begin{array}{l}2073.04 \\
2074.54 \\
2090.11 \\
2090.62 \\
2090.90\end{array}$ & $\begin{array}{l}1 \\
1 \\
1\end{array}$ & $\begin{array}{r}25 \\
2 \\
2 \\
2 \\
15\end{array}$ & $\begin{array}{l}\text { I I I } \\
\text { II } \\
\text { I } \\
\text { II } \\
\text { II }\end{array}$ & $\begin{array}{l}2153.56 \\
2154.06 \\
2155.86 \\
2157.09 \\
2159.38\end{array}$ & $\begin{array}{l}1 \\
1 \\
1 \\
1\end{array}$ & 5 & $\begin{array}{l}\text { II } \\
\text { I } \\
\text { I } \\
\text { I } \\
\text { I }\end{array}$ & $\begin{array}{l}2179.42 \\
2180.96 \\
2181.06 \\
2181.15 \\
2184.31\end{array}$ & $\begin{array}{l}1 \\
1 \\
1 \\
2 \\
1\end{array}$ & $\begin{array}{l}3 \\
1 \\
3\end{array}$ & $\begin{array}{l}\text { I I } \\
\text { I } \\
\text { I } \\
\text { I I } \\
\text { I }\end{array}$ \\
\hline $\begin{array}{l}2091.27 \\
2091.62 \\
2099.47 \\
2103.61 \\
2105.90\end{array}$ & $\begin{array}{l}1 \\
1 \\
1 \\
1 \\
1 \mathrm{~h}\end{array}$ & $\begin{array}{l}15 \\
15 \\
3 w \\
2 w\end{array}$ & $\begin{array}{l}\text { I } \\
\text { II } \\
\text { II I } \\
\text { III } \\
\text { II I }\end{array}$ & $\begin{array}{l}2159.78 \\
2160.86 \\
2161.19 \\
2161.54 \\
2162.76\end{array}$ & $\begin{array}{l}1 \\
1 \\
1 \\
1 \\
1\end{array}$ & & $\begin{array}{l}\text { I } \\
\text { I } \\
\text { I } \\
\text { I } \\
\text { I }\end{array}$ & $\begin{array}{l}2184.66 \\
2184.74 \\
2185.47 \\
2185.63 \\
2186.88\end{array}$ & $\begin{array}{l}1 \\
1 \\
1 \\
1\end{array}$ & $\begin{array}{l}3 \\
3\end{array}$ & $\begin{array}{l}\text { I I } \\
\text { I I } \\
\text { I } \\
\text { I } \\
\text { I }\end{array}$ \\
\hline $\begin{array}{l}2108.37 \\
2110.15 \\
2111.69 \\
2113.98 \\
2114.63\end{array}$ & $\begin{array}{l}2 \\
1 \\
2 \\
1 \\
1\end{array}$ & $\begin{array}{l}3 \\
2 \\
3\end{array}$ & $\begin{array}{l}\text { I I } \\
\text { I I } \\
\text { I I } \\
\text { I } \\
\text { I }\end{array}$ & $\begin{array}{l}2162.82 \\
2163.43 \\
2165.18 \\
2165.89 \\
2166.21\end{array}$ & $\begin{array}{l}1 \\
1 \\
1\end{array}$ & $\begin{array}{r}50 \\
2\end{array}$ & $\begin{array}{l}\text { II I } \\
\text { II } \\
\text { I } \\
\text { I } \\
\text { I }\end{array}$ & $\begin{array}{l}2187.61 \\
2189.64 \\
2190.78 \\
2192.01 \\
2192.33\end{array}$ & $\begin{array}{l}5 \\
1 \\
1\end{array}$ & $\begin{array}{r}10 \\
3 \\
2 \\
\\
50\end{array}$ & $\begin{array}{l}\text { II } \\
\text { II } \\
\text { I I } \\
\text { I } \\
\text { II I }\end{array}$ \\
\hline $\begin{array}{l}2115.26 \\
2116.36 \\
2117.53 \\
2121.64 \\
2123.00\end{array}$ & $\begin{array}{l}1 \\
1 \\
2 \\
2\end{array}$ & $\begin{array}{l}5 \\
5 \\
3 \\
2\end{array}$ & $\begin{array}{l}\text { I I I } \\
\text { I } \\
\text { II } \\
\text { II } \\
\text { I I }\end{array}$ & $\begin{array}{l}2166.48 \\
2166.63 \\
2167.24 \\
2167.56 \\
2167.80\end{array}$ & $\begin{array}{l}1 \\
1 \\
1 \\
1 \\
2\end{array}$ & 2 & $\begin{array}{l}\text { I } \\
\text { I } \\
\text { I } \\
\text { I } \\
\text { II }\end{array}$ & $\begin{array}{l}2192.92 \\
2193.19 \\
2193.92 \\
2194.10 \\
2194.64\end{array}$ & $\begin{array}{l}1 \\
1 \\
1 \\
2 \\
1\end{array}$ & 1 & $\begin{array}{l}\text { I } \\
\text { I } \\
\text { I } \\
\text { II } \\
\text { I }\end{array}$ \\
\hline $\begin{array}{l}2124.11 \\
2126.59 \\
2127.28 \\
2127.89 \\
2128.20\end{array}$ & $\begin{array}{l}2 \\
1 \\
1 \\
1 \\
2\end{array}$ & 2 & $\begin{array}{l}\text { I } \\
\text { I } \\
\text { I } \\
\text { I } \\
\text { II }\end{array}$ & $\begin{array}{l}2169.25 \\
2169.47 \\
2169.57 \\
2170.01 \\
2170.28\end{array}$ & $\begin{array}{l}1 \\
1 \\
1 \\
2\end{array}$ & $\begin{array}{l}2 \\
5 \mathrm{w}\end{array}$ & $\begin{array}{l}\text { I } \\
\text { I } \\
\text { II } \\
\text { I } \\
\text { I I I }\end{array}$ & $\begin{array}{l}2194.90 \\
2195.19 \\
2196.10 \\
2196.47 \\
2196.65\end{array}$ & $\begin{array}{l}1 \\
1 \\
1 \\
1 \\
1\end{array}$ & & $\begin{array}{l}\text { I } \\
\text { I } \\
\text { I } \\
\text { I } \\
\text { I }\end{array}$ \\
\hline $\begin{array}{l}2128.55 \\
2129.25 \\
2129.36 \\
2132.76 \\
2134.69\end{array}$ & $\begin{array}{l}1 \\
1 \\
2 \\
2 \\
1\end{array}$ & $\begin{array}{l}1 \\
4 \\
5 \\
1\end{array}$ & $\begin{array}{l}\text { I I } \\
\text { I } \\
\text { II } \\
\text { II } \\
\text { I I }\end{array}$ & $\begin{array}{l}2171.23 \\
2171.57 \\
2172.00 \\
2172.47 \\
2172.69\end{array}$ & $\begin{array}{l}1 \\
1 \\
1 \\
1 \\
1\end{array}$ & 3 & $\begin{array}{l}\text { II } \\
\text { I } \\
\text { I } \\
\text { I } \\
\text { I }\end{array}$ & $\begin{array}{l}2197.16 \\
2197.68 \\
2198.20 \\
2198.63 \\
2198.73\end{array}$ & $\begin{array}{l}1 \\
2 \\
1 \\
2\end{array}$ & $\begin{array}{r}3 \\
50\end{array}$ & $\begin{array}{l}\text { I } \\
\text { I I } \\
\text { I } \\
\text { I I I } \\
\text { I }\end{array}$ \\
\hline
\end{tabular}


Table 1. Wavelengths of Thorium Spectra - Continued

\begin{tabular}{|c|c|c|c|c|c|c|c|c|c|c|c|}
\hline \multirow{2}{*}{$\begin{array}{l}\text { Wave- } \\
\text { length }\end{array}$} & \multicolumn{2}{|c|}{ Intensity } & \multirow{2}{*}{$\begin{array}{l}\text { Spec- } \\
\text { trum }\end{array}$} & \multirow{2}{*}{$\begin{array}{l}\text { Wave- } \\
\text { length }\end{array}$} & \multicolumn{2}{|c|}{ Intensity } & \multirow{2}{*}{$\begin{array}{l}\text { Spec- } \\
\text { trum }\end{array}$} & \multirow{2}{*}{$\begin{array}{l}\text { Wave- } \\
\text { length }\end{array}$} & \multicolumn{2}{|c|}{ Intensity } & \multirow{2}{*}{$\begin{array}{l}\text { Spec- } \\
\text { trum }\end{array}$} \\
\hline & Lamp & Spark & & & Lamp & Spark & & & Lamp & Spark & \\
\hline $\begin{array}{l}2199.75 \\
2200.18 \\
2200.48 \\
2200.97 \\
2201.17\end{array}$ & $\begin{array}{r}10 \\
2 \\
1 \\
1\end{array}$ & $\begin{array}{r}100 \\
1\end{array}$ & $\begin{array}{l}\text { II I } \\
\text { I } \\
\text { I } \\
\text { I } \\
\text { I }\end{array}$ & $\begin{array}{l}2221.55 \\
2222.14 \\
2222.20 \\
2222.27 \\
2222.60\end{array}$ & $\begin{array}{l}2 \\
2 \\
1 \\
1 \\
1\end{array}$ & 25 & $\begin{array}{l}\text { I I I } \\
\text { I } \\
\text { I } \\
\text { I } \\
\text { I I I }\end{array}$ & $\begin{array}{l}2259.60 \\
2261.75 \\
2261.91 \\
2263.40 \\
2264.65\end{array}$ & $\begin{array}{l}1 \\
1 \\
3 \\
1\end{array}$ & $\begin{array}{l}5 w \\
5\end{array}$ & $\begin{array}{l}\text { I I I } \\
\text { I } \\
\text { I } \\
\text { I I } \\
\text { I }\end{array}$ \\
\hline $\begin{array}{l}2201.28 \\
2201.59 \\
2201.72 \\
2201.92 \\
2202.20\end{array}$ & $\begin{array}{l}1 \\
1 \\
1 \\
1 \\
2\end{array}$ & & $\begin{array}{l}\text { I } \\
\text { I } \\
\text { I } \\
\text { I } \\
\text { I }\end{array}$ & $\begin{array}{l}2223.37 \\
2223.57 \\
2223.61 \\
2223.69 \\
2224.23\end{array}$ & $\begin{array}{l}2 \\
1 \\
1 \\
2\end{array}$ & $\begin{array}{r}20 \\
5\end{array}$ & $\begin{array}{l}\text { I } \\
\text { I } \\
\text { I I I } \\
\text { I } \\
\text { I I }\end{array}$ & $\begin{array}{l}2265.53 \\
2265.93 \\
2267.30 \\
2267.85 \\
2268.13\end{array}$ & $\begin{array}{l}1 \\
2 \\
1 \\
1\end{array}$ & $\begin{array}{l}5 \\
2 \\
4\end{array}$ & $\begin{array}{l}\text { I } \\
\text { II } \\
\text { I I I } \\
\text { I } \\
\text { II }\end{array}$ \\
\hline $\begin{array}{l}2202.79 \\
2203.15 \\
2203.87 \\
2204.16 \\
2205.40\end{array}$ & $\begin{array}{l}1 \\
1 \\
1 \\
1 \\
1\end{array}$ & & $\begin{array}{l}\text { I } \\
\text { I } \\
\text { I } \\
\text { I } \\
\text { I }\end{array}$ & $\begin{array}{l}2224.29 \\
2224.68 \\
2225.00 \\
2225.09 \\
2225.025\end{array}$ & $\begin{array}{l}1 \\
1 \\
1 \\
1\end{array}$ & 10 & $\begin{array}{l}\text { I I I } \\
\text { I } \\
\text { I } \\
\text { I } \\
\text { I I }\end{array}$ & $\begin{array}{l}2268.35 \\
2268.76 \\
2269.12 \\
2269.28 \\
2269.59\end{array}$ & $\begin{array}{l}1 \\
1 \\
1 \\
1 \\
1\end{array}$ & & $\begin{array}{l}\text { I } \\
\text { I } \\
\text { I } \\
\text { I } \\
\text { I }\end{array}$ \\
\hline $\begin{array}{l}2205.97 \\
2206.06 \\
2206.45 \\
2206.65 \\
2207.22\end{array}$ & $\begin{array}{l}1 \\
1 \\
1 \\
2\end{array}$ & 75 & $\begin{array}{l}\text { I } \\
\text { I } \\
\text { I } \\
\text { I I I } \\
\text { I }\end{array}$ & $\begin{array}{l}2226.41 \\
2227.12 \\
2227.29 \\
2228.90 \\
2229.96\end{array}$ & $\begin{array}{l}2 \\
2 \\
2\end{array}$ & $\begin{array}{r}3 \\
3 \\
2 \\
20\end{array}$ & $\begin{array}{l}\text { I I } \\
\text { I I } \\
\text { II } \\
\text { I I } \\
\text { I }\end{array}$ & $\begin{array}{l}2270.22 \\
2270.63 \\
2271.93 \\
2272.91 \\
2273.95\end{array}$ & $\begin{array}{l}1 \\
2 \\
1 \\
1 \\
1\end{array}$ & $\begin{array}{l}1 \\
6 w \\
2 \\
2\end{array}$ & $\begin{array}{l}\text { I I } \\
\text { I I I } \\
\text { I I } \\
\text { I } \\
\text { I I }\end{array}$ \\
\hline $\begin{array}{l}2207.58 \\
2207.98 \\
2208.05 \\
2208.33 \\
2208.54\end{array}$ & $\begin{array}{l}2 \\
2 \\
2 \\
1 \\
2\end{array}$ & $\begin{array}{l}2 \\
5\end{array}$ & $\begin{array}{l}\text { II } \\
\text { I } \\
\text { I I I } \\
\text { I } \\
\text { I }\end{array}$ & $\begin{array}{l}2230.36 \\
2230.85 \\
2231.25 \\
2231.35 \\
2232.20\end{array}$ & $\begin{array}{l}4 \\
1 \\
1 \mathrm{~h} \\
1\end{array}$ & $\begin{array}{l}10 \\
2 \\
6\end{array}$ & $\begin{array}{l}\text { II } \\
\text { I } \\
\text { II } \\
\text { II } \\
\text { I }\end{array}$ & $\begin{array}{l}2274.19 \\
2275.50 \\
2276.14 \\
2276.51 \\
2276.99\end{array}$ & $\begin{array}{l}1 \\
1 \\
1 \\
1\end{array}$ & 10 & $\begin{array}{l}\text { I } \\
\text { I } \\
\text { I } \\
\text { II } \\
\text { I }\end{array}$ \\
\hline $\begin{array}{l}2208.98 \\
2209.07 \\
2209.41 \\
2209.71 \\
2210.64\end{array}$ & $\begin{array}{l}1 \\
1 \\
1 \\
1 \\
2\end{array}$ & $\begin{array}{l}2 \\
2 \\
2 \\
5\end{array}$ & $\begin{array}{l}\text { II } \\
\text { II } \\
\text { II } \\
\text { I } \\
\text { II }\end{array}$ & $\begin{array}{l}2233.28 \\
2233.36 \\
2234.22 \\
2234.51 \\
2234.74\end{array}$ & 1 & $\begin{array}{l}2 \\
10 \mathrm{w} \\
10 \mathrm{w} \\
2\end{array}$ & $\begin{array}{l}\text { II } \\
\text { II I } \\
\text { III } \\
\text { I } \\
\text { III }\end{array}$ & $\begin{array}{l}2277.54 \\
2278.82 \\
2279.79 \\
2280.43 \\
2280.82\end{array}$ & $\begin{array}{l}2 \\
1 \\
5 \\
2\end{array}$ & $\begin{array}{l}30 \\
12\end{array}$ & $\begin{array}{l}\text { I } \\
\text { III } \\
\text { I } \\
\text { II } \\
\text { I }\end{array}$ \\
\hline $\begin{array}{l}2210.89 \\
2211.75 \\
2212.42 \\
2212.48 \\
2212.86\end{array}$ & $\begin{array}{r}10 \\
4 \\
1 \\
1 \\
2\end{array}$ & 3 & $\begin{array}{l}\text { I } \\
\text { I } \\
\text { I } \\
\text { I } \\
\text { I I }\end{array}$ & $\begin{array}{l}2235.76 \\
2236.07 \\
2238.31 \\
2239.76 \\
2241.55\end{array}$ & $\begin{array}{l}1 \\
2 \\
1 \\
5 \\
1\end{array}$ & 10 & $\begin{array}{l}\text { I } \\
\text { I } \\
\text { I } \\
\text { II } \\
\text { I }\end{array}$ & $\begin{array}{l}2281.55 \\
2281.97 \\
2282.40 \\
2282.77 \\
2283.39\end{array}$ & $\begin{array}{l}4 \\
1 \\
2 \\
1\end{array}$ & $\begin{array}{r}200 \\
3 \\
4\end{array}$ & $\begin{array}{l}\text { III I } \\
\text { II } \\
\text { I } \\
\text { II } \\
\text { I }\end{array}$ \\
\hline $\begin{array}{l}2213.12 \\
2213.83 \\
2214.55 \\
2215.12 \\
2216.03\end{array}$ & $\begin{array}{l}1 \\
1 \\
1 \\
2\end{array}$ & $\begin{array}{l}3 \\
2 \\
4 w\end{array}$ & $\begin{array}{l}\text { I } \\
\text { I } \\
\text { I I } \\
\text { II } \\
\text { I I I }\end{array}$ & $\begin{array}{l}2242.53 \\
2243.28 \\
2244.43 \\
2244.90 \\
2247.27\end{array}$ & $\begin{array}{l}1 \\
3 \\
1 \\
1\end{array}$ & $\begin{array}{l}1 \\
5 \\
2 \\
2 \\
5\end{array}$ & $\begin{array}{l}\text { I I } \\
\text { I I } \\
\text { I I } \\
\text { I I } \\
\text { I I }\end{array}$ & $\begin{array}{l}2283.66 \\
2283.97 \\
2284.27 \\
2284.62 \\
2285.31\end{array}$ & $\begin{array}{l}1 \\
1 \\
1 \\
1 \\
2\end{array}$ & $\begin{array}{r}30 \\
3 \\
4\end{array}$ & $\begin{array}{l}\text { I } \\
\text { I } \\
\text { I I } \\
\text { I I I } \\
\text { I I }\end{array}$ \\
\hline $\begin{array}{l}2216.47 \\
2216.67 \\
2216.78 \\
2217.17 \\
2218.05\end{array}$ & $\begin{array}{l}1 \\
5 \\
1 \\
1 \\
2\end{array}$ & $\begin{array}{l}1 \\
1 \\
2\end{array}$ & $\begin{array}{l}\text { I I } \\
\text { I } \\
\text { I I } \\
\text { I } \\
\text { I }\end{array}$ & $\begin{array}{l}2247.70 \\
2249.58 \\
2251.48 \\
2251.97 \\
2253.35\end{array}$ & $\begin{array}{l}2 \\
1 \mathrm{~h} \\
1\end{array}$ & $\begin{array}{r}10 \\
4 \\
3\end{array}$ & $\begin{array}{l}\text { I } \\
\text { II } \\
\text { II I } \\
\text { II } \\
\text { I }\end{array}$ & $\begin{array}{l}2285.65 \\
2287.60 \\
2288.02 \\
2289.69 \\
2290.50\end{array}$ & $\begin{array}{r}1 \\
2 \\
20 \\
2 \\
2\end{array}$ & $\begin{array}{r}100 \\
1\end{array}$ & $\begin{array}{l}\text { I } \\
\text { II I } \\
\text { I } \\
\text { I I } \\
\text { I }\end{array}$ \\
\hline $\begin{array}{l}2219.18 \\
2219.87 \\
2220.07 \\
2220.24 \\
2221.09\end{array}$ & $\begin{array}{l}1 \\
1 \\
1 \\
1 \\
2\end{array}$ & 50 & $\begin{array}{l}\text { I } \\
\text { I } \\
\text { I } \\
\text { I } \\
\text { I I I }\end{array}$ & $\begin{array}{l}2253.77 \\
2254.26 \\
2254.96 \\
2257.22 \\
2257.54\end{array}$ & $\begin{array}{l}1 \\
1 \\
1 \\
1\end{array}$ & $\begin{array}{l}2 \\
5\end{array}$ & $\begin{array}{l}\text { I } \\
\text { I } \\
\text { I } \\
\text { II I } \\
\text { III }\end{array}$ & $\begin{array}{l}2290.64 \\
2291.65 \\
2291.86 \\
2292.58 \\
2292.68\end{array}$ & $\begin{array}{r}2 \\
10 \\
1 \\
2\end{array}$ & $\begin{array}{r}5 \\
150\end{array}$ & $\begin{array}{l}\text { II } \\
\text { II I } \\
\text { I } \\
\text { I } \\
\text { I }\end{array}$ \\
\hline
\end{tabular}


Table 1. Wavelengths of Thorium Spectra - Continued

\begin{tabular}{|c|c|c|c|c|c|c|c|c|c|c|c|}
\hline \multirow{2}{*}{$\begin{array}{l}\text { Wave- } \\
\text { length }\end{array}$} & \multicolumn{2}{|c|}{ Intensity } & \multirow{2}{*}{$\begin{array}{l}\text { Spec- } \\
\text { trum }\end{array}$} & \multirow{2}{*}{$\begin{array}{l}\text { Wave- } \\
\text { length }\end{array}$} & \multicolumn{2}{|c|}{ Intensity } & \multirow{2}{*}{$\begin{array}{l}\text { Spec- } \\
\text { trum }\end{array}$} & \multirow{2}{*}{$\begin{array}{l}\text { Wave- } \\
\text { length }\end{array}$} & \multicolumn{2}{|c|}{ Intensity } & \multirow{2}{*}{$\begin{array}{l}\text { Spec- } \\
\text { trum }\end{array}$} \\
\hline & Lamp & Spark & & & Lamp & Spark & & & Lamp & Spark & \\
\hline $\begin{array}{l}2295.15 \\
2295.76 \\
2296.08 \\
2297.26 \\
2298.21\end{array}$ & $\begin{array}{l}2 \\
1\end{array}$ & $\begin{array}{l}12 \\
25 \\
10 w\end{array}$ & $\begin{array}{l}\text { I } \\
\text { I } \\
\text { IV } \\
\text { III } \\
\text { I I I }\end{array}$ & $\begin{array}{l}2325.97 \\
2326.93 \\
2327.28 \\
2328.30 \\
2328.53\end{array}$ & $\begin{array}{l}1 \\
6 \\
1 \\
1 \\
2\end{array}$ & 10 & $\begin{array}{l}\text { I } \\
\text { I I } \\
\text { I } \\
\text { I } \\
\text { I I }\end{array}$ & $\begin{array}{l}2348.60 \\
2348.82 \\
2349.33 \\
2349.50 \\
2349.68\end{array}$ & $\begin{array}{l}50 \\
2 \\
2 \\
1 \mathrm{~h} \\
3\end{array}$ & $\begin{array}{l}2 \\
1 \\
4\end{array}$ & $\begin{array}{l}\text { I } \\
\text { I } \\
\text { II } \\
\text { I I } \\
\text { II }\end{array}$ \\
\hline $\begin{array}{l}2298.86 \\
2299.29 \\
2300.70 \\
2300.77 \\
2301.22\end{array}$ & $\begin{array}{r}10 \\
2 \\
1 \\
1\end{array}$ & $\begin{array}{r}10 \\
5 \\
200\end{array}$ & $\begin{array}{l}\text { II } \\
\text { I } \\
\text { I } \\
\text { II } \\
\text { I I I }\end{array}$ & $\begin{array}{l}2328.60 \\
2329.06 \\
2329.35 \\
2329.82 \\
2330.23\end{array}$ & $\begin{array}{l}2 \\
1 \\
4 \\
3\end{array}$ & $\begin{array}{r}20 \\
20 \\
\\
2\end{array}$ & $\begin{array}{l}\text { I I I } \\
\text { I I } \\
\text { I } \\
\text { I I } \\
\text { I }\end{array}$ & $\begin{array}{l}2349.91 \\
2350.33 \\
2351.74 \\
2352.98 \\
2354.02\end{array}$ & $\begin{array}{c}10 w \\
2 \\
20 \\
7\end{array}$ & $\begin{array}{r}8 \\
3 \\
50 \\
10\end{array}$ & $\begin{array}{l}\text { I I } \\
\text { I I } \\
\text { I I I } \\
\text { I } \\
\text { I I }\end{array}$ \\
\hline $\begin{array}{l}2301.42 \\
2302.90 \\
2304.53 \\
2305.47 \\
2306.34\end{array}$ & $\begin{array}{r}20 \\
10 \\
2 \\
3 \\
2\end{array}$ & $\begin{array}{l}2 \\
5 \\
1\end{array}$ & $\begin{array}{l}\text { I } \\
\text { I } \\
\text { II } \\
\text { II } \\
\text { II }\end{array}$ & $\begin{array}{l}2330.63 \\
2330.80 \\
2331.07 \\
2331.83 \\
2332.28\end{array}$ & $\begin{array}{l}1 \\
5 \\
2 \\
5 \\
1\end{array}$ & $\begin{array}{r}1 \\
12 \\
6\end{array}$ & $\begin{array}{l}\text { II } \\
\text { II } \\
\text { I I } \\
\text { I } \\
\text { I }\end{array}$ & $\begin{array}{l}2354.16 \\
2354.27 \\
2354.99 \\
2355.24 \\
2355.53\end{array}$ & $\begin{array}{l}3 \\
2 \\
1 \\
4 \\
2\end{array}$ & $\begin{array}{l}3 \\
2 \\
5\end{array}$ & $\begin{array}{l}\text { I } \\
\text { I I } \\
\text { I I } \\
\text { I I } \\
\text { I }\end{array}$ \\
\hline $\begin{array}{l}2306.57 \\
2306.78 \\
2307.07 \\
2307.15 \\
2307.58\end{array}$ & $\begin{array}{r}10 \\
2 \\
1 \\
10\end{array}$ & $\begin{array}{l}1 \\
10 \mathrm{w} \\
10 \\
4\end{array}$ & $\begin{array}{l}\text { I } \\
\text { II } \\
\text { II I } \\
\text { II } \\
\text { II }\end{array}$ & $\begin{array}{l}2332.35 \\
2333.26 \\
2333.87 \\
2334.21 \\
2334.36\end{array}$ & $\begin{array}{r}10 \\
3 \\
2 \\
2 \\
10\end{array}$ & $\begin{array}{l}5 \\
3 \\
1\end{array}$ & $\begin{array}{l}\text { I } \\
\text { II } \\
\text { I I } \\
\text { I } \\
\text { I }\end{array}$ & $\begin{array}{l}2355.75 \\
2355.89 \\
2355.94 \\
2356.19 \\
2356.40\end{array}$ & $\begin{array}{r}1 \\
2 \\
10 \\
10\end{array}$ & $\begin{array}{r}30 \\
4 \\
2\end{array}$ & $\begin{array}{l}\text { I } \\
\text { I I I } \\
\text { I } \\
\text { I I } \\
\text { I }\end{array}$ \\
\hline $\begin{array}{l}2308.26 \\
2308.60 \\
2309.13 \\
2309.42 \\
2310.87\end{array}$ & $\begin{array}{l}1 \\
2 \\
1 \\
2 \\
1\end{array}$ & $\begin{array}{l}3 \\
2\end{array}$ & $\begin{array}{l}\text { I } \\
\text { II } \\
\text { I } \\
\text { II } \\
\text { II }\end{array}$ & $\begin{array}{l}2335.44 \\
2335.53 \\
2336.06 \\
2336.20 \\
2337.34\end{array}$ & $\begin{array}{c}10 \\
1 \\
1\end{array}$ & $5 w$ & $\begin{array}{l}\text { I } \\
\text { I I I } \\
\text { I } \\
\text { I } \\
\text { II I }\end{array}$ & $\begin{array}{l}2356.75 \\
2357.07 \\
2357.21 \\
2357.42 \\
2357.51\end{array}$ & $\begin{array}{r}10 \\
10 \\
20 \\
2\end{array}$ & $\begin{array}{r}8 \\
50\end{array}$ & $\begin{array}{l}\text { I I } \\
\text { I } \\
\text { I I I } \\
\text { I } \\
\text { I }\end{array}$ \\
\hline $\begin{array}{l}2311.54 \\
2311.58 \\
2312.46 \\
2313.35 \\
2313.67\end{array}$ & $\begin{array}{l}2 \\
2\end{array}$ & $\begin{array}{r}20 \\
5 \\
2 \\
1\end{array}$ & $\begin{array}{l}\text { II I } \\
\text { II } \\
\text { II } \\
\text { I } \\
\text { I }\end{array}$ & $\begin{array}{l}2337.46 \\
2338.33 \\
2338.43 \\
2339.18 \\
2339.37\end{array}$ & $\begin{array}{l}1 \\
1 \\
1 \\
1 \\
1\end{array}$ & 1 & $\begin{array}{l}\text { I } \\
\text { I } \\
\text { I } \\
\text { I } \\
\text { II }\end{array}$ & $\begin{array}{l}2357.99 \\
2359.74 \\
2360.42 \\
2360.53 \\
2361.63\end{array}$ & $\begin{array}{r}4 \\
1 \\
1 \\
20 \\
4\end{array}$ & 2 & $\begin{array}{l}\text { I } \\
\text { I I } \\
\text { I } \\
\text { I } \\
\text { I }\end{array}$ \\
\hline $\begin{array}{l}2314.24 \\
2314.51 \\
2315.09 \\
2316.01 \\
2316.64\end{array}$ & $\begin{array}{l}1 \\
1 \\
1 \\
1\end{array}$ & $3 w$ & $\begin{array}{l}\text { I I I } \\
\text { I } \\
\text { I } \\
\text { I I } \\
\text { I }\end{array}$ & $\begin{array}{l}2339.62 \\
2339.92 \\
2340.64 \\
2340.88 \\
2341.41\end{array}$ & $\begin{array}{l}1 \\
1 \\
1 \\
3 \mathrm{w}\end{array}$ & $\begin{array}{r}2 \\
50\end{array}$ & $\begin{array}{l}\text { II } \\
\text { I } \\
\text { II I } \\
\text { I } \\
\text { I }\end{array}$ & $\begin{array}{l}2362.16 \\
2362.62 \\
2362.76 \\
2363.08 \\
2363.12\end{array}$ & $\begin{array}{r}3 \\
20 \\
1 \\
1\end{array}$ & $\begin{array}{r}5 \\
1 \\
100\end{array}$ & $\begin{array}{l}\text { I I } \\
\text { I I I } \\
\text { I } \\
\text { I I I } \\
\text { I }\end{array}$ \\
\hline $\begin{array}{l}2317.16 \\
2317.45 \\
2317.74 \\
2317.94 \\
2318.61\end{array}$ & $\begin{array}{r}1 \\
1 \\
10 \\
20\end{array}$ & $\begin{array}{l}2 \\
4 \\
6 \\
5\end{array}$ & $\begin{array}{l}\text { II } \\
\text { I } \\
\text { II } \\
\text { II } \\
\text { III }\end{array}$ & $\begin{array}{l}2342.36 \\
2342.46 \\
2342.66 \\
2342.87 \\
2343.24\end{array}$ & $\begin{array}{l}1 \\
2 \\
1 \\
2 \\
1\end{array}$ & $\begin{array}{l}1 \\
1 \\
2 \\
1\end{array}$ & $\begin{array}{l}\text { II } \\
\text { II } \\
\text { I } \\
\text { II } \\
\text { II }\end{array}$ & $\begin{array}{l}2363.43 \\
2364.02 \\
2364.19 \\
2364.62 \\
2365.59\end{array}$ & $\begin{array}{r}2 \\
2 \\
10 \\
1 \\
2\end{array}$ & $\begin{array}{c}18 \\
1\end{array}$ & $\begin{array}{l}\text { I } \\
\text { I } \\
\text { II } \\
\text { II } \\
\text { I }\end{array}$ \\
\hline $\begin{array}{l}2319.55 \\
2321.49 \\
2321.62 \\
2323.80 \\
2324.42\end{array}$ & $\begin{array}{r}10 \\
1 \\
2 \\
1\end{array}$ & 200 & $\begin{array}{l}\text { I I I } \\
\text { I } \\
\text { I } \\
\text { I } \\
\text { I }\end{array}$ & $\begin{array}{l}2343.48 \\
2344.28 \\
2345.35 \\
2345.91 \\
2345.98\end{array}$ & $\begin{array}{r}2 \\
1 \\
10\end{array}$ & $\begin{array}{r}3 \\
2 \\
2 \\
15\end{array}$ & $\begin{array}{l}\text { II } \\
\text { II I } \\
\text { I } \\
\text { II } \\
\text { II }\end{array}$ & $\begin{array}{l}2366.04 \\
2366.39 \\
2366.98 \\
2367.72 \\
2367.84\end{array}$ & $\begin{array}{r}40 \\
20 \\
75 \\
2 \\
2\end{array}$ & $\begin{array}{r}100 \\
125 \\
1 \\
2\end{array}$ & $\begin{array}{l}\text { II } \\
\text { I } \\
\text { II } \\
\text { II } \\
\text { II }\end{array}$ \\
\hline $\begin{array}{l}2324.73 \\
2325.17 \\
2325.42 \\
2325.66 \\
2325.74\end{array}$ & $\begin{array}{r}10 \\
1 \\
1 \\
1\end{array}$ & $\begin{array}{l}1 \\
1\end{array}$ & $\begin{array}{l}\text { III } \\
\text { I } \\
\text { I } \\
\text { II } \\
\text { II }\end{array}$ & $\begin{array}{l}2346.63 \\
2347.02 \\
2347.16 \\
2348.21 \\
2348.29\end{array}$ & $\begin{array}{r}10 \\
1 \\
1\end{array}$ & $\begin{array}{l}3 \\
2 \\
2\end{array}$ & $\begin{array}{l}\text { I } \\
\text { II } \\
\text { I } \\
\text { II } \\
\text { II }\end{array}$ & $\begin{array}{l}2368.05 \\
2368.48 \\
2368.96 \\
2369.27 \\
2370.04\end{array}$ & $\begin{array}{r}50 \\
20 \\
1 \\
1 \\
5\end{array}$ & $\begin{array}{r}10 \\
40 \\
3\end{array}$ & $\begin{array}{l}\text { I I } \\
\text { I } \\
\text { I II } \\
\text { I } \\
\text { I I }\end{array}$ \\
\hline
\end{tabular}


Table 1. Wavelengths of Thorium Spectra - Continued

\begin{tabular}{|c|c|c|c|c|c|c|c|c|c|c|c|}
\hline \multirow{2}{*}{$\begin{array}{l}\text { Wave- } \\
\text { length }\end{array}$} & \multicolumn{2}{|c|}{ Intensity } & \multirow{2}{*}{$\begin{array}{l}\text { Spec- } \\
\text { trum }\end{array}$} & \multirow{2}{*}{$\begin{array}{l}\text { Wave- } \\
\text { length }\end{array}$} & \multicolumn{2}{|c|}{ Intensity } & \multirow{2}{*}{$\begin{array}{l}\text { Spec- } \\
\text { trum }\end{array}$} & \multirow{2}{*}{$\begin{array}{l}\text { Wave- } \\
\text { length }\end{array}$} & \multicolumn{2}{|c|}{ Intensity } & \multirow{2}{*}{$\begin{array}{l}\text { Spec- } \\
\text { trum }\end{array}$} \\
\hline & Lamp & Spark & & & Lamp & Spark & & & Lamp & Spark & \\
\hline $\begin{array}{l}2370.39 \\
2370.85 \\
2371.16 \\
2371.43 \\
2371.62\end{array}$ & $\begin{array}{r}5 \\
10 \\
20 \\
10\end{array}$ & $\begin{array}{r}75 \\
2\end{array}$ & $\begin{array}{l}\text { I } \\
\text { I } \\
\text { I } \\
\text { I II } \\
\text { I }\end{array}$ & $\begin{array}{l}2394.53 \\
2394.95 \\
2395.22 \\
2395.63 \\
2395.69\end{array}$ & $\begin{array}{c}1 \\
5 \\
2 \mathrm{~h} \\
10 \\
3\end{array}$ & $\begin{array}{r}2 \\
1 \\
2 \\
25 \\
15\end{array}$ & $\begin{array}{l}\text { I I } \\
\text { I } \\
\text { I I } \\
\text { I I } \\
\text { I I }\end{array}$ & $\begin{array}{l}2410.67 \\
2411.08 \\
2411.16 \\
2411.30 \\
2411.51\end{array}$ & $\begin{array}{r}10 \\
1 \\
2 \\
50 \\
5\end{array}$ & $\begin{array}{r}2 \\
2 \\
75\end{array}$ & $\begin{array}{l}\text { I } \\
\text { II } \\
\text { II } \\
\text { II } \\
\text { I }\end{array}$ \\
\hline $\begin{array}{l}2372.05 \\
2372.35 \\
2372.63 \\
2373.07 \\
2373.13\end{array}$ & $\begin{array}{c}4 \\
1 \\
50 \\
1 \\
2 \mathrm{w}\end{array}$ & $\begin{array}{l}5 \\
1 \\
1 \\
2\end{array}$ & $\begin{array}{l}\text { II } \\
\text { I I } \\
\text { I } \\
\text { II } \\
\text { I }\end{array}$ & $\begin{array}{l}2395.77 \\
2397.10 \\
2397.25 \\
2397.35 \\
2397.51\end{array}$ & $\begin{array}{r}1 \\
4 \\
2 \\
1 \\
10\end{array}$ & $\begin{array}{l}4 \\
2 \\
1 \\
3\end{array}$ & $\begin{array}{l}\text { I } \\
\text { I I } \\
\text { II } \\
\text { I I } \\
\text { II }\end{array}$ & $\begin{array}{l}2411.73 \\
2412.07 \\
2412.45 \\
2413.13 \\
2413.41\end{array}$ & $\begin{array}{c}20 \\
2 \mathrm{~h} \\
5 \\
1 \\
50\end{array}$ & $\begin{array}{r}2 \\
300\end{array}$ & $\begin{array}{l}\text { I } \\
\text { II } \\
\text { I } \\
\text { I } \\
\text { II }\end{array}$ \\
\hline $\begin{array}{l}2373.72 \\
2373.84 \\
2373.99 \\
2374.73 \\
2375.07\end{array}$ & $\begin{array}{r}2 \\
25 \\
5 \\
20 \\
30\end{array}$ & $\begin{array}{r}2 \\
50 \\
2 \\
50\end{array}$ & $\begin{array}{l}\text { II } \\
\text { II } \\
\text { II } \\
\text { I } \\
\text { II }\end{array}$ & $\begin{array}{l}2397.72 \\
2397.78 \\
2397.88 \\
2398.28 \\
2398.50\end{array}$ & $\begin{array}{r}5 \\
20 \\
10 \\
1\end{array}$ & $\begin{array}{c}5 \\
20 w \\
4\end{array}$ & $\begin{array}{l}\text { I I } \\
\text { I I I } \\
\text { I } \\
\text { II I } \\
\text { I }\end{array}$ & $\begin{array}{l}2413.49 \\
2413.84 \\
2414.36 \\
2414.86 \\
2415.31\end{array}$ & $\begin{array}{l}10 \\
10 \\
15 \\
20\end{array}$ & 400 & $\begin{array}{l}\text { III I } \\
\text { I } \\
\text { I } \\
\text { II } \\
\text { I }\end{array}$ \\
\hline $\begin{array}{l}2375.83 \\
2376.36 \\
2376.52 \\
2377.04 \\
2377.84\end{array}$ & $\begin{array}{r}25 \\
2 \\
10 \\
5 \\
75\end{array}$ & $\begin{array}{r}75 \\
1 \\
2 \\
125\end{array}$ & $\begin{array}{l}\text { II I } \\
\text { I I } \\
\text { I } \\
\text { I } \\
\text { I I }\end{array}$ & $\begin{array}{l}2398.60 \\
2398.75 \\
2399.09 \\
2399.24 \\
2400.28\end{array}$ & $\begin{array}{r}1 \\
1 \\
1 \\
1 \\
10\end{array}$ & $\begin{array}{l}3 \\
1 \\
1 \\
5 \\
2\end{array}$ & $\begin{array}{l}\text { I I } \\
\text { I I } \\
\text { I I } \\
\text { I I } \\
\text { I }\end{array}$ & $\begin{array}{l}2415.60 \\
2416.08 \\
2416.28 \\
2416.95 \\
2417.09\end{array}$ & $\begin{array}{r}10 \\
5 \\
2 \\
20 \\
20\end{array}$ & $\begin{array}{r}2 \\
1 \\
1 \\
10\end{array}$ & $\begin{array}{l}\text { I } \\
\text { I I } \\
\text { I I } \\
\text { I } \\
\text { I I }\end{array}$ \\
\hline $\begin{array}{l}2378.61 \\
2379.75 \\
2380.03 \\
2381.49 \\
2382.48\end{array}$ & $\begin{array}{r}15 \\
2 \\
2 \\
15\end{array}$ & $\begin{array}{r}10 \\
5 \\
1 \\
400 \\
10\end{array}$ & $\begin{array}{l}\text { I I } \\
\text { I I } \\
\text { I I } \\
\text { I I I } \\
\text { I I }\end{array}$ & $\begin{array}{l}2400.66 \\
2400.82 \\
2400.95 \\
2401.14 \\
2401.57\end{array}$ & $\begin{array}{r}10 \\
2 \\
1 \\
5 \\
1\end{array}$ & $\begin{array}{l}4 \\
2 \\
5\end{array}$ & $\begin{array}{l}\text { II } \\
\text { I I } \\
\text { I } \\
\text { I I } \\
\text { I }\end{array}$ & $\begin{array}{l}2417.17 \\
2417.22 \\
2417.49 \\
2418.08 \\
2418.30\end{array}$ & $\begin{array}{r}1 \\
10 \\
10 \\
15 \\
2\end{array}$ & $\begin{array}{r}20 \\
2\end{array}$ & $\begin{array}{l}\text { I } \\
\text { I } \\
\text { I } \\
\text { II } \\
\text { I I }\end{array}$ \\
\hline $\begin{array}{l}2382.90 \\
2384.36 \\
2385.59 \\
2386.18 \\
2386.23\end{array}$ & $\begin{array}{r}30 \\
30 \\
15 \\
5 \\
4\end{array}$ & $\begin{array}{r}50 \\
10 \\
5\end{array}$ & $\begin{array}{l}\text { I } \\
\text { I I } \\
\text { II } \\
\text { II } \\
\text { I }\end{array}$ & $\begin{array}{l}2401.64 \\
2401.73 \\
2402.66 \\
2403.12 \\
2403.21\end{array}$ & $\begin{array}{l}5 \\
1 \mathrm{~h} \\
20 \\
15\end{array}$ & $\begin{array}{r}5 \\
1 \\
1 \\
40 \\
3\end{array}$ & $\begin{array}{l}\text { I I } \\
\text { I I } \\
\text { I } \\
\text { I I } \\
\text { I I }\end{array}$ & $\begin{array}{l}2418.63 \\
2418.75 \\
2419.46 \\
2419.56 \\
2419.80\end{array}$ & $\begin{array}{l}1 \\
2 \\
1\end{array}$ & 3 & $\begin{array}{l}\text { I I } \\
\text { I } \\
\text { I } \\
\text { I } \\
\text { I I }\end{array}$ \\
\hline $\begin{array}{l}2386.51 \\
2386.81 \\
2388.08 \\
2388.13 \\
2388.83\end{array}$ & $\begin{array}{l}10 \\
20 \\
10 \\
20 \\
10\end{array}$ & $\begin{array}{r}50 \\
2 \\
30 \\
3\end{array}$ & $\begin{array}{l}\text { I } \\
\text { I I } \\
\text { I } \\
\text { II } \\
\text { I I }\end{array}$ & $\begin{array}{l}2403.40 \\
2403.60 \\
2403.73 \\
2404.17 \\
2404.51\end{array}$ & $\begin{array}{l}5 \\
2 \\
2 \mathrm{~h} \\
50 \\
50\end{array}$ & $\begin{array}{r}1 \\
2 \\
3 \\
75 \\
50\end{array}$ & $\begin{array}{l}\text { I } \\
\text { II } \\
\text { II } \\
\text { II } \\
\text { II }\end{array}$ & $\begin{array}{l}2419.98 \\
2420.18 \\
2421.54 \\
2421.83 \\
2422.25\end{array}$ & $\begin{array}{r}20 \\
10 \\
1 \\
1 \\
10\end{array}$ & 15 & $\begin{array}{l}\text { II } \\
\text { I } \\
\text { I } \\
\text { I } \\
\text { II }\end{array}$ \\
\hline $\begin{array}{l}2389.02 \\
2389.23 \\
2389.80 \\
2390.29 \\
2390.39\end{array}$ & $\begin{array}{c}1 \\
10 \\
1 \\
1 \mathrm{~h} \\
2\end{array}$ & $\begin{array}{l}5 \\
1 \\
1 \\
1\end{array}$ & $\begin{array}{l}\text { I } \\
\text { II } \\
\text { I I } \\
\text { I I } \\
\text { I I }\end{array}$ & $\begin{array}{l}2405.58 \\
2405.85 \\
2406.43 \\
2406.69 \\
2406.98\end{array}$ & $\begin{array}{r}10 \\
1 \\
20 \\
20 \\
2\end{array}$ & $\begin{array}{r}4 \\
2 \\
20 \\
15 \\
3\end{array}$ & $\begin{array}{l}\text { II } \\
\text { II } \\
\text { II } \\
\text { II } \\
\text { I I }\end{array}$ & $\begin{array}{l}2422.46 \\
2422.71 \\
2423.00 \\
2423.33 \\
2423.57\end{array}$ & $\begin{array}{l}10 \\
10 \\
30 \\
5 \\
10\end{array}$ & $\begin{array}{r}2 \\
50 \\
3 \\
4\end{array}$ & $\begin{array}{l}\text { I } \\
\text { I } \\
\text { II } \\
\text { II } \\
\text { II }\end{array}$ \\
\hline $\begin{array}{l}2391.00 \\
2391.38 \\
2391.53 \\
2391.95 \\
2392.94\end{array}$ & $\begin{array}{r}3 \\
20 \\
\\
5 \\
2\end{array}$ & $\begin{array}{r}400 \\
2\end{array}$ & $\begin{array}{l}\text { I } \\
\text { I } \\
\text { II I } \\
\text { I } \\
\text { II }\end{array}$ & $\begin{array}{l}2407.13 \\
2407.58 \\
2408.23 \\
2408.43 \\
2408.67\end{array}$ & $\begin{array}{l}20 \\
30 \\
10 \\
1 \\
10\end{array}$ & $\begin{array}{l}10 \\
20\end{array}$ & $\begin{array}{l}\text { II } \\
\text { II } \\
\text { I } \\
\text { I } \\
\text { II }\end{array}$ & $\begin{array}{l}2423.67 \\
2424.03 \\
2424.54 \\
2424.90 \\
2425.20\end{array}$ & $\begin{array}{r}50 \\
20 \\
10 \\
5\end{array}$ & $\begin{array}{c}100 \\
10 \\
150 \\
2 \mathrm{w}\end{array}$ & $\begin{array}{l}\text { II } \\
\text { II } \\
\text { III } \\
\text { III } \\
\text { I }\end{array}$ \\
\hline $\begin{array}{l}2393.11 \\
2393.58 \\
2393.79 \\
2394.25 \\
2394.30\end{array}$ & $\begin{array}{r}40 \\
10 \\
5 \\
2 \\
2\end{array}$ & $\begin{array}{r}75 \\
8 \\
2 \\
2\end{array}$ & $\begin{array}{l}\text { I I } \\
\text { I I } \\
\text { I } \\
\text { II } \\
\text { II }\end{array}$ & $\begin{array}{l}2409.02 \\
2409.24 \\
2409.46 \\
2409.59 \\
2410.07\end{array}$ & $\begin{array}{r}1 \\
15 \\
1 \\
20 \\
10\end{array}$ & $\begin{array}{r}10 \\
2 \\
15 \\
12\end{array}$ & $\begin{array}{l}\text { I } \\
\text { II } \\
\text { II } \\
\text { II } \\
\text { II }\end{array}$ & $\begin{array}{l}2425.44 \\
2425.54 \\
2425.73 \\
2426.15 \\
2426.38\end{array}$ & $\begin{array}{c}1 \mathrm{~h} \\
1 \\
10 \\
5 \\
1\end{array}$ & $\begin{array}{l}2 \\
2 \\
2\end{array}$ & $\begin{array}{l}\text { II } \\
\text { I } \\
\text { II } \\
\text { II } \\
\text { I }\end{array}$ \\
\hline
\end{tabular}


Table 1. Wavelengths of Thorium Spectra - Continued

\begin{tabular}{|c|c|c|c|c|c|c|c|c|c|c|c|}
\hline \multirow{2}{*}{$\begin{array}{l}\text { Wave- } \\
\text { length }\end{array}$} & \multicolumn{2}{|c|}{ Intensity } & \multirow{2}{*}{$\begin{array}{l}\text { Spec- } \\
\text { trum }\end{array}$} & \multirow{2}{*}{$\begin{array}{l}\text { Wave- } \\
\text { length }\end{array}$} & \multicolumn{2}{|c|}{ Intensity } & \multirow{2}{*}{$\begin{array}{l}\text { Spec- } \\
\text { trum }\end{array}$} & \multirow{2}{*}{$\begin{array}{l}\text { Wave- } \\
\text { length }\end{array}$} & \multicolumn{2}{|c|}{ Intensity } & \multirow{2}{*}{$\begin{array}{l}\text { Spec- } \\
\text { trum }\end{array}$} \\
\hline & Lamp & Spark & & & Lamp & Spark & & & Lamp & Spark & \\
\hline $\begin{array}{l}2426.92 \\
2427.18 \\
2427.47 \\
2427.58 \\
2427.74\end{array}$ & $\begin{array}{l}5 \\
2 \\
2 \\
2 \\
1\end{array}$ & 2 & $\begin{array}{l}\text { I I } \\
\text { I } \\
\text { I } \\
\text { I } \\
\text { I }\end{array}$ & $\begin{array}{l}2438.54 \\
2438.61 \\
2438.77 \\
2439.43 \\
2439.74\end{array}$ & $\begin{array}{l}5 \\
2 \\
1 \\
5 \\
1\end{array}$ & $\begin{array}{l}2 \\
3 \\
1\end{array}$ & $\begin{array}{l}\text { II } \\
\text { II } \\
\text { I } \\
\text { I } \\
\text { I }\end{array}$ & $\begin{array}{l}2451.33 \\
2451.47 \\
2451.56 \\
2451.72 \\
2451.98\end{array}$ & $\begin{array}{r}2 \\
4 \\
10 \\
20 \\
5\end{array}$ & 1 & $\begin{array}{l}\text { I } \\
\text { I } \\
\text { I } \\
\text { I } \\
\text { I }\end{array}$ \\
\hline $\begin{array}{l}2427.98 \\
2428.02 \\
2428.14 \\
2428.57 \\
2428.78\end{array}$ & $\begin{array}{r}10 \\
1 \\
10 \\
5 \\
1\end{array}$ & $30 \mathrm{w}$ & $\begin{array}{l}\text { I I I } \\
\text { I } \\
\text { I } \\
\text { I } \\
\text { I I I }\end{array}$ & $\begin{array}{l}2439.91 \\
2441.01 \\
2441.26 \\
2441.43 \\
2441.55\end{array}$ & $\begin{array}{l}2 \\
2 \\
1 \\
1 \\
2\end{array}$ & $\begin{array}{r}1 \\
200\end{array}$ & $\begin{array}{l}\text { I I } \\
\text { I } \\
\text { I I I } \\
\text { I } \\
\text { I }\end{array}$ & $\begin{array}{l}2452.27 \\
2452.46 \\
2452.74 \\
2452.91 \\
2453.04\end{array}$ & $\begin{array}{r}30 \\
10 \\
2 \\
2\end{array}$ & $\begin{array}{r}4 \\
5 \\
10 \\
2\end{array}$ & $\begin{array}{l}\text { II } \\
\text { I } \\
\text { II } \\
\text { II } \\
\text { I }\end{array}$ \\
\hline $\begin{array}{l}2429.05 \\
2429.40 \\
2429.68 \\
2430.33 \\
2430.48\end{array}$ & $\begin{array}{r}10 \\
5 \\
10 \\
1 \\
5\end{array}$ & $\begin{array}{l}8 \\
4\end{array}$ & $\begin{array}{l}\text { I I } \\
\text { I I } \\
\text { I } \\
\text { I } \\
\text { I }\end{array}$ & $\begin{array}{l}2441.66 \\
2441.99 \\
2442.92 \\
2443.15 \\
2443.34\end{array}$ & $\begin{array}{l}1 \\
5 \\
1 \\
5 \\
1\end{array}$ & $\frac{1}{2}$ & $\begin{array}{l}\text { I } \\
\text { I } \\
\text { II } \\
\text { II } \\
\text { I }\end{array}$ & $\begin{array}{l}2453.26 \\
2453.74 \\
2454.16 \\
2454.34 \\
2454.47\end{array}$ & $\begin{array}{c}20 \\
10 \\
10 \\
2 \\
10 \mathrm{w}\end{array}$ & $\begin{array}{l}4 \\
3 \\
5\end{array}$ & $\begin{array}{l}\text { I } \\
\text { I } \\
\text { I I } \\
\text { I } \\
\text { I I }\end{array}$ \\
\hline $\begin{array}{l}2430.96 \\
2431.05 \\
2431.15 \\
2431.71 \\
2431.86\end{array}$ & $\begin{array}{r}5 \\
1 \\
30 \\
40\end{array}$ & $\begin{array}{r}20 \\
300\end{array}$ & $\begin{array}{l}\text { I } \\
\text { I } \\
\text { II } \\
\text { II I } \\
\text { I }\end{array}$ & $\begin{array}{l}2443.42 \\
2443.68 \\
2443.96 \\
2444.04 \\
2444.46\end{array}$ & $\begin{array}{l}3 \mathrm{~h} \\
20 \\
25 \\
2 \\
25\end{array}$ & $\begin{array}{r}2 \\
4 \\
20 \\
20\end{array}$ & $\begin{array}{l}\text { I I } \\
\text { I } \\
\text { I I } \\
\text { I } \\
\text { I I }\end{array}$ & $\begin{array}{l}2454.52 \\
2454.67 \\
2454.90 \\
2455.50 \\
2455.74\end{array}$ & $\begin{array}{r}2 \\
5 \\
10 \\
4 \\
10\end{array}$ & $\begin{array}{r}3 \\
5 \\
10 \\
12\end{array}$ & $\begin{array}{l}\text { I I } \\
\text { I I } \\
\text { I I } \\
\text { I } \\
\text { I I }\end{array}$ \\
\hline $\begin{array}{l}2432.08 \\
2432.13 \\
2432.26 \\
2432.61 \\
2432.85\end{array}$ & $\begin{array}{r}10 \\
2 \\
2 \\
2 \\
75\end{array}$ & 100 & $\begin{array}{l}\text { I } \\
\text { I } \\
\text { I } \\
\text { I } \\
\text { I I }\end{array}$ & $\begin{array}{l}2444.70 \\
2444.94 \\
2445.06 \\
2445.20 \\
2445.44\end{array}$ & $\begin{array}{r}1 \\
10 \\
1 \\
5 \\
10\end{array}$ & 2 & $\begin{array}{l}\text { I } \\
\text { I } \\
\text { I } \\
\text { I } \\
\text { I }\end{array}$ & $\begin{array}{l}2455.91 \\
2455.97 \\
2456.30 \\
2456.39 \\
2456.86\end{array}$ & $\begin{array}{r}2 \\
1 \\
150 \\
20 \\
50\end{array}$ & $\begin{array}{r}2 \\
200 \\
15 \\
40\end{array}$ & $\begin{array}{l}\text { I I } \\
\text { I } \\
\text { I I } \\
\text { I I } \\
\text { I I }\end{array}$ \\
\hline $\begin{array}{l}2433.33 \\
2433.63 \\
2433.83 \\
2433.95 \\
2434.11\end{array}$ & $\begin{array}{r}1 \\
5 \\
10 \\
5 \\
2\end{array}$ & $\begin{array}{l}8 \\
1 \mathrm{~h} \\
5 \\
2\end{array}$ & $\begin{array}{l}\text { I } \\
\text { II } \\
\text { I } \\
\text { II } \\
\text { II }\end{array}$ & $\begin{array}{l}2445.56 \\
2445.76 \\
2445.91 \\
2446.18 \\
2446.36\end{array}$ & $\begin{array}{r}3 \\
2 \\
1 \\
5 \\
10\end{array}$ & $\begin{array}{r}10 \\
1 \\
2 \\
5\end{array}$ & $\begin{array}{l}\text { II } \\
\text { I I } \\
\text { I } \\
\text { II } \\
\text { I I }\end{array}$ & $\begin{array}{l}2457.04 \\
2457.24 \\
2457.45 \\
2457.75 \\
2458.01\end{array}$ & $\begin{array}{r}2 \\
2 \\
10 \\
20 \\
20\end{array}$ & $\begin{array}{r}2 \\
2 \\
20 \\
8\end{array}$ & $\begin{array}{l}\text { II } \\
\text { II } \\
\text { I } \\
\text { II } \\
\text { I I }\end{array}$ \\
\hline $\begin{array}{l}2434.23 \\
2434.43 \\
2434.72 \\
2434.94 \\
2435.15\end{array}$ & $\begin{array}{r}2 \\
1 \\
2 \\
1 \\
20\end{array}$ & $\begin{array}{l}1 \\
2 \\
5\end{array}$ & $\begin{array}{l}\text { II } \\
\text { I } \\
\text { II } \\
\text { I } \\
\text { I }\end{array}$ & $\begin{array}{l}2446.48 \\
2446.60 \\
2446.84 \\
2447.16 \\
2447.26\end{array}$ & $\begin{array}{l}2 \\
1 \mathrm{~h} \\
10 \\
5 \\
1\end{array}$ & $\begin{array}{l}2 \\
4 \\
1 \\
1\end{array}$ & $\begin{array}{l}\text { II } \\
\text { II } \\
\text { II } \\
\text { I } \\
\text { II }\end{array}$ & $\begin{array}{l}2458.41 \\
2458.61 \\
2458.77 \\
2459.01 \\
2459.44\end{array}$ & $\begin{array}{c}5 \\
10 \\
2 \\
50 \\
5 \mathrm{~h}\end{array}$ & $\begin{array}{r}1 \\
2 \\
40 \\
15\end{array}$ & $\begin{array}{l}\text { I } \\
\text { I } \\
\text { I I } \\
\text { II } \\
\text { I I }\end{array}$ \\
\hline $\begin{array}{l}2435.23 \\
2435.78 \\
2435.95 \\
2436.13 \\
2436.34\end{array}$ & $\begin{array}{l}2 \\
3 \\
5 \\
2 \mathrm{~h} \\
20\end{array}$ & $\begin{array}{r}2 \\
2 \\
2 \\
10\end{array}$ & $\begin{array}{l}\text { II } \\
\text { II } \\
\text { I } \\
\text { II } \\
\text { II }\end{array}$ & $\begin{array}{l}2447.46 \\
2447.74 \\
2447.85 \\
2447.98 \\
2448.47\end{array}$ & $\begin{array}{r}5 \\
1 \\
10 \\
5 \\
10\end{array}$ & $\begin{array}{l}1 \\
4 \\
3 \\
4\end{array}$ & $\begin{array}{l}\text { I } \\
\text { II } \\
\text { I } \\
\text { II } \\
\text { II }\end{array}$ & $\begin{array}{l}2459.67 \\
2459.90 \\
2460.15 \\
2460.30 \\
2460.46\end{array}$ & $\begin{array}{c}2 w \\
2 \\
10 \\
2 \\
1\end{array}$ & $\begin{array}{l}2 \\
3 \\
2 \\
1\end{array}$ & $\begin{array}{l}\text { I I } \\
\text { I I } \\
\text { I I } \\
\text { I I } \\
\text { I I }\end{array}$ \\
\hline $\begin{array}{l}2436.43 \\
2436.62 \\
2436.72 \\
2437.11 \\
2437.54\end{array}$ & $\begin{array}{r}10 \\
1 \\
2 \\
10 \\
30\end{array}$ & 75 & $\begin{array}{l}\text { I } \\
\text { I } \\
\text { I } \\
\text { I } \\
\text { II }\end{array}$ & $\begin{array}{l}2448.70 \\
2449.09 \\
2449.28 \\
2449.51 \\
2449.77\end{array}$ & $\begin{array}{r}15 \\
20 \\
10 \\
1 \\
1\end{array}$ & $\begin{array}{r}15 \\
30 \\
2 \\
1\end{array}$ & $\begin{array}{l}\text { I I } \\
\text { I I } \\
\text { I } \\
\text { I } \\
\text { II }\end{array}$ & $\begin{array}{l}2460.58 \\
2460.79 \\
2460.89 \\
2461.21 \\
2461.59\end{array}$ & $\begin{array}{r}2 \\
2 \\
5 \\
20 \\
5\end{array}$ & $\begin{array}{r}2 \\
2 \\
1 \\
25\end{array}$ & $\begin{array}{l}\text { II } \\
\text { II } \\
\text { I } \\
\text { II } \\
\text { I }\end{array}$ \\
\hline $\begin{array}{l}2437.67 \\
2437.75 \\
2438.04 \\
2438.11 \\
2438.38\end{array}$ & $\begin{array}{r}1 \\
1 \\
2 \\
5 \\
15\end{array}$ & $\begin{array}{r}3 \\
2 \\
10\end{array}$ & $\begin{array}{l}\text { I } \\
\text { I } \\
\text { II } \\
\text { II } \\
\text { II }\end{array}$ & $\begin{array}{l}2449.86 \\
2450.03 \\
2450.15 \\
2450.39 \\
2450.79\end{array}$ & $\begin{array}{r}20 \\
1 \\
1 \\
20 \\
40\end{array}$ & $\begin{array}{r}2 \\
1 \\
1 \\
50\end{array}$ & $\begin{array}{l}\text { I } \\
\text { II } \\
\text { II } \\
\text { I } \\
\text { II }\end{array}$ & $\begin{array}{l}2461.65 \\
2461.89 \\
2462.33 \\
2462.85 \\
2463.24\end{array}$ & $\begin{array}{r}1 \\
5 \\
10 \\
30 \\
1\end{array}$ & $\begin{array}{r}1 \\
4 \\
2 \\
10\end{array}$ & $\begin{array}{l}\text { II } \\
\text { II } \\
\text { I } \\
\text { II } \\
\text { I }\end{array}$ \\
\hline
\end{tabular}


Table 1. Wavelengths of Thorium Spectra - Continued

\begin{tabular}{|c|c|c|c|c|c|c|c|c|c|c|c|}
\hline \multirow{2}{*}{$\begin{array}{l}\text { Wave- } \\
\text { length }\end{array}$} & \multicolumn{2}{|c|}{ Intensity } & \multirow{2}{*}{$\begin{array}{l}\text { Spec- } \\
\text { trum }\end{array}$} & \multirow{2}{*}{$\begin{array}{l}\text { Wave- } \\
\text { length }\end{array}$} & \multicolumn{2}{|c|}{ Intensity } & \multirow{2}{*}{$\begin{array}{l}\text { Spec- } \\
\text { trum }\end{array}$} & \multirow{2}{*}{$\begin{array}{l}\text { Wave- } \\
\text { length }\end{array}$} & \multicolumn{2}{|c|}{ Intensity } & \multirow{2}{*}{$\begin{array}{l}\text { Spec } \\
\text { trum }\end{array}$} \\
\hline & Lamp & Spark & & & Lamp & Spark & & & Lamp & Spark & \\
\hline $\begin{array}{l}2463.38 \\
2463.54 \\
2463.68 \\
2463.88 \\
2464.04\end{array}$ & $\begin{array}{l}5 \\
5 \\
1 \mathrm{~h} \\
10\end{array}$ & 300 & $\begin{array}{l}\text { I } \\
\text { I } \\
\text { III } \\
\text { II } \\
\text { I }\end{array}$ & $\begin{array}{l}2476.88 \\
2476.96 \\
2477.09 \\
2477.29 \\
2477.46\end{array}$ & $\begin{array}{r}10 \\
5 \\
5 \\
5 \\
10\end{array}$ & $\begin{array}{r}6 \\
20 \\
5 \\
1\end{array}$ & $\begin{array}{l}\text { II } \\
\text { II } \\
\text { II } \\
\text { I } \\
\text { I }\end{array}$ & $\begin{array}{l}2490.10 \\
2490.84 \\
2491.13 \\
2491.53 \\
2492.19\end{array}$ & $\begin{array}{r}15 \\
2 \\
2 \\
5 \\
20\end{array}$ & $\begin{array}{r}12 \\
1 \\
4 \\
20\end{array}$ & $\begin{array}{l}\text { II } \\
\text { II } \\
\text { I } \\
\text { II } \\
\text { II }\end{array}$ \\
\hline $\begin{array}{l}2464.20 \\
2464.29 \\
2464.41 \\
2464.49 \\
2464.70\end{array}$ & $\begin{array}{l}1 \\
2 \\
1 \mathrm{~h} \\
2 \\
1\end{array}$ & $\begin{array}{l}1 \\
1\end{array}$ & $\begin{array}{l}\text { I } \\
\text { I } \\
\text { II } \\
\text { I } \\
\text { II }\end{array}$ & $\begin{array}{l}2477.57 \\
2477.78 \\
2477.88 \\
2477.93 \\
2478.47\end{array}$ & $\begin{array}{l}10 \\
2 \mathrm{w} \\
2 \\
10\end{array}$ & $\begin{array}{l}60 \\
20\end{array}$ & $\begin{array}{l}\text { I } \\
\text { I } \\
\text { II } \\
\text { I } \\
\text { II }\end{array}$ & $\begin{array}{l}2492.53 \\
2492.82 \\
2493.03 \\
2493.25 \\
2493.62\end{array}$ & $\begin{array}{r}10 \\
2 \\
10 \\
10 \\
10\end{array}$ & $\begin{array}{r}4 \\
3 \\
10 \\
5\end{array}$ & $\begin{array}{l}\text { II } \\
\text { I } \\
\text { II } \\
\text { II } \\
\text { I I }\end{array}$ \\
\hline $\begin{array}{l}2464.83 \\
2465.09 \\
2465.21 \\
2465.32 \\
2465.62\end{array}$ & $\begin{array}{r}10 \\
2 \\
2 \\
1\end{array}$ & $\begin{array}{l}6 \\
2\end{array}$ & $\begin{array}{l}\text { II } \\
\text { I } \\
\text { III } \\
\text { I } \\
\text { I }\end{array}$ & $\begin{array}{l}2478.70 \\
2479.31 \\
2479.54 \\
2479.78 \\
2480.11\end{array}$ & $\begin{array}{c}30 \mathrm{w} \\
1 \\
20 \\
5 \\
1\end{array}$ & $\begin{array}{l}7 \\
2\end{array}$ & $\begin{array}{l}\text { I } \\
\text { I I } \\
\text { I } \\
\text { I } \\
\text { I I }\end{array}$ & $\begin{array}{l}2493.97 \\
2494.42 \\
2494.61 \\
2494.76 \\
2494.87\end{array}$ & $\begin{array}{r}10 \\
4 \\
50 \\
5 \\
1\end{array}$ & $\begin{array}{r}5 \\
2 \\
75 \\
4\end{array}$ & $\begin{array}{l}\text { II } \\
\text { I I } \\
\text { II } \\
\text { I I } \\
\text { I }\end{array}$ \\
\hline $\begin{array}{l}2465.96 \\
2466.13 \\
2466.73 \\
2466.85 \\
2467.04\end{array}$ & $\begin{array}{r}20 \\
75 \\
1 \\
5 \\
10\end{array}$ & $\begin{array}{r}6 \\
100\end{array}$ & $\begin{array}{l}\text { II } \\
\text { I I } \\
\text { I } \\
\text { I } \\
\text { I }\end{array}$ & $\begin{array}{l}2480.94 \\
2481.15 \\
2481.40 \\
2481.62 \\
2481.81\end{array}$ & $\begin{array}{c}2 \\
5 \\
40 \\
1 \mathrm{w} \\
10\end{array}$ & $\begin{array}{r}40 \\
8\end{array}$ & $\begin{array}{l}\text { I } \\
\text { I } \\
\text { II } \\
\text { I } \\
\text { II }\end{array}$ & $\begin{array}{l}2494.91 \\
2495.35 \\
2495.56 \\
2495.80 \\
2496.01\end{array}$ & $\begin{array}{r}2 \\
50 \\
5 \\
1 \\
10\end{array}$ & $\begin{array}{r}1 \\
100 \\
2 \\
3\end{array}$ & $\begin{array}{l}\text { II } \\
\text { II } \\
\text { II } \\
\text { I } \\
\text { II }\end{array}$ \\
\hline $\begin{array}{l}2467.27 \\
2467.41 \\
2467.63 \\
2468.15 \\
2468.35\end{array}$ & $\begin{array}{r}2 \\
20 \\
50 \\
1\end{array}$ & $\begin{array}{r}4 \\
100 \\
75\end{array}$ & $\begin{array}{l}\text { I } \\
\text { I } \\
\text { I I I } \\
\text { II } \\
\text { I }\end{array}$ & $\begin{array}{l}2482.11 \\
2482.20 \\
2482.46 \\
2482.63 \\
2482.73\end{array}$ & $\begin{array}{l}5 \\
2 \\
5 \\
2 \mathrm{~h} \\
1\end{array}$ & $\begin{array}{l}3 \\
5 \\
2\end{array}$ & $\begin{array}{l}\text { II } \\
\text { I } \\
\text { II } \\
\text { II } \\
\text { I }\end{array}$ & $\begin{array}{l}2496.16 \\
2496.34 \\
2497.24 \\
2497.56 \\
2497.72\end{array}$ & $\begin{array}{r}10 \\
1 \\
15 \\
5\end{array}$ & $\begin{array}{r}1 \\
400 \\
400\end{array}$ & $\begin{array}{l}\text { I } \\
\text { I } \\
\text { II I } \\
\text { II I } \\
\text { I }\end{array}$ \\
\hline $\begin{array}{l}2468.71 \\
2468.91 \\
2469.18 \\
2469.28 \\
2469.34\end{array}$ & $\begin{array}{c}1 \mathrm{w} \\
1 \\
20 \\
2 \\
1\end{array}$ & 4 & $\begin{array}{l}\text { I } \\
\text { I } \\
\text { I } \\
\text { I } \\
\text { I }\end{array}$ & $\begin{array}{l}2482.89 \\
2483.54 \\
2483.71 \\
2483.95 \\
2484.08\end{array}$ & $\begin{array}{r}5 \\
15 \\
20 \\
10 \\
5\end{array}$ & $\begin{array}{r}5 \\
15 \\
10 \\
5\end{array}$ & $\begin{array}{l}\text { II } \\
\text { II } \\
\text { I } \\
\text { II } \\
\text { II }\end{array}$ & $\begin{array}{l}2498.40 \\
2498.86 \\
2499.68 \\
2499.71 \\
2499.97\end{array}$ & $\begin{array}{r}50 \\
100 \\
30 \\
1\end{array}$ & $\begin{array}{r}50 \\
70 \\
2 \\
6\end{array}$ & $\begin{array}{l}\text { II I } \\
\text { II } \\
\text { II } \\
\text { I } \\
\text { I }\end{array}$ \\
\hline $\begin{array}{l}2469.73 \\
2470.15 \\
2470.59 \\
2470.86 \\
2470.99\end{array}$ & $\begin{array}{r}1 \\
20 \\
40 \\
1 \\
5\end{array}$ & $\begin{array}{r}12 \\
75 \\
1 \\
4\end{array}$ & $\begin{array}{l}\text { I } \\
\text { II } \\
\text { II } \\
\text { I } \\
\text { II }\end{array}$ & $\begin{array}{l}2484.18 \\
2484.32 \\
2484.61 \\
2484.74 \\
2485.05\end{array}$ & $\begin{array}{r}2 \\
2 \\
5 \\
1 \\
30\end{array}$ & $\begin{array}{r}2 \\
2 \\
3 \\
50\end{array}$ & $\begin{array}{l}\text { I I } \\
\text { I I } \\
\text { I I } \\
\text { I } \\
\text { II }\end{array}$ & $\begin{array}{l}2500.13 \\
2500.44 \\
2500.79 \\
2501.12 \\
2501.68\end{array}$ & $\begin{array}{r}10 \\
5 \\
10 \\
10 \\
10\end{array}$ & 400 & $\begin{array}{l}\text { I } \\
\text { I } \\
\text { I } \\
\text { III } \\
\text { I }\end{array}$ \\
\hline $\begin{array}{l}2471.16 \\
2471.45 \\
2471.67 \\
2471.92 \\
2472.50\end{array}$ & $\begin{array}{r}5 \\
1 \\
30 \\
10 \\
1\end{array}$ & $\begin{array}{r}3 \\
1 \\
25 \\
5\end{array}$ & $\begin{array}{l}\text { II } \\
\text { I I } \\
\text { II } \\
\text { I I } \\
\text { I }\end{array}$ & $\begin{array}{l}2485.45 \\
2485.62 \\
2485.76 \\
2486.12 \\
2486.38\end{array}$ & $\begin{array}{r}1 \\
1 \\
5 \\
10 \\
2\end{array}$ & $\begin{array}{r}5 \\
12 \\
2\end{array}$ & $\begin{array}{l}\text { I } \\
\text { I } \\
\text { II } \\
\text { II } \\
\text { II }\end{array}$ & $\begin{array}{l}2502.04 \\
2502.14 \\
2502.33 \\
2502.46 \\
2502.87\end{array}$ & $\begin{array}{r}5 \\
1 \\
10 \\
20 \\
50\end{array}$ & $\begin{array}{r}1 \\
2 \\
3 \\
30\end{array}$ & $\begin{array}{l}\text { I } \\
\text { II } \\
\text { I } \\
\text { I } \\
\text { II }\end{array}$ \\
\hline $\begin{array}{l}2473.68 \\
2473.81 \\
2473.98 \\
2474.53 \\
2475.01\end{array}$ & $\begin{array}{r}10 \\
2 \\
10 \\
20 \\
10\end{array}$ & $\begin{array}{r}200 \\
2\end{array}$ & $\begin{array}{l}\text { I } \\
\text { I } \\
\text { II I } \\
\text { I } \\
\text { I }\end{array}$ & $\begin{array}{l}2487.05 \\
2487.18 \\
2487.31 \\
2487.49 \\
2488.37\end{array}$ & $\begin{array}{r}2 \\
2 \\
10 \\
2 \\
1\end{array}$ & 8 & $\begin{array}{l}\text { I } \\
\text { I } \\
\text { II } \\
\text { I } \\
\text { I }\end{array}$ & $\begin{array}{l}2503.02 \\
2503.23 \\
2503.31 \\
2503.553 \\
2503.729\end{array}$ & $\begin{array}{l}10 \\
10 \\
1 \\
20 \\
10\end{array}$ & $\begin{array}{l}1 \\
4\end{array}$ & $\begin{array}{l}\text { I } \\
\text { II } \\
\text { I } \\
\text { I } \\
\text { I }\end{array}$ \\
\hline $\begin{array}{l}2475.31 \\
2475.38 \\
2475.73 \\
2476.13 \\
2476.38\end{array}$ & $\begin{array}{r}2 \\
10 \\
5 \\
10\end{array}$ & 150 & $\begin{array}{l}\text { I I I } \\
\text { I } \\
\text { I } \\
\text { I } \\
\text { I }\end{array}$ & $\begin{array}{l}2488.55 \\
2488.76 \\
2488.89 \\
2489.22 \\
2489.62\end{array}$ & $\begin{array}{c}10 \\
2 \\
5 \mathrm{w} \\
3 \\
75\end{array}$ & 100 & $\begin{array}{l}\text { II } \\
\text { I } \\
\text { I } \\
\text { I } \\
\text { II }\end{array}$ & $\begin{array}{l}2503.853 \\
2504.284 \\
2504.694 \\
2505.263 \\
2505.388\end{array}$ & $\begin{array}{r}1 \\
75 \\
2 \\
2 \\
2\end{array}$ & $\begin{array}{c}100 \\
2 \\
1 \mathrm{~h} \\
1 \mathrm{~h}\end{array}$ & $\begin{array}{l}\text { II } \\
\text { II } \\
\text { II } \\
\text { II } \\
\text { II }\end{array}$ \\
\hline
\end{tabular}


Table 1. Wavelengths of Thorium Spectra - Continued

\begin{tabular}{|c|c|c|c|c|c|c|c|c|c|c|c|}
\hline \multirow{2}{*}{$\begin{array}{l}\text { Wave- } \\
\text { length }\end{array}$} & \multicolumn{2}{|c|}{ Intensity } & \multirow{2}{*}{$\begin{array}{l}\text { Spec- } \\
\text { trum }\end{array}$} & \multirow{2}{*}{$\begin{array}{l}\text { Wave- } \\
\text { length }\end{array}$} & \multicolumn{2}{|c|}{ Intensity } & \multirow{2}{*}{$\begin{array}{l}\text { Spec- } \\
\text { trum }\end{array}$} & \multirow{2}{*}{$\begin{array}{l}\text { Wave- } \\
\text { length }\end{array}$} & \multicolumn{2}{|c|}{ Intensity } & \multirow{2}{*}{$\begin{array}{l}\text { Spec- } \\
\text { trum }\end{array}$} \\
\hline & Lamp & Spark & & & Lamp & Spark & & & Lamp & Spark & \\
\hline $\begin{array}{l}2505.601 \\
2506.012 \\
2506.266 \\
2506.348 \\
2507.490\end{array}$ & $\begin{array}{r}75 \\
2 \\
10 \\
8 \\
2\end{array}$ & $\begin{array}{l}75 \\
1 \mathrm{~h} \\
3 \\
4\end{array}$ & $\begin{array}{l}\text { II } \\
\text { I I } \\
\text { I } \\
\text { I I } \\
\text { I }\end{array}$ & $\begin{array}{l}2522.454 \\
2522.767 \\
2522.959 \\
2523.400 \\
2523.749\end{array}$ & $\begin{array}{r}10 \\
30 \\
15 \\
2 \\
10\end{array}$ & $\begin{array}{l}2 \\
8 \\
20 \\
10 \mathrm{w}\end{array}$ & $\begin{array}{l}\text { I } \\
\text { I } \\
\text { II } \\
\text { II } \\
\text { I }\end{array}$ & $\begin{array}{l}2540.348 \\
2540.720 \\
2541.033 \\
2541.707 \\
2541.907\end{array}$ & $\begin{array}{l}15 \\
5 \mathrm{~h} \\
5 \\
10 \\
1\end{array}$ & $\begin{array}{r}20 \\
4\end{array}$ & $\begin{array}{l}\text { I I } \\
\text { I I } \\
\text { I } \\
\text { I } \\
\text { I I I }\end{array}$ \\
\hline $\begin{array}{l}2507.926 \\
2508.313 \\
2508.734 \\
2509.584 \\
2509.699\end{array}$ & $\begin{array}{r}50 \\
15 \\
5 \\
15 \\
5\end{array}$ & $\begin{array}{r}50 \\
15 \\
15 \\
2\end{array}$ & $\begin{array}{l}\text { II } \\
\text { I I } \\
\text { I } \\
\text { II } \\
\text { II }\end{array}$ & $\begin{array}{l}2524.219 \\
2524.506 \\
2525.389 \\
2525.927 \\
2526.225\end{array}$ & $\begin{array}{r}2 \\
2 \\
15 \\
15\end{array}$ & $\begin{array}{l}5 \\
4 \\
5 \mathrm{w} \\
20 \\
15\end{array}$ & $\begin{array}{l}\text { II } \\
\text { I I } \\
\text { II } \\
\text { II } \\
\text { II }\end{array}$ & $\begin{array}{l}2542.649 \\
2542.880 \\
2543.215 \\
2543.340 \\
2544.370\end{array}$ & $\begin{array}{r}25 \\
1 \\
10 \\
20 \\
3\end{array}$ & $\begin{array}{l}40 \\
8 \\
3 \\
3\end{array}$ & $\begin{array}{l}\text { II } \\
\text { I } \\
\text { I I } \\
\text { I } \\
\text { I I }\end{array}$ \\
\hline $\begin{array}{l}2509.964 \\
2510.126 \\
2511.156 \\
2511.408 \\
2511.592\end{array}$ & $\begin{array}{r}25 \\
2 \\
15 \\
10\end{array}$ & $\begin{array}{c}30 \\
1 \\
10 \\
3 \\
15 \mathrm{w}\end{array}$ & $\begin{array}{l}\text { II } \\
\text { II } \\
\text { II } \\
\text { II } \\
\text { I I I }\end{array}$ & $\begin{array}{l}2526.338 \\
2526.818 \\
2527.878 \\
2528.361 \\
2528.676\end{array}$ & $\begin{array}{r}15 \\
20 \\
30 \\
\\
5\end{array}$ & $\begin{array}{r}20 \\
10 \\
300\end{array}$ & $\begin{array}{l}\text { II } \\
\text { I } \\
\text { I } \\
\text { II I } \\
\text { I }\end{array}$ & $\begin{array}{l}2544.427 \\
2544.608 \\
2544.717 \\
2544.914 \\
2545.101\end{array}$ & $\begin{array}{r}30 \\
5 \\
1 \\
10\end{array}$ & 400 & $\begin{array}{l}\text { I } \\
\text { I } \\
\text { I } \\
\text { I } \\
\text { I I I }\end{array}$ \\
\hline $\begin{array}{l}2511.838 \\
2512.202 \\
2512.465 \\
2512.520 \\
2512.714\end{array}$ & $\begin{array}{l}5 \\
5 \\
5 \\
5\end{array}$ & $300^{2 h}$ & $\begin{array}{l}\text { I } \\
\text { I } \\
\text { I } \\
\text { III } \\
\text { III }\end{array}$ & $\begin{array}{l}2529.330 \\
2529.759 \\
2529.978 \\
2530.079 \\
2530.398\end{array}$ & $\begin{array}{r}2 \\
10 \\
20 \\
20\end{array}$ & $\begin{array}{r}200 \\
3\end{array}$ & $\begin{array}{l}\text { I } \\
\text { I } \\
\text { I II } \\
\text { I } \\
\text { I }\end{array}$ & $\begin{array}{l}2545.340 \\
2545.742 \\
2546.057 \\
2546.263 \\
2546.960\end{array}$ & $\begin{array}{r}40 \\
20 \\
20 \\
5 \\
10\end{array}$ & $\begin{array}{l}25 \\
15\end{array}$ & $\begin{array}{l}\text { II } \\
\text { I I } \\
\text { I } \\
\text { I } \\
\text { I }\end{array}$ \\
\hline $\begin{array}{l}2512.819 \\
2513.210 \\
2513.335 \\
2513.621 \\
2513.930\end{array}$ & $\begin{array}{r}30 \\
1 \\
5 \\
1 \\
1\end{array}$ & & $\begin{array}{l}\text { I } \\
\text { I } \\
\text { I } \\
\text { I } \\
\text { I }\end{array}$ & $\begin{array}{l}2531.648 \\
2531.891 \\
2532.433 \\
2533.224 \\
2533.525\end{array}$ & $\begin{array}{r}5 \\
10 \\
30 \\
10 \\
20\end{array}$ & $\begin{array}{r}20 \\
100 \\
15\end{array}$ & $\begin{array}{l}\text { I } \\
\text { II } \\
\text { II } \\
\text { II } \\
\text { I }\end{array}$ & $\begin{array}{l}2547.134 \\
2547.466 \\
2547.900 \\
2548.138 \\
2549.011\end{array}$ & $\begin{array}{r}4 \\
5 \\
75 \\
40 \\
15\end{array}$ & $\begin{array}{l}75 \\
30\end{array}$ & $\begin{array}{l}\text { I } \\
\text { I } \\
\text { II } \\
\text { II } \\
\text { I }\end{array}$ \\
\hline $\begin{array}{l}2514.108 \\
2514.319 \\
2514.751 \\
2514.866 \\
2515.014\end{array}$ & $\begin{array}{r}1 \\
12 \\
10 \\
10 \\
1\end{array}$ & 300 & $\begin{array}{l}\text { I } \\
\text { I I I } \\
\text { I } \\
\text { I } \\
\text { I }\end{array}$ & $\begin{array}{l}2533.632 \\
2533.873 \\
2533.988 \\
2534.096 \\
2534.196\end{array}$ & $\begin{array}{r}1 \\
10 \\
10 \\
10 \\
1\end{array}$ & $\begin{array}{l}5 \mathrm{w} \\
15 \\
15 \\
1\end{array}$ & $\begin{array}{l}\text { I I } \\
\text { I I } \\
\text { I } \\
\text { II } \\
\text { I I }\end{array}$ & $\begin{array}{l}2549.094 \\
2549.135 \\
2549.531 \\
2549.985 \\
2550.261\end{array}$ & $\begin{array}{r}1 \\
5 \\
20 \\
10\end{array}$ & $\begin{array}{r}100 \\
400 \\
5\end{array}$ & $\begin{array}{l}\text { I } \\
\text { I I I } \\
\text { I I I } \\
\text { I } \\
\text { I }\end{array}$ \\
\hline $\begin{array}{l}2515.571 \\
2515.703 \\
2516.428 \\
2516.592 \\
2517.939\end{array}$ & $\begin{array}{r}2 \\
1 \\
30 \\
5 \\
10\end{array}$ & $\begin{array}{l}50 \\
10\end{array}$ & $\begin{array}{l}\text { I } \\
\text { I } \\
\text { II } \\
\text { I } \\
\text { I I }\end{array}$ & $\begin{array}{l}2534.403 \\
2534.582 \\
2534.916 \\
2535.118 \\
2535.722\end{array}$ & $\begin{array}{r}2 \\
10 \\
2 \\
2 \\
1\end{array}$ & $\begin{array}{r}2 \\
15 \\
4\end{array}$ & $\begin{array}{l}\text { I I } \\
\text { I I } \\
\text { I I I } \\
\text { I } \\
\text { I }\end{array}$ & $\begin{array}{l}2550.374 \\
2550.747 \\
2550.987 \\
2551.240 \\
2551.347\end{array}$ & $\begin{array}{r}4 \\
10 \\
10 \\
20 \\
5\end{array}$ & $\begin{array}{l}2 \mathrm{~h} \\
15\end{array}$ & $\begin{array}{l}\text { I } \\
\text { I } \\
\text { I } \\
\text { I }\end{array}$ \\
\hline $\begin{array}{l}2519.069 \\
2519.368 \\
2519.559 \\
2519.861 \\
2520.118\end{array}$ & $\begin{array}{r}10 \\
2 \\
5 \\
10 \\
20\end{array}$ & $\begin{array}{r}2 \\
15\end{array}$ & $\begin{array}{l}\text { I I } \\
\text { I } \\
\text { I } \\
\text { I } \\
\text { II }\end{array}$ & $\begin{array}{l}2535.871 \\
2536.487 \\
2536.546 \\
2536.668 \\
2537.358\end{array}$ & $\begin{array}{r}15 \\
20 \\
5 \\
30 \\
10\end{array}$ & $\begin{array}{r}25 \\
400\end{array}$ & $\begin{array}{l}\text { I I } \\
\text { I } \\
\text { I I I } \\
\text { I } \\
\text { I }\end{array}$ & $\begin{array}{l}2551.560 \\
2551.711 \\
2551.934 \\
2552.150 \\
2552.275\end{array}$ & $\begin{array}{r}5 \\
5 \\
10 \\
10 \\
5\end{array}$ & $\begin{array}{l}2 \mathrm{~h} \\
1 \\
5 \\
1 \\
2\end{array}$ & $\begin{array}{l}\text { I I } \\
\text { I } \\
\text { II } \\
\text { I } \\
\text { II }\end{array}$ \\
\hline $\begin{array}{l}2520.304 \\
2520.444 \\
2520.658 \\
2520.859 \\
2521.240\end{array}$ & $\begin{array}{r}20 \\
2 \\
75 \\
5 \\
2\end{array}$ & $\begin{array}{r}5 \\
100 \\
2\end{array}$ & $\begin{array}{l}\text { I } \\
\text { I } \\
\text { II } \\
\text { I } \\
\text { I }\end{array}$ & $\begin{array}{l}2537.548 \\
2537.766 \\
2538.446 \\
2538.684 \\
2538.811\end{array}$ & $\begin{array}{r}20 \\
10 \\
2 \\
5 \\
1\end{array}$ & $\begin{array}{l}10 \\
1 \\
2 \mathrm{~h}\end{array}$ & $\begin{array}{l}\text { I } \\
\text { II } \\
\text { I } \\
\text { I } \\
\text { I I }\end{array}$ & $\begin{array}{l}2552.378 \\
2552.692 \\
2552.911 \\
2553.254 \\
2553.456\end{array}$ & $\begin{array}{c}10 \\
2 \\
1 \mathrm{~h} \\
10 \\
5\end{array}$ & $\begin{array}{r}1 \\
10\end{array}$ & $\begin{array}{l}\text { I } \\
\text { I } \\
\text { II } \\
\text { II } \\
\text { I }\end{array}$ \\
\hline $\begin{array}{l}2521.303 \\
2521.771 \\
2521.906 \\
2522.059 \\
2522.106\end{array}$ & $\begin{array}{r}5 \\
10 \\
2 \\
2 \\
5\end{array}$ & 12 & $\begin{array}{l}\text { I } \\
\text { II } \\
\text { I } \\
\text { I } \\
\text { I }\end{array}$ & $\begin{array}{l}2538.935 \\
2539.207 \\
2539.375 \\
2539.858 \\
2540.170\end{array}$ & $\begin{array}{r}20 \\
2 \\
10 \\
10\end{array}$ & $\begin{array}{l}25 \\
10 \\
20 w\end{array}$ & $\begin{array}{l}\text { II } \\
\text { I } \\
\text { II } \\
\text { III } \\
\text { I }\end{array}$ & $\begin{array}{l}2553.818 \\
2554.151 \\
2554.390 \\
2554.651 \\
2554.747\end{array}$ & $\begin{array}{r}10 \\
2 \\
30 \\
30\end{array}$ & 500 & $\begin{array}{l}\text { II } \\
\text { I } \\
\text { I } \\
\text { I } \\
\text { III }\end{array}$ \\
\hline
\end{tabular}


Table 1. Wavelengths of Thorium Spectra - Continued

\begin{tabular}{|c|c|c|c|c|c|c|c|c|c|c|c|}
\hline \multirow{2}{*}{$\begin{array}{l}\text { Wave- } \\
\text { length }\end{array}$} & \multicolumn{2}{|c|}{ Intensity } & \multirow{2}{*}{$\begin{array}{l}\text { Spec- } \\
\text { trum }\end{array}$} & \multirow{2}{*}{$\begin{array}{l}\text { Wave- } \\
\text { length }\end{array}$} & \multicolumn{2}{|c|}{ Intensity } & \multirow{2}{*}{$\begin{array}{l}\text { Spec. } \\
\text { trum }\end{array}$} & \multirow{2}{*}{$\begin{array}{l}\text { Wave- } \\
\text { length }\end{array}$} & \multicolumn{2}{|c|}{ Intensity } & \multirow{2}{*}{$\begin{array}{l}\text { Spec- } \\
\text { trum }\end{array}$} \\
\hline & $a m p$ & Spark & & & Lamp & Spark & & & Lamp & Spark & \\
\hline $\begin{array}{l}2554.924 \\
2555.170 \\
2555.219 \\
2555.481 \\
2555.661\end{array}$ & $\begin{array}{r}5 \\
75 \\
\\
5 \\
5\end{array}$ & $\begin{array}{l}300 \\
100 \\
6 \mathrm{~h}\end{array}$ & $\begin{array}{l}\text { I } \\
\text { II } \\
\text { III } \\
\text { II } \\
\text { I }\end{array}$ & $\begin{array}{l}2568.404 \\
2568.661 \\
2568.984 \\
2569.240 \\
2569.480\end{array}$ & $\begin{array}{r}1 \\
10 \\
1 \\
5 \\
5\end{array}$ & 4 & $\begin{array}{l}I \\
I I \\
I \\
I \\
I\end{array}$ & $\begin{array}{l}2583.451 \\
2584.054 \\
2584.357 \\
2585.010 \\
2585.248\end{array}$ & $\begin{array}{l}50 \\
2 \mathrm{w} \\
1 \\
2 \\
5\end{array}$ & $\begin{array}{l}3 w \\
2\end{array}$ & $\begin{array}{l}\text { I } \\
\text { I } \\
\text { III } \\
\text { I } \\
\text { II }\end{array}$ \\
\hline $\begin{array}{l}2555.894 \\
2556.092 \\
2556.479 \\
2556.739 \\
2557.024\end{array}$ & $\begin{array}{r}10 \\
20 \\
15 \\
2 \\
10\end{array}$ & $\begin{array}{r}1 \\
10 \\
3\end{array}$ & $\begin{array}{l}\text { I } \\
\text { I } \\
\text { II } \\
\text { I } \\
\text { I }\end{array}$ & $\begin{array}{l}2569.699 \\
2569.845 \\
2570.090 \\
2570.256 \\
2570.663\end{array}$ & $\begin{array}{r}2 \\
20 \\
1 \\
2 \\
15\end{array}$ & $2 \mathrm{~h}$ & $\begin{array}{l}\text { I } \\
\text { I } \\
\text { I } \\
\text { I } \\
\text { I I }\end{array}$ & $\begin{array}{l}2585.728 \\
2586.146 \\
2587.063 \\
2587.237 \\
2587.865\end{array}$ & $\begin{array}{r}5 \\
25 \\
2 \\
20 \\
5\end{array}$ & $\begin{array}{l}50 \\
25 \mathrm{w} \\
2\end{array}$ & $\begin{array}{l}\text { I } \\
\text { II } \\
\text { I } \\
\text { III } \\
\text { II }\end{array}$ \\
\hline $\begin{array}{l}2557.475 \\
2557.917 \\
2558.338 \\
2558.522 \\
2558.884\end{array}$ & $\begin{array}{r}2 \\
10 \\
20 \\
2 \\
10\end{array}$ & $\begin{array}{l}1 \\
6\end{array}$ & $\begin{array}{l}\text { I } \\
\text { I } \\
\text { I } \\
\text { I } \\
\text { I }\end{array}$ & $\begin{array}{l}2571.463 \\
2571.611 \\
2572.346 \\
2572.457 \\
2572.744\end{array}$ & $\begin{array}{r}20 \\
10 \\
1 \\
5 \\
20\end{array}$ & 500 & $\begin{array}{l}\text { I } \\
\text { III } \\
\text { I } \\
I \\
I\end{array}$ & $\begin{array}{l}2587.908 \\
2588.916 \\
2589.065 \\
2589.480 \\
2589.618\end{array}$ & $\begin{array}{r}2 \\
25 \\
100 \\
10 \\
3\end{array}$ & $\begin{array}{c}2 \mathrm{w} \\
30 \\
200 \\
10 \\
3 \mathrm{~h}\end{array}$ & $\begin{array}{l}\text { II } \\
\text { II } \\
\text { II } \\
\text { II } \\
\text { II }\end{array}$ \\
\hline $\begin{array}{l}2559.113 \\
2559.175 \\
2559.240 \\
2559.364 \\
2559.418\end{array}$ & $\begin{array}{l}2 \\
2 \mathrm{~h} \\
2 \\
2\end{array}$ & 150 & $\begin{array}{l}\text { I } \\
\text { I I I } \\
\text { I } \\
\text { I } \\
\text { I }\end{array}$ & $\begin{array}{l}2573.139 \\
2573.634 \\
2573.784 \\
2573.865 \\
2574.485\end{array}$ & $\begin{array}{r}40 \\
20 \\
1 \\
5 \\
50\end{array}$ & $\begin{array}{r}3 \\
6 \\
1 \\
100\end{array}$ & $\begin{array}{l}\text { I } \\
\text { II } \\
\text { II } \\
\text { I } \\
\text { II }\end{array}$ & $\begin{array}{l}2589.857 \\
2590.065 \\
2590.225 \\
2590.481 \\
2590.829\end{array}$ & $\begin{array}{r}10 \\
10 \\
15 \\
4 \\
10\end{array}$ & $\begin{array}{l}10 \\
5 \\
20 \\
4 \mathrm{w}\end{array}$ & $\begin{array}{l}\text { II } \\
\text { II } \\
\text { II } \\
\text { II } \\
\text { I }\end{array}$ \\
\hline $\begin{array}{l}2559.475 \\
2559.792 \\
2560.034 \\
2560.108 \\
2560.396\end{array}$ & $\begin{array}{r}2 \\
20 \\
1 \\
5 \\
20\end{array}$ & $\begin{array}{l}6 \\
2 \mathrm{w} \\
2 \mathrm{~h}\end{array}$ & $\begin{array}{l}\text { I } \\
\text { I } \\
\text { II } \\
\text { II } \\
\text { I }\end{array}$ & $\begin{array}{l}2574.959 \\
2575.095 \\
2575.416 \\
2576.210 \\
2576.327\end{array}$ & $\begin{array}{r}10 \\
5 \\
5 \\
5 \\
50\end{array}$ & $\begin{array}{r}2 \\
50\end{array}$ & $\begin{array}{l}\text { I } \\
\text { I } \\
\text { I } \\
\text { II } \\
\text { I I }\end{array}$ & $\begin{array}{l}2591.003 \\
2591.224 \\
2591.369 \\
2591.668 \\
2592.246\end{array}$ & $\begin{array}{r}10 \\
15 \\
5 \\
5 \\
2\end{array}$ & $\begin{array}{r}25 \\
2 \\
2\end{array}$ & $\begin{array}{l}\text { I } \\
\text { III } \\
\text { II } \\
\text { I } \\
\text { III }\end{array}$ \\
\hline $\begin{array}{l}2560.557 \\
2560.934 \\
2561.147 \\
2561.288 \\
2561.611\end{array}$ & $\begin{array}{l}10 \\
20 \\
10 \\
10\end{array}$ & $\begin{array}{c}20 \\
4 \\
15 \mathrm{w}\end{array}$ & $\begin{array}{l}\text { I } \\
\text { II } \\
\text { II } \\
\text { II I } \\
\text { I }\end{array}$ & $\begin{array}{l}2576.688 \\
2576.905 \\
2577.045 \\
2577.285 \\
2577.892\end{array}$ & $\begin{array}{r}125 \\
20 \\
20 \\
20 \\
10\end{array}$ & $\begin{array}{r}100 \\
10 \\
5 \\
2 \\
10\end{array}$ & $\begin{array}{l}\text { II } \\
\text { II } \\
\text { I } \\
\text { I } \\
\text { II }\end{array}$ & $\begin{array}{l}2592.542 \\
2592.765 \\
2593.084 \\
2593.565 \\
2593.877\end{array}$ & $\begin{array}{r}15 \\
5 \\
15 \\
10 \\
20\end{array}$ & $\begin{array}{r}15 \\
8 \\
15\end{array}$ & $\begin{array}{l}\text { II } \\
\text { II } \\
\text { II } \\
\text { I } \\
\text { I }\end{array}$ \\
\hline $\begin{array}{l}2561.939 \\
2562.223 \\
2562.332 \\
2562.680 \\
2562.939\end{array}$ & $\begin{array}{r}50 \\
10 \\
30 \\
10 \\
3\end{array}$ & 75 & $\begin{array}{l}\text { II } \\
\text { I } \\
\text { I } \\
\text { II } \\
\text { I }\end{array}$ & $\begin{array}{l}2578.250 \\
2578.434 \\
2578.630 \\
2578.956 \\
2579.437\end{array}$ & $\begin{array}{r}5 \\
5 \\
10 \\
15 \\
75\end{array}$ & $\begin{array}{c}2 \mathrm{~h} \\
8 \mathrm{w} \\
10 \mathrm{w} \\
12 \\
100\end{array}$ & $\begin{array}{l}\text { II } \\
\text { II } \\
\text { II } \\
\text { II } \\
\text { II }\end{array}$ & $\begin{array}{l}2594.341 \\
2594.656 \\
2595.031 \\
2595.622 \\
2596.414\end{array}$ & $\begin{array}{r}7 \\
10 \\
50 \\
5 \\
10\end{array}$ & $\begin{array}{c}5 \mathrm{~h} \\
10 \\
100 \\
5\end{array}$ & $\begin{array}{l}\text { II } \\
\text { II } \\
\text { II } \\
\text { I } \\
\text { I I }\end{array}$ \\
\hline $\begin{array}{l}2563.097 \\
2563.256 \\
2564.110 \\
2564.314 \\
2564.387\end{array}$ & $\begin{array}{r}5 \\
5 \\
10 \\
2 \\
1\end{array}$ & $1 \mathrm{~h}$ & $\begin{array}{l}\text { II } \\
\text { I } \\
\text { I } \\
\text { I } \\
\text { II I }\end{array}$ & $\begin{array}{l}2579.762 \\
2580.350 \\
2580.700 \\
2581.019 \\
2581.181\end{array}$ & $\begin{array}{r}2 \\
20 \\
40 \\
5 \\
4\end{array}$ & $\begin{array}{r}2 \\
30 \\
75 \\
1 \\
4\end{array}$ & $\begin{array}{l}\text { II } \\
\text { II } \\
\text { II } \\
\text { I } \\
\text { II }\end{array}$ & $\begin{array}{l}2596.744 \\
2597.048 \\
2597.326 \\
2597.648 \\
2597.750\end{array}$ & $\begin{array}{r}50 \\
250 \\
\\
10 \\
20\end{array}$ & $\begin{array}{r}12 \\
200 \\
2000\end{array}$ & $\begin{array}{l}\text { I } \\
\text { II } \\
\text { II I } \\
\text { I } \\
\text { I }\end{array}$ \\
\hline $\begin{array}{l}2564.797 \\
2565.597 \\
2566.594 \\
2566.923 \\
2567.467\end{array}$ & $\begin{array}{r}10 \\
250 \\
125 \\
15 \\
10\end{array}$ & $\begin{array}{r}4 \\
200 \\
100 \\
10\end{array}$ & $\begin{array}{l}\text { II } \\
\text { II } \\
\text { II } \\
\text { II } \\
\text { I }\end{array}$ & $\begin{array}{l}2581.457 \\
2581.811 \\
2581.976 \\
2582.108 \\
2502.351\end{array}$ & $\begin{array}{r}5 \\
20 \\
5 \\
10 \\
5\end{array}$ & $\begin{array}{c}6 \\
10 w\end{array}$ & $\begin{array}{l}\text { I } \\
\text { I } \\
\text { I } \\
\text { I I } \\
\text { I }\end{array}$ & $\begin{array}{l}2598.063 \\
2598.208 \\
2598.506 \\
2598.732 \\
2598.840\end{array}$ & $\begin{array}{r}5 \\
10 \\
1 \\
15 \\
20\end{array}$ & 10 & $\begin{array}{l}\text { I } \\
\text { I } \\
\text { I } \\
\text { II } \\
\text { I }\end{array}$ \\
\hline $\begin{array}{l}2567.650 \\
2567.792 \\
2567.850 \\
2567.940 \\
2568.235\end{array}$ & $\begin{array}{l}20 \\
20\end{array}$ & 200 & $\begin{array}{l}I \\
I \\
I I I \\
I \\
I\end{array}$ & $\begin{array}{l}2582.612 \\
2582.939 \\
2583.129 \\
2583.295 \\
2583.360\end{array}$ & $\begin{array}{r}2 \\
20 \\
5 \\
1\end{array}$ & $\begin{array}{l}2 \\
2\end{array}$ & $\begin{array}{l}\text { II } \\
\text { I } \\
\text { I } \\
\text { I } \\
\text { III }\end{array}$ & $\begin{array}{l}2599.739 \\
2600.080 \\
2600.330 \\
2600.506 \\
2600.638\end{array}$ & $\begin{array}{r}30 \\
5 \\
10 \\
10\end{array}$ & 1500 & $\begin{array}{l}\text { II } \\
\text { I } \\
\text { I } \\
\text { I } \\
\text { III }\end{array}$ \\
\hline
\end{tabular}


Table 1. Wavelengths of Thorium Spectra - Continued

\begin{tabular}{|c|c|c|c|c|c|c|c|c|c|c|c|}
\hline \multirow{2}{*}{$\begin{array}{l}\text { Wave- } \\
\text { length }\end{array}$} & \multicolumn{2}{|c|}{ Intensity } & \multirow{2}{*}{$\begin{array}{l}\text { Spec- } \\
\text { trum }\end{array}$} & \multirow{2}{*}{$\begin{array}{l}\text { Wave- } \\
\text { length }\end{array}$} & \multicolumn{2}{|c|}{ Intensity } & \multirow{2}{*}{$\begin{array}{l}\text { Spec- } \\
\text { trum }\end{array}$} & \multirow{2}{*}{$\begin{array}{l}\text { Wave- } \\
\text { length }\end{array}$} & \multicolumn{2}{|c|}{ Intensity } & \multirow{2}{*}{$\begin{array}{l}\text { Spec- } \\
\text { trum }\end{array}$} \\
\hline & Lamp & Spark & & & Lamp & Spark & & & Lamp & Spark t & \\
\hline $\begin{array}{l}2600.893 \\
2601.318 \\
2601.624 \\
2601.820 \\
2602.143\end{array}$ & $\begin{array}{r}250 \\
20 \\
30 \\
5\end{array}$ & $\begin{array}{l}200 \\
10 \mathrm{w} \\
10 \\
20\end{array}$ & $\begin{array}{l}\text { I I } \\
\text { I I I } \\
\text { I I } \\
\text { I I } \\
\text { I }\end{array}$ & $\begin{array}{l}2617.116 \\
2617.832 \\
2618.903 \\
2618.996 \\
2619.520\end{array}$ & $\begin{array}{r}30 \\
30 \\
150 \\
125 \\
5\end{array}$ & $\begin{array}{r}20 \\
40 \\
300 \\
150\end{array}$ & $\begin{array}{l}\text { II } \\
\text { I I } \\
\text { I I } \\
\text { II } \\
\text { I }\end{array}$ & $\begin{array}{l}2633.623 \\
2633.795 \\
2634.114 \\
2634.162 \\
2634.322\end{array}$ & $\begin{array}{r}5 \\
5 \\
10 \\
10 \\
10\end{array}$ & $\begin{array}{r}1 \\
3 \\
12 \\
2\end{array}$ & $\begin{array}{l}\text { I } \\
\text { II } \\
\text { II } \\
\text { I } \\
\text { I }\end{array}$ \\
\hline $\begin{array}{l}2602.289 \\
2602.605 \\
2602.803 \\
2603.013 \\
2603.294\end{array}$ & $\begin{array}{r}10 \\
5 \\
2 \\
30 \\
10\end{array}$ & $\begin{array}{r}20 \\
2\end{array}$ & $\begin{array}{l}\text { I } \\
\text { I } \\
\text { I } \\
\text { II } \\
\text { I }\end{array}$ & $\begin{array}{l}2619.593 \\
2619.746 \\
2620.219 \\
2620.375 \\
2620.801\end{array}$ & $\begin{array}{r}5 \\
5 \\
5 \\
20 \\
10\end{array}$ & $\begin{array}{l}5 \mathrm{~h} \\
1 \\
20\end{array}$ & $\begin{array}{l}\text { II } \\
\text { I } \\
\text { I } \\
\text { II } \\
\text { I }\end{array}$ & $\begin{array}{l}2634.463 \\
2634.642 \\
2634.938 \\
2635.037 \\
2635.151\end{array}$ & $\begin{array}{l}10 \\
10 \\
40 \\
40 \\
40\end{array}$ & $\begin{array}{l}4 \\
3\end{array}$ & $\begin{array}{l}I \\
I \\
I \\
I \\
I\end{array}$ \\
\hline $\begin{array}{l}2603.556 \\
2603.727 \\
2603.911 \\
2604.231 \\
2604.491\end{array}$ & $\begin{array}{l}20 \\
10 \\
40 \\
10 \\
10\end{array}$ & $\begin{array}{r}10 \\
50 \\
2\end{array}$ & $\begin{array}{l}\text { II } \\
\text { I } \\
\text { II } \\
\text { I } \\
\text { I }\end{array}$ & $\begin{array}{l}2620.939 \\
2621.372 \\
2621.510 \\
2622.353 \\
2622.689\end{array}$ & $\begin{array}{l}30 \\
30 \\
20 \\
50 \\
30\end{array}$ & $\begin{array}{r}50 \\
100 \\
20 \\
150 \\
30\end{array}$ & $\begin{array}{l}\text { II } \\
\text { II } \\
\text { II } \\
\text { II } \\
\text { I I }\end{array}$ & $\begin{array}{l}2635.296 \\
2635.415 \\
2635.895 \\
2636.082 \\
2636.230\end{array}$ & $\begin{array}{l}30 \\
30 \\
\\
20 \\
10\end{array}$ & $\begin{array}{r}25 \\
15 \\
500 \\
\\
12\end{array}$ & $\begin{array}{l}\text { II } \\
\text { II } \\
\text { III } \\
\text { I } \\
\text { II }\end{array}$ \\
\hline $\begin{array}{l}2604.712 \\
2604.956 \\
2605.210 \\
2605.672 \\
2605.997\end{array}$ & $\begin{array}{r}2 \\
4 \\
5 \\
5 \\
40\end{array}$ & $1 \mathrm{~h}$ & $\begin{array}{l}\text { I } \\
\text { II } \\
\text { I } \\
\text { I } \\
\text { II }\end{array}$ & $\begin{array}{l}2623.100 \\
2623.447 \\
2623.797 \\
2624.020 \\
2624.644\end{array}$ & $\begin{array}{r}75 \\
250 \\
30 \\
20 \\
125\end{array}$ & $\begin{array}{r}3000 \\
4 \\
10\end{array}$ & $\begin{array}{l}\text { I } \\
\text { I I } \\
\text { I } \\
\text { I } \\
\text { I }\end{array}$ & $\begin{array}{l}2636.412 \\
2636.675 \\
2636.876 \\
2637.097 \\
2637.407\end{array}$ & $\begin{array}{l}20 \\
10 \\
50 \\
20 \\
10\end{array}$ & $\begin{array}{r}5 \\
10 \\
8\end{array}$ & $\begin{array}{l}\text { II } \\
\text { I } \\
\text { I } \\
\text { II } \\
\text { I }\end{array}$ \\
\hline $\begin{array}{l}2606.418 \\
2606.673 \\
2607.476 \\
2607.775 \\
2608.079\end{array}$ & $\begin{array}{l}20 \\
10 \\
40 \\
20 \\
10\end{array}$ & $\begin{array}{l}30 \\
20\end{array}$ & $\begin{array}{l}\text { I } \\
\text { I } \\
\text { II } \\
\text { II } \\
\text { I }\end{array}$ & $\begin{array}{l}2624.799 \\
2625.106 \\
2625.252 \\
2625.735 \\
2626.095\end{array}$ & $\begin{array}{r}20 \\
50 \\
20 \\
300 \\
30\end{array}$ & $\begin{array}{r}1 \\
3000 \\
40\end{array}$ & $\begin{array}{l}\text { I } \\
\text { I } \\
\text { I } \\
\text { II } \\
\text { II }\end{array}$ & $\begin{array}{l}2637.474 \\
2637.696 \\
2638.161 \\
2638.603 \\
2638.779\end{array}$ & $\begin{array}{r}5 \\
50 \\
2 \\
10\end{array}$ & $\begin{array}{c}500 \mathrm{w} \\
40 \\
200\end{array}$ & $\begin{array}{l}\text { I } \\
\text { III } \\
\text { II } \\
\text { III } \\
\text { I }\end{array}$ \\
\hline $\begin{array}{l}2608.321 \\
2609.225 \\
2609.857 \\
2610.040 \\
2610.404\end{array}$ & $\begin{array}{c}50 \\
20 \\
150 \\
2 \mathrm{~h} \\
10\end{array}$ & $\begin{array}{r}50 \\
500 \\
150\end{array}$ & $\begin{array}{l}\text { I I } \\
\text { I I I } \\
\text { I I } \\
\text { I } \\
\text { I }\end{array}$ & $\begin{array}{l}2626.398 \\
2626.695 \\
2627.157 \\
2627.558 \\
2628.014\end{array}$ & $\begin{array}{r}100 \\
20 \\
10 \\
50 \\
10\end{array}$ & $\begin{array}{r}200 \\
4 \\
2 \\
75\end{array}$ & $\begin{array}{l}\text { II } \\
\text { I } \\
\text { I } \\
\text { II }\end{array}$ & $\begin{array}{l}2639.075 \\
2639.510 \\
2639.886 \\
2640.267 \\
2640.397\end{array}$ & $\begin{array}{r}20 \\
200 \\
50 \\
100 \\
50\end{array}$ & $\begin{array}{r}2 \\
250 \\
100 \\
125 \\
50\end{array}$ & $\begin{array}{l}\text { I } \\
\text { II } \\
\text { II } \\
\text { II } \\
\text { II }\end{array}$ \\
\hline $\begin{array}{l}2611.138 \\
2611.271 \\
2611.425 \\
2611.615 \\
2611.691\end{array}$ & $\begin{array}{r}30 \\
40 \\
40 \\
50 \\
100\end{array}$ & $\begin{array}{r}50 \\
4 \\
4 \\
8 \\
125\end{array}$ & $\begin{array}{l}\text { II } \\
\text { I } \\
\text { I } \\
\text { I } \\
\text { II }\end{array}$ & $\begin{array}{l}2628.541 \\
2628.808 \\
2628.924 \\
2629.069 \\
2629.378\end{array}$ & $\begin{array}{r}10 \\
300 \\
2 \\
20 \\
2\end{array}$ & $\begin{array}{c}500 \\
2 \\
2 \mathrm{~h} \\
1 \mathrm{~h}\end{array}$ & $\begin{array}{l}\text { I } \\
\text { I I } \\
\text { II } \\
\text { I } \\
\text { II }\end{array}$ & $\begin{array}{l}2640.644 \\
2640.868 \\
2640.977 \\
2641.202 \\
2641.488\end{array}$ & $\begin{array}{r}20 \\
40 \\
5 \\
2 \\
500\end{array}$ & $\begin{array}{r}1 \\
2 \\
1500\end{array}$ & $\begin{array}{l}\text { I } \\
\text { I } \\
\text { I } \\
\text { I } \\
\text { II }\end{array}$ \\
\hline $\begin{array}{l}2612.534 \\
2612.696 \\
2612.876 \\
2613.077 \\
2613.319\end{array}$ & $\begin{array}{l}50 \\
20 \\
30 \\
10 \\
10\end{array}$ & $\begin{array}{r}8 \\
20 \\
50 \\
\\
1\end{array}$ & $\begin{array}{l}\text { I } \\
\text { II } \\
\text { II } \\
\text { I } \\
\text { I }\end{array}$ & $\begin{array}{l}2629.614 \\
2630.015 \\
2630.277 \\
2630.670 \\
2630.824\end{array}$ & $\begin{array}{r}100 \\
250 \\
10 \\
10 \\
50\end{array}$ & $\begin{array}{r}125 \\
30 \\
6 \\
5 \\
75\end{array}$ & $\begin{array}{l}\text { II } \\
\text { II } \\
\text { II } \\
\text { II } \\
\text { II }\end{array}$ & $\begin{array}{l}2641.820 \\
2642.397 \\
2642.606 \\
2643.052 \\
2643.274\end{array}$ & $\begin{array}{r}2 \\
20 \\
50 \\
10 \\
50\end{array}$ & $\begin{array}{r}15 \\
300 \\
5 \\
75\end{array}$ & $\begin{array}{l}\text { I } \\
\text { II } \\
\text { II } \\
\text { II } \\
\text { II }\end{array}$ \\
\hline $\begin{array}{l}2613.652 \\
2614.175 \\
2614.363 \\
2614.956 \\
2615.337\end{array}$ & $\begin{array}{r}10 \\
50 \\
5 \\
5 \\
30\end{array}$ & $\begin{array}{l}2 \mathrm{~h} \\
2 \\
5 \mathrm{~h} \\
50\end{array}$ & $\begin{array}{l}\text { I } \\
\text { I } \\
\text { II } \\
\text { II } \\
\text { II }\end{array}$ & $\begin{array}{l}2631.576 \\
2631.884 \\
2632.050 \\
2632.464 \\
2632.546\end{array}$ & $\begin{array}{l}20 \\
40 \\
20 \\
50 \\
75\end{array}$ & $\begin{array}{r}2 \\
6 \\
2 \\
40 \\
25\end{array}$ & $\begin{array}{l}\text { I } \\
\text { I } \\
\text { I } \\
\text { II } \\
\text { II }\end{array}$ & $\begin{array}{l}2643.460 \\
2644.459 \\
2644.681 \\
2644.768 \\
2645.668\end{array}$ & $\begin{array}{l}50 \\
20 \\
50 \\
20 \\
10\end{array}$ & $\begin{array}{r}75 \\
4 \\
100 \\
40\end{array}$ & $\begin{array}{l}\text { II } \\
\text { I } \\
\text { II } \\
\text { II } \\
\text { I }\end{array}$ \\
\hline $\begin{array}{l}2615.635 \\
2615.857 \\
2616.123 \\
2616.632 \\
2616.856\end{array}$ & $\begin{array}{r}40 \\
5 \\
40 \\
20 \\
20\end{array}$ & $\begin{array}{r}8 \\
50 \\
20 \\
20\end{array}$ & $\begin{array}{l}\text { I } \\
\text { I } \\
\text { II } \\
\text { II } \\
\text { I I }\end{array}$ & $\begin{array}{l}2632.647 \\
2632.851 \\
2632.926 \\
2633.123 \\
2633.330\end{array}$ & $\begin{array}{r}40 \\
75 \\
50 \\
5 \\
300\end{array}$ & $\begin{array}{r}15 \\
75 \\
500\end{array}$ & $\begin{array}{l}\text { II } \\
\text { I } \\
\text { II } \\
\text { I } \\
\text { II }\end{array}$ & $\begin{array}{l}2646.171 \\
2646.248 \\
2646.470 \\
2646.715 \\
2646.889\end{array}$ & $\begin{array}{r}10 \\
10 \\
5 \\
10 \\
20\end{array}$ & $\begin{array}{l}2 \\
2 \\
4\end{array}$ & $\begin{array}{l}\text { I } \\
\text { I } \\
\text { II } \\
\text { I } \\
\text { I }\end{array}$ \\
\hline
\end{tabular}


Table 1. Wavelengths of Thorium Spectra - Continued

\begin{tabular}{|c|c|c|c|c|c|c|c|c|c|c|c|}
\hline \multirow{2}{*}{$\begin{array}{l}\text { Wave- } \\
\text { length }\end{array}$} & \multicolumn{2}{|c|}{ Intensity } & \multirow{2}{*}{$\begin{array}{l}\text { Spec- } \\
\text { trum }\end{array}$} & \multirow{2}{*}{$\begin{array}{l}\text { Wave- } \\
\text { length }\end{array}$} & \multicolumn{2}{|c|}{ Intensity } & \multirow{2}{*}{$\begin{array}{l}\text { Spec- } \\
\text { trum }\end{array}$} & \multirow{2}{*}{$\begin{array}{l}\text { Wave- } \\
\text { length }\end{array}$} & \multicolumn{2}{|c|}{ Intensity } & \multirow{2}{*}{$\begin{array}{l}\text { Spec } \\
\text { trum }\end{array}$} \\
\hline & Lamp & Spark & & & Lamp & Spark & & & Lamp & Spark & \\
\hline $\begin{array}{l}2646.928 \\
2647.078 \\
2647.375 \\
2647.503 \\
2647.838\end{array}$ & $\begin{array}{r}2 \\
5 \\
20 \\
20 \\
2\end{array}$ & $\begin{array}{c}6 \\
20 \\
20 \\
1 \mathrm{~h}\end{array}$ & $\begin{array}{l}\text { I I } \\
\text { I } \\
\text { I I } \\
\text { I I } \\
\text { I I }\end{array}$ & $\begin{array}{l}2658.665 \\
2659.206 \\
2659.274 \\
2659.433 \\
2659.854\end{array}$ & $\begin{array}{r}300 \\
20 \\
10 \\
40 \\
50\end{array}$ & $\begin{array}{r}500 \\
1 \\
1 \\
4 \\
100\end{array}$ & $\begin{array}{l}\text { II } \\
\text { I } \\
\text { I } \\
\text { I } \\
\text { I I }\end{array}$ & $\begin{array}{l}2671.050 \\
2671.471 \\
2671.738 \\
2672.202 \\
2672.380\end{array}$ & $\begin{array}{r}20 \\
50 \\
40 \\
5\end{array}$ & $\begin{array}{c}100 \\
50 \mathrm{w} \\
4\end{array}$ & $\begin{array}{l}\text { I } \\
\text { II } \\
\text { II I } \\
\text { I } \\
\text { I }\end{array}$ \\
\hline $\begin{array}{l}2648.211 \\
2648.403 \\
2648.653 \\
2648.751 \\
2649.128\end{array}$ & $\begin{array}{l}50 \\
50 \\
30 \\
20 \\
50\end{array}$ & $\begin{array}{l}100 \\
40 \\
30 \\
10 \mathrm{~h}\end{array}$ & $\begin{array}{l}\text { I I } \\
\text { I } \\
\text { I I } \\
\text { I I } \\
\text { I }\end{array}$ & $\begin{array}{l}2660.599 \\
2660.965 \\
2661.089 \\
2661.388 \\
2661.728\end{array}$ & $\begin{array}{r}50 \\
20 \\
10 \\
100 \\
5\end{array}$ & $\begin{array}{r}75 \\
10 \\
2 \\
200 \\
5\end{array}$ & $\begin{array}{l}\text { II } \\
\text { I I } \\
\text { I } \\
\text { I I } \\
\text { I I }\end{array}$ & $\begin{array}{l}2672.576 \\
2672.666 \\
2672.733 \\
2672.945 \\
2673.279\end{array}$ & $\begin{array}{l}40 \\
20 \\
50 \\
50 \\
40\end{array}$ & $\begin{array}{r}10 \\
5 \\
10 \\
40 \\
4\end{array}$ & $\begin{array}{l}\text { II } \\
\text { I } \\
\text { II } \\
\text { II } \\
\text { I }\end{array}$ \\
\hline $\begin{array}{l}2649.476 \\
2649.875 \\
2650.585 \\
2650.692 \\
2650.834\end{array}$ & $\begin{array}{r}125 \\
200 \\
750 \\
5 \\
5\end{array}$ & $\begin{array}{r}250 \\
500 \\
750 \\
2 \\
1\end{array}$ & $\begin{array}{l}\text { I I } \\
\text { II } \\
\text { I I } \\
\text { I I } \\
\text { I }\end{array}$ & $\begin{array}{l}2661.975 \\
2662.343 \\
2662.859 \\
2663.192 \\
2663.333\end{array}$ & $\begin{array}{r}5 \\
150 \\
150 \\
20 \\
50\end{array}$ & $\begin{array}{r}2 \\
250 \\
250 \\
5\end{array}$ & $\begin{array}{l}\text { I I } \\
\text { II } \\
\text { I I } \\
\text { I } \\
\text { I }\end{array}$ & $\begin{array}{l}2673.397 \\
2673.486 \\
2673.708 \\
2673.917 \\
2674.028\end{array}$ & $\begin{array}{l}40 \\
10 \\
40 \\
20\end{array}$ & $\begin{array}{r}700 \\
10\end{array}$ & $\begin{array}{l}\text { I } \\
\text { I } \\
\text { I I I } \\
\text { I } \\
\text { I }\end{array}$ \\
\hline $\begin{array}{l}2651.131 \\
2651.384 \\
2651.510 \\
2651.712 \\
2651.909\end{array}$ & $\begin{array}{r}5 \\
5 \\
40 \\
50 \\
40\end{array}$ & $\begin{array}{r}1 \\
10 \\
75 \\
2\end{array}$ & $\begin{array}{l}\text { I } \\
\text { I } \\
\text { I } \\
\text { I I } \\
\text { I }\end{array}$ & $\begin{array}{l}2663.530 \\
2663.659 \\
2663.950 \\
2664.059 \\
2664.211\end{array}$ & $\begin{array}{r}2 \\
5 \\
40 \\
50 \\
30\end{array}$ & $\begin{array}{r}100 \\
30\end{array}$ & $\begin{array}{l}\text { I } \\
\text { I } \\
\text { I } \\
\text { I I } \\
\text { I I }\end{array}$ & $\begin{array}{l}2674.263 \\
2674.320 \\
2674.568 \\
2674.751 \\
2674.974\end{array}$ & $\begin{array}{r}40 \\
50 \\
20 \\
40 \\
4\end{array}$ & $\begin{array}{c}20 \mathrm{w} \\
5 \\
15 \\
4\end{array}$ & $\begin{array}{l}\text { II } \\
\text { I } \\
\text { I } \\
\text { I I } \\
\text { I I }\end{array}$ \\
\hline $\begin{array}{l}2652.065 \\
2652.605 \\
2652.740 \\
2652.830 \\
2653.167\end{array}$ & $\begin{array}{l}50 \\
40 \\
10 \\
1 \\
30\end{array}$ & $\begin{array}{r}75 \\
10 \\
1 \\
100\end{array}$ & $\begin{array}{l}\text { II } \\
\text { I } \\
\text { II } \\
\text { II } \\
\text { I I }\end{array}$ & $\begin{array}{l}2664.362 \\
2664.670 \\
2664.986 \\
2665.120 \\
2665.274\end{array}$ & $\begin{array}{r}40 \\
2 \\
20 \\
5 \\
20\end{array}$ & $\begin{array}{l}3 \\
3\end{array}$ & $\begin{array}{l}\text { I } \\
\text { I I } \\
\text { I } \\
\text { I } \\
\text { I I }\end{array}$ & $\begin{array}{l}2675.126 \\
2675.232 \\
2675.428 \\
2675.674 \\
2675.899\end{array}$ & $\begin{array}{l}40 \\
10 \\
10 \\
75 \\
20\end{array}$ & 200 & $\begin{array}{l}\text { I } \\
\text { I } \\
\text { I } \\
\text { I I } \\
\text { I }\end{array}$ \\
\hline $\begin{array}{l}2653.339 \\
2653.576 \\
2653.730 \\
2653.925 \\
2654.090\end{array}$ & $\begin{array}{l}5 \\
2 \\
5 \\
2 \\
1\end{array}$ & 2 & $\begin{array}{l}\text { I } \\
\text { I } \\
\text { I } \\
\text { I } \\
\text { II }\end{array}$ & $\begin{array}{l}2665.546 \\
2665.889 \\
2666.138 \\
2666.257 \\
2666.413\end{array}$ & $\begin{array}{r}2 \\
40 \\
2 \\
20 \\
5\end{array}$ & $\begin{array}{r}100 \\
8 \\
4\end{array}$ & $\begin{array}{l}\text { I } \\
\text { I I } \\
\text { I } \\
\text { I I } \\
\text { I I }\end{array}$ & $\begin{array}{l}2676.132 \\
2676.432 \\
2676.578 \\
2676.624 \\
2676.771\end{array}$ & $\begin{array}{r}2 \\
20 \\
40 \\
30 \\
2\end{array}$ & $\begin{array}{l}40 \\
40 \\
50\end{array}$ & $\begin{array}{l}\text { I } \\
\text { I I } \\
\text { II } \\
\text { I I } \\
\text { I }\end{array}$ \\
\hline $\begin{array}{l}2654.264 \\
2654.447 \\
2654.565 \\
2654.760 \\
2655.030\end{array}$ & $\begin{array}{r}5 \\
50 \\
2 \\
20 \\
5\end{array}$ & $\begin{array}{l}1 \\
4\end{array}$ & $\begin{array}{l}\text { I } \\
\text { I } \\
\text { I I } \\
\text { I } \\
\text { I }\end{array}$ & $\begin{array}{l}2666.551 \\
2666.714 \\
2666.813 \\
2666.905 \\
2667.080\end{array}$ & $\begin{array}{r}50 \\
10 \\
5 \\
50\end{array}$ & $\begin{array}{r}500 \\
50\end{array}$ & $\begin{array}{l}\text { I } \\
\text { I } \\
\text { I } \\
\text { I I I } \\
\text { I I }\end{array}$ & $\begin{array}{l}2677.150 \\
2677.267 \\
2677.353 \\
2677.540 \\
2677.668\end{array}$ & $\begin{array}{r}5 \\
2 \\
20 \\
30 \\
40\end{array}$ & $\begin{array}{r}2 \\
4 \\
20\end{array}$ & $\begin{array}{l}\text { II } \\
\text { I } \\
\text { I } \\
\text { I I } \\
\text { I }\end{array}$ \\
\hline $\begin{array}{l}2655.307 \\
2655.420 \\
2655.535 \\
2655.890 \\
2656.266\end{array}$ & $\begin{array}{l}20 \\
20 \\
20 \\
20 \\
30\end{array}$ & $\begin{array}{r}20 \\
1 \\
20 \\
40\end{array}$ & $\begin{array}{l}\text { I } \\
\text { I I } \\
\text { I } \\
\text { II } \\
\text { I I }\end{array}$ & $\begin{array}{l}2667.539 \\
2667.771 \\
2667.998 \\
2668.152 \\
2668.315\end{array}$ & $\begin{array}{l}40 \\
20 \\
50 \\
20\end{array}$ & $\begin{array}{r}50 \\
500\end{array}$ & $\begin{array}{l}\text { I I } \\
\text { I } \\
\text { I I I } \\
\text { I } \\
\text { I }\end{array}$ & $\begin{array}{l}2677.871 \\
2678.064 \\
2678.165 \\
2678.405 \\
2678.509\end{array}$ & $\begin{array}{r}5 \\
10 \\
40 \\
10\end{array}$ & $\begin{array}{c}5 \mathrm{w} \\
10 \mathrm{w} \\
4\end{array}$ & $\begin{array}{l}\text { I I } \\
\text { I I I } \\
\text { I } \\
\text { I } \\
\text { I }\end{array}$ \\
\hline $\begin{array}{l}2656.533 \\
2656.982 \\
2657.114 \\
2657.365 \\
2657.618\end{array}$ & $\begin{array}{c}10 \\
2 \\
20 \\
3 w \\
2\end{array}$ & $\begin{array}{r}1 \\
75\end{array}$ & $\begin{array}{l}\text { I } \\
\text { I I } \\
\text { I I } \\
\text { I } \\
\text { I }\end{array}$ & $\begin{array}{l}2668.544 \\
2668.692 \\
2669.062 \\
2669.226 \\
2669.446\end{array}$ & $\begin{array}{r}50 \\
10 \\
40 \\
2 \\
50\end{array}$ & $\begin{array}{r}6 \\
2 \\
1 \\
100\end{array}$ & $\begin{array}{l}\text { I } \\
\text { I } \\
\text { I } \\
\text { I } \\
\text { I I }\end{array}$ & $\begin{array}{l}2678.637 \\
2678.760 \\
2678.933 \\
2679.379 \\
2679.690\end{array}$ & $\begin{array}{r}10 \\
10 \\
40 \\
10 \\
5\end{array}$ & $\begin{array}{r}2 \\
40 \\
1\end{array}$ & $\begin{array}{l}\text { I } \\
\text { I } \\
\text { II } \\
\text { I } \\
\text { I }\end{array}$ \\
\hline $\begin{array}{l}2657.785 \\
2657.880 \\
2658.011 \\
2658.217 \\
2658.488\end{array}$ & $\begin{array}{r}2 \\
20 \\
5 \\
20 \\
5\end{array}$ & $\begin{array}{r}10 \\
1 \\
20 \\
3\end{array}$ & $\begin{array}{l}\text { I } \\
\text { I I } \\
\text { I } \\
\text { II } \\
\text { II }\end{array}$ & $\begin{array}{l}2669.699 \\
2670.061 \\
2670.281 \\
2670.630 \\
2670.859\end{array}$ & $\begin{array}{l}40 \\
50 \\
50 \\
20 \\
10\end{array}$ & $\begin{array}{r}10 \\
1 \\
10\end{array}$ & $\begin{array}{l}\text { II } \\
\text { I } \\
\text { I } \\
\text { II } \\
\text { I }\end{array}$ & $\begin{array}{l}2679.884 \\
2680.032 \\
2680.208 \\
2680.686 \\
2680.980\end{array}$ & $\begin{array}{r}40 \\
2 \\
30 \\
100\end{array}$ & $\begin{array}{r}1 \\
30 \\
2000\end{array}$ & $\begin{array}{l}\text { I } \\
\text { I } \\
\text { I I } \\
\text { I } \\
\text { I I I }\end{array}$ \\
\hline
\end{tabular}


Table 1. Wavelengths of Thorium Spectra - Continued

\begin{tabular}{|c|c|c|c|c|c|c|c|c|c|c|c|}
\hline \multirow{2}{*}{$\begin{array}{l}\text { Wave- } \\
\text { length }\end{array}$} & \multicolumn{2}{|c|}{ Intensity } & \multirow{2}{*}{$\begin{array}{l}\text { Spec- } \\
\text { trum }\end{array}$} & \multirow{2}{*}{$\begin{array}{l}\text { Wave- } \\
\text { length }\end{array}$} & \multicolumn{2}{|c|}{ Intensity } & \multirow{2}{*}{$\begin{array}{l}\text { Spec- } \\
\text { trum }\end{array}$} & \multirow{2}{*}{$\begin{array}{l}\text { Wave- } \\
\text { length }\end{array}$} & \multicolumn{2}{|c|}{ Intensity } & \multirow{2}{*}{$\begin{array}{l}\text { Spec- } \\
\text { trum }\end{array}$} \\
\hline & Lamp & Spark & & & Lamp & Spark & & & Lamp & Spark & \\
\hline $\begin{array}{l}2681.244 \\
2681.406 \\
2681.569 \\
2681.879 \\
2682.021\end{array}$ & $\begin{array}{l}10 \\
10 \\
40 \\
20 \\
10\end{array}$ & 3 & $\begin{array}{l}\text { I } \\
\text { I } \\
\text { I } \\
\text { I } \\
\text { II }\end{array}$ & $\begin{array}{l}2692.187 \\
2692.421 \\
2693.104 \\
2693.297 \\
2693.430\end{array}$ & $\begin{array}{r}2 \\
2000 \\
30 \\
20 \\
100\end{array}$ & $\begin{array}{r}750 \\
20\end{array}$ & $\begin{array}{l}\text { I } \\
\text { II } \\
\text { I I } \\
\text { I } \\
\text { I }\end{array}$ & $\begin{array}{l}2705.090 \\
2705.384 \\
2705.653 \\
2705.849 \\
2706.110\end{array}$ & $\begin{array}{l}20 \\
50 \\
50 \\
20 \\
20\end{array}$ & 10 & $\begin{array}{l}\text { I I } \\
\text { I } \\
\text { I } \\
\text { I } \\
\text { I }\end{array}$ \\
\hline $\begin{array}{l}2682.388 \\
2682.700 \\
2682.855 \\
2682.988 \\
2683.118\end{array}$ & $\begin{array}{l}40 \\
20 \\
10 \\
10 \\
10\end{array}$ & $\begin{array}{l}2 \\
1 \\
1\end{array}$ & $\begin{array}{l}\text { I } \\
\text { I } \\
\text { I } \\
\text { I } \\
\text { I }\end{array}$ & $\begin{array}{l}2693.624 \\
2693.965 \\
2694.036 \\
2694.346 \\
2694.686\end{array}$ & $\begin{array}{l}10 \\
30 \\
10 \\
10 \\
50\end{array}$ & $\begin{array}{c}10 \\
30 \\
200 \mathrm{w} \\
8 \\
40\end{array}$ & $\begin{array}{l}\text { II } \\
\text { I I } \\
\text { IV } \\
\text { II } \\
\text { I I }\end{array}$ & $\begin{array}{l}2706.178 \\
2706.410 \\
2707.001 \\
2707.217 \\
2707.386\end{array}$ & $\begin{array}{l}20 \\
75 \\
20 \\
10 \\
40\end{array}$ & $\begin{array}{r}75 \\
1\end{array}$ & $\begin{array}{l}\text { I } \\
\text { I I } \\
\text { I } \\
\text { I } \\
\text { I }\end{array}$ \\
\hline $\begin{array}{l}2683.278 \\
2683.486 \\
2683.615 \\
2683.723 \\
2683.955\end{array}$ & $\begin{array}{l}50 \\
10 \mathrm{w} \\
5 \mathrm{w} \\
5 \\
10\end{array}$ & 1 & $\begin{array}{l}\text { I } \\
\text { I } \\
\text { I } \\
\text { I } \\
\text { I }\end{array}$ & $\begin{array}{l}2695.024 \\
2695.212 \\
2695.555 \\
2695.816 \\
2695.986\end{array}$ & $\begin{array}{r}50 \\
150 \\
500 \\
100 \\
5\end{array}$ & $\begin{array}{r}30 \\
200 \\
1000 \\
100\end{array}$ & $\begin{array}{l}\text { II } \\
\text { II } \\
\text { II } \\
\text { II } \\
\text { I }\end{array}$ & $\begin{array}{l}2707.461 \\
2707.541 \\
2708.181 \\
2708.340 \\
2708.950\end{array}$ & $\begin{array}{r}40 \\
40 \\
700 \\
\\
50\end{array}$ & $\begin{array}{r}40 \\
150 \\
1000\end{array}$ & $\begin{array}{l}\text { I } \\
\text { II } \\
\text { II } \\
\text { II I } \\
\text { I }\end{array}$ \\
\hline $\begin{array}{l}2684.289 \\
2684.677 \\
2685.081 \\
2685.589 \\
2685.836\end{array}$ & $\begin{array}{r}3000 \\
50 \\
40 \\
100 \\
20\end{array}$ & $\begin{array}{r}2000 \\
20 \\
10 \\
15\end{array}$ & $\begin{array}{l}\text { I I } \\
\text { II } \\
\text { II } \\
\text { I } \\
\text { I }\end{array}$ & $\begin{array}{l}2696.179 \\
2696.466 \\
2696.718 \\
2696.827 \\
2697.546\end{array}$ & $\begin{array}{r}50 \\
5 \\
10 \\
100 \\
150\end{array}$ & $\begin{array}{r}40 \\
30 \\
300 \\
250\end{array}$ & $\begin{array}{l}\text { II } \\
\text { I } \\
\text { II } \\
\text { II } \\
\text { II }\end{array}$ & $\begin{array}{l}2709.044 \\
2709.200 \\
2709.413 \\
2709.542 \\
2709.616\end{array}$ & $\begin{array}{l}20 \\
20 \\
50 \\
75 \\
10\end{array}$ & 20 & $\begin{array}{l}\text { I } \\
\text { I } \\
\text { I I } \\
\text { I } \\
\text { I }\end{array}$ \\
\hline $\begin{array}{l}2685.972 \\
2686.107 \\
2686.185 \\
2686.328 \\
2686.501\end{array}$ & $\begin{array}{r}20 \\
40 \\
20 \\
5 \\
20\end{array}$ & 500 & $\begin{array}{l}\text { I } \\
\text { I } \\
\text { I I I } \\
\text { I } \\
\text { I }\end{array}$ & $\begin{array}{l}2697.729 \\
2697.989 \\
2698.130 \\
2698.458 \\
2698.743\end{array}$ & $\begin{array}{r}40 \\
75 \\
20 \\
5 \\
250\end{array}$ & $\begin{array}{r}3 \\
3 \\
500\end{array}$ & $\begin{array}{l}\text { I } \\
\text { I } \\
\text { I } \\
\text { I } \\
\text { I I }\end{array}$ & $\begin{array}{l}2709.869 \\
2709.936 \\
2710.048 \\
2710.481 \\
2710.904\end{array}$ & $\begin{array}{l}30 \\
20 \\
20 \\
50 \\
10\end{array}$ & $\begin{array}{r}20 \\
100 \\
1\end{array}$ & $\begin{array}{l}\text { I I } \\
\text { I } \\
\text { I } \\
\text { II } \\
\text { I }\end{array}$ \\
\hline $\begin{array}{l}2686.797 \\
2687.133 \\
2687.512 \\
2687.615 \\
2787.666\end{array}$ & $\begin{array}{r}20 \\
500 \\
50 \\
40 \\
200\end{array}$ & $\begin{array}{r}500 \\
20 \\
200\end{array}$ & $\begin{array}{l}\text { I } \\
\text { II } \\
\text { I I } \\
\text { I } \\
\text { II }\end{array}$ & $\begin{array}{l}2698.860 \\
2699.015 \\
2699.469 \\
2700.602 \\
2700.787\end{array}$ & $\begin{array}{r}2 \\
100 \\
40 \\
50 \\
40\end{array}$ & $\begin{array}{r}8 \\
1 \\
20 \\
2\end{array}$ & $\begin{array}{l}\text { I } \\
\text { I } \\
\text { I } \\
\text { II } \\
\text { I }\end{array}$ & $\begin{array}{l}2711.464 \\
2712.087 \\
2712.389 \\
2712.768 \\
2712.923\end{array}$ & $\begin{array}{l}100 \\
40 \\
40 \mathrm{w} \\
40 \\
20\end{array}$ & $\begin{array}{r}200 \\
75 \\
\\
50 \\
10\end{array}$ & $\begin{array}{l}\text { II } \\
\text { II } \\
\text { I } \\
\text { II } \\
\text { I I }\end{array}$ \\
\hline $\begin{array}{l}2687.725 \\
2687.912 \\
2687.986 \\
2688.342 \\
2688.570\end{array}$ & $\begin{array}{r}20 \\
10 \\
5 \\
100 \\
10\end{array}$ & $\begin{array}{r}1 \\
200\end{array}$ & $\begin{array}{l}\text { I } \\
\text { I } \\
\text { I } \\
\text { I I } \\
\text { I }\end{array}$ & $\begin{array}{l}2700.991 \\
2701.185 \\
2701.274 \\
2701.348 \\
2701.410\end{array}$ & $\begin{array}{r}20 \\
10 \\
20 \\
4\end{array}$ & $50 w$ & $\begin{array}{l}\text { II } \\
\text { I } \\
\text { I } \\
\text { I } \\
\text { I }\end{array}$ & $\begin{array}{l}2713.251 \\
2713.487 \\
2713.842 \\
2714.148 \\
2714.247\end{array}$ & $\begin{array}{r}50 \\
20 \\
100 \\
20 \\
20\end{array}$ & $\begin{array}{l}100 \\
2 \mathrm{~h}\end{array}$ & $\begin{array}{l}\text { II } \\
\text { I } \\
\text { I } \\
\text { I } \\
\text { I }\end{array}$ \\
\hline $\begin{array}{l}2688.677 \\
2688.894 \\
2689.376 \\
2689.612 \\
2689.715\end{array}$ & $\begin{array}{r}50 \\
40 \\
20 \\
5 \\
10\end{array}$ & $\begin{array}{r}50 \\
1 \\
10\end{array}$ & $\begin{array}{l}\text { II } \\
\text { I } \\
\text { II } \\
\text { I } \\
\text { I }\end{array}$ & $\begin{array}{l}2701.596 \\
2701.827 \\
2702.062 \\
2702.202 \\
2702.388\end{array}$ & $\begin{array}{r}40 \\
150 \\
40 \\
20 \\
10\end{array}$ & $\begin{array}{r}200 \\
4 \\
2\end{array}$ & $\begin{array}{l}\text { I } \\
\text { II } \\
\text { I } \\
\text { I } \\
\text { I }\end{array}$ & $\begin{array}{l}2714.617 \\
2715.089 \\
2715.303 \\
2715.613 \\
2716.320\end{array}$ & $\begin{array}{r}100 \\
100 \\
75 \\
50 \\
200\end{array}$ & $\begin{array}{r}200 \\
200 \\
20 \\
10 \\
500\end{array}$ & $\begin{array}{l}\text { I I } \\
\text { I I } \\
\text { I I } \\
\text { II } \\
\text { I I }\end{array}$ \\
\hline $\begin{array}{l}2689.829 \\
2689.910 \\
2690.079 \\
2690.310 \\
2690.979\end{array}$ & $\begin{array}{l}10 \\
10 \\
20 \\
50 \\
15\end{array}$ & $\begin{array}{r}10 \\
100 \\
20\end{array}$ & $\begin{array}{l}\text { I } \\
\text { I } \\
\text { II } \\
\text { II } \\
\text { II }\end{array}$ & $\begin{array}{l}2702.616 \\
2702.845 \\
2702.921 \\
2703.193 \\
2703.477\end{array}$ & $\begin{array}{l}10 \\
40 \\
30 \\
75 \\
40\end{array}$ & $\begin{array}{r}30 \\
30 \\
3\end{array}$ & $\begin{array}{l}\text { I } \\
\text { I } \\
\text { II } \\
\text { II } \\
\text { I }\end{array}$ & $\begin{array}{l}2716.589 \\
2717.023 \\
2717.506 \\
2717.640 \\
2717.775\end{array}$ & $\begin{array}{l}75 \\
20 \\
20 \\
10 \\
50\end{array}$ & $\begin{array}{c}50 \\
1\end{array}$ & $\begin{array}{l}\text { II } \\
\text { I } \\
\text { I } \\
\text { I } \\
\text { I }\end{array}$ \\
\hline $\begin{array}{l}2691.030 \\
2691.184 \\
2691.369 \\
2691.511 \\
2691.872\end{array}$ & $\begin{array}{r}100 \\
75 \\
50 \\
20 \\
30\end{array}$ & $\begin{array}{c}50 \\
200 \\
10 \\
1 \\
3 \mathrm{w}\end{array}$ & $\begin{array}{l}\text { II } \\
\text { II } \\
\text { I I } \\
\text { I } \\
\text { I }\end{array}$ & $\begin{array}{l}2703.736 \\
2703.962 \\
2704.488 \\
2704.752 \\
2704.903\end{array}$ & $\begin{array}{r}2 \\
1000 \\
50 \\
20 \\
20\end{array}$ & $\begin{array}{r}300 \\
30\end{array}$ & $\begin{array}{l}\text { I } \\
\text { II } \\
\text { II } \\
\text { I } \\
\text { I }\end{array}$ & $\begin{array}{l}2718.421 \\
2718.720 \\
2718.887 \\
2719.191 \\
2719.303\end{array}$ & $\begin{array}{r}10 \\
40 \\
3 \\
20 \\
20\end{array}$ & $\begin{array}{r}15 \\
2\end{array}$ & $\begin{array}{l}\text { I } \\
\text { I I } \\
\text { I } \\
\text { I } \\
\text { I }\end{array}$ \\
\hline
\end{tabular}


Table 1. Wavelengths of Thorium Spectra - Continued

\begin{tabular}{|c|c|c|c|c|c|c|c|c|c|c|c|}
\hline \multirow{2}{*}{$\begin{array}{l}\text { Wave- } \\
\text { length }\end{array}$} & \multicolumn{2}{|c|}{ Intensity } & \multirow{2}{*}{$\begin{array}{l}\text { Spec- } \\
\text { trum }\end{array}$} & \multirow{2}{*}{$\begin{array}{l}\text { Wave- } \\
\text { length }\end{array}$} & \multicolumn{2}{|c|}{ Intensity } & \multirow{2}{*}{$\begin{array}{l}\text { Spec- } \\
\text { trum }\end{array}$} & \multirow{2}{*}{$\begin{array}{l}\text { Wave- } \\
\text { length }\end{array}$} & \multicolumn{2}{|c|}{ Intensity } & \multirow{2}{*}{$\begin{array}{l}\text { Spec } \\
\text { trum }\end{array}$} \\
\hline & Lamp & Spark & & & Lamp & Spark & & & Lamp & Spark & \\
\hline $\begin{array}{l}2719.455 \\
2719.936 \\
2720.349 \\
2720.467 \\
2720.632\end{array}$ & $\begin{array}{r}50 \\
100 \\
20 \\
30 \\
20\end{array}$ & $\begin{array}{r}100 \\
200 \\
40\end{array}$ & $\begin{array}{l}\text { I I } \\
\text { I I } \\
\text { I } \\
\text { I I } \\
\text { I }\end{array}$ & $\begin{array}{l}2736.564 \\
2736.722 \\
2736.978 \\
2737.434 \\
2737.865\end{array}$ & $\begin{array}{r}75 \\
75 \\
40 \\
100 \\
75\end{array}$ & $\begin{array}{r}1 \\
20 \\
200 \\
30\end{array}$ & $\begin{array}{l}\text { I } \\
\text { I I } \\
\text { I } \\
\text { II } \\
\text { I I }\end{array}$ & $\begin{array}{l}2751.758 \\
2752.168 \\
2753.093 \\
2753.346 \\
2753.540\end{array}$ & $\begin{array}{r}50 \\
750 \\
100 \\
40 \\
40\end{array}$ & $\begin{array}{r}25 \\
2000 \\
150 \\
50\end{array}$ & $\begin{array}{l}\text { I I } \\
\text { I I } \\
\text { I I } \\
\text { I } \\
\text { I I }\end{array}$ \\
\hline $\begin{array}{l}2720.826 \\
2721.175 \\
2721.375 \\
2721.697 \\
2722.218\end{array}$ & $\begin{array}{r}40 \\
10 \\
500 \\
50\end{array}$ & $\begin{array}{r}8 \\
100 \\
750 \\
3\end{array}$ & $\begin{array}{l}\text { II } \\
\text { I } \\
\text { I I I } \\
\text { I I } \\
\text { I }\end{array}$ & $\begin{array}{l}2738.104 \\
2738.222 \\
2738.331 \\
2738.815 \\
2739.132\end{array}$ & $\begin{array}{r}20 \\
10 \\
100 \\
10 \\
5\end{array}$ & $\begin{array}{r}75 \\
75 \\
5\end{array}$ & $\begin{array}{l}\text { I } \\
\text { I } \\
\text { II } \\
\text { II } \\
\text { II }\end{array}$ & $\begin{array}{l}2753.823 \\
2754.034 \\
2754.246 \\
2754.514 \\
2754.633\end{array}$ & $\begin{array}{l}10 \\
20 \\
30 \\
30 \\
20\end{array}$ & $\begin{array}{l}20 \\
10 \\
10\end{array}$ & $\begin{array}{l}\text { I } \\
\text { I } \\
\text { I I } \\
\text { I I } \\
\text { I I }\end{array}$ \\
\hline $\begin{array}{l}2722.383 \\
2723.317 \\
2723.827 \\
2723.950 \\
2724.510\end{array}$ & $\begin{array}{r}100 \\
50 \\
40 \\
3 \\
50\end{array}$ & $\begin{array}{r}300 \\
200 \\
3\end{array}$ & $\begin{array}{l}\text { II } \\
\text { II } \\
\text { I } \\
\text { I } \\
\text { I }\end{array}$ & $\begin{array}{l}2739.344 \\
2739.765 \\
2740.208 \\
2740.476 \\
2740.709\end{array}$ & $\begin{array}{l}10 \\
50 \\
15 \\
50 \\
20\end{array}$ & $\begin{array}{r}10 \\
200 \\
2\end{array}$ & $\begin{array}{l}\text { I } \\
\text { I } \\
\text { I I } \\
\text { I I } \\
\text { I }\end{array}$ & $\begin{array}{l}2754.782 \\
2754.886 \\
2755.051 \\
2755.332 \\
2755.453\end{array}$ & $\begin{array}{r}40 \\
40 \\
75 \\
40 \\
5\end{array}$ & $\begin{array}{r}1 \\
3 \\
15\end{array}$ & $\begin{array}{l}\text { I } \\
\text { I } \\
\text { I } \\
\text { I } \\
\text { I }\end{array}$ \\
\hline $\begin{array}{l}2724.886 \\
2725.350 \\
2725.561 \\
2726.185 \\
2726.320\end{array}$ & $\begin{array}{r}100 \\
50 \\
10 \\
50\end{array}$ & $\begin{array}{r}200 \\
500 \\
40\end{array}$ & $\begin{array}{l}\text { II I } \\
\text { I I I } \\
\text { II } \\
\text { I } \\
\text { I }\end{array}$ & $\begin{array}{l}2740.976 \\
2741.470 \\
2741.772 \\
2742.222 \\
2742.409\end{array}$ & $\begin{array}{l}20 \\
50 \\
10 \\
10 \\
20\end{array}$ & 10 & $\begin{array}{l}\text { II } \\
\text { I } \\
\text { I } \\
I \\
I\end{array}$ & $\begin{array}{l}2755.958 \\
2756.095 \\
2756.450 \\
2756.513 \\
2756.705\end{array}$ & $\begin{array}{r}75 \\
2 \\
5 \\
5 \\
2\end{array}$ & 200 & $\begin{array}{l}\text { II I } \\
\text { I } \\
\text { I } \\
\text { I } \\
\text { I }\end{array}$ \\
\hline $\begin{array}{l}2726.491 \\
2726.672 \\
2727.246 \\
2727.402 \\
2728.183\end{array}$ & $\begin{array}{r}150 \\
5 \\
50 \\
20 \\
20\end{array}$ & $\begin{array}{r}200 \\
100 \\
2\end{array}$ & $\begin{array}{l}\text { I I } \\
\text { I } \\
\text { II } \\
\text { I } \\
\text { I }\end{array}$ & $\begin{array}{l}2742.566 \\
2743.075 \\
2743.927 \\
2744.031 \\
2744.136\end{array}$ & $\begin{array}{r}20 \\
300 \\
50 \\
\\
10\end{array}$ & $\begin{array}{c}800 \\
10 \\
100 \mathrm{w}\end{array}$ & $\begin{array}{l}\text { I } \\
\text { II } \\
\text { II } \\
\text { II I } \\
\text { I }\end{array}$ & $\begin{array}{l}2756.853 \\
2757.073 \\
2757.172 \\
2757.365 \\
2757.643\end{array}$ & $\begin{array}{r}40 \\
20 \\
10 \\
50 \\
2\end{array}$ & $\begin{array}{r}5 \\
500 \\
40\end{array}$ & $\begin{array}{l}\text { I } \\
\text { I } \\
\text { I I I } \\
\text { II } \\
\text { I }\end{array}$ \\
\hline $\begin{array}{l}2728.459 \\
2728.689 \\
2728.913 \\
2729.040 \\
2729.333\end{array}$ & $\begin{array}{r}10 \\
40 \\
75 \\
40 \\
500\end{array}$ & $\begin{array}{r}5 \\
75 \\
1 \\
2000\end{array}$ & $\begin{array}{l}\text { I } \\
\text { I } \\
\text { II } \\
\text { I } \\
\text { II }\end{array}$ & $\begin{array}{l}2744.247 \\
2744.367 \\
2744.404 \\
2744.987 \\
2745.200\end{array}$ & $\begin{array}{l}40 \\
20 \\
10 \\
10 \\
10\end{array}$ & 5 & $\begin{array}{l}\text { I } \\
\text { I } \\
\text { I } \\
\text { I I } \\
\text { I }\end{array}$ & $\begin{array}{l}2757.882 \\
2758.066 \\
2758.229 \\
2758.956 \\
2759.067\end{array}$ & $\begin{array}{r}20 \\
20 \\
10 \\
40 \\
2\end{array}$ & 30 & $\begin{array}{l}\text { I } \\
\text { I } \\
\text { I } \\
\text { II } \\
\text { I }\end{array}$ \\
\hline $\begin{array}{l}2730.272 \\
2730.637 \\
2730.810 \\
2731.207 \\
2731.584\end{array}$ & $\begin{array}{r}150 \\
20 \\
10 \\
20 \\
100\end{array}$ & 100 & $\begin{array}{l}\text { I I } \\
\text { I } \\
\text { I } \\
\text { I } \\
\text { I I }\end{array}$ & $\begin{array}{l}2745.348 \\
2745.524 \\
2745.847 \\
2746.022 \\
2746.135\end{array}$ & $\begin{array}{c}4 \mathrm{w} \\
125 \\
10 \\
20 \\
10\end{array}$ & $\begin{array}{r}15 \\
5 \\
1\end{array}$ & $\begin{array}{l}\text { I } \\
\text { I } \\
\text { I I } \\
\text { I } \\
\text { I }\end{array}$ & $\begin{array}{l}2759.228 \\
2759.411 \\
2759.716 \\
2759.858 \\
2760.071\end{array}$ & $\begin{array}{r}2 \\
200 \\
75 \\
10 \\
3\end{array}$ & $\begin{array}{r}500 \\
30\end{array}$ & $\begin{array}{l}\text { I } \\
\text { I I } \\
\text { I I } \\
\text { I } \\
\text { I }\end{array}$ \\
\hline $\begin{array}{l}2731.689 \\
2731.919 \\
2732.701 \\
2732.819 \\
2733.204\end{array}$ & $\begin{array}{r}15 \\
75 \\
100 \\
500 \\
75\end{array}$ & $\begin{array}{c}50 \mathrm{w} \\
50 \\
75 \\
750 \\
75\end{array}$ & $\begin{array}{l}\text { I I I } \\
\text { II } \\
\text { II } \\
\text { II } \\
\text { I I }\end{array}$ & $\begin{array}{l}2746.785 \\
2747.158 \\
2747.583 \\
2747.849 \\
2748.124\end{array}$ & $\begin{array}{r}40 \\
100 \\
50 \\
75 \\
10\end{array}$ & $\begin{array}{r}200 \\
50 \\
100 \\
8\end{array}$ & $\begin{array}{l}\text { I } \\
\text { II } \\
\text { II } \\
\text { II } \\
\text { II }\end{array}$ & $\begin{array}{l}2760.245 \\
2760.401 \\
2761.016 \\
2761.413 \\
2761.499\end{array}$ & $\begin{array}{r}5 \\
250 \\
40 \\
2 \\
10\end{array}$ & $\begin{array}{r}400 \\
3\end{array}$ & $\begin{array}{l}\text { I } \\
\text { II } \\
\text { I } \\
\text { I } \\
\text { I }\end{array}$ \\
\hline $\begin{array}{l}2733.686 \\
2733.765 \\
2733.904 \\
2734.417 \\
2734.540\end{array}$ & $\begin{array}{l}10 \\
10 \\
10 \\
75 \\
40 w\end{array}$ & 75 & $\begin{array}{l}\text { I } \\
\text { I } \\
\text { I } \\
\text { II } \\
\text { I }\end{array}$ & $\begin{array}{l}2748.405 \\
2748.807 \\
2749.340 \\
2749.531 \\
27+9.711\end{array}$ & $\begin{array}{r}100 \\
100 \\
25 \\
150 \\
50\end{array}$ & $\begin{array}{r}10 \\
200 \\
10 \\
250 \\
160\end{array}$ & $\begin{array}{l}\text { I } \\
\text { II } \\
\text { II } \\
\text { II } \\
\text { II }\end{array}$ & $\begin{array}{l}2761.671 \\
2762.381 \\
2762.456 \\
2762.631 \\
2763.617\end{array}$ & $\begin{array}{r}50 \\
100 \\
30 \\
500\end{array}$ & $\begin{array}{l}50 \mathrm{w} \\
40 \\
20 \\
800\end{array}$ & $\begin{array}{l}\text { I I I } \\
\text { I I } \\
\text { I } \\
\text { I I } \\
\text { I I }\end{array}$ \\
\hline $\begin{array}{l}2735.058 \\
2735.205 \\
2735.835 \\
2736.173 \\
2736.440\end{array}$ & $\begin{array}{r}40 \\
20 \\
75 \\
5 \\
75\end{array}$ & $\begin{array}{l}50 \\
20 \\
30 \\
5 \mathrm{w} \\
25\end{array}$ & $\begin{array}{l}\text { II } \\
\text { II } \\
\text { I I } \\
\text { I I } \\
\text { I I }\end{array}$ & $\begin{array}{l}2750.087 \\
2750.520 \\
2750.655 \\
2751.032 \\
2751.570\end{array}$ & $\begin{array}{l}40 \\
20 \\
30 \\
20 \mathrm{w} \\
75\end{array}$ & $\begin{array}{r}30 \\
2 \\
20 \\
150\end{array}$ & $\begin{array}{l}\text { II } \\
\text { I } \\
\text { II } \\
\text { I } \\
\text { I I }\end{array}$ & $\begin{array}{l}2763.888 \\
2764.176 \\
2764.643 \\
2764.830 \\
2765.123\end{array}$ & $\begin{array}{c}5 \\
20 \mathrm{~s} \\
200 \\
10 \\
200\end{array}$ & $\begin{array}{r}1 \\
400 \\
400\end{array}$ & $\begin{array}{l}\text { I } \\
\text { I } \\
\text { II } \\
\text { I } \\
\text { II }\end{array}$ \\
\hline
\end{tabular}


Table 1. Wavelengths of Thorium Spectra - Continued

\begin{tabular}{|c|c|c|c|c|c|c|c|c|c|c|c|}
\hline \multirow{2}{*}{$\begin{array}{l}\text { Wave- } \\
\text { length }\end{array}$} & \multicolumn{2}{|c|}{ Intensity } & \multirow{2}{*}{$\begin{array}{l}\text { Spec- } \\
\text { trum }\end{array}$} & \multirow{2}{*}{$\begin{array}{l}\text { Wave- } \\
\text { length }\end{array}$} & \multicolumn{2}{|c|}{ Intensity } & \multirow{2}{*}{$\begin{array}{l}\text { Spec- } \\
\text { trum }\end{array}$} & \multirow{2}{*}{$\begin{array}{l}\text { Wave- } \\
\text { length }\end{array}$} & \multicolumn{2}{|c|}{ Intensity } & \multirow{2}{*}{$\begin{array}{l}\text { Spec- } \\
\text { trum }\end{array}$} \\
\hline & Lamp & Spark & & & Lamp & Spark & & & Lamp & Spark & \\
\hline $\begin{array}{l}2765.555 \\
2765.686 \\
2765.870 \\
2765.966 \\
2766.062\end{array}$ & $\begin{array}{r}75 \\
40 \\
5 \\
10 \\
5\end{array}$ & 1000 & $\begin{array}{l}\text { I } \\
\text { I } \\
\text { II I } \\
\text { I } \\
\text { I }\end{array}$ & $\begin{array}{l}2778.034 \\
2778.230 \\
2778.407 \\
2778.708 \\
2779.146\end{array}$ & $\begin{array}{c}50 \\
20 \\
150 \\
5 \mathrm{w}\end{array}$ & $\begin{array}{r}40 \\
50 \\
1 \\
200\end{array}$ & $\begin{array}{l}\text { II } \\
\text { II I } \\
\text { I } \\
\text { II } \\
\text { I }\end{array}$ & $\begin{array}{l}2791.669 \\
2791.989 \\
2792.364 \\
2792.687 \\
2793.060\end{array}$ & $\begin{array}{l}20 \\
40 \mathrm{w} \\
40 \\
30 \\
10\end{array}$ & 20 & $\begin{array}{l}\text { II } \\
\text { I } \\
\text { I } \\
\text { II } \\
\text { I }\end{array}$ \\
\hline $\begin{array}{l}2766.459 \\
2766.563 \\
2766.630 \\
2766.914 \\
2766.980\end{array}$ & $\begin{array}{r}5 \\
20 \\
30 \\
10 \\
20\end{array}$ & 2 & $\begin{array}{l}\text { I } \\
\text { I } \\
\text { I } \\
\text { I } \\
\text { I }\end{array}$ & $\begin{array}{l}2779.358 \\
2779.471 \\
2779.663 \\
2779.832 \\
2780.031\end{array}$ & $\begin{array}{r}10 \\
50 \\
20 \\
150 \\
5\end{array}$ & $\begin{array}{r}3 \\
20 \\
5\end{array}$ & $\begin{array}{l}\text { I } \\
\text { I } \\
\text { II } \\
\text { I } \\
\text { I }\end{array}$ & $\begin{array}{l}2793.236 \\
2793.570 \\
2793.696 \\
2794.007 \\
2794.261\end{array}$ & $\begin{array}{r}5 \\
50 \\
50 \\
50 \\
400\end{array}$ & $\begin{array}{r}50 \\
40 \\
500\end{array}$ & $\begin{array}{l}\text { I } \\
\text { II } \\
\text { I } \\
\text { II } \\
\text { II }\end{array}$ \\
\hline $\begin{array}{l}2767.361 \\
2767.678 \\
2767.882 \\
2768.094 \\
2768.248\end{array}$ & $\begin{array}{r}50 \\
5 \\
40 \\
10 \\
20\end{array}$ & $\begin{array}{l}40 \\
10\end{array}$ & $\begin{array}{l}\text { II } \\
\text { I } \\
\text { I } \\
\text { I } \\
\text { I }\end{array}$ & $\begin{array}{l}2780.415 \\
2780.701 \\
2781.001 \\
2781.197 \\
2781.358\end{array}$ & $\begin{array}{r}75 \\
200 \\
50 \\
20 \\
20\end{array}$ & $\begin{array}{r}150 \\
300 \\
2 \\
20\end{array}$ & $\begin{array}{l}\text { II } \\
\text { II } \\
\text { I } \\
\text { I } \\
\text { I I }\end{array}$ & $\begin{array}{l}2794.460 \\
2794.650 \\
2794.811 \\
2794.956 \\
2795.198\end{array}$ & $\begin{array}{l}10 \\
10 \\
40 \\
20 \\
40\end{array}$ & $\begin{array}{r}1 \\
50\end{array}$ & $\begin{array}{l}\text { I } \\
\text { I } \\
\text { I } \\
\text { I } \\
\text { II }\end{array}$ \\
\hline $\begin{array}{l}2768.411 \\
2768.532 \\
2768.851 \\
2769.340 \\
2769.781\end{array}$ & $\begin{array}{c}20 \\
20 \mathrm{~s} \\
1000 \\
5 \mathrm{w} \\
10\end{array}$ & 2000 & $\begin{array}{l}\text { I } \\
\text { I } \\
\text { II } \\
\text { I } \\
\text { I }\end{array}$ & $\begin{array}{l}2782.369 \\
2782.844 \\
2783.060 \\
2783.176 \\
2783.492\end{array}$ & $\begin{array}{r}100 \\
5 \\
150 \\
10 \\
200\end{array}$ & $\begin{array}{r}40 \\
3 \\
200 \\
400\end{array}$ & $\begin{array}{l}\text { II } \\
\text { II } \\
\text { II } \\
\text { I } \\
\text { I I }\end{array}$ & $\begin{array}{l}2795.357 \\
2795.913 \\
2796.202 \\
2796.518 \\
2797.023\end{array}$ & $\begin{array}{r}10 \\
5 \\
5 \\
50 \\
200\end{array}$ & $\begin{array}{r}50 \\
400\end{array}$ & $\begin{array}{l}\text { I } \\
\text { I } \\
\text { I } \\
\text { II } \\
\text { I I }\end{array}$ \\
\hline $\begin{array}{l}2769.970 \\
2770.132 \\
2770.653 \\
2770.825 \\
2771.255\end{array}$ & $\begin{array}{r}10 \\
50 \\
5 \\
1000 \\
40\end{array}$ & $\begin{array}{r}10 \\
800\end{array}$ & $\begin{array}{l}\text { I } \\
\text { I } \\
\text { I } \\
\text { I I } \\
\text { I }\end{array}$ & $\begin{array}{l}2783.651 \\
2783.795 \\
2783.921 \\
2784.060 \\
2784.344\end{array}$ & $\begin{array}{r}40 \\
10 \\
20 \\
125\end{array}$ & $\begin{array}{l}250 \\
300\end{array}$ & $\begin{array}{l}\text { I } \\
\text { I } \\
\text { I } \\
\text { II } \\
\text { II I }\end{array}$ & $\begin{array}{l}2797.740 \\
2798.266 \\
2798.493 \\
2798.665 \\
2798.892\end{array}$ & $\begin{array}{r}150 \\
125 \\
20 \\
100 \\
30\end{array}$ & $\begin{array}{r}250 \\
250 \\
30 \\
100 \\
20\end{array}$ & $\begin{array}{l}\text { II } \\
\text { I I } \\
\text { I I } \\
\text { I I } \\
\text { I I }\end{array}$ \\
\hline $\begin{array}{l}2771.493 \\
2772.013 \\
2772.725 \\
2772.828 \\
2773.024\end{array}$ & $\begin{array}{r}200 \\
100 \\
20 \\
5 \\
150\end{array}$ & $\begin{array}{r}100 \\
100 \\
10 \\
4 \\
18\end{array}$ & $\begin{array}{l}\text { II } \\
\text { II } \\
\text { II } \\
\text { II I } \\
\text { I I }\end{array}$ & $\begin{array}{l}2784.495 \\
2784.979 \\
2785.438 \\
2785.571 \\
2785.619\end{array}$ & $\begin{array}{r}40 \\
100 \\
5 \\
30 \\
50\end{array}$ & $\begin{array}{c}200 \\
3 \\
20 \mathrm{w} \\
200\end{array}$ & $\begin{array}{l}\text { I } \\
\text { II } \\
\text { II } \\
\text { II } \\
\text { I I }\end{array}$ & $\begin{array}{l}2739.114 \\
2799.425 \\
2799.543 \\
2799.678 \\
2799.883\end{array}$ & $\begin{array}{r}200 \\
100 \\
20 \\
5 \\
40\end{array}$ & $\begin{array}{r}750 \\
1\end{array}$ & $\begin{array}{l}\text { II } \\
\text { I } \\
\text { I } \\
\text { I } \\
\text { I }\end{array}$ \\
\hline $\begin{array}{l}2773.518 \\
2773.955 \\
2774.074 \\
2774.492 \\
2774.855\end{array}$ & $\begin{array}{r}40 \\
100 \\
100 \\
40 \\
100\end{array}$ & $\begin{array}{r}4 \\
200 \\
200 \\
100\end{array}$ & $\begin{array}{l}\text { I } \\
\text { II } \\
\text { II } \\
\text { I } \\
\text { II }\end{array}$ & $\begin{array}{l}2786.245 \\
2786.678 \\
2786.916 \\
2787.133 \\
2787.867\end{array}$ & $\begin{array}{r}50 \\
50 \\
100 \\
150 \\
75\end{array}$ & $\begin{array}{r}150 \\
40 \\
200 \\
300\end{array}$ & $\begin{array}{l}\text { II } \\
\text { II } \\
\text { II } \\
\text { II } \\
\text { I }\end{array}$ & $\begin{array}{l}2800.242 \\
2800.569 \\
2801.730 \\
2801.944 \\
2802.276\end{array}$ & $\begin{array}{r}50 \\
300 \\
50 \\
50 \\
50\end{array}$ & $\begin{array}{r}100 \\
400 \\
10 \\
60 \\
100\end{array}$ & $\begin{array}{l}\text { I I } \\
\text { II } \\
\text { I } \\
\text { II } \\
\text { II }\end{array}$ \\
\hline $\begin{array}{l}2775.014 \\
2775.080 \\
2775.238 \\
2775.474 \\
2775.804\end{array}$ & $\begin{array}{r}40 \\
100 \\
150 \\
5\end{array}$ & 300 & $\begin{array}{l}\text { I } \\
\text { III } \\
\text { I } \\
\text { II } \\
\text { I }\end{array}$ & $\begin{array}{l}2788.081 \\
2788.487 \\
2788.682 \\
2788.854 \\
2789.075\end{array}$ & $\begin{array}{r}5 \\
50 \\
75 \\
20 \\
20\end{array}$ & $\begin{array}{r}4 \\
250 \\
10\end{array}$ & $\begin{array}{l}\text { I I } \\
\text { I } \\
\text { II } \\
\text { II } \\
\text { I }\end{array}$ & $\begin{array}{l}2803.373 \\
2803.582 \\
2803.742 \\
2803.860 \\
2804.213\end{array}$ & $\begin{array}{r}100 \\
5 \\
5 \\
5 \\
30\end{array}$ & $\begin{array}{c}200 \\
2 \mathrm{~h} \\
1 \\
40\end{array}$ & $\begin{array}{l}\text { II } \\
\text { I } \\
\text { II } \\
\text { II } \\
\text { II }\end{array}$ \\
\hline $\begin{array}{l}2775.982 \\
2776.197 \\
2776.451 \\
2776.068 \\
2776.734\end{array}$ & $\begin{array}{r}20 \\
100 \\
20 \\
100 \\
10\end{array}$ & 6 & $\begin{array}{l}\text { I } \\
\text { I } \\
\text { I } \\
\text { I } \\
\text { I }\end{array}$ & $\begin{array}{l}2789.405 \\
2789.529 \\
2789.701 \\
2789.990 \\
2790.422\end{array}$ & $\begin{array}{r}10 \\
40 \\
20 \\
125\end{array}$ & $\begin{array}{c}100 \mathrm{w} \\
3 \\
1 \\
400\end{array}$ & $\begin{array}{l}\text { I } \\
\text { III } \\
\text { I } \\
\text { I } \\
\text { II }\end{array}$ & $\begin{array}{l}2804.580 \\
2804.658 \\
2804.908 \\
2805.234 \\
2805.506\end{array}$ & $\begin{array}{r}10 \\
20 \\
125 \\
10 \\
150\end{array}$ & $\begin{array}{r}8 \\
20 \\
10 \\
1\end{array}$ & $\begin{array}{l}\text { II } \\
\text { II } \\
\text { I } \\
\text { I } \\
\text { I }\end{array}$ \\
\hline $\begin{array}{l}2776.825 \\
2777.036 \\
2777.203 \\
2777.457 \\
2777.798\end{array}$ & $\begin{array}{r}20 \\
20 \\
20 \\
100\end{array}$ & $\begin{array}{r}500 \\
2 \\
100\end{array}$ & $\begin{array}{l}\text { III } \\
\text { I } \\
\text { I } \\
\text { I } \\
\text { II }\end{array}$ & $\begin{array}{l}2790.857 \\
2791.013 \\
2791.287 \\
2791.439 \\
2791.478\end{array}$ & $\begin{array}{r}40 \\
200 \\
50 \\
50 \\
40\end{array}$ & $\begin{array}{r}1 \\
400 \\
2 \\
100\end{array}$ & $\begin{array}{l}\text { I } \\
\text { II } \\
\text { I } \\
\text { II } \\
\text { I }\end{array}$ & $\begin{array}{l}2805.724 \\
2805.994 \\
2806.495 \\
2806.633 \\
2806.870\end{array}$ & $\begin{array}{l}20 \\
50 \\
20 \\
20 \\
20\end{array}$ & 10 & $\begin{array}{l}\text { I } \\
\text { I } \\
\text { I } \\
\text { I } \\
\text { I I }\end{array}$ \\
\hline
\end{tabular}


Table 1. Wavelengths of Thorium Spectra - Continued

\begin{tabular}{|c|c|c|c|c|c|c|c|c|c|c|c|}
\hline \multirow{2}{*}{$\begin{array}{l}\text { Wave- } \\
\text { length }\end{array}$} & \multicolumn{2}{|c|}{ Intensity } & \multirow{2}{*}{$\begin{array}{l}\text { Spec- } \\
\text { trum }\end{array}$} & \multirow{2}{*}{$\begin{array}{l}\text { Wave- } \\
\text { length }\end{array}$} & \multicolumn{2}{|c|}{ Intensity } & \multirow{2}{*}{$\begin{array}{l}\text { Spec- } \\
\text { trum }\end{array}$} & \multirow{2}{*}{$\begin{array}{l}\text { Wave- } \\
\text { length }\end{array}$} & \multicolumn{2}{|c|}{ Intensity } & \multirow{2}{*}{$\begin{array}{l}\text { Spec } \\
\text { trum }\end{array}$} \\
\hline & Lamp & Spark & & & Lamp & Spark & & & Lamp & Spark & \\
\hline $\begin{array}{l}2807.037 \\
2807.316 \\
2807.707 \\
2807.826 \\
2808.016\end{array}$ & $\begin{array}{r}50 \\
10 \\
150 \\
30 \\
40\end{array}$ & $\begin{array}{r}10 \\
100 \\
400\end{array}$ & $\begin{array}{l}\text { I } \\
\text { I } \\
\text { II } \\
\text { II } \\
\text { I }\end{array}$ & $\begin{array}{l}2824.098 \\
2824.233 \\
2824.540 \\
2824.701 \\
2825.171\end{array}$ & $\begin{array}{l}20 \\
50 \\
50 \\
\\
40\end{array}$ & $\begin{array}{r}4 \\
1 \\
500 \\
20\end{array}$ & $\begin{array}{l}\text { I } \\
\text { I } \\
\text { I } \\
\text { II I } \\
\text { II }\end{array}$ & $\begin{array}{l}2839.079 \\
2839.234 \\
2839.341 \\
2839.606 \\
2839.971\end{array}$ & $\begin{array}{r}100 \\
300 \\
200 \\
200 \\
40\end{array}$ & $\begin{array}{r}2 \\
400 \\
150 \\
300\end{array}$ & $\begin{array}{l}\text { I } \\
\text { II } \\
\text { II } \\
\text { II } \\
\text { I }\end{array}$ \\
\hline $\begin{array}{l}2808.315 \\
2808.618 \\
2808.857 \\
2808.990 \\
2809.272\end{array}$ & $\begin{array}{r}50 \\
40 \\
50 \\
500 \\
40\end{array}$ & $\begin{array}{r}50 \\
1000\end{array}$ & $\begin{array}{l}\text { I } \\
\text { I } \\
\text { II } \\
\text { I I } \\
\text { I }\end{array}$ & $\begin{array}{l}2825.378 \\
2825.941 \\
2826.546 \\
2826.862 \\
2827.279\end{array}$ & $\begin{array}{r}75 \\
100 \\
40 \\
500 \\
80\end{array}$ & $\begin{array}{r}30 \\
125 \\
1500 \\
120\end{array}$ & $\begin{array}{l}\text { I I } \\
\text { I I } \\
\text { I } \\
\text { I I } \\
\text { I I }\end{array}$ & $\begin{array}{l}2840.154 \\
2840.390 \\
2840.834 \\
2841.164 \\
2841.380\end{array}$ & $\begin{array}{r}250 \\
25 \\
10 \\
250 \\
100\end{array}$ & 500 & $\begin{array}{l}\text { II } \\
\text { I } \\
\text { I } \\
\text { II } \\
\text { I }\end{array}$ \\
\hline $\begin{array}{l}2809.997 \\
2810.362 \\
2810.570 \\
2811.158 \\
2811.407\end{array}$ & $\begin{array}{l}40 \\
20 \\
10 \\
30 \\
30\end{array}$ & $\begin{array}{r}20 \\
10 \\
500\end{array}$ & $\begin{array}{l}\text { I } \\
\text { II } \\
\text { I I } \\
\text { I } \\
\text { I I I }\end{array}$ & $\begin{array}{l}2827.758 \\
2827.987 \\
2828.212 \\
2828.496 \\
2829.124\end{array}$ & $\begin{array}{r}100 \\
100 \\
150 \\
150 \\
50\end{array}$ & $\begin{array}{l}200 \\
200\end{array}$ & $\begin{array}{l}\text { I I } \\
\text { I I } \\
\text { I } \\
\text { I } \\
\text { I }\end{array}$ & $\begin{array}{l}2841.574 \\
2841.808 \\
2842.660 \\
2842.813 \\
2843.053\end{array}$ & $\begin{array}{r}20 \\
75 \\
100 \\
1000 \\
75\end{array}$ & $\begin{array}{r}150 \\
75 \\
2000 \\
75\end{array}$ & $\begin{array}{l}\text { I } \\
\text { I I } \\
\text { I I } \\
\text { I I } \\
\text { I I }\end{array}$ \\
\hline $\begin{array}{l}2811.737 \\
2812.284 \\
2812.421 \\
2813.423 \\
2814.322\end{array}$ & $\begin{array}{r}50 \\
20 \\
50 \\
40 \\
200\end{array}$ & $\begin{array}{r}6 \\
5 \\
100 \\
200\end{array}$ & $\begin{array}{l}\text { I } \\
\text { II } \\
\text { II } \\
\text { I } \\
\text { II }\end{array}$ & $\begin{array}{l}2829.348 \\
2829.441 \\
2829.736 \\
2829.936 \\
2830.447\end{array}$ & $\begin{array}{r}50 \\
50 \\
125 \\
75 \\
200\end{array}$ & $\begin{array}{r}1 \\
100 \\
1 \\
200 \\
500\end{array}$ & $\begin{array}{l}\text { I } \\
\text { II } \\
\text { I } \\
\text { II } \\
\text { II }\end{array}$ & $\begin{array}{l}2843.302 \\
2843.418 \\
2843.559 \\
2843.642 \\
2843.936\end{array}$ & $\begin{array}{r}75 \\
20 \\
2 \\
20 \\
50\end{array}$ & $\begin{array}{r}100 \\
30 \\
2\end{array}$ & $\begin{array}{l}\text { II } \\
\text { II } \\
\text { I I } \\
\text { I } \\
\text { I }\end{array}$ \\
\hline $\begin{array}{l}2814.467 \\
2814.566 \\
2815.047 \\
2815.301 \\
2816.073\end{array}$ & $\begin{array}{r}40 \\
100 \\
40 \\
20 \\
125\end{array}$ & $\begin{array}{r}30 \\
150 \\
50 \\
10 \\
200\end{array}$ & $\begin{array}{l}\text { II } \\
\text { II } \\
\text { II } \\
\text { II } \\
\text { I I }\end{array}$ & $\begin{array}{l}2830.675 \\
2830.960 \\
2831.195 \\
2831.384 \\
2831.672\end{array}$ & $\begin{array}{r}40 \\
40 \\
100 \\
40 \\
30\end{array}$ & $\begin{array}{r}2 \\
2 \\
125 \\
20\end{array}$ & $\begin{array}{l}\text { I } \\
\text { I } \\
\text { I I } \\
\text { I } \\
\text { II }\end{array}$ & $\begin{array}{l}2844.237 \\
2844.352 \\
2845.188 \\
2845.826 \\
2846.044\end{array}$ & $\begin{array}{l}30 \\
50 \mathrm{w} \\
100 \\
50 \\
75\end{array}$ & $\begin{array}{r}1 \\
1 \\
200 \\
200\end{array}$ & $\begin{array}{l}\text { I } \\
\text { I } \\
\text { II } \\
\text { II } \\
\text { I }\end{array}$ \\
\hline $\begin{array}{l}2816.919 \\
2817.138 \\
2817.899 \\
2818.043 \\
2818.493\end{array}$ & $\begin{array}{r}50 \\
75 \\
5 \\
20 \\
20\end{array}$ & $\begin{array}{l}100 \\
150\end{array}$ & $\begin{array}{l}\text { II } \\
\text { I I } \\
\text { I } \\
\text { I } \\
\text { I I }\end{array}$ & $\begin{array}{l}2832.009 \\
2832.314 \\
2832.576 \\
2833.046 \\
2833.329\end{array}$ & $\begin{array}{r}40 \\
300 \\
10 \\
75 \\
100\end{array}$ & $\begin{array}{r}10 \\
400\end{array}$ & $\begin{array}{l}\text { II } \\
\text { II } \\
\text { I } \\
\text { I } \\
\text { II }\end{array}$ & $\begin{array}{l}2846.376 \\
2846.530 \\
2847.026 \\
2847.230 \\
2847.359\end{array}$ & $\begin{array}{r}40 \\
75 \\
5 \\
20 \\
200\end{array}$ & 200 & $\begin{array}{l}\text { I } \\
\text { II } \\
\text { I } \\
\text { I } \\
\text { I I }\end{array}$ \\
\hline $\begin{array}{l}2818.865 \\
2818.966 \\
2819.087 \\
2819.328 \\
2820.337\end{array}$ & $\begin{array}{r}10 \\
10 \\
50 \\
200 \\
100\end{array}$ & $\begin{array}{l}500 \\
300\end{array}$ & $\begin{array}{l}\text { I I } \\
\text { I } \\
\text { I } \\
\text { II } \\
\text { I I }\end{array}$ & $\begin{array}{l}2833.532 \\
2833.621 \\
2833.888 \\
2834.004 \\
2834.494\end{array}$ & $\begin{array}{r}75 \\
3 \\
20 \\
20 \\
75\end{array}$ & 1000 & $\begin{array}{l}\text { II } \\
\text { I } \\
\text { I } \\
\text { I } \\
\text { I I }\end{array}$ & $\begin{array}{l}2847.622 \\
2848.023 \\
2848.085 \\
2848.248 \\
2848.495\end{array}$ & $\begin{array}{l}30 \\
50 \\
40 \\
50 \\
75\end{array}$ & $\begin{array}{r}20 \\
200\end{array}$ & $\begin{array}{l}\text { II } \\
\text { II } \\
\text { I } \\
\text { I } \\
\text { I }\end{array}$ \\
\hline $\begin{array}{l}2820.712 \\
2820.760 \\
2821.072 \\
2821.175 \\
2821.314\end{array}$ & $\begin{array}{r}20 \\
5 \\
50 \\
75 \\
50\end{array}$ & 2 & $\begin{array}{l}\text { I } \\
\text { I } \\
\text { I } \\
\text { I } \\
\text { I }\end{array}$ & $\begin{array}{l}2834.778 \\
2835.290 \\
2835.637 \\
2835.775 \\
2836.048\end{array}$ & $\begin{array}{r}100 \\
20 \\
20 \\
10 \\
500\end{array}$ & $\begin{array}{r}125 \\
1 \\
2 \\
500\end{array}$ & $\begin{array}{l}\text { II } \\
\text { I } \\
\text { I } \\
\text { I } \\
\text { II }\end{array}$ & $\begin{array}{l}2848.684 \\
2849.159 \\
2849.315 \\
2849.574 \\
2849.705\end{array}$ & $\begin{array}{r}5 \\
50 \\
40 \\
\\
5\end{array}$ & $\begin{array}{l}75 \\
5 \mathrm{~h}\end{array}$ & $\begin{array}{l}\text { I } \\
\text { I I } \\
\text { I } \\
\text { I I } \\
\text { I }\end{array}$ \\
\hline $\begin{array}{l}2821.611 \\
2822.026 \\
2822.183 \\
2822.260 \\
2822.380\end{array}$ & $\begin{array}{r}125 \\
200 \\
75 \\
5 \\
40\end{array}$ & $\begin{array}{c}200 \\
500 \\
40 \\
50 \mathrm{~h}\end{array}$ & $\begin{array}{l}\text { I I } \\
\text { I I } \\
\text { I I } \\
\text { I } \\
\text { I I }\end{array}$ & $\begin{array}{l}2836.207 \\
2836.441 \\
2836.628 \\
2837.299 \\
2837.636\end{array}$ & $\begin{array}{r}100 \\
400 \\
2 \\
2000 \\
10\end{array}$ & $\begin{array}{c}2 \\
100 \\
100 \mathrm{w} \\
4000 \\
5\end{array}$ & $\begin{array}{l}\text { I } \\
\text { II } \\
\text { III } \\
I I \\
\text { II }\end{array}$ & $\begin{array}{l}2849.930 \\
2850.005 \\
2850.611 \\
2850.819 \\
2851.261\end{array}$ & $\begin{array}{r}20 \\
2 \\
75 \\
40 \\
800\end{array}$ & 1500 & $\begin{array}{l}\text { I } \\
\text { I } \\
\text { I } \\
\text { I } \\
\text { I I }\end{array}$ \\
\hline $\begin{array}{l}2822.566 \\
2822.689 \\
2822.832 \\
2823.132 \\
2823.554\end{array}$ & $\begin{array}{l}40 \\
50 \\
50 \\
50 \\
75\end{array}$ & $\begin{array}{r}100 \\
50 \\
40 \\
125 \\
100\end{array}$ & $\begin{array}{l}\text { II } \\
\text { I I } \\
\text { I I } \\
\text { I I } \\
\text { I I }\end{array}$ & $\begin{array}{l}2837.867 \\
2838.243 \\
2838.486 \\
2838.587 \\
2838.872\end{array}$ & $\begin{array}{r}5 \\
30 \\
100 \\
20 \\
20\end{array}$ & 10 & $\begin{array}{l}\text { I } \\
\text { I I } \\
\text { I } \\
\text { II } \\
\text { I }\end{array}$ & $\begin{array}{l}2851.436 \\
2851.554 \\
2852.496 \\
2852.990 \\
2853.562\end{array}$ & $\begin{array}{l}75 \\
100 \\
75 \\
10 \\
10 \mathrm{w}\end{array}$ & $\begin{array}{r}200 \\
500 \\
10\end{array}$ & $\begin{array}{l}\text { I I } \\
\text { I } \\
\text { II } \\
\text { II } \\
\text { I }\end{array}$ \\
\hline
\end{tabular}


Table 1. Wavelengths of Thorium Spectra - Continued

\begin{tabular}{|c|c|c|c|c|c|c|c|c|c|c|c|}
\hline \multirow{2}{*}{$\begin{array}{l}\text { Wave- } \\
\text { length }\end{array}$} & \multicolumn{2}{|c|}{ Intensity } & \multirow{2}{*}{$\begin{array}{l}\text { Spec- } \\
\text { trum }\end{array}$} & \multirow{2}{*}{$\begin{array}{l}\text { Wave- } \\
\text { length }\end{array}$} & \multicolumn{2}{|c|}{ Intensity } & \multirow{2}{*}{$\begin{array}{l}\text { Spec- } \\
\text { trum }\end{array}$} & \multirow{2}{*}{$\begin{array}{l}\text { Wave- } \\
\text { length }\end{array}$} & \multicolumn{2}{|c|}{ Intensity } & \multirow{2}{*}{$\begin{array}{l}\text { Spec- } \\
\text { trum }\end{array}$} \\
\hline & Lamp & Spark & & & Lamp & Spark & & & Lamp & Spark & \\
\hline $\begin{array}{l}2854.138 \\
2854.346 \\
2854.614 \\
2854.917 \\
2855.890\end{array}$ & $\begin{array}{r}150 \\
75 \\
50 \\
150 \\
50\end{array}$ & $\begin{array}{r}800 \\
40 \\
200 \\
300\end{array}$ & $\begin{array}{l}\text { I I } \\
\text { I } \\
\text { I I } \\
\text { I I } \\
\text { I I }\end{array}$ & $\begin{array}{l}2867.056 \\
2867.109 \\
2867.252 \\
2867.440 \\
2867.690\end{array}$ & $\begin{array}{r}30 \\
10 \\
40 \\
40 \\
2\end{array}$ & $200 w$ & $\begin{array}{l}\text { I I } \\
\text { I } \\
\text { I } \\
\text { I } \\
\text { I I }\end{array}$ & $\begin{array}{l}2880.152 \\
2880.627 \\
2881.135 \\
2882.001 \\
2882.336\end{array}$ & $\begin{array}{r}40 \\
100 \\
150 \\
100 \\
30\end{array}$ & $\begin{array}{r}300 \\
400 \\
20\end{array}$ & $\begin{array}{l}\text { I } \\
\text { I } \\
\text { I I } \\
\text { I I } \\
\text { I I }\end{array}$ \\
\hline $\begin{array}{l}2856.150 \\
2856.234 \\
2856.302 \\
2856.421 \\
2857.137\end{array}$ & $\begin{array}{r}5 \\
20 \\
10 \\
40 \\
100\end{array}$ & $\begin{array}{l}5 \\
1\end{array}$ & $\begin{array}{l}\text { I I } \\
\text { I } \\
\text { I } \\
\text { I } \\
\text { I }\end{array}$ & $\begin{array}{l}2867.925 \\
2868.250 \\
2868.440 \\
2868.679 \\
2868.960\end{array}$ & $\begin{array}{r}20 \\
10 \\
100 \\
75 \\
20\end{array}$ & $\begin{array}{r}5 \\
50 \\
300\end{array}$ & $\begin{array}{l}\text { I } \\
\text { I } \\
\text { I I } \\
\text { I I } \\
\text { I }\end{array}$ & $\begin{array}{l}2882.508 \\
2882.772 \\
2883.254 \\
2883.434 \\
2883.611\end{array}$ & $\begin{array}{r}100 \\
50 \\
75 \\
20 \\
100\end{array}$ & $\begin{array}{r}250 \\
5 \\
100\end{array}$ & $\begin{array}{l}\text { I I } \\
\text { I } \\
\text { I } \\
\text { I I } \\
\text { I I }\end{array}$ \\
\hline $\begin{array}{l}2857.493 \\
2857.789 \\
2857.880 \\
2858.308 \\
2858.559\end{array}$ & $\begin{array}{c}150 \\
10 \mathrm{w} \\
4 \\
10 \\
5\end{array}$ & 500 & $\begin{array}{l}\text { I I } \\
\text { I } \\
\text { I } \\
\text { I } \\
\text { I }\end{array}$ & $\begin{array}{l}2869.044 \\
2869.398 \\
2869.536 \\
2869.917 \\
2870.050\end{array}$ & $\begin{array}{r}5 \\
5 \\
20 \\
100 \\
50\end{array}$ & $\begin{array}{l}5 \mathrm{hh} \\
500 \\
100\end{array}$ & $\begin{array}{l}\text { I } \\
\text { I } \\
\text { II } \\
\text { I I } \\
\text { I I }\end{array}$ & $\begin{array}{l}2883.717 \\
2884.291 \\
2885.046 \\
2885.697 \\
2885.964\end{array}$ & $\begin{array}{r}75 \\
1500 \\
900 \\
10 \\
40\end{array}$ & $\begin{array}{r}2000 \\
1800 \\
40\end{array}$ & $\begin{array}{l}\text { I } \\
\text { I I } \\
\text { I I } \\
\text { I } \\
\text { I I }\end{array}$ \\
\hline $\begin{array}{l}2858.914 \\
2858.958 \\
2859.120 \\
2859.219 \\
2859.603\end{array}$ & $\begin{array}{r}100 \\
20 \\
5 \\
20 \\
40\end{array}$ & $\begin{array}{r}200 \\
30\end{array}$ & $\begin{array}{l}\text { I I } \\
\text { I I } \\
\text { I } \\
\text { I } \\
\text { I }\end{array}$ & $\begin{array}{l}2870.403 \\
2870.815 \\
2871.097 \\
2871.728 \\
2871.979\end{array}$ & $\begin{array}{c}1500 \\
100 \\
40 \\
30 \\
20 \mathrm{w}\end{array}$ & $\begin{array}{r}2500 \\
200 \\
30 \\
2000\end{array}$ & $\begin{array}{l}\text { I I } \\
\text { I I } \\
\text { I I } \\
\text { I I I } \\
\text { I }\end{array}$ & $\begin{array}{l}2886.236 \\
2886.496 \\
2886.644 \\
2886.973 \\
2887.310\end{array}$ & $\begin{array}{r}150 \\
150 \\
20 \\
200 \\
1\end{array}$ & $\begin{array}{l}300 \\
300\end{array}$ & $\begin{array}{l}\text { I I } \\
\text { I I } \\
\text { I } \\
\text { I } \\
\text { I I I }\end{array}$ \\
\hline $\begin{array}{l}2859.768 \\
2860.048 \\
2860.488 \\
2860.777 \\
2860.901\end{array}$ & $\begin{array}{r}75 \\
40 \\
150 \\
75 \\
3\end{array}$ & 202 & $\begin{array}{l}\text { I } \\
\text { I I I } \\
\text { I } \\
\text { I } \\
\text { I }\end{array}$ & $\begin{array}{l}2872.345 \\
2872.880 \\
2873.156 \\
2873.460 \\
2873.717\end{array}$ & $\begin{array}{r}5 \\
5 \\
10 \\
5 \\
120\end{array}$ & $\begin{array}{c}4 \mathrm{w} \\
1 \\
1 \\
300\end{array}$ & $\begin{array}{l}\text { I I } \\
\text { I } \\
\text { I } \\
\text { I } \\
\text { I I }\end{array}$ & $\begin{array}{l}2887.814 \\
2888.389 \\
2888.545 \\
2888.771 \\
2888.909\end{array}$ & $\begin{array}{r}500 \\
20 \\
20 \\
20 \\
10\end{array}$ & 1000 & $\begin{array}{l}\text { I I } \\
\text { I } \\
\text { I } \\
\text { I } \\
\text { II I }\end{array}$ \\
\hline $\begin{array}{l}2861.060 \\
2861.270 \\
2861.338 \\
2861.416 \\
2862.011\end{array}$ & $\begin{array}{r}5 \\
10 \\
200 \\
500 \\
50\end{array}$ & $\begin{array}{r}5 \\
300 \\
750 \\
30\end{array}$ & $\begin{array}{l}\text { I } \\
\text { I I } \\
\text { I I } \\
\text { I I } \\
\text { I I }\end{array}$ & $\begin{array}{l}2873.985 \\
2874.154 \\
2874.337 \\
2874.425 \\
2874.626\end{array}$ & $\begin{array}{r}5 \\
5 \\
5 \\
4 \\
75\end{array}$ & 300 & $\begin{array}{l}\text { I } \\
\text { I } \\
\text { I } \\
\text { I } \\
\text { I I }\end{array}$ & $\begin{array}{l}2889.205 \\
2889.280 \\
2889.482 \\
2889.635 \\
2889.749\end{array}$ & $\begin{array}{r}20 \\
20 \\
10 \\
5 \\
5\end{array}$ & 6 & $\begin{array}{l}\text { II } \\
\text { I } \\
\text { I } \\
\text { I } \\
\text { I }\end{array}$ \\
\hline $\begin{array}{l}2862.282 \\
2862.530 \\
2862.610 \\
2862.880 \\
2863.144\end{array}$ & $\begin{array}{r}5 \\
40 \\
100 \\
10 \\
20\end{array}$ & $\begin{array}{l}20 \\
40 w\end{array}$ & $\begin{array}{l}\text { I } \\
\text { I I } \\
\text { I I } \\
\text { I } \\
\text { I }\end{array}$ & $\begin{array}{l}2874.738 \\
2874.902 \\
2874.950 \\
2875.466 \\
2875.638\end{array}$ & $\begin{array}{r}2 \\
75 \\
\\
50 \\
100\end{array}$ & $\begin{array}{l}200 \\
10 \mathrm{w} \\
500\end{array}$ & $\begin{array}{l}\text { I } \\
\text { II } \\
\text { I I } \\
\text { I } \\
\text { I I }\end{array}$ & $\begin{array}{l}2890.080 \\
2890.195 \\
2890.432 \\
2890.493 \\
2890.880\end{array}$ & $\begin{array}{r}50 \\
1 \\
2 \\
2 \\
5\end{array}$ & $\begin{array}{l}4 \\
3 \\
4 \\
3 \\
8\end{array}$ & $\begin{array}{l}\text { I } \\
\text { I I } \\
\text { I I } \\
\text { I I } \\
\text { I I }\end{array}$ \\
\hline $\begin{array}{l}2863.508 \\
2863.677 \\
2864.049 \\
2864.144 \\
2864.284\end{array}$ & $\begin{array}{l}40 \\
50 \\
40 \\
10 \\
20\end{array}$ & 1 & $\begin{array}{l}\text { I } \\
\text { I } \\
\text { I } \\
\text { I } \\
\text { I }\end{array}$ & $\begin{array}{l}2876.032 \\
2876.413 \\
2876.646 \\
2876.753 \\
2876.958\end{array}$ & $\begin{array}{r}50 \\
150 \\
20 \\
20 \\
5\end{array}$ & $\begin{array}{r}400 \\
10\end{array}$ & $\begin{array}{l}\text { I } \\
\text { I I } \\
\text { I } \\
\text { II } \\
\text { I }\end{array}$ & $\begin{array}{l}2891.249 \\
2891.725 \\
2891.815 \\
2892.171 \\
2892.415\end{array}$ & $\begin{array}{r}200 \\
100 \\
100 \\
75 \\
1\end{array}$ & $\begin{array}{l}200 \\
200 \\
200 \\
200\end{array}$ & $\begin{array}{l}\text { I I } \\
\text { I I } \\
\text { I I } \\
\text { I I } \\
\text { I }\end{array}$ \\
\hline $\begin{array}{l}2864.654 \\
2864.980 \\
2865.237 \\
2865.485 \\
2865.608\end{array}$ & $\begin{array}{c}150 \\
10 \mathrm{w} \\
100 \\
5 \\
5\end{array}$ & $\begin{array}{l}500 \\
150\end{array}$ & $\begin{array}{l}\text { II } \\
\text { I } \\
\text { I I } \\
\text { I } \\
\text { I }\end{array}$ & $\begin{array}{l}2877.059 \\
2877.387 \\
2877.540 \\
2877.810 \\
2877.967\end{array}$ & $\begin{array}{c}5 \\
40 \\
20 \\
1 \\
20 \mathrm{w}\end{array}$ & 30 & $\begin{array}{l}\text { I } \\
\text { II } \\
\text { I } \\
\text { I } \\
\text { I }\end{array}$ & $\begin{array}{l}2892.778 \\
2892.816 \\
2892.904 \\
2893.076 \\
2893.150\end{array}$ & $\begin{array}{r}2 \\
2 \\
20 \\
20 \\
10\end{array}$ & 20 & $\begin{array}{l}\text { I } \\
\text { I I } \\
\text { I } \\
\text { I } \\
\text { I I }\end{array}$ \\
\hline $\begin{array}{l}2865.732 \\
2866.238 \\
2866.401 \\
2866.646 \\
2866.790\end{array}$ & $\begin{array}{r}40 \\
40 \\
100 \\
100 \\
4\end{array}$ & $\begin{array}{l}100 \\
150\end{array}$ & $\begin{array}{l}\text { I } \\
\text { I } \\
\text { I I } \\
\text { I I } \\
\text { I }\end{array}$ & $\begin{array}{l}2878.638 \\
2879.191 \\
2879.378 \\
2879.530 \\
2880.056\end{array}$ & $\begin{array}{r}100 \\
100 \\
5 \\
200 \\
20\end{array}$ & $\begin{array}{r}50 \\
100 \\
200\end{array}$ & $\begin{array}{l}\text { I I } \\
\text { I I } \\
\text { I } \\
\text { I I } \\
\text { I }\end{array}$ & $\begin{array}{l}2893.749 \\
2893.869 \\
2894.487 \\
2894.985 \\
2895.135\end{array}$ & $\begin{array}{l}10 \\
10 \mathrm{w} \\
30 \\
3 \\
400\end{array}$ & $\begin{array}{r}5 \\
30 \\
200\end{array}$ & $\begin{array}{l}\text { I I } \\
\text { I } \\
\text { I I } \\
\text { I } \\
\text { I I }\end{array}$ \\
\hline
\end{tabular}


Table 1. Wavelengths of Thorium Spectra - Continued

\begin{tabular}{|c|c|c|c|c|c|c|c|c|c|c|c|}
\hline \multirow{2}{*}{$\begin{array}{l}\text { Wave- } \\
\text { length }\end{array}$} & \multicolumn{2}{|c|}{ Intensity } & \multirow{2}{*}{$\begin{array}{l}\text { Spec- } \\
\text { trum }\end{array}$} & \multirow{2}{*}{$\begin{array}{l}\text { Wave- } \\
\text { length }\end{array}$} & \multicolumn{2}{|c|}{ Intensity } & \multirow{2}{*}{$\begin{array}{l}\text { Spec- } \\
\text { trum }\end{array}$} & \multirow{2}{*}{$\begin{array}{l}\text { Wave- } \\
\text { length }\end{array}$} & \multicolumn{2}{|c|}{ Intensity } & \multirow{2}{*}{$\begin{array}{l}\text { Spec- } \\
\text { trum }\end{array}$} \\
\hline & $a m p$ & Spark & & & I & Spark & & & Lan & Spark & \\
\hline $\begin{array}{l}2895.264 \\
2895.738 \\
2895.941 \\
2896.002 \\
2896.312\end{array}$ & $\begin{array}{r}5 \\
50 \\
5 \\
50 \\
50\end{array}$ & $\begin{array}{r}15 \\
5\end{array}$ & $\begin{array}{l}\text { I } \\
\text { I I } \\
\text { II } \\
\text { I } \\
\text { I }\end{array}$ & $\begin{array}{l}2908.690 \\
2908.979 \\
2909.103 \\
2909.297 \\
2909.375\end{array}$ & $\begin{array}{l}4 \\
20 \\
20 \mathrm{w} \\
5 \\
5\end{array}$ & $\begin{array}{l}10 \\
30\end{array}$ & $\begin{array}{l}\text { I I } \\
\text { I I } \\
\text { I } \\
\text { I } \\
\text { I }\end{array}$ & $\begin{array}{l}2923.952 \\
2924.104 \\
2924.571 \\
2925.054 \\
2925.525\end{array}$ & $\begin{array}{r}20 \\
75 \\
75 \\
800 \\
75\end{array}$ & $\begin{array}{r}1000 \\
150 \\
75 \\
1500 \\
15\end{array}$ & $\begin{array}{l}\text { I I I } \\
\text { I I } \\
\text { I I } \\
\text { I I } \\
\text { I }\end{array}$ \\
\hline $\begin{array}{l}2896.423 \\
2896.756 \\
2897.069 \\
2897.484 \\
2897.617\end{array}$ & $\begin{array}{r}10 \\
2 \\
400 \\
20 \\
20\end{array}$ & $\begin{array}{r}1000 \\
600 \\
1\end{array}$ & $\begin{array}{l}\text { I } \\
\text { II I } \\
\text { II } \\
\text { I } \\
\text { I }\end{array}$ & $\begin{array}{l}2909.454 \\
2909.767 \\
2909.974 \\
2910.157 \\
2910.596\end{array}$ & $\begin{array}{c}30 \mathrm{w} \\
150 \\
20 \\
\\
600\end{array}$ & $\begin{array}{l}40 \mathrm{w} \\
100 \\
10 \mathrm{w} \\
600\end{array}$ & $\begin{array}{l}\text { II } \\
\text { II } \\
\text { I } \\
\text { II I } \\
\text { I I }\end{array}$ & $\begin{array}{l}2925.648 \\
2926.342 \\
2926.431 \\
2926.813 \\
2927.258\end{array}$ & $\begin{array}{l}50 \\
30 \\
20 \\
10\end{array}$ & $\begin{array}{r}100 \\
5 \\
20 \\
10\end{array}$ & $\begin{array}{l}\text { I I I } \\
\text { I } \\
\text { I I } \\
\text { I } \\
\text { I }\end{array}$ \\
\hline $\begin{array}{l}2898.260 \\
2898.354 \\
2898.553 \\
2898.950 \\
2899.375\end{array}$ & $\begin{array}{r}200 \\
5 \\
75 \\
50 \\
100\end{array}$ & $\begin{array}{c}100 \mathrm{w} \\
50\end{array}$ & $\begin{array}{l}\text { II I } \\
\text { I } \\
\text { I } \\
\text { I I I } \\
\text { I I }\end{array}$ & $\begin{array}{l}2910.720 \\
2911.320 \\
2912.009 \\
2912.151 \\
2912.665\end{array}$ & $\begin{array}{r}40 \\
300 \\
400 \\
5 \\
50\end{array}$ & $\begin{array}{l}20 \mathrm{~h} \\
500 \\
400 \\
75\end{array}$ & $\begin{array}{l}\text { I I } \\
\text { I I } \\
\text { I I } \\
\text { I } \\
\text { I I }\end{array}$ & $\begin{array}{l}2927.373 \\
2927.473 \\
2928.254 \\
2928.699 \\
2928.994\end{array}$ & $\begin{array}{r}5 \\
10 \\
1000 \\
100 \\
5\end{array}$ & $\begin{array}{l}1200 \\
3000 \mathrm{w}\end{array}$ & $\begin{array}{l}\text { I } \\
\text { I } \\
\text { I I } \\
\text { I I I } \\
\text { I }\end{array}$ \\
\hline $\begin{array}{l}2899.723 \\
2900.064 \\
2900.117 \\
2900.360 \\
2900.542\end{array}$ & $\begin{array}{l}600 \\
20 \mathrm{w} \\
30 \\
30 \\
2\end{array}$ & 1200 & $\begin{array}{l}\text { I I } \\
\text { I } \\
\text { I } \\
\text { I } \\
\text { I }\end{array}$ & $\begin{array}{l}2912.761 \\
2912.985 \\
2913.704 \\
2914.348 \\
2914.498\end{array}$ & $\begin{array}{l}75 \\
10 \\
40 \\
75 \\
75\end{array}$ & $\begin{array}{r}200 \\
40 \\
100 \\
100\end{array}$ & $\begin{array}{l}\text { I I } \\
\text { I } \\
\text { I I } \\
\text { I I } \\
\text { I I }\end{array}$ & $\begin{array}{l}2929.287 \\
2929.775 \\
2930.185 \\
2930.250 \\
2930.904\end{array}$ & $\begin{array}{r}200 \\
5 \\
5 \\
5 \\
150\end{array}$ & $\begin{array}{l}250 \\
5 \mathrm{w} \\
250\end{array}$ & $\begin{array}{l}\text { I I } \\
\text { I } \\
\text { I I } \\
\text { I } \\
\text { II }\end{array}$ \\
\hline $\begin{array}{l}2900.648 \\
2900.766 \\
2901.063 \\
2901.328 \\
2901.380\end{array}$ & $\begin{array}{r}50 \\
50 \\
50 \\
50 \\
2\end{array}$ & $2 \mathrm{~h}$ & $\begin{array}{l}\text { I } \\
\text { I } \\
\text { I } \\
\text { I } \\
\text { I }\end{array}$ & $\begin{array}{l}2914.583 \\
2915.470 \\
2915.753 \\
2916.367 \\
2916.481\end{array}$ & $\begin{array}{r}75 \\
20 \\
5 \\
150 \\
75\end{array}$ & $\begin{array}{r}100 \\
20\end{array}$ & $\begin{array}{l}\text { I I } \\
\text { I I } \\
\text { I } \\
\text { I } \\
\text { I I }\end{array}$ & $\begin{array}{l}2931.273 \\
2931.518 \\
2931.652 \\
2931.772 \\
2932.151\end{array}$ & $\begin{array}{r}75 \\
20 \\
50 \\
75 \\
100\end{array}$ & $\begin{array}{r}2 \\
75\end{array}$ & $\begin{array}{l}\text { I } \\
\text { I } \\
\text { I } \\
\text { I } \\
\text { I I }\end{array}$ \\
\hline $\begin{array}{l}2901.760 \\
2902.156 \\
2902.474 \\
2902.708 \\
2903.044\end{array}$ & $\begin{array}{l}10 \\
20 \\
20 \\
75 \\
75\end{array}$ & $\begin{array}{r}1 \\
2 \\
50\end{array}$ & $\begin{array}{l}\text { I } \\
\text { I } \\
\text { I } \\
\text { I } \\
\text { II }\end{array}$ & $\begin{array}{l}0.828 \\
0.946 \\
7.034 \\
7.411 \\
7.781\end{array}$ & $\begin{array}{r}4 \\
4 \\
200 \\
600 \\
100\end{array}$ & $\begin{array}{l}400 \\
800 \\
400\end{array}$ & $\begin{array}{l}\text { I } \\
\text { I } \\
\text { I I } \\
\text { I I } \\
\text { II }\end{array}$ & $\begin{array}{l}2932.314 \\
2932.524 \\
2932.642 \\
2932.870 \\
2933.095\end{array}$ & $\begin{array}{r}20 \\
75 \\
100 \\
5 \\
200\end{array}$ & $\begin{array}{l}1 \\
100 \mathrm{w} \\
100 \\
300\end{array}$ & $\begin{array}{l}\text { I } \\
\text { I I I } \\
\text { I I } \\
\text { I } \\
\text { I I }\end{array}$ \\
\hline $\begin{array}{l}2903.164 \\
2903.300 \\
2903.564 \\
2904.266 \\
2904.440\end{array}$ & $\begin{array}{r}250 \\
4 \\
100 \\
75 \\
30\end{array}$ & $\begin{array}{l}400 \mathrm{w} \\
200 \\
150 \\
40\end{array}$ & $\begin{array}{l}\text { II } \\
\text { I } \\
\text { II } \\
\text { II } \\
\text { II }\end{array}$ & $\begin{array}{l}2918.433 \\
2918.585 \\
2918.916 \\
2919.171\end{array}$ & $\begin{array}{r}150 \\
4 \\
2 \\
50 \\
75\end{array}$ & 400 & $\begin{array}{l}\text { I I } \\
\text { I } \\
\text { I } \\
\text { I } \\
\text { II }\end{array}$ & $\begin{array}{l}2934.008 \\
2934.134 \\
2934.532 \\
2934.989 \\
2935.653\end{array}$ & $\begin{array}{r}75 \\
200 \\
50 \\
150 \\
100\end{array}$ & $\begin{array}{r}25 \\
300 \\
20 \\
150 \\
150\end{array}$ & $\begin{array}{l}\text { I I } \\
\text { I I } \\
\text { I I } \\
\text { I I } \\
\text { I I }\end{array}$ \\
\hline $\begin{array}{l}2904.700 \\
2905.009 \\
2905.096 \\
2905.227 \\
2905.316\end{array}$ & $\begin{array}{r}40 \\
5 \\
50 \\
20 \\
20\end{array}$ & $\begin{array}{c}30 \\
100 \\
20 \mathrm{~h}\end{array}$ & $\begin{array}{l}\text { I I } \\
\text { I I } \\
\text { I I } \\
\text { I } \\
\text { I }\end{array}$ & $\begin{array}{l}2919.444 \\
2919.837 \\
2920.050 \\
2920.364 \\
2920.937\end{array}$ & $\begin{array}{r}50 \\
300 \\
40 \\
100 \\
200\end{array}$ & $\begin{array}{r}50 \\
500 \\
30 \\
200 \\
300\end{array}$ & $\begin{array}{l}\text { I I } \\
\text { II } \\
\text { II } \\
\text { I I } \\
\text { I I }\end{array}$ & & $\begin{array}{r}75 \\
75 \\
300 \\
600 \\
10\end{array}$ & $\begin{array}{r}75 \\
75 \\
700 \\
1000 \\
5\end{array}$ & $\begin{array}{l}\text { I I } \\
\text { I I } \\
\text { I I } \\
\text { I I } \\
\text { I }\end{array}$ \\
\hline $\begin{array}{l}2905.738 \\
2905.924 \\
2906.120 \\
2907.223 \\
2907.450\end{array}$ & $\begin{array}{r}10 \\
200 \\
200 \\
50 \\
50\end{array}$ & $\begin{array}{r}15 \\
400 \\
400\end{array}$ & $\begin{array}{l}\text { I I } \\
\text { I I } \\
\text { I I } \\
\text { I } \\
\text { I }\end{array}$ & $\begin{array}{l}.083 \\
.364 \\
.558 \\
.623 \\
.598\end{array}$ & $\begin{array}{r}100 \\
100 \\
50 \\
200 \\
300\end{array}$ & $\begin{array}{l}10 \\
10 \\
10 \\
40 \\
30\end{array}$ & $\begin{array}{l}\text { I I } \\
\text { II } \\
\text { I I } \\
\text { II } \\
\text { I I }\end{array}$ & $\begin{array}{l}2937.435 \\
2938.098 \\
2938.220 \\
2938.325 \\
2939.081\end{array}$ & $\begin{array}{r}125 \\
125 \\
10 \\
5\end{array}$ & $\begin{array}{l}500 \\
100 \\
300 \\
10 \mathrm{~h}\end{array}$ & $\begin{array}{l}\text { I I } \\
\text { I I } \\
\text { I I I } \\
\text { I I } \\
\text { I }\end{array}$ \\
\hline $\begin{array}{l}2907.544 \\
2907.774 \\
2908.160 \\
2908.360 \\
2908.506\end{array}$ & $\begin{array}{r}100 \\
10 \\
200 \\
75\end{array}$ & $\begin{array}{c}5 \\
100 \\
2 \mathrm{w} \\
400\end{array}$ & $\begin{array}{l}\text { II I } \\
\text { II } \\
\text { I } \\
\text { II } \\
\text { I }\end{array}$ & $\begin{array}{l}2922.795 \\
2922.988 \\
2923.379 \\
2923.480 \\
2923.639\end{array}$ & $\begin{array}{c}50 \\
125 \\
50 \\
4 \mathrm{w} \\
50\end{array}$ & $\begin{array}{l}150 \\
125\end{array}$ & $\begin{array}{l}\text { I I } \\
\text { I I } \\
\text { I } \\
\text { I } \\
\text { I }\end{array}$ & $\begin{array}{l}2939.567 \\
2939.616 \\
2940.080 \\
2940.301 \\
2940.581\end{array}$ & $\begin{array}{r}150 \\
150 \\
400 \\
40 \\
150\end{array}$ & $\begin{array}{r}400 \\
400 \\
\\
12 \\
150\end{array}$ & $\begin{array}{l}\text { II } \\
\text { II } \\
\text { I } \\
\text { II } \\
\text { II }\end{array}$ \\
\hline
\end{tabular}


Table 1. Wavelengths of Thorium Spectra - Continued

\begin{tabular}{|c|c|c|c|c|c|c|c|c|c|c|c|}
\hline \multirow{2}{*}{$\begin{array}{l}\text { Wave- } \\
\text { length }\end{array}$} & \multicolumn{2}{|c|}{ Intensity } & \multirow{2}{*}{$\begin{array}{l}\text { Spec- } \\
\text { trum }\end{array}$} & \multirow{2}{*}{$\begin{array}{l}\text { Wave- } \\
\text { length }\end{array}$} & \multicolumn{2}{|c|}{ Intensity } & \multirow{2}{*}{$\begin{array}{l}\text { Spec- } \\
\text { trum }\end{array}$} & \multirow{2}{*}{$\begin{array}{l}\text { Wave- } \\
\text { length }\end{array}$} & \multicolumn{2}{|c|}{ Intensity } & \multirow{2}{*}{$\begin{array}{l}\text { Spec } \\
\text { trum }\end{array}$} \\
\hline & & & & & & & & & & & \\
\hline $\begin{array}{l}2940.676 \\
2940.862 \\
2941.314 \\
2941.886 \\
2942.625\end{array}$ & $\begin{array}{r}150 \\
5 \\
125 \\
100 \\
250\end{array}$ & $\begin{array}{l}250 \\
150 \\
150 \\
500\end{array}$ & $\begin{array}{l}\text { II } \\
\text { II } \\
\text { II } \\
\text { II } \\
\text { II }\end{array}$ & $\begin{array}{l}2953.568 \\
2953.604 \\
2954.361 \\
2954.555 \\
2954.883\end{array}$ & $\begin{array}{r}50 \\
5 \\
10 \\
4 \\
100\end{array}$ & $\begin{array}{r}50 \\
2 \\
200\end{array}$ & $\begin{array}{l}\text { II } \\
\text { I } \\
\text { I } \\
\text { I } \\
\text { II }\end{array}$ & $\begin{array}{l}2966.640 \\
2967.229 \\
2967.816 \\
2968.007 \\
2968.320\end{array}$ & $\begin{array}{c}4 \\
40 \\
50 \\
5 \mathrm{w} \\
5\end{array}$ & $\begin{array}{r}4 \\
30 \\
4\end{array}$ & $\begin{array}{l}\text { II } \\
\text { II } \\
\text { I } \\
\text { I } \\
\text { I }\end{array}$ \\
\hline $\begin{array}{l}2942.857 \\
2942.987 \\
2943.088 \\
2943.235 \\
2943.340\end{array}$ & $\begin{array}{r}1500 \\
50 \\
30 \\
5 \\
3\end{array}$ & 1700 & $\begin{array}{l}\text { II } \\
\text { I } \\
\text { I } \\
\text { I } \\
\text { I }\end{array}$ & $\begin{array}{l}2955.033 \\
2955.603 \\
2955.850 \\
2956.244 \\
2956.630\end{array}$ & $\begin{array}{r}200 \\
200 \\
100 \\
10 \\
40\end{array}$ & $\begin{array}{r}400 \\
300 \\
200 \\
5 \\
50\end{array}$ & $\begin{array}{l}\text { I I } \\
\text { I I } \\
\text { I I } \\
\text { I I } \\
\text { I I }\end{array}$ & $\begin{array}{l}2968.687 \\
2969.062 \\
2969.377 \\
2969.823 \\
2970.016\end{array}$ & $\begin{array}{r}1000 \\
100 \\
125 \\
300 \\
5\end{array}$ & $\begin{array}{r}1400 \\
100 \\
125 \\
250 \\
4\end{array}$ & $\begin{array}{l}\text { II } \\
\text { II } \\
\text { II } \\
\text { II } \\
\text { II }\end{array}$ \\
\hline $\begin{array}{l}2943.440 \\
2943.717 \\
2943.848 \\
2943.953 \\
2944.382\end{array}$ & $\begin{array}{r}3 \\
50 \\
5 \\
50 \\
4\end{array}$ & 20 & $\begin{array}{l}\text { I } \\
\text { I } \\
\text { I } \\
\text { II } \\
\text { I }\end{array}$ & $\begin{array}{l}2956.822 \\
2957.065 \\
2957.580 \\
2957.920 \\
2958.141\end{array}$ & $\begin{array}{r}10 \\
40 \\
600 \\
250 \\
125\end{array}$ & $\begin{array}{r}40 \\
1000 \\
200 \\
400\end{array}$ & $\begin{array}{l}\text { I } \\
\text { II } \\
\text { I I } \\
\text { II } \\
\text { I I }\end{array}$ & $\begin{array}{l}2970.080 \\
2970.573 \\
2970.628 \\
2970.816 \\
2971.102\end{array}$ & $\begin{array}{l}50 \\
50 \\
75 \\
75\end{array}$ & $\begin{array}{l}20 \\
50 \\
30 \\
10 \mathrm{w}\end{array}$ & $\begin{array}{l}\text { I I } \\
\text { I } \\
\text { II } \\
\text { II } \\
\text { I II }\end{array}$ \\
\hline $\begin{array}{l}2944.463 \\
2944.615 \\
2944.948 \\
2945.343 \\
2945.504\end{array}$ & $\begin{array}{r}120 \\
50 \\
125 \\
5 \\
5\end{array}$ & $\begin{array}{l}250 \\
12 \\
250 \\
10 \mathrm{w}\end{array}$ & $\begin{array}{l}\text { I I } \\
\text { I } \\
\text { I I } \\
\text { II I } \\
\text { I }\end{array}$ & $\begin{array}{l}2958.371 \\
2958.566 \\
2958.830 \\
2959.139 \\
2959.277\end{array}$ & $\begin{array}{l}50 \\
50 \\
10 \\
50 \\
50\end{array}$ & $\begin{array}{r}1 \\
30\end{array}$ & $\begin{array}{l}\text { I } \\
\text { I I } \\
\text { I } \\
\text { I } \\
\text { I I }\end{array}$ & $\begin{array}{l}2971.479 \\
2971.721 \\
2971.970 \\
2972.223 \\
2972.442\end{array}$ & $\begin{array}{r}600 \\
5 \\
5 \\
300 \\
2\end{array}$ & 400 & $\begin{array}{l}\text { II } \\
\text { I } \\
\text { I } \\
\text { II } \\
\text { I }\end{array}$ \\
\hline $\begin{array}{l}2945.889 \\
2946.255 \\
2946.539 \\
2946.610 \\
2946.927\end{array}$ & $\begin{array}{r}30 \\
100 \\
5 \\
30 \\
50\end{array}$ & $\begin{array}{l}30 \mathrm{~h} \\
100 \\
250 \\
10\end{array}$ & $\begin{array}{l}\text { II } \\
\text { II } \\
\text { I } \\
\text { II } \\
\text { I }\end{array}$ & $\begin{array}{l}2959.448 \\
2959.863 \\
2960.167 \\
2960.368 \\
2960.529\end{array}$ & $\begin{array}{c}5 \\
50 \\
2 \\
2 \mathrm{~h} \\
20\end{array}$ & $\begin{array}{r}2 \\
75\end{array}$ & $\begin{array}{l}\text { II } \\
\text { I I } \\
\text { I } \\
\text { I } \\
\text { II }\end{array}$ & $\begin{array}{l}2972.884 \\
2973.530 \\
2974.009 \\
2974.088 \\
2974.409\end{array}$ & $\begin{array}{r}75 \\
300 \\
900 \\
400 \\
50\end{array}$ & $\begin{array}{r}50 \\
600 \\
1000 \\
50 \\
30\end{array}$ & $\begin{array}{l}\text { I I } \\
\text { II } \\
\text { I I } \\
\text { I } \\
\text { II }\end{array}$ \\
\hline $\begin{array}{l}2947.230 \\
2947.461 \\
2947.840 \\
2948.367 \\
2948.513\end{array}$ & $\begin{array}{r}75 \\
25 \\
20 \\
50 \\
5\end{array}$ & $\begin{array}{l}30 \\
50 \mathrm{w}\end{array}$ & $\begin{array}{l}\text { I } \\
\text { I } \\
\text { II } \\
\text { I I } \\
\text { I }\end{array}$ & $\begin{array}{l}2960.774 \\
2960.986 \\
2961.263 \\
2961.380 \\
2961.476\end{array}$ & $\begin{array}{r}75 \\
10 \\
2 \\
2 \\
20\end{array}$ & 1000 & $\begin{array}{l}\text { I I } \\
\text { I } \\
\text { I } \\
\text { I } \\
\text { II I }\end{array}$ & $\begin{array}{l}2974.846 \\
2974.930 \\
2975.051 \\
2975.488 \\
2975.591\end{array}$ & $\begin{array}{r}10 \\
5 \\
5 \\
10\end{array}$ & 1000 & $\begin{array}{l}\text { I } \\
\text { III } \\
\text { I } \\
\text { I } \\
\text { I }\end{array}$ \\
\hline $\begin{array}{l}2948.778 \\
2949.065 \\
2949.234 \\
2949.543 \\
2949.934\end{array}$ & $\begin{array}{r}50 \\
400 \\
100 \\
100 \\
300\end{array}$ & $\begin{array}{r}75 \\
900 \\
100 \\
15 \\
500\end{array}$ & $\begin{array}{l}\text { II } \\
\text { I I } \\
\text { II } \\
\text { I } \\
\text { II }\end{array}$ & $\begin{array}{l}2961.532 \\
2961.775 \\
2961.798 \\
2961.938 \\
2962.016\end{array}$ & $\begin{array}{r}5 \\
15 \\
5 \\
100\end{array}$ & $\begin{array}{r}200 \\
10\end{array}$ & $\begin{array}{l}\text { I } \\
\text { I I I } \\
\text { I I } \\
\text { I I } \\
\text { I }\end{array}$ & $\begin{array}{l}2976.017 \\
2976.097 \\
2976.490 \\
2976.044 \\
2977.023\end{array}$ & $\begin{array}{r}300 \\
75 \\
10 \\
125 \\
25\end{array}$ & $\begin{array}{r}125 \\
1\end{array}$ & $\begin{array}{l}\text { II } \\
\text { I } \\
\text { I } \\
\text { II } \\
\text { I }\end{array}$ \\
\hline $\begin{array}{l}2950.433 \\
2950.752 \\
2950.864 \\
2951.061 \\
2951.206\end{array}$ & $\begin{array}{l}300 \\
20 \mathrm{w} \\
5 \\
10 \\
200\end{array}$ & $\begin{array}{r}400 \\
30 \\
\\
5 \\
400\end{array}$ & $\begin{array}{l}\text { I I } \\
\text { I I } \\
\text { I } \\
\text { II } \\
\text { I I }\end{array}$ & $\begin{array}{l}2962.563 \\
2963.048 \\
2963.442 \\
2963.612 \\
2963.874\end{array}$ & $\begin{array}{r}5 \\
5 \\
5 \\
100 \\
100\end{array}$ & $\begin{array}{r}5 \\
150 \\
150\end{array}$ & $\begin{array}{l}\text { I } \\
\text { I I } \\
\text { I } \\
\text { II } \\
\text { I I }\end{array}$ & $\begin{array}{l}2977.300 \\
2978.321 \\
2978.476 \\
2978.652 \\
2978.716\end{array}$ & $\begin{array}{r}100 \\
75 \\
50\end{array}$ & $3000 \mathrm{w}$ & $\begin{array}{l}\text { I I I } \\
\text { I } \\
\text { I } \\
\text { I } \\
\text { I I I }\end{array}$ \\
\hline $\begin{array}{l}2951.567 \\
2951.902 \\
2952.125 \\
2952.482 \\
2952.586\end{array}$ & $\begin{array}{r}120 \\
50 \\
100 \\
5 \\
10\end{array}$ & $\begin{array}{r}150 \\
50 \\
125 \\
10\end{array}$ & $\begin{array}{l}\text { I I } \\
\text { I I } \\
\text { I I } \\
\text { I } \\
\text { I I }\end{array}$ & $\begin{array}{l}2964.001 \\
2964.111 \\
2964.572 \\
2964.918 \\
2965.114\end{array}$ & $\begin{array}{r}100 \\
125 \\
5 \\
400 \\
120\end{array}$ & $\begin{array}{r}600 \\
75\end{array}$ & $\begin{array}{l}\text { I I } \\
\text { I I } \\
\text { I } \\
\text { I I } \\
\text { I I }\end{array}$ & $\begin{array}{l}2978.789 \\
2979.031 \\
2979.483 \\
2979.840 \\
2980.102\end{array}$ & $\begin{array}{r}75 \\
125 \\
2 \\
75 \\
25\end{array}$ & 2 & $\begin{array}{l}\text { I } \\
\text { I } \\
\text { I } \\
\text { I } \\
\text { I }\end{array}$ \\
\hline $\begin{array}{l}2952.745 \\
2952.808 \\
2953.050 \\
2953.124 \\
2953.454\end{array}$ & $\begin{array}{r}5 \\
5 \\
25 \\
10 \\
10\end{array}$ & 1 & $\begin{array}{l}\text { I } \\
\text { I } \\
\text { I } \\
\text { I } \\
\text { I }\end{array}$ & $\begin{array}{l}2965.495 \\
2966.054 \\
2966.148 \\
2966.418 \\
2966.542\end{array}$ & $\begin{array}{r}400 \\
10 \\
100 \\
50 \\
2\end{array}$ & $\begin{array}{l}600 \\
75 \\
20 \\
2 \mathrm{~h}\end{array}$ & $\begin{array}{l}\text { I I } \\
\text { I } \\
\text { I I } \\
\text { I I } \\
\text { I I }\end{array}$ & $\begin{array}{l}2980.326 \\
2981.329 \\
2981.400 \\
2981.478 \\
2981.837\end{array}$ & $\begin{array}{r}200 \\
300 \\
10 \\
300 \\
400\end{array}$ & $\begin{array}{l}300 \\
400 \\
5 \mathrm{~h} \\
400 \\
400\end{array}$ & $\begin{array}{l}\text { I I } \\
\text { I I } \\
\text { I I } \\
\text { I I } \\
\text { I I }\end{array}$ \\
\hline
\end{tabular}


Table 1. Wavelengths of Thorium Spectra - Continued

\begin{tabular}{|c|c|c|c|c|c|c|c|c|c|c|c|}
\hline \multirow{2}{*}{$\begin{array}{l}\text { Wave- } \\
\text { length }\end{array}$} & \multicolumn{2}{|c|}{ Intensity } & \multirow{2}{*}{$\begin{array}{l}\text { Spec- } \\
\text { trum }\end{array}$} & \multirow{2}{*}{$\begin{array}{l}\text { Wave- } \\
\text { length }\end{array}$} & \multicolumn{2}{|c|}{ Intensity } & \multirow{2}{*}{$\begin{array}{l}\text { Spec- } \\
\text { trum }\end{array}$} & \multirow{2}{*}{$\begin{array}{l}\text { Wave- } \\
\text { length }\end{array}$} & \multicolumn{2}{|c|}{ Intensity } & \multirow{2}{*}{$\begin{array}{l}\text { Spec- } \\
\text { trum }\end{array}$} \\
\hline & amp & Spark & & & Lamp & Spark & & & Lamp & Spark & \\
\hline $\begin{array}{l}2981.997 \\
2982.033 \\
2982.193 \\
2982.430 \\
2982.707\end{array}$ & $\begin{array}{c}100 \\
100 \\
75 \\
50 \mathrm{~h}\end{array}$ & $\begin{array}{r}30 \\
20 \\
1 \\
20\end{array}$ & $\begin{array}{l}\text { II } \\
\text { I } \\
\text { I } \\
\text { III }\end{array}$ & $\begin{array}{l}2997.819 \\
2997.948 \\
2998.132 \\
2998.605 \\
2999.095\end{array}$ & $\begin{array}{r}50 \\
5 \\
100 \\
50 \\
300\end{array}$ & $\begin{array}{c}50 \\
4 \mathrm{~h} \\
20 \\
1200\end{array}$ & $\begin{array}{l}\text { II } \\
\text { I } \\
\text { I } \\
\text { I I } \\
\text { II }\end{array}$ & $\begin{array}{l}3015.191 \\
3015.363 \\
3015.725 \\
3016.137 \\
3016.248\end{array}$ & $\begin{array}{r}10 \\
20 \\
150 \\
20 \\
20\end{array}$ & 300 & $\begin{array}{l}\text { I } \\
\text { I } \\
\text { I I } \\
\text { I } \\
\text { I }\end{array}$ \\
\hline $\begin{array}{l}2983.020 \\
2983.213 \\
2983.559 \\
2983.662 \\
2983.804\end{array}$ & $\begin{array}{r}100 \\
50 \\
5 \\
100 \\
450\end{array}$ & $\begin{array}{r}150 \\
20 \\
10 \\
20 \\
700\end{array}$ & $\begin{array}{l}\text { II I } \\
\text { II } \\
\text { II } \\
\text { I } \\
\text { II }\end{array}$ & $\begin{array}{l}2999.275 \\
2999.797 \\
3000.339 \\
3000.460 \\
3000.612\end{array}$ & $\begin{array}{r}125 \\
150 \\
150 \\
5 \\
10\end{array}$ & $\begin{array}{l}125 \\
250\end{array}$ & $\begin{array}{l}\text { II } \\
\text { II } \\
\text { I } \\
\text { I } \\
\text { I }\end{array}$ & $\begin{array}{l}3016.327 \\
3016.457 \\
3016.751 \\
3017.135 \\
3017.441\end{array}$ & $\begin{array}{r}5 \\
3 \\
40 \\
150 \\
40\end{array}$ & $\begin{array}{c}10 \mathrm{w} \\
\mathrm{I} \mathrm{h} \\
\mathrm{I} \\
300 \\
20\end{array}$ & $\begin{array}{l}\text { I I } \\
\text { I } \\
\text { I } \\
\text { II } \\
\text { II }\end{array}$ \\
\hline $\begin{array}{l}2984.088 \\
2984.408 \\
2984.627 \\
2984.764 \\
2985.242\end{array}$ & $\begin{array}{r}20 \\
100 \\
50 \\
100 \\
600\end{array}$ & $\begin{array}{r}100 \\
75 \\
800\end{array}$ & $\begin{array}{l}\text { I } \\
\text { II } \\
\text { I } \\
\text { II } \\
\text { II }\end{array}$ & $\begin{array}{l}3000.939 \\
3001.266 \\
3001.753 \\
3002.392 \\
3002.684\end{array}$ & $\begin{array}{r}250 \\
500 \\
50 \\
800 \\
150\end{array}$ & $\begin{array}{l}250 \\
600 \\
800\end{array}$ & $\begin{array}{l}\text { I } \\
\text { II } \\
I \\
I \\
I\end{array}$ & $\begin{array}{l}3018.489 \\
3018.630 \\
3019.414 \\
3019.617 \\
3019.875\end{array}$ & $\begin{array}{r}200 \\
3 \\
200 \\
30 \\
50\end{array}$ & $\begin{array}{r}400 \\
\\
300 \\
15\end{array}$ & $\begin{array}{l}\text { II } \\
\text { I } \\
\text { II } \\
\text { I I } \\
\text { I }\end{array}$ \\
\hline $\begin{array}{l}2985.748 \\
2985.798 \\
2985.963 \\
2986.080 \\
2986.381\end{array}$ & $\begin{array}{l}10 \\
10 \\
10 \\
5\end{array}$ & $\begin{array}{r}1 \\
2000\end{array}$ & $\begin{array}{l}\text { I } \\
\text { I } \\
\text { I } \\
\text { II I } \\
\text { I }\end{array}$ & $\begin{array}{l}3003.203 \\
3003.595 \\
3003.850 \\
3004.247 \\
3004.635\end{array}$ & $\begin{array}{r}100 \\
50 \\
40 \\
75 \\
50\end{array}$ & $\begin{array}{l}40 \\
40\end{array}$ & $\begin{array}{l}\text { I } \\
\text { I } \\
\text { II } \\
\text { I } \\
\text { II }\end{array}$ & $\begin{array}{l}3020.050 \\
3020.318 \\
3021.476 \\
3022.096 \\
3022.413\end{array}$ & $\begin{array}{r}2 \\
150 \\
150 \\
2\end{array}$ & $\begin{array}{l}150 \mathrm{w} \\
2 \mathrm{~h} \\
300 \\
300\end{array}$ & $\begin{array}{l}\text { II I } \\
\text { I I } \\
\text { I I } \\
\text { II } \\
\text { I }\end{array}$ \\
\hline $\begin{array}{l}2986.449 \\
2986.770 \\
2986.830 \\
2987.317 \\
2987.674\end{array}$ & $\begin{array}{r}5 \\
300 \\
10 \\
5 \\
200\end{array}$ & 300 & $\begin{array}{l}\text { I } \\
\text { II } \\
\text { I } \\
\text { I } \\
\text { I }\end{array}$ & $\begin{array}{l}3006.001 \\
3006.167 \\
3006.635 \\
3006.929 \\
3007.474\end{array}$ & $\begin{array}{r}100 \\
100 \\
75 \\
300 \\
5\end{array}$ & 600 & $\begin{array}{l}\text { II } \\
\text { I } \\
\text { I } \\
\text { II } \\
\text { I }\end{array}$ & $\begin{array}{l}3022.512 \\
3023.040 \\
3023.400 \\
3023.564 \\
3023.642\end{array}$ & $\begin{array}{r}10 \\
4 \\
4 \\
5 \\
50\end{array}$ & 5 & $\begin{array}{l}\text { I } \\
\text { I } \\
\text { I } \\
\text { I I } \\
\text { I }\end{array}$ \\
\hline $\begin{array}{l}2988.235 \\
2989.274 \\
2989.552 \\
2989.710 \\
2989.898\end{array}$ & $\begin{array}{r}1400 \\
20 \\
5 \\
5 \\
100\end{array}$ & $\begin{array}{r}1800 \\
2 \\
200\end{array}$ & $\begin{array}{l}\text { II } \\
\text { I } \\
\text { II } \\
\text { I } \\
\text { II }\end{array}$ & $\begin{array}{l}3007.624 \\
3007.796 \\
3007.951 \\
3008.272 \\
3008.497\end{array}$ & $\begin{array}{r}300 \\
75 \\
75 \\
75 \\
200\end{array}$ & $\begin{array}{r}400 \\
100 \\
75 \\
75 \\
300\end{array}$ & $\begin{array}{l}\text { II } \\
\text { I I } \\
\text { II } \\
\text { II } \\
\text { II }\end{array}$ & $\begin{array}{l}3023.784 \\
3024.460 \\
3024.675 \\
3024.877 \\
3025.124\end{array}$ & $\begin{array}{r}5 \\
5 \\
200 \\
40 \\
50\end{array}$ & $\begin{array}{l}5 \mathrm{~h} \\
200 \\
50\end{array}$ & $\begin{array}{l}\text { I } \\
\text { II } \\
\text { II } \\
\text { I } \\
\text { II }\end{array}$ \\
\hline $\begin{array}{l}2990.719 \\
2991.054 \\
2991.685 \\
2992.261 \\
2992.606\end{array}$ & $\begin{array}{r}1 \\
600 \\
300 \\
10 \\
5\end{array}$ & $\begin{array}{r}600 \\
500 \\
10\end{array}$ & $\begin{array}{l}\text { I } \\
\text { II } \\
\text { II } \\
\text { II } \\
\text { I }\end{array}$ & $\begin{array}{l}3008.917 \\
3009.550 \\
3009.763 \\
3010.000 \\
3010.138\end{array}$ & $\begin{array}{r}75 \\
10 \\
150 \\
50 \\
20\end{array}$ & $\begin{array}{r}200 \\
75\end{array}$ & $\begin{array}{l}\text { I } \\
\text { I } \\
\text { II } \\
\text { II } \\
\text { I }\end{array}$ & $\begin{array}{l}3025.387 \\
3025.446 \\
3026.149 \\
3026.572 \\
3027.229\end{array}$ & $\begin{array}{r}10 \\
200 \\
30 \\
400 \\
100\end{array}$ & $\begin{array}{r}10 \\
50 \\
10 \\
700 \\
30\end{array}$ & $\begin{array}{l}\text { II } \\
\text { II } \\
\text { II } \\
\text { II } \\
\text { II }\end{array}$ \\
\hline $\begin{array}{l}2992.724 \\
2993.071 \\
2993.395 \\
2993.648 \\
2993.814\end{array}$ & $\begin{array}{r}5 \\
50 \\
10 \\
300 \\
300\end{array}$ & $\begin{array}{r}4 \\
500 \\
1000\end{array}$ & $\begin{array}{l}\text { I } \\
\text { I } \\
\text { I I } \\
\text { I I } \\
\text { I I }\end{array}$ & $\begin{array}{l}3010.410 \\
3010.729 \\
3010.898 \\
3011.297 \\
3011.592\end{array}$ & $\begin{array}{r}6 \\
100 \\
10 \\
10 \\
100\end{array}$ & $\begin{array}{r}1 \\
10 \\
50\end{array}$ & $\begin{array}{l}\text { I } \\
\text { I } \\
\text { II } \\
\text { I } \\
\text { II }\end{array}$ & $\begin{array}{l}3027.595 \\
3027.883 \\
3028.573 \\
3028.821 \\
3029.130\end{array}$ & $\begin{array}{r}75 \\
250 \\
40 \\
4\end{array}$ & $\begin{array}{l}20 \\
40 \mathrm{w} \\
500\end{array}$ & $\begin{array}{l}\text { II } \\
\text { II I } \\
\text { II } \\
\text { I } \\
\text { I }\end{array}$ \\
\hline $\begin{array}{l}2994.583 \\
2995.167 \\
2995.271 \\
2995.514 \\
2995.815\end{array}$ & $\begin{array}{r}20 \\
100 \\
100 \\
50\end{array}$ & $\begin{array}{r}5 \\
150 \\
100 \\
2000\end{array}$ & $\begin{array}{l}\text { I I } \\
\text { I } \\
\text { I I } \\
\text { I I } \\
\text { I I I }\end{array}$ & $\begin{array}{l}3011.988 \\
3012.704 \\
3012.987 \\
3013.597 \\
3013.774\end{array}$ & $\begin{array}{r}4 \\
100 \\
10 \\
100 \\
4\end{array}$ & $\begin{array}{r}150 \\
5 \\
125\end{array}$ & $\begin{array}{l}\text { I } \\
\text { I I } \\
\text { I I } \\
\text { I I } \\
\text { I }\end{array}$ & $\begin{array}{l}3029.402 \\
3029.604 \\
3030.025 \\
3030.484 \\
3030.712\end{array}$ & $\begin{array}{r}10 \\
5 \\
10 \\
300 \\
20\end{array}$ & $\begin{array}{l}4 \\
2 \\
3\end{array}$ & $\begin{array}{l}\text { II } \\
\text { I } \\
\text { II } \\
\text { I } \\
\text { I }\end{array}$ \\
\hline $\begin{array}{l}2995.959 \\
2996.220 \\
2996.559 \\
2996.986 \\
2997.194\end{array}$ & $\begin{array}{r}150 \\
50 \\
10 \\
400 \\
150\end{array}$ & $\begin{array}{l}50 \\
600 \\
100\end{array}$ & $\begin{array}{l}\text { I } \\
\text { II } \\
\text { I } \\
\text { II } \\
\text { II }\end{array}$ & $\begin{array}{l}3014.207 \\
3014.406 \\
3014.577 \\
3014.632 \\
3014.918\end{array}$ & $\begin{array}{r}10 \\
50 \\
40 \\
40 \\
100\end{array}$ & $\begin{array}{r}40 \\
20 \\
125\end{array}$ & $\begin{array}{l}\text { I } \\
\text { II } \\
\text { I I } \\
\text { I } \\
\text { II }\end{array}$ & $\begin{array}{l}3030.864 \\
3031.195 \\
3031.286 \\
3031.699 \\
3031.953\end{array}$ & $\begin{array}{l}150 \\
150 \\
150 \\
300 \\
300\end{array}$ & $\begin{array}{r}200 \\
8 \\
150 \\
50 \\
400\end{array}$ & $\begin{array}{l}\text { I I } \\
\text { I } \\
\text { I I } \\
\text { I I } \\
\text { I I }\end{array}$ \\
\hline
\end{tabular}


Table 1. Wavelengths of Thorium Spectra - Continued

\begin{tabular}{|c|c|c|c|c|c|c|c|c|c|c|c|}
\hline \multirow{2}{*}{$\begin{array}{l}\text { Wave- } \\
\text { lingth }\end{array}$} & \multicolumn{2}{|c|}{ Intensity } & \multirow{2}{*}{$\begin{array}{l}\text { Spec- } \\
\text { trum }\end{array}$} & \multirow{2}{*}{$\begin{array}{l}\text { Wave- } \\
\text { length }\end{array}$} & \multicolumn{2}{|c|}{ Intensity } & \multirow{2}{*}{$\begin{array}{l}\text { Spec- } \\
\text { trum }\end{array}$} & \multirow{2}{*}{$\begin{array}{l}\text { Wave- } \\
\text { length }\end{array}$} & \multicolumn{2}{|c|}{ Intensity } & \multirow{2}{*}{$\begin{array}{l}\text { Spec- } \\
\text { trum }\end{array}$} \\
\hline & Lamp & Spark & & & Lamp & Spark & & & Lamp & Spark & \\
\hline $\begin{array}{l}3032.004 \\
3032.558 \\
3032.891 \\
3033.135 \\
3033.299\end{array}$ & $\begin{array}{r}100 \\
2 \\
40 \\
3\end{array}$ & $\begin{array}{c}25 \\
4 \\
100 \mathrm{w} \\
30 \mathrm{w}\end{array}$ & $\begin{array}{l}\text { I } \\
\text { I } \\
\text { I } \\
\text { I I I } \\
\text { II }\end{array}$ & $\begin{array}{l}3048.481 \\
3049.093 \\
3049.650 \\
3049.858 \\
3050.310\end{array}$ & $\begin{array}{r}30 \\
1500 \\
200 \\
200 \\
5\end{array}$ & $\begin{array}{r}1 \\
2000 \\
400 \\
400\end{array}$ & $\begin{array}{l}\text { I } \\
\text { II } \\
\text { I I } \\
\text { I I } \\
\text { I I }\end{array}$ & $\begin{array}{l}3069.066 \\
3069.256 \\
3069.657 \\
3069.832 \\
3070.012\end{array}$ & $\begin{array}{r}75 \\
250 \\
20 \\
20 \\
50\end{array}$ & $\begin{array}{r}300 \\
2\end{array}$ & $\begin{array}{l}\text { I } \\
\text { II } \\
\text { I } \\
I \\
I\end{array}$ \\
\hline $\begin{array}{l}3034.063 \\
3034.318 \\
3034.636 \\
3034.886 \\
3035.109\end{array}$ & $\begin{array}{r}600 \\
50 \\
200 \\
40 \\
400\end{array}$ & $\begin{array}{r}1000 \\
50 \\
10 \\
600\end{array}$ & $\begin{array}{l}\text { II } \\
\text { II } \\
\text { I } \\
\text { I } \\
\text { II }\end{array}$ & $\begin{array}{l}3050.627 \\
3050.983 \\
3051.786 \\
3052.120 \\
3052.403\end{array}$ & $\begin{array}{r}150 \\
200 \\
200 \\
40 \\
75\end{array}$ & $\begin{array}{l}400 \\
400 \\
100\end{array}$ & $\begin{array}{l}\text { I } \\
\text { II } \\
\text { I I } \\
\text { I } \\
\text { II }\end{array}$ & $\begin{array}{l}3070.294 \\
3070.816 \\
3070.942 \\
3071.185 \\
3071.621\end{array}$ & $\begin{array}{r}20 \\
600 \\
300 \\
20 \\
20\end{array}$ & $\begin{array}{l}600 \\
300\end{array}$ & $\begin{array}{l}\text { I } \\
\text { II } \\
\text { II } \\
\text { I } \\
\text { I }\end{array}$ \\
\hline $\begin{array}{l}3035.529 \\
3035.753 \\
3036.090 \\
3036.912 \\
3037.107\end{array}$ & $\begin{array}{r}100 \\
100 \\
2 \\
100 \\
50\end{array}$ & $\begin{array}{r}100 \\
125 \\
100 \\
50\end{array}$ & $\begin{array}{l}\text { I I } \\
\text { I I } \\
\text { I } \\
\text { II } \\
\text { II }\end{array}$ & $\begin{array}{l}3052.774 \\
3053.456 \\
3053.528 \\
3053.765 \\
3053.842\end{array}$ & $\begin{array}{r}75 \\
10 \\
75 \\
5 \\
20\end{array}$ & $\begin{array}{c}50 \mathrm{~h} \\
2\end{array}$ & $\begin{array}{l}\text { II } \\
\text { I } \\
\text { I } \\
\text { I } \\
\text { I }\end{array}$ & $\begin{array}{l}3071.776 \\
3072.114 \\
3072.824 \\
3073.430 \\
3073.807\end{array}$ & $\begin{array}{r}10 \\
600 \\
500 \\
75 \\
20\end{array}$ & $\begin{array}{r}600 \\
500 \\
100 \\
1\end{array}$ & $\begin{array}{l}\text { I } \\
\text { II } \\
\text { II } \\
\text { II } \\
\text { I }\end{array}$ \\
\hline $\begin{array}{l}3037.762 \\
3038.007 \\
3038.598 \\
3038.814 \\
3038.984\end{array}$ & $\begin{array}{r}10 \\
125 \\
400 \\
40 \\
20\end{array}$ & $\begin{array}{r}6 \\
150 \\
500 \\
40\end{array}$ & $\begin{array}{l}\text { II } \\
\text { II } \\
\text { II } \\
\text { II } \\
\text { I }\end{array}$ & $\begin{array}{l}3054.944 \\
3055.502 \\
3055.696 \\
3055.814 \\
3056.097\end{array}$ & $\begin{array}{r}3 \\
10 \\
5 \\
30 \\
75\end{array}$ & $\begin{array}{r}1 \\
120\end{array}$ & $\begin{array}{l}\text { II } \\
\text { I } \\
\text { I } \\
\text { I } \\
\text { II }\end{array}$ & $\begin{array}{l}3074.091 \\
3074.249 \\
3074.515 \\
3074.902 \\
3075.056\end{array}$ & $\begin{array}{c}40 \\
5 \mathrm{w} \\
40 \\
5 \\
100\end{array}$ & 300 & $\begin{array}{l}\text { I } \\
\text { I } \\
\text { I } \\
\text { I } \\
\text { I I }\end{array}$ \\
\hline $\begin{array}{l}3039.416 \\
3039.526 \\
3039.652 \\
3040.047 \\
3040.630\end{array}$ & $\begin{array}{r}50 \\
10 \\
4 \\
100 \\
10\end{array}$ & $\begin{array}{r}30 \\
4 \\
75\end{array}$ & $\begin{array}{l}\text { II } \\
\text { II } \\
\text { I } \\
\text { II } \\
\text { I }\end{array}$ & $\begin{array}{l}3056.282 \\
3056.441 \\
3056.527 \\
3056.686 \\
3056.841\end{array}$ & $\begin{array}{r}20 \\
75 \\
5 \\
40 \\
75\end{array}$ & 100 & $\begin{array}{l}\text { I } \\
\text { II } \\
\text { I } \\
\text { I } \\
\text { II }\end{array}$ & $\begin{array}{l}3075.283 \\
3075.525 \\
3075.835 \\
3076.030 \\
3076.407\end{array}$ & $\begin{array}{l}20 \\
20 \\
50 \\
50 \\
50\end{array}$ & $\begin{array}{r}3 \\
30 \\
75 \\
1\end{array}$ & $\begin{array}{l}\text { I } \\
\text { I } \\
\text { II } \\
\text { II } \\
\text { I }\end{array}$ \\
\hline $\begin{array}{l}3040.875 \\
3041.508 \\
3041.934 \\
3042.302 \\
3042.633\end{array}$ & $\begin{array}{r}3 \\
100 \\
50 \\
40 \\
40\end{array}$ & $\begin{array}{r}100 \\
30\end{array}$ & $\begin{array}{l}\text { I } \\
\text { II } \\
\text { I } \\
\text { II } \\
\text { I }\end{array}$ & $\begin{array}{l}3057.630 \\
3057.888 \\
3058.023 \\
3058.126 \\
3058.422\end{array}$ & $\begin{array}{r}150 \\
75 \\
125 \\
75 \\
200\end{array}$ & $\begin{array}{r}150 \\
100 \\
\\
50 \\
400\end{array}$ & $\begin{array}{l}\text { II } \\
\text { II } \\
\text { I } \\
\text { II } \\
\text { II }\end{array}$ & $\begin{array}{l}3077.338 \\
3077.715 \\
3077.931 \\
3078.828 \\
3078.980\end{array}$ & $\begin{array}{r}200 \\
200 \\
100 \\
2000\end{array}$ & $\begin{array}{r}400 \\
100 \\
3000 \\
2000\end{array}$ & $\begin{array}{l}\text { II } \\
\text { I } \\
\text { II } \\
\text { II } \\
\text { II I }\end{array}$ \\
\hline $\begin{array}{l}3042.796 \\
3043.060 \\
3043.243 \\
3043.601 \\
3044.030\end{array}$ & $\begin{array}{r}40 \\
200 \\
150 \\
20 \\
1\end{array}$ & $\begin{array}{r}250 \\
150 \\
6\end{array}$ & $\begin{array}{l}\text { I } \\
\text { II } \\
\text { II } \\
\text { II } \\
\text { I }\end{array}$ & $\begin{array}{l}3059.695 \\
3060.177 \\
3060.440 \\
3061.246 \\
3061.698\end{array}$ & $\begin{array}{l}50 \\
300 \\
600 \\
75 \mathrm{w} \\
150\end{array}$ & $\begin{array}{c}900 \\
2 \\
100 \mathrm{w} \\
1200\end{array}$ & $\begin{array}{l}\text { I } \\
\text { II } \\
\text { I } \\
\text { II } \\
\text { II }\end{array}$ & $\begin{array}{l}3079.566 \\
3079.887 \\
3080.216 \\
3080.830 \\
3081.023\end{array}$ & $\begin{array}{r}75 \\
125 \\
1000 \\
75 \\
40\end{array}$ & $\begin{array}{r}3 \\
125 \\
1000 \\
25 \\
20\end{array}$ & $\begin{array}{l}\text { I } \\
\text { II } \\
\text { II } \\
\text { II } \\
\text { II }\end{array}$ \\
\hline $\begin{array}{l}3044.336 \\
3044.583 \\
3044.744 \\
3045.060 \\
3045.317\end{array}$ & $\begin{array}{l}10 \\
20 \\
50 \\
50 \\
10\end{array}$ & $\begin{array}{l}60 \\
40\end{array}$ & $\begin{array}{l}\text { I } \\
\text { I } \\
\text { II } \\
\text { II } \\
\text { I }\end{array}$ & $\begin{array}{l}3061.811 \\
3062.456 \\
3063.027 \\
3063.107 \\
3065.030\end{array}$ & $\begin{array}{r}30 \\
200 \\
1000 \\
10 \\
100\end{array}$ & $\begin{array}{c}1000 \mathrm{w} \\
20 \mathrm{w} \\
125\end{array}$ & $\begin{array}{l}\text { I } \\
\text { I } \\
\text { I } \\
\text { II } \\
\text { II }\end{array}$ & $\begin{array}{l}3081.657 \\
3081.978 \\
3082.167 \\
3082.504 \\
3082.998\end{array}$ & $\begin{array}{l}100 \\
200 \\
200 \\
100 \\
150\end{array}$ & $\begin{array}{l}150 \\
300 \\
150 \\
\\
125\end{array}$ & $\begin{array}{l}\text { I I } \\
\text { I I } \\
\text { II } \\
\text { I } \\
\text { II }\end{array}$ \\
\hline $\begin{array}{l}3045.557 \\
3045.764 \\
3046.030 \\
3046.142 \\
3046.438\end{array}$ & $\begin{array}{r}500 \\
20 \\
50 \\
50 \\
20\end{array}$ & 600 & $\begin{array}{l}\text { II } \\
\text { I } \\
\text { I } \\
\text { II } \\
\text { I }\end{array}$ & $\begin{array}{l}3065.315 \\
3065.681 \\
3065.928 \\
3066.297 \\
3066.410\end{array}$ & $\begin{array}{r}10 \\
200 \\
400 \\
100\end{array}$ & $\begin{array}{l}300 \\
200 \\
150\end{array}$ & $\begin{array}{l}\text { I } \\
\text { I } \\
\text { II } \\
\text { II I } \\
\text { II }\end{array}$ & $\begin{array}{l}3083.294 \\
3083.605 \\
3083.834 \\
3084.506 \\
3085.465\end{array}$ & $\begin{array}{r}100 \\
40 \\
50 \\
40 \\
40\end{array}$ & $\begin{array}{c}800 \mathrm{w} \\
4 \\
8 \\
10 \\
50\end{array}$ & $\begin{array}{l}\text { III } \\
\text { I } \\
\text { II } \\
\text { II } \\
\text { II }\end{array}$ \\
\hline $\begin{array}{l}3046.952 \\
3047.192 \\
3047.813 \\
3048.027 \\
3048.229\end{array}$ & $\begin{array}{r}400 \\
5 \\
200 \\
10 \\
30\end{array}$ & $\begin{array}{r}600 \\
1 \\
10 \\
20\end{array}$ & $\begin{array}{l}\text { II } \\
\text { I } \\
\text { I } \\
\text { II } \\
\text { II }\end{array}$ & $\begin{array}{l}3066.797 \\
3067.726 \\
3068.256 \\
3068.904 \\
3068.972\end{array}$ & $\begin{array}{r}10 \\
1000 \\
50 \\
800 \\
200\end{array}$ & $\begin{array}{c}2000 \mathrm{w} \\
50 \\
100 \\
300\end{array}$ & $\begin{array}{l}\text { I } \\
\text { II } \\
\text { II } \\
\text { I } \\
\text { II }\end{array}$ & $\begin{array}{l}3085.774 \\
3086.200 \\
3086.353 \\
3086.560 \\
3086.855\end{array}$ & $\begin{array}{l}50 \\
2 \mathrm{w} \\
20 \\
50 \\
40\end{array}$ & $\begin{array}{l}10 \\
40\end{array}$ & $\begin{array}{l}\text { I } \\
\text { II } \\
\text { II } \\
\text { II } \\
\text { I }\end{array}$ \\
\hline
\end{tabular}


Table 1. Wavelengths of Thorium Spectra - Continued

\begin{tabular}{|c|c|c|c|c|c|c|c|c|c|c|c|}
\hline \multirow{2}{*}{$\begin{array}{l}\text { Wave- } \\
\text { length }\end{array}$} & \multicolumn{2}{|c|}{ Intensity } & \multirow{2}{*}{$\begin{array}{l}\text { Spec- } \\
\text { trum }\end{array}$} & \multirow{2}{*}{$\begin{array}{l}\text { Wave- } \\
\text { length }\end{array}$} & \multicolumn{2}{|c|}{ Intensity } & \multirow{2}{*}{$\begin{array}{l}\text { Spec- } \\
\text { trum }\end{array}$} & \multirow{2}{*}{$\begin{array}{l}\text { Wave- } \\
\text { length }\end{array}$} & \multicolumn{2}{|c|}{ Intensity } & \multirow{2}{*}{$\begin{array}{l}\text { Spec- } \\
\text { trum }\end{array}$} \\
\hline & Lamp & Spark & & & Lamp & Spark & & & & & \\
\hline $\begin{array}{l}3086.919 \\
3087.270 \\
3087.410 \\
3087.679 \\
3087.996\end{array}$ & $\begin{array}{r}5 \\
5 \\
75 \\
50 \\
40\end{array}$ & $\begin{array}{l}5 \\
2 \\
3\end{array}$ & $\begin{array}{l}\text { I } \\
\text { II } \\
\text { I } \\
\text { I } \\
\text { I }\end{array}$ & $\begin{array}{l}3107.035 \\
3107.345 \\
3107.622 \\
3107.735 \\
3108.299\end{array}$ & $\begin{array}{r}400 \\
50 \\
10 \\
10 \\
1500\end{array}$ & $\begin{array}{r}800 \\
75 \\
\\
2000\end{array}$ & $\begin{array}{l}\text { I I } \\
\text { I I } \\
\text { I } \\
\text { I } \\
\text { II }\end{array}$ & $\begin{array}{l}3125.741 \\
3125.866 \\
3126.055 \\
3126.258 \\
3126.404\end{array}$ & $\begin{array}{r}500 \\
200 \\
100 \\
40 \\
40\end{array}$ & $\begin{array}{l}500 \\
150 \\
200\end{array}$ & $\begin{array}{l}\text { II } \\
\text { I I } \\
\text { I I } \\
\text { I } \\
\text { I }\end{array}$ \\
\hline $\begin{array}{l}3088.473 \\
3088.835 \\
3089.627 \\
3090.091 \\
3090.650\end{array}$ & $\begin{array}{r}400 \\
75 \\
100 \\
250 \\
30\end{array}$ & $\begin{array}{r}600 \\
75 \\
400 \\
250 \\
10\end{array}$ & $\begin{array}{l}\text { II I } \\
\text { I I } \\
\text { II } \\
\text { II } \\
\text { I I }\end{array}$ & $\begin{array}{l}3108.460 \\
3110.026 \\
3110.826 \\
3110.944 \\
3111.062\end{array}$ & $\begin{array}{r}20 \\
400 \\
75 \\
20 \\
10\end{array}$ & $\begin{array}{r}1200 \\
25\end{array}$ & $\begin{array}{l}\text { I } \\
\text { I I } \\
\text { II } \\
\text { I } \\
\text { I }\end{array}$ & $\begin{array}{l}3126.616 \\
3126.924 \\
3127.041 \\
3127.212 \\
3127.555\end{array}$ & $\begin{array}{r}10 \\
20 \\
20 \\
200 \\
100\end{array}$ & $\begin{array}{l}400 \\
100\end{array}$ & $\begin{array}{l}\text { I } \\
\text { I } \\
\text { I } \\
\text { II } \\
\text { I I }\end{array}$ \\
\hline $\begin{array}{l}3091.642 \\
3091.849 \\
3092.061 \\
3092.326 \\
3092.825\end{array}$ & $\begin{array}{r}75 \\
20 \\
40 \\
100 \\
125\end{array}$ & $\begin{array}{r}100 \\
1 \\
125\end{array}$ & $\begin{array}{l}\text { II } \\
\text { I } \\
\text { I } \\
\text { II } \\
\text { I }\end{array}$ & $\begin{array}{l}3111.273 \\
3111.428 \\
3111.767 \\
3111.830 \\
3112.088\end{array}$ & $\begin{array}{r}100 \\
100 \\
100 \\
75 \\
100\end{array}$ & $\begin{array}{r}100 \\
25 \\
30 \\
200\end{array}$ & $\begin{array}{l}\text { I } \\
\text { I I } \\
\text { II } \\
\text { I I } \\
\text { I I }\end{array}$ & $\begin{array}{l}3127.718 \\
3127.856 \\
3128.309 \\
3128.582 \\
3129.279\end{array}$ & $\begin{array}{l}40 \\
50 \\
50 \mathrm{w} \\
50 \\
50\end{array}$ & $\begin{array}{l}100 \mathrm{w} \\
200 \\
200\end{array}$ & $\begin{array}{l}\text { I } \\
\text { II } \\
\text { I } \\
\text { II } \\
\text { II }\end{array}$ \\
\hline $\begin{array}{l}3093.048 \\
3093.717 \\
3094.011 \\
3094.701 \\
3095.043\end{array}$ & $\begin{array}{r}75 \\
125 \\
100 \\
50 \\
10\end{array}$ & $\begin{array}{r}100 \\
2 \\
1 \\
75\end{array}$ & $\begin{array}{l}\text { II } \\
\text { I } \\
\text { I } \\
\text { II } \\
\text { I }\end{array}$ & $\begin{array}{l}3112.400 \\
3112.925 \\
3113.246 \\
3113.421 \\
3113.878\end{array}$ & $\begin{array}{l}20 \\
75 \\
75 \mathrm{w} \\
1\end{array}$ & $\begin{array}{c}1000 \\
50 \\
6 \\
50 \mathrm{w}\end{array}$ & $\begin{array}{l}\text { I I I } \\
\text { I } \\
\text { II } \\
\text { I } \\
\text { III }\end{array}$ & $\begin{array}{l}3129.977 \\
3130.266 \\
3130.426 \\
3130.793 \\
3131.072\end{array}$ & $\begin{array}{r}50 \\
50 \\
50 \\
75 \\
200\end{array}$ & $\begin{array}{r}400 \\
20 \\
\\
50 \\
750\end{array}$ & $\begin{array}{l}\text { I I } \\
\text { I I } \\
\text { I } \\
\text { II } \\
\text { I I }\end{array}$ \\
\hline $\begin{array}{l}3095.616 \\
3095.747 \\
3096.266 \\
3096.428 \\
3096.864\end{array}$ & $\begin{array}{r}75 \\
40 \\
100 \\
400 \\
50\end{array}$ & $\begin{array}{l}40 \\
40 \\
150 \\
300 \\
30 \mathrm{~h}\end{array}$ & $\begin{array}{l}\text { II } \\
\text { I I } \\
\text { I I } \\
\text { I I } \\
\text { I I }\end{array}$ & $\begin{array}{l}3114.054 \\
3114.268 \\
3115.191 \\
3115.413 \\
3115.539\end{array}$ & $\begin{array}{r}150 \\
100 \\
40 \\
20 \\
50\end{array}$ & $\begin{array}{l}200 \\
200 \\
10 \mathrm{w} \\
40\end{array}$ & $\begin{array}{l}\text { I I } \\
\text { I I } \\
\text { I I } \\
\text { I I } \\
\text { I }\end{array}$ & $\begin{array}{l}3132.249 \\
3132.389 \\
3132.824 \\
3133.620 \\
3133.983\end{array}$ & $\begin{array}{r}20 \\
75 \\
20 \\
100 \\
100\end{array}$ & $\begin{array}{r}40 \\
25 \\
10 \\
500\end{array}$ & $\begin{array}{l}\text { II } \\
\text { I I } \\
\text { I } \\
\text { II } \\
\text { I }\end{array}$ \\
\hline $\begin{array}{l}3097.137 \\
3097.270 \\
3097.956 \\
3098.119 \\
3098.328\end{array}$ & $\begin{array}{r}20 \\
300 \\
\\
20 \\
50\end{array}$ & $\begin{array}{r}20 \\
300 \\
500\end{array}$ & $\begin{array}{l}\text { I I } \\
\text { I I } \\
\text { I I I } \\
\text { I } \\
\text { I }\end{array}$ & $\begin{array}{l}3115.742 \\
3115.943 \\
3116.282 \\
3116.483 \\
3117.152\end{array}$ & $\begin{array}{r}100 \\
5 \\
1000 \\
200 \\
100\end{array}$ & $\begin{array}{r}300 \\
1000 \\
400 \\
200\end{array}$ & $\begin{array}{l}\text { II } \\
\text { II } \\
\text { II } \\
\text { II } \\
\text { II }\end{array}$ & $\begin{array}{l}3134.206 \\
3134.425 \\
3134.718 \\
3134.889 \\
3135.084\end{array}$ & $\begin{array}{r}50 \\
200 \\
20 \\
40 \\
30\end{array}$ & $\begin{array}{l}1000 \\
300\end{array}$ & $\begin{array}{l}\text { I } \\
\text { II } \\
\text { I } \\
\text { I } \\
\text { I I }\end{array}$ \\
\hline $\begin{array}{l}3098.390 \\
3098.725 \\
3099.735 \\
3099.871 \\
3100.792\end{array}$ & $\begin{array}{r}40 \\
100 \\
100 \\
100 \\
75\end{array}$ & $\begin{array}{r}125 \\
100 \\
30\end{array}$ & $\begin{array}{l}\text { I } \\
\text { I } \\
\text { I I } \\
\text { I I } \\
\text { I I }\end{array}$ & $\begin{array}{l}3117.689 \\
3118.473 \\
3118.909 \\
3119.365 \\
3119.517\end{array}$ & $\begin{array}{r}200 \\
40 \\
10 \\
300 \\
1000\end{array}$ & $\begin{array}{r}600 \\
40 \\
\\
250 \\
2500\end{array}$ & $\begin{array}{l}\text { II } \\
\text { II } \\
\text { I } \\
\text { II } \\
\text { II }\end{array}$ & $\begin{array}{l}3135.163 \\
3136.217 \\
3136.709 \\
3136.834 \\
3137.171\end{array}$ & $\begin{array}{r}40 \\
700 \\
50 \\
350 \\
40\end{array}$ & $\begin{array}{r}4 \\
100 \\
200 \\
\\
200\end{array}$ & $\begin{array}{l}\text { I } \\
\text { I } \\
\text { II } \\
\text { I } \\
\text { II }\end{array}$ \\
\hline $\begin{array}{l}3100.932 \\
3101.684 \\
3102.127 \\
3102.490 \\
3102.666\end{array}$ & $\begin{array}{c}75 \\
250 \\
75 \mathrm{w} \\
30 \\
500\end{array}$ & $\begin{array}{r}1500 \\
300\end{array}$ & $\begin{array}{l}\text { II } \\
\text { II } \\
\text { I } \\
\text { I } \\
\text { II }\end{array}$ & $\begin{array}{l}3119.965 \\
3120.158 \\
3120.451 \\
3120.882 \\
3121.009\end{array}$ & $\begin{array}{r}40 \\
100 \\
75 \\
300 \\
100\end{array}$ & $\begin{array}{r}100 \\
75 \\
200\end{array}$ & $\begin{array}{l}\text { I } \\
\text { II } \\
\text { II } \\
\text { I } \\
\text { II }\end{array}$ & $\begin{array}{l}3137.418 \\
3137.940 \\
3138.199 \\
3138.322 \\
3138.585\end{array}$ & $\begin{array}{l}20 \\
20 \\
40 \\
50 \\
10\end{array}$ & 100 & $\begin{array}{l}\text { I } \\
\text { I } \\
\text { I } \\
\text { II } \\
\text { I }\end{array}$ \\
\hline $\begin{array}{l}3103.186 \\
3103.767 \\
3103.998 \\
3104.462 \\
3104.894\end{array}$ & $\begin{array}{c}50 \mathrm{w} \\
20 \\
40 \\
150 \\
30\end{array}$ & $\begin{array}{r}2 \\
150\end{array}$ & $\begin{array}{l}\text { I } \\
\text { I } \\
\text { I } \\
\text { I I } \\
\text { I }\end{array}$ & $\begin{array}{l}3122.107 \\
3122.332 \\
3122.400 \\
3122.571 \\
3122.968\end{array}$ & $\begin{array}{r}2 \\
50 \\
5 \\
1000\end{array}$ & $\begin{array}{c}50 \\
500 \mathrm{w} \\
3000\end{array}$ & $\begin{array}{l}\text { II } \\
\text { I } \\
\text { II I } \\
\text { I } \\
\text { II }\end{array}$ & $\begin{array}{l}3139.235 \\
3139.301 \\
3139.692 \\
3139.890 \\
3140.070\end{array}$ & $\begin{array}{r}20 \\
2000 \\
10 \\
150 \\
20\end{array}$ & $\begin{array}{r}1000 \\
150 \\
30\end{array}$ & $\begin{array}{l}\text { I } \\
\text { I I } \\
\text { I } \\
\text { II } \\
\text { I I }\end{array}$ \\
\hline $\begin{array}{l}3104.959 \\
3105.055 \\
3105.480 \\
3105.753 \\
3106.683\end{array}$ & $\begin{array}{l}100 \\
200 \\
100 \\
700 \\
150\end{array}$ & $\begin{array}{r}400 \\
400 \\
1200 \\
200\end{array}$ & $\begin{array}{l}\text { I } \\
\text { I I } \\
\text { I I } \\
\text { II } \\
\text { I I }\end{array}$ & $\begin{array}{l}3123.154 \\
3124.002 \\
3124.390 \\
3125.210 \\
3125.513\end{array}$ & $\begin{array}{r}50 \\
100 \\
400 \\
300 \\
800\end{array}$ & $\begin{array}{r}75 \\
2000 \\
1000 \\
2400\end{array}$ & $\begin{array}{l}\text { I } \\
\text { I I } \\
\text { II } \\
\text { I I } \\
\text { I I }\end{array}$ & $\begin{array}{l}3140.274 \\
3140.489 \\
3140.944 \\
3141.333 \\
3141.841\end{array}$ & $\begin{array}{r}700 \\
75 \\
50 \\
40 \\
100\end{array}$ & $\begin{array}{r}75 \\
30 \\
100 \\
50 \\
250\end{array}$ & $\begin{array}{l}\text { I } \\
\text { I I } \\
\text { I I } \\
\text { I I } \\
\text { I I }\end{array}$ \\
\hline
\end{tabular}


Table 1. Wavelengths of Thorium Spectra - Continued

\begin{tabular}{|c|c|c|c|c|c|c|c|c|c|c|c|}
\hline \multirow{2}{*}{$\begin{array}{l}\text { Wave- } \\
\text { length }\end{array}$} & \multicolumn{2}{|c|}{ Intensity } & \multirow{2}{*}{$\begin{array}{l}\text { Spec- } \\
\text { trum }\end{array}$} & \multirow{2}{*}{$\begin{array}{l}\text { Wave- } \\
\text { length }\end{array}$} & \multicolumn{2}{|c|}{ Intensity } & \multirow{2}{*}{$\begin{array}{l}\text { Spec- } \\
\text { trum }\end{array}$} & \multirow{2}{*}{$\begin{array}{l}\text { Wave- } \\
\text { length }\end{array}$} & \multicolumn{2}{|c|}{ Intensity } & \multirow{2}{*}{$\begin{array}{l}\text { Spec } \\
\text { trum }\end{array}$} \\
\hline & $9 m$ & Spark & & & $\mathrm{amp}$ & Spark & & & $\mathrm{am}$ & Spar & \\
\hline $\begin{array}{l}3142.341 \\
3142.434 \\
3142.533 \\
3142.686 \\
3142.838\end{array}$ & $\begin{array}{r}30 \\
40 \\
100 \\
75 \\
150\end{array}$ & 2000 & $\begin{array}{l}\text { I I I } \\
\text { I } \\
\text { I } \\
\text { I } \\
\text { I I }\end{array}$ & $\begin{array}{l}3160.819 \\
3161.070 \\
3161.159 \\
3161.360 \\
3161.684\end{array}$ & $\begin{array}{l}50 \\
50 \\
40 \mathrm{~h} \\
200 \\
150\end{array}$ & $\begin{array}{l}75 \mathrm{w} \\
40 \\
\\
400\end{array}$ & $\begin{array}{l}\text { II } \\
\text { II } \\
\text { I } \\
\text { I } \\
\text { II }\end{array}$ & $\begin{array}{l}3177.880 \\
3178.246 \\
3178.618 \\
3178.783 \\
3179.049\end{array}$ & $\begin{array}{r}75 \\
600 \\
75 \\
75 \\
500\end{array}$ & $\begin{array}{c}200 \mathrm{w} \\
50 \\
150 \\
1000\end{array}$ & $\begin{array}{l}\text { II } \\
\text { I } \\
\text { I } \\
\text { II } \\
\text { I I }\end{array}$ \\
\hline $\begin{array}{l}3143.425 \\
3144.128 \\
3144.500 \\
3144.695 \\
3145.275\end{array}$ & $\begin{array}{r}2 \\
75 \\
40 \\
75 \\
50\end{array}$ & $\begin{array}{r}20 \\
75 \\
100\end{array}$ & $\begin{array}{l}\text { I } \\
\text { I } \\
\text { II } \\
\text { II } \\
\text { II }\end{array}$ & $\begin{array}{l}3162.071 \\
3162.576 \\
3162.835 \\
3163.079 \\
3163.239\end{array}$ & $\begin{array}{r}10 \\
75 \\
100 \\
40 \\
40\end{array}$ & $\begin{array}{c}50 \mathrm{w} \\
700 \\
30 \\
40\end{array}$ & $\begin{array}{l}\text { I } \\
\text { I I } \\
\text { I I } \\
\text { I I } \\
\text { I I }\end{array}$ & $\begin{array}{l}3180.199 \\
3181.185 \\
3181.663 \\
3181.780 \\
3182.042\end{array}$ & $\begin{array}{c}2000 \\
100 \\
400 \mathrm{w} \\
2 \\
20\end{array}$ & $\begin{array}{r}5000 \\
700 \\
20 \\
2\end{array}$ & $\begin{array}{l}\text { II } \\
\text { I I } \\
\text { I } \\
\text { II } \\
\text { I }\end{array}$ \\
\hline $\begin{array}{l}3145.636 \\
3146.044 \\
3146.303 \\
3146.806 \\
3147.083\end{array}$ & $\begin{array}{r}400 \\
200 \\
100 \\
40 \\
20\end{array}$ & $\begin{array}{r}100 \\
2000\end{array}$ & $\begin{array}{l}\text { I } \\
\text { II } \\
\text { I } \\
\text { II } \\
\text { I I }\end{array}$ & $\begin{array}{l}3163.495 \\
3163.708 \\
3163.866 \\
3164.301 \\
3164.483\end{array}$ & $\begin{array}{r}20 \\
100 \\
40 \\
75 \\
100\end{array}$ & $\begin{array}{r}20 \\
700\end{array}$ & $\begin{array}{l}\text { I } \\
\text { I } \\
\text { I } \\
\text { II } \\
\text { II }\end{array}$ & $\begin{array}{l}3182.402 \\
3182.638 \\
3183.142 \\
3183.375 \\
3183.552\end{array}$ & $\begin{array}{r}200 \\
250 \\
20 \\
40 \\
150\end{array}$ & $\begin{array}{r}300 \\
400 \\
40 \\
\\
200\end{array}$ & $\begin{array}{l}\text { I I } \\
\text { II } \\
\text { I I } \\
\text { I } \\
\text { II }\end{array}$ \\
\hline $\begin{array}{l}3147.447 \\
3147.737 \\
3148.056 \\
3148.226 \\
3149.409\end{array}$ & $\begin{array}{r}150 \\
40 \\
\\
50 \\
20\end{array}$ & 500 & $\begin{array}{l}\text { I } \\
\text { I } \\
\text { I I I } \\
\text { I } \\
\text { I }\end{array}$ & $\begin{array}{l}3165.036 \\
3165.623 \\
3165.820 \\
3166.094 \\
3166.190\end{array}$ & $\begin{array}{l}50 \\
75 \\
250 \\
300 \\
20 \mathrm{w}\end{array}$ & $\begin{array}{r}3 \\
300 \\
500 \\
600\end{array}$ & $\begin{array}{l}\text { I } \\
\text { I I } \\
\text { I I } \\
\text { I I } \\
\text { I }\end{array}$ & $\begin{array}{l}3183.790 \\
3183.977 \\
3184.264 \\
3184.607 \\
3184.946\end{array}$ & $\begin{array}{r}100 \\
75 \\
200 \\
50 \\
500\end{array}$ & $\begin{array}{r}200 \\
100 \\
\\
75 \\
1000\end{array}$ & $\begin{array}{l}\text { I I } \\
\text { I I } \\
\text { I } \\
\text { II } \\
\text { II }\end{array}$ \\
\hline $\begin{array}{l}3149.599 \\
3149.963 \\
3150.457 \\
3151.017 \\
3151.182\end{array}$ & $\begin{array}{r}20 \\
50 \\
300 \\
50 \\
75\end{array}$ & $\begin{array}{r}75 \\
800\end{array}$ & $\begin{array}{l}\text { I } \\
\text { II } \\
\text { II } \\
\text { I } \\
\text { I }\end{array}$ & $\begin{array}{l}.811 \\
.553 \\
3.010 \\
.315 \\
3.724\end{array}$ & $\begin{array}{l}75 \\
200 \\
150 \\
20 \mathrm{w} \\
20\end{array}$ & $\begin{array}{c}10 \\
500 \\
4 \\
10 \\
10 \mathrm{~h}\end{array}$ & $\begin{array}{l}\text { I } \\
\text { I I } \\
\text { I } \\
\text { II } \\
\text { I I }\end{array}$ & $\begin{array}{l}3185.629 \\
3186.421 \\
3186.994 \\
3187.399 \\
3187.466\end{array}$ & $\begin{array}{l}75 \mathrm{w} \\
10 \mathrm{w} \\
100 \\
300 \\
20\end{array}$ & $\begin{array}{r}20 \\
200 \\
75 \\
5\end{array}$ & $\begin{array}{l}\text { II } \\
\text { I } \\
\text { II } \\
\text { II } \\
\text { II }\end{array}$ \\
\hline $\begin{array}{l}3151.647 \\
3151.799 \\
3152.285 \\
3152.391 \\
3153.209\end{array}$ & $\begin{array}{l}100 \\
50 \mathrm{~h} \\
50 \\
50 \\
50\end{array}$ & $\begin{array}{l}600 \\
200 h\end{array}$ & $\begin{array}{l}\text { I I } \\
\text { I I } \\
\text { I } \\
\text { I } \\
\text { I I }\end{array}$ & $\begin{array}{l}3168.905 \\
3168.972 \\
3169.324 \\
3169.808 \\
3169.937\end{array}$ & $\begin{array}{r}30 \\
10 \\
150 \\
50 \\
40\end{array}$ & $\begin{array}{c}600 \\
75 w \\
2\end{array}$ & $\begin{array}{l}\text { I } \\
\text { I } \\
\text { I I } \\
\text { I I } \\
\text { I }\end{array}$ & $\begin{array}{l}3188.230 \\
3188.292 \\
3189.040 \\
3189.558 \\
3189.710\end{array}$ & $\begin{array}{r}500 \\
200 \\
5 \\
50 \\
125\end{array}$ & $\begin{array}{r}1000 \\
500 \\
2\end{array}$ & $\begin{array}{l}\text { I I } \\
\text { I I } \\
\text { II } \\
\text { I } \\
\text { I }\end{array}$ \\
\hline $\begin{array}{l}3153.632 \\
3153.754 \\
3154.026 \\
3154.299 \\
3154.768\end{array}$ & $\begin{array}{r}50 \\
20 \\
10 \\
1000 \\
800\end{array}$ & $\begin{array}{r}1 \\
5 \\
1200 \\
1500\end{array}$ & $\begin{array}{l}\text { I } \\
\text { I } \\
\text { I } \\
\text { I I } \\
\text { I I }\end{array}$ & $\begin{array}{l}3170.419 \\
3171.268 \\
3171.692 \\
3172.110 \\
3172.499\end{array}$ & $\begin{array}{r}100 \\
400 \\
100 \\
100 \\
50\end{array}$ & $\begin{array}{r}200 \\
20 \\
200 \\
200 \\
150\end{array}$ & $\begin{array}{l}\text { II } \\
\text { I } \\
\text { II } \\
\text { I I } \\
\text { I I }\end{array}$ & $\begin{array}{l}3190.068 \\
3190.171 \\
3190.352 \\
3190.638 \\
3190.720\end{array}$ & $\begin{array}{r}300 \\
300 \\
200 \\
2 \\
100\end{array}$ & $\begin{array}{r}600 \\
400 \\
150 \\
4 \\
200\end{array}$ & $\begin{array}{l}\text { I I } \\
\text { I I } \\
\text { I I } \\
\text { I I } \\
\text { I I }\end{array}$ \\
\hline $\begin{array}{l}3155.583 \\
3155.833 \\
3156.392 \\
3156.848 \\
3157.220\end{array}$ & $\begin{array}{r}50 \\
200 \\
200 \\
75 \\
200\end{array}$ & $\begin{array}{r}20 \\
500 \\
300 \\
10 \\
200\end{array}$ & $\begin{array}{l}\text { I I } \\
\text { I I } \\
\text { I I } \\
\text { I } \\
\text { I I }\end{array}$ & $\begin{array}{l}3173.057 \\
3173.211 \\
3173.426 \\
3174.020 \\
3174.198\end{array}$ & $\begin{array}{r}40 \\
75 \\
400 \\
50 \\
150\end{array}$ & $\begin{array}{r}2 \\
150 \\
20 \\
\\
500\end{array}$ & $\begin{array}{l}\text { I } \\
\text { II } \\
\text { I } \\
\text { I } \\
\text { II }\end{array}$ & $\begin{array}{l}3191.085 \\
3191.207 \\
3191.838 \\
3192.106 \\
3192.573\end{array}$ & $\begin{array}{r}150 \\
150 \\
50 \\
100 \\
400\end{array}$ & $\begin{array}{r}125 \\
150 \\
50 \\
100 \\
8\end{array}$ & $\begin{array}{l}\text { II } \\
\text { II } \\
\text { II } \\
\text { I I } \\
\text { I }\end{array}$ \\
\hline $\begin{array}{l}3157.333 \\
3157.506 \\
3157.912 \\
3158.391 \\
3158.615\end{array}$ & $\begin{array}{c}5 \mathrm{w} \\
40 \\
75 \\
75 \\
100\end{array}$ & $\begin{array}{r}50 \\
6 \\
200\end{array}$ & $\begin{array}{l}\text { I } \\
\text { I } \\
\text { I I } \\
\text { I } \\
\text { I I }\end{array}$ & $\begin{array}{l}3174.451 \\
3174.832 \\
3175.045 \\
3175.728 \\
3176.163\end{array}$ & $\begin{array}{r}75 \\
100 \\
10 \\
500 \\
200\end{array}$ & $\begin{array}{r}200 \\
5 \\
2000\end{array}$ & $\begin{array}{l}\text { II } \\
\text { I } \\
\text { II } \\
\text { II } \\
\text { I }\end{array}$ & $\begin{array}{l}3192.848 \\
3193.162 \\
3193.361 \\
3193.781 \\
3194.088\end{array}$ & $\begin{array}{r}20 \\
150 \\
150 \\
150 \\
50\end{array}$ & $\begin{array}{l}200 \\
200\end{array}$ & $\begin{array}{l}\text { I } \\
\text { II } \\
\text { I I } \\
\text { I } \\
\text { I }\end{array}$ \\
\hline $\begin{array}{l}3158.859 \\
3159.070 \\
3159.701 \\
3160.170 \\
3160.356\end{array}$ & $\begin{array}{r}40 \\
100 \\
75 \\
75 \\
40\end{array}$ & $\begin{array}{r}4 \\
100 \\
50 \\
75 \\
10\end{array}$ & $\begin{array}{l}\text { I } \\
\text { I I } \\
\text { I I } \\
\text { I I } \\
\text { I I }\end{array}$ & $\begin{array}{l}3176.504 \\
3177.194 \\
3177.397 \\
3177.490 \\
3177.770\end{array}$ & $\begin{array}{r}100 \\
100 \\
75 \\
50 \\
50\end{array}$ & $\begin{array}{r}500 \\
300 \\
10 \\
10 \\
4\end{array}$ & $\begin{array}{l}\text { I I } \\
\text { II } \\
\text { I I } \\
\text { I I } \\
\text { I I }\end{array}$ & $\begin{array}{l}3194.247 \\
3194.843 \\
3195.162 \\
3195.316 \\
3195.687\end{array}$ & $\begin{array}{r}20 \\
10 \\
100 \\
100 \\
400\end{array}$ & $\begin{array}{c}20 \mathrm{~h} \\
10 \mathrm{~h} \\
40 \\
150 \\
15\end{array}$ & $\begin{array}{l}\text { II } \\
\text { II } \\
\text { II } \\
\text { II } \\
\text { I }\end{array}$ \\
\hline
\end{tabular}


Table 1. Wavelengths of Thorium Spectra - Continued

\begin{tabular}{|c|c|c|c|c|c|c|c|c|c|c|c|}
\hline \multirow{2}{*}{$\begin{array}{l}\text { Wave- } \\
\text { length }\end{array}$} & \multicolumn{2}{|c|}{ Intensity } & \multirow{2}{*}{$\begin{array}{l}\text { Spec- } \\
\text { trum }\end{array}$} & \multirow{2}{*}{$\begin{array}{l}\text { Wave- } \\
\text { length }\end{array}$} & \multicolumn{2}{|c|}{ Intensity } & \multirow{2}{*}{$\begin{array}{l}\text { Spec- } \\
\text { trum }\end{array}$} & \multirow{2}{*}{$\begin{array}{l}\text { Wave- } \\
\text { length }\end{array}$} & \multicolumn{2}{|c|}{ Intensity } & \multirow{2}{*}{$\begin{array}{l}\text { Spec } \\
\text { trum }\end{array}$} \\
\hline & Lamp & Spark & & & Lamp & & & & & spa & \\
\hline $\begin{array}{l}3196.606 \\
3196.754 \\
3197.567 \\
3197.703 \\
3198.052\end{array}$ & $\begin{array}{l}40 \\
20 \\
75 \\
75 \\
40\end{array}$ & $\begin{array}{c}1 \\
75 \mathrm{w} \\
50\end{array}$ & $\begin{array}{l}\text { I } \\
\text { I } \\
\text { II } \\
\text { II } \\
\text { I }\end{array}$ & $\begin{array}{l}3215.101 \\
3215.782 \\
3215.899 \\
3216.340 \\
3216.532\end{array}$ & $\begin{array}{r}10 \\
400 \\
100 \\
5 \\
40\end{array}$ & $\begin{array}{r}50 \\
2\end{array}$ & $\begin{array}{l}\text { I } \\
\text { I } \\
\text { I } \\
\text { I } \\
\text { I }\end{array}$ & $\begin{array}{l}3232.130 \\
3232.312 \\
3232.732 \\
3232.801 \\
3233.347\end{array}$ & $\begin{array}{r}800 \\
300 \\
75 \\
75 \\
100\end{array}$ & $\begin{array}{r}100 \\
30 \\
100\end{array}$ & $\begin{array}{l}\text { I } \\
\text { I } \\
\text { II } \\
\text { II } \\
\text { I I }\end{array}$ \\
\hline $\begin{array}{l}3198.229 \\
3198.482 \\
3198.704 \\
3198.962 \\
3200.550\end{array}$ & $\begin{array}{r}300 \\
300 \\
75 \\
200 \\
20\end{array}$ & $\begin{array}{r}400 \\
300 \\
150 \\
400 \\
20\end{array}$ & $\begin{array}{l}\text { II } \\
\text { I I } \\
\text { II } \\
\text { II } \\
\text { II }\end{array}$ & $\begin{array}{l}3216.634 \\
3216.781 \\
3216.990 \\
3217.258 \\
3217.450\end{array}$ & $\begin{array}{r}50 \\
75 \\
150 \\
40 \\
500\end{array}$ & $\begin{array}{r}1000 \\
3 \\
500\end{array}$ & $\begin{array}{l}\text { II I } \\
\text { I } \\
\text { I } \\
\text { I } \\
\text { II }\end{array}$ & $\begin{array}{l}3233.625 \\
3234.511 \\
3234.902 \\
3235.001 \\
3235.387\end{array}$ & $\begin{array}{r}100 \\
50 \\
50 \\
400 \\
50\end{array}$ & $\begin{array}{l}50 \\
75 \\
75\end{array}$ & $\begin{array}{l}\text { II } \\
\text { I } \\
\text { II } \\
\text { I } \\
\text { II }\end{array}$ \\
\hline $\begin{array}{l}3200.729 \\
3201.186 \\
3201.485 \\
3202.135 \\
3202.523\end{array}$ & $\begin{array}{r}20 \\
75 \\
5 \\
75 \\
100\end{array}$ & $\begin{array}{r}150 \\
75 \\
2\end{array}$ & $\begin{array}{l}\text { I } \\
\text { II } \\
\text { I } \\
\text { II } \\
\text { I }\end{array}$ & $\begin{array}{l}3217.729 \\
3217.847 \\
3218.118 \\
3218.315 \\
3218.750\end{array}$ & $\begin{array}{r}400 \\
150 \\
50 \\
150 \\
75\end{array}$ & $\begin{array}{r}10 \\
75 \\
150 \\
100\end{array}$ & $\begin{array}{l}\text { I } \\
\text { II } \\
\text { I } \\
\text { II } \\
\text { II }\end{array}$ & $\begin{array}{l}3235.846 \\
3236.575 \\
3236.777 \\
3237.232 \\
3237.514\end{array}$ & $\begin{array}{r}1200 \\
150 \\
50 \\
100 \\
20\end{array}$ & $\begin{array}{r}1800 \\
1 \\
2 \\
500 \\
2\end{array}$ & $\begin{array}{l}\text { II } \\
\text { I } \\
\text { I } \\
\text { II } \\
\text { I }\end{array}$ \\
\hline $\begin{array}{l}3203.085 \\
3203.232 \\
3203.611 \\
3203.878 \\
3204.176\end{array}$ & $\begin{array}{r}40 \\
150 \\
150 \\
150 \\
75\end{array}$ & $\begin{array}{r}2 \\
200 \\
125 \\
125 \\
75\end{array}$ & $\begin{array}{l}\text { I } \\
\text { II } \\
\text { II } \\
\text { II } \\
\text { II }\end{array}$ & $\begin{array}{l}3218.867 \\
3219.482 \\
3219.938 \\
3220.352 \\
3220.980\end{array}$ & $\begin{array}{l}200 \\
20 \\
5 \mathrm{w} \\
600 \\
300\end{array}$ & 200 & $\begin{array}{l}\text { II } \\
\text { I } \\
\text { I } \\
\text { II } \\
\text { I }\end{array}$ & $\begin{array}{l}3238.119 \\
3238.799 \\
3238.940 \\
3239.288 \\
3239.914\end{array}$ & $\begin{array}{r}2000 \\
50 \\
400 \\
300 \\
10\end{array}$ & $\begin{array}{r}2500 \\
50 \\
20 \\
400\end{array}$ & $\begin{array}{l}\text { II } \\
\text { I I } \\
\text { I } \\
\text { I I } \\
\text { I }\end{array}$ \\
\hline $\begin{array}{l}3204.466 \\
3205.067 \\
3205.285 \\
3205.475 \\
3205.646\end{array}$ & $\begin{array}{r}40 \\
40 \\
100 \\
50 \\
150\end{array}$ & $\begin{array}{r}3 \\
2 \\
100 \\
8 \\
8\end{array}$ & $\begin{array}{l}\text { I } \\
\text { I } \\
\text { II } \\
\text { I } \\
\text { I }\end{array}$ & $\begin{array}{l}3221.288 \\
3221.593 \\
3221.869 \\
3222.805 \\
3223.166\end{array}$ & $\begin{array}{r}1000 \\
75 \\
200 \\
200 \\
75\end{array}$ & $\begin{array}{r}3000 \\
150 \\
30 \\
75\end{array}$ & $\begin{array}{l}\text { II } \\
\text { I } \\
\text { II } \\
\text { I } \\
\text { II }\end{array}$ & $\begin{array}{l}3240.210 \\
3240.331 \\
3240.484 \\
3240.645 \\
3241.118\end{array}$ & $\begin{array}{r}30 \\
30 \\
300 \\
400 \\
600\end{array}$ & $\begin{array}{r}10 \\
400 \\
2 \\
800\end{array}$ & $\begin{array}{l}\text { I } \\
\text { II } \\
\text { II } \\
\text { I } \\
\text { II }\end{array}$ \\
\hline $\begin{array}{l}3206.178 \\
3206.458 \\
3206.938 \\
3207.192 \\
3207.783\end{array}$ & $\begin{array}{r}200 \\
40 \\
150 \\
75 \\
150\end{array}$ & $\begin{array}{r}200 \\
2 \\
200 \\
75 \\
250\end{array}$ & $\begin{array}{l}\text { II } \\
\text { I } \\
\text { II } \\
\text { II } \\
\text { II }\end{array}$ & $\begin{array}{l}3223.281 \\
3223.489 \\
3223.798 \\
3223.909 \\
3224.999\end{array}$ & $\begin{array}{r}75 \\
5 \\
150 \\
40 \\
100\end{array}$ & $\begin{array}{r}15 \\
100 \\
1\end{array}$ & $\begin{array}{l}\text { II } \\
\text { I } \\
\text { II } \\
\text { I } \\
\text { I }\end{array}$ & $\begin{array}{l}3241.541 \\
3242.160 \\
3242.257 \\
3242.381 \\
3243.041\end{array}$ & $\begin{array}{r}200 \\
50 \\
200 \\
150 \\
300\end{array}$ & $\begin{array}{r}150 \\
250 \\
50 \\
200\end{array}$ & $\begin{array}{l}\text { II I } \\
\text { I } \\
\text { II } \\
\text { II } \\
\text { I I }\end{array}$ \\
\hline $\begin{array}{l}3207.940 \\
3208.029 \\
3208.515 \\
3209.330 \\
3209.752\end{array}$ & $\begin{array}{r}150 \\
125 \\
100 \\
100 \\
40\end{array}$ & $\begin{array}{r}200 \\
250 \\
\\
100 \\
1\end{array}$ & $\begin{array}{l}\text { II } \\
\text { II } \\
\text { I } \\
\text { I I } \\
\text { I }\end{array}$ & $\begin{array}{l}3225.419 \\
3225.660 \\
3225.901 \\
3226.122 \\
3226.318\end{array}$ & $\begin{array}{l}800 \\
400 \\
400 \\
300 \\
100\end{array}$ & $\begin{array}{r}1200 \\
40 \\
75 \\
200\end{array}$ & $\begin{array}{l}\text { II } \\
\text { II } \\
\text { I } \\
\text { II } \\
\text { I }\end{array}$ & $\begin{array}{l}3243.574 \\
3243.763 \\
3243.910 \\
3244.041 \\
3244.243\end{array}$ & $\begin{array}{r}40 \\
20 \\
75 \\
200 \\
40\end{array}$ & 10 & $\begin{array}{l}\text { I } \\
\text { II } \\
\text { I } \\
\text { I } \\
\text { I }\end{array}$ \\
\hline $\begin{array}{l}3209.997 \\
3210.308 \\
3210.785 \\
3211.196 \\
3211.475\end{array}$ & $\begin{array}{r}100 \\
400 \\
100 \\
400 \\
2\end{array}$ & $\begin{array}{r}4 \\
600 \\
1 \\
4 \\
2\end{array}$ & $\begin{array}{l}\text { I } \\
\text { II } \\
\text { I } \\
\text { I } \\
\text { II }\end{array}$ & $\begin{array}{l}3226.414 \\
3227.001 \\
3227.476 \\
3227.584 \\
3227.784\end{array}$ & $\begin{array}{r}200 \\
300 \\
20 \\
50 \\
400\end{array}$ & $\begin{array}{l}200 \\
200\end{array}$ & $\begin{array}{l}\text { II } \\
\text { II } \\
\text { I } \\
\text { I } \\
\text { II }\end{array}$ & $\begin{array}{l}3244.453 \\
3244.880 \\
3245.770 \\
3246.002 \\
3246.149\end{array}$ & $\begin{array}{l}800 \\
75 \\
600 \\
70 \\
50 \mathrm{w}\end{array}$ & $\begin{array}{r}40 \\
1500\end{array}$ & $\begin{array}{l}\text { I } \\
\text { I } \\
\text { II } \\
\text { I } \\
\text { I }\end{array}$ \\
\hline $\begin{array}{l}3212.005 \\
3212.133 \\
3212.337 \\
3212.810 \\
3213.105\end{array}$ & $\begin{array}{r}300 \\
10 \\
20 \\
100 \\
40\end{array}$ & $\begin{array}{r}200 \\
2 \\
100 \\
2\end{array}$ & $\begin{array}{l}\text { II } \\
\text { II } \\
\text { I } \\
\text { II } \\
\text { I }\end{array}$ & $\begin{array}{l}3228.093 \\
3228.338 \\
3228.526 \\
3229.013 \\
322 \% .673\end{array}$ & $\begin{array}{c}50 \\
10 \\
40 \\
1500 \\
50 \mathrm{w}\end{array}$ & $2500^{2}$ & $\begin{array}{l}\text { I } \\
\text { I } \\
\text { I } \\
\text { I I }\end{array}$ & $\begin{array}{l}3246.583 \\
3246.802 \\
3247.210 \\
3247.323 \\
3247.591\end{array}$ & $\begin{array}{l}100 \\
100 \\
100 \\
20 \\
300\end{array}$ & $\begin{array}{r}100 \\
75\end{array}$ & $\begin{array}{l}\text { II } \\
\text { II } \\
\text { I } \\
\text { I } \\
\text { II }\end{array}$ \\
\hline $\begin{array}{l}3213.225 \\
3213.576 \\
3214.079 \\
3214.382 \\
3214.644\end{array}$ & $\begin{array}{r}40 \\
400 \\
400 \\
400 \\
200\end{array}$ & $\begin{array}{r}600 \\
20 \\
4 \\
2\end{array}$ & $\begin{array}{l}\text { I } \\
\text { I I } \\
\text { I } \\
\text { I } \\
\text { I }\end{array}$ & $\begin{array}{l}3229.979 \\
3230.372 \\
3230.862 \\
3231.467 \\
3232.036\end{array}$ & $\begin{array}{r}300 \\
40 \\
300 \\
100 \\
100\end{array}$ & $\begin{array}{r}75 \\
500 \\
3 \\
500\end{array}$ & $\begin{array}{l}\text { I } \\
\text { I } \\
\text { II } \\
\text { I } \\
\text { III }\end{array}$ & $\begin{array}{l}3248.380 \\
3248.537 \\
3248.606 \\
3248.690 \\
3248.900\end{array}$ & $\begin{array}{r}75 \\
400 \\
10 \\
150 \\
200\end{array}$ & $\begin{array}{r}75 \\
400 \\
50 \\
300\end{array}$ & $\begin{array}{l}\text { I I } \\
\text { I I } \\
\text { I } \\
\text { II } \\
\text { I I }\end{array}$ \\
\hline
\end{tabular}


Table 1. Wavelengths of Thorium Spectra - Continued

\begin{tabular}{|c|c|c|c|c|c|c|c|c|c|c|c|}
\hline \multirow{2}{*}{$\begin{array}{l}\text { Wave- } \\
\text { length }\end{array}$} & \multicolumn{2}{|c|}{ Intensity } & \multirow{2}{*}{$\begin{array}{l}\text { Spec- } \\
\text { trum }\end{array}$} & \multirow{2}{*}{$\begin{array}{l}\text { Wave- } \\
\text { length }\end{array}$} & \multicolumn{2}{|c|}{ Intensity } & \multirow{2}{*}{$\begin{array}{l}\text { Spec- } \\
\text { trum }\end{array}$} & \multirow{2}{*}{$\begin{array}{l}\text { Wave- } \\
\text { length }\end{array}$} & \multicolumn{2}{|c|}{ Intensity } & \multirow{2}{*}{$\begin{array}{l}\text { Spec- } \\
\text { trum }\end{array}$} \\
\hline & $a m p$ & Spark & & & $\mathrm{amp}$ & Spark & & & Lamp & Spark & \\
\hline $\begin{array}{l}3249.333 \\
3249.866 \\
3249.920 \\
3250.189 \\
3251.306\end{array}$ & $\begin{array}{r}75 \\
700 \\
5 \\
150 \\
10\end{array}$ & $\begin{array}{r}30 \\
4 \\
200 \\
15\end{array}$ & $\begin{array}{l}\text { I } \\
\text { I } \\
\text { I I } \\
\text { I I } \\
\text { I I }\end{array}$ & $\begin{array}{l}3272.047 \\
3272.300 \\
3272.567 \\
3273.894 \\
3274.392\end{array}$ & $\begin{array}{r}800 \\
20 \\
600 \\
200\end{array}$ & $\begin{array}{c}12 \\
10 \mathrm{w} \\
20 \\
1200 \\
100\end{array}$ & $\begin{array}{l}\text { I } \\
\text { I I I } \\
\text { I I } \\
\text { I I } \\
\text { I I }\end{array}$ & $\begin{array}{l}3293.944 \\
3294.231 \\
3294.565 \\
3294.745 \\
3294.991\end{array}$ & $\begin{array}{l}400 \\
300 \\
150 \\
200 \\
300\end{array}$ & $\begin{array}{r}500 \\
10 \\
150 \\
100 \\
400\end{array}$ & $\begin{array}{l}\text { II } \\
\text { I } \\
\text { II } \\
\text { II } \\
\text { II }\end{array}$ \\
\hline $\begin{array}{l}3251.586 \\
3251.919 \\
3252.738 \\
3252.959 \\
3253.687\end{array}$ & $\begin{array}{c}10 \mathrm{w} \\
2000 \\
200 \\
200 \\
400\end{array}$ & $\begin{array}{r}75 \\
800 \\
800 \\
30\end{array}$ & $\begin{array}{l}\text { I } \\
\text { I I } \\
\text { I I } \\
\text { I I } \\
\text { I }\end{array}$ & $\begin{array}{l}3275.059 \\
3275.248 \\
3276.182 \\
3276.298 \\
3276.543\end{array}$ & $\begin{array}{r}400 \\
50 \\
200 \\
50 \\
40\end{array}$ & $\begin{array}{r}1600 \\
600\end{array}$ & $\begin{array}{l}\text { II } \\
\text { I } \\
\text { II } \\
\text { I } \\
\text { I }\end{array}$ & $\begin{array}{l}3295.320 \\
3295.525 \\
3296.600 \\
3297.361 \\
3297.831\end{array}$ & $\begin{array}{l}400 \\
400 \\
200 \\
350 \\
400\end{array}$ & $\begin{array}{l}500 \\
500 \\
800 \\
500 \\
500\end{array}$ & $\begin{array}{l}\text { II } \\
\text { II } \\
\text { II } \\
\text { II } \\
\text { II }\end{array}$ \\
\hline $\begin{array}{l}3253.869 \\
3254.069 \\
3254.583 \\
3254.816 \\
3255.512\end{array}$ & $\begin{array}{r}400 \\
300 \\
50 \\
400 \\
400\end{array}$ & $\begin{array}{r}75 \\
2 \\
600 \\
600\end{array}$ & $\begin{array}{l}\text { I } \\
\text { I } \\
\text { I } \\
\text { I I } \\
\text { I I }\end{array}$ & $\begin{array}{l}3276.706 \\
3277.060 \\
3277.690 \\
3277.910 \\
3278.188\end{array}$ & $\begin{array}{r}150 \\
300 \\
75 \\
100 \\
100\end{array}$ & $\begin{array}{r}2 \\
100 \\
100\end{array}$ & $\begin{array}{l}\text { I } \\
\text { I } \\
\text { I } \\
\text { II } \\
\text { I I }\end{array}$ & $\begin{array}{l}3298.038 \\
3298.689 \\
3298.799 \\
3299.356 \\
3299.664\end{array}$ & $\begin{array}{l}400 \\
75 \\
200 \\
20 \mathrm{~h} \\
75\end{array}$ & 30 & $\begin{array}{l}\text { I } \\
\text { I } \\
\text { I } \\
\text { I } \\
\text { I I }\end{array}$ \\
\hline $\begin{array}{l}3255.922 \\
3256.277 \\
3256.690 \\
3257.160 \\
3257.374\end{array}$ & $\begin{array}{r}200 \\
1500 \\
50 \\
250 \\
1300\end{array}$ & $\begin{array}{r}4500 \\
500 \\
10\end{array}$ & $\begin{array}{l}\text { I } \\
\text { I I } \\
\text { I } \\
\text { I I } \\
\text { I }\end{array}$ & $\begin{array}{l}3278.746 \\
3279.017 \\
3280.374 \\
3280.732 \\
3280.958\end{array}$ & $\begin{array}{r}300 \\
40 \\
800 \\
150 \\
10\end{array}$ & $\begin{array}{r}200 \\
1600 \\
5 \\
5\end{array}$ & $\begin{array}{l}\text { I I } \\
\text { I } \\
\text { I I } \\
\text { I } \\
\text { I I }\end{array}$ & $\begin{array}{l}3300.254 \\
3300.611 \\
3300.754 \\
3301.263 \\
3301.347\end{array}$ & $\begin{array}{r}50 \\
200 \\
40 \\
600 \\
100\end{array}$ & $\begin{array}{r}200 \\
1000\end{array}$ & $\begin{array}{l}\text { I } \\
\text { II } \\
\text { I } \\
\text { I I } \\
\text { I }\end{array}$ \\
\hline $\begin{array}{l}3257.932 \\
3258.110 \\
3258.741 \\
3259.061 \\
3259.237\end{array}$ & $\begin{array}{l}200 \\
600 \\
100 \\
800 \\
100\end{array}$ & $\begin{array}{r}250 \\
50 \\
\\
5 \\
125\end{array}$ & $\begin{array}{l}\text { I I } \\
\text { I } \\
\text { I } \\
\text { I } \\
\text { I I }\end{array}$ & $\begin{array}{l}3281.037 \\
3281.178 \\
3281.270 \\
3281.408 \\
3281.967\end{array}$ & $\begin{array}{l}400 \\
75 \mathrm{w} \\
75 \\
200 \\
150\end{array}$ & $\begin{array}{r}5 \\
100 \\
400 \\
100\end{array}$ & $\begin{array}{l}\text { I } \\
\text { I } \\
\text { II } \\
\text { II } \\
\text { I I }\end{array}$ & $\begin{array}{l}3301.634 \\
3302.163 \\
3303.257 \\
3303.480 \\
3304.238 \mathrm{i}\end{array}$ & $\begin{array}{l}500 \\
200 \\
100 \\
200 \\
3000\end{array}$ & $\begin{array}{r}100 \\
2 \\
50 \\
50\end{array}$ & $\begin{array}{l}\text { II } \\
\text { I } \\
\text { I } \\
\text { I I } \\
\text { I }\end{array}$ \\
\hline $\begin{array}{l}3259.615 \\
3260.922 \\
3261.113 \\
3261.543 \\
3262.668\end{array}$ & $\begin{array}{r}300 \\
200 \\
75 \\
350 \\
3000\end{array}$ & $\begin{array}{r}500 \\
600 \\
50 \\
400 \\
3000\end{array}$ & $\begin{array}{l}\text { I I } \\
\text { I I } \\
\text { I I } \\
\text { I I } \\
\text { I I }\end{array}$ & $\begin{array}{l}3282.120 \\
3282.384 \\
3282.600 \\
3282.967 \\
3283.644\end{array}$ & $\begin{array}{l}100 \\
50 \mathrm{w} \\
200 \\
200 \\
100\end{array}$ & $\begin{array}{r}125 \\
400 \\
250 \\
1\end{array}$ & $\begin{array}{l}\text { II } \\
\text { I } \\
\text { II } \\
\text { I I } \\
\text { I }\end{array}$ & $\begin{array}{l}3305.301 \\
3305.590 \\
3306.047 \\
3307.388 \\
3307.554\end{array}$ & $\begin{array}{l}400 \mathrm{~s} \\
40 \\
40 \\
20 \mathrm{~h} \\
20 \mathrm{~h}\end{array}$ & $\begin{array}{r}2 \\
50 \\
75\end{array}$ & $\begin{array}{l}\text { I } \\
\text { II } \\
\text { II } \\
\text { I } \\
\text { I }\end{array}$ \\
\hline $\begin{array}{l}3263.030 \\
3263.183 \\
3263.624 \\
3263.762 \\
3264.123\end{array}$ & $\begin{array}{l}300 \\
200 \\
100 \\
100 \\
200\end{array}$ & $\begin{array}{r}400 \\
30 \\
100 \\
50 \\
200\end{array}$ & $\begin{array}{l}\text { I I } \\
\text { I } \\
\text { I I } \\
\text { I I } \\
\text { I I }\end{array}$ & $\begin{array}{l}3284.172 \\
3285.096 \\
3285.519 \\
3285.748 \\
3286.578\end{array}$ & $\begin{array}{r}40 \\
300 \\
900 \\
400\end{array}$ & $\begin{array}{r}500 \\
2 \\
2 \\
5 \\
600\end{array}$ & $\begin{array}{l}\text { I I I } \\
\text { I } \\
\text { I } \\
\text { I } \\
\text { I I }\end{array}$ & $\begin{array}{l}3308.060 \\
3308.454 \\
3309.135 \\
3309.365 \mathrm{i} \\
3310.238\end{array}$ & $\begin{array}{r}200 \\
40 \\
300 \\
800 \\
400\end{array}$ & $\begin{array}{r}300 \\
2 \\
600 \\
6 \\
800\end{array}$ & $\begin{array}{l}\text { II } \\
\text { I } \\
\text { II } \\
\text { I } \\
\text { II }\end{array}$ \\
\hline $\begin{array}{l}3264.441 \\
3265.582 \\
3266.319 \\
3266.625 \\
3266.998\end{array}$ & $\begin{array}{l}300 \\
300 \\
150 \\
400 \\
400\end{array}$ & $\begin{array}{r}400 \\
400 \\
200 \\
75 \\
800\end{array}$ & $\begin{array}{l}\text { I I } \\
\text { I I } \\
\text { I I } \\
\text { I } \\
\text { I I }\end{array}$ & $\begin{array}{l}3287.076 \\
3287.789 \mathrm{i} \\
3288.156 \\
3288.376 \\
3289.494\end{array}$ & $\begin{array}{r}10 \\
1600 \\
200 \\
300 \\
125\end{array}$ & $\begin{array}{r}10 \\
2000 \\
200 \\
4\end{array}$ & $\begin{array}{l}\text { I I } \\
\text { I I } \\
\text { I I } \\
\text { I } \\
\text { I }\end{array}$ & $\begin{array}{l}3310.481 \\
3310.634 \\
3310.805 \\
3310.912 \\
3311.390\end{array}$ & $\begin{array}{r}200 \\
250 \\
75 \\
40 \\
100\end{array}$ & $\begin{array}{r}400 \\
40 \\
125\end{array}$ & $\begin{array}{l}\text { I I } \\
\text { I } \\
\text { II } \\
\text { I } \\
\text { II }\end{array}$ \\
\hline $\begin{array}{l}3267.435 \\
3267.612 \\
3268.440 \\
3268.758 \\
3269.475\end{array}$ & $\begin{array}{r}20 \\
10 \\
150 \\
100 \\
300\end{array}$ & $\begin{array}{r}50 \\
30 \\
400\end{array}$ & $\begin{array}{l}\text { I } \\
\text { I } \\
\text { I I } \\
\text { I I } \\
\text { I I }\end{array}$ & $\begin{array}{l}3289.626 \\
3290.116 \\
3290.233 \\
3291.028 \\
3291.441\end{array}$ & $\begin{array}{l}100 \\
400 \\
75 \\
50 \mathrm{w} \\
20 \mathrm{w}\end{array}$ & 100 & $\begin{array}{l}\text { I } \\
\text { I I } \\
\text { I } \\
\text { I } \\
\text { I I }\end{array}$ & $\begin{array}{l}3312.070 \\
3312.342 \\
3312.661 \\
3313.064 \\
3313.424\end{array}$ & $\begin{array}{r}100 \\
20 \\
75 \\
400 \\
100\end{array}$ & $\begin{array}{r}150 \\
75 \\
8\end{array}$ & $\begin{array}{l}\text { I I } \\
\text { I } \\
\text { I I } \\
\text { I } \\
\text { I I }\end{array}$ \\
\hline $\begin{array}{l}3270.097 \\
3270.233 \\
3270.826 \\
3271.533 \\
3271.881\end{array}$ & $\begin{array}{l}100 \\
100 \\
600 \\
125 \\
600\end{array}$ & $\begin{array}{r}100 \\
200 \\
10\end{array}$ & $\begin{array}{l}\text { I } \\
\text { II } \\
\text { I } \\
\text { I } \\
\text { I }\end{array}$ & $\begin{array}{l}3291.744 \\
3292.520 \mathrm{i} \\
3292.924 \\
3293.584 \\
3293.692\end{array}$ & $\begin{array}{r}3000 \\
3000 \\
75 \\
400 \\
150\end{array}$ & $\begin{array}{r}3000 \\
2500 \\
2 \\
400 \\
200\end{array}$ & $\begin{array}{l}\text { II } \\
\text { I I } \\
\text { I } \\
\text { II } \\
\text { I I }\end{array}$ & $\begin{array}{l}3313.642 \\
3313.975 \\
3314.805 \\
3315.186 \\
3315.536\end{array}$ & $\begin{array}{r}600 \\
100 \\
400 \\
100 \\
75\end{array}$ & $\begin{array}{r}600 \\
1000 \\
150 \\
100\end{array}$ & $\begin{array}{l}\text { I I } \\
\text { I } \\
\text { II } \\
\text { I I } \\
\text { II }\end{array}$ \\
\hline
\end{tabular}


Table 1. Wavelengths of Thorium Spectra - Continued

\begin{tabular}{|c|c|c|c|c|c|c|c|c|c|c|c|}
\hline \multirow{2}{*}{$\begin{array}{l}\text { Wave- } \\
\text { length }\end{array}$} & \multicolumn{2}{|c|}{ Intensity } & \multirow{2}{*}{$\begin{array}{l}\text { Spec- } \\
\text { trum }\end{array}$} & \multirow{2}{*}{$\begin{array}{l}\text { Wave- } \\
\text { length }\end{array}$} & \multicolumn{2}{|c|}{ Intensity } & \multirow{2}{*}{$\begin{array}{l}\text { Spec- } \\
\text { trum }\end{array}$} & \multirow{2}{*}{$\begin{array}{l}\text { Wave- } \\
\text { length }\end{array}$} & \multicolumn{2}{|c|}{ Intensity } & \multirow{2}{*}{$\begin{array}{l}\text { Spec- } \\
\text { trum }\end{array}$} \\
\hline & Lamp & Spark & & & Lamp & Spark & & & Lamp & Spark & \\
\hline $\begin{array}{l}3315.826 \\
3316.204 \\
3316.758 \\
3317.242 \\
3317.551\end{array}$ & $\begin{array}{l}200 \\
200 \\
20 \\
10 \mathrm{~h} \\
200\end{array}$ & $\begin{array}{r}40 \\
200\end{array}$ & $\begin{array}{l}\text { I I } \\
\text { I I } \\
\text { I } \\
\text { I } \\
\text { I I }\end{array}$ & $\begin{array}{l}3337.279 \\
3337.468 \\
3337.870 \mathrm{i} \\
3338.393 \\
3338.582\end{array}$ & $\begin{array}{r}300 \\
100 \\
2000 \\
600 \\
50\end{array}$ & $\begin{array}{r}20 \\
150 \\
75 \\
10\end{array}$ & $\begin{array}{l}\text { I } \\
\text { II } \\
\text { II } \\
\text { II } \\
\text { I I }\end{array}$ & $\begin{array}{l}3355.250 \\
3355.378 \\
3355.545 \\
3356.678 \\
3356.823\end{array}$ & $\begin{array}{r}300 \\
75 \\
200 \\
75 \\
300\end{array}$ & $\begin{array}{r}50 \\
100 \\
50\end{array}$ & $\begin{array}{l}\text { II } \\
\text { I } \\
\text { I I } \\
\text { I } \\
\text { I I }\end{array}$ \\
\hline $\begin{array}{l}3317.734 \\
3318.232 \\
3318.376 \\
3318.965 \\
3319.127\end{array}$ & $\begin{array}{r}200 \\
40 \\
200 \\
200 \\
300\end{array}$ & $\begin{array}{r}250 \\
30 \\
250\end{array}$ & $\begin{array}{l}\text { I I } \\
\text { I I } \\
\text { I } \\
\text { II } \\
\text { I }\end{array}$ & $\begin{array}{l}3338.818 \\
3339.234 \\
3339.435 \\
3340.005 \\
3340.318\end{array}$ & $\begin{array}{r}100 \\
75 \\
100 \\
100 \\
125\end{array}$ & 20 & $\begin{array}{l}\text { II } \\
\text { I } \\
\text { I } \\
\text { I } \\
\text { I }\end{array}$ & $\begin{array}{l}3357.047 \\
3357.237 \\
3357.392 \\
3357.739 \\
3358.080\end{array}$ & $\begin{array}{r}75 \\
100 \\
20 \\
40 \\
40\end{array}$ & $\begin{array}{l}20 \\
30 \\
20 \\
10 \\
10\end{array}$ & $\begin{array}{l}\text { II } \\
\text { I I } \\
\text { I I } \\
\text { II } \\
\text { I I }\end{array}$ \\
\hline $\begin{array}{l}3319.602 \\
3319.904 \\
3320.292 \\
3320.469 \\
3320.873\end{array}$ & $\begin{array}{r}200 \\
400 \\
400 \\
400 \\
40\end{array}$ & $\begin{array}{l}100 \\
200 \\
400\end{array}$ & $\begin{array}{l}\text { I I } \\
\text { I } \\
\text { II } \\
\text { I } \\
\text { I }\end{array}$ & $\begin{array}{l}3340.712 \\
3342.068 \\
3342.179 \\
3342.376 \\
3342.885\end{array}$ & $\begin{array}{l}400 \\
200 \\
40 \\
75 \mathrm{w} \\
50 \mathrm{~h}\end{array}$ & $\begin{array}{r}3 \\
50\end{array}$ & $\begin{array}{l}\text { I } \\
\text { II } \\
\text { I } \\
\text { I } \\
\text { I }\end{array}$ & $\begin{array}{l}3358.601 i \\
3359.056 \\
3359.604 \\
3359.750 \\
3360.146\end{array}$ & $\begin{array}{r}2000 \\
100 \\
100 \\
200 \\
200\end{array}$ & $\begin{array}{r}200 \\
20 \\
3 \\
10 \\
20\end{array}$ & $\begin{array}{l}\text { II } \\
\text { II } \\
\text { I } \\
\text { II } \\
\text { II }\end{array}$ \\
\hline $\begin{array}{l}3321.047 \\
3321.437 \\
3321.568 \\
3322.091 \\
3322.450\end{array}$ & $\begin{array}{r}100 \\
2000 \\
75 \\
400 \\
100\end{array}$ & $\begin{array}{r}1600 \\
75 \\
3 \\
125\end{array}$ & $\begin{array}{l}\text { I } \\
\text { I I } \\
\text { I I } \\
\text { I } \\
\text { I I }\end{array}$ & $\begin{array}{l}3343.156 \\
3343.257 \\
3343.337 \\
3343.494 \\
3343.610\end{array}$ & $\begin{array}{l}400 \\
150 \\
400 \\
200 \\
400\end{array}$ & $\begin{array}{l}20 \\
60\end{array}$ & $\begin{array}{l}\text { I } \\
\text { II } \\
\text { I } \\
\text { II } \\
\text { I }\end{array}$ & $\begin{array}{l}3360.367 \\
3360.997 \\
3361.193 \\
3361.612 \\
3361.728\end{array}$ & $\begin{array}{l}400 \\
400 \\
350 \\
300 \\
400\end{array}$ & $\begin{array}{r}40 \\
8 \\
3 \\
200 \\
200\end{array}$ & $\begin{array}{l}\text { II } \\
\text { I } \\
\text { I } \\
\text { I I } \\
\text { I I }\end{array}$ \\
\hline $\begin{array}{l}3322.945 \\
3324.752 \mathrm{i} \\
3325.120 \mathrm{i} \\
3326.452 \\
3326.775\end{array}$ & $\begin{array}{r}350 \\
2000 \\
3000 \\
400 \\
100\end{array}$ & $\begin{array}{r}1 \\
2000 \\
3000 \\
500\end{array}$ & $\begin{array}{l}\text { I } \\
\text { II } \\
\text { II } \\
\text { I I } \\
\text { I }\end{array}$ & $\begin{array}{l}3343.799 \\
3344.300 \\
3344.871 \\
3345.162 \\
3345.359\end{array}$ & $\begin{array}{r}200 \\
150 \\
500 \\
200 \\
20\end{array}$ & $\begin{array}{r}15 \\
20 \\
50 \\
\\
5\end{array}$ & $\begin{array}{l}\text { II } \\
\text { I I } \\
\text { II } \\
\text { I } \\
\text { II }\end{array}$ & $\begin{array}{l}3361.874 \\
3362.180 \\
3362.497 \\
3362.667 \\
3363.061\end{array}$ & $\begin{array}{r}75 \\
200 \\
400 \\
200 \\
250\end{array}$ & $\begin{array}{l}30 \\
20 \\
20 \\
10 \\
15\end{array}$ & $\begin{array}{l}\text { I I } \\
\text { I I } \\
\text { I I } \\
\text { I I } \\
\text { I I }\end{array}$ \\
\hline $\begin{array}{l}3327.182 \\
3327.498 \\
3327.705 \\
3328.246 \\
3328.630\end{array}$ & $\begin{array}{r}50 \\
40 \\
50 \\
200 \\
20\end{array}$ & $\begin{array}{r}300 \\
20 \\
50 \\
2\end{array}$ & $\begin{array}{l}\text { I I } \\
\text { I } \\
\text { I I } \\
\text { I I } \\
\text { I }\end{array}$ & $\begin{array}{l}3345.828 \\
3345.992 \\
3346.552 \\
3346.975 \\
3347.247\end{array}$ & $\begin{array}{r}150 \\
100 \\
400 \\
300 \\
40\end{array}$ & $\begin{array}{r}4 \\
50 \\
15\end{array}$ & $\begin{array}{l}\text { I } \\
\text { I } \\
\text { II } \\
\text { I } \\
\text { II }\end{array}$ & $\begin{array}{l}3363.300 \\
3363.700 \\
3364.132 \\
3364.381 \\
3364.689\end{array}$ & $\begin{array}{r}10 \\
400 \\
100 \\
100 \\
1200\end{array}$ & $\begin{array}{r}25 \\
15 \\
100\end{array}$ & $\begin{array}{l}\text { I } \\
\text { II } \\
\text { I } \\
\text { II } \\
\text { II }\end{array}$ \\
\hline $\begin{array}{l}3328.967 \\
3329.723 \\
3330.477 \mathrm{i} \\
3330.964 \\
3331.188\end{array}$ & $\begin{array}{r}100 \\
400 \\
1800 \\
200 \\
100\end{array}$ & $\begin{array}{r}15 \\
20 \\
100 \\
15 \\
40\end{array}$ & $\begin{array}{l}\text { II } \\
\text { I } \\
\text { I } \\
\text { II } \\
\text { I I }\end{array}$ & $\begin{array}{l}3347.405 \\
3347.574 \\
3347.988 \\
3348.297 \\
3348.776\end{array}$ & $\begin{array}{r}150 \\
150 \\
500 \\
75 \\
3000\end{array}$ & 15 & $\begin{array}{l}\text { I } \\
\text { I I } \\
\text { I } \\
\text { I } \\
\text { I }\end{array}$ & $\begin{array}{l}3365.129 \\
3365.331 \\
3365.615 \\
3365.975 \\
3366.529\end{array}$ & $\begin{array}{r}400 \\
800 \\
40 \\
100 \\
300\end{array}$ & $\begin{array}{r}20 \\
10 \\
300\end{array}$ & $\begin{array}{l}\text { I } \\
\text { I } \\
\text { I I } \\
\text { I } \\
\text { I I }\end{array}$ \\
\hline $\begin{array}{l}3331.723 \\
3332.079 \\
3332.400 \\
3332.473 \\
3333.127\end{array}$ & $\begin{array}{r}200 \\
100 \\
100 \\
100 \\
1000\end{array}$ & $\begin{array}{l}20 \\
30 \\
10\end{array}$ & $\begin{array}{l}\text { I } \\
\text { II } \\
\text { I I } \\
\text { I } \\
\text { I }\end{array}$ & $\begin{array}{l}3348.963 \\
3349.381 \\
3349.838 \\
3350.355 \\
3351.228 \mathrm{i}\end{array}$ & $\begin{array}{l}800 \\
200 \mathrm{w} \\
100 \mathrm{w} \\
200 \\
2500\end{array}$ & $\begin{array}{r}100 \\
50 \\
10 \\
75 \\
300\end{array}$ & $\begin{array}{l}\text { I I } \\
\text { I I } \\
\text { I I } \\
\text { I I } \\
\text { I I }\end{array}$ & $\begin{array}{l}3366.706 \\
3367.018 \\
3367.578 \\
3367.810 \\
3369.000\end{array}$ & $\begin{array}{l}100 \\
40 \mathrm{~h} \\
350 \\
1200 \\
200 \mathrm{w}\end{array}$ & $\begin{array}{r}200 \\
8\end{array}$ & $\begin{array}{l}\text { II } \\
\text { I } \\
\text { I } \\
\text { II } \\
\text { I }\end{array}$ \\
\hline $\begin{array}{l}3334.041 \\
3334.608 \\
3335.056 \\
3335.228 \\
3335.688\end{array}$ & $\begin{array}{l}100 \mathrm{w} \\
3000 \\
400 \\
250 \\
200\end{array}$ & $\begin{array}{r}20 \\
500 \\
200\end{array}$ & $\begin{array}{l}\text { II } \\
\text { II } \\
\text { II } \\
\text { I } \\
\text { I }\end{array}$ & $\begin{array}{l}3351.575 \\
3351.832 \\
3352.023 \\
3352.389 \\
3352.736\end{array}$ & $\begin{array}{r}150 \\
50 \\
200 \\
50 \\
50\end{array}$ & $\begin{array}{l}50 \\
10 \\
10\end{array}$ & $\begin{array}{l}\text { II } \\
\text { II } \\
\text { I } \\
\text { I I } \\
\text { I }\end{array}$ & $\begin{array}{l}3370.150 \\
3370.380 \\
3370.739 \\
3370.888 \\
3371.465\end{array}$ & $\begin{array}{c}100 \mathrm{w} \\
75 \\
80 \\
80 \\
100\end{array}$ & $\begin{array}{r}10 \\
4 \\
20\end{array}$ & $\begin{array}{l}\text { I } \\
\text { II } \\
\text { I } \\
\text { I } \\
\text { I I }\end{array}$ \\
\hline $\begin{array}{l}3336.064 \\
3336.154 \\
3336.568 \\
3336.772 \\
3337.147\end{array}$ & $\begin{array}{r}40 \\
100 \\
75 \\
100 \\
400\end{array}$ & $\begin{array}{l}75 \\
50\end{array}$ & $\begin{array}{l}\text { I I } \\
\text { I } \\
\text { I } \\
\text { II } \\
\text { I I }\end{array}$ & $\begin{array}{l}3353.136 \\
3353.950 \\
3354.177 \\
3354.611 \\
3355.101\end{array}$ & $\begin{array}{l}200 \\
300 \\
300 \\
300 \\
300\end{array}$ & $\begin{array}{r}50 \\
100 \\
400 \\
300 \\
100\end{array}$ & $\begin{array}{l}\text { II } \\
\text { II } \\
\text { I I } \\
\text { I I } \\
\text { I }\end{array}$ & $\begin{array}{l}3371.790 \\
3372.300 \\
3372.686 \\
3372.814 \\
3373.056\end{array}$ & $\begin{array}{r}400 \\
60 \\
200 \\
100 \\
80\end{array}$ & $\begin{array}{r}200 \\
8 \\
30 \\
5\end{array}$ & $\begin{array}{l}\text { II } \\
\text { I } \\
\text { II } \\
\text { I } \\
\text { I }\end{array}$ \\
\hline
\end{tabular}


Table 1. Wavelengths of Thorium Spectra - Continued

\begin{tabular}{|c|c|c|c|c|c|c|c|c|c|c|c|}
\hline \multirow{2}{*}{$\begin{array}{l}\text { Wave- } \\
\text { length }\end{array}$} & \multicolumn{2}{|c|}{ Intensity } & \multirow{2}{*}{$\begin{array}{l}\text { Spec- } \\
\text { trum }\end{array}$} & \multirow{2}{*}{$\begin{array}{l}\text { Wave- } \\
\text { length }\end{array}$} & \multicolumn{2}{|c|}{ Intensity } & \multirow{2}{*}{$\begin{array}{l}\text { Spec- } \\
\text { trum }\end{array}$} & \multirow{2}{*}{$\begin{array}{l}\text { Wave- } \\
\text { length }\end{array}$} & \multicolumn{2}{|c|}{ Intensity } & \multirow{2}{*}{$\begin{array}{l}\text { Spec } \\
\text { trum }\end{array}$} \\
\hline & Lamp & Spark & & & Lamp & Spark & & & Lamp & Spark & \\
\hline $\begin{array}{l}3373.491 \\
3374.578 \\
3374.975 \mathrm{i} \\
3375.598 \\
3376.374\end{array}$ & $\begin{array}{r}700 \\
200 \\
1600 \\
300 \\
250\end{array}$ & $\begin{array}{c}100 \\
75 \\
15 \\
5 \\
2 w\end{array}$ & $\begin{array}{l}\text { II } \\
\text { II } \\
\text { I } \\
\text { I } \\
\text { I }\end{array}$ & $\begin{array}{l}3397.076 \\
3397.517 \\
3398.099 \\
3398.533 \\
3399.017\end{array}$ & $\begin{array}{r}50 \\
1600 \\
80 \\
1600 \\
150\end{array}$ & $\begin{array}{l}10 \\
15 \\
\\
20\end{array}$ & $\begin{array}{l}\text { II } \\
\text { I } \\
\text { I } \\
\text { I } \\
\text { II }\end{array}$ & $\begin{array}{l}3419.172 \\
3420.177 \\
3420.369 \\
3421.210 \mathrm{i} \\
3421.669\end{array}$ & $\begin{array}{r}300 \\
40 \\
140 \\
2500 \\
50\end{array}$ & $\begin{array}{r}200 \\
1 \\
200\end{array}$ & $\begin{array}{l}\text { II } \\
\text { I } \\
\text { I } \\
\text { I } \\
\text { I }\end{array}$ \\
\hline $\begin{array}{l}3376.842 \\
3377.478 \\
3377.660 \\
3377.973 \\
3378.566\end{array}$ & $\begin{array}{l}200 \\
200 \mathrm{w} \\
75 \\
20 \mathrm{~h} \\
400\end{array}$ & $\begin{array}{r}20 \\
600 \\
10 \\
5 \\
150\end{array}$ & $\begin{array}{l}\text { II } \\
\text { II I } \\
\text { II } \\
\text { II } \\
\text { II }\end{array}$ & $\begin{array}{l}3399.322 \\
3400.312 \\
3400.624 \\
3400.978 \\
3401.689\end{array}$ & $\begin{array}{r}5 \\
20 \\
80 \\
40 \\
400\end{array}$ & $\begin{array}{r}3 \\
20 \\
1 \\
10 \\
75\end{array}$ & $\begin{array}{l}\text { I I } \\
\text { I I } \\
\text { I } \\
\text { I I } \\
\text { I I }\end{array}$ & $\begin{array}{l}3422.651 \\
3423.114 \\
3423.987 \\
3425.150 \\
3425.408\end{array}$ & $\begin{array}{r}400 \\
300 \\
1600 \\
400 \\
160\end{array}$ & $\begin{array}{r}100 \\
75 \\
50 \\
125\end{array}$ & $\begin{array}{l}\text { II } \\
\text { I I } \\
\text { I } \\
\text { I I } \\
\text { I }\end{array}$ \\
\hline $\begin{array}{l}3379.093 \\
3379.300 \\
3379.669 \\
3379.963 \\
3380.715\end{array}$ & $\begin{array}{r}100 \\
20 \\
100 \\
20 \\
20\end{array}$ & $\begin{array}{r}5 \\
5 \\
20 \\
4\end{array}$ & $\begin{array}{l}\text { I I } \\
\text { II } \\
\text { II } \\
\text { I I } \\
\text { I }\end{array}$ & $\begin{array}{l}3402.021 \\
3402.701 \\
3403.279 \\
3403.881 \\
3404.016\end{array}$ & $\begin{array}{r}250 \\
1500 \\
500 \\
125 \\
125\end{array}$ & $\begin{array}{r}50 \\
500 \\
100\end{array}$ & $\begin{array}{l}\text { I I } \\
\text { I I } \\
\text { I I } \\
\text { I } \\
\text { I }\end{array}$ & $\begin{array}{l}3425.593 \\
3425.955 \\
3426.129 \\
3427.074 \\
3427.444\end{array}$ & $\begin{array}{r}100 \\
200 \\
100 \\
400 \\
40\end{array}$ & $\begin{array}{l}30 \\
20 \\
10 \\
10\end{array}$ & $\begin{array}{l}\text { I } \\
\text { I I } \\
\text { I I } \\
\text { I } \\
\text { I I }\end{array}$ \\
\hline $\begin{array}{l}3380.860 \mathrm{i} \\
3381.349 \\
3381.902 \\
3383.113 \\
3383.582\end{array}$ & $\begin{array}{r}900 \\
400 \\
80 \\
400 \\
100\end{array}$ & $\begin{array}{r}6 \\
50 \\
200\end{array}$ & $\begin{array}{l}\text { I } \\
\text { I I } \\
\text { I } \\
\text { I I } \\
\text { I }\end{array}$ & $\begin{array}{l}3404.632 \\
3405.558 \mathrm{i} \\
3406.230 \\
3406.540 \\
3406.825\end{array}$ & $\begin{array}{r}300 \\
1400 \\
400 \\
50 \\
40\end{array}$ & $\begin{array}{l}75 \\
40 \\
50\end{array}$ & $\begin{array}{l}\text { II } \\
\text { I } \\
\text { II } \\
\text { I } \\
\text { I }\end{array}$ & $\begin{array}{l}3427.984 \\
3428.625 \\
3428.720 \\
3428.998 \\
3429.384\end{array}$ & $\begin{array}{l}150 \\
300 \\
250 \\
600 \\
200\end{array}$ & $\begin{array}{r}15 \\
2 \\
10 \\
15\end{array}$ & $\begin{array}{l}\text { II } \\
\text { I } \\
\text { I } \\
\text { I } \\
\text { I I }\end{array}$ \\
\hline $\begin{array}{l}3383.959 \\
3384.395 \\
3384.983 \\
3385.531 \mathrm{i} \\
3386.498\end{array}$ & $\begin{array}{r}5 \\
150 \\
75 \\
800 \\
400\end{array}$ & $\begin{array}{r}30 \\
2 \\
30 \\
200 \\
200\end{array}$ & $\begin{array}{l}\text { I I } \\
\text { I } \\
\text { I I } \\
\text { I I } \\
\text { I I }\end{array}$ & $\begin{array}{l}3407.617 \\
3407.821 \\
3408.630 \\
3408.745 \\
3409.083\end{array}$ & $\begin{array}{r}75 \\
200 \\
75 \\
200 \\
40\end{array}$ & $\begin{array}{r}20 \\
10 \\
10 \\
5\end{array}$ & $\begin{array}{l}\text { II } \\
\text { I } \\
\text { II } \\
\text { I } \\
\text { II }\end{array}$ & $\begin{array}{l}3429.568 \\
3429.884 \\
3430.324 \\
3430.536 \\
3431.008\end{array}$ & $\begin{array}{r}200 \\
400 \\
50 \\
150 \\
150\end{array}$ & $\begin{array}{r}20 \\
8 \\
20 \\
20\end{array}$ & $\begin{array}{l}\text { I } \\
\text { II } \\
\text { II } \\
\text { II } \\
\text { II }\end{array}$ \\
\hline $\begin{array}{l}3386.756 \\
3387.129 \\
3387.463 \\
3387.911 \\
3388.145\end{array}$ & $\begin{array}{r}75 \\
50 \\
150 \\
800 \\
75\end{array}$ & $\begin{array}{r}20 \\
10 \\
5\end{array}$ & $\begin{array}{l}\text { I I } \\
\text { I I } \\
\text { I } \\
\text { I } \\
\text { I }\end{array}$ & $\begin{array}{l}3409.264 \\
3410.064 \\
3410.702 \\
3410.823 \\
3411.369\end{array}$ & $\begin{array}{r}250 \\
300 \\
20 \\
20 \\
200\end{array}$ & 200 & $\begin{array}{l}\text { II } \\
\text { I } \\
\text { I } \\
\text { I } \\
\text { II }\end{array}$ & $\begin{array}{l}3431.251 \\
3431.805 \\
3432.065 \\
3432.270 \\
3432.378\end{array}$ & $\begin{array}{r}200 \\
200 \\
50 \\
40 \\
75\end{array}$ & $\begin{array}{l}100 \\
15 \mathrm{~h}\end{array}$ & $\begin{array}{l}\text { I } \\
\text { II } \\
\text { I I } \\
\text { I } \\
\text { I }\end{array}$ \\
\hline $\begin{array}{l}3388.576 \\
3389.041 \\
3389.456 \\
3389.646 \\
3390.365\end{array}$ & $\begin{array}{l}300 \\
350 \\
900 \\
300 \\
400\end{array}$ & $\begin{array}{l}50 \\
10 \\
75 \\
20\end{array}$ & $\begin{array}{l}\text { II } \\
\text { I } \\
\text { I } \\
\text { II } \\
\text { II }\end{array}$ & $\begin{array}{l}3411.769 \\
3412.158 \\
3412.398 \\
3413.013 i \\
3413.385\end{array}$ & $\begin{array}{r}400 \\
125 \\
100 \\
1800 \\
200\end{array}$ & $\begin{array}{l}100 \\
50 \\
12 \\
20\end{array}$ & $\begin{array}{l}\text { II } \\
\text { I } \\
\text { II } \\
\text { I } \\
\text { II }\end{array}$ & $\begin{array}{l}3432.860 \\
3433.203 \\
3433.999 \mathrm{i} \\
3434.252 \\
3434.736\end{array}$ & $\begin{array}{r}40 \\
50 \\
3000 \\
200 \\
900\end{array}$ & $\begin{array}{r}300 \\
30\end{array}$ & $\begin{array}{l}\text { I } \\
\text { I } \\
\text { II } \\
\text { I } \\
\text { I }\end{array}$ \\
\hline $\begin{array}{l}3390.805 \\
3392.035 \mathrm{i} \\
3392.947 \\
3393.232 \\
3393.406\end{array}$ & $\begin{array}{r}200 \\
6000 \\
100 \\
100 \\
200\end{array}$ & $\begin{array}{r}18 \\
=30 \\
15 \\
15 \\
2\end{array}$ & $\begin{array}{l}\text { II } \\
\text { II } \\
\text { II } \\
\text { I I } \\
\text { I }\end{array}$ & $\begin{array}{l}3413.627 \\
3413.918 \\
3414.506 \\
3414.915 \\
3415.121\end{array}$ & $\begin{array}{l}150 \\
100 \\
200 \\
75 \\
40 \mathrm{~h}\end{array}$ & $\begin{array}{r}2 \\
5 \\
100\end{array}$ & $\begin{array}{l}\text { I } \\
\text { I } \\
\text { I I } \\
\text { I } \\
\text { I }\end{array}$ & $\begin{array}{l}3435.170 \\
3435.372 \\
3435.980 \\
3436.708 \\
3437.017\end{array}$ & $\begin{array}{l}200 \\
1251 \\
2000 \\
1200 \\
150\end{array}$ & $\begin{array}{r}2 \\
2 \\
200 \\
50 \\
20\end{array}$ & $\begin{array}{l}\text { I } \\
\text { I } \\
\text { II } \\
\text { II } \\
\text { II }\end{array}$ \\
\hline $\begin{array}{l}3393.986 \\
3394.130 \\
3394.579 \\
3394.793 \\
3395.095\end{array}$ & $\begin{array}{r}200 \\
200 \\
150 \\
300 \\
80\end{array}$ & $\begin{array}{r}100 \\
50 \\
100 \\
5\end{array}$ & $\begin{array}{l}\text { I } \\
\text { I I } \\
\text { I I } \\
\text { I I } \\
\text { I }\end{array}$ & $\begin{array}{l}3415.474 \\
3415.884 \\
3416.402 \\
3416.586 \\
3417.099\end{array}$ & $\begin{array}{r}50 \\
200 \\
100 \\
100 \\
50\end{array}$ & $\begin{array}{l}25 \\
25 \\
10\end{array}$ & $\begin{array}{l}\text { I } \\
\text { II } \\
\text { II } \\
\text { I } \\
\text { II }\end{array}$ & $\begin{array}{l}3437.309 \\
3437.677 \\
3437.780 \\
3437.983 \\
3438.955\end{array}$ & $\begin{array}{r}2500 \\
20 \\
50 \\
40 \\
600\end{array}$ & $\begin{array}{r}30 \\
10 \\
200\end{array}$ & $\begin{array}{l}\text { I } \\
\text { I } \\
\text { I I } \\
\text { I } \\
\text { I I }\end{array}$ \\
\hline $\begin{array}{l}3395.368 \\
3395.658 \\
3395.979 \\
3396.409 \\
3396.727 \mathrm{i}\end{array}$ & $\begin{array}{l}200 \\
100 w \\
200 \\
200 \\
1400\end{array}$ & $\begin{array}{l}30 \\
20 \\
10 \\
20 \\
40\end{array}$ & $\begin{array}{l}\text { II } \\
\text { I I } \\
\text { II } \\
\text { II } \\
\text { I }\end{array}$ & $\begin{array}{l}3417.487 \\
3417.668 \\
3417.704 \\
3418.769 \\
3418.937\end{array}$ & $\begin{array}{r}250 \\
15 \\
100 \\
300 \\
300\end{array}$ & $\begin{array}{r}3 \\
20 \\
100 \\
100\end{array}$ & $\begin{array}{l}\text { I } \\
\text { II } \\
\text { II } \\
\text { II } \\
\text { I I }\end{array}$ & $\begin{array}{l}3439.403 \\
3439.722 \\
3440.428 \\
3441.012 \\
3441.361\end{array}$ & $\begin{array}{r}500 \\
1000 \\
80 \\
300 \\
250\end{array}$ & $\begin{array}{r}300 \\
50 \\
50\end{array}$ & $\begin{array}{l}\text { I } \\
\text { I I } \\
\text { I } \\
\text { I I } \\
\text { I I }\end{array}$ \\
\hline
\end{tabular}


Table 1. Wavelengths of Thorium Spectra - Continued

\begin{tabular}{|c|c|c|c|c|c|c|c|c|c|c|c|}
\hline \multirow{2}{*}{$\begin{array}{l}\text { Wave- } \\
\text { length }\end{array}$} & \multicolumn{2}{|c|}{ Intensity } & \multirow{2}{*}{$\begin{array}{l}\text { Spec- } \\
\text { trum }\end{array}$} & \multirow{2}{*}{$\begin{array}{l}\text { Wave- } \\
\text { length }\end{array}$} & \multicolumn{2}{|c|}{ Intensity } & \multirow{2}{*}{$\begin{array}{l}\text { Spec- } \\
\text { trum }\end{array}$} & \multirow{2}{*}{$\begin{array}{l}\text { Wave- } \\
\text { length }\end{array}$} & \multicolumn{2}{|c|}{ Intensity } & \multirow{2}{*}{$\begin{array}{l}\text { Spec- } \\
\text { trum }\end{array}$} \\
\hline & Lamp & & & & & Spa & & & & & \\
\hline $\begin{array}{l}3441.509 \\
3442.579 \mathrm{i} \\
3443.111 \\
3443.381 \\
3443.506\end{array}$ & $\begin{array}{l}250 \\
800 \\
250 \\
200 \\
150 \mathrm{w}\end{array}$ & $\begin{array}{r}10 \\
100\end{array}$ & $\begin{array}{l}\text { I } \\
\text { I } \\
\text { II } \\
\text { I } \\
\text { I }\end{array}$ & $\begin{array}{l}3459.632 \\
3461.017 \\
3461.216 \\
3461.561 \\
3462.850 \mathrm{i}\end{array}$ & $\begin{array}{r}125 \\
1600 \\
1600 \\
100 \\
2000\end{array}$ & $\begin{array}{r}10 \\
30 \\
20 \\
1 \\
200\end{array}$ & $\begin{array}{l}\text { II } \\
\text { I } \\
\text { I } \\
\text { I } \\
\text { II }\end{array}$ & $\begin{array}{l}3476.887 \\
3477.699 \\
3478.125 \\
3478.461 \\
3478.602\end{array}$ & $\begin{array}{l}75 \\
200 \\
200 \\
200 \\
150 \mathrm{w}\end{array}$ & $\begin{array}{r}6 \\
50 \\
50 \\
50\end{array}$ & $\begin{array}{l}\text { I I } \\
\text { I I } \\
\text { I I } \\
\text { I I } \\
\text { I }\end{array}$ \\
\hline $\begin{array}{l}3444.003 \\
3444.250 \\
3444.790 \\
3445.213 \\
3445.377\end{array}$ & $\begin{array}{r}150 \\
125 \\
75 \\
250 \\
200\end{array}$ & $\begin{array}{l}40 \\
25 \\
75 \\
50\end{array}$ & $\begin{array}{l}\text { II } \\
\text { I } \\
\text { I } \\
\text { II } \\
\text { II }\end{array}$ & $\begin{array}{l}3463.580 \\
3463.719 \\
3464.417 \\
3464.470 \\
3464.571\end{array}$ & $\begin{array}{r}75 \\
1500 \\
100 \\
50 \\
20\end{array}$ & $\begin{array}{r}5 \\
200 \\
5 \\
2 \\
1\end{array}$ & $\begin{array}{l}\text { II } \\
\text { II } \\
\text { II } \\
\text { I } \\
\text { I }\end{array}$ & $\begin{array}{l}3479.028 \\
3479.172 \mathrm{i} \\
3479.675 \\
3480.048 \\
3480.792\end{array}$ & $\begin{array}{l}150 \\
800 \\
350 \\
500 \\
300\end{array}$ & $\begin{array}{r}100 \\
200 \\
10 \\
2 \\
5\end{array}$ & $\begin{array}{l}\text { II } \\
\text { I I } \\
\text { I } \\
\text { I } \\
\text { I I }\end{array}$ \\
\hline $\begin{array}{l}3445.732 \\
3446.532 \\
3446.962 \\
3447.169 \\
3447.610\end{array}$ & $\begin{array}{l}300 \\
5001 \\
50 \\
50 \\
300\end{array}$ & $\begin{array}{r}80 \\
50 \\
4 \\
2 \\
40\end{array}$ & $\begin{array}{l}\text { I I } \\
\text { I I } \\
\text { I I } \\
\text { I } \\
\text { I I }\end{array}$ & $\begin{array}{l}3464.720 \\
3464.862 \\
3465.027 \\
3465.060 \\
3465.465\end{array}$ & $\begin{array}{l}10 \\
200 \mathrm{w} \\
150 \\
40 \\
50\end{array}$ & $\begin{array}{r}10 \\
1 \\
1\end{array}$ & $\begin{array}{l}\text { I } \\
\text { I } \\
\text { II } \\
\text { I } \\
\text { I }\end{array}$ & $\begin{array}{l}3480.994 \\
3481.597 \\
3482.278 \\
3482.546 \\
3482.762\end{array}$ & $\begin{array}{r}40 \\
100 \\
200 \\
200 \\
150\end{array}$ & $\begin{array}{r}8 \\
1 \\
25 \\
25\end{array}$ & $\begin{array}{l}\text { I I } \\
\text { I } \\
\text { I } \\
\text { I I } \\
\text { I I }\end{array}$ \\
\hline $\begin{array}{l}3447.833 \\
3448.946 \\
3449.278 \\
3449.478 \\
3449.643\end{array}$ & $\begin{array}{r}100 \\
400 \\
200 \\
75 \\
500\end{array}$ & $\begin{array}{r}40 \\
100\end{array}$ & $\begin{array}{l}\text { I } \\
\text { I } \\
\text { II } \\
\text { I } \\
\text { I I }\end{array}$ & $\begin{array}{l}3465.771 \\
3465.915 \\
3466.082 \\
3466.190 \\
3466.523\end{array}$ & $\begin{array}{r}400 \\
200 \\
50 \\
100 \\
800\end{array}$ & $\begin{array}{r}200 \\
15 \\
2 \\
2 \\
4\end{array}$ & $\begin{array}{l}\text { I I } \\
\text { I I } \\
\text { I } \\
\text { I } \\
\text { I }\end{array}$ & $\begin{array}{l}3483.110 \\
3483.163 \\
3483.246 \\
3483.402 \\
3484.072\end{array}$ & $\begin{array}{l}200 \\
100 \\
200 \\
200 \\
300\end{array}$ & $\begin{array}{r}1 \\
20 \\
2 \\
2 \\
40\end{array}$ & $\begin{array}{l}\text { I } \\
\text { I I } \\
\text { I } \\
\text { I } \\
\text { I I }\end{array}$ \\
\hline $\begin{array}{l}3449.942 \\
3450.250 \\
3450.704 \\
3450.944 \\
3451.297\end{array}$ & $\begin{array}{r}200 \\
50 \\
75 \\
150 \\
100\end{array}$ & $\begin{array}{r}50 \\
5 \\
50\end{array}$ & $\begin{array}{l}\text { II } \\
\text { I } \\
\text { II } \\
\text { I I } \\
\text { I }\end{array}$ & $\begin{array}{l}3466.639 \\
3466.886 \\
3467.237 \\
3467.360 \\
3467.658\end{array}$ & $\begin{array}{l}1000 \\
100 \\
150 \\
50 \\
50 \mathrm{w}\end{array}$ & $\begin{array}{l}5 \\
6 \\
1\end{array}$ & $\begin{array}{l}\text { I } \\
\text { I I } \\
\text { I } \\
\text { I } \\
\text { I }\end{array}$ & $\begin{array}{l}3484.408 \\
3484.562 \\
3484.737 \\
3484.947 \\
3485.204\end{array}$ & $\begin{array}{r}75 \\
100 \\
300 \\
200 \\
500\end{array}$ & 20 & $\begin{array}{l}\text { I I } \\
\text { I } \\
\text { I } \\
\text { I } \\
\text { I I }\end{array}$ \\
\hline $\begin{array}{l}3451.702 \mathrm{i} \\
3452.191 \\
3452.677 \\
3452.910 \\
3453.511\end{array}$ & $\begin{array}{r}900 \\
75 \\
200 \\
300 \\
800\end{array}$ & $\begin{array}{r}20 \\
100 \\
2 \\
12\end{array}$ & $\begin{array}{l}\text { I } \\
\text { I } \\
\text { II } \\
\text { I } \\
\text { I }\end{array}$ & $\begin{array}{l}3467.923 \\
3468.219 \mathrm{i} \\
3468.705 \\
3469.340 \\
3469.918\end{array}$ & $\begin{array}{r}125 \\
2000 \\
200 \\
1200 \\
1200\end{array}$ & $\begin{array}{r}30 \\
75 \\
10 \\
20 \\
300\end{array}$ & $\begin{array}{l}\text { I I } \\
\text { I I } \\
\text { I I } \\
\text { I } \\
\text { I I }\end{array}$ & $\begin{array}{l}3485.465 \\
3485.711 \\
3486.536 \\
3486.828 \\
3486.966\end{array}$ & $\begin{array}{r}150 \\
100 \\
3500 \\
200 \\
200\end{array}$ & $\begin{array}{r}10 \\
100 \\
2 \\
10\end{array}$ & $\begin{array}{l}\text { II } \\
\text { I } \\
\text { I } \\
\text { I } \\
\text { I I }\end{array}$ \\
\hline $\begin{array}{l}3453.907 \\
3454.197 \\
3454.676 \\
3454.955 \\
3454.990\end{array}$ & $\begin{array}{r}75 \\
150 \\
75 \\
50 \\
15\end{array}$ & $\begin{array}{r}8 \\
15 \\
20 \\
15 \\
2\end{array}$ & $\begin{array}{l}\text { I I } \\
\text { I I } \\
\text { I I } \\
\text { I I } \\
\text { I I }\end{array}$ & $\begin{array}{l}3470.557 \\
3470.998 \\
3471.207 \\
3471.952 \\
3472.687\end{array}$ & $\begin{array}{r}300 \\
300 \\
1200 \\
500 \\
200\end{array}$ & $\begin{array}{l}3 \\
1 \\
6 \\
4 \\
2\end{array}$ & $\begin{array}{l}\text { I } \\
\text { I } \\
\text { I } \\
\text { I } \\
\text { I }\end{array}$ & $\begin{array}{l}3487.071 \\
3487.836 \\
3487.974 \\
3488.121 \\
3488.330\end{array}$ & $\begin{array}{l}150 \\
200 \\
200 \\
100 \\
100\end{array}$ & $\begin{array}{l}30 \\
40\end{array}$ & $\begin{array}{l}\text { I I } \\
\text { I I } \\
\text { I } \\
\text { I } \\
\text { I }\end{array}$ \\
\hline $\begin{array}{l}3455.111 \\
3455.265 \\
3455.597 \\
3455.922 \\
3456.429\end{array}$ & $\begin{array}{r}25 \\
100 \\
400 \\
75 \\
200\end{array}$ & $\begin{array}{r}3 \\
20 \\
10 \\
4\end{array}$ & $\begin{array}{l}\text { II } \\
\text { I I } \\
\text { I } \\
\text { I I } \\
\text { I }\end{array}$ & $\begin{array}{l}3472.841 \\
3473.027 \\
3473.409 \\
3473.540 \\
3473.770\end{array}$ & $\begin{array}{l}100 \\
350 \\
150 \\
400 \\
150\end{array}$ & $\begin{array}{r}10 \\
20 \\
2 \\
10\end{array}$ & $\begin{array}{l}\text { I } \\
\text { I I } \\
\text { I I } \\
\text { I } \\
\text { I I }\end{array}$ & $\begin{array}{l}3488.474 \\
3488.830 \\
3489.187 \\
3489.499 \\
3489.713\end{array}$ & $\begin{array}{l}100 \\
800 \\
800 \\
8001 \\
200\end{array}$ & $\begin{array}{r}2 \\
4 \\
10\end{array}$ & $\begin{array}{l}\text { I } \\
\text { I } \\
\text { I } \\
\text { I } \\
\text { I }\end{array}$ \\
\hline $\begin{array}{l}3456.673 \\
2456.920 \\
3457.062 \\
3457.423 \\
3457.675\end{array}$ & $\begin{array}{r}300 \\
50 \\
800 \\
100 \\
100\end{array}$ & $\begin{array}{r}3 \\
15 \\
1 \\
10\end{array}$ & $\begin{array}{l}\text { I } \\
\text { I I } \\
\text { I } \\
\text { I } \\
\text { I I }\end{array}$ & $\begin{array}{l}3473.941 \\
3474.273 \\
3474.522 \\
3474.708 \\
3475.169\end{array}$ & $\begin{array}{l}50 \mathrm{w} \\
200 \\
200 \\
175 \\
50\end{array}$ & 15 & $\begin{array}{l}\text { I } \\
\text { I I } \\
\text { I } \\
\text { I } \\
\text { I }\end{array}$ & $\begin{array}{l}3489.823 \\
3490.265 \\
3490.450 \\
3491.571 \\
3491.894\end{array}$ & $\begin{array}{l}100 \\
200 \\
300 \\
300 \\
600\end{array}$ & $\begin{array}{r}30 \\
40 \\
50 \\
50 \\
1\end{array}$ & $\begin{array}{l}\text { II } \\
\text { I I } \\
\text { II } \\
\text { I I } \\
\text { I }\end{array}$ \\
\hline $\begin{array}{l}3457.810 \\
3457.897 \\
3458.136 \\
3458.754 \\
3459.481\end{array}$ & $\begin{array}{r}50 \\
75 \\
200 \\
50 \\
150\end{array}$ & 10 & $\begin{array}{l}\text { I } \\
\text { II } \\
\text { I } \\
\text { I } \\
\text { I I }\end{array}$ & $\begin{array}{l}3475.533 \\
3475.946 \\
3476.237 \\
3476.377 \\
3476.545\end{array}$ & $\begin{array}{l}150 \\
400 \mathrm{w} \\
100 \\
400 \\
200\end{array}$ & $\begin{array}{r}25 \\
3 \\
1 \\
30\end{array}$ & $\begin{array}{l}\text { II } \\
\text { I } \\
\text { I } \\
\text { I } \\
\text { I I }\end{array}$ & $\begin{array}{l}3492.117 \\
3492.317 \\
3492.357 \\
3492.460 \\
3492.674\end{array}$ & $\begin{array}{l}100 \\
100 \\
100 \mathrm{w} \\
100 \mathrm{w} \\
100\end{array}$ & $\begin{array}{r}1 \\
1 \\
1 \\
1 \\
15\end{array}$ & $\begin{array}{l}\text { I } \\
\text { I } \\
\text { I } \\
\text { I } \\
\text { I I }\end{array}$ \\
\hline
\end{tabular}


Table 1. Wavelengths of Thorium Spectra - Continued

\begin{tabular}{|c|c|c|c|c|c|c|c|c|c|c|c|}
\hline \multirow{2}{*}{$\begin{array}{l}\text { Wave- } \\
\text { length }\end{array}$} & \multicolumn{2}{|c|}{ Intensity } & \multirow{2}{*}{$\begin{array}{l}\text { Spec- } \\
\text { trum }\end{array}$} & \multirow{2}{*}{$\begin{array}{l}\text { Wave- } \\
\text { length }\end{array}$} & \multicolumn{2}{|c|}{ Intensity } & \multirow{2}{*}{$\begin{array}{l}\text { Spec- } \\
\text { trum }\end{array}$} & \multirow{2}{*}{$\begin{array}{l}\text { Wave- } \\
\text { length }\end{array}$} & \multicolumn{2}{|c|}{ Intensity } & \multirow{2}{*}{$\begin{array}{l}\text { Spec- } \\
\text { trum }\end{array}$} \\
\hline & & Spark & & & Lamp & Spark & & & Lan & Spark & \\
\hline $\begin{array}{l}3492.893 \\
3492.943 \\
3493.073 \\
3493.241 \\
3493.518 \mathrm{i}\end{array}$ & $\begin{array}{r}200 \\
400 \\
100 \\
200 \\
2000\end{array}$ & $\begin{array}{r}1 \\
10 \\
20 \\
150\end{array}$ & $\begin{array}{l}\text { I } \\
\text { I } \\
\text { II } \\
\text { II } \\
\text { II }\end{array}$ & $\begin{array}{l}3506.641 \\
3506.853 \\
3507.183 \\
3507.507 \\
3507.568\end{array}$ & $\begin{array}{r}600 \\
200 \\
500 \\
600 \\
10\end{array}$ & 500 & $\begin{array}{l}\text { I } \\
\text { II } \\
\text { I } \\
\text { I II }\end{array}$ & $\begin{array}{l}3519.111 \\
3519.693 \\
3520.134 \\
3520.680 \\
3520.704\end{array}$ & $\begin{array}{l}100 \\
200 \\
100 \\
100 \\
400\end{array}$ & $\begin{array}{r}2 \\
40 \\
10\end{array}$ & $\begin{array}{l}\text { I } \\
\text { II } \\
\text { I } \\
\text { II } \\
\text { I }\end{array}$ \\
\hline $\begin{array}{l}3494.172 \\
3494.556 \\
3494.801 \\
3495.146 \\
3495.699\end{array}$ & $\begin{array}{l}100 \mathrm{w} \\
100 \\
300 \\
100 \\
900\end{array}$ & $\begin{array}{c}20 w \\
2 \\
1 \\
1\end{array}$ & $\begin{array}{l}\text { I } \\
\text { II } \\
\text { I } \\
\text { I } \\
\text { I }\end{array}$ & $\begin{array}{l}3507.807 \\
3508.077 \\
3508.183 \\
3508.353 \\
3508.684\end{array}$ & $\begin{array}{l}500 \\
600 \mathrm{w} \\
200 \\
400 \\
200\end{array}$ & & $\begin{array}{l}\text { I } \\
\text { I } \\
\text { I } \\
\text { I } \\
\text { I }\end{array}$ & $\begin{array}{l}3521.062 \\
3521.509 \\
3521.919 \\
3522.268 \\
3522.399\end{array}$ & $\begin{array}{r}500 \\
100 \\
800 \\
100 \\
50\end{array}$ & $\begin{array}{r}3 \\
1 \\
200 \\
1\end{array}$ & $\begin{array}{l}\text { I } \\
\text { I } \\
\text { II } \\
\text { I } \\
\text { I }\end{array}$ \\
\hline $\begin{array}{l}3496.051 \\
3496.810 \\
3497.008 \\
3497.260 \\
3497.458\end{array}$ & $\begin{array}{l}500 \\
500 \\
200 \\
500 \\
200\end{array}$ & $\begin{array}{r}1 \\
2 \\
40 \\
5 \\
2\end{array}$ & $\begin{array}{l}\text { I } \\
\text { I } \\
\text { I I } \\
\text { I } \\
\text { I }\end{array}$ & $\begin{array}{l}3509.094 \\
3509.189 \\
3509.803 \\
3509.946 \\
3510.308\end{array}$ & $\begin{array}{r}500 \\
100 \\
50 \\
200 \\
200\end{array}$ & $\begin{array}{r}10 \\
10 \\
3\end{array}$ & $\begin{array}{l}\text { I } \\
\text { II } \\
\text { II } \\
\text { I } \\
\text { I }\end{array}$ & $\begin{array}{l}3522.565 \\
3522.605 \\
3522.658 \\
3523.021 \\
3523.197\end{array}$ & $\begin{array}{r}50 \\
100 \\
200 \\
300 \\
200\end{array}$ & & $\begin{array}{l}\text { I } \\
\text { I } \\
\text { I } \\
\text { I } \\
\text { I }\end{array}$ \\
\hline $\begin{array}{l}3497.682 \\
3498.001 \\
3498.621 \mathrm{i} \\
3498.957 \\
3498.989\end{array}$ & $\begin{array}{l}200 \\
300 \\
900 \\
400 \\
300\end{array}$ & $\begin{array}{r}30 \\
50 \\
8 \\
50\end{array}$ & $\begin{array}{l}\text { II } \\
\text { II } \\
\text { I } \\
\text { I } \\
\text { II }\end{array}$ & $\begin{array}{l}3510.534 \\
3510.731 \\
3511.004 \\
3511.157 \mathrm{i} \\
3511.286\end{array}$ & $\begin{array}{r}250 \\
400 \\
50 \\
1000 \\
100\end{array}$ & $\begin{array}{r}25 \\
50 \\
\\
2 \\
5\end{array}$ & $\begin{array}{l}\text { II } \\
\text { II } \\
\text { I } \\
\text { I } \\
\text { II }\end{array}$ & $\begin{array}{l}3523.306 \\
3523.513 \\
3523.682 \\
3523.758 \\
3524.180\end{array}$ & $\begin{array}{l}75 \\
500 \mathrm{w} \\
100 \\
400 \\
300\end{array}$ & 2 & $\begin{array}{l}\text { I } \\
\text { I } \\
\text { I } \\
\text { I } \\
\text { I }\end{array}$ \\
\hline $\begin{array}{l}3499.821 \\
3499.983 \\
3500.097 \\
3500.263 \\
3500.532\end{array}$ & $\begin{array}{l}300 \\
500 \\
500 \\
300 \mathrm{w} \\
300\end{array}$ & $\begin{array}{r}40 \\
2 \\
25\end{array}$ & $\begin{array}{l}\text { I } \\
\text { II } \\
\text { I } \\
\text { I } \\
\text { I }\end{array}$ & $\begin{array}{l}3511.559 \\
3511.673 \\
3512.347 \\
3512.747 \\
3513.213\end{array}$ & $\begin{array}{r}500 \\
1000 \\
100 \\
400 \\
300\end{array}$ & $\begin{array}{l}100 \\
500 \mathrm{w} \\
40 \\
20\end{array}$ & $\begin{array}{l}\text { I I } \\
\text { I I } \\
\text { I } \\
\text { II } \\
\text { I I }\end{array}$ & $\begin{array}{l}3524.365 \\
3524.706 \\
3524.905 \\
3525.123 \\
3525.626\end{array}$ & $\begin{array}{r}50 \\
300 \\
50 \\
50 \\
100\end{array}$ & $\begin{array}{l}15 \\
10\end{array}$ & $\begin{array}{l}\text { I } \\
\text { I } \\
\text { I } \\
\text { II } \\
\text { I I }\end{array}$ \\
\hline $\begin{array}{l}3500.578 \\
3500.835 \\
3501.002 \\
3501.217 \\
3501.458\end{array}$ & $\begin{array}{l}300 \\
200 \\
200 \\
200 \\
600\end{array}$ & 75 & $\begin{array}{l}\text { I } \\
\text { I } \\
\text { I } \\
\text { I } \\
\text { I I }\end{array}$ & $\begin{array}{l}3513.292 \\
3513.438 \\
3513.759 \\
3514.286 \\
3514.526\end{array}$ & $\begin{array}{l}200 \\
200 \\
100 \\
300 \\
700\end{array}$ & $\begin{array}{r}2 \\
4 \\
75\end{array}$ & $\begin{array}{l}\text { I } \\
\text { I } \\
\text { II } \\
\text { I } \\
\text { II }\end{array}$ & $\begin{array}{l}3526.041 \\
3526.249 \\
3526.640 \\
3526.761 \\
3527.006\end{array}$ & $\begin{array}{l}200 \\
150 \\
500 \\
500 \\
100\end{array}$ & $\begin{array}{r}25 \\
2 \\
4\end{array}$ & $\begin{array}{l}\text { I } \\
\text { I I } \\
\text { I } \\
\text { I } \\
\text { I }\end{array}$ \\
\hline $\begin{array}{l}3501.626 \\
3501.735 \\
3501.867 \\
3501.942 \\
3502.150\end{array}$ & $\begin{array}{l}200 \\
200 \\
500 \mathrm{w} \\
300 \\
75\end{array}$ & $\begin{array}{r}1 \\
1 \\
25\end{array}$ & $\begin{array}{l}\text { I } \\
\text { I } \\
\text { I } \\
\text { II } \\
\text { I }\end{array}$ & $\begin{array}{l}3514.622 \\
3514.962 \\
3515.222 \\
3515.323 \\
3515.425\end{array}$ & $\begin{array}{r}75 \\
500 \\
200 \\
500 \\
75\end{array}$ & 10 & $\begin{array}{l}\text { I } \\
\text { II } \\
\text { I } \\
\text { I } \\
\text { II }\end{array}$ & $\begin{array}{l}3527.324 \\
3527.795 \\
3528.088 \\
3528.408 \\
3528.816\end{array}$ & $\begin{array}{r}200 \\
250 \\
50 \\
350 \\
500\end{array}$ & $\begin{array}{c}6 \mathrm{w} \\
2 \\
100 \mathrm{w}\end{array}$ & $\begin{array}{l}\text { I } \\
\text { I } \\
\text { I I } \\
\text { I } \\
\text { I I }\end{array}$ \\
\hline $\begin{array}{l}3502.769 \\
3502.954 \\
3503.124 \\
3503.611 \\
3503.785 \mathrm{i}\end{array}$ & $\begin{array}{l}500 \\
600 \\
600 \\
400 \\
500\end{array}$ & $\begin{array}{r}75 \\
3\end{array}$ & $\begin{array}{l}\text { I I } \\
\text { I } \\
\text { I } \\
\text { I I } \\
\text { I }\end{array}$ & $\begin{array}{l}3515.550 \\
3515.710 \\
3515.851 \\
3515.927 \\
3516.003\end{array}$ & $\begin{array}{l}75 \\
200 \\
150 \\
100 \\
100 \mathrm{w}\end{array}$ & $\begin{array}{l}4 \\
8 \\
8\end{array}$ & $\begin{array}{l}\text { II } \\
\text { II } \\
\text { II } \\
\text { I } \\
\text { I }\end{array}$ & $\begin{array}{l}3528.953 \\
3529.385 \\
3529.531 \\
3529.679 \\
3530.143\end{array}$ & $\begin{array}{r}600 \\
500 \\
50 \\
400 \\
20\end{array}$ & $\begin{array}{r}150 \\
1 \\
2 \\
6\end{array}$ & $\begin{array}{l}\text { I I } \\
\text { I } \\
\text { I } \\
\text { I } \\
\text { I I }\end{array}$ \\
\hline $\begin{array}{l}3504.033 \\
3504.242 \\
3504.574 \\
3504.631 \\
3504.849\end{array}$ & $\begin{array}{l}200 \\
200 \\
200 \mathrm{w} \\
200 \\
200\end{array}$ & 10 & $\begin{array}{l}\text { II } \\
\text { I } \\
\text { I } \\
\text { I } \\
\text { I }\end{array}$ & $\begin{array}{l}3516.359 \\
3516.580 \\
3516.828 \\
3517.256 \\
3517.455\end{array}$ & $\begin{array}{l}500 \\
400 \\
500 \\
100 \\
100\end{array}$ & $\begin{array}{r}25 \\
2 \\
100\end{array}$ & $\begin{array}{l}\text { II } \\
\text { I } \\
\text { II } \\
\text { I } \\
\text { I }\end{array}$ & $\begin{array}{l}3530.521 \\
3530.741 \\
3531.038 \\
3531.134 \\
3531.282\end{array}$ & $\begin{array}{r}500 \\
100 \\
50 \\
100 \\
200\end{array}$ & $\begin{array}{l}40 \\
10\end{array}$ & $\begin{array}{l}\text { II } \\
\text { II } \\
\text { I } \\
\text { I } \\
\text { I }\end{array}$ \\
\hline $\begin{array}{l}3504.991 \\
3505.485 \\
3506.044 \\
3506.127 \\
3506.340\end{array}$ & $\begin{array}{r}400 \\
300 \\
50 \\
50 \\
300\end{array}$ & $\begin{array}{l}50 \\
10\end{array}$ & $\begin{array}{l}\text { I } \\
\text { I I } \\
\text { I } \\
\text { I I } \\
\text { I }\end{array}$ & $\begin{array}{l}3517.960 \\
3518.086 \\
3518.139 \\
3518.4031 \\
3518.889\end{array}$ & $\begin{array}{l}200 \mathrm{w} \\
100 \\
400 \\
1000 \\
400\end{array}$ & $\begin{array}{r}8 \\
50\end{array}$ & $\begin{array}{l}\text { I } \\
\text { I } \\
\text { I } \\
\text { I } \\
\text { I I }\end{array}$ & $\begin{array}{l}3531.438 \\
3531.643 \\
3531.870 \\
3531.925 \\
3532.095\end{array}$ & $\begin{array}{r}1000 \\
125 \\
150 \\
150 \\
100\end{array}$ & $\begin{array}{r}4 \\
15 \\
50 \\
75\end{array}$ & $\begin{array}{l}\text { I } \\
\text { I I } \\
\text { I I } \\
\text { II } \\
\text { I }\end{array}$ \\
\hline
\end{tabular}


Table 1. Wavelengths of Thorium Spectra - Continued

\begin{tabular}{|c|c|c|c|c|c|c|c|c|c|c|c|}
\hline \multirow{2}{*}{$\begin{array}{l}\text { Wave- } \\
\text { length }\end{array}$} & \multicolumn{2}{|c|}{ Intensity } & \multirow{2}{*}{$\begin{array}{l}\text { Spec- } \\
\text { trum }\end{array}$} & \multirow{2}{*}{$\begin{array}{l}\text { Wave- } \\
\text { length }\end{array}$} & \multicolumn{2}{|c|}{ Intensity } & \multirow{2}{*}{$\begin{array}{l}\text { Spec- } \\
\text { trum }\end{array}$} & \multirow{2}{*}{$\begin{array}{l}\text { Wave- } \\
\text { length }\end{array}$} & \multicolumn{2}{|c|}{ Intensity } & \multirow{2}{*}{$\begin{array}{l}\text { Spec- } \\
\text { trum }\end{array}$} \\
\hline & $\operatorname{Lamp}$ & Spark & & & Lamp & Spark & & & & Spark & \\
\hline $\begin{array}{l}3532.500 \\
3532.998 \\
3533.614 \\
3533.714 \\
3534.227\end{array}$ & $\begin{array}{r}50 \\
20 \\
100 \\
100 \\
300\end{array}$ & $\begin{array}{r}4 \\
10\end{array}$ & $\begin{array}{l}\text { I } \\
\text { I I } \\
\text { I } \\
\text { I I } \\
\text { I }\end{array}$ & $\begin{array}{l}3546.801 \\
3546.943 \\
3547.344 \\
3547.460 \\
3547.927\end{array}$ & $\begin{array}{c}50 \mathrm{w} \\
50 \mathrm{w} \\
1000 \\
50 \\
50\end{array}$ & & $\begin{array}{l}\text { I } \\
\text { I } \\
\text { I } \\
\text { I } \\
\text { I }\end{array}$ & $\begin{array}{l}3561.777 \\
3562.251 \\
3562.529 \\
3562.599 \\
3563.302\end{array}$ & $\begin{array}{l}500 \\
200 \\
100 \\
200 \\
300\end{array}$ & 7 & $\begin{array}{l}\text { I } \\
\text { I } \\
\text { I } \\
\text { I } \\
\text { I }\end{array}$ \\
\hline $\begin{array}{l}3534.374 \\
3534.646 \\
3534.909 \\
3535.247 \\
3535.375\end{array}$ & $\begin{array}{r}100 \\
200 \\
100 \\
200 \\
50\end{array}$ & $\begin{array}{l}1 \\
3\end{array}$ & $\begin{array}{l}\text { I } \\
\text { I } \\
\text { I } \\
\text { I } \\
\text { I I }\end{array}$ & $\begin{array}{l}3548.664 \\
3549.060 \\
3549.282 \\
3549.597 \\
3549.735\end{array}$ & $\begin{array}{r}200 \\
50 \\
100 \\
1200 \\
200\end{array}$ & $\begin{array}{r}3 \\
50\end{array}$ & $\begin{array}{l}\text { I } \\
\text { I } \\
\text { I } \\
\text { I } \\
\text { I I }\end{array}$ & $\begin{array}{l}3563.372 \\
3563.705 \\
3563.911 \\
3563.972 \\
3564.069\end{array}$ & $\begin{array}{r}800 \\
100 \\
250 \\
\\
50\end{array}$ & $\begin{array}{l}2 \\
20 \\
50 \mathrm{w}\end{array}$ & $\begin{array}{l}\text { I } \\
\text { I } \\
\text { I I } \\
\text { I I I } \\
\text { I }\end{array}$ \\
\hline $\begin{array}{l}3536.014 \\
3536.631 \\
3536.978 \\
3537.153 \\
3537.552\end{array}$ & $\begin{array}{l}600 \\
100 \\
100 \\
450 \\
100\end{array}$ & $\begin{array}{r}25 \\
25 \\
100\end{array}$ & $\begin{array}{l}\text { I } \\
\text { II } \\
\text { I } \\
\text { I I } \\
\text { I }\end{array}$ & $\begin{array}{l}3550.045 \\
3550.291 \\
3550.718 \\
3550.783 \\
3550.881\end{array}$ & $\begin{array}{l}100 \\
300 \\
400 \\
300 \\
200\end{array}$ & $\begin{array}{r}50 \\
2 \\
1\end{array}$ & $\begin{array}{l}\text { I } \\
\text { I I } \\
\text { I } \\
\text { I } \\
\text { I }\end{array}$ & $\begin{array}{l}3564.233 \\
3564.502 \\
3564.601 \\
3564.715 \\
3565.044\end{array}$ & $\begin{array}{l}300 \\
150 \\
200 \\
300 \\
100\end{array}$ & $\begin{array}{r}3 \\
50\end{array}$ & $\begin{array}{l}\text { I } \\
\text { I } \\
\text { I } \\
\text { I I } \\
\text { I }\end{array}$ \\
\hline $\begin{array}{l}3537.812 \\
3538.210 \\
3538.502 \\
3538.772 \\
3538.835\end{array}$ & $\begin{array}{r}50 \\
200 \\
100 \\
100 \\
100\end{array}$ & $1000 \mathrm{w}$ & $\begin{array}{l}\text { I } \\
\text { I } \\
\text { I } \\
\text { I I I } \\
\text { I }\end{array}$ & $\begin{array}{l}3551.401 \mathrm{i} \\
3551.738 \\
3551.881 \\
3552.698 \\
3553.110\end{array}$ & $\begin{array}{r}1000 \\
100 \\
100 \\
50 \\
400\end{array}$ & $\begin{array}{r}15 \\
20 \\
100\end{array}$ & $\begin{array}{l}\text { I } \\
\text { I } \\
\text { I I } \\
\text { I } \\
\text { I I }\end{array}$ & $\begin{array}{l}3565.114 \\
3565.176 \\
3565.393 \\
3565.824 \\
3566.193\end{array}$ & $\begin{array}{r}200 \\
150 \\
500 \\
500 \\
50\end{array}$ & $\begin{array}{c}4 \\
20 \\
2 \\
2 w\end{array}$ & $\begin{array}{l}\text { I } \\
\text { I } \\
\text { I } \\
\text { I } \\
\text { I }\end{array}$ \\
\hline $\begin{array}{l}3538.936 \\
3539.321 \\
3539.587 \mathbf{i} \\
3539.846 \\
3540.049\end{array}$ & $\begin{array}{r}50 \\
800 \\
4000 \\
150 \\
100\end{array}$ & $\begin{array}{l}150 \\
100\end{array}$ & $\begin{array}{l}\text { I } \\
\text { I I } \\
\text { I I } \\
\text { I } \\
\text { I }\end{array}$ & $\begin{array}{l}3553.379 \\
3553.953 \\
3554.336 \\
3554.471 \\
3554.648\end{array}$ & $\begin{array}{r}300 \\
50 \\
150 \\
50 \\
10\end{array}$ & 15 & $\begin{array}{l}\text { I I } \\
\text { I } \\
\text { I } \\
\text { I } \\
\text { I I }\end{array}$ & $\begin{array}{l}3566.542 \\
3567.055 \\
3567.264 \mathrm{i} \\
3567.642 \\
3567.694\end{array}$ & $\begin{array}{r}100 \\
200 \\
1200 \\
200 \\
250\end{array}$ & $\begin{array}{l}30 \\
20 \\
30\end{array}$ & $\begin{array}{l}\text { I } \\
\text { II } \\
\text { I } \\
\text { I } \\
\text { I I }\end{array}$ \\
\hline $\begin{array}{l}3540.323 \\
3540.510 \\
3540.526 \\
3541.116 \\
3541.384\end{array}$ & $\begin{array}{r}20 \\
100 \\
50 \\
50 \\
50\end{array}$ & 5 & $\begin{array}{l}\text { I I } \\
\text { I } \\
\text { I } \\
\text { I } \\
\text { I }\end{array}$ & $\begin{array}{l}3555.015 \\
3555.104 \\
3555.706 \\
3555.836 \\
3556.318\end{array}$ & $\begin{array}{l}1500 \\
200 \\
500 \\
200 \mathrm{w} \\
200\end{array}$ & $\begin{array}{r}10 \\
20 \\
4 \\
25\end{array}$ & $\begin{array}{l}\text { I } \\
\text { I I } \\
\text { I } \\
\text { I } \\
\text { I I }\end{array}$ & $\begin{array}{l}3567.966 \\
3568.056 \\
3569.055 \\
3569.193 \\
3569.624\end{array}$ & $\begin{array}{l}250 \\
50 \mathrm{w} \\
50 \\
100 \mathrm{~s} \\
250\end{array}$ & 30 & $\begin{array}{l}\text { II } \\
\text { I } \\
\text { I } \\
\text { I } \\
\text { I I }\end{array}$ \\
\hline $\begin{array}{l}3541.623 \\
3541.973 \\
3542.209 \\
3542.500 \\
3542.629\end{array}$ & $\begin{array}{l}250 \\
100 \\
150 \\
500 \\
200\end{array}$ & $\begin{array}{r}50 \\
15 \\
3 \\
2 \\
20\end{array}$ & $\begin{array}{l}\text { I I } \\
\text { I I } \\
\text { I } \\
\text { I } \\
\text { I I }\end{array}$ & $\begin{array}{l}3556.773 \\
3556.969 \\
3557.037 \\
3557.467 \\
3557.692\end{array}$ & $\begin{array}{r}50 \\
150 \\
50 \\
400 \\
20\end{array}$ & 100 & $\begin{array}{l}\text { I } \\
\text { I } \\
\text { I } \\
\text { I I } \\
\text { I }\end{array}$ & $\begin{array}{l}3569.820 \\
3569.930 \\
3570.358 \\
3570.522 \\
3570.826\end{array}$ & $\begin{array}{c}1000 \\
10 \\
500 \\
250 \\
1001\end{array}$ & $\begin{array}{l}5 \\
3 \\
2 \\
1\end{array}$ & $\begin{array}{l}\text { I } \\
\text { I I } \\
\text { I } \\
\text { I } \\
\text { I }\end{array}$ \\
\hline $\begin{array}{l}3543.025 \\
3543.136 \\
3543.202 \\
3544.018 \mathrm{i} \\
3544.258\end{array}$ & $\begin{array}{c}150 \\
50 \mathrm{w} \\
50 \\
1500 \\
250\end{array}$ & 10 & $\begin{array}{l}\text { I } \\
\text { I } \\
\text { I } \\
\text { I } \\
\text { I }\end{array}$ & $\begin{array}{l}3557.724 \\
3557.811 \\
3558.346 \\
3558.794 \\
3558.843\end{array}$ & $\begin{array}{l}100 \\
150 \\
100 \\
50 \\
100\end{array}$ & $\begin{array}{l}2 \\
3 \\
1 \\
2\end{array}$ & $\begin{array}{l}\text { I } \\
\text { I } \\
\text { I } \\
\text { I } \\
\text { I }\end{array}$ & $\begin{array}{l}3571.008 \\
3571.573 \\
3571.814 \\
3572.070 \\
3572.393\end{array}$ & $\begin{array}{r}300 \\
400 \\
100 \\
300 \\
1000\end{array}$ & $\begin{array}{r}75 \\
2 \\
2 \\
350\end{array}$ & $\begin{array}{l}\text { I } \\
\text { II } \\
\text { I } \\
\text { I } \\
\text { II }\end{array}$ \\
\hline $\begin{array}{l}3544.364 \\
3544.474 \\
3544.779 \\
3544.851 \\
3544.995\end{array}$ & $\begin{array}{r}50 \\
100 \\
150 \\
150 \\
500\end{array}$ & $\begin{array}{r}6 \\
1 \\
1 \\
30\end{array}$ & $\begin{array}{l}\text { I I } \\
\text { I } \\
\text { I } \\
\text { I } \\
\text { I I }\end{array}$ & $\begin{array}{l}3559.449 \mathrm{i} \\
3559.609 \\
3559.734 \\
3559.951 \\
3560.018\end{array}$ & $\begin{array}{r}2500 \\
300 \\
250 \\
300 \\
300\end{array}$ & $\begin{array}{r}300 \\
20 \\
30 \\
300\end{array}$ & $\begin{array}{l}\text { I I } \\
\text { I } \\
\text { I I } \\
\text { I I } \\
\text { I }\end{array}$ & $\begin{array}{l}3573.217 \\
3573.509 \\
3574.179 \\
3574.288 \\
3574.479\end{array}$ & $\begin{array}{l}1000 \\
500 \\
400 \mathrm{w} \\
200 \\
200\end{array}$ & $\begin{array}{r}100 \\
50\end{array}$ & $\begin{array}{l}\text { I I } \\
\text { I I } \\
\text { I } \\
\text { I } \\
\text { I }\end{array}$ \\
\hline $\begin{array}{l}3545.183 \\
3545.288 \\
3545.394 \\
3545.961 \\
3546.256\end{array}$ & $\begin{array}{l}200 \\
100 \\
100 \\
500 \\
200\end{array}$ & $\begin{array}{l}10 \\
10 \\
20\end{array}$ & $\begin{array}{l}\text { I I } \\
\text { I I } \\
\text { I I } \\
\text { I } \\
\text { I I }\end{array}$ & $\begin{array}{l}3560.688 \\
3560.848 \\
3560.890 \\
3561.287 \\
3561.404\end{array}$ & $\begin{array}{l}300 \\
75 \\
300 \\
50 \mathrm{~h} \\
250\end{array}$ & 10 & $\begin{array}{l}\text { I } \\
\text { I I } \\
\text { I } \\
\text { I } \\
\text { I }\end{array}$ & $\begin{array}{l}3574.615 \\
3575.121 \\
3575.324 \\
3576.486 \\
3576.557 \mathrm{i}\end{array}$ & $\begin{array}{c}100 \mathrm{~h} \\
1000 \\
750 \\
200 \\
1000\end{array}$ & $\begin{array}{r}1 \\
5 \\
200 \\
10 \\
40\end{array}$ & $\begin{array}{l}\text { I } \\
\text { I } \\
\text { II } \\
\text { I } \\
\text { I }\end{array}$ \\
\hline
\end{tabular}


Table 1. Wavelengths of Thorium Spectra - Continued

\begin{tabular}{|c|c|c|c|c|c|c|c|c|c|c|c|}
\hline \multirow{2}{*}{$\begin{array}{l}\text { Wave- } \\
\text { length }\end{array}$} & \multicolumn{2}{|c|}{ Intensity } & \multirow{2}{*}{$\begin{array}{l}\text { Spec- } \\
\text { trum }\end{array}$} & \multirow{2}{*}{$\begin{array}{l}\text { Wave- } \\
\text { length }\end{array}$} & \multicolumn{2}{|c|}{ Intensity } & \multirow{2}{*}{$\begin{array}{l}\text { Spec- } \\
\text { trum }\end{array}$} & \multirow{2}{*}{$\begin{array}{l}\text { Wave- } \\
\text { length }\end{array}$} & \multicolumn{2}{|c|}{ Intensity } & \multirow{2}{*}{$\begin{array}{l}\text { Spec- } \\
\text { trum }\end{array}$} \\
\hline & amp & Spark & & & Lamp & Spark & & & Lamp & Spark & \\
\hline $\begin{array}{l}3577.140 \\
3577.217 \\
3577.746 \\
3578.168 \\
3578.525\end{array}$ & $\begin{array}{r}50 \\
400 \\
100 \\
500 \\
100\end{array}$ & $\begin{array}{l}50 \\
25\end{array}$ & $\begin{array}{l}\text { I } \\
\text { I I } \\
\text { I } \\
\text { I I } \\
\text { I }\end{array}$ & $\begin{array}{l}3591.907 \\
3592.114 \\
3592.487 \\
3592.779 \mathrm{i} \\
3593.400\end{array}$ & $\begin{array}{r}20 \\
75 \\
50 \\
2000 \\
20\end{array}$ & $\begin{array}{r}2 \\
4 \\
10 \\
2\end{array}$ & $\begin{array}{l}\text { I I } \\
\text { I } \\
\text { I I } \\
\text { I } \\
\text { I }\end{array}$ & $\begin{array}{l}3605.821 \\
3606.092 \\
3606.210 \\
3606.613 \\
3606.693\end{array}$ & $\begin{array}{r}750 \\
125 \\
75 \\
40\end{array}$ & $\begin{array}{c}150 w \\
3\end{array}$ & $\begin{array}{l}\text { I } \\
\text { I } \\
\text { III } \\
\text { I } \\
\text { I }\end{array}$ \\
\hline $\begin{array}{l}3578.951 \\
3579.347 \\
3579.411 \\
3579.452 \\
3579.545\end{array}$ & $\begin{array}{r}800 \\
800 \\
200 \\
800 \\
1000\end{array}$ & $\begin{array}{r}3 \\
150 \\
50\end{array}$ & $\begin{array}{l}\text { I } \\
\text { II } \\
\text { II } \\
\text { I } \\
\text { I }\end{array}$ & $\begin{array}{l}3593.877 \\
3594.100 \\
3594.721 \\
3594.988 \\
3595.250\end{array}$ & $\begin{array}{r}150 \\
100 \\
100 \\
400 \\
4\end{array}$ & $\begin{array}{r}125 \\
8 \\
3 \\
6\end{array}$ & $\begin{array}{l}\text { I I } \\
\text { II } \\
\text { I } \\
\text { I } \\
\text { I I I }\end{array}$ & $\begin{array}{l}3606.790 \\
3607.028 \\
3607.393 \\
3607.653 \\
3607.726\end{array}$ & $\begin{array}{l}75 \\
75 \\
75 \\
20 \\
40\end{array}$ & $\begin{array}{r}15 \\
5\end{array}$ & $\begin{array}{l}\text { I } \\
\text { I I } \\
\text { I I } \\
\text { I } \\
\text { I }\end{array}$ \\
\hline $\begin{array}{l}3579.668 \\
3579.900 \\
3580.046 \\
3580.227 \\
3581.295\end{array}$ & $\begin{array}{l}200 \\
100 \mathrm{w} \\
25 \\
1000 \\
200\end{array}$ & $\begin{array}{r}2 \\
50 \\
3\end{array}$ & $\begin{array}{l}\text { I } \\
\text { I } \\
\text { II } \\
\text { I I } \\
\text { I }\end{array}$ & $\begin{array}{l}3595.330 \\
3595.611 \\
3595.969 \\
3596.308 \\
3596.535\end{array}$ & $\begin{array}{l}100 \\
500 \\
50 \\
20 \mathrm{~h} \\
50\end{array}$ & $\begin{array}{l}20 \\
10\end{array}$ & $\begin{array}{l}\text { II } \\
\text { I } \\
\text { I } \\
\text { I } \\
\text { I }\end{array}$ & $\begin{array}{l}3607.910 \\
3607.986 \\
3608.377 \\
3609.223 \\
3609.443\end{array}$ & $\begin{array}{r}40 \\
20 \\
1200 \\
200 \\
3000\end{array}$ & $\begin{array}{r}2 \\
5 \\
100 \\
500\end{array}$ & $\begin{array}{l}\text { I } \\
\text { I } \\
\text { I } \\
\text { II } \\
\text { I I }\end{array}$ \\
\hline $\begin{array}{l}3581.347 \\
3581.753 \\
3581.837 \\
3582.008 \\
3582.389\end{array}$ & $\begin{array}{l}100 \\
800 \\
300 \mathrm{w} \\
1000 \\
200\end{array}$ & $\begin{array}{r}30 \\
2\end{array}$ & $\begin{array}{l}\text { I } \\
\text { I } \\
\text { I } \\
\text { I I } \\
\text { I }\end{array}$ & $\begin{array}{l}3596.903 \\
3597.019 \\
3597.495 \\
3597.700 \\
3598.120 \mathrm{i}\end{array}$ & $\begin{array}{r}50 \\
40 \\
250 \\
50 \\
2000\end{array}$ & $\begin{array}{r}6 \\
2 \\
4 \\
\\
20\end{array}$ & $\begin{array}{l}\text { I I } \\
\text { I } \\
\text { I } \\
\text { I } \\
\text { I }\end{array}$ & $\begin{array}{l}3610.039 \\
3610.131 \\
3610.395 \\
3610.648 \\
3610.792\end{array}$ & $\begin{array}{l}200 \\
150 \\
200 \\
150 \\
150\end{array}$ & $\begin{array}{r}75 \\
100 \\
150 \\
2 \\
25\end{array}$ & $\begin{array}{l}\text { I I } \\
\text { I I } \\
\text { I I } \\
\text { I } \\
\text { I I }\end{array}$ \\
\hline $\begin{array}{l}3582.486 \\
3583.037 \\
3583.107 \\
3583.294 \\
3583.610\end{array}$ & $\begin{array}{r}200 \\
600 \\
1000 \\
500 \\
100\end{array}$ & $\begin{array}{r}1 \\
50 \\
1 \\
1 \\
1\end{array}$ & $\begin{array}{l}\text { I } \\
\text { I I } \\
\text { I } \\
\text { I } \\
\text { I }\end{array}$ & $\begin{array}{l}3598.523 \\
3598.692 \\
3599.034 \\
3599.357 \\
3599.725\end{array}$ & $\begin{array}{r}100 \\
125 \\
50 \\
75 \\
200\end{array}$ & $\begin{array}{r}6 \\
15 \\
2\end{array}$ & $\begin{array}{l}\text { I } \\
\text { II } \\
\text { I I } \\
\text { I }\end{array}$ & $\begin{array}{l}3611.163 \\
3611.259 \\
3611.513 \\
3611.655 \\
3611.757\end{array}$ & $\begin{array}{r}100 \\
40 \\
20 \\
20 \\
20\end{array}$ & & $\begin{array}{l}\text { I } \\
\text { I } \\
\text { I } \\
\text { I } \\
\text { I }\end{array}$ \\
\hline $\begin{array}{l}3583.931 \\
3584.175 \mathrm{i} \\
3585.054 \\
3585.246 \\
3585.552\end{array}$ & $\begin{array}{l}100 \\
800 \\
400 \\
500 \\
100\end{array}$ & $\begin{array}{r}10 \\
100 \\
5\end{array}$ & $\begin{array}{l}\text { I } \\
\text { I } \\
\text { I I } \\
\text { I } \\
\text { I I }\end{array}$ & $\begin{array}{l}3599.879 \\
3600.202 \\
3600.434 \\
3600.610 \\
3600.824\end{array}$ & $\begin{array}{r}75 \\
50 \\
250 \\
50 \\
50\end{array}$ & $\begin{array}{l}8 \\
2 \\
2 \\
6\end{array}$ & $\begin{array}{l}\text { II } \\
\text { I } \\
\text { I } \\
\text { I } \\
\text { I I }\end{array}$ & $\begin{array}{l}3612.117 \\
3612.427 \mathrm{i} \\
3612.865 \\
3613.089 \\
3613.542\end{array}$ & $\begin{array}{c}40 \mathrm{w} \\
1400 \\
500 \\
20 \\
50\end{array}$ & $\begin{array}{l}3 \\
20 \\
10\end{array}$ & $\begin{array}{l}\text { I I } \\
\text { I } \\
\text { I } \\
\text { I I } \\
\text { I }\end{array}$ \\
\hline $\begin{array}{l}3585.764 \\
3585.885 \\
3586.481 \\
3586.698 \\
3586.959\end{array}$ & $\begin{array}{r}100 \\
100 \\
100 \\
50 \\
30\end{array}$ & $\begin{array}{l}25 \\
10\end{array}$ & $\begin{array}{l}\text { I I } \\
\text { I I } \\
\text { I } \\
\text { I } \\
\text { I I }\end{array}$ & $\begin{array}{l}3601.044 \\
3601.298 \\
3601.611 \\
3601.772 \\
3601.986\end{array}$ & $\begin{array}{r}600 \\
100 \\
50 \\
125 \\
40\end{array}$ & 300 & $\begin{array}{l}\text { I I } \\
\text { I } \\
\text { I } \\
\text { I } \\
\text { I I }\end{array}$ & $\begin{array}{l}3613.616 \\
3613.779 \\
3614.009 \\
3614.211 \\
3614.350\end{array}$ & $\begin{array}{r}20 \\
200 \\
600 \\
500 \\
500\end{array}$ & $\begin{array}{r}2 \\
20 \\
100 \\
3 \\
3\end{array}$ & $\begin{array}{l}\text { I I } \\
\text { I I } \\
\text { I I } \\
\text { I } \\
\text { I }\end{array}$ \\
\hline $\begin{array}{l}3587.097 \\
3587.837 \\
3588.223 \\
3588.303 \\
3589.146\end{array}$ & $\begin{array}{l}100 \\
100 \\
100 \\
125 \\
125\end{array}$ & $\begin{array}{l}25 \\
25 \\
75\end{array}$ & $\begin{array}{l}\text { I } \\
\text { I } \\
\text { I I } \\
\text { I I } \\
\text { I I }\end{array}$ & $\begin{array}{l}3602.301 \\
3602.485 \\
3602.653 \\
3602.910 \\
3603.037\end{array}$ & $\begin{array}{l}40 \\
10 \\
20 \mathrm{w} \\
75 \\
100\end{array}$ & 10 & $\begin{array}{l}\text { I } \\
\text { I I } \\
\text { I } \\
\text { I } \\
\text { I I }\end{array}$ & $\begin{array}{l}3615.132 \mathrm{i} \\
3615.366 \\
3615.845 \\
3616.176 \\
3616.697\end{array}$ & $\begin{array}{r}2000 \\
75 \\
100 \\
125 \\
400\end{array}$ & $\begin{array}{r}125 \\
6 \\
1 \\
1 \\
12\end{array}$ & $\begin{array}{l}\text { I I } \\
\text { I I } \\
\text { I } \\
\text { I } \\
\text { I I }\end{array}$ \\
\hline $\begin{array}{l}3589.362 \\
3589.746 \\
3589.995 \\
3590.354 \\
3590.527\end{array}$ & $\begin{array}{c}400 \\
1200 \\
200 \\
20 \mathrm{~h} \\
40\end{array}$ & $\begin{array}{r}150 \\
15 \\
2 \\
10\end{array}$ & $\begin{array}{l}\text { I I } \\
\text { I } \\
\text { I } \\
\text { I } \\
\text { I I }\end{array}$ & $\begin{array}{l}3603.208 \\
3603.360 \\
3603.489 \\
3603.620 \\
3603.686\end{array}$ & $\begin{array}{r}500 \\
150 \\
40 \\
75 \\
75\end{array}$ & $\begin{array}{r}200 \\
100 \\
5\end{array}$ & $\begin{array}{l}\text { I I } \\
\text { I I } \\
\text { I } \\
\text { I I } \\
\text { I }\end{array}$ & $\begin{array}{l}3617.020 \\
3617.116 \\
3617.665 \\
3617.743 \\
3617.815\end{array}$ & $\begin{array}{r}800 \\
1200 \\
125 \\
40 \\
75\end{array}$ & $\begin{array}{r}200 \\
300 \\
3\end{array}$ & $\begin{array}{l}\text { II } \\
\text { I I } \\
\text { I } \\
\text { II } \\
\text { I }\end{array}$ \\
\hline $\begin{array}{l}3590.933 \\
3591.058 \\
3591.256 \\
3591.452 \\
3591.621\end{array}$ & $\begin{array}{r}125 \\
200 \\
100 \\
1000 \\
30\end{array}$ & 200 & $\begin{array}{l}\text { I } \\
\text { I I } \\
\text { I } \\
\text { I } \\
\text { I I }\end{array}$ & $\begin{array}{l}3604.049 \\
3604.480 \\
3604.680 \\
3605.197 \\
3605.649\end{array}$ & $\begin{array}{l}500 \mathrm{~d} \\
75 \\
800 \\
500 \\
100\end{array}$ & $\begin{array}{r}4 \\
2 \\
15\end{array}$ & $\begin{array}{l}\text { I } \\
\text { I } \\
\text { I } \\
\text { I } \\
\text { I I }\end{array}$ & $\begin{array}{l}3617.941 \\
3618.362 \\
3618.590 \\
3618.756 \\
3619.210\end{array}$ & $\begin{array}{r}20 \\
800 \\
50 \\
200 \\
200\end{array}$ & $\begin{array}{l}2 \\
8 \\
2 \\
1\end{array}$ & $\begin{array}{l}\text { I } \\
\text { I } \\
\text { I } \\
\text { I } \\
\text { I }\end{array}$ \\
\hline
\end{tabular}

$5466370-60-3$ 
Table 1. Wavelengths of Thorium Spectra - Continued

\begin{tabular}{|c|c|c|c|c|c|c|c|c|c|c|c|}
\hline \multirow{2}{*}{$\begin{array}{l}\text { Wave- } \\
\text { length }\end{array}$} & \multicolumn{2}{|c|}{ Intensity } & \multirow{2}{*}{$\begin{array}{l}\text { Spec- } \\
\text { trum }\end{array}$} & \multirow{2}{*}{$\begin{array}{l}\text { Wave- } \\
\text { length }\end{array}$} & \multicolumn{2}{|c|}{ Intensity } & \multirow{2}{*}{$\begin{array}{l}\text { Spec- } \\
\text { trum }\end{array}$} & \multirow{2}{*}{$\begin{array}{l}\text { Wave- } \\
\text { length }\end{array}$} & \multicolumn{2}{|c|}{ Intensity } & \multirow{2}{*}{$\begin{array}{l}\text { Spec- } \\
\text { trum }\end{array}$} \\
\hline & Lamp & Spark & & & Lamp & Spark & & & Lamp & Spar & \\
\hline $\begin{array}{l}3619.345 \\
3619.622 \\
3619.708 \\
3619.924 \\
3620.046\end{array}$ & $\begin{array}{l}20 \\
50 \\
75 \\
40 \\
40\end{array}$ & $\begin{array}{r}2 \\
25\end{array}$ & $\begin{array}{l}\text { I } \\
\text { I } \\
\text { II } \\
I \\
I\end{array}$ & $\begin{array}{l}3630.477 \\
3630.613 \\
3630.844 \\
3630.986 \\
3631.151\end{array}$ & $\begin{array}{l}20 \\
40 \\
40 \mathrm{w} \\
40 \\
40\end{array}$ & $\begin{array}{l}4 \\
1 \\
5\end{array}$ & $\begin{array}{l}\text { I } \\
\text { I I } \\
\text { I } \\
\text { I } \\
\text { I I }\end{array}$ & $\begin{array}{l}3641.400 \\
3641.581 \\
3641.841 \\
3642.249 \mathrm{i} \\
3642.569\end{array}$ & $\begin{array}{r}40 \\
20 \\
100 \\
2200 \\
400\end{array}$ & $\begin{array}{r}20 \\
10 \\
3\end{array}$ & $\begin{array}{l}\text { I } \\
\text { I } \\
\text { I I } \\
\text { I } \\
\text { I }\end{array}$ \\
\hline $\begin{array}{l}3620.287 \\
3620.372 \\
3620.499 \\
3620.557 \\
3620.837\end{array}$ & $\begin{array}{r}200 \\
200 \\
40 \\
40 \\
250\end{array}$ & $\begin{array}{r}75 \\
10 \\
2\end{array}$ & $\begin{array}{l}\text { I } \\
\text { II } \\
\text { I I } \\
\text { I } \\
\text { I }\end{array}$ & $\begin{array}{l}3631.703 \\
3631.861 \\
3632.116 \\
3632.225 \\
3632.624\end{array}$ & $\begin{array}{r}40 \\
100 \\
75 \\
50 \\
100\end{array}$ & 30 & $\begin{array}{l}\text { I } \\
\text { I } \\
\text { I } \\
\text { I } \\
\text { I I }\end{array}$ & $\begin{array}{l}3642.889 \\
3643.026 \\
3643.513 \\
3643.825 \\
3644.014\end{array}$ & $\begin{array}{l}40 \\
40 \mathrm{w} \\
800 \\
20 \mathrm{w} \\
40\end{array}$ & 6 & $\begin{array}{l}\text { I I } \\
\text { I } \\
\text { I } \\
\text { I } \\
\text { I }\end{array}$ \\
\hline $\begin{array}{l}3621.118 \\
3622.282 \\
3622.331 \\
3622.731 \\
3622.795 \mathrm{i}\end{array}$ & $\begin{array}{r}1200 \\
125 \\
1000 \\
40 \\
800\end{array}$ & $\begin{array}{r}200 \\
5 \\
5 \\
4\end{array}$ & $\begin{array}{l}\text { II } \\
\text { I } \\
\text { I } \\
\text { I I } \\
\text { I }\end{array}$ & $\begin{array}{l}3632.830 \mathrm{i} \\
3633.079 \\
3633.244 \\
3633.370 \\
3633.683\end{array}$ & $\begin{array}{c}1000 \\
40 \\
40 \mathrm{~s} \\
50 \\
40\end{array}$ & $\begin{array}{r}10 \\
3\end{array}$ & $\begin{array}{l}\text { I } \\
\text { II } \\
\text { I } \\
\text { II } \\
\text { I }\end{array}$ & $\begin{array}{l}3644.352 \\
3644.716 \\
3644.989 \\
3645.251 \\
3645.364\end{array}$ & $\begin{array}{r}100 \\
50 \\
100 \\
4 \\
200\end{array}$ & $\begin{array}{r}20 \\
5 \\
2 \\
2\end{array}$ & $\begin{array}{l}\text { I I } \\
\text { I I } \\
\text { I } \\
\text { I I } \\
\text { I }\end{array}$ \\
\hline $\begin{array}{l}3622.932 \\
3623.052 \\
3623.768 \\
3623.980 \\
3624.471\end{array}$ & $\begin{array}{r}20 \\
10 \\
50 \\
75 \\
400\end{array}$ & $\begin{array}{r}4 \\
4 \\
2 \\
75 \\
50\end{array}$ & $\begin{array}{l}\text { II } \\
\text { I I } \\
\text { I } \\
\text { I I } \\
\text { I I }\end{array}$ & $\begin{array}{l}3633.735 \\
3633.908 \\
3634.221 \\
3634.580 \\
3634.907\end{array}$ & $\begin{array}{r}20 \\
50 \\
100 \\
1700 \\
100\end{array}$ & $\begin{array}{r}1 \\
25 \\
15\end{array}$ & $\begin{array}{l}I \\
I \\
I I \\
I \\
I\end{array}$ & $\begin{array}{l}3645.569 \\
3645.707 \\
3645.885 \\
3645.951 \\
3646.271\end{array}$ & $\begin{array}{l}100 \\
200 \\
20 \mathrm{~h} \\
40 \\
150\end{array}$ & 2 & $\begin{array}{l}\text { I } \\
\text { I } \\
\text { I } \\
\text { I } \\
\text { I }\end{array}$ \\
\hline $\begin{array}{l}3624.895 \\
3625.027 \\
3625.147 \\
3625.625 \\
3625.889\end{array}$ & $\begin{array}{l}400 \\
400 \\
200 \\
500 \\
150\end{array}$ & $\begin{array}{r}150 \\
100 \\
3 \\
100 \\
75\end{array}$ & $\begin{array}{l}\text { II I } \\
\text { II } \\
\text { I } \\
\text { II } \\
\text { II }\end{array}$ & $\begin{array}{l}3635.242 \\
3635.422 \\
3635.944 \\
3636.176 \\
3636.288\end{array}$ & $\begin{array}{r}100 \\
200 \\
1900 \\
100 \\
50\end{array}$ & $\begin{array}{r}30 \\
200 \\
15 \\
20\end{array}$ & $\begin{array}{l}\text { I I } \\
\text { I I } \\
\text { I } \\
\text { I I } \\
\text { I }\end{array}$ & $\begin{array}{l}3646.580 \\
3646.882 \\
3647.047 \\
3647.304 \\
3647.375\end{array}$ & $\begin{array}{l}20 \\
100 \\
20 \mathrm{w} \\
100 \\
100\end{array}$ & $\begin{array}{r}20 \\
2 \\
10 \\
10\end{array}$ & $\begin{array}{l}\text { I } \\
\text { I I } \\
\text { I I } \\
\text { I I } \\
\text { I I }\end{array}$ \\
\hline $\begin{array}{l}3625.927 \\
3626.085 \\
3626.473 \\
3626.558 \\
3626.619\end{array}$ & $\begin{array}{l}250 \\
75 \\
100 \\
100 \mathrm{~h} \\
100 \mathrm{~h}\end{array}$ & $\begin{array}{r}50 \\
5 \\
1 \\
1 \\
1\end{array}$ & $\begin{array}{l}\text { I I } \\
\text { I } \\
\text { I } \\
\text { I } \\
\text { I }\end{array}$ & $\begin{array}{l}3636.350 \\
3636.566 \\
3636.831 \\
3637.557 \\
3637.684\end{array}$ & $\begin{array}{r}40 \\
100 \\
400 \\
150 \\
50\end{array}$ & $\begin{array}{r}2 \\
20 \\
2 \\
25 \\
3\end{array}$ & $\begin{array}{l}\text { I } \\
\text { I I } \\
\text { I } \\
\text { I I } \\
\text { I }\end{array}$ & $\begin{array}{l}3647.646 \\
3648.171 \\
3648.420 \\
3648.635 \\
3648.852\end{array}$ & $\begin{array}{r}200 \\
100 \\
150 \\
50 \\
100\end{array}$ & $\begin{array}{r}50 \\
15 \\
20 \\
4 \\
3\end{array}$ & $\begin{array}{l}\text { I I } \\
\text { I I } \\
\text { I I } \\
\text { I I } \\
\text { I }\end{array}$ \\
\hline $\begin{array}{l}3626.940 \\
3627.073 \\
3627.152 \\
3627.264 \\
3627.339\end{array}$ & $\begin{array}{r}150 \\
50 \\
50 \\
75 \\
20\end{array}$ & $\begin{array}{r}4 \\
2 \\
3 \\
10\end{array}$ & $\begin{array}{l}\text { I } \\
\text { I } \\
\text { I I } \\
\text { II } \\
\text { I }\end{array}$ & $\begin{array}{l}3637.748 \\
3638.000 \\
3638.098 \\
3638.146 \\
3638.316\end{array}$ & $\begin{array}{r}40 \\
50 \\
50 \\
50 \\
400\end{array}$ & $3 w$ & $\begin{array}{l}\text { I I } \\
\text { I } \\
\text { I } \\
\text { I } \\
\text { I }\end{array}$ & $\begin{array}{l}3649.248 \\
3649.733 \\
3650.193 \\
3650.440 \\
3650.518\end{array}$ & $\begin{array}{r}600 \\
1200 \\
50 \\
75 \\
75\end{array}$ & $\begin{array}{r}150 \\
20 \\
\\
3 \\
5\end{array}$ & $\begin{array}{l}\text { I I } \\
\text { I } \\
\text { I } \\
\text { I } \\
\text { I I }\end{array}$ \\
\hline $\begin{array}{l}3627.589 \\
3627.647 \\
3627.721 \\
3627.792 \\
3628.023\end{array}$ & $\begin{array}{l}50 \\
20 \\
20 \\
40 \\
75\end{array}$ & $\begin{array}{r}10 \\
5 \\
3\end{array}$ & $\begin{array}{l}\text { II } \\
\text { I } \\
\text { I } \\
\text { I } \\
\text { I }\end{array}$ & $\begin{array}{l}3638.642 \\
3639.445 \\
3639.606 \\
3639.866 \\
3639.994\end{array}$ & $\begin{array}{l}500 \\
750 \\
10 \\
400 \\
40 \mathrm{~h}\end{array}$ & $\begin{array}{c}4 \\
125 \\
4 w \\
10\end{array}$ & $\begin{array}{l}\text { I } \\
\text { I I } \\
\text { I I } \\
\text { I } \\
\text { I }\end{array}$ & $\begin{array}{l}3650.765 \\
3651.570 \\
3651.827 \\
3652.166 \\
3652.537\end{array}$ & $\begin{array}{r}200 \\
125 \\
40 \\
1000 \\
150\end{array}$ & $\begin{array}{r}75 \\
6 \\
200 \\
50\end{array}$ & $\begin{array}{l}\text { I I } \\
\text { I I } \\
\text { I } \\
\text { I I } \\
\text { I I }\end{array}$ \\
\hline $\begin{array}{l}3628.124 \\
3628.397 \\
3628.556 \\
3628.862 \\
3628.923\end{array}$ & $\begin{array}{l}50 \\
20 \\
40 \mathrm{~s} \\
50 \\
20\end{array}$ & & $\begin{array}{l}\text { I } \\
\text { I } \\
\text { I } \\
\text { I } \\
\text { I }\end{array}$ & $\begin{array}{l}3640.035 \\
3640.182 \\
3640.470 \\
3640.566 \\
3640.881\end{array}$ & $\begin{array}{r}50 \\
20 \\
5 \\
75 \\
40\end{array}$ & 10 & $\begin{array}{l}\text { I } \\
\text { II } \\
\text { I } \\
\text { I } \\
\text { II }\end{array}$ & $\begin{array}{l}3652.870 \\
3653.093 \\
3653.373 \\
3653.601 \\
3653.812\end{array}$ & $\begin{array}{r}40 \\
50 \\
75 \\
100 \\
20\end{array}$ & 10 & $\begin{array}{l}\text { I } \\
\text { I } \\
\text { I } \\
\text { I I } \\
\text { I }\end{array}$ \\
\hline $\begin{array}{l}3629.007 \\
3629.269 \\
3629.338 \\
3629.376 \\
3629.850\end{array}$ & $\begin{array}{r}50 \\
40 \\
50 \\
40 \\
200\end{array}$ & $\begin{array}{r}2 \\
40 \\
2\end{array}$ & $\begin{array}{l}\text { I } \\
\text { I } \\
\text { I I } \\
\text { I } \\
\text { I }\end{array}$ & $\begin{array}{l}3640.922 \\
3640.959 \\
3641.109 \\
3641.286 \\
3641.367\end{array}$ & $\begin{array}{l}75 \\
50 \\
75 \\
50 \\
40\end{array}$ & $\begin{array}{l}15 \\
12\end{array}$ & $\begin{array}{l}\text { I } \\
\text { I } \\
\text { II } \\
\text { II } \\
\text { I }\end{array}$ & $\begin{array}{l}3653.918 \\
3654.139 \\
3654.464 \\
3654.583 \\
3655.029\end{array}$ & $\begin{array}{r}20 \\
20 \\
300 \\
75 \\
20\end{array}$ & $\begin{array}{c}1 \\
4 \\
151 \\
2\end{array}$ & $\begin{array}{l}\text { I } \\
\text { I } \\
\text { I } \\
\text { I I } \\
\text { I }\end{array}$ \\
\hline
\end{tabular}


Table 1. Wavelengths of Thorium Spectra - Continued

\begin{tabular}{|c|c|c|c|c|c|c|c|c|c|c|c|}
\hline \multirow{2}{*}{$\begin{array}{l}\text { Wave- } \\
\text { length }\end{array}$} & \multicolumn{2}{|c|}{ Intensity } & \multirow{2}{*}{$\begin{array}{l}\text { Spec- } \\
\text { trum }\end{array}$} & \multirow{2}{*}{$\begin{array}{l}\text { Wave- } \\
\text { length }\end{array}$} & \multicolumn{2}{|c|}{ Intensity } & \multirow{2}{*}{$\begin{array}{l}\text { Spec- } \\
\text { trum }\end{array}$} & \multirow{2}{*}{$\begin{array}{l}\text { Wave- } \\
\text { length }\end{array}$} & \multicolumn{2}{|c|}{ Intensity } & \multirow{2}{*}{$\begin{array}{l}\text { Spec } \\
\text { trum }\end{array}$} \\
\hline & & & & & Lam & Spark & & & Lamp & Spark & \\
\hline $\begin{array}{l}3655.127 \\
3655.331 \\
3655.839 \\
3655.962 \\
3656.047\end{array}$ & $\begin{array}{l}250 \\
40 \mathrm{w} \\
40 \\
50 \\
40\end{array}$ & 2 & $\begin{array}{l}\text { I } \\
\text { I } \\
\text { I } \\
\text { I } \\
\text { I }\end{array}$ & $\begin{array}{l}3668.140 \mathrm{i} \\
3668.391 \\
3668.834 \\
3668.913 \\
3669.330\end{array}$ & $\begin{array}{r}1000 \\
75 \\
40 \\
75 \\
50\end{array}$ & $\begin{array}{r}20 \\
1\end{array}$ & $\begin{array}{l}\text { I } \\
\text { I } \\
\text { I } \\
I \\
I\end{array}$ & $\begin{array}{l}3681.105 \\
3681.178 \\
3681.263 \\
3681.574 \\
3681.882\end{array}$ & $\begin{array}{r}40 \\
75 \\
50 \\
20 \\
200\end{array}$ & $\begin{array}{r}3 \\
20\end{array}$ & $\begin{array}{l}\text { I I } \\
\text { II } \\
\text { I } \\
\text { I } \\
\text { II }\end{array}$ \\
\hline $\begin{array}{l}3656.200 \\
3656.694 \mathrm{i} \\
3657.058 \\
3657.296 \\
3657.526\end{array}$ & $\begin{array}{c}125 \\
1000 \\
125 \\
40 \mathrm{w} \\
40\end{array}$ & $\begin{array}{r}50 \\
4 \\
1 \\
6\end{array}$ & $\begin{array}{l}\text { I I } \\
\text { I } \\
\text { I } \\
\text { I } \\
\text { I I }\end{array}$ & $\begin{array}{l}3669.406 \\
3669.642 \\
3669.969 \mathrm{i} \\
3670.063 \\
3670.520\end{array}$ & $\begin{array}{r}40 \\
200 \\
750 \\
250 \\
50\end{array}$ & $\begin{array}{r}12 \\
1 \\
15 \\
50 \\
1\end{array}$ & $\begin{array}{l}\text { I I } \\
\text { I } \\
\text { I } \\
\text { I I } \\
\text { I }\end{array}$ & $\begin{array}{l}3682.178 \\
3682.291 \\
3682.348 \\
3682.4861 \\
3682.838\end{array}$ & $\begin{array}{r}100 \\
50 \\
75 \\
1000 \\
10\end{array}$ & $\begin{array}{r}1 \\
1 \\
15 \\
8 \\
5\end{array}$ & $\begin{array}{l}\text { I } \\
\text { I } \\
\text { I I } \\
\text { I } \\
\text { I I }\end{array}$ \\
\hline $\begin{array}{l}3657.644 \\
3658.064 \\
3658.228 \\
3658.540 \\
3658.808\end{array}$ & $\begin{array}{r}200 \\
600 \\
200 \\
5 \\
1000\end{array}$ & $\begin{array}{r}150 \\
100 \\
5\end{array}$ & $\begin{array}{l}\text { I } \\
\text { II } \\
\text { I I } \\
\text { I } \\
\text { I }\end{array}$ & $\begin{array}{l}3670.635 \\
3671.029 \\
3671.165 \\
3671.255 \\
3671.536\end{array}$ & $\begin{array}{l}100 \\
20 \mathrm{w} \\
40 \\
50 \\
700\end{array}$ & $\begin{array}{r}10 \\
8 \\
20\end{array}$ & $\begin{array}{l}\text { II } \\
\text { II } \\
\text { I I } \\
\text { I } \\
\text { I }\end{array}$ & $\begin{array}{l}3683.186 \\
3683.312 \\
3683.491 \\
3683.722 \\
3683.849\end{array}$ & $\begin{array}{r}40 \\
75 \\
125 \\
20 \\
20\end{array}$ & $\begin{array}{r}10 \\
2 \\
3\end{array}$ & $\begin{array}{l}\text { I } \\
\text { I I } \\
\text { I I } \\
\text { I I } \\
\text { I }\end{array}$ \\
\hline $\begin{array}{l}3659.509 \\
3659.631 \\
3660.098 \\
3660.335 \\
3660.502\end{array}$ & $\begin{array}{l}1500 \\
1500 \\
50 \mathrm{w} \\
50 \\
200\end{array}$ & $\begin{array}{c}200 \mathrm{w} \\
50 \\
2 \\
1 \\
2\end{array}$ & $\begin{array}{l}\text { I I } \\
\text { I } \\
\text { I I } \\
\text { I } \\
\text { I }\end{array}$ & $\begin{array}{l}3671.787 \\
3671.881 \\
3671.973 \\
3672.299 \\
3672.517\end{array}$ & $\begin{array}{r}20 \\
40 \\
20 \\
400 \\
300\end{array}$ & $\begin{array}{l}3 \\
2\end{array}$ & $\begin{array}{l}\text { I } \\
\text { I } \\
\text { I } \\
\text { I }\end{array}$ & $\begin{array}{l}3683.936 \\
3684.363 \\
3684.731 \\
3684.935 \\
3685.587\end{array}$ & $\begin{array}{r}50 \\
50 \\
40 \\
400 \\
100\end{array}$ & $\begin{array}{r}8 \\
2 \\
10\end{array}$ & $\begin{array}{l}\text { I I } \\
\text { I } \\
\text { I } \\
\text { I } \\
\text { I }\end{array}$ \\
\hline $\begin{array}{l}3660.930 \\
3661.470 \\
3661.590 \\
3661.621 \\
3661.981\end{array}$ & $\begin{array}{r}10 \\
40 \\
200 \\
500 \\
150\end{array}$ & $\begin{array}{c}200 \\
125 \mathrm{w} \\
1\end{array}$ & $\begin{array}{l}\text { I } \\
\text { I I I } \\
\text { I } \\
\text { I I } \\
\text { I }\end{array}$ & $\begin{array}{l}3672.947 \\
3673.104 \\
3673.262 \\
3673.792 \\
3674.008\end{array}$ & $\begin{array}{r}75 \\
100 \\
100 \\
200 \\
200\end{array}$ & $\begin{array}{r}12 \\
50 \\
100 \\
2\end{array}$ & $\begin{array}{l}\text { I I } \\
\text { I } \\
\text { I I } \\
\text { I I } \\
\text { I }\end{array}$ & $\begin{array}{l}3685.868 \\
3686.284 \\
3686.398 \\
3686.981 \\
3687.196\end{array}$ & $\begin{array}{r}100 \\
50 \\
40 \\
100 \\
150\end{array}$ & 12 & $\begin{array}{l}\text { II } \\
\text { I } \\
\text { I } \\
\text { I I } \\
\text { I }\end{array}$ \\
\hline $\begin{array}{l}3662.188 \\
3662.286 \\
3662.571 \\
3662.637 \\
3662.753\end{array}$ & $\begin{array}{r}50 \\
50 \\
40 \\
20 \\
125\end{array}$ & $\begin{array}{l}3 \\
2 \\
2\end{array}$ & $\begin{array}{l}\text { II } \\
\text { I } \\
\text { I } \\
\text { II } \\
\text { I }\end{array}$ & $\begin{array}{l}3674.089 \\
3674.417 \\
3674.650 \\
3674.890 \\
3675.136\end{array}$ & $\begin{array}{r}250 \\
40 \\
20 \\
300 \\
400\end{array}$ & $\begin{array}{l}1 \\
2 \\
3\end{array}$ & I & $\begin{array}{l}3687.500 \\
3687.666 \\
3687.977 \\
3688.199 \\
3688.265\end{array}$ & $\begin{array}{r}75 \\
100 \\
500 \\
50 \\
40\end{array}$ & $\begin{array}{r}20 \\
30 \\
3 \\
4 \\
2\end{array}$ & $\begin{array}{l}\text { II } \\
\text { I I } \\
\text { I } \\
\text { I I } \\
\text { I I }\end{array}$ \\
\hline $\begin{array}{l}3662.964 \\
3663.197 \\
3663.552 \\
3663.702 \\
3663.967\end{array}$ & $\begin{array}{r}50 \\
100 \\
75 \\
800 \\
40\end{array}$ & $\begin{array}{r}50 \\
250\end{array}$ & $\begin{array}{l}\text { I } \\
\text { I I } \\
\text { I } \\
\text { I I } \\
\text { I }\end{array}$ & $\begin{array}{l}3675.568 \\
3675.788 \\
3675.961 \\
3676.149 \\
3676.689\end{array}$ & $\begin{array}{r}1000 \\
400 \\
400 \\
300 \\
100\end{array}$ & $\begin{array}{r}200 \\
2 \\
2 \\
3 \\
15\end{array}$ & $\begin{array}{l}\text { I } \\
\text { I } \\
\text { I } \\
\text { II }\end{array}$ & $\begin{array}{l}3688.664 \\
3688.759 \\
3689.571 \\
3689.731 \\
3690.115\end{array}$ & $\begin{array}{r}50 \\
1000 \\
50 \\
75 \\
500\end{array}$ & 200 & $\begin{array}{l}\text { I } \\
\text { I I } \\
\text { I } \\
\text { I } \\
\text { I I }\end{array}$ \\
\hline $\begin{array}{l}3664.108 \\
3664.589 \\
3665.043 \\
3665.181 \\
3665.449\end{array}$ & $\begin{array}{r}150 \\
100 \\
40 \\
75 \\
100\end{array}$ & 10 & $\begin{array}{l}\text { I } \\
\text { I } \\
\text { I } \\
\text { I }\end{array}$ & $\begin{array}{l}3676.822 \\
3676.961 \\
3677.087 \\
3677.308 \\
3677.744\end{array}$ & $\begin{array}{l}50 \\
20 w \\
40 \\
75 \\
75\end{array}$ & $\begin{array}{r}3 \\
3 \\
25\end{array}$ & $\begin{array}{l}\text { I } \\
\text { I } \\
\text { I I } \\
\text { I } \\
\text { I I }\end{array}$ & $\begin{array}{l}3690.490 \\
3690.624 \\
3691.413 \\
3691.615 \\
3691.881\end{array}$ & $\begin{array}{r}300 \\
1000 \\
700 \\
500 \\
1300\end{array}$ & $\begin{array}{r}50 \\
15 \\
8 \\
5 \\
3\end{array}$ & $\begin{array}{l}\text { I I } \\
\text { I } \\
\text { I } \\
\text { I } \\
\text { I }\end{array}$ \\
\hline $\begin{array}{l}3665.625 \\
3665.725 \\
3665.905 \\
3666.144 \\
3666.374\end{array}$ & $\begin{array}{r}75 \\
100 \\
20 \\
20 \\
40\end{array}$ & 25 & $\begin{array}{l}\text { I } \\
\text { II } \\
\text { I } \\
\text { I } \\
\text { II }\end{array}$ & $\begin{array}{l}3677.917 \\
3678.043 \\
3678.272 \\
3678.478 \\
3678.772\end{array}$ & $\begin{array}{r}100 \\
150 \\
40 \\
400 \\
40\end{array}$ & $\begin{array}{r}20 \\
200 \\
10 \\
3 \\
6\end{array}$ & $\begin{array}{l}\text { I I } \\
\text { I I } \\
\text { I I } \\
\text { I } \\
\text { I I }\end{array}$ & $\begin{array}{l}3692.076 \\
3692.390 \\
3692.5661 \\
3693.247 \\
3693.533\end{array}$ & $\begin{array}{r}200 \\
1200 \\
100 \\
100\end{array}$ & $\begin{array}{r}40 \\
500 \\
4 \\
8\end{array}$ & $\begin{array}{l}\text { II } \\
\text { I I I } \\
\text { I } \\
\text { I } \\
\text { II }\end{array}$ \\
\hline $\begin{array}{l}3666.802 \\
3666.984 \\
3667.312 \\
3667.459 \\
3667.621\end{array}$ & $\begin{array}{r}40 \\
900 \\
20 \\
40 \\
150\end{array}$ & $\begin{array}{l}3 \\
7\end{array}$ & $\begin{array}{l}\text { I } \\
\text { I } \\
\text { I } \\
\text { I } \\
\text { I }\end{array}$ & $\begin{array}{l}3679.131 \\
3679.551 \\
3679.715 \\
3680.443 \\
3680.604\end{array}$ & $\begin{array}{l}400 \\
400 \mathrm{~h} \\
500 \\
400 \\
250\end{array}$ & $\begin{array}{r}3 \\
10 \\
200 \\
3 \\
2\end{array}$ & $\begin{array}{l}\text { I } \\
\text { I } \\
\text { I I } \\
\text { I } \\
\text { I }\end{array}$ & $\begin{array}{l}3693.697 \\
3693.809 \\
3693.905 \\
3693.990 \\
3694.177\end{array}$ & $\begin{array}{l}125 \\
200 \\
200 \\
300 \\
800\end{array}$ & $\begin{array}{r}4 \\
3 \\
100 \\
15 \\
2\end{array}$ & $\begin{array}{l}\text { I } \\
\text { I } \\
\text { I I } \\
\text { I I } \\
\text { I }\end{array}$ \\
\hline
\end{tabular}


Table 1. Wavelengths of Thorium Spectra - Continued

\begin{tabular}{|c|c|c|c|c|c|c|c|c|c|c|c|}
\hline \multirow{2}{*}{$\begin{array}{l}\text { Wave- } \\
\text { length }\end{array}$} & \multicolumn{2}{|c|}{ Intensity } & \multirow{2}{*}{$\begin{array}{l}\text { Spec- } \\
\text { trum }\end{array}$} & \multirow{2}{*}{$\begin{array}{l}\text { Wave- } \\
\text { length }\end{array}$} & \multicolumn{2}{|c|}{ Intensity } & \multirow{2}{*}{$\begin{array}{l}\text { Spec- } \\
\text { trum }\end{array}$} & \multirow{2}{*}{$\begin{array}{l}\text { Wave- } \\
\text { length }\end{array}$} & \multicolumn{2}{|c|}{ Intensity } & \multirow{2}{*}{$\begin{array}{l}\text { Spec- } \\
\text { trum }\end{array}$} \\
\hline & $a m p$ & Spark & & & Lamp & Spark & & & Lamp & Spark & \\
\hline $\begin{array}{l}3694.243 \\
3694.365 \\
3694.785 \\
3695.050 \\
3695.289\end{array}$ & $\begin{array}{r}75 \\
400 \\
50 \\
30 \\
600\end{array}$ & $\begin{array}{l}4 \\
3 \\
4 \\
3\end{array}$ & $\begin{array}{l}\text { I } \\
\text { I } \\
\text { I } \\
\text { I I } \\
\text { I }\end{array}$ & $\begin{array}{l}3704.968 \\
3705.942 \\
3706.054 \\
3706.222 \\
3706.409\end{array}$ & $\begin{array}{l}75 \\
50 \\
50 \\
40 w \\
75\end{array}$ & $\begin{array}{r}10 \\
8 \\
7 \\
5\end{array}$ & $\begin{array}{l}\text { I I } \\
\text { I I } \\
\text { I I } \\
\text { I } \\
\text { I I }\end{array}$ & $\begin{array}{l}3716.765 \\
3716.940 \\
3716.986 \\
3717.069 \\
3717.383\end{array}$ & $\begin{array}{l}50 \\
50 \\
50 \\
50 \\
75 \mathrm{w}\end{array}$ & 3 & $\begin{array}{l}\text { II } \\
\text { I } \\
\text { I } \\
\text { I } \\
\text { I I }\end{array}$ \\
\hline $\begin{array}{l}3695.742 \\
3695.976 \\
3696.255 \\
3696.310 \\
3696.403\end{array}$ & $\begin{array}{r}20 \\
250 \\
20 \\
2 \\
40\end{array}$ & 100 & $\begin{array}{l}\text { I } \\
\text { I I } \\
\text { I } \\
\text { I } \\
\text { I }\end{array}$ & $\begin{array}{l}3706.771 \\
3706.996 \\
3707.430 \\
3707.467 \\
3707.600\end{array}$ & $\begin{array}{r}2400 \\
400 \\
100 \\
100 \\
75\end{array}$ & $\begin{array}{l}30 \\
12 \\
10\end{array}$ & $\begin{array}{l}\text { I } \\
\text { I } \\
\text { II } \\
\text { I } \\
\text { I }\end{array}$ & $\begin{array}{l}3717.508 \\
3717.609 \\
3717.830 \\
3718.164 \\
3718.450\end{array}$ & $\begin{array}{r}10 \\
10 \\
100 \\
100 \\
10\end{array}$ & $\begin{array}{l}30 \\
18\end{array}$ & $\begin{array}{l}\text { I } \\
\text { I } \\
\text { II } \\
\text { I I } \\
\text { I }\end{array}$ \\
\hline $\begin{array}{l}3696.526 \\
3696.647 \\
3696.757 \\
3697.032 \\
3697.198\end{array}$ & $\begin{array}{r}75 \\
150 \\
100 \\
150 \\
100\end{array}$ & $\begin{array}{r}30 \\
1 \\
30\end{array}$ & $\begin{array}{l}\text { I } \\
\text { I I } \\
\text { I } \\
\text { II } \\
\text { I }\end{array}$ & $\begin{array}{l}3707.719 \\
3708.393 \\
3708.482 \\
3708.571 \\
3708.663\end{array}$ & $\begin{array}{l}75 \\
75 \\
40 \\
40 \\
40\end{array}$ & 4 & $\begin{array}{l}\text { I } \\
\text { I } \\
\text { I } \\
\text { I } \\
\text { I I }\end{array}$ & $\begin{array}{l}3718.651 \\
3719.011 \\
3719.147 \\
3719.435 \mathrm{i} \\
3719.694\end{array}$ & $\begin{array}{r}100 \\
50 \\
10 \\
3000 \\
50\end{array}$ & $\begin{array}{r}10 \\
3\end{array}$ & $\begin{array}{l}\text { I I } \\
\text { I I } \\
\text { I } \\
\text { I } \\
\text { I I }\end{array}$ \\
\hline $\begin{array}{l}3697.503 \\
3697.744 \\
3698.106 \\
3698.297 \\
3698.608\end{array}$ & $\begin{array}{r}50 \\
200 \\
1300 \\
100 \\
50\end{array}$ & $\begin{array}{r}1 \\
8 \\
30\end{array}$ & $\begin{array}{l}\text { I } \\
\text { I } \\
\text { I } \\
\text { I I } \\
\text { I }\end{array}$ & $\begin{array}{l}3708.757 \\
3708.884 \\
3709.400 \\
3709.625 \\
3709.853\end{array}$ & $\begin{array}{r}100 \\
40 \\
20 \\
75 \\
125 \mathrm{w}\end{array}$ & $\begin{array}{r}15 \\
6\end{array}$ & $\begin{array}{l}\text { II } \\
\text { I } \\
\text { I } \\
\text { I I } \\
\text { I }\end{array}$ & $\begin{array}{l}3719.960 \\
3720.307 \\
3720.694 \\
3720.998 \\
3721.216\end{array}$ & $\begin{array}{r}600 \\
300 \\
75 \\
100 \\
400\end{array}$ & $\begin{array}{r}300 \\
200 \\
10 \\
2 \\
3\end{array}$ & $\begin{array}{l}\text { I I } \\
\text { I I } \\
\text { I I } \\
\text { I } \\
\text { I }\end{array}$ \\
\hline $\begin{array}{l}3698.750 \\
3699.049 \\
3699.181 \\
3699.352 \\
3699.580\end{array}$ & $\begin{array}{r}75 \\
100 \\
200 \\
100 \\
5\end{array}$ & $\begin{array}{r}10 \\
2 \\
1\end{array}$ & $\begin{array}{l}\text { I I } \\
\text { I } \\
\text { I } \\
\text { I } \\
\text { I }\end{array}$ & $\begin{array}{l}3710.074 \\
3710.221 \\
3710.288 \\
3710.549 \\
3710.668\end{array}$ & $\begin{array}{l}50 \\
50 \\
100 \\
40 \\
40 \mathrm{w}\end{array}$ & 5 & $\begin{array}{l}\text { I } \\
\text { I } \\
\text { I } \\
\text { I I } \\
\text { I }\end{array}$ & $\begin{array}{l}3721.344 \\
3721.484 \\
3721.821 \\
3722.113 \\
3722.818\end{array}$ & $\begin{array}{c}1001 \\
75 \\
1500 \\
1000 \\
75\end{array}$ & $\begin{array}{r}5 \\
300 \\
200\end{array}$ & $\begin{array}{l}\text { I } \\
\text { I } \\
\text { II } \\
\text { I I } \\
\text { I }\end{array}$ \\
\hline $\begin{array}{l}3699.882 \\
3700.311 \\
3700.456 \\
3700.521 \\
3700.767\end{array}$ & $\begin{array}{l}500 \\
125 \\
50 \mathrm{~h} \\
20 \\
125\end{array}$ & $\begin{array}{r}5 \\
30 \\
2 \\
100\end{array}$ & $\begin{array}{l}\text { I } \\
\text { I I } \\
\text { I } \\
\text { I } \\
\text { I I }\end{array}$ & $\begin{array}{l}3710.823 \\
3710.942 \\
3711.304 \mathrm{i} \\
3711.622 \\
3711.819\end{array}$ & $\begin{array}{l}40 \\
50 \\
600 \\
600 \\
125 d\end{array}$ & $\begin{array}{r}150 \\
6 \\
1\end{array}$ & $\begin{array}{l}\text { I } \\
\text { I } \\
\text { II } \\
\text { I } \\
\text { I }\end{array}$ & $\begin{array}{l}3723.287 \\
3723.656 \\
3723.927 \\
3724.171 \\
3724.296\end{array}$ & $\begin{array}{l}100 \\
100 \\
100 \\
20 \mathrm{~h} \\
40\end{array}$ & $\begin{array}{l}30 \\
10\end{array}$ & $\begin{array}{l}\text { I I } \\
\text { I I } \\
\text { I } \\
\text { I } \\
\text { I }\end{array}$ \\
\hline $\begin{array}{l}3700.978 \mathrm{i} \\
3701.585 \\
3701.873 \\
3702.065 \\
3702.135\end{array}$ & $\begin{array}{l}300 \\
75 \mathrm{w} \\
75 \\
100 \\
75\end{array}$ & 10 & $\begin{array}{l}\text { I } \\
\text { I } \\
\text { I } \\
\text { I I } \\
\text { I }\end{array}$ & $\begin{array}{l}3711.921 \\
3712.185 \\
3712.297 \\
3712.368 \\
3712.540\end{array}$ & $\begin{array}{r}100 \\
50 \\
20 \\
75 \\
400\end{array}$ & 75 & $\begin{array}{l}\text { I } \\
\text { I } \\
\text { I } \\
\text { I I }\end{array}$ & $\begin{array}{l}3724.494 \\
3724.662 \\
3724.729 \\
3724.935 \\
3725.162\end{array}$ & $\begin{array}{r}100 \\
100 \\
150 \\
20 \\
50\end{array}$ & $\begin{array}{r}4 \\
4 \\
100\end{array}$ & $\begin{array}{l}\text { I } \\
\text { I } \\
\text { I I } \\
\text { I } \\
\text { I }\end{array}$ \\
\hline $\begin{array}{l}3702.251 \\
3702.470 \\
3702.627 \\
3702.867 \\
3703.233\end{array}$ & $\begin{array}{l}20 \mathrm{~h} \\
50 \\
20 \\
125 \\
500\end{array}$ & $\begin{array}{r}75 \\
3\end{array}$ & $\begin{array}{l}\text { I } \\
\text { I } \\
\text { I } \\
\text { I I } \\
\text { I }\end{array}$ & $\begin{array}{l}3713.020 \\
3713.164 \\
3713.404 \\
3713.638 \\
3713.887\end{array}$ & $\begin{array}{l}20 \mathrm{w} \\
20 \\
20 \\
20 \mathrm{w} \\
75\end{array}$ & $\begin{array}{l}1 \\
2 \\
1 \\
4\end{array}$ & $\begin{array}{l}\text { I } \\
\text { I } \\
\text { I } \\
\text { I } \\
\text { I I }\end{array}$ & $\begin{array}{l}3725.391 \\
3725.721 \\
3725.866 \\
3725.961 \\
3726.094\end{array}$ & $\begin{array}{r}500 \\
100 \\
1.00 \\
75 \\
20\end{array}$ & 4 & $\begin{array}{l}\text { I } \\
\text { I } \\
\text { I } \\
\text { I } \\
\text { I I }\end{array}$ \\
\hline $\begin{array}{l}3703.460 \\
3703.776 \\
3703.908 \\
3703.986 \\
3704.085\end{array}$ & $\begin{array}{r}30 \\
600 \\
200 \\
200 \\
200\end{array}$ & $\begin{array}{r}4 \\
20 \\
20 \\
30\end{array}$ & $\begin{array}{l}\text { I } \\
\text { I } \\
\text { I I } \\
\text { I I } \\
\text { I I }\end{array}$ & $\begin{array}{l}3714.072 \\
3714.295 \\
3714.329 \\
3714.689 \\
3715.080\end{array}$ & $\begin{array}{l}20 \\
40 \\
40 \\
50 \\
75 \mathrm{~s}\end{array}$ & $\begin{array}{l}3 \\
4 \\
3\end{array}$ & $\begin{array}{l}\text { I } \\
\text { I I } \\
\text { I I } \\
\text { I } \\
\text { I }\end{array}$ & $\begin{array}{l}3726.191 \\
3726.721 \\
3726.836 \\
3727.154 \\
3727.253\end{array}$ & $\begin{array}{r}100 \\
600 \\
20 \\
400 \\
300\end{array}$ & $\begin{array}{r}4 \\
100 \\
2 \\
2\end{array}$ & $\begin{array}{l}\text { I } \\
\text { I I } \\
\text { I } \\
\text { I } \\
\text { I }\end{array}$ \\
\hline $\begin{array}{l}3704.141 \\
3704.341 \\
3704.577 \\
3704.713 \\
3704.866\end{array}$ & $\begin{array}{l}150 \\
75 \\
50 \mathrm{w} \\
75 \mathrm{w} \\
400\end{array}$ & $\begin{array}{l}6 \\
2 \\
2\end{array}$ & $\begin{array}{l}\text { I } \\
\text { I I } \\
\text { I } \\
\text { I } \\
\text { I }\end{array}$ & $\begin{array}{l}3715.558 \\
3715.717 \\
3715.866 \\
3716.216 \\
3716.576\end{array}$ & $\begin{array}{l}250 \\
50 \mathrm{w} \\
100 \\
40 \\
200\end{array}$ & $\begin{array}{l}3 \\
3 \\
1 \\
3 \\
3\end{array}$ & $\begin{array}{l}\text { I } \\
\text { I I } \\
\text { I } \\
\text { I I } \\
\text { I }\end{array}$ & $\begin{array}{l}3727.356 \\
3727.902 \mathrm{i} \\
3728.131 \\
3728.495 \\
3728.572\end{array}$ & $\begin{array}{r}300 \\
800 \\
10 \\
10 \\
10\end{array}$ & $\begin{array}{r}2 \\
10 \\
2\end{array}$ & $\begin{array}{l}\text { I } \\
\text { I } \\
\text { II } \\
\text { I } \\
\text { I }\end{array}$ \\
\hline
\end{tabular}


Table 1. Wavelengths of Thorium Spectra - Continued

\begin{tabular}{|c|c|c|c|c|c|c|c|c|c|c|c|}
\hline \multirow{2}{*}{$\begin{array}{l}\text { Wave- } \\
\text { length }\end{array}$} & \multicolumn{2}{|c|}{ Intensity } & \multirow{2}{*}{$\begin{array}{l}\text { Spec- } \\
\text { trum }\end{array}$} & \multirow{2}{*}{$\begin{array}{l}\text { Wave- } \\
\text { length }\end{array}$} & \multicolumn{2}{|c|}{ Intensity } & \multirow{2}{*}{$\begin{array}{l}\text { Spec- } \\
\text { trum }\end{array}$} & \multirow{2}{*}{$\begin{array}{l}\text { Wave- } \\
\text { length }\end{array}$} & \multicolumn{2}{|c|}{ Intensity } & \multirow{2}{*}{$\begin{array}{l}\text { Spec- } \\
\text { trum }\end{array}$} \\
\hline & Lamp & Spark & & & Lamp & Spark & & & Lamp & Spark & \\
\hline $\begin{array}{l}3728.668 \\
3728.733 \\
3728.916 \\
3728.986 \\
3729.124\end{array}$ & $\begin{array}{l}20 \\
40 \\
100 \mathrm{w} \\
10 \\
20\end{array}$ & $2 \mathrm{~h}$ & $\begin{array}{l}\text { I } \\
\text { I } \\
\text { I } \\
\text { II } \\
\text { I }\end{array}$ & $\begin{array}{l}3741.885 \\
3742.028 \\
3742.066 \\
3742.234 \\
3742.279\end{array}$ & $\begin{array}{l}100 \mathrm{~h} \\
100 \\
50 \\
100 \\
50\end{array}$ & $\begin{array}{r}1 \\
1 \\
1 \\
10\end{array}$ & $\begin{array}{l}\text { I } \\
\text { I } \\
\text { I } \\
\text { II } \\
\text { I }\end{array}$ & $\begin{array}{l}3755.810 \\
3755.986 \\
3756.291 \\
3756.450 \\
3756.557\end{array}$ & $\begin{array}{l}1 \\
250 \\
600 \\
100 \\
50 \mathrm{w}\end{array}$ & $\begin{array}{r}2 \\
3 \\
25 \\
1\end{array}$ & $\begin{array}{l}\text { II } \\
\text { I } \\
\text { II } \\
\text { I } \\
\text { I }\end{array}$ \\
\hline $\begin{array}{l}3729.257 \\
3729.684 \\
3729.949 \\
3730.371 \\
3730.620\end{array}$ & $\begin{array}{l}10 \mathrm{w} \\
50 \\
300 \\
900 \\
20\end{array}$ & $\begin{array}{l}3 \\
5 \\
3\end{array}$ & $\begin{array}{l}\text { I } \\
\text { I } \\
\text { I } \\
\text { I } \\
\text { I I }\end{array}$ & $\begin{array}{l}3742.585 \\
3742.737 \\
3742.923 \mathrm{i} \\
3743.172 \\
3743.512\end{array}$ & $\begin{array}{c}50 \mathrm{w} \\
10 \\
1100 \\
20 \mathrm{w} \\
250\end{array}$ & $\begin{array}{l}20 \\
50\end{array}$ & $\begin{array}{l}\text { I } \\
\text { I } \\
\text { I } \\
\text { I } \\
\text { I I }\end{array}$ & $\begin{array}{l}3756.756 \\
3756.891 \\
3757.385 \\
3757.695 \\
3758.380\end{array}$ & $\begin{array}{r}100 \\
20 \\
10 \\
1000 \\
15\end{array}$ & $\begin{array}{l}6 \\
2 \mathrm{~h} \\
20 \\
2\end{array}$ & $\begin{array}{l}\text { II } \\
\text { II } \\
\text { I } \\
\text { I }\end{array}$ \\
\hline $\begin{array}{l}3730.749 \\
3730.885 \\
3731.262 \\
3731.421 \\
3731.686\end{array}$ & $\begin{array}{l}150 \\
20 \\
200 \\
300 \\
20 \mathrm{w}\end{array}$ & $\begin{array}{r}200 \\
3 \\
50\end{array}$ & $\begin{array}{l}\text { II } \\
\text { II } \\
\text { I } \\
\text { II } \\
\text { I }\end{array}$ & $\begin{array}{l}3743.806 \\
3743.898 \\
3744.537 \\
3744.662 \\
3744.743\end{array}$ & $\begin{array}{r}150 \\
30 \\
75 \\
100 \\
150\end{array}$ & $\begin{array}{r}5 \\
20\end{array}$ & $\begin{array}{l}\text { II } \\
\text { I } \\
\text { I } \\
\text { II } \\
\text { II }\end{array}$ & $\begin{array}{l}3758.465 \\
3758.702 \\
3758.822 \\
3759.266 \\
3759.312\end{array}$ & $\begin{array}{l}600 \\
200 \\
60 \mathrm{w} \\
150 \\
300\end{array}$ & $\begin{array}{r}5 \\
2 \\
3 \\
25\end{array}$ & $\begin{array}{l}\text { I } \\
\text { I } \\
\text { II } \\
\text { I } \\
\text { I }\end{array}$ \\
\hline $\begin{array}{l}3731.811 \\
3732.252 \\
3732.539 \\
3732.659 \\
3732.727\end{array}$ & $\begin{array}{l}40 \mathrm{w} \\
100 \\
100 \\
75 \\
50\end{array}$ & $\begin{array}{l}1 \\
8 \\
2\end{array}$ & $\begin{array}{l}\text { I } \\
\text { I } \\
\text { I } \\
\text { I I } \\
\text { II }\end{array}$ & $\begin{array}{l}3744.850 \\
3744.912 \\
3745.182 \\
3745.660 \\
3745.978\end{array}$ & $\begin{array}{r}50 \\
150 \\
200 \\
350 \\
900\end{array}$ & $\begin{array}{r}2 \\
4 \\
200\end{array}$ & $\begin{array}{l}\text { I } \\
\text { I } \\
\text { I } \\
\text { I } \\
\text { I I }\end{array}$ & $\begin{array}{l}3759.429 \\
3759.883 \\
3760.280 \\
3760.803 \\
3761.104\end{array}$ & $\begin{array}{r}300 \\
75 \\
250 \\
50 \\
500\end{array}$ & $\begin{array}{r}8 \\
50 \\
12\end{array}$ & $\begin{array}{l}\text { I } \\
\text { I } \\
\text { II } \\
\text { I } \\
\text { I }\end{array}$ \\
\hline $\begin{array}{l}3732.978 \\
3733.664 \\
3733.945 \\
3734.122 \\
3734.416\end{array}$ & $\begin{array}{l}500 \\
500 \\
20 \\
20 \mathrm{w} \\
40\end{array}$ & $\begin{array}{l}4 \\
5 \\
1 \\
2\end{array}$ & $\begin{array}{l}\text { I } \\
\text { I } \\
\text { I } \\
\text { I } \\
\text { I }\end{array}$ & $\begin{array}{l}3746.226 \\
3746.329 \\
3746.464 \\
3746.669 \\
3746.794\end{array}$ & $\begin{array}{r}50 \\
30 \\
250 \\
20 \\
30\end{array}$ & $\begin{array}{r}4 \\
20\end{array}$ & $\begin{array}{l}\text { II } \\
\text { I } \\
\text { II } \\
\text { I } \\
\text { I }\end{array}$ & $\begin{array}{l}3761.214 \\
3761.314 \\
3761.466 \\
3761.522 \\
3761.700\end{array}$ & $\begin{array}{l}40 \\
300 \\
300 \\
100 \\
150\end{array}$ & $\begin{array}{l}3 \\
3 \\
3 \\
1 \\
2\end{array}$ & $\begin{array}{l}\text { I } \\
\text { I } \\
\text { I } \\
\text { I } \\
\text { I }\end{array}$ \\
\hline $\begin{array}{l}3734.593 \\
3735.359 \\
3735.510 \\
3735.835 \\
3736.499\end{array}$ & $\begin{array}{l}200 \\
50 \mathrm{w} \\
100 \\
30 \\
125\end{array}$ & $\begin{array}{r}75 \\
12 \\
2\end{array}$ & $\begin{array}{l}\text { I I } \\
\text { I } \\
\text { II } \\
\text { I } \\
\text { I }\end{array}$ & $\begin{array}{l}3747.176 \\
3747.235 \\
3747.538 \\
3748.272 \\
3748.824\end{array}$ & $\begin{array}{r}50 \\
40 \\
900 \\
100 \\
100\end{array}$ & $\begin{array}{r}4 \\
300 \\
40\end{array}$ & $\begin{array}{l}\text { I } \\
\text { I } \\
\text { II } \\
\text { I I } \\
\text { I }\end{array}$ & $\begin{array}{l}3761.886 \\
3761.960 \\
3762.148 \\
3762.258 \\
3762.403\end{array}$ & $\begin{array}{l}50 w \\
5 \\
10 w \\
75 \\
75\end{array}$ & 2 & $\begin{array}{l}\text { I } \\
\text { I } \\
\text { I } \\
\text { I } \\
\text { I }\end{array}$ \\
\hline $\begin{array}{l}3736.935 \\
3737.509 \\
3737.630 \\
3737.930 \\
3738.106\end{array}$ & $\begin{array}{r}100 \\
700 \\
10 \\
50 \\
20\end{array}$ & $\begin{array}{r}30 \\
6 \\
6 \\
5 \\
4\end{array}$ & $\begin{array}{l}\text { II } \\
\text { I } \\
\text { II } \\
\text { II } \\
\text { II }\end{array}$ & $\begin{array}{l}3748.970 \\
3749.085 \\
3749.410 \\
3749.621 \\
3749.744\end{array}$ & $\begin{array}{r}25 \\
250 \\
10 \\
50 \\
50\end{array}$ & $\begin{array}{r}10 \\
2 \\
3\end{array}$ & $\begin{array}{l}\text { I I } \\
\text { I } \\
\text { I } \\
\text { I } \\
\text { I }\end{array}$ & $\begin{array}{l}3762.495 \\
3762.879 \\
3762.935 \mathrm{i} \\
3763.320 \\
3763.573\end{array}$ & $\begin{array}{r}10 \\
1500 \\
1200 \\
250 \\
100\end{array}$ & $\begin{array}{r}1 \\
300 \\
75 \\
20\end{array}$ & $\begin{array}{l}\text { I } \\
\text { II } \\
\text { I } \\
\text { II } \\
\text { II }\end{array}$ \\
\hline $\begin{array}{l}3738.366 \\
3738.434 \\
3738.741 \\
3738.836 \\
3739.185\end{array}$ & $\begin{array}{r}75 \\
50 \\
10 \\
500 \\
75\end{array}$ & $\begin{array}{l}20 w \\
100\end{array}$ & $\begin{array}{l}\text { I } \\
\text { I } \\
\text { II } \\
\text { II } \\
\text { I }\end{array}$ & $\begin{array}{l}3750.149 \\
3750.492 \\
3750.667 \\
3751.018 \\
3751.122\end{array}$ & $\begin{array}{l}20 \\
300 \\
40 \mathrm{w} \\
300 \\
300\end{array}$ & $\begin{array}{l}2 \\
5 w \\
2 \\
3\end{array}$ & $\begin{array}{l}\text { I } \\
\text { I } \\
\text { II } \\
\text { I } \\
\text { I }\end{array}$ & $\begin{array}{l}3763.660 \\
3764.051 \\
3764.189 \\
3764.655 \\
3764.801\end{array}$ & $\begin{array}{r}40 \\
100 \\
100 \\
50 \\
50\end{array}$ & $\begin{array}{r}4 \\
15 \\
3\end{array}$ & $\begin{array}{l}\text { II } \\
\text { II } \\
\text { I } \\
\text { I } \\
\text { I }\end{array}$ \\
\hline $\begin{array}{l}3739.292 \\
3739.395 \\
3739.680 \\
3739.940 \\
3740.073\end{array}$ & $\begin{array}{l}20 \mathrm{w} \\
10 \mathrm{~h} \\
150 \\
30 \\
30 \mathrm{~h}\end{array}$ & & $\begin{array}{l}\text { I } \\
\text { I } \\
\text { I } \\
\text { I } \\
\text { I }\end{array}$ & $\begin{array}{l}3751.758 \\
3751.882 \\
3752.028 \\
3752.128 \\
3752.5691\end{array}$ & $\begin{array}{c}10 \\
20 \\
10 \mathrm{~h} \\
20 \\
3500\end{array}$ & 300 & $\begin{array}{l}\text { II } \\
\text { I } \\
\text { I } \\
\text { I } \\
\text { II }\end{array}$ & $\begin{array}{l}3765.241 \\
3765.412 \\
3765.537 \\
3765.693 \\
3766.076\end{array}$ & $\begin{array}{r}800 \\
500 \\
4 \\
100 \\
200\end{array}$ & $\begin{array}{l}3 \\
2 \\
2\end{array}$ & $\begin{array}{l}\text { I } \\
\text { I } \\
\text { II } \\
\text { I }\end{array}$ \\
\hline $\begin{array}{l}3740.407 \\
3740.739 \\
3740.867 \\
3741.194 \\
3741.675\end{array}$ & $\begin{array}{r}20 \\
30 \\
300 \\
3000 \\
30\end{array}$ & $\begin{array}{r}4 \\
75 \\
500 \\
2\end{array}$ & $\begin{array}{l}\text { II } \\
\text { I } \\
\text { II } \\
\text { II } \\
\text { II }\end{array}$ & $\begin{array}{l}3753.241 \\
3753.453 \\
3754.031 \\
3754.593 \\
3755.210\end{array}$ & $\begin{array}{r}250 \\
20 \\
400 \\
600 \\
900\end{array}$ & $\begin{array}{r}3 \\
4 \\
50 \\
20\end{array}$ & $\begin{array}{l}\text { I } \\
\text { I } \\
\text { I } \\
\text { II } \\
\text { I }\end{array}$ & $\begin{array}{l}3766.445 \\
3767.210 \\
3767.468 \\
3767.548 \\
3767.899\end{array}$ & $\begin{array}{r}100 \\
10 \\
50 \\
50 \\
500\end{array}$ & $\begin{array}{r}5 \\
200\end{array}$ & $\begin{array}{l}\text { I } \\
\text { II } \\
\text { I } \\
\text { I } \\
\text { II }\end{array}$ \\
\hline
\end{tabular}


Table 1. Wavelengths of Thorium Spectra - Continued

\begin{tabular}{|c|c|c|c|c|c|c|c|c|c|c|c|}
\hline \multirow{2}{*}{$\begin{array}{l}\text { Wave- } \\
\text { length }\end{array}$} & \multicolumn{2}{|c|}{ Intensity } & \multirow{2}{*}{$\begin{array}{l}\text { Spec- } \\
\text { trum }\end{array}$} & \multirow{2}{*}{$\begin{array}{l}\text { Wave- } \\
\text { length }\end{array}$} & \multicolumn{2}{|c|}{ Intensity } & \multirow{2}{*}{$\begin{array}{l}\text { Spec- } \\
\text { trum }\end{array}$} & \multirow{2}{*}{$\begin{array}{l}\text { Wave- } \\
\text { length }\end{array}$} & \multicolumn{2}{|c|}{ Intensity } & \multirow{2}{*}{$\begin{array}{l}\text { Spec } \\
\text { trum }\end{array}$} \\
\hline & Lamp & Spark & & & Lamp & & & & & & \\
\hline $\begin{array}{l}3768.436 \\
3768.673 \\
3768.775 \\
3768.930 \\
3769.095\end{array}$ & $\begin{array}{r}250 \\
40 \\
40 \\
20 \\
20\end{array}$ & 20 & $\begin{array}{l}\text { II } \\
\text { I } \\
\text { I } \\
\text { I } \\
\text { I }\end{array}$ & $\begin{array}{l}3780.524 \\
3780.966 \mathbf{i} \\
3781.314 \\
3781.801 \\
3782.103\end{array}$ & $\begin{array}{r}20 \\
350 \\
180 \\
20 \\
75\end{array}$ & $\begin{array}{r}10 \\
5 \\
10\end{array}$ & $\begin{array}{l}\text { I } \\
\text { I } \\
\text { I } \\
\text { I } \\
\text { I I }\end{array}$ & $\begin{array}{l}3793.203 \\
3793.305 \\
3793.497 \\
3793.816 \\
3794.150\end{array}$ & $\begin{array}{r}10 \\
20 \\
200 \\
250 \\
400\end{array}$ & $\begin{array}{r}1 \\
10 \\
1 \\
100\end{array}$ & $\begin{array}{l}\text { I } \\
\text { I } \\
\text { I I } \\
\text { I } \\
\text { I I }\end{array}$ \\
\hline $\begin{array}{l}3769.253 \\
3769.592 \\
3770.054 \\
3770.377 \\
3770.557\end{array}$ & $\begin{array}{r}50 \\
600 \\
1300 \\
10 \\
5 \mathrm{~h}\end{array}$ & $\begin{array}{r}3 \\
15\end{array}$ & $\begin{array}{l}\text { I } \\
\text { I } \\
\text { I } \\
\text { I } \\
\text { I }\end{array}$ & $\begin{array}{l}3782.325 \\
3783.008 \\
3783.289 \\
3783.490 \\
3783.657\end{array}$ & $\begin{array}{r}20 \\
500 \\
500 \\
100 \\
300\end{array}$ & $\begin{array}{r}300 \\
100 \\
\\
30\end{array}$ & $\begin{array}{l}\text { I } \\
\text { II } \\
\text { II } \\
\text { I } \\
\text { I I }\end{array}$ & $\begin{array}{l}3794.309 \\
3794.689 \\
3795.380 \\
3795.675 \\
3796.047\end{array}$ & $\begin{array}{r}40 \\
400 \\
500 \\
50 \\
100\end{array}$ & $\begin{array}{r}10 \\
2 \\
30 \\
4\end{array}$ & $\begin{array}{l}\text { I I } \\
\text { I } \\
\text { I I } \\
\text { I I } \\
\text { I }\end{array}$ \\
\hline $\begin{array}{l}3770.608 \\
3770.718 \\
3770.845 \\
3770.928 \\
3771.370 \mathrm{i}\end{array}$ & $\begin{array}{c}10 \\
50 \mathrm{w} \\
20 \\
20 \mathrm{~h} \\
1500\end{array}$ & 4 & $\begin{array}{l}\text { I } \\
\text { I } \\
\text { I } \\
\text { I } \\
\text { I }\end{array}$ & $\begin{array}{l}3783.743 \\
3784.129 \\
3784.402 \\
3784.567 \\
3784.788\end{array}$ & $\begin{array}{l}30 \\
50 \mathrm{w} \\
50 \\
250 \\
100\end{array}$ & $\begin{array}{l}2 \\
1\end{array}$ & $\begin{array}{l}\text { I I } \\
\text { I } \\
\text { I } \\
\text { I } \\
\text { I }\end{array}$ & $\begin{array}{l}3796.187 \\
3796.226 \\
3796.333 \\
3796.371 \\
3796.681\end{array}$ & $\begin{array}{r}40 \\
20 \\
10 \\
20 \\
100\end{array}$ & 2 & $\begin{array}{l}\text { I } \\
\text { I } \\
\text { I } \\
\text { I } \\
\text { I }\end{array}$ \\
\hline $\begin{array}{l}3771.617 \\
3771.960 \\
3772.234 \\
3772.419 \\
3772.648\end{array}$ & $\begin{array}{r}400 \\
10 \\
200 \\
20 \\
500\end{array}$ & 20 & $\begin{array}{l}\text { I } \\
\text { I } \\
\text { I I } \\
\text { I } \\
\text { I }\end{array}$ & $\begin{array}{l}3785.145 \\
3785.277 \\
3785.600 \mathbf{i} \\
3785.906 \\
3786.452\end{array}$ & $\begin{array}{r}50 \\
125 \\
1000 \\
100 \\
10\end{array}$ & $\begin{array}{l}300 \\
200\end{array}$ & $\begin{array}{l}\text { I } \\
\text { I } \\
\text { I I } \\
\text { II } \\
\text { I }\end{array}$ & $\begin{array}{l}3796.724 \\
3796.937 \\
3796.991 \\
3797.201 \\
3797.450\end{array}$ & $\begin{array}{l}400 \\
100 \mathrm{~h} \\
50 \\
400 \\
150\end{array}$ & $\begin{array}{r}2 \\
75\end{array}$ & $\begin{array}{l}\text { I } \\
\text { I } \\
\text { I } \\
\text { I } \\
\text { I I }\end{array}$ \\
\hline $\begin{array}{l}3773.046 \\
3773.306 \\
3773.458 \\
3773.744 \\
3774.194\end{array}$ & $\begin{array}{r}100 \\
100 \\
50 \\
600 \\
400\end{array}$ & $\begin{array}{l}20 \\
20\end{array}$ & $\begin{array}{l}\text { II } \\
\text { I } \\
\text { I } \\
\text { II } \\
\text { I I }\end{array}$ & $\begin{array}{l}3786.876 \\
3787.105 \\
3787.213 \\
3787.472 \\
3787.634\end{array}$ & $\begin{array}{r}300 \\
50 \\
20 \\
150 \\
150\end{array}$ & $\begin{array}{r}30 \\
3 \\
30 \\
1\end{array}$ & $\begin{array}{l}\text { I I } \\
\text { I I } \\
\text { I } \\
\text { II } \\
\text { I }\end{array}$ & $\begin{array}{l}3797.712 \\
3798.030 \\
3798.099 \\
3798.444 \\
3798.768\end{array}$ & $\begin{array}{l}150 \\
2 \mathrm{~h} \\
400 \\
10 \\
50\end{array}$ & $\begin{array}{l}2 \mathrm{~h} \\
6 \\
6\end{array}$ & $\begin{array}{l}\text { I I } \\
\text { I } \\
\text { I } \\
\text { I I } \\
\text { I }\end{array}$ \\
\hline $\begin{array}{l}3774.729 \\
3774.930 \\
3775.156 \\
3775.311 \\
3775.418\end{array}$ & $\begin{array}{r}250 \\
2 \\
200 \\
100 \\
200\end{array}$ & $\begin{array}{l}1 \\
6 \\
1\end{array}$ & $\begin{array}{l}\text { I } \\
\text { I } \\
\text { I } \\
\text { I I } \\
\text { I }\end{array}$ & $\begin{array}{l}3787.754 \\
3788.352 \\
3788.744 \\
3789.113 \\
3789.164\end{array}$ & $\begin{array}{r}40 \\
300 \\
10 \\
1000 \\
1500\end{array}$ & $\begin{array}{r}30 \\
100\end{array}$ & $\begin{array}{l}\text { I } \\
\text { II } \\
\text { I } \\
\text { II } \\
\text { I }\end{array}$ & $\begin{array}{l}3799.162 \\
3799.263 \\
3799.384 \\
3800.104 \\
3800.194\end{array}$ & $\begin{array}{r}30 \\
50 \\
100 \\
2 \\
300\end{array}$ & $\begin{array}{l}2 \mathrm{~h} \\
2 \\
2\end{array}$ & $\begin{array}{l}\text { I } \\
\text { I I } \\
\text { I } \\
\text { I I } \\
\text { I }\end{array}$ \\
\hline $\begin{array}{l}3775.905 \\
3776.272 \\
3776.496 \\
3776.614 \\
3777.180\end{array}$ & $\begin{array}{l}200 \\
600 \\
100 \\
200 \\
100\end{array}$ & $\begin{array}{r}30 \\
6 \\
6\end{array}$ & $\begin{array}{l}\text { II } \\
\text { I } \\
\text { I } \\
\text { II } \\
\text { I }\end{array}$ & $\begin{array}{l}3789.395 \\
3789.516 \\
3789.552 \\
3789.601 \\
3789.654\end{array}$ & $\begin{array}{l}50 \\
20 \\
15 \\
15 \\
20\end{array}$ & $2 w$ & $\begin{array}{l}\text { II } \\
\text { I } \\
\text { I } \\
\text { I I } \\
\text { I }\end{array}$ & $\begin{array}{l}3800.365 \\
3800.585 \\
3800.874 \\
3800.924 \\
3801.150\end{array}$ & $\begin{array}{r}300 \\
150 \\
20 \\
20 \\
10\end{array}$ & $\begin{array}{r}25 \\
150\end{array}$ & $\begin{array}{l}\text { II } \\
\text { II } \\
\text { I } \\
\text { I } \\
\text { I }\end{array}$ \\
\hline $\begin{array}{l}3777.283 \\
3777.407 \\
3777.553 \\
3777.743 \\
3777.818\end{array}$ & $\begin{array}{l}40 \\
200 \\
100 \mathrm{w} \\
75 \\
50\end{array}$ & $\frac{1}{2 h}$ & $\begin{array}{l}\text { II } \\
\text { I } \\
\text { I } \\
\text { I } \\
\text { I I }\end{array}$ & $\begin{array}{l}3789.948 \\
3790.089 \\
3790.271 \\
3790.350 \\
3790.787\end{array}$ & $\begin{array}{r}50 \\
50 \\
20 \\
500 \\
1400\end{array}$ & $\begin{array}{r}30 \\
75 \\
\\
4 \\
20\end{array}$ & $\begin{array}{l}\text { II } \\
\text { II } \\
\text { I } \\
\text { I } \\
\text { I }\end{array}$ & $\begin{array}{l}3801.320 \\
3801.441 \\
3801.567 \\
3801.914 \\
3802.143\end{array}$ & $\begin{array}{r}75 \\
900 \\
500 \\
100 \\
150\end{array}$ & $\begin{array}{r}10 \\
8 \\
6 \\
25 \\
25\end{array}$ & $\begin{array}{l}\text { II } \\
\text { I } \\
\text { I } \\
\text { II } \\
\text { I I }\end{array}$ \\
\hline $\begin{array}{l}3778.045 \\
3778.356 \\
3778.518 \\
3778.750 \\
3778.910\end{array}$ & $\begin{array}{r}75 \\
20 \\
20 \\
100 \\
50\end{array}$ & $\begin{array}{l}1 \\
2 \mathrm{~h} \\
3 \\
1\end{array}$ & $\begin{array}{l}\text { I } \\
\text { I I } \\
\text { I } \\
\text { I } \\
\text { I }\end{array}$ & $\begin{array}{l}3791.034 \\
3791.215 \\
3791.293 \\
3791.507 \\
3791.970\end{array}$ & $\begin{array}{r}20 \\
75 \\
250 \\
150 \\
100\end{array}$ & 150 & $\begin{array}{l}\text { I } \\
\text { I } \\
\text { II } \\
\text { I } \\
\text { I I }\end{array}$ & $\begin{array}{l}3802.416 \\
3802.620 \\
3802.723 \\
3802.865 \\
3803.075 i\end{array}$ & $\begin{array}{r}200 \\
20 \\
40 \\
200 \\
4000\end{array}$ & $\begin{array}{r}2 \\
30\end{array}$ & $\begin{array}{l}\text { I } \\
\text { I } \\
\text { I } \\
\text { I } \\
\text { I }\end{array}$ \\
\hline $\begin{array}{l}3778.980 \\
3779.562 \\
3779.693 \\
3780.149 \\
3780.288\end{array}$ & $\begin{array}{c}75 \\
150 \\
25 \mathrm{w} \\
75 \\
40\end{array}$ & $\begin{array}{l}1 \\
2 \\
1 \\
3\end{array}$ & $\begin{array}{l}\text { I } \\
\text { I } \\
\text { I I } \\
\text { I } \\
\text { I I }\end{array}$ & $\begin{array}{l}3792.096 \\
3792.382 \\
3792.726 \\
3792.953 \\
3793.092\end{array}$ & $\begin{array}{l}20 \\
300 \\
500 \\
125 \mathrm{w} \\
200\end{array}$ & $\begin{array}{l}10 \\
4 \\
8 \mathrm{w} \\
1\end{array}$ & $\begin{array}{l}\text { I } \\
\text { I } \\
\text { I } \\
\text { I I } \\
\text { I }\end{array}$ & $\begin{array}{l}3803.607 \\
3803.837 \\
3803.979 \\
3804.120 \\
3804.326\end{array}$ & $\begin{array}{l}50 \mathrm{w} \\
150 \\
600 \\
200 \\
150\end{array}$ & $\begin{array}{r}2 \\
5 \\
10\end{array}$ & $\begin{array}{l}\text { I } \\
\text { I } \\
\text { I } \\
\text { I } \\
\text { I I }\end{array}$ \\
\hline
\end{tabular}


Table 1. Wavelengths of Thorium Spectra - Continued

\begin{tabular}{|c|c|c|c|c|c|c|c|c|c|c|c|}
\hline \multirow{2}{*}{$\begin{array}{l}\text { Wave- } \\
\text { length }\end{array}$} & \multicolumn{2}{|c|}{ Intensity } & \multirow{2}{*}{$\begin{array}{l}\text { Spec- } \\
\text { trum }\end{array}$} & \multirow{2}{*}{$\begin{array}{l}\text { Wave- } \\
\text { length }\end{array}$} & \multicolumn{2}{|c|}{ Intensity } & \multirow{2}{*}{$\begin{array}{l}\text { Spec- } \\
\text { trum }\end{array}$} & \multirow{2}{*}{$\begin{array}{l}\text { Wave- } \\
\text { length }\end{array}$} & \multicolumn{2}{|c|}{ Intensity } & \multirow{2}{*}{$\begin{array}{l}\text { Spec- } \\
\text { trum }\end{array}$} \\
\hline & Lamp & & & & Lam & Spark & & & & Spark & \\
\hline $\begin{array}{l}3804.697 \\
3804.994 \\
3805.328 \\
3805.814 \\
3805.962\end{array}$ & $\begin{array}{r}200 \\
200 \\
200 \\
700 \\
50\end{array}$ & $\begin{array}{l}12 \mathrm{w} \\
20 \\
100 \\
125 \\
100\end{array}$ & $\begin{array}{l}\text { II } \\
\text { I I } \\
\text { I I } \\
\text { I I } \\
\text { I I }\end{array}$ & $\begin{array}{l}3818.093 \\
3818.686 \mathrm{i} \\
3819.112 \\
3819.161 \\
3819.279\end{array}$ & $\begin{array}{l}100 \\
500 \\
150 \\
301 \\
30\end{array}$ & $\begin{array}{l}4 \\
5 \\
1 \\
3\end{array}$ & $\begin{array}{l}\text { II } \\
\text { I } \\
\text { I } \\
\text { I } \\
\text { II }\end{array}$ & $\begin{array}{l}3831.356 \\
3831.644 \\
3831.737 \\
3831.959 \\
3832.580\end{array}$ & $\begin{array}{l}20 \\
150 \\
400 \\
20 \mathrm{w} \\
50\end{array}$ & 200 & $\begin{array}{l}\text { I } \\
\text { I } \\
\text { I I } \\
\text { I } \\
\text { I }\end{array}$ \\
\hline $\begin{array}{l}3806.058 \\
3806.146 \\
3806.258 \\
3806.516 \\
3806.878\end{array}$ & $\begin{array}{r}20 \\
100 \\
30 \\
50 \\
40\end{array}$ & $8 w$ & $\begin{array}{l}\text { I } \\
\text { I } \\
\text { I I } \\
\text { I } \\
\text { I }\end{array}$ & $\begin{array}{l}3819.331 \\
3819.617 \\
3819.898 \\
3819.935 \\
3820.093\end{array}$ & $\begin{array}{r}50 \\
40 \\
200 \\
40 \\
75\end{array}$ & 30 & $\begin{array}{l}\text { II } \\
\text { I } \\
\text { I } \\
\text { II } \\
\text { I }\end{array}$ & $\begin{array}{l}3832.782 \\
3832.970 \\
3833.086 \\
3833.224 \\
3833.691\end{array}$ & $\begin{array}{r}200 \\
75 \\
400 \\
50 \\
75\end{array}$ & $\begin{array}{r}30 \\
20 \\
4 \\
3 \\
12\end{array}$ & $\begin{array}{l}\text { II } \\
\text { II } \\
\text { I } \\
\text { II } \\
\text { II }\end{array}$ \\
\hline $\begin{array}{l}3807.268 \\
3807.532 \\
3807.677 \\
3807.871 \\
3808.125\end{array}$ & $\begin{array}{c}200 \\
15 \\
100 \mathrm{w} \\
1200 \\
300\end{array}$ & $\begin{array}{r}2 \\
2 \\
5 \\
300 \\
75\end{array}$ & $\begin{array}{l}\text { I } \\
\text { I I } \\
\text { I } \\
\text { I I } \\
\text { I I }\end{array}$ & $\begin{array}{l}3820.786 \\
3821.425 \\
3821.749 \\
3822.152 \\
3822.704\end{array}$ & $\begin{array}{r}1200 \\
1000 \\
100 \\
1000 \\
100\end{array}$ & $\begin{array}{r}200 \\
3 \\
250 \\
1\end{array}$ & $\begin{array}{l}\text { I } \\
\text { I I } \\
\text { I } \\
\text { I I } \\
\text { I }\end{array}$ & $\begin{array}{l}3834.477 \\
3834.887 \\
3835.068 \\
3835.348 \\
3835.409\end{array}$ & $\begin{array}{l}40 \\
75 \\
40 \mathrm{w} \\
100 \\
125\end{array}$ & $\begin{array}{l}1 \\
1\end{array}$ & $\begin{array}{l}\text { I } \\
\text { I } \\
\text { I } \\
\text { I } \\
\text { I }\end{array}$ \\
\hline $\begin{array}{l}3808.518 \\
3808.612 \\
3808.689 \\
3809.014 \\
3809.125\end{array}$ & $\begin{array}{r}5 \\
400 \\
5 \\
10 \\
50\end{array}$ & $\begin{array}{r}2 \\
10 \\
3\end{array}$ & $\begin{array}{l}\text { I I } \\
\text { I } \\
\text { I I } \\
\text { I } \\
\text { I }\end{array}$ & $\begin{array}{l}3822.855 \\
3823.066 \\
3823.318 \\
3823.458 \\
3823.582\end{array}$ & $\begin{array}{l}200 \\
600 \\
125 \\
125 \\
400\end{array}$ & $\begin{array}{r}2 \\
4 \\
20 \\
125\end{array}$ & $\begin{array}{l}\text { I } \\
\text { I } \\
\text { II } \\
\text { I } \\
\text { II }\end{array}$ & $\begin{array}{l}3835.709 \\
3835.958 \\
3836.208 \\
3836.538 \\
3836.582\end{array}$ & $\begin{array}{r}100 \\
100 \\
30 \\
400 \\
400\end{array}$ & $\begin{array}{l}2 \\
1 \\
4 \\
5\end{array}$ & $\begin{array}{l}\text { I } \\
\text { I } \\
\text { I } \\
\text { I } \\
\text { I }\end{array}$ \\
\hline $\begin{array}{l}3809.253 \\
3809.446 \\
3809.830 \\
3810.290 \\
3810.496\end{array}$ & $\begin{array}{l}40 \mathrm{w} \\
40 \\
400 \\
50 \\
100\end{array}$ & $\begin{array}{r}3 \\
125 \\
2 \\
4\end{array}$ & $\begin{array}{l}\text { I } \\
\text { I I } \\
\text { I I } \\
\text { I I } \\
\text { I I }\end{array}$ & $\begin{array}{l}3823.862 \\
3823.979 \\
3824.066 \\
3824.538 \\
3824.751\end{array}$ & $\begin{array}{l}30 \mathrm{w} \\
50 \\
20 \\
40 \\
40\end{array}$ & 1 & $\begin{array}{l}\text { I } \\
\text { I } \\
\text { I } \\
\text { I } \\
\text { I }\end{array}$ & $\begin{array}{l}3836.709 \\
3837.018 \\
3837.291 \\
3837.337 \\
3837.806\end{array}$ & $\begin{array}{l}50 \\
40 \\
75 \\
75 \\
40\end{array}$ & 10 & $\begin{array}{l}\text { I } \\
\text { I } \\
\text { I } \\
\text { I } \\
\text { II }\end{array}$ \\
\hline $\begin{array}{l}3810.688 \\
3810.990 \\
3811.349 \\
3811.754 \\
3811.963\end{array}$ & $\begin{array}{r}40 \\
700 \\
100 \\
75 \\
30\end{array}$ & $\begin{array}{r}20 \\
20 \\
2\end{array}$ & $\begin{array}{l}\text { I } \\
\text { I } \\
\text { I I } \\
\text { I } \\
\text { I I }\end{array}$ & $\begin{array}{l}3825.027 \\
3825.123 \\
3825.370 \\
3825.516 \\
3826.363\end{array}$ & $\begin{array}{r}300 \\
1200 \\
50 \\
75 \\
400\end{array}$ & $\begin{array}{r}200 \\
30\end{array}$ & $\begin{array}{l}\text { II } \\
\text { I } \\
\text { I } \\
\text { I } \\
\text { I }\end{array}$ & $\begin{array}{l}3837.870 \\
3838.631 \\
3838.787 \\
3838.856 \\
3839.409\end{array}$ & $\begin{array}{l}2000 \\
10 \mathrm{~h} \\
50 \mathrm{w} \\
10 \mathrm{~h} \\
75\end{array}$ & 100 & $\begin{array}{l}\text { I } \\
\text { I } \\
\text { I } \\
\text { I } \\
\text { II }\end{array}$ \\
\hline $\begin{array}{l}3812.035 \\
3812.128 \\
3812.396 \\
3812.665 \\
3812.825\end{array}$ & $\begin{array}{l}50 \\
20 \\
200 \\
50 \mathrm{w} \\
20\end{array}$ & 5 & $\begin{array}{l}\text { I } \\
\text { I } \\
\text { I } \\
\text { I } \\
\text { I }\end{array}$ & $\begin{array}{l}3826.468 \\
3826.942 \\
3827.269 \\
3827.498 \\
3828.385 \mathrm{i}\end{array}$ & $\begin{array}{r}2 \\
400 \\
50 \\
50 \\
3200\end{array}$ & $150^{2}$ & $\begin{array}{l}\text { I I } \\
\text { I I } \\
\text { I } \\
\text { I } \\
\text { I }\end{array}$ & $\begin{array}{l}3839.694 \mathrm{i} \\
3839.738 \\
3840.618 \\
3840.799 \\
3841.352\end{array}$ & $\begin{array}{r}2500 \\
500 \\
75 \\
600 \\
40\end{array}$ & $\begin{array}{r}300 \\
1 \\
4 \\
3\end{array}$ & $\begin{array}{l}\text { I } \\
\text { II } \\
\text { I } \\
\text { I } \\
\text { I I }\end{array}$ \\
\hline $\begin{array}{l}3813.067 \mathrm{i} \\
3813.319 \\
3813.663 \\
3813.809 \\
3814.011\end{array}$ & $\begin{array}{r}1200 \\
40 \\
200 \\
400 \\
50\end{array}$ & $\begin{array}{r}400 \\
10 \\
15 \\
8\end{array}$ & $\begin{array}{l}\text { II } \\
\text { II } \\
\text { I I } \\
\text { I } \\
\text { I }\end{array}$ & $\begin{array}{l}3828.858 \\
3828.962 \\
3829.032 \\
3829.180 \\
3829.273\end{array}$ & $\begin{array}{r}20 \\
50 \\
75 \\
40 \\
8\end{array}$ & & $\begin{array}{l}\text { I } \\
\text { I } \\
\text { I } \\
\text { I } \\
\text { I }\end{array}$ & $\begin{array}{l}3841.463 \\
3841.507 \\
3841.638 \\
3841.960 \mathrm{i} \\
3842.552\end{array}$ & $\begin{array}{r}10 \\
10 \\
20 \\
1200 \\
150\end{array}$ & 300 & $\begin{array}{l}\text { I } \\
\text { I } \\
\text { I } \\
\text { II } \\
\text { I }\end{array}$ \\
\hline $\begin{array}{l}3815.018 \\
3815.563 \\
3816.161 \\
3816.557 \\
3816.724\end{array}$ & $\begin{array}{r}200 \\
300 \\
200 \\
50 \\
50\end{array}$ & $\begin{array}{r}100 \\
4 \\
1 \\
10\end{array}$ & $\begin{array}{l}\text { I I } \\
\text { I } \\
\text { I } \\
\text { I I } \\
\text { I }\end{array}$ & $\begin{array}{l}3829.333 \\
3829.419 \\
3829.650 \\
3829.833 \\
3830.059\end{array}$ & $\begin{array}{r}400 \\
200 \\
10 \\
50 \\
400\end{array}$ & $\begin{array}{c}20 w \\
20 w \\
\\
4 w \\
6\end{array}$ & $\begin{array}{l}\text { I I } \\
\text { I I } \\
\text { I } \\
\text { I I } \\
\text { I }\end{array}$ & $\begin{array}{l}3842.745 \\
3842.807 \\
3842.898 \\
3843.018 \\
3843.415\end{array}$ & $\begin{array}{r}40 \\
30 \\
800 \\
400 \\
20\end{array}$ & $\begin{array}{l}20 \\
4 \\
2 \mathrm{~h}\end{array}$ & $\begin{array}{l}\text { I } \\
\text { I } \\
\text { I I } \\
\text { I } \\
\text { II }\end{array}$ \\
\hline $\begin{array}{l}3816.787 \\
3817.113 \\
3817.290 \\
3817.361 \\
3817.473\end{array}$ & $\begin{array}{l}50 \\
100 \\
50 \mathrm{w} \\
100 \\
800\end{array}$ & $\begin{array}{l}75 \\
35\end{array}$ & $\begin{array}{l}\text { I I } \\
\text { I } \\
\text { I } \\
\text { I } \\
\text { I }\end{array}$ & $\begin{array}{l}3830.304 \\
3830.506 \\
3830.770 \\
3830.828 \\
3830.934\end{array}$ & $\begin{array}{r}20 \\
50 \\
800 \\
4 \\
4\end{array}$ & $\begin{array}{c}151 \\
1 \\
1\end{array}$ & $\begin{array}{l}\text { I } \\
\text { I I } \\
\text { I } \\
\text { I I } \\
\text { I I }\end{array}$ & $\begin{array}{l}3843.509 \\
3843.885 \\
3844.184 \\
3844.567 \\
3844.934\end{array}$ & $\begin{array}{l}150 \\
20 \mathrm{w} \\
20 \mathrm{w} \\
20 \mathrm{~h} \\
200\end{array}$ & 1 & $\begin{array}{l}\text { I } \\
\text { I } \\
\text { I } \\
\text { I } \\
\text { I }\end{array}$ \\
\hline
\end{tabular}


Table 1. Wavelengths of Thorium Spectra - Continued

\begin{tabular}{|c|c|c|c|c|c|c|c|c|c|c|c|}
\hline \multirow{2}{*}{$\begin{array}{l}\text { Wave- } \\
\text { length }\end{array}$} & \multicolumn{2}{|c|}{ Intensity } & \multirow{2}{*}{$\begin{array}{l}\text { Spec- } \\
\text { trum }\end{array}$} & \multirow{2}{*}{$\begin{array}{l}\text { Wave- } \\
\text { length }\end{array}$} & \multicolumn{2}{|c|}{ Intensity } & \multirow{2}{*}{$\begin{array}{l}\text { Spec- } \\
\text { trum }\end{array}$} & \multirow{2}{*}{$\begin{array}{l}\text { Wave- } \\
\text { length }\end{array}$} & \multicolumn{2}{|c|}{ Intensity } & \multirow{2}{*}{$\begin{array}{l}\text { Spec- } \\
\text { trum }\end{array}$} \\
\hline & Lamp & Spark & & & Lamp & Spark & & & amp & Spark & \\
\hline $\begin{array}{l}3845.092 \\
3845.224 \\
3845.299 \\
3845.534 \\
3845.800\end{array}$ & $\begin{array}{r}200 \\
30 \\
30 \\
125 \\
20\end{array}$ & $\begin{array}{r}1 \\
1 \\
75\end{array}$ & $\begin{array}{l}\text { I } \\
\text { I } \\
\text { I } \\
\text { II } \\
\text { I }\end{array}$ & $\begin{array}{l}3856.621 \\
3856.755 \\
3856.818 \\
3857.106 \\
3857.195\end{array}$ & $\begin{array}{r}100 \\
40 \\
10 \\
40 \\
10\end{array}$ & 2 & $\begin{array}{l}\text { I } \\
\text { I } \\
\text { I } \\
\text { I } \\
\text { I }\end{array}$ & $\begin{array}{l}3870.367 \\
3870.687 \\
3870.766 \\
3870.921 \\
3871.072\end{array}$ & $\begin{array}{l}40 w \\
40 \\
40 \\
50 \\
75\end{array}$ & $\begin{array}{l}4 \\
5\end{array}$ & $\begin{array}{l}\text { I } \\
\text { I } \\
\text { I } \\
\text { II } \\
\text { I I }\end{array}$ \\
\hline $\begin{array}{l}3846.039 \\
3846.105 \\
3846.169 \\
3846.248 \\
3846.627\end{array}$ & $\begin{array}{r}20 \\
20 \\
15 \\
125 \\
200\end{array}$ & $\begin{array}{r}100 \\
4\end{array}$ & $\begin{array}{l}\text { I } \\
\text { I } \\
\text { I } \\
\text { I I } \\
\text { I }\end{array}$ & $\begin{array}{l}3857.351 \\
3857.508 \\
3857.917 \\
3858.359 \\
3858.910\end{array}$ & $\begin{array}{r}75 \\
150 \\
10 \\
200 \\
200\end{array}$ & $\begin{array}{r}10 \\
20 \\
\\
2 \\
2\end{array}$ & $\begin{array}{l}\text { I I } \\
\text { I I } \\
\text { I } \\
\text { I } \\
\text { I }\end{array}$ & $\begin{array}{l}3871.243 \\
3871.456 \\
3871.613 \\
3871.917 \\
3872.017\end{array}$ & $\begin{array}{l}50 \\
200 \\
125 \\
20 \mathrm{w} \\
10 \mathrm{~h}\end{array}$ & $\begin{array}{r}4 \\
30 \\
20\end{array}$ & $\begin{array}{l}\text { I I } \\
\text { I I } \\
\text { I I } \\
\text { I } \\
\text { I }\end{array}$ \\
\hline $\begin{array}{l}3846.761 \\
3846.880 \\
3847.453 \\
3847.618 \\
3848.179\end{array}$ & $\begin{array}{r}30 \\
350 \\
10 \\
150 \\
20\end{array}$ & $\begin{array}{l}2 \\
2\end{array}$ & $\begin{array}{l}\text { I } \\
\text { I } \\
\text { I } \\
\text { I } \\
\text { I }\end{array}$ & $\begin{array}{l}3859.457 \\
3859.846 \\
3859.908 \\
3860.649 \\
3861.047\end{array}$ & $\begin{array}{r}10 \\
500 \\
40 \\
75 \\
50\end{array}$ & $\begin{array}{r}200 \\
30\end{array}$ & $\begin{array}{l}\text { I } \\
\text { I I } \\
\text { I I } \\
\text { I } \\
\text { I }\end{array}$ & $\begin{array}{l}3872.358 \\
3872.718 \\
3873.146 \\
3873.470 \\
3873.818\end{array}$ & $\begin{array}{r}100 \\
500 \\
200 \\
500 \\
2000\end{array}$ & $\begin{array}{r}30 \\
300 \\
3 \\
30 \\
150\end{array}$ & $\begin{array}{l}\text { II } \\
\text { I I } \\
\text { I } \\
\text { I } \\
\text { I }\end{array}$ \\
\hline $\begin{array}{l}3848.355 \\
3848.628 \\
3849.060 \\
3849.180 \\
3849.739\end{array}$ & $\begin{array}{c}20 \mathrm{w} \\
100 \\
75 \\
4 \\
20\end{array}$ & $50 \mathrm{w}$ & $\begin{array}{l}\text { I } \\
\text { I } \\
\text { I } \\
\text { I I I } \\
\text { I }\end{array}$ & $\begin{array}{l}3861.149 \\
3861.360 \\
3861.509 \\
3861.544 \\
3861.578\end{array}$ & $\begin{array}{l}20 \\
30 \\
75 \\
50 \\
50\end{array}$ & 50 & $\begin{array}{l}\text { I } \\
\text { I } \\
\text { I } \\
\text { I I } \\
\text { I }\end{array}$ & $\begin{array}{l}3873.947 \\
3874.239 \\
3874.861 \\
3874.956 \\
3875.370\end{array}$ & $\begin{array}{r}20 \\
800 \\
1800 \\
300 \\
1500\end{array}$ & $\begin{array}{r}4 \\
25 \\
4 \\
25\end{array}$ & $\begin{array}{l}\text { II } \\
\text { I } \\
\text { I } \\
\text { I } \\
\text { I }\end{array}$ \\
\hline $\begin{array}{l}3849.908 \\
3850.128 \\
3850.278 \\
3850.686 \\
3851.073\end{array}$ & $\begin{array}{l}150 \\
300 \\
125 \\
75 \mathrm{w} \\
125\end{array}$ & $\begin{array}{r}75 \\
75 \\
\\
1\end{array}$ & $\begin{array}{l}\text { I } \\
\text { I I } \\
\text { II } \\
\text { I } \\
\text { I }\end{array}$ & $\begin{array}{l}3862.055 \\
3862.418 \\
3862.567 \\
3862.663 \\
3863.406 \mathrm{i}\end{array}$ & $\begin{array}{r}50 \\
400 \\
20 \\
400 \\
1200\end{array}$ & $\begin{array}{r}4 \\
50 \\
2 \\
150\end{array}$ & $\begin{array}{l}\text { II } \\
\text { II } \\
\text { II } \\
\text { I } \\
\text { II }\end{array}$ & $\begin{array}{l}3875.500 \\
3875.642 \\
3875.957 \\
3876.211 \\
3876.258\end{array}$ & $\begin{array}{r}5 \\
200 \\
75 \\
20 \\
10\end{array}$ & $\begin{array}{l}2 \\
3 \\
1\end{array}$ & $\begin{array}{l}\text { II } \\
\text { I } \\
\text { I } \\
\text { I } \\
\text { I }\end{array}$ \\
\hline $\begin{array}{l}3851.153 \\
3851.296 \\
3851.667 \\
3852.135 \\
3852.585\end{array}$ & $\begin{array}{c}150 \\
4 \mathrm{w} \\
30 \\
600 \\
20\end{array}$ & 12 & $\begin{array}{l}\text { I } \\
\text { I } \\
\text { I } \\
\text { I } \\
\text { I }\end{array}$ & $\begin{array}{l}3863.856 \\
3864.114 \\
3864.766 \\
3864.835 \\
3864.976\end{array}$ & $\begin{array}{r}50 \\
10 \\
75 \\
100 \\
125\end{array}$ & 3 & $\begin{array}{l}\text { I } \\
\text { I } \\
\text { I } \\
\text { I } \\
\text { I }\end{array}$ & $\begin{array}{l}3876.494 \\
3876.550 \\
3876.802 \\
3876.944 \\
3877.235\end{array}$ & $\begin{array}{l}20 \mathrm{w} \\
10 \\
10 \mathrm{w} \\
10 \mathrm{w} \\
40\end{array}$ & $2 w$ & $\begin{array}{l}\text { II I } \\
\text { I } \\
\text { I } \\
\text { I } \\
\text { I }\end{array}$ \\
\hline $\begin{array}{l}3852.717 \\
3852.958 \\
3853.360 \\
3853.434 \\
3853.565\end{array}$ & $\begin{array}{l}40 \\
300 \\
30 \\
10 \mathrm{w} \\
40\end{array}$ & 50 & $\begin{array}{l}\text { I } \\
\text { II } \\
\text { I } \\
\text { I } \\
\text { I }\end{array}$ & $\begin{array}{l}3865.813 \\
3866.066 \\
3866.366 \\
3866.776 \\
3866.904\end{array}$ & $\begin{array}{l}10 \mathrm{w} \\
125 \\
20 \mathrm{w} \\
20 \\
400\end{array}$ & $\begin{array}{l}50 \\
50\end{array}$ & $\begin{array}{l}\text { I } \\
\text { II } \\
\text { I } \\
\text { I } \\
\text { I I }\end{array}$ & $\begin{array}{l}3877.458 \\
3877.597 \\
3878.663 \\
3879.263 \\
3879.445\end{array}$ & $\begin{array}{l}250 \\
200 \\
150 \\
400 \\
150\end{array}$ & $\begin{array}{l}3 \\
2 \\
4 \\
3\end{array}$ & $\begin{array}{l}\text { I } \\
\text { I } \\
\text { I } \\
\text { I } \\
\text { I }\end{array}$ \\
\hline $\begin{array}{l}3853.641 \\
3853.793 \\
3853.830 \\
3853.984 \\
3854.125\end{array}$ & $\begin{array}{l}10 \\
50 \\
125 \\
75 \mathrm{w} \\
100 \mathrm{w}\end{array}$ & 1 & $\begin{array}{l}\text { I } \\
\text { I } \\
\text { I } \\
\text { I } \\
\text { I }\end{array}$ & $\begin{array}{l}3867.266 \\
3867.575 \\
3867.848 \\
3867.966 \\
3868.251\end{array}$ & $\begin{array}{l}10 \\
125 \\
75 \\
75 \mathrm{w} \\
125\end{array}$ & $\begin{array}{l}8 \\
15 \\
4 \mathrm{w} \\
1\end{array}$ & $\begin{array}{l}\text { I } \\
\text { I I } \\
\text { II } \\
\text { I } \\
\text { I }\end{array}$ & $\begin{array}{l}3879.642 \\
3879.791 \\
3879.977 \\
3880.192 \\
3880.538\end{array}$ & $\begin{array}{c}1000 \\
2 \\
40 \mathrm{w} \\
400 \\
15\end{array}$ & $\begin{array}{r}25 \\
2 \\
2\end{array}$ & $\begin{array}{l}\text { I } \\
\text { I I } \\
\text { I } \\
\text { I } \\
\text { I }\end{array}$ \\
\hline $\begin{array}{l}3854.307 \\
3854.511 \mathrm{i} \\
3854.878 \\
3855.064 \\
3855.602\end{array}$ & $\begin{array}{c}100 \mathrm{w} \\
1200 \\
150 \\
100 \\
30\end{array}$ & $\begin{array}{r}400 \\
75\end{array}$ & $\begin{array}{l}\text { I } \\
\text { II } \\
\text { I I } \\
\text { I } \\
\text { I }\end{array}$ & $\begin{array}{l}3868.382 \\
3868.835 \\
3869.033 \\
3869.222 \\
3869.397\end{array}$ & $\begin{array}{l}75 \\
30 \mathrm{w} \\
20 \mathrm{~h} \\
20 \mathrm{~h} \\
30\end{array}$ & & $\begin{array}{l}\text { I } \\
\text { I } \\
\text { I } \\
\text { I }\end{array}$ & $\begin{array}{l}3881.039 \\
3881.319 \\
3881.496 \\
3881.785 \\
3882.145\end{array}$ & $\begin{array}{l}50 \\
30 \mathrm{w} \\
200 \\
50 \\
200\end{array}$ & $\begin{array}{l}40 \\
50\end{array}$ & $\begin{array}{l}\text { I } \\
\text { I } \\
\text { I I } \\
\text { I } \\
\text { II }\end{array}$ \\
\hline $\begin{array}{l}3855.676 \\
3856.112 \\
3856.190 \\
3856.360 \\
3856.517\end{array}$ & $\begin{array}{r}40 \\
10 \\
10 \\
100 \\
10\end{array}$ & $\begin{array}{l}6 \\
8\end{array}$ & $\begin{array}{l}\text { I } \\
\text { I I } \\
\text { I I } \\
\text { I } \\
\text { I }\end{array}$ & $\begin{array}{l}3869.664 \mathrm{i} \\
3869.970 \\
3870.172 \\
3870.229 \\
3870.271\end{array}$ & $\begin{array}{r}600 \\
75 \\
50 \\
20 \\
20\end{array}$ & $\begin{array}{l}12 \\
50\end{array}$ & $\begin{array}{l}\text { I } \\
\text { II } \\
\text { I } \\
\text { I } \\
\text { I }\end{array}$ & $\begin{array}{l}3882.314 \\
3882.556 \\
3882.788 \\
3882.859 \\
3883.080\end{array}$ & $\begin{array}{l}100 \\
20 \\
20 \mathrm{w} \\
20 \\
20\end{array}$ & & $\begin{array}{l}\text { I } \\
\text { I } \\
\text { I } \\
\text { I } \\
\text { I }\end{array}$ \\
\hline
\end{tabular}


Table 1. Wavelengths of Thorium Spectra - Continued

\begin{tabular}{|c|c|c|c|c|c|c|c|c|c|c|c|}
\hline \multirow{2}{*}{$\begin{array}{l}\text { Wave- } \\
\text { length }\end{array}$} & \multicolumn{2}{|c|}{ Intensity } & \multirow{2}{*}{$\begin{array}{l}\text { Spec- } \\
\text { trum }\end{array}$} & \multirow{2}{*}{$\begin{array}{l}\text { Wave- } \\
\text { length }\end{array}$} & \multicolumn{2}{|c|}{ Intensity } & \multirow{2}{*}{$\begin{array}{l}\text { Spec- } \\
\text { trum }\end{array}$} & \multirow{2}{*}{$\begin{array}{l}\text { Wave- } \\
\text { length }\end{array}$} & \multicolumn{2}{|c|}{ Intensity } & \multirow{2}{*}{$\begin{array}{l}\text { Spec- } \\
\text { trum }\end{array}$} \\
\hline & Lamp & Spark & & & Lamp & Spark & & & Lamp & Spark & \\
\hline $\begin{array}{l}3883.530 \\
3883.766 \\
3884.131 \\
3884.312 \\
3884.522\end{array}$ & $\begin{array}{l}100 \\
125 \\
30 \mathrm{w} \\
20 \\
300\end{array}$ & $\begin{array}{r}1 \\
1 \\
1 \\
1 \\
20\end{array}$ & $\begin{array}{l}\text { I } \\
\text { I } \\
\text { I } \\
\text { I } \\
\text { I I }\end{array}$ & $\begin{array}{l}3896.267 \\
3896.969 \\
3897.309 \\
3897.361 \\
3897.609\end{array}$ & $\begin{array}{l}40 \\
30 \mathrm{w} \\
40 \\
10 \\
40 \mathrm{w}\end{array}$ & & $\begin{array}{l}\text { I } \\
\text { I } \\
\text { I } \\
\text { I } \\
\text { I }\end{array}$ & $\begin{array}{l}3908.023 \\
3908.101 \\
3908.486 \\
3908.744 \\
3908.978\end{array}$ & $\begin{array}{l}40 \mathrm{w} \\
20 \\
30 \\
300 \\
150\end{array}$ & $\begin{array}{r}3 \\
10 \\
1\end{array}$ & $\begin{array}{l}\text { I } \\
\text { I } \\
\text { II } \\
\text { I I } \\
\text { I }\end{array}$ \\
\hline $\begin{array}{l}3884.633 \\
3884.820 \\
3885.060 \\
3885.143 \\
3885.215\end{array}$ & $\begin{array}{r}50 \\
400 \\
75 \\
40 \\
50\end{array}$ & $\begin{array}{r}300 \\
2\end{array}$ & $\begin{array}{l}\text { I } \\
\text { I I } \\
\text { I } \\
\text { I } \\
\text { I }\end{array}$ & $\begin{array}{l}3897.773 \\
3897.866 \\
3898.178 \\
3898.431 \\
3898.502\end{array}$ & $\begin{array}{r}75 \\
50 \\
75 \\
400 \\
300\end{array}$ & $\begin{array}{l}1 \\
2 \\
4\end{array}$ & $\begin{array}{l}\text { I } \\
\text { I } \\
\text { I } \\
\text { I } \\
\text { I }\end{array}$ & $\begin{array}{l}3909.134 \\
3909.584 \\
3909.988 \\
3910.249 \\
3910.331\end{array}$ & $\begin{array}{r}250 \\
10 \\
75 \\
40 \\
20\end{array}$ & 2 & $\begin{array}{l}\text { I } \\
\text { I } \\
\text { I } \\
\text { I } \\
\text { I }\end{array}$ \\
\hline $\begin{array}{l}3885.633 \\
3885.767 \\
3886.000 \\
3886.621 \\
3886.914\end{array}$ & $\begin{array}{c}30 \\
125 \\
50 \mathrm{w} \\
50 \\
700\end{array}$ & $\begin{array}{l}4 \\
6 \\
5 \\
6\end{array}$ & $\begin{array}{l}\text { I I } \\
\text { I I } \\
\text { I } \\
\text { I } \\
\text { I }\end{array}$ & $\begin{array}{l}3898.792 \\
3899.020 \\
3899.314 \\
3899.705 \\
3900.032\end{array}$ & $\begin{array}{l}150 \\
100 \\
50 \\
10 \\
30 \mathrm{w}\end{array}$ & $\begin{array}{r}10 \\
8 \\
1 \\
2\end{array}$ & $\begin{array}{l}\text { I I } \\
\text { I I } \\
\text { I } \\
\text { I I } \\
\text { I }\end{array}$ & $\begin{array}{l}3910.521 \\
3910.617 \\
3910.772 \\
3910.979 \\
3911.168\end{array}$ & $\begin{array}{l}50 \\
40 \mathrm{w} \\
125 \\
200 \\
40\end{array}$ & $\begin{array}{l}1 \\
1\end{array}$ & $\begin{array}{l}\text { I } \\
\text { I } \\
\text { I } \\
\text { I } \\
\text { I }\end{array}$ \\
\hline $\begin{array}{l}3887.010 \\
3887.431 \\
3887.505 \\
3888.055 \\
3888.229\end{array}$ & $\begin{array}{l}700 \\
25 \\
75 \\
50 \mathrm{w} \\
30\end{array}$ & $\begin{array}{l}8 \\
6 \\
3\end{array}$ & $\begin{array}{l}\text { I } \\
\text { II } \\
\text { I } \\
\text { I } \\
\text { I }\end{array}$ & $\begin{array}{l}3900.124 \\
3900.573 \\
3900.876 \\
3901.144 \\
3901.658\end{array}$ & $\begin{array}{r}150 \\
400 \\
1500 \\
125 \\
300\end{array}$ & $\begin{array}{r}40 \\
5 \\
300 \\
100 \\
2\end{array}$ & $\begin{array}{l}\text { I I } \\
\text { I } \\
\text { I I } \\
\text { I I } \\
\text { I }\end{array}$ & $\begin{array}{l}3911.228 \\
3911.300 \\
3911.524 \\
3911.909 \\
3912.278\end{array}$ & $\begin{array}{r}20 \\
50 \\
75 \\
600 \\
300\end{array}$ & $\begin{array}{r}4 \\
2 \\
100\end{array}$ & $\begin{array}{l}\text { I } \\
\text { I I } \\
\text { I } \\
\text { I } \\
\text { I I }\end{array}$ \\
\hline $\begin{array}{l}3888.964 \\
3889.540 \\
3889.608 \\
3889.715 \\
3889.905\end{array}$ & $\begin{array}{l}40 \\
75 \\
40 \\
20 \\
75\end{array}$ & $\begin{array}{l}1 \\
1 \\
1 \\
1\end{array}$ & $\begin{array}{l}\text { I } \\
\text { I } \\
\text { I } \\
\text { I } \\
\text { I }\end{array}$ & $\begin{array}{l}3902.116 \\
3902.166 \\
3902.472 \\
3902.729 \\
3902.794\end{array}$ & $\begin{array}{l}100 \\
150 \\
75 \\
5 \\
75 \mathrm{w}\end{array}$ & $\begin{array}{r}25 \\
6 \\
2\end{array}$ & $\begin{array}{l}\text { I I } \\
\text { I } \\
\text { I I } \\
\text { I I } \\
\text { I }\end{array}$ & $\begin{array}{l}3912.488 \\
3912.735 \\
3913.008 \\
3913.079 \\
3913.301\end{array}$ & $\begin{array}{r}20 \\
30 \\
250 \\
250 \\
20\end{array}$ & $\begin{array}{r}1 \\
100\end{array}$ & $\begin{array}{l}\text { I } \\
\text { I } \\
\text { I I } \\
\text { I } \\
\text { I }\end{array}$ \\
\hline $\begin{array}{l}3890.053 \\
3890.815 \\
3890.979 \\
3891.053 \\
3891.198\end{array}$ & $\begin{array}{r}20 \\
50 \\
4 \\
200 \\
75\end{array}$ & $\begin{array}{r}3 \\
8 \\
30 \\
3\end{array}$ & $\begin{array}{l}\text { I } \\
\text { I I } \\
\text { I I } \\
\text { I I } \\
\text { I I }\end{array}$ & $\begin{array}{l}3903.098 \\
3903.256 \\
3903.476 \\
3903.762 \\
3903.912\end{array}$ & $\begin{array}{r}1500 \\
5 \\
100 \\
40 \\
75\end{array}$ & $\begin{array}{l}6 \\
3 w \\
2\end{array}$ & $\begin{array}{l}\text { I } \\
\text { I I } \\
\text { I } \\
\text { I } \\
\text { I }\end{array}$ & $\begin{array}{l}3913.450 \\
3913.823 \\
3914.164 \\
3914.289 \\
3914.466\end{array}$ & $\begin{array}{l}10 \mathrm{w} \\
300 \\
125 \\
50 \\
40\end{array}$ & $\begin{array}{r}10 \\
1 \\
4\end{array}$ & $\begin{array}{l}\text { I } \\
\text { I I } \\
\text { I } \\
\text { I } \\
\text { I I }\end{array}$ \\
\hline $\begin{array}{l}3891.516 \\
3891.723 \\
3892.301 \\
3892.426 \\
3892.610\end{array}$ & $\begin{array}{r}50 \\
200 \\
200 \\
30 \\
20\end{array}$ & $\begin{array}{r}1 \\
2 \\
20 \\
3\end{array}$ & $\begin{array}{l}\text { I } \\
\text { I } \\
\text { I I } \\
\text { I I } \\
\text { I }\end{array}$ & $\begin{array}{l}3904.001 \\
3904.075 \\
3904.184 \\
3904.416 \\
3904.781\end{array}$ & $\begin{array}{r}50 \\
1000 \\
100 \\
20 \\
50\end{array}$ & $\begin{array}{r}100 \\
2\end{array}$ & $\begin{array}{l}\text { I } \\
\text { II } \\
\text { I } \\
\text { I } \\
\text { I }\end{array}$ & $\begin{array}{l}3914.530 \\
3914.623 \\
3914.787 \\
3914.827 \\
3915.104\end{array}$ & $\begin{array}{l}20 \\
20 \\
50 \\
30 \\
10\end{array}$ & & $\begin{array}{l}\text { I } \\
\text { I } \\
\text { I } \\
\text { I } \\
\text { I }\end{array}$ \\
\hline $\begin{array}{l}3892.704 \\
3893.025 \\
3893.112 \\
3893.337 \\
3893.411\end{array}$ & $\begin{array}{r}100 \\
75 \\
100 \\
75 \\
75\end{array}$ & $\begin{array}{l}1 \\
10 \\
15 \\
6\end{array}$ & $\begin{array}{l}\text { I } \\
\text { I I } \\
\text { I I } \\
\text { I } \\
\text { I I }\end{array}$ & $\begin{array}{l}3905.186 i \\
3905.348 \\
3905.657 \\
3905.801 \\
3905.866\end{array}$ & $\begin{array}{r}1500 \\
10 \\
20 \\
50 \\
10\end{array}$ & $\begin{array}{r}200 \\
50\end{array}$ & $\begin{array}{l}\text { I I } \\
\text { I I } \\
\text { I } \\
\text { I } \\
\text { I }\end{array}$ & $\begin{array}{l}3915.197 \\
3915.289 \\
3915.648 \\
3915.847 \\
3916.416\end{array}$ & $\begin{array}{r}10 \\
75 \\
10 \\
100 \\
500\end{array}$ & $\begin{array}{l}1 \\
1 \\
1 \\
3\end{array}$ & $\begin{array}{l}\text { I } \\
\text { I } \\
\text { I } \\
\text { I } \\
\text { I }\end{array}$ \\
\hline $\begin{array}{l}3893.643 \\
3893.812 \\
3894.012 \\
3894.402 \\
3894.702\end{array}$ & $\begin{array}{r}200 \\
100 \\
20 \\
125 \\
10\end{array}$ & 20 & $\begin{array}{l}\text { I } \\
\text { I } \\
\text { I } \\
\text { I I } \\
\text { I }\end{array}$ & $\begin{array}{l}3905.921 \\
3906.031 \\
3906.307 \\
3906.383 \\
3906.711\end{array}$ & $\begin{array}{l}20 \\
40 \mathrm{w} \\
75 \\
30 \mathrm{~h} \\
20 \mathrm{w}\end{array}$ & $\begin{array}{l}3 w \\
1 \\
1\end{array}$ & $\begin{array}{l}\text { I } \\
\text { II } \\
\text { I } \\
\text { I } \\
\text { I }\end{array}$ & $\begin{array}{l}3916.587 \\
3916.724 \\
3916.788 \\
3916.816 \\
3917.265\end{array}$ & $\begin{array}{r}20 \\
100 \\
200 \\
10 \\
400\end{array}$ & $\begin{array}{c}2 \\
150 \\
2001\end{array}$ & $\begin{array}{l}\text { I } \\
\text { I I } \\
\text { II } \\
\text { I } \\
\text { I }\end{array}$ \\
\hline $\begin{array}{l}3894.896 \\
3895.416 \\
3895.910 \\
3895.986 \\
3896.161\end{array}$ & $\begin{array}{r}125 \\
2400 \\
10 \\
20 \\
40\end{array}$ & $\begin{array}{l}20 \\
75\end{array}$ & $\begin{array}{l}\text { I I } \\
\text { I } \\
\text { I } \\
\text { I } \\
\text { I }\end{array}$ & $\begin{array}{l}3906.823 \\
3907.157 \\
3907.327 \\
3907.542 \\
3907.901\end{array}$ & $\begin{array}{r}30 \\
125 \\
50 \\
150 \\
50\end{array}$ & $\begin{array}{l}3 w \\
2 \\
3 \\
1 \\
30\end{array}$ & $\begin{array}{l}\text { II } \\
\text { I } \\
\text { II } \\
\text { I } \\
\text { I I }\end{array}$ & $\begin{array}{l}3918.070 \\
3918.284 \\
3918.508 \\
3919.018 \\
3919.270\end{array}$ & $\begin{array}{r}400 \\
50 \\
300 \\
800 \\
75\end{array}$ & $\begin{array}{r}50 \\
6 \\
150 \\
15\end{array}$ & $\begin{array}{l}\text { II } \\
\text { II } \\
\text { II } \\
\text { I } \\
\text { I }\end{array}$ \\
\hline
\end{tabular}


Table 1. Wavelengths of Thorium Spectra - Continued

\begin{tabular}{|c|c|c|c|c|c|c|c|c|c|c|c|}
\hline \multirow{2}{*}{$\begin{array}{l}\text { Wave- } \\
\text { length }\end{array}$} & \multicolumn{2}{|c|}{ Intensity } & \multirow{2}{*}{$\begin{array}{l}\text { Spec- } \\
\text { trum }\end{array}$} & \multirow{2}{*}{$\begin{array}{l}\text { Wave- } \\
\text { length }\end{array}$} & \multicolumn{2}{|c|}{ Intensity } & \multirow{2}{*}{$\begin{array}{l}\text { Spec- } \\
\text { trum }\end{array}$} & \multirow{2}{*}{$\begin{array}{l}\text { Wave- } \\
\text { length }\end{array}$} & \multicolumn{2}{|c|}{ Intensity } & \multirow{2}{*}{$\begin{array}{l}\text { Spec } \\
\text { trum }\end{array}$} \\
\hline & & & & & & & & & & & \\
\hline $\begin{array}{l}3919.453 \\
3919.974 \\
3920.054 \\
3920.305 \\
3920.435\end{array}$ & $\begin{array}{l}40 w \\
75 \\
75 \\
150 \\
125\end{array}$ & 100 & $\begin{array}{l}\text { I } \\
\text { I } \\
\text { I I } \\
\text { I }\end{array}$ & $\begin{array}{l}3931.757 \\
3931.998 \\
3932.226 \\
3932.911 \mathrm{i} \\
3933.235\end{array}$ & $\begin{array}{r}10 \\
250 \\
400 \\
1400 \\
200\end{array}$ & $\begin{array}{r}125 \\
150 \\
15\end{array}$ & $\begin{array}{l}\text { I } \\
\text { II } \\
\text { II } \\
\text { I } \\
\text { I }\end{array}$ & $\begin{array}{l}3943.391 \\
3943.604 \\
3943.688 \\
3944.249 \\
3945.134\end{array}$ & $\begin{array}{l}400 \\
150 \\
250 \\
400 \\
150\end{array}$ & $\begin{array}{l}150 \\
150 \\
125\end{array}$ & $\begin{array}{l}\text { I I } \\
\text { I } \\
\text { I I } \\
\text { I } \\
\text { I I }\end{array}$ \\
\hline $\begin{array}{l}3920.542 \\
3920.957 \\
3921.014 \\
3921.167 \\
3921.203\end{array}$ & $\begin{array}{r}100 \\
50 \\
50 \\
10 \\
20\end{array}$ & $\begin{array}{l}4 \mathrm{w} \\
4 \\
4\end{array}$ & $\begin{array}{l}\text { II } \\
\text { II } \\
\text { I I } \\
\text { I } \\
\text { I }\end{array}$ & $\begin{array}{l}3933.355 \\
3933.655 \\
3933.784 \\
3933.965 \\
3934.060\end{array}$ & $\begin{array}{r}75 \\
10 \\
30 \\
75 \\
5\end{array}$ & $\begin{array}{r}200 \\
1\end{array}$ & $\begin{array}{l}\text { I } \\
\text { I I } \\
I \\
\text { I } \\
\text { I }\end{array}$ & $\begin{array}{l}3945.211 \\
3945.508 \\
3945.824 \\
3946.148 \\
3946.392\end{array}$ & $\begin{array}{r}75 \\
1000 \\
500 \\
1500 \\
300\end{array}$ & $\begin{array}{l}250 \\
100 \\
300\end{array}$ & $\begin{array}{l}\text { I } \\
\text { II } \\
\text { II } \\
\text { I I } \\
\text { I }\end{array}$ \\
\hline $\begin{array}{l}3921.392 \\
3921.452 \\
3921.533 \\
3922.097 \\
3922.216\end{array}$ & $\begin{array}{r}50 \\
100 \\
30 \\
50 \\
150\end{array}$ & $\begin{array}{r}4 \\
30\end{array}$ & $\begin{array}{l}\text { II } \\
\text { I } \\
\text { I } \\
\text { I } \\
\text { II }\end{array}$ & $\begin{array}{l}3934.174 \\
3934.271 \\
3935.205 \\
3935.230 \\
3935.479\end{array}$ & $\begin{array}{r}20 \\
200 \\
200 \\
5 \\
40\end{array}$ & $\begin{array}{r}3 \\
2 \\
30\end{array}$ & $\begin{array}{l}\text { II } \\
\text { I } \\
\text { II } \\
\text { I } \\
\text { I }\end{array}$ & $\begin{array}{l}3946.478 \\
3947.132 \\
3947.331 \\
3947.649 \\
3947.794\end{array}$ & $\begin{array}{c}200 \mathrm{w} \\
250 \\
1000 \\
20 \\
75\end{array}$ & $\begin{array}{r}5 \\
10 \\
5\end{array}$ & $\begin{array}{l}\text { I } \\
\text { I } \\
\text { I } \\
\text { I I } \\
\text { I }\end{array}$ \\
\hline $\begin{array}{l}3922.383 \\
3923.596 \\
3923.799 \mathrm{i} \\
3924.399 \\
3924.639\end{array}$ & $\begin{array}{l}125 \\
20 \mathrm{w} \\
400 \\
250 \\
20\end{array}$ & $\begin{array}{l}1 \\
3 \\
1\end{array}$ & $\begin{array}{l}I \\
I \\
I \\
I \\
I\end{array}$ & $\begin{array}{l}3935.631 \\
3935.937 \\
3936.350 \\
3936.586 \\
3936.827\end{array}$ & $\begin{array}{c}100 \\
75 \\
150 \\
40 \mathrm{~s} \\
20\end{array}$ & $\begin{array}{l}30 \\
10 \\
50\end{array}$ & $\begin{array}{l}\text { II } \\
\text { II } \\
\text { II } \\
\text { I } \\
\text { I }\end{array}$ & $\begin{array}{l}3948.027 \\
3948.133 \\
3948.964 \mathrm{i} \\
3949.202 \\
3949.343\end{array}$ & $\begin{array}{r}600 \\
200 \\
1000 \\
10 \\
50\end{array}$ & $\begin{array}{r}3 \\
2 \\
300\end{array}$ & $\begin{array}{l}\text { I } \\
\text { I } \\
\text { I I } \\
\text { I } \\
\text { I }\end{array}$ \\
\hline $\begin{array}{l}3924.984 \\
3925.091 \\
3925.209 \\
3925.587 \\
3925.731\end{array}$ & $\begin{array}{r}5 \\
1000 \\
800 \\
150 \\
50\end{array}$ & $\begin{array}{r}20 \\
40 \\
10 \\
1\end{array}$ & $\begin{array}{l}\text { I I } \\
\text { I } \\
\text { I } \\
\text { I } \\
\text { I }\end{array}$ & $\begin{array}{l}3937.040 \\
3937.285 \\
3937.354 \\
3937.923 \\
3938.086\end{array}$ & $\begin{array}{c}400 \\
30 \\
30 \mathrm{w} \\
200 \\
401\end{array}$ & 150 & $\begin{array}{l}\text { I I } \\
\text { I } \\
\text { I } \\
\text { I I } \\
\text { I }\end{array}$ & $\begin{array}{l}3949.463 \\
3950.394 \\
3950.802 \\
3951.110 \\
3951.546\end{array}$ & $\begin{array}{r}75 \\
1000 \\
100 \\
100 \\
1500\end{array}$ & $\begin{array}{r}300 \\
1 \\
30 \\
300\end{array}$ & $\begin{array}{l}\text { I } \\
\text { II } \\
\text { I } \\
\text { II } \\
\text { I I }\end{array}$ \\
\hline $\begin{array}{l}3926.053 \\
3926.436 \\
3926.704 \\
3926.860 \\
3927.171\end{array}$ & $\begin{array}{l}20 \\
30 \mathrm{w} \\
75 \\
100 \\
200\end{array}$ & $\begin{array}{r}30 \\
125\end{array}$ & $\begin{array}{l}\text { I } \\
\text { I } \\
\text { II } \\
\text { I } \\
\text { I I }\end{array}$ & $\begin{array}{l}3938.145 \\
3938.422 \\
3938.609 \\
3938.686 \\
3938.731\end{array}$ & $\begin{array}{r}40 \\
50 \\
200 \\
100 \\
20\end{array}$ & $\begin{array}{l}3 \\
3 \\
2\end{array}$ & $\begin{array}{l}\text { I } \\
\text { I I } \\
\text { I } \\
\text { I } \\
\text { I I }\end{array}$ & $\begin{array}{l}3952.199 \\
3952.450 \\
3952.759 \\
3952.854 \\
3953.337\end{array}$ & $\begin{array}{r}20 \\
40 \\
500 \\
100 \\
100\end{array}$ & $\begin{array}{l}2 \\
2 \\
2\end{array}$ & $\begin{array}{l}\text { I } \\
\text { I } \\
\text { I } \\
\text { I } \\
\text { I }\end{array}$ \\
\hline $\begin{array}{l}3927.420 \\
3927.799 \\
3928.233 \\
3928.298 \\
3928.484\end{array}$ & $\begin{array}{l}300 \\
100 \\
10 \mathrm{w} \\
40 \\
20 \mathrm{w}\end{array}$ & $\begin{array}{r}200 \\
1\end{array}$ & $\begin{array}{l}\text { I I } \\
\text { I } \\
\text { I } \\
\text { I } \\
\text { I }\end{array}$ & $\begin{array}{l}3938.775 \\
3938.930 \\
3939.152 \\
3939.313 \\
3939.532\end{array}$ & $\begin{array}{r}150 \\
20 \\
150 \\
100 \\
40\end{array}$ & $\begin{array}{l}2 \\
1\end{array}$ & $\begin{array}{l}\text { I } \\
\text { I } \\
\text { I } \\
\text { I }\end{array}$ & $\begin{array}{l}3953.450 \\
3953.673 \\
3953.902 \\
3953.973 \\
3954.067\end{array}$ & $\begin{array}{l}50 \\
50 \\
20 \\
20 \\
75\end{array}$ & $\begin{array}{r}50 \\
1 \\
1\end{array}$ & $\begin{array}{l}\text { II } \\
\text { I } \\
\text { I } \\
\text { I } \\
\text { I }\end{array}$ \\
\hline $\begin{array}{l}3928.693 \\
3928.857 \\
3928.954 \\
3929.288 \\
3929.384\end{array}$ & $\begin{array}{r}50 \\
150 \\
150 \\
100 \\
75\end{array}$ & $\begin{array}{l}4 \\
4 \\
5\end{array}$ & $\begin{array}{l}\text { II } \\
\text { I } \\
\text { I } \\
\text { I } \\
\text { I }\end{array}$ & $\begin{array}{l}3939.790 \\
3940.368 \\
3940.703 \\
3940.833 \\
3941.136\end{array}$ & $\begin{array}{r}50 \\
50 \\
100 \\
100 \\
50\end{array}$ & $\begin{array}{l}12 \\
12\end{array}$ & $\begin{array}{l}\text { I I } \\
\text { I I } \\
\text { I } \\
\text { I } \\
\text { I }\end{array}$ & $\begin{array}{l}3954.133 \\
3954.462 \\
3954.608 \\
3954.724 \\
3954.929\end{array}$ & $\begin{array}{l}75 \\
50 \\
10 \\
75 \\
40\end{array}$ & 4 & $\begin{array}{l}\text { I } \\
\text { I I } \\
\text { I } \\
\text { I } \\
\text { I }\end{array}$ \\
\hline $\begin{array}{l}3929.667 \\
3930.326 \\
3930.649 \\
3930.927 \\
3931.077\end{array}$ & $\begin{array}{r}2500 \\
40 \\
75 \\
10 \\
75\end{array}$ & $\begin{array}{r}300 \\
4\end{array}$ & $\begin{array}{l}\text { I I } \\
\text { I I } \\
\text { I } \\
\text { I } \\
\text { I }\end{array}$ & $\begin{array}{l}3941.226 \\
3941.365 \\
3941.725 \\
3942.067 \\
3942.357\end{array}$ & $\begin{array}{r}40 \\
40 \\
50 \\
350 \\
200\end{array}$ & $\begin{array}{r}2 \\
8 \\
4 \\
40\end{array}$ & $\begin{array}{l}\text { I } \\
\text { I I } \\
\text { I I } \\
\text { I } \\
\text { I }\end{array}$ & $\begin{array}{l}3955.111 \\
3955.168 \\
3955.256 \\
3955.894 \\
3956.013\end{array}$ & $\begin{array}{r}15 \\
800 \\
50 \\
200 \\
200\end{array}$ & $\begin{array}{r}10 \\
3 \\
6\end{array}$ & $\begin{array}{l}\text { I I } \\
\text { I } \\
\text { I I } \\
\text { I } \\
\text { I }\end{array}$ \\
\hline $\begin{array}{l}3931.252 \\
3931.330 \\
3931.425 \\
3931.503 \\
3931.642\end{array}$ & $\begin{array}{l}75 \\
40 \\
40 \\
50 \\
10\end{array}$ & $20 \mathrm{w}$ & $\begin{array}{l}\text { I I } \\
\text { I } \\
\text { I } \\
\text { I } \\
\text { I }\end{array}$ & $\begin{array}{l}3942.554 \\
3942.642 \\
3942.857 \\
3942.902 \\
3943.257\end{array}$ & $\begin{array}{l}300 \\
300 \\
40 \\
100 \mathrm{w} \\
125\end{array}$ & $\begin{array}{r}2 \\
40 \\
8 \\
50\end{array}$ & $\begin{array}{l}\text { I } \\
\text { I I } \\
\text { I I } \\
\text { I } \\
\text { I I }\end{array}$ & $\begin{array}{l}3956.110 \\
3956.176 \\
3956.478 \\
3956.592 \\
3956.685\end{array}$ & $\begin{array}{r}40 \\
30 \\
75 \\
400 \\
400\end{array}$ & $\begin{array}{l}300 \\
200\end{array}$ & $\begin{array}{l}\text { I } \\
\text { I } \\
\text { I } \\
\text { I I } \\
\text { I I }\end{array}$ \\
\hline
\end{tabular}


Table 1. Wavelengths of Thorium Spectra - Continued

\begin{tabular}{|c|c|c|c|c|c|c|c|c|c|c|c|}
\hline \multirow{2}{*}{$\begin{array}{l}\text { Wave- } \\
\text { length }\end{array}$} & \multicolumn{2}{|c|}{ Intensity } & \multirow{2}{*}{$\begin{array}{l}\text { Spec- } \\
\text { trum }\end{array}$} & \multirow{2}{*}{$\begin{array}{l}\text { Wave- } \\
\text { length }\end{array}$} & \multicolumn{2}{|c|}{ Intensity } & \multirow{2}{*}{$\begin{array}{l}\text { Spec- } \\
\text { trum }\end{array}$} & \multirow{2}{*}{$\begin{array}{l}\text { Wave- } \\
\text { length }\end{array}$} & \multicolumn{2}{|c|}{ Intensity } & \multirow{2}{*}{$\begin{array}{l}\text { Spec- } \\
\text { trum }\end{array}$} \\
\hline & Lamp & Spark & & & Lamp & Spark & & & Lamp & Spark & \\
\hline $\begin{array}{l}3956.843 \\
3957.057 \\
3957.157 \\
3957.289 \\
3957.472\end{array}$ & $\begin{array}{r}20 \\
20 \\
100 \\
20 \\
40\end{array}$ & 150 & $\begin{array}{l}\text { I } \\
\text { I } \\
\text { II } \\
\text { I } \\
\text { I }\end{array}$ & $\begin{array}{l}3967.793 \\
3967.921 \\
3968.483 \\
3969.003 \\
3969.335\end{array}$ & $\begin{array}{r}20 \\
10 \\
500 \\
125 \\
10\end{array}$ & $\begin{array}{l}400 \\
40 \\
10 \mathrm{w}\end{array}$ & $\begin{array}{l}\text { I } \\
\text { I } \\
\text { I I } \\
\text { I I } \\
\text { I I }\end{array}$ & $\begin{array}{l}3982.476 \\
3982.604 \\
3982.732 \\
3982.890 \\
3983.007\end{array}$ & $\begin{array}{r}50 \\
25 \\
8 \\
40 \\
25\end{array}$ & 1 & $\begin{array}{l}\text { I } \\
\text { I } \\
\text { I } \\
\text { I } \\
\text { I }\end{array}$ \\
\hline $\begin{array}{l}3957.595 \\
3957.796 \\
3957.889 \\
3958.009 \\
3958.221\end{array}$ & $\begin{array}{l}20 \\
20 \\
10 \\
10 \mathrm{w} \\
40\end{array}$ & & $\begin{array}{l}\text { I } \\
\text { I } \\
\text { I } \\
\text { I } \\
\text { I }\end{array}$ & $\begin{array}{l}3969.523 \\
3969.660 \\
3969.829 \\
3969.927 \\
3970.545\end{array}$ & $\begin{array}{r}10 \\
150 \\
10 \\
20 \\
15\end{array}$ & $\begin{array}{c}20 w \\
4 \\
4\end{array}$ & $\begin{array}{l}\text { I I } \\
\text { I } \\
\text { I I } \\
\text { I } \\
\text { I }\end{array}$ & $\begin{array}{l}3983.168 \\
3983.256 \\
3983.493 \\
3983.836 \\
3984.094\end{array}$ & $\begin{array}{l}15 \\
4 \mathrm{w} \\
4 \mathrm{w} \\
8 \\
75\end{array}$ & 1 & $\begin{array}{l}\text { I } \\
\text { I } \\
\text { I } \\
\text { I } \\
\text { I }\end{array}$ \\
\hline $\begin{array}{l}3958.477 \\
3958.572 \\
3958.929 \\
3959.029 \\
3959.222\end{array}$ & $\begin{array}{l}10 \\
10 \\
40 \\
10 \mathrm{w} \\
100\end{array}$ & $\begin{array}{l}2 \\
6\end{array}$ & $\begin{array}{l}\text { I } \\
\text { I } \\
\text { I } \\
\text { I } \\
\text { I I }\end{array}$ & $\begin{array}{l}3970.650 \\
3970.996 \\
3971.133 \\
3971.583 \\
3971.662\end{array}$ & $\begin{array}{r}5 \\
5 \\
100 \\
10 \\
20\end{array}$ & 1 & $\begin{array}{l}\text { I } \\
\text { I } \\
\text { I } \\
\text { I } \\
\text { I }\end{array}$ & $\begin{array}{l}3984.370 \\
3984.616 \\
3984.873 \\
3985.242 \\
3985.405\end{array}$ & $\begin{array}{r}25 \\
25 \\
150 \\
8 \\
25\end{array}$ & $\begin{array}{r}10 \\
15 \\
2 \\
6\end{array}$ & $\begin{array}{l}\text { I I } \\
\text { II } \\
\text { I } \\
\text { I } \\
\text { I I }\end{array}$ \\
\hline $\begin{array}{l}3959.299 \\
3959.597 \\
3959.799 \\
3960.019 \\
3960.141\end{array}$ & $\begin{array}{r}1000 \\
75 \\
20 \\
20 \\
10\end{array}$ & $\begin{array}{l}6 \\
1 \\
1\end{array}$ & $\begin{array}{l}\text { I } \\
\text { I } \\
\text { I } \\
\text { I } \\
\text { I }\end{array}$ & $\begin{array}{l}3971.831 \\
3972.153 \\
3972.633 \\
3973.196 \\
3973.331\end{array}$ & $\begin{array}{r}10 \\
1400 \\
500 \\
1100 \\
200\end{array}$ & $\begin{array}{r}50 \\
10 \\
15 \\
2\end{array}$ & $\begin{array}{l}\text { I } \\
\text { I } \\
\text { I } \\
\text { I } \\
\text { I }\end{array}$ & $\begin{array}{l}3985.463 \\
3985.735 \\
3986.068 \\
3986.225 \\
3986.408\end{array}$ & $\begin{array}{r}15 \\
8 \\
40 \\
8 \\
15\end{array}$ & 1 & $\begin{array}{l}\text { I } \\
\text { I } \\
\text { I } \\
\text { I } \\
\text { I I }\end{array}$ \\
\hline $\begin{array}{l}3960.268 \\
3960.331 \\
3960.876 \\
3960.956 \\
3961.049\end{array}$ & $\begin{array}{r}100 \\
50 \\
125 \\
10 \\
20\end{array}$ & $\begin{array}{r}50 \\
2 \\
2\end{array}$ & $\begin{array}{l}\text { I } \\
\text { II } \\
\text { I } \\
\text { I } \\
\text { I }\end{array}$ & $\begin{array}{l}3973.608 \\
3973.686 \\
3973.995 \\
3974.223 \\
3974.393\end{array}$ & $\begin{array}{c}50 \\
20 \\
20 \\
125 \\
40 \mathrm{~h}\end{array}$ & $\begin{array}{c}2 \mathrm{~h} \\
2 \mathrm{~h} \\
50 \\
4\end{array}$ & $\begin{array}{l}\text { I } \\
\text { I } \\
\text { I } \\
\text { I I } \\
\text { I }\end{array}$ & $\begin{array}{l}3986.608 \\
3986.636 \\
3986.679 \\
3987.075 \\
3987.216\end{array}$ & $\begin{array}{r}15 \\
15 \\
4 \\
15 \\
600\end{array}$ & $\begin{array}{l}2 \\
5 \\
1 \\
2\end{array}$ & $\begin{array}{l}\text { II } \\
\text { I I } \\
\text { I } \\
\text { I I } \\
\text { I }\end{array}$ \\
\hline $\begin{array}{l}3961.294 \\
3961.964 \\
3962.100 \\
3962.195 \\
3962.338\end{array}$ & $\begin{array}{r}20 \\
20 \\
50 \\
100 \\
75\end{array}$ & $\begin{array}{r}2 \\
1 \\
50\end{array}$ & $\begin{array}{l}\text { I } \\
\text { I } \\
\text { I } \\
\text { I } \\
\text { I I }\end{array}$ & $\begin{array}{l}3974.830 \\
3975.220 \\
3975.457 \\
3975.833 \\
3975.920\end{array}$ & $\begin{array}{l}75 \\
100 \\
100 \mathrm{w} \\
100 \mathrm{~h} \\
40\end{array}$ & $\begin{array}{r}25 \\
1 \\
2\end{array}$ & $\begin{array}{l}\text { I } \\
\text { II } \\
\text { I } \\
\text { I } \\
\text { I }\end{array}$ & $\begin{array}{l}3987.480 \\
3987.552 \\
3987.705 \\
3987.781 \\
3987.865\end{array}$ & $\begin{array}{r}15 \\
25 \\
10 \\
4 \\
30\end{array}$ & $\begin{array}{r}4 \\
25\end{array}$ & $\begin{array}{l}\text { II } \\
\text { I } \\
\text { I I } \\
\text { I } \\
\text { I }\end{array}$ \\
\hline $\begin{array}{l}3962.418 \\
3962.785 \\
3962.920 \\
3963.220 \\
3963.467\end{array}$ & $\begin{array}{r}400 \\
30 \\
40 \\
200 \\
200\end{array}$ & $\begin{array}{r}3 \\
100 \\
125\end{array}$ & $\begin{array}{l}\text { I } \\
\text { I } \\
\text { I I } \\
\text { I I } \\
\text { II }\end{array}$ & $\begin{array}{l}3976.159 \\
3976.417 \\
3977.016 \\
3977.790 \\
3978.104\end{array}$ & $\begin{array}{r}30 \\
600 \\
20 \\
10 \\
10\end{array}$ & $\begin{array}{l}150 \\
3 w\end{array}$ & $\begin{array}{l}\text { I } \\
\text { II } \\
\text { I } \\
\text { II } \\
\text { I }\end{array}$ & $\begin{array}{l}3988.008 \\
3988.384 \\
3988.602 \\
3988.849 \\
3989.492\end{array}$ & $\begin{array}{r}400 \\
30 \\
40 \\
75 \\
25\end{array}$ & $\begin{array}{r}300 \\
10 \\
50\end{array}$ & $\begin{array}{l}\text { I I } \\
\text { I } \\
\text { I I } \\
\text { I I } \\
\text { I }\end{array}$ \\
\hline $\begin{array}{l}3964.030 \\
3964.329 \\
3964.737 \\
3964.865 \\
3965.365\end{array}$ & $\begin{array}{l}300 \\
100 \mathrm{w} \\
100 \\
100 \\
5\end{array}$ & 15 & $\begin{array}{l}\text { I } \\
\text { I } \\
\text { I } \\
\text { I I } \\
\text { I }\end{array}$ & $\begin{array}{l}3978.429 \\
3979.042 \\
3979.350 \\
3979.397 \\
3979.610\end{array}$ & $\begin{array}{r}20 \\
300 \\
20 \\
20 \\
4\end{array}$ & 50 & $\begin{array}{l}\text { I } \\
\text { I I } \\
\text { I } \\
\text { I } \\
\text { I }\end{array}$ & $\begin{array}{l}3989.584 \\
3990.012 \\
3990.309 \\
3990.490 \\
3990.809\end{array}$ & $\begin{array}{r}15 \\
150 \\
25 \\
800 \\
30\end{array}$ & 20 & $\begin{array}{l}\text { I } \\
\text { I } \\
\text { I } \\
\text { I } \\
\text { I }\end{array}$ \\
\hline $\begin{array}{l}3965.480 \\
3965.821 \\
3966.050 \\
3966.227 \\
3966.332\end{array}$ & $\begin{array}{l}30 \\
15 \\
10 \\
40 \\
40\end{array}$ & $\begin{array}{l}2 \\
3\end{array}$ & $\begin{array}{l}\text { I } \\
\text { I } \\
\text { I I } \\
\text { I I } \\
\text { I }\end{array}$ & $\begin{array}{l}3979.759 \\
3979.952 \\
3980.089 \mathrm{i} \\
3980.748 \\
3981.107\end{array}$ & $\begin{array}{r}4 \\
100 \\
1100 \\
300 \\
500\end{array}$ & $\begin{array}{r}2 \\
10 \\
200 \\
300\end{array}$ & $\begin{array}{l}\text { I } \\
\text { I } \\
\text { I } \\
\text { I I } \\
\text { I I }\end{array}$ & $\begin{array}{l}3990.892 \\
3991.059 \\
3991.333 \\
3991.607 \\
3991.731\end{array}$ & $\begin{array}{r}50 \\
5 \\
8 \\
10 \\
800\end{array}$ & $\begin{array}{l}3 \\
4 \\
4\end{array}$ & $\begin{array}{l}\text { I } \\
\text { II } \\
\text { I } \\
\text { II } \\
\text { I }\end{array}$ \\
\hline $\begin{array}{l}3966.474 \\
3966.656 \\
3966.964 \\
3967.210 \\
3967.392 \mathrm{i}\end{array}$ & $\begin{array}{r}10 \\
200 \\
40 \\
50 \\
2000\end{array}$ & $\begin{array}{l}50 \\
75 \\
40\end{array}$ & $\begin{array}{l}\text { I } \\
\text { I } \\
\text { II } \\
\text { II } \\
\text { I }\end{array}$ & $\begin{array}{l}3981.363 \\
3981.530 \\
3981.824 \\
3982.099 \\
3982.228\end{array}$ & $\begin{array}{r}20 \\
200 \\
300 \\
100 \\
40\end{array}$ & $\begin{array}{r}15 \\
3 \\
12 \\
6\end{array}$ & $\begin{array}{l}\text { I } \\
\text { I I } \\
\text { I } \\
\text { I I } \\
\text { I I }\end{array}$ & $\begin{array}{l}3992.043 \\
3992.275 \\
3992.616 \\
3992.796 \\
3993.294\end{array}$ & $\begin{array}{r}15 \\
50 \\
8 \\
4 \\
15\end{array}$ & $\begin{array}{r}3 \\
50\end{array}$ & $\begin{array}{l}\text { II } \\
\text { I I } \\
\text { I } \\
\text { I } \\
\text { I }\end{array}$ \\
\hline
\end{tabular}


Table 1. Wavelengths of Thorium Spectra - Continued

\begin{tabular}{|c|c|c|c|c|c|c|c|c|c|c|c|}
\hline \multirow{2}{*}{$\begin{array}{l}\text { Wave- } \\
\text { length }\end{array}$} & \multicolumn{2}{|c|}{ Intensity } & \multirow{2}{*}{$\begin{array}{l}\text { Spec- } \\
\text { trum }\end{array}$} & \multirow{2}{*}{$\begin{array}{l}\text { Wave- } \\
\text { length }\end{array}$} & \multicolumn{2}{|c|}{ Intensity } & \multirow{2}{*}{$\begin{array}{l}\text { Spec- } \\
\text { trum }\end{array}$} & \multirow{2}{*}{$\begin{array}{l}\text { Wave- } \\
\text { length }\end{array}$} & \multicolumn{2}{|c|}{ Intensity } & \multirow{2}{*}{$\begin{array}{l}\text { Spec- } \\
\text { trum }\end{array}$} \\
\hline & Lamp & Spark & & & Lamp & Spark & & & Lamp & Spark & \\
\hline $\begin{array}{l}3993.389 \\
3993.511 \\
3993.720 \\
3994.001 \\
3994.073\end{array}$ & $\begin{array}{l}10 w \\
30 \\
30 \\
4 \\
8\end{array}$ & 10 & $\begin{array}{l}\text { I } \\
\text { I } \\
\text { I I } \\
\text { I } \\
\text { I }\end{array}$ & $\begin{array}{l}4006.373 \\
4006.721 \\
4007.017 \\
4007.509 \\
4007.842\end{array}$ & $\begin{array}{r}100 \\
15 \\
600 \\
25 \\
30\end{array}$ & $\begin{array}{r}75 \\
200 \\
1\end{array}$ & $\begin{array}{l}\text { I I } \\
\text { I } \\
\text { I I } \\
\text { I } \\
\text { I }\end{array}$ & $\begin{array}{l}4021.423 \\
4021.542 \\
4021.750 \\
4022.082 \\
4022.721\end{array}$ & $\begin{array}{l}15 \\
15 \mathrm{w} \\
50 \\
500 \mathrm{w} \\
15\end{array}$ & 100 & $\begin{array}{l}\text { I } \\
\text { I } \\
\text { I } \\
\text { I I } \\
\text { I }\end{array}$ \\
\hline $\begin{array}{l}3994.352 \\
3994.549 \mathrm{i} \\
3995.030 \\
3995.098 \\
3995.188\end{array}$ & $\begin{array}{c}25 \\
1200 \\
20 \mathrm{w} \\
8 \\
8\end{array}$ & $\begin{array}{r}50 \\
200 \\
4 \\
1\end{array}$ & $\begin{array}{l}\text { II } \\
\text { I } \\
\text { I } \\
\text { I } \\
\text { I }\end{array}$ & $\begin{array}{l}4008.210 \mathrm{i} \\
4009.048 \\
4009.528 \\
4009.717 \\
4009.810\end{array}$ & $\begin{array}{r}1600 \\
1600 \\
15 \\
80 \\
80\end{array}$ & $\begin{array}{r}30 \\
50 \\
2 \\
2 \\
2\end{array}$ & $\begin{array}{l}\text { I } \\
\text { I } \\
\text { I I } \\
\text { I } \\
\text { I }\end{array}$ & $\begin{array}{l}4022.889 \\
4023.151 \\
4023.334 \\
4023.538 \\
4023.735\end{array}$ & $\begin{array}{r}10 \\
20 \\
150 \\
15 \\
8\end{array}$ & $\begin{array}{l}2 \\
3 \\
1\end{array}$ & $\begin{array}{l}\text { I } \\
\text { I } \\
\text { I } \\
\text { I I } \\
\text { I }\end{array}$ \\
\hline $\begin{array}{l}3995.240 \\
3995.348 \\
3995.451 \\
3995.597 \\
3995.714\end{array}$ & $\begin{array}{l}4 \\
4 \\
8 \\
8 \\
8\end{array}$ & & $\begin{array}{l}\text { I } \\
\text { I } \\
\text { I } \\
\text { I } \\
\text { I }\end{array}$ & $\begin{array}{l}4010.094 \\
4010.279 \\
4010.419 \\
4010.525 \\
4010.981\end{array}$ & $\begin{array}{r}15 \\
15 \\
15 \\
10 \\
4\end{array}$ & $\begin{array}{l}3 \\
4 \\
2\end{array}$ & $\begin{array}{l}\text { I } \\
\text { I } \\
\text { II } \\
\text { II } \\
\text { I I }\end{array}$ & $\begin{array}{l}4024.174 \\
4024.459 \\
4024.798 \\
4025.000 \\
4025.160\end{array}$ & $\begin{array}{r}25 \\
30 \\
100 \\
4 \\
10\end{array}$ & $\begin{array}{l}8 \\
2 \\
2\end{array}$ & $\begin{array}{l}\text { I } \\
\text { I I } \\
\text { I } \\
\text { I } \\
\text { I }\end{array}$ \\
\hline $\begin{array}{l}3996.061 \\
3996.430 \\
3996.509 \\
3996.668 \\
3996.932\end{array}$ & $\begin{array}{r}600 \\
15 \\
25 \\
75 \\
40\end{array}$ & 300 & $\begin{array}{l}\text { I I } \\
\text { I } \\
\text { I } \\
\text { I } \\
\text { I }\end{array}$ & $\begin{array}{l}4011.090 \\
4011.355 \\
4011.590 \\
4011.743 \\
4012.4951\end{array}$ & $\begin{array}{r}8 \\
50 \\
400 \\
800 \\
2000\end{array}$ & $\begin{array}{r}2 \\
3 \\
50 \\
75\end{array}$ & $\begin{array}{l}\text { I I } \\
\text { I } \\
\text { I } \\
\text { I } \\
\text { I }\end{array}$ & $\begin{array}{l}4025.298 \\
4025.654 \\
4025.948 \\
4026.157 \\
4026.748\end{array}$ & $\begin{array}{r}4 \\
400 \\
10 \\
400 \\
10\end{array}$ & $\begin{array}{r}150 \\
50 \\
2\end{array}$ & $\begin{array}{l}\text { I } \\
\text { I I } \\
\text { I } \\
\text { I I } \\
\text { I }\end{array}$ \\
\hline $\begin{array}{l}3997.015 \\
3997.106 \\
3997.345 \\
3997.466 \\
3997.867\end{array}$ & $\begin{array}{l}50 \\
25 \\
25 \\
40 \\
75\end{array}$ & $\begin{array}{l}10 \\
20\end{array}$ & $\begin{array}{l}\text { I } \\
\text { I } \\
\text { I } \\
\text { I I } \\
\text { I I }\end{array}$ & $\begin{array}{l}4013.023 \\
4013.101 \\
4013.254 \\
4013.912 \\
4014.506\end{array}$ & $\begin{array}{l}10 \\
10 \\
20 \\
75 \\
20\end{array}$ & $\begin{array}{l}30 \\
25\end{array}$ & $\begin{array}{l}\text { I } \\
\text { I } \\
\text { I I } \\
\text { I } \\
\text { I I }\end{array}$ & $\begin{array}{l}4026.814 \\
4027.006 \\
4027.338 \\
4027.400 \\
4027.547\end{array}$ & $\begin{array}{r}8 \\
1000 \\
30 \\
8 \\
30\end{array}$ & $\begin{array}{r}1 \\
8 \\
10 \\
3\end{array}$ & $\begin{array}{l}\text { I } \\
\text { I } \\
\text { I I } \\
\text { I I } \\
\text { I }\end{array}$ \\
\hline $\begin{array}{l}3998.054 \\
3998.404 \\
3998.734 \\
3998.799 \\
3998.954\end{array}$ & $\begin{array}{r}25 \\
40 \\
40 \\
4 \\
125\end{array}$ & 5 & $\begin{array}{l}\text { I } \\
\text { I } \\
\text { I } \\
\text { I I } \\
\text { I }\end{array}$ & $\begin{array}{l}4014.714 \\
4015.101 \\
4015.584 \\
4015.821 \\
4016.110\end{array}$ & $\begin{array}{r}300 \\
15 \\
8 \\
12 \\
30\end{array}$ & $\begin{array}{l}2 \\
2 \\
1 \\
4\end{array}$ & $\begin{array}{l}\text { I } \\
\text { I I } \\
\text { I } \\
\text { I I } \\
\text { I }\end{array}$ & $\begin{array}{l}4027.653 \\
4028.334 \\
4028.696 \\
4028.950 \\
4029.023\end{array}$ & $\begin{array}{r}30 \\
40 \\
125 \\
50 \\
25\end{array}$ & $\begin{array}{r}15 \\
20 \\
2\end{array}$ & $\begin{array}{l}\text { I I } \\
\text { I } \\
\text { I I } \\
\text { I } \\
\text { I I }\end{array}$ \\
\hline $\begin{array}{l}3999.302 \\
3999.943 \\
4000.030 \\
4000.282 \\
4000.438\end{array}$ & $\begin{array}{r}4 \\
10 \\
20 \\
400 \\
10\end{array}$ & 4 & $\begin{array}{l}\text { I } \\
\text { I I } \\
\text { I } \\
\text { I } \\
\text { I }\end{array}$ & $\begin{array}{l}4016.221 \\
4016.297 \\
4016.640 \\
4016.687 \\
4017.064\end{array}$ & $\begin{array}{l}15 \\
12 \\
30 \\
50 \\
50\end{array}$ & 20 & $\begin{array}{l}\text { I } \\
\text { I I } \\
\text { I } \\
\text { I } \\
\text { I }\end{array}$ & $\begin{array}{l}4029.320 \\
4029.518 \\
4029.659 \\
4029.825 \\
4030.298\end{array}$ & $\begin{array}{r}40 \\
20 \\
150 \\
150 \\
200\end{array}$ & $\begin{array}{r}10 \\
5 \\
2 \\
10\end{array}$ & $\begin{array}{l}\text { I I } \\
\text { I } \\
\text { I I } \\
\text { I } \\
\text { I }\end{array}$ \\
\hline $\begin{array}{l}4000.743 \\
4001.060 \\
4001.727 \\
4001.888 \\
4002.367\end{array}$ & $\begin{array}{r}8 \\
800 \\
40 \\
150 \\
25\end{array}$ & $\begin{array}{l}6 \\
2\end{array}$ & $\begin{array}{l}\text { I } \\
\text { I } \\
\text { I I } \\
\text { I } \\
\text { I }\end{array}$ & $\begin{array}{l}4017.133 \\
4017.258 \\
4017.484 \\
4017.725 \\
4018.099\end{array}$ & $\begin{array}{r}50 \\
25 \\
15 \\
8 \\
400\end{array}$ & $\begin{array}{r}1 \\
15 \\
2\end{array}$ & $\begin{array}{l}\text { I } \\
\text { I } \\
\text { I I } \\
\text { I } \\
\text { I }\end{array}$ & $\begin{array}{l}4030.839 \\
4031.079 \\
4031.331 \\
4031.478 \\
4031.655\end{array}$ & $\begin{array}{c}1800 \\
30 \mathrm{w} \\
50 \\
15 \\
8\end{array}$ & $\begin{array}{l}35 \\
20 \\
10\end{array}$ & $\begin{array}{l}\text { I } \\
\text { I I } \\
\text { I I } \\
\text { I } \\
\text { I }\end{array}$ \\
\hline $\begin{array}{l}4003.105 \\
4003.307 \\
4003.570 \\
4004.186 \\
405.547\end{array}$ & $\begin{array}{l}100 \\
200 \\
200 \\
8 w \\
4\end{array}$ & $\begin{array}{r}100 \\
200 \\
2 \\
1\end{array}$ & $\begin{array}{l}\text { I I } \\
\text { I I } \\
\text { I } \\
\text { I } \\
\text { I }\end{array}$ & $\begin{array}{l}4019.1291 \\
4019.779 \\
4019.857 \\
4020.013 \\
4020.096\end{array}$ & $\begin{array}{r}1200 \\
10 \\
50 \\
8 \\
15\end{array}$ & $\begin{array}{r}500 \\
1 \\
1 \\
4\end{array}$ & $\begin{array}{l}\text { I I } \\
\text { I } \\
\text { I } \\
\text { I } \\
\text { I I }\end{array}$ & $\begin{array}{l}4031.774 \\
4032.020 \\
4032.248 \\
4032.455 \\
4032.595\end{array}$ & $\begin{array}{l}10 \\
15 \mathrm{w} \\
15 \mathrm{w} \\
600 \\
800\end{array}$ & $\begin{array}{c}2 \mathrm{w} \\
1 \\
2 \\
200 \\
125\end{array}$ & $\begin{array}{l}\text { II } \\
\text { I } \\
\text { II } \\
\text { I I } \\
\text { I }\end{array}$ \\
\hline $\begin{array}{l}4004.630 \\
4005.085 \\
4005.527 \\
4005.568 \\
4005.953\end{array}$ & $\begin{array}{l}8 \mathrm{w} \\
400^{-} \\
75 \\
40 \\
100\end{array}$ & $\begin{array}{r}3 \\
100 \\
75\end{array}$ & $\begin{array}{l}\text { I } \\
\text { I } \\
\text { II } \\
\text { II } \\
\text { I }\end{array}$ & $\begin{array}{l}4020.354 \\
4020.608 \\
4020.770 \\
4021.005 \\
4021.140\end{array}$ & $\begin{array}{r}150 \\
30 \\
8 \\
10 \\
50\end{array}$ & 5 & $\begin{array}{l}\text { I } \\
\text { I } \\
\text { I } \\
\text { I } \\
\text { I }\end{array}$ & $\begin{array}{l}4032.888 \\
4033.181 \\
4033.480 \\
4033.774 \\
4033.908\end{array}$ & $\begin{array}{r}4 \\
15 \\
8 \\
80 \\
125\end{array}$ & $\begin{array}{l}1 \\
2\end{array}$ & $\begin{array}{l}\text { I } \\
\text { I I } \\
\text { I } \\
\text { I } \\
\text { I }\end{array}$ \\
\hline
\end{tabular}


Table 1. Wavelengths of Thorium Spectra - Continued

\begin{tabular}{|c|c|c|c|c|c|c|c|c|c|c|c|}
\hline \multirow{2}{*}{$\begin{array}{l}\text { Wave- } \\
\text { length }\end{array}$} & \multicolumn{2}{|c|}{ Intensity } & \multirow{2}{*}{$\begin{array}{l}\text { Spec- } \\
\text { trum }\end{array}$} & \multirow{2}{*}{$\begin{array}{l}\text { Wave- } \\
\text { length }\end{array}$} & \multicolumn{2}{|c|}{ Intensity } & \multirow{2}{*}{$\begin{array}{l}\text { Spec- } \\
\text { trum }\end{array}$} & \multirow{2}{*}{$\begin{array}{l}\text { Wave- } \\
\text { length }\end{array}$} & \multicolumn{2}{|c|}{ Intensity } & \multirow{2}{*}{$\begin{array}{l}\text { Spec- } \\
\text { trum }\end{array}$} \\
\hline & Lamp & Spark & & & Lamp & Spark & & & Lamp & Spark & \\
\hline $\begin{array}{l}4034.247 \\
4034.548 \\
4034.600 \\
4034.895 \\
4034.927\end{array}$ & $\begin{array}{r}125 \\
4 \\
25 \\
75 \\
30\end{array}$ & $\begin{array}{r}100 \\
1 \\
10\end{array}$ & $\begin{array}{l}\text { II } \\
\text { I } \\
\text { I } \\
\text { II } \\
\text { I }\end{array}$ & $\begin{array}{l}4047.578 \\
4048.028 \\
4048.286 \\
4048.434 \\
4048.799\end{array}$ & $\begin{array}{r}15 \\
30 \\
200 \\
200 \\
4\end{array}$ & $\begin{array}{r}25 \\
30 \\
20 \\
100\end{array}$ & $\begin{array}{l}\text { I } \\
\text { II } \\
\text { II } \\
\text { II } \\
\text { I I I }\end{array}$ & $\begin{array}{l}4064.985 \\
4065.247 \\
4065.545 \\
4065.615 \\
4065.683\end{array}$ & $\begin{array}{l}30 \\
15 \\
25 \\
30 \\
15\end{array}$ & 5 & $\begin{array}{l}\text { I } \\
\text { II } \\
\text { I } \\
\text { I } \\
\text { I I }\end{array}$ \\
\hline $\begin{array}{l}4035.349 \\
4035.471 \\
4035.573 \\
4035.730 \\
4036.0481\end{array}$ & $\begin{array}{r}30 \\
15 \\
15 \\
25 \\
1800\end{array}$ & 15 & $\begin{array}{l}\text { I } \\
\text { I } \\
\text { I } \\
\text { I } \\
\text { I }\end{array}$ & $\begin{array}{l}4049.532 \\
4049.833 \\
4049.949 \\
4050.894 \\
4051.126\end{array}$ & $\begin{array}{r}30 \\
15 \\
400 \\
800 \\
15\end{array}$ & $\begin{array}{l}6 \\
8 \mathrm{w} \\
10 \\
4\end{array}$ & $\begin{array}{l}\text { I } \\
\text { I I } \\
\text { I } \\
\text { I } \\
\text { I I }\end{array}$ & $\begin{array}{l}4065.887 \\
4066.001 \\
4066.164 \\
4066.430 \\
4066.818\end{array}$ & $\begin{array}{l}30 \\
15 \\
10 \\
25 \\
50\end{array}$ & $\begin{array}{l}2 \\
5 \\
2 \\
1\end{array}$ & $\begin{array}{l}\text { I } \\
\text { I I } \\
\text { I I } \\
\text { I } \\
\text { I }\end{array}$ \\
\hline $\begin{array}{l}4036.357 \\
4036.561 \\
4036.933 \\
4037.047 \\
4037.127\end{array}$ & $\begin{array}{r}40 \\
400 \\
8 \\
15 \\
4\end{array}$ & $\begin{array}{r}1 \\
200\end{array}$ & $\begin{array}{l}\text { I } \\
\text { I I } \\
\text { I } \\
\text { I } \\
\text { I }\end{array}$ & $\begin{array}{l}4051.198 \\
4051.271 \\
4051.497 \\
4051.899 \\
4052.255\end{array}$ & $\begin{array}{c}4 \\
4 \\
100 \\
15 \\
30 w\end{array}$ & $\begin{array}{l}1 \\
1\end{array}$ & $\begin{array}{l}\text { I } \\
\text { I } \\
\text { I } \\
\text { I } \\
\text { I }\end{array}$ & $\begin{array}{l}4066.956 \\
4067.451 \mathrm{i} \\
4067.590 \\
4067.873 \\
4068.017\end{array}$ & $\begin{array}{r}25 \\
400 \\
10 \\
25 \\
15\end{array}$ & $\begin{array}{r}4 \\
5 \\
10 \\
7\end{array}$ & $\begin{array}{l}\text { I } \\
\text { I } \\
\text { I I } \\
\text { I I } \\
\text { I I }\end{array}$ \\
\hline $\begin{array}{l}4037.256 \\
4037.559 \\
4038.226 \\
4038.453 \\
4038.620\end{array}$ & $\begin{array}{r}125 \\
200 \\
125 \\
40 \\
50\end{array}$ & $\begin{array}{r}25 \\
3 \\
2 \\
1 \\
2\end{array}$ & $\begin{array}{l}\text { II } \\
\text { I } \\
\text { I } \\
\text { I } \\
\text { II }\end{array}$ & $\begin{array}{l}4052.441 \\
4052.810 \\
4053.525 \\
4054.180 \\
4054.295\end{array}$ & $\begin{array}{r}15 \\
15 \\
1000 \\
8 \\
200\end{array}$ & $\begin{array}{r}3 \\
1 \\
25 \\
2 \\
1\end{array}$ & $\begin{array}{l}\text { II } \\
\text { I } \\
\text { I } \\
\text { I I } \\
\text { I }\end{array}$ & $\begin{array}{l}4068.469 \\
4068.709 \\
4069.203 \\
4069.457 \\
4069.753\end{array}$ & $\begin{array}{r}30 \\
20 \\
750 \\
400 \\
8\end{array}$ & $\begin{array}{r}4 \\
400 \\
3\end{array}$ & $\begin{array}{l}\text { I } \\
\text { II } \\
\text { II } \\
\text { I } \\
\text { II }\end{array}$ \\
\hline $\begin{array}{l}4038.955 \\
4039.018 \\
4039.265 \\
4039.430 \\
4039.862\end{array}$ & $\begin{array}{r}50 \\
25 \\
15 \\
125 \\
800\end{array}$ & $\begin{array}{l}10 \\
10\end{array}$ & $\begin{array}{l}\text { I } \\
\text { I } \\
\text { I } \\
\text { II } \\
\text { I }\end{array}$ & $\begin{array}{l}4054.767 \\
4055.231 \\
4055.907 \\
4056.005 \\
4056.395\end{array}$ & $\begin{array}{r}8 \\
30 \\
25 \\
25 \\
15\end{array}$ & $\begin{array}{r}2 \\
15\end{array}$ & $\begin{array}{l}\text { I I } \\
\text { I I } \\
\text { I } \\
\text { I } \\
\text { I }\end{array}$ & $\begin{array}{l}4070.039 \\
4070.112 \\
4070.235 \\
4070.673 \\
4070.755\end{array}$ & $\begin{array}{l}30 \\
15 \\
75 \\
15 \\
40\end{array}$ & $\begin{array}{l}2 \\
3\end{array}$ & $\begin{array}{l}\text { I } \\
\text { I } \\
\text { I } \\
\text { II } \\
\text { I }\end{array}$ \\
\hline $\begin{array}{l}4040.388 \\
4040.912 \\
4041.203 \\
4041.544 \\
4041.806\end{array}$ & $\begin{array}{r}30 \\
150 \\
300 \\
15 \\
15\end{array}$ & $\begin{array}{r}10 \\
3 \\
150 \\
4\end{array}$ & $\begin{array}{l}\text { II } \\
\text { I } \\
\text { II } \\
\text { I } \\
\text { I I }\end{array}$ & $\begin{array}{l}4056.635 \\
4057.023 \\
4057.120 \\
4057.328 \\
4057.474\end{array}$ & $\begin{array}{r}30 \\
15 \\
15 \\
40 \\
8\end{array}$ & $\begin{array}{r}2 \\
5 \\
10\end{array}$ & $\begin{array}{l}\text { II } \\
\text { I I } \\
\text { I } \\
\text { I I } \\
\text { I }\end{array}$ & $\begin{array}{l}4071.314 \\
4071.548 \\
4071.743 \\
4072.172 \\
4072.629\end{array}$ & $\begin{array}{r}15 \\
40 \\
400 \\
8 \\
400\end{array}$ & $\begin{array}{l}8 \\
2 \\
3\end{array}$ & $\begin{array}{l}\text { I } \\
\text { I } \\
\text { I } \\
\text { I I } \\
\text { I }\end{array}$ \\
\hline $\begin{array}{l}4041.974 \\
4042.060 \\
4042.260 \\
4042.642 \\
4042.977\end{array}$ & $\begin{array}{r}15 \\
15 \\
15 \\
8 \\
30\end{array}$ & 50 & $\begin{array}{l}\text { I } \\
\text { I } \\
\text { I } \\
\text { I } \\
\text { I I }\end{array}$ & $\begin{array}{l}4057.777 \\
4057.938 \\
4058.317 \\
4058.675 \\
4059.253 \mathrm{i}\end{array}$ & $\begin{array}{r}25 \\
50 \\
8 \\
8 \\
1000\end{array}$ & $\begin{array}{r}30 \\
1\end{array}$ & $\begin{array}{l}\text { I I } \\
\text { I } \\
\text { I } \\
\text { I } \\
\text { I }\end{array}$ & $\begin{array}{l}4072.910 \\
4072.999 \\
4073.170 \\
4073.278 \\
4073.709\end{array}$ & $\begin{array}{r}30 \\
15 \\
8 \\
8 \\
200\end{array}$ & 50 & $\begin{array}{l}\text { II } \\
\text { I } \\
\text { I } \\
\text { I } \\
\text { II }\end{array}$ \\
\hline $\begin{array}{l}4043.123 \\
4043.395 \mathrm{i} \\
4043.893 \\
4044.227 \\
4044.692\end{array}$ & $\begin{array}{r}40 \\
800 \\
8 \\
4 \\
15\end{array}$ & $\begin{array}{l}50 \\
20\end{array}$ & $\begin{array}{l}\text { I I } \\
\text { I } \\
\text { I } \\
\text { I } \\
\text { I }\end{array}$ & $\begin{array}{l}4059.808 \\
4059.890 \\
4060.058 \\
4061.618 \\
4061.767\end{array}$ & $\begin{array}{l}15 \mathrm{~h} \\
250 \\
20 \\
40 \\
15\end{array}$ & $\begin{array}{r}15 \\
1\end{array}$ & $\begin{array}{l}\text { I } \\
\text { II } \\
\text { I } \\
\text { I } \\
\text { I }\end{array}$ & $\begin{array}{l}4073.855 \\
4074.239 \\
4074.434 \\
4074.642 \\
4074.795\end{array}$ & $\begin{array}{r}100 \\
30 \\
25 \\
100 \\
8\end{array}$ & $\begin{array}{l}2 \\
2 \mathrm{~h} \\
50\end{array}$ & $\begin{array}{l}\text { I } \\
\text { II } \\
\text { I } \\
\text { II } \\
\text { I }\end{array}$ \\
\hline $\begin{array}{l}4044.924 \\
4045.223 \\
4045.612 \\
4045.731 \\
4046.246\end{array}$ & $\begin{array}{r}75 \\
30 \\
75 \\
4 \\
40\end{array}$ & $\begin{array}{r}1 \\
30 \\
1\end{array}$ & $\begin{array}{l}\text { I } \\
\text { I } \\
\text { II } \\
\text { I } \\
\text { I }\end{array}$ & $\begin{array}{l}4061.870 \\
4062.076 \\
4062.290 \\
4062.650 \\
4062.745\end{array}$ & $\begin{array}{r}15 \\
25 \\
8 \\
15 \\
8\end{array}$ & $\frac{1}{2 h}$ & $\begin{array}{l}\text { I } \\
\text { I } \\
\text { I I } \\
\text { I I } \\
\text { I }\end{array}$ & $\begin{array}{l}4074.927 \\
4075.050 \\
4075.503 \\
4075.707 \\
4075.903\end{array}$ & $\begin{array}{r}10 \\
5 \\
400 \\
30 \\
125\end{array}$ & $\begin{array}{l}2 \\
1 \\
3 \\
8 \\
1\end{array}$ & $\begin{array}{l}\text { II } \\
\text { I I } \\
\text { I } \\
\text { II } \\
\text { I }\end{array}$ \\
\hline $\begin{array}{l}4046.621 \\
4046.822 \\
4047.052 \\
4047.323 \\
4047.464\end{array}$ & $\begin{array}{r}8 \\
30 \\
15 \\
4 \\
15\end{array}$ & & $\begin{array}{l}\text { I } \\
\text { I } \\
\text { I } \\
\text { I } \\
\text { I }\end{array}$ & $\begin{array}{l}4063.059 \\
4063.403 \\
4063.670 \\
4064.325 \\
4064.598\end{array}$ & $\begin{array}{r}8 \\
800 \\
20 \\
400 \\
15\end{array}$ & $\begin{array}{r}2 \\
50 \\
5 \\
10 \\
3\end{array}$ & $\begin{array}{l}\text { I I } \\
\text { I I } \\
\text { I I } \\
\text { I } \\
\text { I I }\end{array}$ & $\begin{array}{l}4076.610 \\
4076.875 \\
4077.257 \\
4077.620 \\
4077.865\end{array}$ & $\begin{array}{r}4 \\
8 \\
4 \\
30 \\
25\end{array}$ & $2 w$ & $\begin{array}{l}\text { I I } \\
\text { I } \\
\text { I } \\
\text { I } \\
\text { I }\end{array}$ \\
\hline
\end{tabular}


Table 1. Wavelengths of Thorium Spectra - Continued

\begin{tabular}{|c|c|c|c|c|c|c|c|c|c|c|c|}
\hline \multirow{2}{*}{$\begin{array}{l}\text { Wave- } \\
\text { length }\end{array}$} & \multicolumn{2}{|c|}{ Intensity } & \multirow{2}{*}{$\begin{array}{l}\text { Spec- } \\
\text { trum }\end{array}$} & \multirow{2}{*}{$\begin{array}{l}\text { Wave- } \\
\text { length }\end{array}$} & \multicolumn{2}{|c|}{ Intensity } & \multirow{2}{*}{$\begin{array}{l}\text { Spec- } \\
\text { trum }\end{array}$} & \multirow{2}{*}{$\begin{array}{l}\text { Wave- } \\
\text { length }\end{array}$} & \multicolumn{2}{|c|}{ Intensity } & \multirow{2}{*}{$\begin{array}{l}\text { Spec- } \\
\text { trum }\end{array}$} \\
\hline & Lamp & Spark & & & Lamp & Spark & & & Lamp & Spark & \\
\hline $\begin{array}{l}4077.977 \\
4078.459 \\
4078.520 \\
4078.654 \\
4078.872\end{array}$ & $\begin{array}{c}30 \\
8 \\
8 \mathrm{~h} \\
15 \\
150\end{array}$ & $\begin{array}{l}1 \\
2 \mathrm{~h} \\
2 \\
3\end{array}$ & $\begin{array}{l}\text { I } \\
\text { II } \\
\text { I } \\
\text { I } \\
\text { I }\end{array}$ & $\begin{array}{l}4090.582 \\
4090.640 \\
4090.789 \\
4091.022 \\
4091.344\end{array}$ & $\begin{array}{r}8 \\
3 \\
5 \\
15 \\
30\end{array}$ & 6 & $\begin{array}{l}\text { I } \\
\text { I } \\
\text { I } \\
\text { I } \\
\text { I I }\end{array}$ & $\begin{array}{l}4103.103 \\
4103.265 \\
4103.456 \\
4103.650 \\
4103.682\end{array}$ & $\begin{array}{r}15 \\
40 \\
25 \\
4 \\
350\end{array}$ & $\begin{array}{r}50 \\
8\end{array}$ & $\begin{array}{l}\text { I } \\
\text { I I } \\
\text { I } \\
\text { I I } \\
\text { I }\end{array}$ \\
\hline $\begin{array}{l}4079.132 \\
4079.258 \\
4079.444 \\
4079.601 \\
4079.695\end{array}$ & $\begin{array}{r}25 \\
25 \\
4 \\
15 \\
30\end{array}$ & 8 & $\begin{array}{l}\text { II } \\
\text { I } \\
\text { I } \\
\text { II } \\
\text { I }\end{array}$ & $\begin{array}{l}4091.529 \\
4091.588 \\
4091.732 \\
4091.911 \\
4091.982\end{array}$ & $\begin{array}{r}10 \\
25 \\
40 \\
8 \\
40\end{array}$ & $\begin{array}{l}4 \\
1\end{array}$ & $\begin{array}{l}\text { I I } \\
\text { I } \\
\text { I } \\
\text { I } \\
\text { I }\end{array}$ & $\begin{array}{l}4103.987 \\
4104.383 \\
4104.837 \\
4105.054 \\
4105.336\end{array}$ & $\begin{array}{r}8 \\
250 \\
125 \\
25 \\
800\end{array}$ & $\begin{array}{r}150 \\
2 \\
300\end{array}$ & $\begin{array}{l}\text { I } \\
\text { I I } \\
\text { I } \\
\text { I } \\
\text { I I }\end{array}$ \\
\hline $\begin{array}{l}4079.894 \\
4080.294 \\
4080.357 \\
4080.704 \\
4080.964\end{array}$ & $\begin{array}{r}25 \\
10 \\
150 \\
400 \\
15\end{array}$ & $\begin{array}{c}101 \\
4 \\
2 \\
3 \\
3\end{array}$ & $\begin{array}{l}\text { I I } \\
\text { I I } \\
\text { I } \\
\text { I } \\
\text { I I }\end{array}$ & $\begin{array}{l}4092.127 \\
4092.316 \\
4092.474 \\
4092.684 \\
4092.824\end{array}$ & $\begin{array}{c}15 \\
8 \\
8 \\
15 \mathrm{w} \\
4 \mathrm{w}\end{array}$ & 1 & $\begin{array}{l}\text { I } \\
\text { I I } \\
\text { I } \\
\text { I } \\
\text { I }\end{array}$ & $\begin{array}{l}4105.913 \\
4106.119 \\
4106.217 \\
4106.351 \\
4106.436\end{array}$ & $\begin{array}{r}150 \\
15 \\
25 \\
25 \\
4\end{array}$ & $\begin{array}{l}75 \\
10\end{array}$ & $\begin{array}{l}\text { I I } \\
\text { I I } \\
\text { I } \\
\text { I } \\
\text { I }\end{array}$ \\
\hline $\begin{array}{l}4081.371 \\
4081.586 \\
4081.847 \\
4082.080 \\
4082.258\end{array}$ & $\begin{array}{r}800 \\
30 \\
25 \\
125 \\
125\end{array}$ & $\begin{array}{r}10 \\
20 \\
4 \\
75\end{array}$ & $\begin{array}{l}\text { I } \\
\text { I } \\
\text { I I } \\
\text { I } \\
\text { I I }\end{array}$ & $\begin{array}{l}4093.389 \\
4093.552 \\
4093.669 \\
4093.999 \\
4094.108\end{array}$ & $\begin{array}{r}30 \\
8 \\
40 \\
4 \\
4\end{array}$ & 6 & $\begin{array}{l}\text { I I } \\
\text { I } \\
\text { I } \\
\text { I } \\
\text { I }\end{array}$ & $\begin{array}{l}4106.525 \\
4106.573 \\
4106.852 \\
4106.944 \\
4107.051\end{array}$ & $\begin{array}{r}8 \\
8 \\
4 \\
8 \\
40\end{array}$ & 2 & $\begin{array}{l}\text { I } \\
\text { I } \\
\text { I } \\
\text { I I } \\
\text { I }\end{array}$ \\
\hline $\begin{array}{l}4082.435 \\
4082.862 \\
4083.006 \\
4083.380 \\
4083.467\end{array}$ & $\begin{array}{r}30 \\
25 \\
4 \\
4 \\
500\end{array}$ & $\begin{array}{l}8 \\
3\end{array}$ & $\begin{array}{l}\text { I } \\
\text { I } \\
\text { I } \\
\text { I I } \\
\text { I }\end{array}$ & $\begin{array}{l}4094.747 \mathrm{i} \\
4095.200 \\
4095.618 \\
4096.072 \\
4096.324\end{array}$ & $\begin{array}{r}1600 \\
8 \\
4 \\
200 \\
25\end{array}$ & 300 & $\begin{array}{l}\text { II } \\
\text { I } \\
\text { I } \\
\text { I } \\
\text { II }\end{array}$ & $\begin{array}{l}4107.291 \\
4107.373 \\
4107.436 \\
4107.795 \\
4107.859\end{array}$ & $\begin{array}{r}75 \\
30 \\
8 \\
25 \\
100\end{array}$ & $\begin{array}{r}2 \\
20 \\
20\end{array}$ & $\begin{array}{l}\text { I } \\
\text { I I } \\
\text { I } \\
\text { I I } \\
\text { I }\end{array}$ \\
\hline $\begin{array}{l}4083.818 \\
4084.084 \\
4084.289 \\
4084.480 \\
4084.618\end{array}$ & $\begin{array}{r}15 \\
25 \\
100 \\
5 \\
40\end{array}$ & 2 & $\begin{array}{l}\text { I } \\
\text { I } \\
\text { I } \\
\text { I I } \\
\text { I }\end{array}$ & $\begin{array}{l}4096.676 \\
4096.817 \\
4096.927 \\
4097.327 \\
4097.680\end{array}$ & $\begin{array}{r}8 \\
8 \\
15 \\
75 \\
2\end{array}$ & $\begin{array}{r}20 \\
8\end{array}$ & $\begin{array}{l}\text { I } \\
\text { I } \\
\text { I } \\
\text { I I } \\
\text { I I }\end{array}$ & $\begin{array}{l}4108.420 \mathrm{i} \\
4108.964 \\
4109.323 \\
4109.713 \\
4110.071\end{array}$ & $\begin{array}{l}800 \\
4 \mathrm{w} \\
400 \\
15 \\
15\end{array}$ & $\begin{array}{r}300 \\
6 \\
4\end{array}$ & $\begin{array}{l}\text { I I } \\
\text { I } \\
\text { I } \\
\text { I } \\
\text { I I }\end{array}$ \\
\hline $\begin{array}{l}4085.041 \\
4085.254 \\
4085.432 \\
4085.809 \\
4086.042\end{array}$ & $\begin{array}{r}300 \\
200 \\
600 \\
15 \\
25\end{array}$ & $\begin{array}{r}500 \\
10 \\
10 \\
15\end{array}$ & $\begin{array}{l}\text { I I } \\
\text { I } \\
\text { I } \\
\text { II } \\
\text { I }\end{array}$ & $\begin{array}{l}4097.742 \\
4098.084 \\
4098.398 \\
4098.614 \\
4098.728\end{array}$ & $\begin{array}{r}800 \\
4 \\
15 \\
8 \\
50\end{array}$ & $\begin{array}{l}4 \\
2 \\
1 \\
1\end{array}$ & $\begin{array}{l}\text { I } \\
\text { I I } \\
\text { I } \\
\text { I } \\
\text { I }\end{array}$ & $\begin{array}{l}4110.165 \\
4110.509 \\
4110.638 \\
4110.826 \\
4110.861\end{array}$ & $\begin{array}{r}4 \\
40 \\
75 \\
400 \\
200\end{array}$ & $\begin{array}{r}100 \\
75 \\
150\end{array}$ & $\begin{array}{l}\text { I } \\
\text { I I } \\
\text { I I } \\
\text { I } \\
\text { I I }\end{array}$ \\
\hline $\begin{array}{l}4086.521 \text { i } \\
4086.676 \\
4086.925 \\
4087.167 \\
4087.284\end{array}$ & $\begin{array}{c}1600 \\
20 \mathrm{w} \\
5 \\
15 \\
350\end{array}$ & $\begin{array}{l}300 \\
30 w\end{array}$ & $\begin{array}{l}\text { I I } \\
\text { I I } \\
\text { I } \\
\text { I } \\
\text { I }\end{array}$ & $\begin{array}{l}4098.936 \\
4099.209 \\
4099.308 \\
4099.552 \\
4099.992\end{array}$ & $\begin{array}{l}125 \\
15 \mathrm{w} \\
8 \\
125 \\
30\end{array}$ & $\begin{array}{r}100 \\
1 \\
2\end{array}$ & $\begin{array}{l}\text { I I } \\
\text { I } \\
\text { I } \\
\text { I } \\
\text { I }\end{array}$ & $\begin{array}{l}4111.129 \\
4111.436 \\
4111.609 \\
4111.827 \\
4112.101\end{array}$ & $\begin{array}{r}25 \\
8 \\
15 \\
4 \\
25\end{array}$ & $\begin{array}{l}6 \\
1 \\
1\end{array}$ & $\begin{array}{l}\text { I I } \\
\text { I I } \\
\text { I } \\
\text { I I } \\
\text { I }\end{array}$ \\
\hline $\begin{array}{l}4087.674 \\
4087.890 \\
4088.200 \\
4088.627 \\
4086.725\end{array}$ & $\begin{array}{c}5 \\
8 \\
5 \mathrm{w} \\
40 \\
400\end{array}$ & 3 & $\begin{array}{l}\text { I } \\
\text { I } \\
\text { I } \\
\text { I } \\
\text { I }\end{array}$ & $\begin{array}{l}4100.341 \mathrm{i} \\
4100.836 \\
4100.911 \\
4101.240 \\
4101.323\end{array}$ & $\begin{array}{r}1100 \\
25 \\
50 \\
15 \\
10\end{array}$ & $\begin{array}{r}15 \\
150\end{array}$ & $\begin{array}{l}\text { I } \\
\text { I I } \\
\text { I } \\
\text { I } \\
\text { II }\end{array}$ & $\begin{array}{l}4112.188 \\
4112.314 \\
4112.388 \\
4112.748 \\
4113.554\end{array}$ & $\begin{array}{r}8 \\
30 \\
15 \\
1700 \\
40\end{array}$ & $\begin{array}{l}50 \\
40 \\
50\end{array}$ & $\begin{array}{l}\text { I } \\
\text { II } \\
\text { I } \\
\text { I } \\
\text { II }\end{array}$ \\
\hline $\begin{array}{l}4089.138 \\
4089.624 \\
4090.036 \\
4090.099 \\
4090.195\end{array}$ & $\begin{array}{r}400 \\
8 \\
8 \\
4 \\
8\end{array}$ & 3 & $\begin{array}{l}\text { I } \\
\text { I } \\
\text { I } \\
\text { I } \\
\text { I }\end{array}$ & $\begin{array}{l}4101.620 \\
4102.290 \\
4102.617 \\
4102.819 \\
4103.005\end{array}$ & $\begin{array}{r}4 \\
25 \\
400 \\
30 \\
15\end{array}$ & 3 & $\begin{array}{l}\text { I } \\
\text { I } \\
\text { I } \\
\text { I } \\
\text { I }\end{array}$ & $\begin{array}{l}4113.869 \\
4114.050 \\
4115.140 \\
4115.262 \\
4115.539\end{array}$ & $\begin{array}{l}30 \\
15 \\
15 \mathrm{w} \\
4 \\
15\end{array}$ & $\begin{array}{l}1 \\
2 \\
8\end{array}$ & $\begin{array}{l}\text { I } \\
\text { I } \\
\text { I } \\
\text { I } \\
\text { I I }\end{array}$ \\
\hline
\end{tabular}


Table 1. Wavelengths of Thorium Spectra - Continued

\begin{tabular}{|c|c|c|c|c|c|c|c|c|c|c|c|}
\hline \multirow{2}{*}{$\begin{array}{l}\text { Wave- } \\
\text { length }\end{array}$} & \multicolumn{2}{|c|}{ Intensity } & \multirow{2}{*}{$\begin{array}{l}\text { Spec- } \\
\text { trum }\end{array}$} & \multirow{2}{*}{$\begin{array}{l}\text { Wave- } \\
\text { length }\end{array}$} & \multicolumn{2}{|c|}{ Intensity } & \multirow{2}{*}{$\begin{array}{l}\text { Spec- } \\
\text { trum }\end{array}$} & \multirow{2}{*}{$\begin{array}{l}\text { Wave- } \\
\text { length }\end{array}$} & \multicolumn{2}{|c|}{ Intensity } & \multirow{2}{*}{$\begin{array}{l}\text { Spec- } \\
\text { trum }\end{array}$} \\
\hline & Lamp & Spark & & & Lamp & Spark & & & Lamp & Spark & \\
\hline $\begin{array}{l}4115.759 \mathrm{i} \\
4116.714 \\
4117.703 \\
4117.838 \\
4118.087\end{array}$ & $\begin{array}{r}800 \\
1600 \\
15 \\
15 \\
8\end{array}$ & $\begin{array}{r}5 \\
500 \\
2 \\
3\end{array}$ & $\begin{array}{l}\text { I } \\
\text { I I } \\
\text { I I } \\
\text { I I } \\
\text { I }\end{array}$ & $\begin{array}{l}4131.001 \\
4131.423 \\
4131.656 \\
4131.710 \\
4132.274\end{array}$ & $\begin{array}{r}800 \\
75 \\
40 \\
50 \\
15\end{array}$ & $\begin{array}{r}15 \\
100\end{array}$ & $\begin{array}{l}\text { I } \\
\text { I I } \\
\text { I } \\
\text { I } \\
\text { I }\end{array}$ & $\begin{array}{l}4143.039 \\
4143.183 \\
4143.332 \\
4143.646 \\
4144.023\end{array}$ & $\begin{array}{r}15 \\
8 \\
25 \\
75 \\
15\end{array}$ & $\begin{array}{l}1 \\
1\end{array}$ & $\begin{array}{l}\text { I } \\
\text { I } \\
\text { I } \\
\text { I } \\
\text { I }\end{array}$ \\
\hline $\begin{array}{l}4118.218 \\
4118.325 \\
4118.483 \\
4118.595 \\
4118.773\end{array}$ & $\begin{array}{r}15 \\
40 \\
200 \\
25 \\
25\end{array}$ & $\begin{array}{l}2 \\
1 \\
4 \\
1\end{array}$ & $\begin{array}{l}\text { I I } \\
\text { I } \\
\text { I } \\
\text { II } \\
\text { I }\end{array}$ & $\begin{array}{l}4132.417 \\
4132.608 \\
4132.753 \mathrm{i} \\
4133.285 \\
4133.602\end{array}$ & $\begin{array}{r}4 \\
40 \\
600 \\
15 \\
15\end{array}$ & 200 & $\begin{array}{l}\text { I } \\
\text { I } \\
\text { I I } \\
\text { I } \\
\text { I }\end{array}$ & $\begin{array}{l}4144.335 \\
4144.448 \\
4144.573 \\
4145.041 \\
4145.292\end{array}$ & $\begin{array}{l}40 \\
15 \\
40 \\
15 \\
30\end{array}$ & $\begin{array}{l}1 \\
1\end{array}$ & $\begin{array}{l}\text { I } \\
\text { I } \\
\text { I } \\
\text { I } \\
\text { I }\end{array}$ \\
\hline $\begin{array}{l}4118.940 \\
4119.390 \\
4119.469 \\
4120.007 \\
4120.367\end{array}$ & $\begin{array}{r}4 \\
4 \\
30 \\
15 \\
15\end{array}$ & $3 w$ & $\begin{array}{l}\text { I } \\
\text { I } \\
\text { I } \\
\text { I I } \\
\text { I }\end{array}$ & $\begin{array}{l}4133.953 \\
4134.055 \\
4134.111 \\
4134.318 \\
4134.607\end{array}$ & $\begin{array}{c}75 \\
75 \\
100 \\
75 \\
15 \mathrm{w}\end{array}$ & $\begin{array}{r}100 \\
2\end{array}$ & $\begin{array}{l}\text { I } \\
\text { I } \\
\text { I I } \\
\text { I } \\
\text { I }\end{array}$ & $\begin{array}{l}4145.838 \\
4145.918 \\
4145.984 \\
4146.063 \\
4146.497\end{array}$ & $\begin{array}{r}50 \\
15 \\
8 \\
8 \\
4\end{array}$ & 2 & $\begin{array}{l}\text { I } \\
\text { I } \\
I \\
I \\
I\end{array}$ \\
\hline $\begin{array}{l}4120.405 \\
4120.831 \\
4121.854 \\
4122.674 \\
4122.962\end{array}$ & $\begin{array}{r}15 \\
8 \\
30 \\
125 \\
50\end{array}$ & $\begin{array}{l}30 \\
50 \\
20\end{array}$ & $\begin{array}{l}\text { I } \\
\text { I } \\
\text { II } \\
\text { I I } \\
\text { I I }\end{array}$ & $\begin{array}{l}4134.828 \\
4134.982 \\
4135.132 \\
4135.286 \\
4135.479\end{array}$ & $\begin{array}{r}4 \\
8 \\
8 \\
4 \\
200\end{array}$ & $1 \mathrm{w}$ & $\begin{array}{l}\text { I } \\
\text { I I } \\
\text { I } \\
\text { I } \\
\text { I }\end{array}$ & $\begin{array}{l}4146.658 \\
4146.964 \\
4147.535 \\
4148.017 \\
4148.169\end{array}$ & $\begin{array}{r}15 \\
25 \\
75 \\
20 \\
200\end{array}$ & $\begin{array}{r}5 \\
1 \\
5 \\
250\end{array}$ & $\begin{array}{l}\text { I I } \\
\text { I } \\
\text { I } \\
\text { I I } \\
\text { I I }\end{array}$ \\
\hline $\begin{array}{l}4123.263 \\
4123.531 \\
4123.597 \\
4123.719 \\
4124.036\end{array}$ & $\begin{array}{r}8 \\
75 \\
40 \\
100 \\
150\end{array}$ & $\begin{array}{r}2 \\
15\end{array}$ & $\begin{array}{l}\text { I I } \\
\text { I I } \\
\text { I } \\
\text { I } \\
\text { I }\end{array}$ & $\begin{array}{l}4135.934 \\
4136.128 \\
4136.283 \\
4136.401 \\
4136.433\end{array}$ & $\begin{array}{r}8 \\
30 \\
125 \\
50 \\
100\end{array}$ & $\begin{array}{r}1 \\
5 \\
50\end{array}$ & $\begin{array}{l}\text { I } \\
\text { I } \\
\text { I } \\
\text { I I } \\
\text { I }\end{array}$ & $\begin{array}{l}4148.344 \\
4148.645 \\
4148.720 \\
4148.828 \\
4149.203\end{array}$ & $\begin{array}{l}50 \\
30 \mathrm{~h} \\
50 \\
40 \\
200\end{array}$ & $\begin{array}{r}100 \\
3 \\
2\end{array}$ & $\begin{array}{l}\text { I I } \\
\text { I I } \\
\text { I } \\
\text { I } \\
\text { I }\end{array}$ \\
\hline $\begin{array}{l}4124.511 \\
4124.664 \\
4124.772 \\
4124.875 \\
4125.462\end{array}$ & $\begin{array}{r}30 \\
40 \\
30 \\
8 \\
4\end{array}$ & $\begin{array}{l}50 \\
50\end{array}$ & $\begin{array}{l}\text { I I } \\
\text { I I } \\
\text { I } \\
\text { I } \\
\text { I }\end{array}$ & $\begin{array}{l}4136.882 \\
4137.013 \\
4137.117 \\
4137.303 \\
4137.607\end{array}$ & $\begin{array}{l}4 \\
8 \\
8 \\
8 w \\
8\end{array}$ & $2 w$ & $\begin{array}{l}\text { I } \\
\text { I } \\
\text { I } \\
\text { I } \\
\text { I I }\end{array}$ & $\begin{array}{l}4149.987 \mathrm{i} \\
4150.072 \\
4150.365 \\
4150.478 \\
4150.590\end{array}$ & $\begin{array}{r}800 \\
40 \\
25 \\
15 \\
15\end{array}$ & $\begin{array}{r}200 \\
4 \\
1\end{array}$ & $\begin{array}{l}\text { II } \\
\text { I } \\
\text { I I } \\
\text { I } \\
\text { I }\end{array}$ \\
\hline $\begin{array}{l}4125.695 \\
4125.958 \\
4126.029 \\
4126.346 \\
4126.753\end{array}$ & $\begin{array}{l}30 \\
15 \\
25 \\
30 \\
25\end{array}$ & 1 & $\begin{array}{l}\text { I } \\
\text { I } \\
\text { I } \\
\text { I } \\
\text { I }\end{array}$ & $\begin{array}{l}4137.880 \\
4138.038 \\
4138.291 \\
4138.765 \\
4138.841\end{array}$ & $\begin{array}{r}8 \\
150 \\
8 \\
30 \\
40\end{array}$ & $\begin{array}{r}4 \\
2 \\
1 \\
15\end{array}$ & $\begin{array}{l}\text { I I } \\
\text { I } \\
\text { I } \\
\text { I } \\
\text { I I }\end{array}$ & $\begin{array}{l}4150.656 \\
4151.141 \\
4151.445 \\
4151.641 \\
4152.139\end{array}$ & $\begin{array}{r}25 \\
40 \\
15 \\
8 \\
15\end{array}$ & $\begin{array}{l}1 \\
4\end{array}$ & $\begin{array}{l}\text { I } \\
\text { I } \\
\text { I I } \\
\text { I } \\
\text { I }\end{array}$ \\
\hline $\begin{array}{l}4127.412 \mathrm{i} \\
4128.043 \\
4128.296 \\
4128.471 \\
4128.608\end{array}$ & $\begin{array}{r}1000 \\
50 \\
8 \\
8 \\
4\end{array}$ & $\begin{array}{r}50 \\
1 \\
4 \\
1\end{array}$ & $\begin{array}{l}\text { I } \\
\text { I } \\
\text { II } \\
\text { I } \\
\text { I }\end{array}$ & $\begin{array}{l}4138.880 \\
4139.307 \\
4139.462 \\
4139.610 \\
4139.756\end{array}$ & $\begin{array}{l}30 \\
15 \mathrm{~h} \\
25 \\
4 \\
8\end{array}$ & 1 & $\begin{array}{l}\text { I } \\
\text { I } \\
I \\
I \\
I\end{array}$ & $\begin{array}{l}4152.203 \\
4153.153 \\
4153.207 \\
4153.576 \\
4153.733\end{array}$ & $\begin{array}{r}25 \\
25 \\
8 \\
25 \\
15\end{array}$ & 4 & $\begin{array}{l}\text { I } \\
\text { I } \\
\text { I } \\
\text { II }\end{array}$ \\
\hline $\begin{array}{l}4128.813 \\
4128.912 \\
4129.071 \\
4129.511 \\
4129.851\end{array}$ & $\begin{array}{c}2 w \\
4 \\
4 \\
100 \\
25\end{array}$ & 2 & $\begin{array}{l}\text { I } \\
\text { I } \\
\text { I } \\
\text { I } \\
\text { I }\end{array}$ & $\begin{array}{l}4140.232 \\
4140.383 \\
4141.108 \\
4141.326 \\
4141.634\end{array}$ & $\begin{array}{r}400 \\
200 \\
4 \\
15 \\
100\end{array}$ & $\begin{array}{r}150 \\
75\end{array}$ & $\begin{array}{l}\text { I I } \\
\text { I I } \\
\text { I } \\
\text { I } \\
\text { I I }\end{array}$ & $\begin{array}{l}4153.861 \\
4153.995 \\
4154.130 \\
4154.374 \\
4154.717\end{array}$ & $\begin{array}{r}15 \\
8 \\
8 \\
25 \\
100\end{array}$ & $\begin{array}{l}1 \\
1 \\
2\end{array}$ & $\begin{array}{l}\text { I } \\
\text { I } \\
\text { I } \\
\text { I } \\
\text { I }\end{array}$ \\
\hline $\begin{array}{l}4129.914 \\
4130.132 \\
4130.170 \\
4130.326 \\
4130.668\end{array}$ & $\begin{array}{r}15 \\
8 \\
8 \\
200 \\
15\end{array}$ & $\begin{array}{l}3 \\
3\end{array}$ & $\begin{array}{l}\text { I I } \\
\text { I } \\
\text { I } \\
\text { I } \\
\text { I I }\end{array}$ & $\begin{array}{l}4141.857 \\
4142.326 \\
4142.475 \\
4142.702 \\
4142.966\end{array}$ & $\begin{array}{r}15 \\
50 \\
100 \\
600 \\
25\end{array}$ & $\begin{array}{r}3 \\
150 \\
200 \\
2\end{array}$ & $\begin{array}{l}\text { I } \\
\text { I } \\
\text { I I } \\
\text { I I } \\
\text { I }\end{array}$ & $\begin{array}{l}4155.004 \\
4155.166 \\
4155.295 \\
4155.473 \\
4155.639\end{array}$ & $\begin{array}{r}15 \\
4 \\
5 \\
5 \\
8\end{array}$ & $\begin{array}{l}4 w \\
2 w\end{array}$ & $\begin{array}{l}\text { I } \\
\text { I } \\
\text { I I } \\
\text { I I } \\
\text { I }\end{array}$ \\
\hline
\end{tabular}


Table 1. Wavelengths of Thorium Spectra - Continued

\begin{tabular}{|c|c|c|c|c|c|c|c|c|c|c|c|}
\hline \multirow{2}{*}{$\begin{array}{l}\text { Wave-- } \\
\text { length }\end{array}$} & \multicolumn{2}{|c|}{ Intensity } & \multirow{2}{*}{$\begin{array}{l}\text { Spec- } \\
\text { trum }\end{array}$} & \multirow{2}{*}{$\begin{array}{l}\text { Wave- } \\
\text { length }\end{array}$} & \multicolumn{2}{|c|}{ Intensity } & \multirow{2}{*}{$\begin{array}{l}\text { Spec- } \\
\text { trum }\end{array}$} & \multirow{2}{*}{$\begin{array}{l}\text { Wave- } \\
\text { length }\end{array}$} & \multicolumn{2}{|c|}{ Intensity } & \multirow{2}{*}{$\begin{array}{l}\text { Spec } \\
\text { trum }\end{array}$} \\
\hline & Lamp & Spark & & & Lamp & & & & & Spark & \\
\hline $\begin{array}{l}4155.725 \\
4155.986 \\
4156.233 \\
4156.521 \\
4157.272\end{array}$ & $\begin{array}{r}8 \\
15 \\
125 \\
400 \\
100\end{array}$ & $\begin{array}{r}75 \\
200 \\
2\end{array}$ & $\begin{array}{l}\text { I } \\
\text { I } \\
\text { I I } \\
\text { I I } \\
\text { I }\end{array}$ & $\begin{array}{l}4168.633 \\
4169.082 \\
4169.213 \\
4169.758 \\
4169.827\end{array}$ & $\begin{array}{l}150 \\
50 \\
75 \\
25 \\
75 \mathrm{~h}\end{array}$ & 100 & $\begin{array}{l}\text { II } \\
\text { I } \\
\text { I } \\
\text { I } \\
\text { I }\end{array}$ & $\begin{array}{l}4182.158 \\
4182.443 \\
4182.709 \\
4182.935 \\
4183.562\end{array}$ & $\begin{array}{l}75 \\
15 \\
50 \mathrm{w} \\
150 \\
50\end{array}$ & 100 & $\begin{array}{l}\text { I I } \\
\text { I } \\
\text { I } \\
\text { I } \\
\text { I I }\end{array}$ \\
\hline $\begin{array}{l}4157.391 \\
4157.541 \\
4157.760 \\
4157.939 \\
4158.095\end{array}$ & $\begin{array}{l}100 \\
25 \\
15 \mathrm{w} \\
8 \\
8\end{array}$ & $\begin{array}{l}1 \\
1\end{array}$ & $\begin{array}{l}\text { I } \\
\text { I } \\
\text { I } \\
\text { I } \\
\text { I }\end{array}$ & $\begin{array}{l}4170.468 \\
4170.536 \\
4170.782 \\
4171.342 \\
4171.970\end{array}$ & $\begin{array}{r}125 \\
500 \\
100 \\
125 \\
25\end{array}$ & $\begin{array}{r}25 \\
10 \\
30 \\
125\end{array}$ & $\begin{array}{l}\text { I I } \\
\text { I } \\
\text { II } \\
\text { I I } \\
\text { I }\end{array}$ & $\begin{array}{l}4183.948 \\
4184.135 \\
4184.277 \\
4184.603 \\
4184.709\end{array}$ & $\begin{array}{r}50 \\
500 \\
100 \\
250 \\
40\end{array}$ & $\begin{array}{r}1 \\
1 \\
8 \\
12\end{array}$ & $\begin{array}{l}\text { I } \\
\text { I } \\
\text { II } \\
\text { I } \\
\text { I I }\end{array}$ \\
\hline $\begin{array}{l}4158.535 \mathrm{i} \\
4159.150 \\
4159.403 \\
4159.659 \\
4160.057\end{array}$ & $\begin{array}{r}800 \\
40 \\
15 \\
500 \\
50\end{array}$ & $\begin{array}{r}15 \\
100\end{array}$ & $\begin{array}{l}\text { I } \\
\text { I } \\
\text { I } \\
\text { II } \\
\text { I }\end{array}$ & $\begin{array}{l}4172.050 \\
4172.203 \\
4172.383 \\
4172.625 \\
4173.304\end{array}$ & $\begin{array}{r}25 \\
8 \\
40 \\
200 \\
15\end{array}$ & & $\begin{array}{l}\text { I } \\
\text { I } \\
\text { I } \\
\text { I } \\
\text { I }\end{array}$ & $\begin{array}{l}4185.042 \\
4185.143 \\
4185.296 \\
4185.471 \\
4185.809\end{array}$ & $\begin{array}{r}30 \\
250 \\
75 \\
200 \\
15\end{array}$ & & $\begin{array}{l}\text { I } \\
\text { I } \\
\text { I } \\
\text { I } \\
\text { I }\end{array}$ \\
\hline $\begin{array}{l}4160.286 \\
4160.351 \\
4160.544 \\
4160.732 \\
4161.108\end{array}$ & $\begin{array}{r}15 \\
50 \\
25 \\
150 \\
70\end{array}$ & $\begin{array}{l}1 \\
2\end{array}$ & $\begin{array}{l}\text { I } \\
\text { I } \\
\text { I } \\
\text { I } \\
\text { I }\end{array}$ & $\begin{array}{l}4173.449 \\
4173.525 \\
4173.620 \\
4173.720 \\
4173.885\end{array}$ & $\begin{array}{r}100 \\
75 \\
4 \\
75 \\
50\end{array}$ & $\begin{array}{l}1 \\
3 \\
1\end{array}$ & $\begin{array}{l}\text { I } \\
\text { I } \\
\text { I } \\
\text { I } \\
\text { I }\end{array}$ & $\begin{array}{l}4185.947 \\
4187.136 \\
4187.308 \\
4188.103 \\
4188.188\end{array}$ & $\begin{array}{r}40 \\
250 \\
25 \\
50 \\
40\end{array}$ & $\begin{array}{l}5 \\
1 \\
2\end{array}$ & $\begin{array}{l}\text { I } \\
\text { I } \\
\text { I } \\
\text { I I } \\
\text { I }\end{array}$ \\
\hline $\begin{array}{l}4161.586 \\
4161.738 \\
4161.887 \\
4162.026 \\
4162.507\end{array}$ & $\begin{array}{r}125 \\
300 \\
75 \\
15 \\
400\end{array}$ & $\begin{array}{l}8 \\
2 \\
4 \\
1 \\
2\end{array}$ & $\begin{array}{l}\text { II } \\
\text { I } \\
\text { II } \\
\text { I } \\
\text { I }\end{array}$ & $\begin{array}{l}4173.953 \\
4174.055 \\
4174.436 \\
4174.650 \\
4174.764\end{array}$ & $\begin{array}{r}40 \\
25 \\
30 \\
30 \\
8\end{array}$ & $\begin{array}{l}2 \\
6\end{array}$ & $\begin{array}{l}\text { I I } \\
\text { I } \\
\text { I I } \\
\text { I } \\
\text { I }\end{array}$ & $\begin{array}{l}4188.581 \\
4188.664 \\
4188.815 \\
4189.562 \\
4189.631\end{array}$ & $\begin{array}{l}75 \\
150 \\
25 \\
350 \mathrm{w} \\
50 \mathrm{w}\end{array}$ & 10 & $\begin{array}{l}\text { I I } \\
\text { I } \\
\text { I } \\
\text { I } \\
\text { I }\end{array}$ \\
\hline $\begin{array}{l}4162.678 \\
4162.775 \\
4163.177 \\
4163.644 \\
4163.729\end{array}$ & $\begin{array}{l}100 \\
150 \mathrm{~h} \\
200 \\
400 \\
200\end{array}$ & $\begin{array}{l}75 \\
20 \\
50 \\
301\end{array}$ & $\begin{array}{l}\text { I I } \\
\text { I } \\
\text { II } \\
\text { I I } \\
\text { I I }\end{array}$ & $\begin{array}{l}4175.320 \\
4175.397 \\
4176.018 \\
4176.331 \\
4176.481\end{array}$ & $\begin{array}{l}30 \\
15 \\
8 \mathrm{w} \\
30 \\
50\end{array}$ & $\begin{array}{r}1 \\
15\end{array}$ & $\begin{array}{l}\text { I } \\
\text { I } \\
\text { I } \\
\text { II } \\
\text { I }\end{array}$ & $\begin{array}{l}4189.726 \\
4189.817 \\
4190.465 \\
4190.623 \\
4190.813\end{array}$ & $\begin{array}{r}30 \\
40 \\
15 \\
150 \\
100\end{array}$ & 1 & $\begin{array}{l}\text { I } \\
\text { I I } \\
\text { I } \\
\text { I } \\
\text { I }\end{array}$ \\
\hline $\begin{array}{l}4163.944 \\
4164.252 \\
4164.394 \\
4164.452 \\
4164.529\end{array}$ & $\begin{array}{l}150 \\
800 \\
15 \\
25 \\
40\end{array}$ & 50 & $\begin{array}{l}\text { I } \\
\text { I I } \\
\text { I } \\
\text { I } \\
\text { I }\end{array}$ & $\begin{array}{l}4176.681 \\
4177.162 \\
4177.727 \\
4178.060 \mathrm{i} \\
4178.845\end{array}$ & $\begin{array}{c}15 \mathrm{w} \\
100 \\
50 \mathrm{w} \\
3000 \\
250\end{array}$ & $\begin{array}{r}1 \\
1 \\
400 \\
1\end{array}$ & $\begin{array}{l}\text { II } \\
\text { I } \\
\text { I } \\
\text { II } \\
\text { I }\end{array}$ & $\begin{array}{l}4191.022 \\
4191.148 \\
4191.820 \\
4191.993 \\
4192.362\end{array}$ & $\begin{array}{r}10 \\
100 \\
150 \\
30 \\
300\end{array}$ & $\begin{array}{r}30 \\
3 \\
2\end{array}$ & $\begin{array}{l}\text { I } \\
\text { I } \\
\text { I I } \\
\text { I I } \\
\text { I }\end{array}$ \\
\hline $\begin{array}{l}4164.685 \\
4164.973 \\
4165.071 \\
4165.340 \\
4165.766 \mathrm{i}\end{array}$ & $\begin{array}{r}25 \\
150 \\
250 \\
15 \\
1000\end{array}$ & $\begin{array}{l}15 \\
12\end{array}$ & $\begin{array}{l}\text { I } \\
\text { I } \\
\text { I } \\
\text { I } \\
\text { I }\end{array}$ & $\begin{array}{l}4179.218 \\
4179.308 \\
4179.618 \\
4179.711 \\
4179.956\end{array}$ & $\begin{array}{l}150 \\
300 \\
250 \\
125 \\
150\end{array}$ & $\begin{array}{r}1 \\
1 \\
100 \\
100\end{array}$ & $\begin{array}{l}\text { I } \\
\text { I } \\
\text { I } \\
\text { I I } \\
\text { I I }\end{array}$ & $\begin{array}{l}4193.017 \mathrm{i} \\
4193.518 \\
4194.084 \\
4194.218 \\
4194.582\end{array}$ & $\begin{array}{r}900 \\
150 \\
100 \\
15 \\
30\end{array}$ & $\begin{array}{r}8 \\
1 \\
20\end{array}$ & $\begin{array}{l}\text { I } \\
\text { I } \\
\text { I I } \\
\text { I } \\
\text { I }\end{array}$ \\
\hline $\begin{array}{l}4166.492 \\
4166.683 \\
4166.903 \\
4167.117 \\
4167.269\end{array}$ & $\begin{array}{r}15 \\
8 \\
15 \\
40 \\
25\end{array}$ & 8 & $\begin{array}{l}\text { I I } \\
\text { I } \\
\text { I } \\
\text { I } \\
\text { I I }\end{array}$ & $\begin{array}{l}4180.326 \\
4180.487 \\
4180.556 \\
4180.716 \\
4180.885\end{array}$ & $\begin{array}{l}100 \\
50 \mathrm{w} \\
15 \\
75 \\
75\end{array}$ & 4 & $\begin{array}{l}\text { I } \\
\text { I } \\
\text { II } \\
\text { I } \\
\text { I }\end{array}$ & $\begin{array}{l}4194.934 \\
4195.560 \\
4195.831 \\
4195.952 \\
4196.287\end{array}$ & $\begin{array}{r}350 \\
500 \\
400 \\
100 \\
50\end{array}$ & $\begin{array}{r}2 \\
100 \\
50 \\
25\end{array}$ & $\begin{array}{l}\text { I } \\
\text { I I } \\
\text { I I } \\
\text { I I } \\
\text { I }\end{array}$ \\
\hline $\begin{array}{l}4167.390 \\
4167.680 \\
4167.862 \\
4168.052 \\
4168.457\end{array}$ & $\begin{array}{r}30 \\
8 \\
100 \\
200 \\
200\end{array}$ & $\begin{array}{l}1 \\
1\end{array}$ & $\begin{array}{l}\text { I } \\
\text { I } \\
\text { I } \\
\text { I } \\
\text { I }\end{array}$ & $\begin{array}{l}4180.954 \\
4181.083 \\
4181.163 \\
4181.344 \\
4181.503\end{array}$ & $\begin{array}{r}75 \\
20 \\
125 \\
15 \\
15\end{array}$ & $\begin{array}{l}2 \\
4 \\
4\end{array}$ & $\begin{array}{l}\text { I } \\
\text { I I } \\
\text { I } \\
\text { I I } \\
\text { I }\end{array}$ & $\begin{array}{l}4196.498 \\
4196.840 \\
4197.013 \\
4197.112 \\
4197.307\end{array}$ & $\begin{array}{l}30 \\
50 \mathrm{w} \\
25 \\
25 \\
15\end{array}$ & & $\begin{array}{l}\text { I } \\
\text { I } \\
\text { I } \\
\text { I } \\
\text { I }\end{array}$ \\
\hline
\end{tabular}


Table 1. Wavelengths of Thorium Spectra - Continued

\begin{tabular}{|c|c|c|c|c|c|c|c|c|c|c|c|}
\hline \multirow{2}{*}{$\begin{array}{l}\text { Wave- } \\
\text { length }\end{array}$} & \multicolumn{2}{|c|}{ Intensity } & \multirow{2}{*}{$\begin{array}{l}\text { Spec- } \\
\text { trum }\end{array}$} & \multirow{2}{*}{$\begin{array}{l}\text { Wave- } \\
\text { length }\end{array}$} & \multicolumn{2}{|c|}{ Intensity } & \multirow{2}{*}{$\begin{array}{l}\text { Spec- } \\
\text { trum }\end{array}$} & \multirow{2}{*}{$\begin{array}{l}\text { Wave- } \\
\text { length }\end{array}$} & \multicolumn{2}{|c|}{ Intensity } & \multirow{2}{*}{$\begin{array}{l}\text { Spec } \\
\text { trum }\end{array}$} \\
\hline & & Spark & & & Lamp & Spark & & & Lamp & Spark & \\
\hline $\begin{array}{l}4197.380 \\
4197.661 \\
4197.723 \\
4197.756 \\
4197.936\end{array}$ & $\begin{array}{r}40 \\
40 \\
10 \\
40 \\
100\end{array}$ & $\begin{array}{r}2 \\
10\end{array}$ & $\begin{array}{l}\text { I } \\
\text { I } \\
\text { II } \\
\text { I } \\
\text { II }\end{array}$ & $\begin{array}{l}4210.298 \\
4210.451 \\
4210.770 \\
4210.924 \\
4211.294\end{array}$ & $\begin{array}{c}5 \\
100 \\
400 \\
1200 \\
15 \mathrm{~h}\end{array}$ & $\begin{array}{r}1 \\
6 \\
10\end{array}$ & $\begin{array}{l}\text { I } \\
\text { I } \\
\text { I } \\
\text { I } \\
\text { I }\end{array}$ & $\begin{array}{l}4223.598 \\
4223.981 \\
4224.246 \\
4224.622 \\
4224.872\end{array}$ & $\begin{array}{r}300 \\
30 \\
200 \\
150 \\
15\end{array}$ & $\begin{array}{r}2 \\
50 \\
20\end{array}$ & $\begin{array}{l}\text { I } \\
\text { I } \\
\text { II } \\
\text { II } \\
\text { I }\end{array}$ \\
\hline $\begin{array}{l}4198.105 \\
4198.653 \\
4198.879 \\
4198.939 \\
4199.020\end{array}$ & $\begin{array}{r}75 \\
100 \\
50 \\
50 \\
40\end{array}$ & 30 & $\begin{array}{l}\text { I } \\
\text { I } \\
\text { I } \\
\text { I } \\
\text { II }\end{array}$ & $\begin{array}{l}4211.513 \\
4211.887 \\
4212.314 \\
4212.454 \\
4213.072\end{array}$ & $\begin{array}{l}100 \\
100 \\
5 \mathrm{w} \\
5 \\
400\end{array}$ & $\begin{array}{r}30 \\
3\end{array}$ & $\begin{array}{l}\text { II } \\
\text { I } \\
\text { I } \\
\text { I } \\
\text { I }\end{array}$ & $\begin{array}{l}4225.102 \\
4225.517 \\
4225.805 \\
4226.049 \\
4226.305\end{array}$ & $\begin{array}{r}10 \\
150 \\
50 \\
50 \\
150\end{array}$ & & $\begin{array}{l}\text { I } \\
\text { I } \\
\text { I } \\
\text { I } \\
\text { I }\end{array}$ \\
\hline $\begin{array}{l}4199.430 \\
4199.632 \\
4200.008 \\
4200.158 \\
4200.392\end{array}$ & $\begin{array}{l}15 \mathrm{w} \\
150 \\
30 \\
15 \\
15 \mathrm{w}\end{array}$ & 1 & $\begin{array}{l}\text { I } \\
\text { I } \\
\text { I } \\
\text { I } \\
\text { I }\end{array}$ & $\begin{array}{l}4213.373 \\
4213.993 \\
4214.539 \\
4214.628 \\
4214.828 \mathrm{i}\end{array}$ & $\begin{array}{r}300 \\
75 \\
50 \\
30 \\
200\end{array}$ & $\begin{array}{r}2 \\
10 \\
7 \\
2\end{array}$ & $\begin{array}{l}\text { I I } \\
\text { I } \\
\text { II } \\
\text { II } \\
\text { I }\end{array}$ & $\begin{array}{l}4226.780 \\
4227.392 \\
4227.650 \\
4228.417 \\
4228.682\end{array}$ & $\begin{array}{r}200 \\
400 \\
100 \\
150 \\
75\end{array}$ & $\begin{array}{l}2 \\
3 \\
2 \\
1 \\
2\end{array}$ & $\begin{array}{l}\text { I } \\
\text { I } \\
\text { I } \\
\text { I } \\
\text { I }\end{array}$ \\
\hline $\begin{array}{l}4200.837 \\
4200.997 \\
4201.607 \\
4201.849 \\
4202.258\end{array}$ & $\begin{array}{r}20 \\
5 \\
100 \\
500 \\
150\end{array}$ & $\begin{array}{r}2 \\
2 \\
3 \\
100\end{array}$ & $\begin{array}{l}\text { I } \\
\text { I I } \\
\text { I } \\
\text { II } \\
\text { I }\end{array}$ & $\begin{array}{l}4215.145 \\
4215.208 \\
4215.374 \\
4215.630 \\
4215.853\end{array}$ & $\begin{array}{r}5 \\
10 \\
5 \\
100 \\
100\end{array}$ & 1 & $\begin{array}{l}\text { I } \\
\text { I } \\
\text { I } \\
\text { I } \\
\text { I }\end{array}$ & $\begin{array}{l}4228.763 \\
4228.990 \\
4229.149 \\
4229.454 \\
4229.768\end{array}$ & $\begin{array}{l}125 w \\
40 \\
500 \\
500 \\
15\end{array}$ & $\begin{array}{r}1 \\
3 \\
2 \\
50\end{array}$ & $\begin{array}{l}\text { I } \\
\text { II } \\
\text { I } \\
\text { II } \\
\text { I }\end{array}$ \\
\hline $\begin{array}{l}4203.060 \\
4203.086 \\
4203.179 \\
4203.292 \\
4203.437\end{array}$ & $\begin{array}{l}50 \\
40 \\
20 \\
20 \\
50\end{array}$ & & $\begin{array}{l}\text { I } \\
\text { I } \\
\text { I } \\
\text { I } \\
\text { I }\end{array}$ & $\begin{array}{l}4216.057 \\
4216.205 \\
4216.369 \\
4216.937 \\
4217.070\end{array}$ & $\begin{array}{l}300 \\
50 \\
150 \\
10 \mathrm{w} \\
30\end{array}$ & $\frac{1}{2}$ & $\begin{array}{l}\text { I } \\
\text { I } \\
\text { I } \\
\text { I } \\
\text { I }\end{array}$ & $\begin{array}{l}4229.863 \\
4229.954 \\
4230.239 \\
4230.427 \mathrm{i} \\
4230.822\end{array}$ & $\begin{array}{l}30 \\
20 \mathrm{w} \\
50 \\
600 \\
150\end{array}$ & $\begin{array}{l}1 \\
4\end{array}$ & $\begin{array}{l}\text { I } \\
\text { I } \\
\text { I } \\
\text { I } \\
\text { I }\end{array}$ \\
\hline $\begin{array}{l}4203.578 \\
4203.890 \\
4204.044 \\
4204.535 \\
4204.684\end{array}$ & $\begin{array}{r}75 \\
100 \\
300 \\
100 \\
20\end{array}$ & $\begin{array}{l}1 \\
2 \\
1\end{array}$ & $\begin{array}{l}\text { I } \\
\text { I } \\
\text { I } \\
\text { I } \\
\text { II }\end{array}$ & $\begin{array}{l}4217.192 \\
4217.227 \\
4217.756 \\
4217.948 \\
4218.192\end{array}$ & $\begin{array}{l}100 \mathrm{w} \\
100 \\
30 \\
10 \\
150\end{array}$ & $\begin{array}{l}10 \\
2 \\
8 w\end{array}$ & $\begin{array}{l}\text { I } \\
\text { II } \\
\text { II } \\
\text { I } \\
\text { I I }\end{array}$ & $\begin{array}{l}4230.963 \\
4231.076 \\
4231.171 \\
4231.751 \\
4231.976\end{array}$ & $\begin{array}{r}30 \\
20 \\
25 \\
100 \\
30\end{array}$ & $\begin{array}{l}5 w \\
2\end{array}$ & $\begin{array}{l}\text { I } \\
\text { I } \\
\text { II } \\
\text { I } \\
\text { I I }\end{array}$ \\
\hline $\begin{array}{l}4204.927 \\
4205.009 \\
4205.128 \\
4205.389 \\
4205.537\end{array}$ & $\begin{array}{l}15 \mathrm{~h} \\
30 \\
100 \\
30 \\
50 \mathrm{w}\end{array}$ & $2 w$ & $\begin{array}{l}\text { II } \\
\text { I } \\
\text { I } \\
\text { I } \\
\text { I }\end{array}$ & $\begin{array}{l}4218.541 \\
4219.000 \\
4219.379 \\
4219.577 \\
4219.680\end{array}$ & $\begin{array}{r}100 \\
20 \\
100 \\
20 \\
25\end{array}$ & $\begin{array}{r}20 \\
2 \\
10 \\
3\end{array}$ & $\begin{array}{l}\text { II } \\
\text { II } \\
\text { II } \\
\text { I } \\
\text { I }\end{array}$ & $\begin{array}{l}4232.533 \\
4232.657 \\
4232.707 \\
4233.293 \\
4233.726\end{array}$ & $\begin{array}{r}75 \\
15 \\
5 \\
300 \\
20\end{array}$ & 50 & $\begin{array}{l}\text { II } \\
\text { I } \\
\text { I } \\
\text { II } \\
\text { I }\end{array}$ \\
\hline $\begin{array}{l}4205.787 \\
4206.053 \\
4206.663 \\
4207.176 \\
4207.322\end{array}$ & $\begin{array}{r}30 \\
75 \\
150 \\
100 \\
5\end{array}$ & $\begin{array}{r}5 \\
50\end{array}$ & $\begin{array}{l}\text { II } \\
\text { I } \\
\text { I I } \\
\text { I } \\
\text { I }\end{array}$ & $\begin{array}{l}4220.075 \\
4220.400 \\
4220.564 \\
4220.733 \\
4220.856\end{array}$ & $\begin{array}{r}750 \\
10 \\
10 \\
150 \\
20\end{array}$ & 50 & $\begin{array}{l}\text { I } \\
\text { I } \\
I \\
I \\
I\end{array}$ & $\begin{array}{l}4233.902 \\
4234.231 \\
4234.302 \\
4234.489 \\
4234.988\end{array}$ & $\begin{array}{l}50 \\
50 \\
50 \\
10 \\
75\end{array}$ & 5 & $\begin{array}{l}\text { I } \\
\text { I } \\
\text { I } \\
\text { I } \\
\text { II }\end{array}$ \\
\hline $\begin{array}{l}4207.480 \\
4207.612 \\
4207.928 \\
4208.082 \\
4208.416\end{array}$ & $\begin{array}{r}4 \\
75 \\
15 \\
15 \\
150\end{array}$ & 2 & $\begin{array}{l}\text { I } \\
\text { I } \\
\text { I } \\
\text { I } \\
\text { I }\end{array}$ & $\begin{array}{l}4221.152 \\
4221.691 \\
4221.840 \\
4222.179 \\
4222.444\end{array}$ & $\begin{array}{l}75 \\
75 \\
5 \\
30 \\
75 \mathrm{w}\end{array}$ & $\begin{array}{l}1 \\
4 \\
1\end{array}$ & $\begin{array}{l}\text { I } \\
\text { I } \\
\text { I } \\
\text { I I } \\
\text { I }\end{array}$ & $\begin{array}{l}4235.095 \\
4235.235 \\
4235.464 \mathrm{i} \\
4236.051 \\
4236.387\end{array}$ & $\begin{array}{l}30 \\
20 \mathrm{w} \\
600 \\
150 \\
75\end{array}$ & $\begin{array}{l}6 \\
3\end{array}$ & $\begin{array}{l}\text { I } \\
\text { I } \\
\text { I } \\
\text { I } \\
\text { I }\end{array}$ \\
\hline $\begin{array}{l}4208.891 \mathrm{i} \\
4209.196 \\
4209.972 \\
4210.090 \\
4210.258\end{array}$ & $\begin{array}{r}3000 \\
100 \\
150 \\
75 \\
10\end{array}$ & $\begin{array}{r}300 \\
2 \\
1 \\
1 .\end{array}$ & $\begin{array}{l}\text { I } \\
\text { I } \\
\text { I } \\
\text { I } \\
\text { I }\end{array}$ & $\begin{array}{l}4222.580 \\
4222.656 \\
4222.751 \\
4222.930 \\
4223.434\end{array}$ & $\begin{array}{l}30 \\
10 \\
100 \\
10 \mathrm{w} \\
400\end{array}$ & $\begin{array}{l}1 \\
5 w \\
2\end{array}$ & $\begin{array}{l}\text { I } \\
\text { I } \\
\text { I I } \\
\text { I } \\
\text { I }\end{array}$ & $\begin{array}{l}4236.720 \\
4236.926 \\
4237.112 \\
4237.184 \\
4237.531\end{array}$ & $\begin{array}{l}10 \\
15 \\
35 \\
30 \\
20\end{array}$ & $10^{1 \mathrm{w}}$ & $\begin{array}{l}\text { I } \\
\text { II } \\
\text { I I } \\
\text { I } \\
\text { I }\end{array}$ \\
\hline
\end{tabular}


Table 1. Wavelengths of Thorium Spectra - Continued

\begin{tabular}{|c|c|c|c|c|c|c|c|c|c|c|c|}
\hline \multirow{2}{*}{$\begin{array}{l}\text { Wave- } \\
\text { length }\end{array}$} & \multicolumn{2}{|c|}{ Intensity } & \multirow{2}{*}{$\begin{array}{l}\text { Spec } \\
\text { trum }\end{array}$} & \multirow{2}{*}{$\begin{array}{l}\text { Wave- } \\
\text { length }\end{array}$} & \multicolumn{2}{|c|}{ Intensity } & \multirow{2}{*}{$\begin{array}{l}\text { Spec- } \\
\text { trum }\end{array}$} & \multirow{2}{*}{$\begin{array}{l}\text { Wave- } \\
\text { length }\end{array}$} & \multicolumn{2}{|c|}{ Intensity } & \multirow{2}{*}{$\begin{array}{l}\text { Spec } \\
\text { trum }\end{array}$} \\
\hline & Lamp & Spark & & & Lamp & Spark & & & & & \\
\hline $\begin{array}{l}4237.652 \\
4238.116 \\
4238.374 \\
4238.538 \\
4238.585\end{array}$ & $\begin{array}{l}10 \\
30 \\
40 \\
20 \\
30\end{array}$ & 1 & $\begin{array}{l}\text { I } \\
\text { I } \\
\text { I } \\
\text { I } \\
\text { I }\end{array}$ & $\begin{array}{l}4252.392 \\
4252.635 \\
4252.801 \\
4253.248 \\
4253.534\end{array}$ & $\begin{array}{c}15 \\
4 \mathrm{~h} \\
4 \mathrm{w} \\
40 \\
800\end{array}$ & $\begin{array}{l}4 \\
6\end{array}$ & $\begin{array}{l}\text { II I } \\
\text { I } \\
\text { I } \\
\text { II }\end{array}$ & $\begin{array}{l}4269.065 \\
4269.941 \\
4270.330 \\
4270.735 \\
4271.087\end{array}$ & $\begin{array}{r}75 \\
200 \\
300 \\
75 \\
250\end{array}$ & $\begin{array}{r}2 \\
50 \\
25\end{array}$ & $\begin{array}{l}\text { I } \\
\text { I } \\
\text { II } \\
\text { I } \\
\text { II }\end{array}$ \\
\hline $\begin{array}{l}4238.975 \\
4239.674 \\
4239.784 \\
4239.855 \\
4239.936\end{array}$ & $\begin{array}{l}25 \\
10 \\
15 \\
5 \mathrm{~h} \\
5 \mathrm{w}\end{array}$ & $\begin{array}{l}3 \\
2 w\end{array}$ & $\begin{array}{l}\text { I I } \\
\text { I I } \\
\text { I } \\
\text { I } \\
\text { I }\end{array}$ & $\begin{array}{l}4253.862 \\
4254.016 \\
4254.378 \\
4254.445 \\
4254.964\end{array}$ & $\begin{array}{r}100 \\
50 \\
10 \\
100 \\
4\end{array}$ & $\begin{array}{r}15 \\
3 \\
15\end{array}$ & $\begin{array}{l}\text { I } \\
\text { II } \\
\text { I } \\
\text { II } \\
\text { I }\end{array}$ & $\begin{array}{l}4271.432 \\
4272.088 \\
4272.137 \\
4272.292 \\
4272.865\end{array}$ & $\begin{array}{r}100 \\
40 \\
20 \\
300 \\
400\end{array}$ & 2 & $\begin{array}{l}\text { I } \\
\text { I } \\
\text { I } \\
\text { I } \\
\text { I }\end{array}$ \\
\hline $\begin{array}{l}4240.592 \\
4240.742 \\
4241.198 \\
4241.972 \\
4242.338\end{array}$ & $\begin{array}{r}100 \\
15 \\
150 \\
20 \\
30\end{array}$ & 20 & $\begin{array}{l}\text { II } \\
\text { I } \\
\text { I } \\
\text { I } \\
\text { II }\end{array}$ & $\begin{array}{l}4255.238 \\
4255.472 \\
4255.780 \\
4256.091 \\
4256.252\end{array}$ & $\begin{array}{r}200 \\
20 \\
200 \\
200 \\
500\end{array}$ & $\begin{array}{r}2 \\
40 \\
4\end{array}$ & $\begin{array}{l}\text { I } \\
\text { I } \\
\text { I } \\
\text { I I } \\
\text { I }\end{array}$ & $\begin{array}{l}4273.3571 \\
4274.025 \\
4274.322 \\
4275.204 \\
4275.348\end{array}$ & $\begin{array}{r}1000 \\
400 \\
100 \\
30 \\
8\end{array}$ & $\begin{array}{r}175 \\
75 \\
40 \\
5\end{array}$ & $\begin{array}{l}\text { II } \\
\text { I I } \\
\text { I I } \\
\text { I I } \\
\text { I }\end{array}$ \\
\hline $\begin{array}{l}4242.724 \\
4243.021 \\
4243.265 \\
4243.926 \\
4244.270\end{array}$ & $\begin{array}{r}75 \\
40 \\
125 \\
400 \\
50\end{array}$ & $\begin{array}{r}6 \\
1 \\
1 \\
50\end{array}$ & $\begin{array}{l}\text { II } \\
\text { I } \\
\text { I } \\
\text { II } \\
\text { I }\end{array}$ & $\begin{array}{l}4257.496 \mathrm{i} \\
4257.893 \\
4258.225 \\
4258.515 \\
4258.986\end{array}$ & $\begin{array}{r}700 \\
125 \\
30 \\
500 \\
75\end{array}$ & $\begin{array}{l}5 \\
2 \\
2\end{array}$ & $\begin{array}{l}I \\
I \\
I \\
I\end{array}$ & $\begin{array}{l}4275.426 \\
4275.566 \\
4275.840 \\
4276.026 \\
4276.281\end{array}$ & $\begin{array}{r}8 \\
20 \\
4 \\
4 \\
15\end{array}$ & & $\begin{array}{l}\text { I } \\
\text { I } \\
\text { I } \\
\text { I } \\
\text { I }\end{array}$ \\
\hline $\begin{array}{l}4244.486 \\
4244.677 \\
4244.819 \\
4245.457 \\
4245.554\end{array}$ & $\begin{array}{l}15 \\
10 \\
30 \\
75 \\
50 \mathrm{~h}\end{array}$ & $\begin{array}{l}2 w \\
3 \\
1\end{array}$ & $\begin{array}{l}\text { I } \\
\text { I } \\
\text { I I } \\
\text { I I } \\
\text { I }\end{array}$ & $\begin{array}{l}4259.497 \\
4259.629 \\
4259.718 \\
4259.850 \\
4259.981\end{array}$ & $\begin{array}{r}125 \\
8 \\
20 \\
15 \\
15\end{array}$ & & $\begin{array}{l}\text { I } \\
\text { I } \\
I \\
I \\
I\end{array}$ & $\begin{array}{l}4276.807 \\
4276.965 \\
4277.3141 \\
4277.938 \\
4278.322\end{array}$ & $\begin{array}{r}500 \\
250 \\
1200 \\
10 \\
500\end{array}$ & $\begin{array}{c}50 \\
20 \mathrm{~h} \\
200 \\
\\
5\end{array}$ & $\begin{array}{l}\text { II } \\
\text { II } \\
\text { I I } \\
\text { I } \\
\text { I }\end{array}$ \\
\hline $\begin{array}{l}4245.656 \\
4245.856 \\
4245.967 \\
4246.091 \\
4246.329\end{array}$ & $\begin{array}{l}5 \\
20 \mathrm{w} \\
10 \\
5 \mathrm{w} \\
50\end{array}$ & 1 & $\begin{array}{l}\text { I } \\
\text { I } \\
\text { I } \\
\text { I } \\
\text { I }\end{array}$ & $\begin{array}{l}4260.335 \\
4260.801 \\
4260.976 \\
4261.273 \\
4261.484\end{array}$ & $\begin{array}{r}800 \\
75 \\
100 \\
40 \\
75\end{array}$ & $\begin{array}{r}3 \\
4 \\
10\end{array}$ & $\begin{array}{l}\text { I } \\
\text { I } \\
\text { I } \\
\text { I I } \\
\text { I I }\end{array}$ & $\begin{array}{l}4278.530 \\
4278.820 \\
4279.062 \\
4279.275 \\
4279.480\end{array}$ & $\begin{array}{r}2 \\
15 \\
100 \\
25 \\
10\end{array}$ & $\begin{array}{l}4 \\
3\end{array}$ & $\begin{array}{l}\text { I I } \\
\text { I } \\
\text { I } \\
\text { I } \\
\text { I }\end{array}$ \\
\hline $\begin{array}{l}4246.803 \\
4247.290 \\
4247.352 \\
4247.604 \\
4247.859\end{array}$ & $\begin{array}{r}40 \\
20 \\
20 \\
125 \\
30\end{array}$ & 10 & $\begin{array}{l}\text { I } \\
\text { I } \\
\text { I } \\
\text { II } \\
\text { I }\end{array}$ & $\begin{array}{l}4261.956 \\
4262.077 \\
4262.611 \\
4262.726 \\
4263.358\end{array}$ & $\begin{array}{r}40 \\
8 \\
200 \\
50 \\
300\end{array}$ & $\begin{array}{r}3 \\
15 \\
75\end{array}$ & $\begin{array}{l}\text { I } \\
\text { I I } \\
\text { I } \\
\text { II } \\
\text { II }\end{array}$ & $\begin{array}{l}4279.636 \\
4279.783 \\
4279.943 \\
4280.236 \\
4280.567\end{array}$ & $\begin{array}{r}30 \\
10 \\
10 \\
25 \\
300\end{array}$ & $\begin{array}{l}8 \\
2\end{array}$ & $\begin{array}{l}\text { I } \\
\text { I } \\
\text { I } \\
\text { II } \\
\text { I }\end{array}$ \\
\hline $\begin{array}{l}4247.993 \\
4248.393 \\
4248.518 \\
4248.729 \\
4248.857\end{array}$ & $\begin{array}{r}400 \\
200 \\
75 \\
30 \\
15\end{array}$ & 75 & $\begin{array}{l}\text { I I } \\
\text { I } \\
\text { I } \\
\text { I } \\
\text { I }\end{array}$ & $\begin{array}{l}4263.739 \\
4264.104 \\
4264.338 \\
4264.400 \\
4264.575\end{array}$ & $\begin{array}{l}20 \mathrm{w} \\
100 \\
100 \mathrm{w} \\
20\end{array}$ & $\begin{array}{r}6 \\
10\end{array}$ & $\begin{array}{l}\text { I } \\
\text { I } \\
\text { I } \\
\text { II I } \\
\text { I }\end{array}$ & $\begin{array}{l}4281.067 \\
4281.414 \\
4282.043 \\
4283.094 \\
4283.515\end{array}$ & $\begin{array}{r}400 \\
400 \\
2000 \\
200 \\
400\end{array}$ & $\begin{array}{r}75 \\
75 \\
150 \\
2 \\
100\end{array}$ & $\begin{array}{l}\text { I I } \\
\text { I I } \\
\text { I I } \\
\text { I } \\
\text { I I }\end{array}$ \\
\hline $\begin{array}{l}4249.163 \\
4249.480 \\
4249.680 \\
4250.312 \\
4251.075\end{array}$ & $\begin{array}{r}10 \\
100 \\
250 \\
1200 \\
15\end{array}$ & $\begin{array}{l}50 \\
75\end{array}$ & $\begin{array}{l}\text { I } \\
\text { I } \\
\text { I I } \\
\text { I } \\
\text { I }\end{array}$ & $\begin{array}{l}4264.879 \\
4264.935 \\
4265.030 \\
4265.489 \\
4265.941\end{array}$ & $\begin{array}{l}10 \\
15 \\
15 \\
50 \\
4 w\end{array}$ & $\begin{array}{l}5 w \\
3 w \\
6 w\end{array}$ & $\begin{array}{l}\text { I } \\
\text { I I } \\
\text { I I } \\
\text { I I } \\
\text { I }\end{array}$ & $\begin{array}{l}4284.071 \\
4284.190 \\
4284.421 \\
4284.512 \\
4284.974\end{array}$ & $\begin{array}{r}8 \\
4 \\
4 \\
20 \\
300\end{array}$ & $\begin{array}{r}6 \\
75\end{array}$ & $\begin{array}{l}\text { I } \\
\text { I } \\
\text { I } \\
\text { I I } \\
\text { I I }\end{array}$ \\
\hline $\begin{array}{l}4251.278 \\
4251.414 \\
4251.516 \\
4251.579 \\
4252.023\end{array}$ & $\begin{array}{l}40 \\
25 w \\
5 w \\
15 \\
25\end{array}$ & 4 & $\begin{array}{l}\text { I } \\
\text { I } \\
\text { I } \\
\text { I I } \\
\text { I }\end{array}$ & $\begin{array}{l}4266.426 \\
4267.224 \\
4267.518 \\
4267.652 \\
4267.716\end{array}$ & $\begin{array}{r}15 \\
15 \\
200 \\
8 \\
20\end{array}$ & $\begin{array}{r}15 \\
3\end{array}$ & $\begin{array}{l}\text { I } \\
\text { I } \\
\text { I I } \\
\text { I } \\
\text { I I }\end{array}$ & $\begin{array}{l}4285.187 \\
4285.403 \\
4285.942 \\
4286.182 \\
4286.215\end{array}$ & $\begin{array}{r}25 \\
10 \\
20 \\
10 \\
200\end{array}$ & 15 & $\begin{array}{l}\text { I I } \\
\text { I } \\
\text { I } \\
\text { I } \\
\text { I }\end{array}$ \\
\hline
\end{tabular}


Table 1. Wavelengths of Thorium Spectra - Continued

\begin{tabular}{|c|c|c|c|c|c|c|c|c|c|c|c|}
\hline \multirow{2}{*}{$\begin{array}{l}\text { Wave- } \\
\text { length }\end{array}$} & \multicolumn{2}{|c|}{ Intensity } & \multirow{2}{*}{$\begin{array}{l}\text { Spec- } \\
\text { trum }\end{array}$} & \multirow{2}{*}{$\begin{array}{l}\text { Wave- } \\
\text { length }\end{array}$} & \multicolumn{2}{|c|}{ Intensity } & \multirow{2}{*}{$\begin{array}{l}\text { Spec- } \\
\text { trum }\end{array}$} & \multirow{2}{*}{$\begin{array}{l}\text { Wave- } \\
\text { length }\end{array}$} & \multicolumn{2}{|c|}{ Intensity } & \multirow{2}{*}{$\begin{array}{l}\text { Spec } \\
\text { trum }\end{array}$} \\
\hline & & & & & Lamp & Spark & & & Lamp & Spark & \\
\hline $\begin{array}{l}4286.700 \\
4287.080 \\
4287.396 \\
4287.682 \\
4287.958\end{array}$ & $\begin{array}{r}75 \\
100 \\
8 \\
25 \\
40\end{array}$ & $\begin{array}{l}8 \\
6\end{array}$ & $\begin{array}{l}\text { II } \\
\text { I } \\
\text { I } \\
\text { II } \\
\text { II }\end{array}$ & $\begin{array}{l}4302.108 \\
4302.211 \\
4303.093 \\
4303.223 \\
4303.380\end{array}$ & $\begin{array}{r}10 \\
20 \\
20 \\
4 \\
30\end{array}$ & $3 \mathrm{~h}$ & $\begin{array}{l}\text { I } \\
\text { II } \\
\text { I } \\
\text { I } \\
\text { II }\end{array}$ & $\begin{array}{l}4318.294 \\
4318.416 \mathrm{i} \\
4319.085 \\
4320.124 \\
4320.449\end{array}$ & $\begin{array}{r}20 \\
700 \\
200 \\
300 \\
100\end{array}$ & $\begin{array}{r}30 \\
6 \\
30 \\
100 \\
40\end{array}$ & $\begin{array}{l}\text { II } \\
\text { I } \\
\text { II } \\
\text { II } \\
\text { II }\end{array}$ \\
\hline $\begin{array}{l}4288.050 \\
4288.476 \\
4288.659 \\
4289.209 \\
4289.408\end{array}$ & $\begin{array}{r}100 \\
200 \\
200 \\
10 \\
15\end{array}$ & $\begin{array}{r}12 \\
1 \\
2\end{array}$ & $\begin{array}{l}\text { II } \\
\text { I } \\
\text { I } \\
\text { I } \\
\text { I }\end{array}$ & $\begin{array}{l}4303.820 \\
4303.991 \\
4304.417 \\
4304.954 \\
4305.324\end{array}$ & $\begin{array}{r}8 \\
40 \\
40 \\
100 \\
10\end{array}$ & $\begin{array}{l}2 \mathrm{~h} \\
1 \\
4\end{array}$ & $\begin{array}{l}\text { I } \\
\text { I } \\
\text { I } \\
\text { I } \\
\text { I I }\end{array}$ & $\begin{array}{l}4320.582 \\
4320.732 \\
4320.972 \\
4321.718 \\
4321.987\end{array}$ & $\begin{array}{r}300 \\
100 \\
50 \\
12 \\
20\end{array}$ & 50 & $\begin{array}{l}\text { II } \\
\text { I } \\
\text { I } \\
\text { I } \\
\text { II }\end{array}$ \\
\hline $\begin{array}{l}4289.653 \\
4290.388 \\
4290.945 \\
4291.378 \\
4291.8101\end{array}$ & $\begin{array}{r}100 \\
100 \\
15 \\
4 \\
400\end{array}$ & $\begin{array}{l}1 \\
6 \\
2 \\
3\end{array}$ & $\begin{array}{l}\text { I } \\
\text { I } \\
\text { I } \\
\text { II } \\
\text { I }\end{array}$ & $\begin{array}{l}4305.493 \\
4305.556 \\
4305.906 \\
4306.368 \\
4307.1761\end{array}$ & $\begin{array}{r}8 \\
10 \\
15 \\
200 \\
700\end{array}$ & $\begin{array}{r}2 \\
40 \\
10\end{array}$ & $\begin{array}{l}\text { I } \\
\text { I } \\
\text { II } \\
\text { I I } \\
\text { I }\end{array}$ & $\begin{array}{l}4322.568 \\
4322.735 \\
4322.954 \\
4323.031 \\
4323.561\end{array}$ & $\begin{array}{r}8 \mathrm{w} \\
10 \\
5 \\
15 \\
4\end{array}$ & $\begin{array}{l}4 \\
6\end{array}$ & $\begin{array}{l}\text { I } \\
\text { III } \\
\text { I } \\
\text { II } \\
\text { I }\end{array}$ \\
\hline $\begin{array}{l}4292.305 \\
4292.465 \\
4292.506 \\
4292.968 \\
4293.070\end{array}$ & $\begin{array}{r}30 \\
4 \\
15 \\
4 \\
2\end{array}$ & $\begin{array}{l}2 \\
3\end{array}$ & $\begin{array}{l}\text { I } \\
\text { I } \\
\text { II } \\
\text { I } \\
\text { II }\end{array}$ & $\begin{array}{l}4308.124 \\
4308.586 \\
4309.104 \\
4309.200 \\
4309.548\end{array}$ & $\begin{array}{r}200 \\
200 \\
8 \\
8 \\
10\end{array}$ & 2 & $\begin{array}{l}\text { I } \\
\text { I } \\
\text { I } \\
\text { I I } \\
\text { I }\end{array}$ & $\begin{array}{l}4324.254 \\
4324.436 \\
4324.628 \\
4324.900 \\
4324.953\end{array}$ & $\begin{array}{r}12 \\
8 \\
8 \\
15 \\
4\end{array}$ & 2 & $\begin{array}{l}\text { I } \\
\text { I } \\
\text { I } \\
\text { II } \\
\text { I }\end{array}$ \\
\hline $\begin{array}{l}4293.429 \\
4293.772 \\
4294.019 \\
4294.719 \\
4295.043\end{array}$ & $\begin{array}{r}15 \\
50 \\
8 \\
100 \\
75\end{array}$ & 100 & $\begin{array}{l}\text { I } \\
\text { I } \\
\text { I } \\
\text { I } \\
\text { II }\end{array}$ & $\begin{array}{l}4309.899 \\
4309.993 \\
4310.637 \\
4310.962 \\
4311.062\end{array}$ & $\begin{array}{r}20 \\
300 \\
12 \\
15 \\
50\end{array}$ & $\begin{array}{r}50 \\
6\end{array}$ & $\begin{array}{l}\text { I } \\
\text { I I } \\
\text { I } \\
\text { I } \\
\text { I }\end{array}$ & $\begin{array}{l}4325.009 \\
4325.278 \\
4326.167 \\
4326.332 \\
4326.514\end{array}$ & $\begin{array}{r}4 \\
125 \\
8 \\
4 \\
12\end{array}$ & 3 & $\begin{array}{l}\text { I } \\
\text { I } \\
\text { I } \\
\text { I } \\
\text { I }\end{array}$ \\
\hline $\begin{array}{l}4295.124 \\
4295.229 \\
4295.580 \\
4295.810 \\
4296.355\end{array}$ & $\begin{array}{l}5 \\
10 \\
75 \\
40 w \\
10\end{array}$ & 2 & $\begin{array}{l}\text { II } \\
\text { I } \\
\text { I } \\
\text { I } \\
\text { I }\end{array}$ & $\begin{array}{l}4311.233 \\
4311.315 \\
4311.584 \\
4311.627 \\
4311.796\end{array}$ & $\begin{array}{r}10 \\
5 \\
200 \\
100 \\
200\end{array}$ & $\begin{array}{l}3 \\
3\end{array}$ & $\begin{array}{l}\text { I } \\
\text { I } \\
\text { I } \\
\text { I } \\
\text { I }\end{array}$ & $\begin{array}{l}4326.676 \\
4327.031 \\
4327.087 \\
4327.124 \\
4327.223\end{array}$ & $\begin{array}{r}12 \\
40 \\
200 \\
40 \\
50\end{array}$ & $\begin{array}{r}20 \\
5\end{array}$ & $\begin{array}{l}\text { I } \\
\text { I } \\
\text { II } \\
\text { I } \\
\text { II }\end{array}$ \\
\hline $\begin{array}{l}4296.574 \\
4296.888 \\
4296.962 \\
4297.304 \\
4297.632\end{array}$ & $\begin{array}{l}15 \\
10 \\
10 \mathrm{w} \\
400 \\
10\end{array}$ & $\begin{array}{l}5 w \\
5 \\
10\end{array}$ & $\begin{array}{l}\text { II } \\
\text { II } \\
\text { I } \\
\text { I } \\
\text { I }\end{array}$ & $\begin{array}{l}4312.994 \\
4313.304 \\
4313.422 \\
4313.476 \\
4313.611\end{array}$ & $\begin{array}{c}1000 \\
40 \\
10 \\
2 \\
12 \mathrm{w}\end{array}$ & $\begin{array}{r}12 \\
15 \\
2\end{array}$ & $\begin{array}{l}\text { I } \\
\text { I I } \\
\text { I } \\
\text { I I } \\
\text { I }\end{array}$ & $\begin{array}{l}4327.441 \\
4327.540 \\
4327.708 \\
4327.979 \\
4328.275\end{array}$ & $\begin{array}{r}10 \\
10 \\
75 \\
12 \\
8\end{array}$ & 4 & $\begin{array}{l}\text { I } \\
\text { I } \\
\text { I } \\
\text { I } \\
\text { I I }\end{array}$ \\
\hline $\begin{array}{l}4297.939 \\
4298.125 \\
4298.182 \\
4298.562 \\
4298.836\end{array}$ & $\begin{array}{r}40 \\
30 \\
6 \\
8 \\
120\end{array}$ & $\begin{array}{r}2 \\
40\end{array}$ & $\begin{array}{l}\text { I } \\
\text { I } \\
\text { I } \\
\text { II } \\
\text { I I }\end{array}$ & $\begin{array}{l}4313.781 \\
4314.023 \\
4314.323 \\
4314.801 \\
4314.945\end{array}$ & $\begin{array}{c}12 \\
15 \\
150 \\
8 \\
5 \mathrm{w}\end{array}$ & & $\begin{array}{l}\text { I } \\
I \\
I \\
I \\
I\end{array}$ & $\begin{array}{l}4328.678 \\
4328.909 \\
4329.086 \\
4329.211 \\
4329.487\end{array}$ & $\begin{array}{r}50 \\
400 \\
5 \\
8 \\
100\end{array}$ & $\begin{array}{r}30 \\
4 \\
30\end{array}$ & $\begin{array}{l}\text { II } \\
\text { I } \\
\text { II } \\
\text { I } \\
\text { II }\end{array}$ \\
\hline $\begin{array}{l}4299.436 \\
4299.631 \\
4299.8391 \\
4300.140 \\
4300.269\end{array}$ & $\begin{array}{r}5 \\
100 \\
600 \\
5 \\
8\end{array}$ & $\begin{array}{l}5 \\
4\end{array}$ & $\begin{array}{l}\text { II } \\
\text { I } \\
\text { I } \\
\text { II } \\
\text { I }\end{array}$ & $\begin{array}{l}4315.254 \mathrm{i} \\
4315.573 \\
4315.709 \\
4315.953 \\
4316.109\end{array}$ & $\begin{array}{r}400 \\
4 \\
12 \\
30 \\
50\end{array}$ & $\begin{array}{r}4 \\
20\end{array}$ & $\begin{array}{l}\text { I } \\
\text { I } \\
\text { I I } \\
\text { I } \\
\text { I }\end{array}$ & $\begin{array}{l}4330.211 \\
4330.403 \\
4330.8441 \\
4331.200 \\
4331.332\end{array}$ & $\begin{array}{r}75 \\
50 \\
300 \\
1 \\
200\end{array}$ & $\begin{array}{l}2 w \\
2 \\
5 w\end{array}$ & $\begin{array}{l}\text { I } \\
\text { I } \\
\text { I } \\
\text { I I } \\
\text { I }\end{array}$ \\
\hline $\begin{array}{l}4300.789 \\
4301.278 \\
4301.570 \\
4301.862 \\
4302.049\end{array}$ & $\begin{array}{l}50 \\
20 \\
10 \\
10 \\
10 \mathrm{w}\end{array}$ & $\begin{array}{l}20 \\
4 \\
5 w\end{array}$ & $\begin{array}{l}\text { II } \\
\text { II } \\
\text { I } \\
\text { II } \\
\text { I }\end{array}$ & $\begin{array}{l}4316.367 \\
4317.221 \\
4317.533 \\
4317.663 \\
4317.844\end{array}$ & $\begin{array}{l}20 \\
10 \\
2 \mathrm{~h} \\
4 \\
75\end{array}$ & 6 & $\begin{array}{l}\text { I } \\
\text { I I } \\
\text { I } \\
\text { I } \\
\text { I }\end{array}$ & $\begin{array}{l}4331.918 \\
4332.331 \\
4333.262 \\
4333.560 \\
4333.640\end{array}$ & $\begin{array}{r}100 \\
125 \\
20 \\
12 \\
12\end{array}$ & 40 & $\begin{array}{l}\text { II } \\
\text { I } \\
\text { I } \\
\text { I } \\
\text { I }\end{array}$ \\
\hline
\end{tabular}


Table 1. Wavelengths of Thorium Spectra - Continued

\begin{tabular}{|c|c|c|c|c|c|c|c|c|c|c|c|}
\hline \multirow{2}{*}{$\begin{array}{l}\text { Wave- } \\
\text { length }\end{array}$} & \multicolumn{2}{|c|}{ Intensity } & \multirow{2}{*}{$\begin{array}{l}\text { Spec- } \\
\text { trum }\end{array}$} & \multirow{2}{*}{$\begin{array}{l}\text { Wave- } \\
\text { length }\end{array}$} & \multicolumn{2}{|c|}{ Intensity } & \multirow{2}{*}{$\begin{array}{l}\text { Spec- } \\
\text { trum }\end{array}$} & \multirow{2}{*}{$\begin{array}{l}\text { Wave- } \\
\text { length }\end{array}$} & \multicolumn{2}{|c|}{ Intensity } & \multirow{2}{*}{$\begin{array}{l}\text { Spec } \\
\text { trum }\end{array}$} \\
\hline & $\operatorname{amp}$ & Spark & & & $\operatorname{amp}$ & Spark & & & Lamp & Spark & \\
\hline $\begin{array}{l}4333.933 \\
4334.249 \\
4334.737 \\
4334.924 \\
4335.320\end{array}$ & $\begin{array}{r}200 \\
20 \\
8 \\
8 \\
100\end{array}$ & $\begin{array}{r}100 \\
4\end{array}$ & $\begin{array}{l}\text { II } \\
\text { I } \\
\text { I } \\
\text { I } \\
\text { II }\end{array}$ & $\begin{array}{l}4351.013 \\
4351.062 \\
4351.274 \\
4351.481 \\
4351.511\end{array}$ & $\begin{array}{l}8 w \\
2 w \\
50 \\
75 \\
20\end{array}$ & 35 & $\begin{array}{l}\text { II } \\
\text { I } \\
\text { I } \\
\text { II } \\
\text { I }\end{array}$ & $\begin{array}{l}4366.960 \\
4367.283 \\
4367.419 \\
4367.732 \\
4367.916\end{array}$ & $\begin{array}{r}60 \\
50 \\
75 \\
4 \\
8\end{array}$ & 20 & $\begin{array}{l}\text { II } \\
\text { I } \\
\text { I } \\
\text { I } \\
\text { I }\end{array}$ \\
\hline $\begin{array}{l}4335.700 \\
4336.464 \\
4336.973 \\
4337.275 \\
4337.840\end{array}$ & $\begin{array}{r}800 \\
50 \\
12 \\
900 \\
2\end{array}$ & $\begin{array}{r}75 \\
10 \\
125 \\
2\end{array}$ & $\begin{array}{l}\text { I } \\
\text { II } \\
\text { I } \\
\text { I } \\
\text { II }\end{array}$ & $\begin{array}{l}4351.895 \\
4352.114 \\
4352.166 \\
4352.611 \\
4352.670\end{array}$ & $\begin{array}{r}4 \\
4 \\
8 \\
100 \\
600\end{array}$ & 75 & $\begin{array}{l}\text { I } \\
\text { I } \\
\text { II } \\
\text { I } \\
\text { II }\end{array}$ & $\begin{array}{l}4368.339 \\
4368.682 \\
4368.995 \\
4369.059 \\
4369.304\end{array}$ & $\begin{array}{r}15 \\
4 \\
10 \\
4 \\
250\end{array}$ & $\begin{array}{l}10 \\
40\end{array}$ & $\begin{array}{l}\text { I } \\
\text { I } \\
\text { I } \\
\text { II } \\
\text { I I }\end{array}$ \\
\hline $\begin{array}{l}4338.098 \\
4338.370 \\
4338.778 \\
4339.011 \\
4339.381\end{array}$ & $\begin{array}{r}350 \\
2 \\
5 \\
8 \\
20\end{array}$ & $\begin{array}{l}2 \\
2 \\
2 w\end{array}$ & $\begin{array}{l}\text { I } \\
\text { I I } \\
\text { I I } \\
\text { I } \\
\text { I }\end{array}$ & $\begin{array}{l}4352.819 \\
4353.389 \\
4353.435 \\
4354.100 \\
4354.481\end{array}$ & $\begin{array}{c}15 \\
100 \\
500 \\
5 \mathrm{~h} \\
400\end{array}$ & $\begin{array}{r}2 \\
75 \\
1 \\
4\end{array}$ & $\begin{array}{l}\text { I I } \\
\text { II } \\
\text { I } \\
\text { I } \\
\text { I }\end{array}$ & $\begin{array}{l}4369.494 \\
4369.598 \\
4369.867 \\
4370.249 \\
4370.469\end{array}$ & $\begin{array}{r}200 \\
8 \\
200 \\
12 \\
8\end{array}$ & & $\begin{array}{l}\text { I } \\
\text { I } \\
\text { I } \\
\text { I } \\
\text { I }\end{array}$ \\
\hline $\begin{array}{l}4339.442 \\
4339.867 \\
4340.456 \\
4340.894 \\
4341.024\end{array}$ & $\begin{array}{r}4 \\
125 \\
4 \\
400 \\
75\end{array}$ & 125 & $\begin{array}{l}\text { I } \\
\text { I } \\
\text { I } \\
\text { I } \\
\text { I I }\end{array}$ & $\begin{array}{l}4355.001 \\
4355.322 \\
4355.521 \\
4356.034 \\
4356.340\end{array}$ & $\begin{array}{r}8 \\
120 \\
200 \\
75 \\
8\end{array}$ & 75 & $\begin{array}{l}\text { I } \\
\text { II } \\
\text { I } \\
\text { I } \\
\text { I }\end{array}$ & $\begin{array}{l}4370.625 \\
4371.136 \\
4371.338 \\
4371.745 \\
4372.149\end{array}$ & $\begin{array}{r}4 \\
20 \\
20 \\
8 \\
8\end{array}$ & $\begin{array}{l}2 \\
2 w\end{array}$ & $\begin{array}{l}\text { I } \\
\text { I } \\
\text { I I } \\
\text { I I } \\
\text { I }\end{array}$ \\
\hline $\begin{array}{l}4342.050 \\
4342.256 \mathrm{i} \\
4342.439 \\
4342.662 \\
4342.952\end{array}$ & $\begin{array}{r}20 \\
300 \\
500 \\
40 \\
4\end{array}$ & $\begin{array}{r}75 \\
3\end{array}$ & $\begin{array}{l}\text { I } \\
\text { I I } \\
\text { I } \\
\text { I } \\
\text { I }\end{array}$ & $\begin{array}{l}4356.546 \\
4356.596 \\
4357.063 \\
4357.614 \\
4357.840\end{array}$ & $\begin{array}{r}12 \\
4 \\
8 \\
125 \\
8\end{array}$ & $\begin{array}{r}1 \\
30\end{array}$ & $\begin{array}{l}\text { I } \\
\text { I } \\
\text { I I } \\
\text { II } \\
\text { I }\end{array}$ & $\begin{array}{l}4372.648 \\
4372.802 \\
4373.042 \\
4373.900 \\
4374.124 \mathrm{i}\end{array}$ & $\begin{array}{c}12 \mathrm{~s} \\
8 \\
200 \\
200 \\
600\end{array}$ & $\begin{array}{r}4 \\
75 \\
8\end{array}$ & $\begin{array}{l}\text { I } \\
\text { I } \\
\text { I } \\
\text { I I } \\
\text { I }\end{array}$ \\
\hline $\begin{array}{l}4343.014 \\
4343.038 \\
4343.378 \\
4343.610 \\
4343.944\end{array}$ & $\begin{array}{r}20 \\
4 \\
30 \\
50 \\
125\end{array}$ & $\begin{array}{l}20 \\
30\end{array}$ & $\begin{array}{l}\text { I } \\
\text { I } \\
\text { I } \\
\text { II } \\
\text { II }\end{array}$ & $\begin{array}{l}4358.330 \\
4358.537 \\
4358.807 \\
4359.119 \\
4359.365\end{array}$ & $\begin{array}{r}400 \\
30 \\
8 \\
4 \\
600\end{array}$ & $\begin{array}{r}4 \\
20 \\
15 \\
8 \\
8\end{array}$ & $\begin{array}{l}\text { I } \\
\text { I I } \\
\text { I I } \\
\text { I I } \\
\text { I }\end{array}$ & $\begin{array}{l}4374.784 \\
4375.012 \\
4375.068 \\
4375.280 \\
4375.398\end{array}$ & $\begin{array}{r}500 \\
8 \\
50 \\
4 \\
4\end{array}$ & $\begin{array}{r}100 \\
2\end{array}$ & $\begin{array}{l}\text { II } \\
\text { I } \\
\text { I } \\
\text { I } \\
\text { I }\end{array}$ \\
\hline $\begin{array}{l}4344.322 \\
4345.049 \\
4345.178 \\
4345.400 \\
4345.637\end{array}$ & $\begin{array}{c}300 \\
30 \\
15 \\
8 \\
75 \mathrm{~h}\end{array}$ & $\begin{array}{l}75 \\
20\end{array}$ & $\begin{array}{l}\text { I I } \\
\text { I I } \\
\text { I } \\
\text { I } \\
\text { I }\end{array}$ & $\begin{array}{l}4359.717 \\
4360.163 \\
4360.706 \\
4361.298 \\
4361.794\end{array}$ & $\begin{array}{r}40 \\
100 \\
20 \\
150 \\
30\end{array}$ & $\begin{array}{r}2 \\
100 \\
30\end{array}$ & $\begin{array}{l}\text { I } \\
\text { I } \\
\text { I } \\
\text { II } \\
\text { I I }\end{array}$ & $\begin{array}{l}4375.592 \\
4375.837 \\
4376.257 \\
4376.529 \\
4377.312\end{array}$ & $\begin{array}{r}25 \\
40 \\
20 \\
200 \\
75\end{array}$ & 100 & $\begin{array}{l}\text { I I } \\
\text { I } \\
\text { I } \\
\text { I } \\
\text { II }\end{array}$ \\
\hline $\begin{array}{l}4345.690 \\
4345.844 \\
4346.432 \\
4346.791 \\
4347.225\end{array}$ & $\begin{array}{c}10 \\
100 \\
500 \\
4 \mathrm{~h} \\
100\end{array}$ & 100 & $\begin{array}{l}\text { I } \\
\text { I } \\
\text { I } \\
\text { I } \\
\text { II }\end{array}$ & $\begin{array}{l}4362.298 \\
4362.358 \\
4362.460 \\
4362.707 \\
4362.770\end{array}$ & $\begin{array}{r}5 \\
12 \\
75 \\
8 \\
75\end{array}$ & 4 & $\begin{array}{l}\text { I } \\
\text { I }\end{array}$ & $\begin{array}{l}4377.474 \\
4377.655 \\
4377.897 \\
4378.177 \mathrm{i} \\
4378.829\end{array}$ & $\begin{array}{r}8 \\
12 \\
4 \\
500 \\
25\end{array}$ & $\begin{array}{r}3 \\
20\end{array}$ & $\begin{array}{l}\text { I } \\
\text { I } \\
\text { I } \\
\text { I } \\
\text { I I }\end{array}$ \\
\hline $\begin{array}{l}4347.636 \\
4348.296 \\
4348.591 \\
4349.071 \\
4349.407\end{array}$ & $\begin{array}{r}20 \\
8 \\
400 \\
400 \\
20\end{array}$ & $\begin{array}{l}12 \\
2 \\
2 \\
1 \mathrm{~h}\end{array}$ & $\begin{array}{l}\text { I } \\
\text { II } \\
\text { I } \\
\text { I } \\
\text { II }\end{array}$ & $\begin{array}{l}4363.104 \\
4363.423 \\
4363.492 \\
4364.033 \\
4364.182\end{array}$ & $\begin{array}{r}8 \\
20 \\
10 \\
30 \\
4\end{array}$ & 4 & $\begin{array}{l}\text { I } \\
\text { I } \\
\text { I } \\
\text { I } \\
\text { I I }\end{array}$ & $\begin{array}{l}4379.008 \\
4379.589 \\
4379.946 \\
4380.289 \\
4380.704\end{array}$ & $\begin{array}{c}10 \\
8 \\
25 \mathrm{w} \\
125 \\
20 \mathrm{~h}\end{array}$ & $\begin{array}{l}3 \mathrm{w} \\
18^{3} \\
2\end{array}$ & $\begin{array}{l}\text { I } \\
\text { II } \\
\text { I I } \\
\text { I } \\
\text { I }\end{array}$ \\
\hline $\begin{array}{l}4349.614 \\
4350.270 \\
4350.382 \\
4350.651 \\
4350.823\end{array}$ & $\begin{array}{r}15 \\
75 \\
8 \\
4 \\
30\end{array}$ & 30 & $\begin{array}{l}\text { I I } \\
\text { I } \\
\text { I } \\
\text { I } \\
\text { I I I }\end{array}$ & $\begin{array}{l}4364.488 \\
4365.016 \\
4365.472 \\
4365.528 \\
4365.930 \mathrm{i}\end{array}$ & $\begin{array}{c}40 \mathrm{~W} \\
50 \\
8 \\
8 \\
600\end{array}$ & 10 & $\begin{array}{l}\text { I } \\
\text { I } \\
\text { I } \\
\text { I } \\
\text { I }\end{array}$ & $\begin{array}{l}4380.864 \\
4381.054 \\
4381.405 \\
4381.860 \mathrm{i} \\
4382.898\end{array}$ & $\begin{array}{r}2 \\
4 \\
350 \\
900 \\
50\end{array}$ & $\begin{array}{r}10 \\
150\end{array}$ & $\begin{array}{l}\text { I } \\
\text { I } \\
\text { I I } \\
\text { I } \\
\text { I }\end{array}$ \\
\hline
\end{tabular}


Table 1. Wavelengths of Thorium Spectra - Continued

\begin{tabular}{|c|c|c|c|c|c|c|c|c|c|c|c|}
\hline \multirow{2}{*}{$\begin{array}{l}\text { Wave- } \\
\text { length }\end{array}$} & \multicolumn{2}{|c|}{ Intensity } & \multirow{2}{*}{$\begin{array}{l}\text { Spec- } \\
\text { trum }\end{array}$} & \multirow{2}{*}{$\begin{array}{l}\text { Wave- } \\
\text { length }\end{array}$} & \multicolumn{2}{|c|}{ Intensity } & \multirow{2}{*}{$\begin{array}{l}\text { Spec- } \\
\text { trum }\end{array}$} & \multirow{2}{*}{$\begin{array}{l}\text { Wave- } \\
\text { length }\end{array}$} & \multicolumn{2}{|c|}{ Intensity } & \multirow{2}{*}{$\begin{array}{l}\text { Spec } \\
\text { trum }\end{array}$} \\
\hline & Lamp & & & & Lamp & Spark & & & Lamp & Spark t & \\
\hline $\begin{array}{l}4383.860 \\
4384.015 \\
4384.129 \\
4384.646 \\
4385.234\end{array}$ & $\begin{array}{r}40 \\
8 \\
8 \\
300 \\
4\end{array}$ & $\begin{array}{l}2 \mathrm{~h} \\
2 \mathrm{~h} \\
2\end{array}$ & $\begin{array}{l}\text { I } \\
\text { I I } \\
\text { I I } \\
\text { I } \\
\text { I }\end{array}$ & $\begin{array}{l}4401.582 \mathrm{i} \\
4402.260 \\
4402.448 \\
4402.605 \\
4402.738\end{array}$ & $\begin{array}{r}400 \\
75 \\
4 \\
12 \\
8\end{array}$ & $\begin{array}{l}20 \\
2 \mathrm{~h}\end{array}$ & $\begin{array}{l}\text { I } \\
\text { I } \\
\text { I } \\
\text { I } \\
\text { I I }\end{array}$ & $\begin{array}{l}4420.752 \\
4420.794 \\
4421.160 \\
4421.546 \\
4421.833\end{array}$ & $\begin{array}{r}5 \\
200 \\
4 \\
200 \\
4\end{array}$ & $3 w$ & $\begin{array}{l}\text { I I } \\
\text { I } \\
\text { I I } \\
\text { I I } \\
\text { I I }\end{array}$ \\
\hline $\begin{array}{l}4385.640 \\
4385.754 \\
4386.116 \\
4386.267 \\
4386.483\end{array}$ & $\begin{array}{r}6 \\
20 \\
8 \\
20 \\
12\end{array}$ & 3 & $\begin{array}{l}\text { I } \\
\text { I } \\
\text { I I } \\
\text { I } \\
\text { I }\end{array}$ & $\begin{array}{l}4402.927 \mathrm{i} \\
4403.428 \\
4403.714 \\
4404.132 \\
4405.711\end{array}$ & $\begin{array}{l}400 \\
12 \mathrm{w} \\
25 \\
75 \\
15\end{array}$ & 2 & $\begin{array}{l}\text { I } \\
\text { I } \\
\text { I } \\
\text { I I }\end{array}$ & $\begin{array}{l}4422.059 \\
4422.789 \\
4422.894 \\
4423.266 \\
4423.723\end{array}$ & $\begin{array}{r}350 \\
10 \\
40 \\
30 \\
200\end{array}$ & $\begin{array}{r}3 \\
10 \\
2\end{array}$ & $\begin{array}{l}\text { I } \\
\text { I I } \\
\text { I } \\
\text { I I } \\
\text { I }\end{array}$ \\
\hline $\begin{array}{l}4386.714 \\
4387.022 \\
4387.102 \\
4387.214 \\
4387.473\end{array}$ & $\begin{array}{r}8 \\
75 \\
75 \\
4 \\
4\end{array}$ & 20 & $\begin{array}{l}\text { I } \\
\text { I I } \\
\text { I I } \\
\text { I } \\
\text { I }\end{array}$ & $\begin{array}{l}4405.847 \\
4405.988 \\
4406.421 \\
4406.771 \\
4406.903\end{array}$ & $\begin{array}{r}4 \\
4 \\
20 \\
5 \\
12\end{array}$ & $\begin{array}{c}10 w \\
2\end{array}$ & $\begin{array}{l}\text { I } \\
\text { I } \\
\text { I I } \\
\text { I I } \\
\text { I }\end{array}$ & $\begin{array}{l}4423.930 \\
4424.237 \\
4424.546 \\
4424.837 \\
4425.117\end{array}$ & $\begin{array}{r}40 \\
20 \\
15 \\
200 \\
5\end{array}$ & 30 & $\begin{array}{l}\text { I I } \\
\text { I } \\
\text { I } \\
\text { I } \\
\text { I I }\end{array}$ \\
\hline $\begin{array}{l}4387.741 \\
4388.100 \\
4388.561 \\
4388.663 \\
4388.792\end{array}$ & $\begin{array}{r}125 \\
40 \\
4 \\
4 \\
2\end{array}$ & 175 & $\begin{array}{l}\text { I I } \\
\text { I } \\
\text { I } \\
\text { I } \\
\text { I }\end{array}$ & $\begin{array}{l}4407.276 \\
4407.500 \\
4407.893 \\
4408.474 \\
4408.883 \mathrm{i}\end{array}$ & $\begin{array}{r}12 \\
4 \\
4 \\
200 \\
600\end{array}$ & $\begin{array}{r}40 \\
5\end{array}$ & $\begin{array}{l}\text { I } \\
\text { I } \\
\text { I } \\
\text { I I } \\
\text { I }\end{array}$ & $\begin{array}{l}4425.449 \\
4425.984 \\
4426.189 \\
4426.519 \\
4426.834\end{array}$ & $\begin{array}{r}100 \\
100 \\
12 \\
12 \\
4\end{array}$ & $\begin{array}{r}125 \\
2\end{array}$ & $\begin{array}{l}\text { I } \\
\text { II } \\
\text { I I } \\
\text { I } \\
\text { I }\end{array}$ \\
\hline $\begin{array}{l}4388.975 \\
4389.482 \\
4389.668 \\
4389.916 \\
4390.167\end{array}$ & $\begin{array}{r}75 \\
8 \\
15 \\
8 \\
15\end{array}$ & 1 & $\begin{array}{l}\text { I } \\
\text { I } \\
\text { I } \\
\text { I } \\
\text { I }\end{array}$ & $\begin{array}{l}4409.015 \\
4409.740 \\
4409.901 \\
4410.410 \\
4410.477\end{array}$ & $\begin{array}{r}8 \\
50 \\
10 \\
125\end{array}$ & 125 & $\begin{array}{l}\text { I I } \\
\text { I } \\
\text { I } \\
\text { I } \\
\text { I I }\end{array}$ & $\begin{array}{l}4427.645 \\
4428.547 \\
4428.887 \\
4429.261 \\
4429.618\end{array}$ & $\begin{array}{r}125 \\
150 \\
125 \\
20 \\
50\end{array}$ & $\begin{array}{r}50 \\
12 \\
7 \\
4\end{array}$ & $\begin{array}{l}\text { I I } \\
\text { I } \\
\text { I } \\
\text { I I } \\
\text { I }\end{array}$ \\
\hline $\begin{array}{l}4391.110 \mathrm{i} \\
4391.530 \\
4391.938 \\
4392.478 \\
4392.977\end{array}$ & $\begin{array}{c}3000 \\
50 \\
10 \\
75 \mathrm{~h} \\
400\end{array}$ & 400 & $\begin{array}{l}\text { I I } \\
\text { I } \\
\text { I } \\
\text { I } \\
\text { I }\end{array}$ & $\begin{array}{l}4411.145 \\
4411.250 \\
4411.606 \\
4412.556 \\
4412.741\end{array}$ & $\begin{array}{c}20 \mathrm{~W} \\
8 \\
50 \\
600 \\
800\end{array}$ & $\begin{array}{r}10 \\
400\end{array}$ & $\begin{array}{l}\text { I } \\
\text { I } \\
\text { I I } \\
\text { I } \\
\text { I I }\end{array}$ & $\begin{array}{l}4429.815 \\
4429.969 \\
4430.149 \\
4432.249 \\
4432.963 \mathrm{i}\end{array}$ & $\begin{array}{r}40 \\
8 \\
30 \\
200 \\
600\end{array}$ & $\begin{array}{r}4 \\
150\end{array}$ & $\begin{array}{l}\text { I } \\
\text { I } \\
\text { I } \\
\text { I } \\
\text { I I }\end{array}$ \\
\hline $\begin{array}{l}4393.761 \\
4394.260 \\
4394.448 \\
4394.551 \\
4394.900\end{array}$ & $\begin{array}{r}400 \\
8 \\
15 \\
15 \\
250\end{array}$ & 100 & $\begin{array}{l}\text { I } \\
\text { I } \\
\text { I } \\
\text { I } \\
\text { I I }\end{array}$ & $\begin{array}{l}4412.909 \\
4413.369 \\
4413.634 \\
4414.525 \\
4414.778\end{array}$ & $\begin{array}{r}400 \\
40 \\
50 \\
400 \\
20\end{array}$ & $\begin{array}{r}100 \\
1 \\
4\end{array}$ & $\begin{array}{l}\text { I } \\
\text { I I } \\
\text { I } \\
\text { I } \\
\text { I }\end{array}$ & $\begin{array}{l}4433.482 \\
4434.067 \\
4434.321 \\
4434.825 \\
4435.290\end{array}$ & $\begin{array}{r}150 \\
50 \\
30 \\
75 \\
30\end{array}$ & $\begin{array}{r}2 \\
10 \\
5\end{array}$ & $\begin{array}{l}\text { I } \\
\text { I I } \\
\text { I I } \\
\text { I I } \\
\text { I }\end{array}$ \\
\hline $\begin{array}{l}4395.513 \\
4395.675 \\
4396.145 \\
4396.480 \\
4397.014\end{array}$ & $\begin{array}{r}4 \\
4 \\
50 \\
250 \\
50\end{array}$ & 75 & $\begin{array}{l}\text { I } \\
\text { I } \\
\text { I } \\
\text { I I } \\
\text { I }\end{array}$ & $\begin{array}{l}4415.530 \\
4416.236 \\
4416.842 \\
4417.215 \\
4417.358\end{array}$ & $\begin{array}{c}40 \mathrm{w} \\
400 \\
200 \mathrm{~h} \\
4 \\
8\end{array}$ & $\begin{array}{r}100 \\
2\end{array}$ & $\begin{array}{l}\text { I } \\
\text { I I } \\
\text { I } \\
\text { I } \\
\text { I }\end{array}$ & $\begin{array}{l}4436.057 \\
4436.284 \\
4436.549 \\
4436.984 \\
4437.435\end{array}$ & $\begin{array}{r}125 \\
150 \\
150 \\
100 \\
75\end{array}$ & $\begin{array}{r}40 \\
40 \\
100\end{array}$ & $\begin{array}{l}\text { I I } \\
\text { I I } \\
\text { I I } \\
\text { I } \\
\text { I }\end{array}$ \\
\hline $\begin{array}{l}4397.295 \\
4397.914 \\
4398.245 \\
4398.642 \\
4399.096\end{array}$ & $\begin{array}{c}2 \\
400 \\
10 \mathrm{w} \\
4 \\
300\end{array}$ & $\begin{array}{r}1 \\
125\end{array}$ & $\begin{array}{l}\text { I } \\
\text { I I } \\
\text { I } \\
\text { I } \\
\text { I I }\end{array}$ & $\begin{array}{l}4417.504 \\
4417.762 \\
4417.980 \\
4418.417 \\
4418.666\end{array}$ & $\begin{array}{r}12 \\
4 \\
12 \\
40 \\
40\end{array}$ & $30^{3 \mathrm{~h}}$ & $\begin{array}{l}\text { I } \\
\text { I } \\
\text { I } \\
\text { I I } \\
\text { I I }\end{array}$ & $\begin{array}{l}4437.718 \\
4438.739 \\
4439.117 \\
4439.848 \\
4440.276\end{array}$ & $\begin{array}{l}25 \\
200 \\
400 \\
20 \\
400 \mathrm{w}\end{array}$ & $\begin{array}{r}2 \\
30 \\
150\end{array}$ & $\begin{array}{l}\text { I I } \\
\text { I } \\
\text { I I } \\
\text { I } \\
\text { I }\end{array}$ \\
\hline $\begin{array}{l}4399.403 \\
4399.969 \\
4400.386 \\
4401.177 \\
4401.215\end{array}$ & $\begin{array}{r}60 \\
200 \\
40 \\
4 \\
20\end{array}$ & $\begin{array}{r}10 \\
15 \\
2\end{array}$ & $\begin{array}{l}\text { I } \\
\text { I } \\
\text { I I } \\
\text { I } \\
\text { I I }\end{array}$ & $\begin{array}{l}4419.015 \\
4419.309 \\
4419.776 \\
4420.135 \\
4420.264\end{array}$ & $\begin{array}{r}40 \\
5 \\
50 \\
40 \\
75\end{array}$ & $\begin{array}{r}20 \\
2 \\
2 \\
2\end{array}$ & $\begin{array}{l}\text { I I } \\
\text { I I } \\
\text { I } \\
\text { I } \\
\text { I }\end{array}$ & $\begin{array}{l}4440.577 \\
4440.858 \\
4441.601 \\
4442.512 \\
4443.070\end{array}$ & $\begin{array}{r}300 \\
400 \\
300 \\
8 \\
200\end{array}$ & $\begin{array}{r}60 \\
150\end{array}$ & $\begin{array}{l}\text { I I } \\
\text { I I } \\
\text { I } \\
\text { I } \\
\text { I }\end{array}$ \\
\hline
\end{tabular}


Table 1. Wavelengths of Thorium Spectra - Continued

\begin{tabular}{|c|c|c|c|c|c|c|c|c|c|c|c|}
\hline \multirow{2}{*}{$\begin{array}{l}\text { Wave- } \\
\text { length }\end{array}$} & \multicolumn{2}{|c|}{ Intensity } & \multirow{2}{*}{$\begin{array}{l}\text { Spec- } \\
\text { trum }\end{array}$} & \multirow{2}{*}{$\begin{array}{l}\text { Wave- } \\
\text { length }\end{array}$} & \multicolumn{2}{|c|}{ Intensity } & \multirow{2}{*}{$\begin{array}{l}\text { Spec- } \\
\text { trum }\end{array}$} & \multirow{2}{*}{$\begin{array}{l}\text { Wave- } \\
\text { length }\end{array}$} & \multicolumn{2}{|c|}{ Intensity } & \multirow{2}{*}{$\begin{array}{l}\text { Spec- } \\
\text { trum }\end{array}$} \\
\hline & Lamp & Spark & & & Lamp & Spark & & & Lamp & Spark & \\
\hline $\begin{array}{l}4443.649 \\
4443.764 \\
4443.916 \\
4445.014 \\
4445.308 i\end{array}$ & $\begin{array}{r}75 \\
30 \\
15 \\
200 \\
300\end{array}$ & $\begin{array}{r}30 \\
2\end{array}$ & $\begin{array}{l}\text { I } \\
\text { I } \\
\text { I } \\
\text { II } \\
\text { I }\end{array}$ & $\begin{array}{l}4463.825 \\
4464.120 \\
4464.708 \\
4464.852 \\
4465.968\end{array}$ & $\begin{array}{l}125 \\
15 \\
20 \mathrm{w} \\
20 \\
100\end{array}$ & 8 & $\begin{array}{l}\text { I } \\
\text { I } \\
\text { I } \\
\text { I } \\
\text { II }\end{array}$ & $\begin{array}{l}4485.783 \\
4486.653 \\
4486.897 \\
4487.496 \\
4488.317\end{array}$ & $\begin{array}{l}150 \\
200 \\
200 \\
300 \\
150\end{array}$ & $\begin{array}{r}40 \\
150 \\
2 \\
200\end{array}$ & $\begin{array}{l}\text { II } \\
\text { II } \\
\text { I } \\
\text { II } \\
\text { I }\end{array}$ \\
\hline $\begin{array}{l}4445.889 \\
4446.442 \\
4446.576 \\
4446.775 \\
4447.124\end{array}$ & $\begin{array}{r}200 \\
50 \\
40\end{array}$ & $\begin{array}{l}30 \mathrm{w} \\
15 \mathrm{w}\end{array}$ & $\begin{array}{l}\text { I } \\
\text { I } \\
\text { I } \\
\text { I I I } \\
\text { I I I }\end{array}$ & $\begin{array}{l}4465.3411 \\
4466.619 \\
4467.396 \\
4467.747 \\
4468.327\end{array}$ & $\begin{array}{r}300 \\
30 \\
4 \\
20 \\
150\end{array}$ & $\begin{array}{r}250 \\
4 \\
6 \\
2\end{array}$ & $\begin{array}{l}\text { II } \\
\text { I } \\
\text { I } \\
\text { II } \\
\text { I }\end{array}$ & $\begin{array}{l}4488.680 \\
4489.506 \\
4489.696 \\
4490.209 \\
4490.678\end{array}$ & $\begin{array}{r}400 \\
200 \\
15 \\
50\end{array}$ & $\begin{array}{l}200 \\
10 \mathrm{w} \\
15\end{array}$ & $\begin{array}{l}\text { I I } \\
\text { I I I } \\
\text { I } \\
\text { I I } \\
\text { I }\end{array}$ \\
\hline $\begin{array}{l}4447.221 \\
4447.834 \\
4448.545 \\
4449.431 \\
4449.884\end{array}$ & $\begin{array}{r}100 \\
400 \\
50 \\
12 \\
6\end{array}$ & $\begin{array}{r}100 \\
10 \\
1\end{array}$ & $\begin{array}{l}\text { I } \\
\text { II } \\
\text { II } \\
I \\
I\end{array}$ & $\begin{array}{l}4469.185 \\
4469.530 \\
4470.010 \\
4470.984 \\
4471.774\end{array}$ & $\begin{array}{r}30 \\
400 \\
2 \\
150 \\
20\end{array}$ & $\begin{array}{r}3 \\
4 \\
30\end{array}$ & $\begin{array}{l}\text { I } \\
\text { I } \\
\text { II } \\
I \\
\text { II }\end{array}$ & $\begin{array}{l}4491.065 \\
4491.374 \\
4491.712 \\
4491.890 \\
4492.126\end{array}$ & $\begin{array}{r}6 \\
12 \\
8 \\
5 \\
40\end{array}$ & $\begin{array}{l}10 \\
20 \\
10\end{array}$ & $\begin{array}{l}\text { I } \\
\text { I I } \\
\text { II } \\
\text { II } \\
\text { I }\end{array}$ \\
\hline $\begin{array}{l}4450.773 \\
4451.052 \\
4451.347 \\
4451.729 \\
4452.564\end{array}$ & $\begin{array}{r}200 \\
10 \\
50 \\
20 \\
200\end{array}$ & $\begin{array}{r}2 \\
15\end{array}$ & $\begin{array}{l}\text { I } \\
\text { I I } \\
\text { I } \\
\text { I } \\
\text { I }\end{array}$ & $\begin{array}{l}4471.884 \\
4471.985 \\
4472.250 \\
4472.618 \\
4472.839\end{array}$ & $\begin{array}{r}6 \\
10 \\
200 \\
15 \\
40\end{array}$ & $\begin{array}{r}1 \\
40\end{array}$ & $\begin{array}{l}\text { I } \\
\text { I } \\
\text { II } \\
\text { I } \\
\text { I }\end{array}$ & $\begin{array}{l}4492.237 \\
4492.579 \\
4492.693 \\
4493.3341 \\
4494.071\end{array}$ & $\begin{array}{r}25 \\
15 \\
20 \\
1200 \\
8\end{array}$ & $\begin{array}{r}30 \\
3\end{array}$ & $\begin{array}{l}\text { I I } \\
\text { I } \\
\text { I } \\
\text { I } \\
\text { I }\end{array}$ \\
\hline $\begin{array}{l}4453.187 \\
4453.614 \\
4453.856 \\
4454.144 \\
4454.510\end{array}$ & $\begin{array}{r}15 \\
10 \\
4 \\
30 \\
30\end{array}$ & 4 & $\begin{array}{l}\text { II } \\
\text { I } \\
\text { I } \\
\text { I } \\
\text { II }\end{array}$ & $\begin{array}{l}4474.071 \\
4474.397 \\
4474.845 \\
4475.219 \\
4475.760\end{array}$ & $\begin{array}{r}300 \\
4 \\
200 \\
250 \\
40\end{array}$ & 125 & $\begin{array}{l}\text { II } \\
\text { I } \\
\text { I } \\
\text { I } \\
\text { I }\end{array}$ & $\begin{array}{l}4494.705 \\
4494.965 \\
4495.244 \\
4495.490 \\
4495.604\end{array}$ & $\begin{array}{l}20 \\
20 \\
20 \\
20 \\
10\end{array}$ & $\begin{array}{r}15 \\
30 \\
2\end{array}$ & $\begin{array}{l}\text { I } \\
\text { I I } \\
\text { I I } \\
\text { I I } \\
\text { I }\end{array}$ \\
\hline $\begin{array}{l}4454.748 \\
4455.020 \\
4455.278 \\
4455.730 \\
4455.859\end{array}$ & $\begin{array}{l}8 \\
20 \\
20 \\
20 \\
80\end{array}$ & $\begin{array}{r}75 \\
10 \\
6\end{array}$ & $\begin{array}{l}\text { II } \\
\text { II } \\
\text { II } \\
\text { II } \\
\text { I }\end{array}$ & $\begin{array}{l}4476.903 \\
4477.040 \\
4477.218 \\
4477.471 \\
4477.608\end{array}$ & $\begin{array}{r}10 \\
4 \\
8 \\
4 \\
2\end{array}$ & $\begin{array}{l}2 \\
2\end{array}$ & $\begin{array}{l}\text { II } \\
\text { II } \\
\text { I } \\
\text { I } \\
\text { I }\end{array}$ & $\begin{array}{l}4496.318 \\
4496.646 \\
4496.749 \\
4497.921 \\
4498.151\end{array}$ & $\begin{array}{r}200 \\
20 \\
200 \\
10\end{array}$ & $\begin{array}{c}200 \\
2 \mathrm{w} \\
3 \\
1\end{array}$ & $\begin{array}{l}\text { II } \\
\text { I } \\
\text { II } \\
\text { I } \\
\text { I }\end{array}$ \\
\hline $\begin{array}{l}4456.480 \\
4456.678 \\
4456.715 \\
4457.071 \\
4457.219\end{array}$ & $\begin{array}{r}10 \\
10 \\
100 \\
100\end{array}$ & $\begin{array}{l}25 \\
15\end{array}$ & $\begin{array}{l}\text { I } \\
\text { II } \\
\text { I } \\
\text { I I } \\
\text { I }\end{array}$ & $\begin{array}{l}4478.413 \\
4478.587 \\
4478.782 \\
4479.052 \\
4479.164\end{array}$ & $\begin{array}{l}30 \\
40 \\
10 \\
12 \\
20\end{array}$ & 20 & $\begin{array}{l}\text { I } \\
\text { I } \\
\text { I } \\
\text { II }\end{array}$ & $\begin{array}{l}4498.957 \\
4499.399 \\
4499.993 \\
4500.578 \\
4501.112\end{array}$ & $\begin{array}{l}800 \\
150 \mathrm{w} \\
500 \\
30 \mathrm{~h} \\
40\end{array}$ & $\begin{array}{r}20 \\
200 \\
2 \\
1\end{array}$ & $\begin{array}{l}\text { I } \\
\text { I } \\
I \\
I \\
I\end{array}$ \\
\hline $\begin{array}{l}4458.0021 \\
4458.737 \\
4459.531 \\
4459.967 \\
4460.196\end{array}$ & $\begin{array}{r}600 \\
75 \\
80 \\
8 \\
8\end{array}$ & 20 & $\begin{array}{l}\text { I } \\
\text { I } \\
\text { I } \\
\text { I } \\
\text { I }\end{array}$ & $\begin{array}{l}4479.643 \\
4480.284 \\
4480.557 \\
4480.818 \\
4481.155\end{array}$ & $\begin{array}{r}200 \\
10 \\
8 \\
300\end{array}$ & $\begin{array}{l}75 \\
15\end{array}$ & $\begin{array}{l}\text { I } \\
\text { I } \\
\text { I } \\
\text { II } \\
\text { II I }\end{array}$ & $\begin{array}{l}4501.284 \\
4502.378 \\
4503.008 \\
4503.208 \\
4504.277\end{array}$ & $\begin{array}{r}4 \\
10 \\
8 \\
10 \\
20\end{array}$ & 1 & $\begin{array}{l}\text { I } \\
\text { I } \\
\text { I } \\
\text { I } \\
\text { I }\end{array}$ \\
\hline $\begin{array}{l}4460.346 \\
4461.083 \\
4461.238 \\
4461.508 \\
4461.715\end{array}$ & $\begin{array}{r}5 \\
600 \\
50 \\
50\end{array}$ & $100^{3 w}$ & $\begin{array}{l}\text { I } \\
\text { II } \\
\text { I } \\
\text { I } \\
\text { I I }\end{array}$ & $\begin{array}{l}4481.192 \\
4481.641 \\
4482.169 \mathrm{i} \\
4482.771 \\
4482.903\end{array}$ & $\begin{array}{r}15 \\
20 \\
300 \\
8 \\
2\end{array}$ & 20 & $\begin{array}{l}\text { I } \\
\text { II } \\
\text { I } \\
\text { I } \\
\text { I }\end{array}$ & $\begin{array}{l}4504.641 \\
4505.221 \\
4506.493 \\
4507.434 \\
4508.037\end{array}$ & $\begin{array}{r}50 \\
400 \\
200 \\
30 \\
80\end{array}$ & $\begin{array}{r}1 \\
15 \\
4 \\
1\end{array}$ & $\begin{array}{l}\text { I } \\
\text { I } \\
\text { I } \\
\text { I } \\
\text { I }\end{array}$ \\
\hline $\begin{array}{l}4462.490 \\
4462.741 \\
4463.067 \\
4463.197 \\
4463.674\end{array}$ & $\begin{array}{r}4 \\
5 \\
8 \\
8 \\
75\end{array}$ & $\begin{array}{r}10 \\
2\end{array}$ & $\begin{array}{l}\text { I } \\
\text { I } \\
\text { I I } \\
\text { II } \\
\text { I }\end{array}$ & $\begin{array}{l}4483.350 \\
4484.054 \\
4484.270 \\
4484.614 \\
4484.966\end{array}$ & $\begin{array}{r}250 \\
75 \\
15 \\
6 \\
4\end{array}$ & $\begin{array}{r}2 \\
100 \\
2 \\
1\end{array}$ & $\begin{array}{l}\text { I } \\
\text { I I } \\
\text { II } \\
\text { I } \\
\text { I }\end{array}$ & $\begin{array}{l}4508.146 \\
4508.622 \\
4508.841 \\
4509.593 \\
4509.971\end{array}$ & $\begin{array}{r}30 \\
40 \\
4 \\
4 \\
20\end{array}$ & $\begin{array}{r}12 \\
12 \\
3 \\
2 \\
2\end{array}$ & $\begin{array}{l}\text { II } \\
\text { I I } \\
\text { II } \\
\text { II } \\
\text { I I }\end{array}$ \\
\hline
\end{tabular}


Table 1. Wavelengths of Thorium Spectra - Continued

\begin{tabular}{|c|c|c|c|c|c|c|c|c|c|c|c|}
\hline \multirow{2}{*}{$\begin{array}{l}\text { Wave- } \\
\text { length }\end{array}$} & \multicolumn{2}{|c|}{ Intensity } & \multirow{2}{*}{$\begin{array}{l}\text { Spec- } \\
\text { trum }\end{array}$} & \multirow{2}{*}{$\begin{array}{l}\text { Wave- } \\
\text { length }\end{array}$} & \multicolumn{2}{|c|}{ Intersity } & \multirow{2}{*}{$\begin{array}{l}\text { Spec- } \\
\text { trum }\end{array}$} & \multirow{2}{*}{$\begin{array}{l}\text { Wave- } \\
\text { length }\end{array}$} & \multicolumn{2}{|c|}{ Intensity } & \multirow{2}{*}{$\begin{array}{l}\text { Spec- } \\
\text { trum }\end{array}$} \\
\hline & Lamp & Spark & & & Lamp & Spark & & & $\operatorname{Lamp}$ & Spark & \\
\hline $\begin{array}{l}4510.526 \mathrm{i} \\
4511.293 \\
4511.872 \\
4512.496 \\
4513.232\end{array}$ & $\begin{array}{r}800 \\
5 \\
4 \\
250 \\
200\end{array}$ & $\begin{array}{r}125 \\
2\end{array}$ & $\begin{array}{l}\text { II } \\
\text { I } \\
\text { I } \\
\text { II } \\
\text { I }\end{array}$ & $\begin{array}{l}4534.122 \\
4534.407 \\
4535.254 i \\
4535.987 \\
4536.267\end{array}$ & $\begin{array}{r}250 \\
10 \\
300 \\
30 \\
5\end{array}$ & $\begin{array}{r}250 \\
8\end{array}$ & $\begin{array}{l}\text { II } \\
\text { I } \\
\text { I } \\
\text { I } \\
\text { I }\end{array}$ & $\begin{array}{l}4554.425 \\
4554.583 \\
4554.687 \\
4554.815 \\
4555.523\end{array}$ & $\begin{array}{r}10 \\
2 \\
2 \\
2 \\
10\end{array}$ & 200 & $\begin{array}{l}\text { I } \\
\text { I } \\
\text { I } \\
\text { II } \\
\text { I I }\end{array}$ \\
\hline $\begin{array}{l}4513.688 \\
4514.047 \\
4514.550 \\
4515.121 \\
4515.644\end{array}$ & $\begin{array}{r}300 \\
20 \\
10 \\
300 \\
10\end{array}$ & 25 & $\begin{array}{l}\text { I } \\
\text { I } \\
\text { I } \\
\text { I } \\
\text { I }\end{array}$ & $\begin{array}{l}4536.425 \\
4536.762 \\
4536.872 \\
4537.085 \\
4537.760\end{array}$ & $\begin{array}{r}5 \\
5 \\
5 \\
200 \\
4\end{array}$ & 300 & $\begin{array}{l}\text { I I } \\
\text { I } \\
\text { I } \\
\text { I I } \\
\text { I }\end{array}$ & $\begin{array}{l}4555.700 \\
4555.813 \mathrm{i} \\
4556.217 \\
4556.366 \\
4556.802\end{array}$ & $\begin{array}{r}500 \\
6 \\
8 \\
6\end{array}$ & $\begin{array}{r}200 \\
4\end{array}$ & $\begin{array}{l}\text { I I I } \\
\text { I } \\
\text { I } \\
\text { I } \\
\text { I I }\end{array}$ \\
\hline $\begin{array}{l}4515.981 \\
4516.542 \\
4517.040 \\
4517.458 \\
4517.658\end{array}$ & $\begin{array}{r}250 \\
20 \\
50 \\
6 \\
10\end{array}$ & $\begin{array}{r}200 \\
4\end{array}$ & $\begin{array}{l}\text { I I } \\
\text { I } \\
\text { II } \\
\text { I } \\
\text { I }\end{array}$ & $\begin{array}{l}4538.116 \\
4538.279 \\
4538.837 \\
4539.232 \\
4539.736\end{array}$ & $\begin{array}{r}2 \\
1 \\
18 \\
6 \\
3\end{array}$ & $\begin{array}{r}1 \\
50\end{array}$ & $\begin{array}{l}\text { I } \\
\text { I I } \\
\text { I I } \\
\text { I } \\
\text { I }\end{array}$ & $\begin{array}{l}4557.056 \\
4557.557 \\
4558.349 \\
4559.059 \\
4559.313\end{array}$ & $\begin{array}{r}2 \\
6 \\
150 \\
20 \\
10\end{array}$ & $\begin{array}{l}2 \\
1\end{array}$ & $\begin{array}{l}\text { I } \\
\text { I } \\
\text { I } \\
\text { I } \\
\text { I }\end{array}$ \\
\hline $\begin{array}{l}4517.899 \\
4518.170 \\
4518.356 \\
4518.641 \\
4519.260\end{array}$ & $\begin{array}{r}6 \\
15 \\
40 \\
200\end{array}$ & $\begin{array}{c}200 \mathrm{w} \\
30 \\
2\end{array}$ & $\begin{array}{l}\text { I } \\
\text { I I I } \\
\text { I } \\
\text { I I } \\
\text { I }\end{array}$ & $\begin{array}{l}4540.408 \\
4541.003 \\
4541.359 \\
4541.523 \\
4541.621\end{array}$ & $\begin{array}{r}75 \\
300 \\
3 \\
4 \\
4\end{array}$ & 200 & $\begin{array}{l}\text { II } \\
\text { I } \\
\text { I } \\
\text { I } \\
\text { II }\end{array}$ & $\begin{array}{l}4559.662 \\
4559.799 \\
4560.069 \\
4560.166 \\
4560.396\end{array}$ & $\begin{array}{l}5 \\
2 \\
2 \\
3 \\
7\end{array}$ & $\begin{array}{l}2 \\
2\end{array}$ & $\begin{array}{l}\text { I } \\
\text { I I } \\
\text { I I } \\
\text { I } \\
\text { I }\end{array}$ \\
\hline $\begin{array}{l}4519.750 \\
4520.034 \\
4520.919 \\
4521.219 \\
4521.732\end{array}$ & $\begin{array}{r}150 \\
2 \\
500 \\
40\end{array}$ & $\begin{array}{r}12 \\
3 \\
30 \\
250\end{array}$ & $\begin{array}{l}\text { I I } \\
\text { I I } \\
\text { I I } \\
\text { I } \\
\text { I }\end{array}$ & $\begin{array}{l}4542.430 \\
4542.750 \\
4543.212 \\
4543.421 \\
4543.667\end{array}$ & $\begin{array}{r}3 \\
4 \\
25 \\
30 \\
2\end{array}$ & $\begin{array}{r}6 \\
75\end{array}$ & $\begin{array}{l}\text { I I } \\
\text { I } \\
\text { II } \\
\text { I } \\
\text { I }\end{array}$ & $\begin{array}{l}4560.668 \\
4561.361 \\
4562.088 \\
4562.472 \\
4563.282\end{array}$ & $\begin{array}{r}6 \\
500 \\
100 \\
5 \\
300\end{array}$ & $\begin{array}{c}3 \\
7 \\
3 \\
20 \mathrm{~h} \\
500\end{array}$ & $\begin{array}{l}\text { I I } \\
\text { I } \\
\text { I } \\
\text { II } \\
\text { I I }\end{array}$ \\
\hline $\begin{array}{l}4522.315 \\
4522.784 \\
4523.472 \\
4524.143 \\
4524.600\end{array}$ & $\begin{array}{r}30 \\
50 \\
12 \\
30 \\
4\end{array}$ & $\begin{array}{r}100 \\
1 \\
2\end{array}$ & $\begin{array}{l}\text { I } \\
\text { I I } \\
\text { I } \\
\text { I } \\
\text { II }\end{array}$ & $\begin{array}{l}4543.865 \\
4544.523 \\
4544.896 \\
4545.362 \\
4545.452\end{array}$ & $\begin{array}{r}6 \\
250 \\
10 \\
2 \\
2\end{array}$ & $\begin{array}{r}200 \\
4\end{array}$ & $\begin{array}{l}\text { I } \\
\text { II } \\
\text { II } \\
\text { I } \\
\text { I }\end{array}$ & $\begin{array}{l}4563.662 \\
4564.186 \\
4564.845 \\
4565.797 \\
4566.245\end{array}$ & $\begin{array}{r}300 \\
20 \\
30 \\
25 \\
2\end{array}$ & $\begin{array}{l}40 \\
60\end{array}$ & $\begin{array}{l}\text { I } \\
\text { I I } \\
\text { I } \\
\text { I } \\
\text { I I }\end{array}$ \\
\hline $\begin{array}{l}4524.854 \\
4525.102 \\
4525.444 \\
4525.758 \\
4526.044\end{array}$ & $\begin{array}{r}30 \\
50 \\
10 \\
8 \\
15\end{array}$ & $\begin{array}{r}40 \\
100\end{array}$ & $\begin{array}{l}\text { I I } \\
\text { II } \\
\text { I } \\
\text { I } \\
\text { I I }\end{array}$ & $\begin{array}{l}4545.839 \\
4545.917 \\
4546.144 \\
4546.700 \\
4546.843\end{array}$ & $\begin{array}{r}350 \\
5 \\
25 \\
8\end{array}$ & $\begin{array}{r}25 \\
4 \\
12 \\
20\end{array}$ & $\begin{array}{l}\text { II } \\
\text { I } \\
\text { I I } \\
\text { I I } \\
\text { I }\end{array}$ & $\begin{array}{l}4566.660 \\
4566.959 \\
4567.249 \\
4568.142 \\
4568.525\end{array}$ & $\begin{array}{c}25 \\
3 \\
160 \mathrm{~h} \\
30 \\
12\end{array}$ & $\begin{array}{r}35 \\
2\end{array}$ & $\begin{array}{l}\text { I I } \\
\text { I } \\
\text { I } \\
\text { I } \\
\text { I }\end{array}$ \\
\hline $\begin{array}{l}4526.921 \\
4527.182 \\
4527.728 \\
4528.034 \\
4528.419\end{array}$ & $\begin{array}{r}12 \\
3 \\
18 \\
30 \\
12\end{array}$ & $\begin{array}{c}5 w \\
35 \\
1\end{array}$ & $\begin{array}{l}\text { I } \\
\text { I I } \\
\text { I I } \\
\text { I } \\
\text { I I }\end{array}$ & $\begin{array}{l}4547.098 \\
4547.268 \\
4547.938 \\
4548.344 \\
4548.983\end{array}$ & $\begin{array}{r}70 \\
100 \\
2 \\
100 \\
10\end{array}$ & 2 & $\begin{array}{l}\text { I } \\
\text { I } \\
\text { I } \\
\text { I } \\
\text { I }\end{array}$ & $\begin{array}{l}4569.051 \\
4569.155 \\
4569.719 \\
4570.972 \mathrm{i} \\
4571.203\end{array}$ & $\begin{array}{r}12 \\
10 \\
50 \\
500 \\
40\end{array}$ & 2 & $\begin{array}{l}\text { II } \\
\text { I } \\
\text { I } \\
\text { I } \\
\text { I }\end{array}$ \\
\hline $\begin{array}{l}4528.977 \\
4529.497 \\
4529.933 \\
4530.320 \\
4530.648\end{array}$ & $\begin{array}{r}12 \\
30 \\
10 \\
160 \\
5\end{array}$ & $\begin{array}{r}15 \\
75 \\
\\
5 \\
4\end{array}$ & $\begin{array}{l}\text { I I } \\
\text { I I } \\
\text { I } \\
\text { I } \\
\text { I I }\end{array}$ & $\begin{array}{l}4549.852 \\
4550.184 \\
4550.419 \\
4550.451 \\
4551.490\end{array}$ & $\begin{array}{r}6 \\
3 \\
8 \\
8 \\
160\end{array}$ & $\begin{array}{l}10 \\
75\end{array}$ & $\begin{array}{l}\text { I } \\
\text { I } \\
\text { II } \\
\text { I } \\
\text { I I }\end{array}$ & $\begin{array}{l}4571.533 \\
4572.656 \\
4572.953 \\
4573.259 \\
4573.709\end{array}$ & $\begin{array}{r}12 \\
15 \\
4 \\
6 \\
200\end{array}$ & 1 & $\begin{array}{l}\text { I } \\
\text { I } \\
\text { I I } \\
\text { I } \\
\text { I }\end{array}$ \\
\hline $\begin{array}{l}4531.196 \\
4531.706 \\
4532.261 \\
4533.080 \\
4533.292\end{array}$ & $\begin{array}{l}10 \\
100 \\
175 \\
150 \\
150\end{array}$ & $\begin{array}{l}200 \\
300 \\
250\end{array}$ & $\begin{array}{l}\text { I } \\
\text { I I } \\
\text { I I } \\
\text { I } \\
\text { I I }\end{array}$ & $\begin{array}{l}4551.731 \\
4551.834 \\
4552.158 \\
4553.021 \\
4553.850\end{array}$ & $\begin{array}{r}2 \\
6 \\
250 \\
250 \\
50\end{array}$ & $\begin{array}{r}3 \\
200 \\
75\end{array}$ & $\begin{array}{l}\text { I } \\
\text { I } \\
\text { I } \\
\text { I I } \\
\text { I I }\end{array}$ & $\begin{array}{l}4574.039 \\
4574.308 \\
4575.267 \\
4575.507 \\
4576.496\end{array}$ & $\begin{array}{c}100 \\
50 \\
15 \\
50 \\
30 \mathrm{w}\end{array}$ & $\begin{array}{l}2 \\
5\end{array}$ & $\begin{array}{l}\text { I } \\
\text { I } \\
\text { II } \\
\text { I } \\
\text { I }\end{array}$ \\
\hline
\end{tabular}


Table 1. Wavelengths of Thorium Spectra - Continued

\begin{tabular}{|c|c|c|c|c|c|c|c|c|c|c|c|}
\hline \multirow{2}{*}{$\begin{array}{l}\text { Wave- } \\
\text { length }\end{array}$} & \multicolumn{2}{|c|}{ Intensity } & \multirow{2}{*}{$\begin{array}{l}\text { Spec- } \\
\text { trum }\end{array}$} & \multirow{2}{*}{$\begin{array}{l}\text { Wave- } \\
\text { length }\end{array}$} & \multicolumn{2}{|c|}{ Intensity } & \multirow{2}{*}{$\begin{array}{l}\text { Spec- } \\
\text { trum }\end{array}$} & \multirow{2}{*}{$\begin{array}{l}\text { Wave- } \\
\text { length }\end{array}$} & \multicolumn{2}{|c|}{ Intensity } & \multirow{2}{*}{$\begin{array}{l}\text { Spec- } \\
\text { trum }\end{array}$} \\
\hline & Lamp & Spark & & & Lamp & Spark & & & & Spark & \\
\hline $\begin{array}{l}4576.737 \\
4577.126 \\
4577.587 \\
4577.841 \\
4578.834\end{array}$ & $\begin{array}{l}20 \\
10 \\
20 \\
40 d \\
15\end{array}$ & & $\begin{array}{l}\text { I } \\
\text { I } \\
\text { I } \\
\text { I } \\
\text { I }\end{array}$ & $\begin{array}{l}4599.718 \\
4600.885 \\
4601.383 \\
4601.564 \\
4601.969\end{array}$ & $\begin{array}{l}15 \\
25 \mathrm{~h} \\
8 \\
3 \\
4\end{array}$ & 5 & $\begin{array}{l}\text { I } \\
\text { I } \\
\text { I } \\
\text { I } \\
\text { I I }\end{array}$ & $\begin{array}{l}4623.551 \\
4623.897 \\
4624.141 \\
4624.310 \\
4624.689\end{array}$ & $\begin{array}{l}4 \\
15 \\
15 \\
15 \\
12\end{array}$ & $\begin{array}{r}100 \\
75\end{array}$ & $\begin{array}{l}\text { I } \\
\text { II } \\
\text { I I } \\
\text { I } \\
\text { I }\end{array}$ \\
\hline $\begin{array}{l}4579.019 \\
4579.331 \\
4579.839 \\
4580.402 \\
4580.765\end{array}$ & $\begin{array}{l}15 \\
25 \\
40 \\
12 \\
10\end{array}$ & $\begin{array}{l}2 \\
2\end{array}$ & $\begin{array}{l}\text { I I } \\
\text { I } \\
\text { I } \\
\text { I } \\
\text { I I }\end{array}$ & $\begin{array}{l}4602.904 \\
4603.160 \\
4603.944 \\
4604.133 \\
4604.668\end{array}$ & $\begin{array}{r}75 \\
175 \\
4 \\
2 \\
5\end{array}$ & $\begin{array}{l}75 \\
6 \\
3 \\
5\end{array}$ & $\begin{array}{l}\text { I I } \\
\text { I } \\
\text { I I } \\
\text { I I } \\
\text { I I }\end{array}$ & $\begin{array}{l}4625.044 \\
4625.286 \\
4625.503 \\
4626.139 \\
4626.648\end{array}$ & $\begin{array}{r}30 \\
15 \\
18 \\
1 \\
8\end{array}$ & $\begin{array}{r}125 \\
3 \\
35\end{array}$ & $\begin{array}{l}\text { I I } \\
\text { I } \\
\text { I } \\
\text { I I } \\
\text { I I }\end{array}$ \\
\hline $\begin{array}{l}4581.195 \\
4581.582 \\
4582.441 \\
4582.536 \\
4582.782\end{array}$ & $\begin{array}{r}160 \\
50 \\
10 \\
4 \\
4\end{array}$ & $\begin{array}{r}3 \\
10 \\
8 \\
4\end{array}$ & $\begin{array}{l}\text { I } \\
\text { I I } \\
\text { II } \\
\text { I } \\
\text { II }\end{array}$ & $\begin{array}{l}4605.233 \\
4605.433 \\
4605.839 \\
4606.308 \\
4606.515\end{array}$ & $\begin{array}{r}6 \\
1 \\
7 \\
2 \\
100\end{array}$ & $\begin{array}{l}25 \\
5 \mathrm{~h} \\
75\end{array}$ & $\begin{array}{l}\text { I I } \\
\text { I } \\
\text { I } \\
\text { I I } \\
\text { I I }\end{array}$ & $\begin{array}{l}4627.092 \\
4627.299 \\
4627.641 \\
4627.876 \\
4628.215\end{array}$ & $\begin{array}{r}4 \\
125 \\
6 \\
5 \\
125\end{array}$ & $\begin{array}{r}1 \\
1 \\
20 \\
1\end{array}$ & $\begin{array}{l}\text { I I } \\
\text { I } \\
\text { I I } \\
\text { I } \\
\text { I }\end{array}$ \\
\hline $\begin{array}{l}4583.080 \\
4583.668 \\
4583.886 \\
4584.371 \\
4584.662\end{array}$ & $\begin{array}{l}6 \\
15 \\
10 \\
50 \\
20\end{array}$ & $\begin{array}{l}12 \\
75\end{array}$ & $\begin{array}{l}\text { I } \\
\text { II } \\
\text { I } \\
\text { I I } \\
\text { I }\end{array}$ & $\begin{array}{l}4607.399 \\
4607.683 \\
4607.953 \\
4608.630 \\
4609.382\end{array}$ & $\begin{array}{r}40 \\
10 \\
125 \\
75 \\
125\end{array}$ & $\begin{array}{r}1 \\
200\end{array}$ & $\begin{array}{l}\text { I } \\
\text { I } \\
\text { I } \\
\text { I } \\
\text { I I }\end{array}$ & $\begin{array}{l}4628.568 \\
4629.031 \\
4629.355 \\
4629.771 \\
4630.351\end{array}$ & $\begin{array}{r}30 \\
8 \\
20 \\
25 \\
5\end{array}$ & $\begin{array}{l}20 \\
25 \\
50 \\
30 \\
40\end{array}$ & $\begin{array}{l}\text { I I } \\
\text { I I } \\
\text { I I } \\
\text { I I } \\
\text { I I }\end{array}$ \\
\hline $\begin{array}{l}4585.138 \\
4585.414 \\
4586.063 \\
4586.279 \\
4586.620\end{array}$ & $\begin{array}{l}12 \mathrm{w} \\
1 \\
20 \\
20 \\
20\end{array}$ & $\begin{array}{r}2 \\
75 \\
25\end{array}$ & $\begin{array}{l}\text { I } \\
\text { I } \\
\text { I I } \\
\text { I I } \\
\text { I I }\end{array}$ & $\begin{array}{l}4609.872 \\
4610.648 \\
4611.873 \\
4612.183 \\
4612.558\end{array}$ & $\begin{array}{r}75 \\
125 \\
200 \\
3 \\
200\end{array}$ & $\begin{array}{r}150 \\
3 \\
150 \\
2 \\
50\end{array}$ & $\begin{array}{l}\text { I I } \\
\text { I } \\
\text { I I } \\
\text { I I } \\
\text { I I }\end{array}$ & $\begin{array}{l}4630.639 \\
4631.035 \\
4631.156 \\
4631.761 \mathrm{i} \\
4632.520\end{array}$ & $\begin{array}{c}4 \\
8 \\
3 \mathrm{w} \\
300 \\
4\end{array}$ & $100 \mathrm{w}$ & $\begin{array}{l}\text { I I } \\
\text { I } \\
\text { I } \\
\text { I I } \\
\text { I }\end{array}$ \\
\hline $\begin{array}{l}4587.219 \\
4587.763 \\
4588.218 \\
4588.427 \mathrm{i} \\
4589.122\end{array}$ & $\begin{array}{r}5 \\
18 \\
400 \\
100\end{array}$ & $\begin{array}{r}4 \\
15 \\
100 \\
5 \\
400\end{array}$ & $\begin{array}{l}\text { I I } \\
\text { I I } \\
\text { I I I } \\
\text { I } \\
\text { I I }\end{array}$ & $\begin{array}{l}4613.484 \\
4613.624 \\
4614.000 \\
4614.185 \\
4614.288\end{array}$ & $\begin{array}{r}5 \\
125 \\
4 \\
5 \\
2\end{array}$ & $\begin{array}{c}50 \\
20 \\
1\end{array}$ & $\begin{array}{l}\text { I I } \\
\text { I } \\
\text { I I } \\
\text { I } \\
\text { I I }\end{array}$ & $\begin{array}{l}4633.772 \\
4633.827 \\
4635.408 \\
4636.458 \\
4636.767\end{array}$ & $\begin{array}{l}300 \\
5 \\
4 \mathrm{w} \\
2\end{array}$ & 75 & $\begin{array}{l}\text { I } \\
\text { I I } \\
\text { I } \\
\text { I } \\
\text { I }\end{array}$ \\
\hline $\begin{array}{l}4589.665 \\
4591.070 \\
4591.418 \\
4591.591 \\
4592.122\end{array}$ & $\begin{array}{l}250 \\
2 \mathrm{w} \\
4 \\
3 \\
4\end{array}$ & 125 & $\begin{array}{l}\text { I I } \\
\text { I } \\
\text { I } \\
\text { I } \\
\text { I I }\end{array}$ & $\begin{array}{l}4615.034 \\
4615.338 \\
4615.654 \\
4615.892 \\
4616.454\end{array}$ & $\begin{array}{r}125 \\
70 \\
10 \\
12 \\
15\end{array}$ & $\begin{array}{l}2 \\
2 \\
1\end{array}$ & $\begin{array}{l}\text { I } \\
\text { I } \\
\text { I } \\
\text { I } \\
\text { I }\end{array}$ & $\begin{array}{l}4637.192 \\
4638.135 \\
4638.687 \\
4639.090 \\
4639.526\end{array}$ & $\begin{array}{l}20 \\
60 \mathrm{~h} \\
100 \\
3 \\
75\end{array}$ & $\begin{array}{r}2 \\
40\end{array}$ & $\begin{array}{l}\text { I } \\
\text { I } \\
\text { I } \\
\text { I I } \\
\text { I }\end{array}$ \\
\hline $\begin{array}{l}4592.283 \\
4592.673 \\
4593.286 \\
4593.501 \\
4593.649\end{array}$ & $\begin{array}{r}5 \\
400 \\
20 \\
150\end{array}$ & $\begin{array}{r}30 \\
100 \\
30 \\
20\end{array}$ & $\begin{array}{l}\text { I } \\
\text { I } \\
\text { I I } \\
\text { I I I } \\
\text { I }\end{array}$ & $\begin{array}{l}4617.303 \\
4617.916 \\
4618.316 \\
4618.932 \\
4619.520\end{array}$ & $\begin{array}{r}10 \\
12 \\
9 \\
7 \\
400\end{array}$ & 300 & $\begin{array}{l}\text { I } \\
\text { I } \\
\text { I } \\
\text { I } \\
\text { I I }\end{array}$ & $\begin{array}{l}4639.709 \\
4640.048 \\
4641.232 \\
4641.618 \\
4642.048\end{array}$ & $\begin{array}{l}100 \\
500 \\
250 \\
4 \mathrm{w} \\
15\end{array}$ & $\begin{array}{r}200 \\
200 \\
150 \\
\\
20\end{array}$ & $\begin{array}{l}\text { I I } \\
\text { I I } \\
\text { I I } \\
\text { I } \\
\text { I I }\end{array}$ \\
\hline $\begin{array}{l}4593.975 \\
4595.420 \mathrm{i} \\
4596.312 \\
4596.986 \\
4577.273\end{array}$ & $\begin{array}{l}10 \\
600 \\
125 \mathrm{w} \\
2 \mathrm{w} \\
2 \mathrm{w}\end{array}$ & $\begin{array}{l}20 \\
30 \\
2 \\
2 \mathrm{w}\end{array}$ & $\begin{array}{l}\text { II } \\
\text { I } \\
\text { I } \\
\text { II } \\
\text { I }\end{array}$ & $\begin{array}{l}4620.248 \\
4620.454 \\
4620.721 \\
4621.168 \\
4621.660\end{array}$ & $\begin{array}{c}50 \\
35 \\
12 \\
200 \\
10 \mathrm{w}\end{array}$ & $\begin{array}{r}12 \\
3 \\
2\end{array}$ & $\begin{array}{l}\text { I } \\
\text { I } \\
\text { I I } \\
\text { I } \\
\text { I }\end{array}$ & $\begin{array}{l}4642.273 \\
4642.590 \\
4642.860 \\
4643.563 \\
4644.350\end{array}$ & $\begin{array}{c}1 \\
1 \\
2 \mathrm{w} \\
80 \\
3\end{array}$ & 6 & $\begin{array}{l}\text { I } \\
\text { I } \\
\text { I } \\
\text { I } \\
\text { II }\end{array}$ \\
\hline $\begin{array}{l}4597.904 \\
4598.058 \\
4598.438 \\
4598.942 \\
4599.353\end{array}$ & $\begin{array}{c}4 s \\
2 \\
20 \\
7 \\
5\end{array}$ & 1 & $\begin{array}{l}\text { I } \\
\text { I } \\
\text { I } \\
\text { I } \\
\text { II }\end{array}$ & $\begin{array}{l}4621.867 \\
4622.110 \\
4622.767 \\
4623.119 \\
4623.416\end{array}$ & $\begin{array}{r}3 \\
20 \\
9 \\
10 \\
4\end{array}$ & 4 & $\begin{array}{l}\text { I } \\
\text { I } \\
\text { I } \\
\text { I } \\
\text { I }\end{array}$ & $\begin{array}{l}4644.712 \\
4645.495 \\
4646.151 \\
4646.688 \\
4647.256\end{array}$ & $\begin{array}{r}200 \\
10 \\
4 \\
250 \\
200\end{array}$ & $\begin{array}{r}3 \\
20 \\
4 \\
2 \\
2\end{array}$ & $\begin{array}{l}\text { I } \\
\text { II } \\
\text { II } \\
\text { I } \\
\text { I }\end{array}$ \\
\hline
\end{tabular}


Table 1. Wavelengths of Thorium Spectra - Continued

\begin{tabular}{|c|c|c|c|c|c|c|c|c|c|c|c|}
\hline \multirow{2}{*}{$\begin{array}{l}\text { Wave- } \\
\text { length }\end{array}$} & \multicolumn{2}{|c|}{ Intensity } & \multirow{2}{*}{$\begin{array}{l}\text { Spec- } \\
\text { trum }\end{array}$} & \multirow{2}{*}{$\begin{array}{l}\text { Wave- } \\
\text { length }\end{array}$} & \multicolumn{2}{|c|}{ Intensity } & \multirow{2}{*}{$\begin{array}{l}\text { Spec- } \\
\text { trum }\end{array}$} & \multirow{2}{*}{$\begin{array}{l}\text { Wave- } \\
\text { length }\end{array}$} & \multicolumn{2}{|c|}{ Intensity } & \multirow{2}{*}{$\begin{array}{l}\text { Spec } \\
\text { trum }\end{array}$} \\
\hline & Lamp & park & & & Lamp & Spark & & & Lamp & Spark & \\
\hline $\begin{array}{l}4647.938 \\
4648.169 \\
4648.366 \\
4648.445 \\
4648.865\end{array}$ & $\begin{array}{r}20 \\
2 \\
8 \\
10 \\
4\end{array}$ & 51 & $\begin{array}{l}\text { II } \\
\text { I } \\
\text { I } \\
\text { I } \\
\text { I I }\end{array}$ & $\begin{array}{l}4669.353 \\
4669.684 \\
4669.975 \\
4670.930 \\
4671.531\end{array}$ & $\begin{array}{r}30 \\
20 \\
400 \\
75 \\
1\end{array}$ & $\begin{array}{l}2 \mathrm{w} \\
1 \\
15 \\
2\end{array}$ & $\begin{array}{l}\text { I } \\
\text { I } \\
\text { I } \\
\text { I } \\
\text { I }\end{array}$ & $\begin{array}{l}4694.090 \\
4694.486 \\
4694.898 \\
4695.038 \\
4695.457\end{array}$ & $\begin{array}{r}400 \\
10 \\
400 \\
175\end{array}$ & $\begin{array}{r}500 \\
125 \\
4\end{array}$ & $\begin{array}{l}\text { I I } \\
\text { I } \\
\text { I I I } \\
\text { I } \\
\text { I }\end{array}$ \\
\hline $\begin{array}{l}4649.517 \\
4649.977 \\
4650.235 \\
4650.627 \\
4650.820\end{array}$ & $\begin{array}{r}5 \\
60 \\
150 \\
15 \\
50\end{array}$ & 50 & $\begin{array}{l}\text { I } \\
\text { I } \\
\text { I } \\
\text { I } \\
\text { I I }\end{array}$ & $\begin{array}{l}4671.735 \\
4671.901 \\
4672.443 \\
4673.052 \\
4673.661 \mathrm{i}\end{array}$ & $\begin{array}{r}2 \\
1 \\
75 \\
70 \\
600\end{array}$ & $\begin{array}{r}1 \\
10 \\
20\end{array}$ & $\begin{array}{l}\text { I } \\
\text { I } \\
\text { I I } \\
\text { I } \\
\text { I }\end{array}$ & $\begin{array}{l}4695.821 \\
4696.685 \\
4696.808 \\
4697.190 \\
4697.396\end{array}$ & $\begin{array}{l}8 \\
30 \\
20 \mathrm{~h} \\
8\end{array}$ & $\begin{array}{l}4 \\
1 \\
2\end{array}$ & $\begin{array}{l}\text { I I } \\
\text { I } \\
\text { I } \\
\text { I } \\
\text { I I }\end{array}$ \\
\hline $\begin{array}{l}4650.934 \\
4651.101 \\
4651.555 \\
4651.985 \\
4652.407\end{array}$ & $\begin{array}{r}50 \\
20 \\
500 \\
100 \\
3\end{array}$ & $\begin{array}{r}30 \\
300 \\
30 \\
2\end{array}$ & $\begin{array}{l}\text { I I } \\
\text { I } \\
\text { I I } \\
\text { I I } \\
\text { I I }\end{array}$ & $\begin{array}{l}4674.230 \\
4674.774 \\
4675.050 \\
4675.373 \\
4676.058\end{array}$ & $\begin{array}{r}3 \\
10 \\
3 \\
80 \\
350\end{array}$ & $600 w$ & $\begin{array}{l}\text { I } \\
\text { I I } \\
\text { I } \\
\text { I } \\
\text { I I }\end{array}$ & $\begin{array}{l}4697.597 \\
4698.240 \\
4698.764 \\
4699.104 \\
4699.466\end{array}$ & $\begin{array}{r}9 \\
100 \\
50 \\
10 \\
5\end{array}$ & $\begin{array}{r}10 \\
100 \\
8 \\
5\end{array}$ & $\begin{array}{l}\text { I } \\
\text { I } \\
\text { I I } \\
\text { I I } \\
\text { I I }\end{array}$ \\
\hline $\begin{array}{l}4652.819 \\
4653.080 \\
4654.013 \\
4655.032 \\
4655.200\end{array}$ & $\begin{array}{r}10 \\
3 \\
15 \\
50 \\
150\end{array}$ & $\begin{array}{l}6 \\
21 \\
2 \\
3\end{array}$ & $\begin{array}{l}\text { II } \\
\text { I I } \\
\text { I } \\
\text { I } \\
\text { I }\end{array}$ & $\begin{array}{l}4676.489 \\
4676.970 \\
4678.234 \\
4678.617 \\
4679.015\end{array}$ & $\begin{array}{r}10 \\
75 \\
100 \\
10 \\
10\end{array}$ & $\begin{array}{l}8 \\
15 \\
10 \\
10\end{array}$ & $\begin{array}{l}\text { I } \\
\text { I I } \\
\text { I I } \\
\text { I I } \\
\text { I I }\end{array}$ & $\begin{array}{l}4699.664 \\
4700.141 \\
4700.771 \\
4700.955 \\
4701.440\end{array}$ & $\begin{array}{c}80 \\
200 \\
100 \\
20 \\
12 \mathrm{w}\end{array}$ & $\begin{array}{r}5 \\
200 \\
20\end{array}$ & $\begin{array}{l}\text { I } \\
\text { I I } \\
\text { I } \\
\text { I I } \\
\text { I }\end{array}$ \\
\hline $\begin{array}{l}4655.724 \\
4656.178 \\
4656.552 \\
4656.730 \\
4657.336\end{array}$ & $\begin{array}{r}1 \\
20 \\
20 \\
8 \\
6\end{array}$ & $\begin{array}{c}3 \\
1 \\
10\end{array}$ & $\begin{array}{l}\text { I I } \\
\text { I } \\
\text { I } \\
\text { II } \\
\text { I }\end{array}$ & $\begin{array}{l}4679.529 \\
4679.599 \\
4679.710 \\
4680.244 \\
4680.396\end{array}$ & $\begin{array}{c}3 \mathrm{~h} \\
6 \mathrm{w} \\
2 \\
150 \\
20\end{array}$ & $\begin{array}{l}3 \\
1\end{array}$ & $\begin{array}{l}\text { I } \\
\text { I } \\
\text { I I } \\
\text { I } \\
\text { I }\end{array}$ & $\begin{array}{l}4701.854 \\
4702.315 \\
4702.603 \\
4702.772 \\
4703.202\end{array}$ & $\begin{array}{r}40 \\
50 \\
10 \\
5 \\
25\end{array}$ & $\begin{array}{r}30 \\
3\end{array}$ & $\begin{array}{l}\text { I } \\
\text { I I } \\
\text { II } \\
\text { I } \\
\text { I }\end{array}$ \\
\hline $\begin{array}{l}4658.128 \\
4658.385 \\
4658.516 \\
4658.720 \\
4659.566\end{array}$ & $\begin{array}{r}2 \\
30 \\
30 \\
2 \\
150\end{array}$ & $\begin{array}{r}12 \\
10 \\
2\end{array}$ & $\begin{array}{l}\text { I } \\
\text { I } \\
\text { I I } \\
\text { I I } \\
\text { I }\end{array}$ & $\begin{array}{l}4680.647 \\
4681.188 \\
4682.242 \\
4682.733 \\
4683.385\end{array}$ & $\begin{array}{l}250 \\
100 \\
175 \\
20 \\
250\end{array}$ & $\begin{array}{r}40 \\
20 \\
5\end{array}$ & $\begin{array}{l}\text { I } \\
\text { I I } \\
\text { I } \\
\text { I } \\
\text { I }\end{array}$ & $\begin{array}{l}4703.990 \mathrm{i} \\
4704.499 \\
4704.658 \\
4704.933 \\
4705.294\end{array}$ & $\begin{array}{r}500 \\
5 \\
10 \\
3 \\
10\end{array}$ & $\begin{array}{r}8 \\
4 \\
10 \\
6\end{array}$ & $\begin{array}{l}\text { I } \\
\text { II } \\
\text { I I } \\
\text { I } \\
\text { I }\end{array}$ \\
\hline $\begin{array}{l}4660.392 \\
4661.125 \\
4661.405 \\
4661.581 \\
4661.716\end{array}$ & $\begin{array}{r}100 \\
8 \\
12 \\
6 \\
3\end{array}$ & 100 & $\begin{array}{l}\text { I } \\
\text { I } \\
\text { I I } \\
\text { I } \\
\text { I }\end{array}$ & $\begin{array}{l}4684.113 \\
4684.373 \\
4684.641 \\
4684.813 \\
4685.087\end{array}$ & $\begin{array}{r}15 \\
10 \\
8 \\
3 \\
6\end{array}$ & $\begin{array}{r}18 \\
1\end{array}$ & $\begin{array}{l}\text { I I } \\
\text { I } \\
\text { I } \\
\text { I } \\
\text { I }\end{array}$ & $\begin{array}{l}4705.765 \\
4706.253 \\
4706.572 \\
4706.682 \\
4707.041\end{array}$ & $\begin{array}{r}400 \\
100 \\
20 \\
4 \\
15\end{array}$ & $\begin{array}{l}500 \\
200\end{array}$ & $\begin{array}{l}\text { I I } \\
\text { I I } \\
\text { I } \\
\text { I } \\
\text { I }\end{array}$ \\
\hline $\begin{array}{l}4661.948 \\
4662.215 \\
4662.332 \\
4662.593 \\
4663.202 \mathrm{i}\end{array}$ & $\begin{array}{r}4 \\
2 \\
2 \\
7 \\
200\end{array}$ & 3 & $\begin{array}{l}\text { I } \\
\text { I I } \\
\text { I } \\
\text { I } \\
\text { I }\end{array}$ & $\begin{array}{l}4685.511 \\
4685.973 \\
4686.194 \mathrm{i} \\
4686.579 \\
4688.303\end{array}$ & $\begin{array}{r}20 \\
400 \\
20 \\
15\end{array}$ & $\begin{array}{r}30 \\
5 \\
3 \\
25 \\
15\end{array}$ & $\begin{array}{l}\text { I I } \\
\text { I I } \\
\text { I } \\
\text { I I } \\
\text { II }\end{array}$ & $\begin{array}{l}4707.583 \\
4707.786 \\
4708.100 \\
4708.287 \\
4708.884\end{array}$ & $\begin{array}{r}3 \\
20 \\
40 \\
175 \\
2\end{array}$ & $\begin{array}{r}5 \\
30 \\
80\end{array}$ & $\begin{array}{l}\text { I I } \\
\text { I } \\
\text { I I } \\
\text { I } \\
\text { I }\end{array}$ \\
\hline $\begin{array}{l}4664.308 \\
4664.540 \\
4664.796 \\
4664.972 \\
4665.711\end{array}$ & $\begin{array}{r}2 \\
2 \\
6 \\
20 \\
20\end{array}$ & 3 & $\begin{array}{l}\text { I } \\
\text { I I } \\
\text { I } \\
\text { I } \\
\text { I }\end{array}$ & $\begin{array}{l}4688.465 \\
4689.200 \\
4690.352 \\
4690.642 \\
4691.068\end{array}$ & $\begin{array}{l}250 \\
200 \mathrm{~d} \\
20 \\
120 \\
100\end{array}$ & $\begin{array}{l}300 \\
200 \\
100 \\
300\end{array}$ & $\begin{array}{l}\text { I } \\
\text { I I } \\
\text { I I } \\
\text { I } \\
\text { I I }\end{array}$ & $\begin{array}{l}4709.354 \\
4710.636 \\
4710.987 \\
4711.130 \\
4711.411\end{array}$ & $\begin{array}{r}6 \\
5 \\
20 \\
2 \\
40\end{array}$ & 8 & $\begin{array}{l}\text { I } \\
\text { I } \\
\text { I } \\
\text { I I } \\
\text { I }\end{array}$ \\
\hline $\begin{array}{l}4666.009 \\
4666.518 \\
4666.793 \\
4668.172 \mathrm{i} \\
4668.944\end{array}$ & $\begin{array}{r}125 \\
75 \\
400 \\
700 \\
3\end{array}$ & $\begin{array}{r}150 \\
6 \\
10\end{array}$ & $\begin{array}{l}\text { I I } \\
\text { I } \\
\text { I } \\
\text { I } \\
\text { I }\end{array}$ & $\begin{array}{l}4691.637 \\
4691.880 \\
4692.486 \\
4693.111 \\
4693.535\end{array}$ & $\begin{array}{r}150 \\
20 \\
2 \\
2 \\
3\end{array}$ & $\begin{array}{l}4 \\
2 \\
3 w\end{array}$ & $\begin{array}{l}\text { I } \\
\text { I } \\
\text { I } \\
\text { I I } \\
\text { I }\end{array}$ & $\begin{array}{l}4711.999 \\
4712.457 \\
4712.842 \\
4713.798 \\
4714.126\end{array}$ & $\begin{array}{r}15 \\
500 \\
150 \\
20 \\
10\end{array}$ & $\begin{array}{r}125 \\
2 \\
10 \\
3\end{array}$ & $\begin{array}{l}\text { I } \\
\text { I } \\
\text { I } \\
\text { I I } \\
\text { I I }\end{array}$ \\
\hline
\end{tabular}


Table 1. Wavelengths of Thorium Spectra - Continued

\begin{tabular}{|c|c|c|c|c|c|c|c|c|c|c|c|}
\hline \multirow{2}{*}{$\begin{array}{l}\text { Wave- } \\
\text { length }\end{array}$} & \multicolumn{2}{|c|}{ Intensity } & \multirow{2}{*}{$\begin{array}{l}\text { Spec- } \\
\text { trum }\end{array}$} & \multirow{2}{*}{$\begin{array}{l}\text { Wave- } \\
\text { length }\end{array}$} & \multicolumn{2}{|c|}{ Intensity } & \multirow{2}{*}{$\begin{array}{l}\text { Spec- } \\
\text { trum }\end{array}$} & \multirow{2}{*}{$\begin{array}{l}\text { Wave- } \\
\text { length }\end{array}$} & \multicolumn{2}{|c|}{ Intensity } & \multirow{2}{*}{$\begin{array}{l}\text { Spec- } \\
\text { trum }\end{array}$} \\
\hline & $a m p$ & Spark & & & $a m p$ & Spark & & & L & Spark & \\
\hline $\begin{array}{l}4714.672 \\
4714.860 \\
4715.432 \\
4716.014 \\
4716.594\end{array}$ & $\begin{array}{r}20 \\
4 \\
125 \\
100 \\
4\end{array}$ & $\begin{array}{r}10 \\
100 \\
30 \\
5\end{array}$ & $\begin{array}{l}\text { I } \\
\text { I I } \\
\text { I I } \\
\text { I I } \\
\text { I I }\end{array}$ & $\begin{array}{l}4734.758 \\
4735.237 \\
4735.346 \\
4735.470 \\
4735.723\end{array}$ & $\begin{array}{l}4 \\
2 \\
6 \\
3 \\
2\end{array}$ & $\begin{array}{l}3 \\
6\end{array}$ & $\begin{array}{l}\text { II } \\
\text { I I } \\
\text { I } \\
\text { I } \\
\text { I I }\end{array}$ & $\begin{array}{r}4753.807 \\
4754.199 \\
14754.379 \\
4754.933 \\
4755.371\end{array}$ & $\begin{array}{l}3 \\
3 \\
2 \\
8 \\
3\end{array}$ & $\begin{array}{l}5 \\
6\end{array}$ & $\begin{array}{l}\text { II } \\
\text { I I } \\
\text { I } \\
\text { I } \\
\text { I }\end{array}$ \\
\hline $\begin{array}{l}4716.859 \\
4717.381 \\
4718.049 \\
4718.606 \\
4719.252\end{array}$ & $\begin{array}{r}12 \\
10 \\
25 \\
200 \\
12\end{array}$ & $\begin{array}{r}300 \\
7\end{array}$ & $\begin{array}{l}\text { I I } \\
\text { I } \\
\text { I } \\
\text { II } \\
\text { I I }\end{array}$ & $\begin{array}{l}4735.989 \\
4736.228 \\
4736.394 \\
4736.515 \\
4736.930\end{array}$ & $\begin{array}{r}70 \\
10 \\
12 \\
10 \\
4\end{array}$ & 75 & $\begin{array}{l}\text { I } \\
\text { I } \\
\text { I I } \\
\text { I } \\
\text { I }\end{array}$ & $\begin{array}{l}4755.630 \\
4755.910 \\
4756.545 \\
4756.687 \\
4756.880\end{array}$ & $\begin{array}{r}1 \\
1 \\
25 \\
50 \\
1\end{array}$ & $\begin{array}{r}3 \\
30 \\
2\end{array}$ & $\begin{array}{l}\text { II } \\
\text { I } \\
\text { I } \\
\text { II } \\
\text { II }\end{array}$ \\
\hline $\begin{array}{l}4719.451 \\
4719.694 \\
4719.977 \\
4720.155 \\
4720.449\end{array}$ & $\begin{array}{l}3 \\
20 \\
70 \\
18 \\
70\end{array}$ & 250 & $\begin{array}{l}\text { I } \\
\text { I } \\
\text { I I } \\
\text { I } \\
\text { I }\end{array}$ & $\begin{array}{l}4737.644 \\
4737.908 \\
4738.460 \\
4738.845 \\
4739.109\end{array}$ & $\begin{array}{r}20 \\
60 \\
10 \\
9 \\
15\end{array}$ & 75 & $\begin{array}{l}\text { I I } \\
\text { I } \\
\text { I } \\
\text { I } \\
\text { I }\end{array}$ & $\begin{array}{l}4757.135 \\
4757.405 \\
4758.123 \\
4758.741 \\
4759.589\end{array}$ & $\begin{array}{l}10 \\
12 \\
350 \mathrm{~d} \\
12 \\
40\end{array}$ & $\begin{array}{r}2 \\
10 \\
30\end{array}$ & $\begin{array}{l}\text { I } \\
\text { I } \\
\text { I } \\
\text { I } \\
\text { I I }\end{array}$ \\
\hline $\begin{array}{l}4720.781 \\
4720.977 \\
4721.274 \\
4721.876 \\
4722.088\end{array}$ & $\begin{array}{r}40 \\
10 \\
150 \\
250\end{array}$ & $\begin{array}{r}1 \\
1 \\
10 \\
5\end{array}$ & $\begin{array}{l}\text { I } \\
\text { I } \\
\text { I } \\
\text { I I } \\
\text { I }\end{array}$ & $\begin{array}{l}4739.673 \\
4739.875 \\
4740.529 \\
4740.934 \\
4741.311\end{array}$ & $\begin{array}{r}150 \\
25 \\
400 \\
100 \\
100\end{array}$ & $\begin{array}{r}3 \\
75 \\
600 \\
50\end{array}$ & $\begin{array}{l}\text { I } \\
\text { II } \\
\text { II } \\
\text { I } \\
\text { I }\end{array}$ & $\begin{array}{l}4759.679 \\
4760.047 \\
4761.111 \\
4761.863 \\
4762.518\end{array}$ & $\begin{array}{r}40 \\
8 \\
400 \\
25 \\
10\end{array}$ & $\begin{array}{r}20 \\
500 \\
40 \\
8\end{array}$ & $\begin{array}{l}\text { I } \\
\text { I I } \\
\text { I I } \\
\text { I I } \\
\text { I I }\end{array}$ \\
\hline $\begin{array}{l}4722.484 \\
4722.954 \\
4723.044 \\
4723.441 \\
4723.782\end{array}$ & $\begin{array}{l}400 \\
600\end{array}$ & $\begin{array}{r}10 \\
8 \\
400 \\
250\end{array}$ & $\begin{array}{l}\text { I } \\
\text { I I I } \\
\text { I I I } \\
\text { II } \\
\text { I }\end{array}$ & $\begin{array}{l}4742.118 \\
4742.258 \\
4742.564 \\
4743.030 \\
4743.127\end{array}$ & $\begin{array}{r}75 \\
75 \\
100 \\
2 \\
3\end{array}$ & $\begin{array}{r}125 \\
2 \\
3\end{array}$ & $\begin{array}{l}\text { I } \\
\text { I I } \\
\text { I } \\
\text { I } \\
\text { I I }\end{array}$ & $\begin{array}{l}4763.749 \\
4763.900 \\
4764.346 \\
4765.603 \\
4766.600 \mathrm{i}\end{array}$ & $\begin{array}{r}15 \\
2 \\
300 \\
100 \\
200\end{array}$ & $\begin{array}{r}18 \\
2 \\
175 \\
100 \\
2\end{array}$ & $\begin{array}{l}\text { I I } \\
\text { I I } \\
\text { I I } \\
\text { I } \\
\text { I }\end{array}$ \\
\hline $\begin{array}{l}4724.133 \\
4724.758 \\
4725.345 \\
4725.763 \\
4725.944\end{array}$ & $\begin{array}{c}5 \\
200 \\
7 \\
8 \mathrm{w} \\
15\end{array}$ & $\begin{array}{r}7 \\
175 \\
7 \\
15\end{array}$ & $\begin{array}{l}\text { II } \\
\text { II } \\
\text { I } \\
\text { I } \\
\text { I I }\end{array}$ & $\begin{array}{l}4743.680 \\
4744.276 \\
4744.476 \\
4744.652 \\
4744.812\end{array}$ & $\begin{array}{r}200 \\
3 \\
2 \\
5 \\
6\end{array}$ & $\begin{array}{r}25 \\
3\end{array}$ & $\begin{array}{l}\text { II } \\
\text { I } \\
\text { I I } \\
\text { I } \\
\text { I }\end{array}$ & $\begin{array}{l}4766.946 \\
4767.493 \\
4768.066 \\
4768.160 \\
4768.760\end{array}$ & $\begin{array}{r}12 \\
3 \\
50 \\
3 \\
10\end{array}$ & $\begin{array}{r}20 \\
4 \\
20 \\
5 \\
15\end{array}$ & $\begin{array}{l}\text { I I } \\
\text { I I } \\
\text { I I } \\
\text { I I } \\
\text { I I }\end{array}$ \\
\hline $\begin{array}{l}4726.319 \\
4726.468 \\
4726.912 \\
4727.443 \\
4727.844\end{array}$ & $\begin{array}{r}30 \\
125 \\
8 \\
5 \\
60\end{array}$ & $\begin{array}{r}8 \\
60 \\
8 \\
2\end{array}$ & $\begin{array}{l}\text { II } \\
\text { II } \\
\text { II } \\
\text { I } \\
\text { I }\end{array}$ & $\begin{array}{l}4745.315 \\
4746.115 \\
4746.370 \\
4746.921 \\
4747.408\end{array}$ & $\begin{array}{c}100 \mathrm{~d} \\
8 \\
10 \\
20 \\
7\end{array}$ & 3 & $\begin{array}{l}\text { I } \\
\text { I } \\
\text { I } \\
\text { I } \\
\text { I }\end{array}$ & $\begin{array}{l}4769.398 \\
4769.540 \\
4769.870 \\
4770.416 \\
4770.590\end{array}$ & $\begin{array}{r}25 \\
2 \\
50 \\
25\end{array}$ & 4 & $\begin{array}{l}\text { I I } \\
\text { I } \\
\text { I } \\
\text { I } \\
\text { I }\end{array}$ \\
\hline $\begin{array}{l}4728.136 \\
4728.570 \\
4729.112 \\
4729.867 \\
4730.087\end{array}$ & $\begin{array}{r}125 \\
15 \\
250 \\
125 \\
20\end{array}$ & $\begin{array}{r}5 \\
150 \\
150\end{array}$ & $\begin{array}{l}\text { I } \\
\text { I } \\
\text { I } \\
\text { II } \\
\text { I }\end{array}$ & $\begin{array}{l}4747.614 \\
4747.981 \\
4748.109 \\
4748.406 \\
4748.583\end{array}$ & $\begin{array}{r}20 \\
2 \\
3 \\
20 \\
20\end{array}$ & 25 & $\begin{array}{l}\text { I I } \\
\text { I } \\
\text { I } \\
\text { I } \\
\text { I I }\end{array}$ & $\begin{array}{l}4770.775 \\
4771.339 \\
4771.504 \\
4771.970 \\
4773.100\end{array}$ & $\begin{array}{r}8 \\
8 \\
4 \\
2 \\
30\end{array}$ & $\begin{array}{r}40 \\
1 \\
2 \\
20\end{array}$ & $\begin{array}{l}\text { I I } \\
\text { I } \\
\text { I } \\
\text { I I } \\
\text { I I }\end{array}$ \\
\hline $\begin{array}{l}4730.653 \\
4730.876 \\
4731.173 \\
4731.614 \\
4732.091\end{array}$ & $\begin{array}{l}50 \\
6 \\
10 \\
3 \mathrm{w} \\
15\end{array}$ & $\begin{array}{l}4 \\
5\end{array}$ & $\begin{array}{l}\text { I } \\
\text { II } \\
\text { II } \\
\text { I } \\
\text { I }\end{array}$ & $\begin{array}{l}4749.188 \\
4749.955 \\
4750.500 \\
4750.750 \\
4751.110\end{array}$ & $\begin{array}{r}200 \\
160 \\
2 \\
2 \\
2\end{array}$ & $\begin{array}{r}10 \\
6 \\
1 \\
1\end{array}$ & $\begin{array}{l}\text { I } \\
\text { I } \\
\text { I } \\
\text { I }\end{array}$ & $\begin{array}{l}4773.250 \\
4774.270 \\
4774.844 \\
4775.072 \\
4775.332\end{array}$ & $\begin{array}{r}100 \\
100 \\
5 \\
4 \\
150\end{array}$ & $\begin{array}{r}500 \\
8 \\
20\end{array}$ & $\begin{array}{l}\text { I } \\
\text { II } \\
\text { I } \\
\text { I I } \\
\text { I }\end{array}$ \\
\hline $\begin{array}{l}4732.659 \\
4733.017 \\
4733.511 \\
4734.049 \\
4734.629\end{array}$ & $\begin{array}{r}20 \\
12 \\
2 \\
80 \\
4\end{array}$ & $\begin{array}{r}100 \\
40\end{array}$ & $\begin{array}{l}\text { II } \\
\text { II } \\
\text { I } \\
\text { I } \\
\text { II }\end{array}$ & $\begin{array}{l}4751.341 \\
4751.861 \\
4752.414 \mathbf{i} \\
4753.240 \\
4753.590\end{array}$ & $\begin{array}{r}10 \\
10 \\
500 \\
3 \\
3\end{array}$ & $\begin{array}{r}10 \\
10 \\
500\end{array}$ & $\begin{array}{l}\text { II } \\
\text { I I } \\
\text { I I } \\
\text { I } \\
\text { I }\end{array}$ & $\begin{array}{l}4775.798 \\
4776.334 \\
4776.786 \\
4777.191 \\
4777.649\end{array}$ & $\begin{array}{r}150 \\
15 \\
20 \\
200 \\
10\end{array}$ & $\begin{array}{r}1 \\
75 \\
10 \\
40\end{array}$ & $\begin{array}{l}\text { I } \\
\text { I } \\
\text { II } \\
\text { I } \\
\text { I I }\end{array}$ \\
\hline
\end{tabular}


Table 1. Wavelengths of Thorium Spectra - Continued

\begin{tabular}{|c|c|c|c|c|c|c|c|c|c|c|c|}
\hline \multirow{2}{*}{$\begin{array}{l}\text { Wave- } \\
\text { length }\end{array}$} & \multicolumn{2}{|c|}{ Intensity } & \multirow{2}{*}{$\begin{array}{l}\text { Spec- } \\
\text { trum }\end{array}$} & \multirow{2}{*}{$\begin{array}{l}\text { Wave- } \\
\text { length }\end{array}$} & \multicolumn{2}{|c|}{ Intensity } & \multirow{2}{*}{$\begin{array}{l}\text { Spec- } \\
\text { trum }\end{array}$} & \multirow{2}{*}{$\begin{array}{l}\text { Wave- } \\
\text { length }\end{array}$} & \multicolumn{2}{|c|}{ Intensity } & \multirow{2}{*}{$\begin{array}{l}\text { Spec } \\
\text { trum }\end{array}$} \\
\hline & Lamp & Spark & & & Lamp & Spark & & & Lamp & Spark & \\
\hline $\begin{array}{l}4778.297 \\
4778.900 \\
4779.596 \\
4779.719 \\
4780.439\end{array}$ & $\begin{array}{c}300 \\
18 \\
100 \\
50 \\
100 \mathrm{~h}\end{array}$ & $\begin{array}{r}10 \\
50 \\
50 \\
2\end{array}$ & $\begin{array}{l}\text { I } \\
\text { I I } \\
\text { I } \\
\text { I } \\
\text { I }\end{array}$ & $\begin{array}{l}4799.764 \\
4800.178 \\
4801.045 \\
4801.334 \\
4802.045\end{array}$ & $\begin{array}{c}10 \\
100 \\
25 \\
7 \\
5 \mathrm{~s}\end{array}$ & $\begin{array}{r}9 \\
75 \\
2\end{array}$ & $\begin{array}{l}\text { I I } \\
\text { I I } \\
\text { I } \\
\text { I } \\
\text { I }\end{array}$ & $\begin{array}{l}4822.016 \\
4822.859 \\
4823.195 \\
4823.607 \\
4823.995\end{array}$ & $\begin{array}{r}30 \\
300 \\
75 \\
250 \\
200\end{array}$ & $\begin{array}{r}100 \\
10 \\
75 \\
20 \\
10\end{array}$ & $\begin{array}{l}\text { I I I } \\
\text { I } \\
\text { I I } \\
\text { I } \\
\text { I }\end{array}$ \\
\hline $\begin{array}{l}4780.765 \\
4781.288 \\
4781.537 \\
4781.623 \\
4781.867\end{array}$ & $\begin{array}{l}75 \\
25 \\
75 \\
20 \\
20\end{array}$ & 30 & \begin{tabular}{l|} 
I I I \\
I \\
I \\
I I \\
I
\end{tabular} & $\begin{array}{l}4802.428 \\
4802.675 \\
4803.488 \\
4803.955 \\
4804.531\end{array}$ & $\begin{array}{r}3 \\
12 \\
75 \\
125 \\
3\end{array}$ & $\begin{array}{r}4 \\
2 \\
500 \\
125\end{array}$ & $\begin{array}{l}\text { II } \\
\text { I } \\
\text { II } \\
\text { II } \\
\text { I }\end{array}$ & $\begin{array}{l}4824.645 \\
4825.042 \\
4825.170 \\
4825.553 \\
4826.012\end{array}$ & $\begin{array}{r}30 \\
25 \\
4 \\
6 \\
25\end{array}$ & 50 & $\begin{array}{l}\text { I I } \\
\text { I } \\
\text { I } \\
\text { I } \\
\text { I }\end{array}$ \\
\hline $\begin{array}{l}4782.230 \\
4782.360 \\
4782.761 \\
4783.642 \\
4783.870\end{array}$ & $\begin{array}{c}6 \mathrm{w} \\
7 \\
180 \\
30 \\
150\end{array}$ & $\begin{array}{r}125 \\
20 \\
40\end{array}$ & $\begin{array}{l}\text { I } \\
\text { I } \\
\text { I I } \\
\text { I I } \\
\text { I }\end{array}$ & $\begin{array}{l}4805.248 \\
4805.575 \\
4806.447 \\
4806.680 \\
4807.102\end{array}$ & $\begin{array}{r}20 \\
18 \\
8 \\
20 \\
10\end{array}$ & $\begin{array}{r}1 \\
20\end{array}$ & $\begin{array}{l}\text { I } \\
\text { I I } \\
\text { I } \\
\text { I } \\
\text { II }\end{array}$ & $\begin{array}{l}4826.378 \\
4826.707 \\
4827.580 \\
4828.470 \\
4828.645\end{array}$ & $\begin{array}{r}10 \\
300 \\
2 \\
10 \\
15\end{array}$ & $\begin{array}{r}30 \\
4 \\
40\end{array}$ & $\begin{array}{l}\text { I I } \\
\text { I } \\
\text { I } \\
\text { I I } \\
\text { I }\end{array}$ \\
\hline $\begin{array}{l}4784.038 \\
4784.921 \\
4785.264 \\
4785.755 \\
4786.528\end{array}$ & $\begin{array}{r}120 \\
125 \\
15 \\
15 \\
150\end{array}$ & $\begin{array}{r}50 \\
3 \\
4 \\
2\end{array}$ & $\begin{array}{l}\text { I } \\
\text { I I } \\
\text { II } \\
\text { I I } \\
\text { I }\end{array}$ & $\begin{array}{l}4808.133 i \\
4808.741 \\
4809.619 \\
4810.515 \\
4810.889\end{array}$ & $\begin{array}{r}350 \\
5 \\
300 \\
10 \\
3\end{array}$ & $\begin{array}{r}12 \\
4 \\
1\end{array}$ & $\begin{array}{l}\text { I } \\
\text { I } \\
\text { I } \\
\text { I } \\
\text { I }\end{array}$ & $\begin{array}{l}4828.945 \\
4829.181 \\
4829.411 \\
4829.809 \\
4829.884\end{array}$ & $\begin{array}{r}4 \\
1 \\
4 \\
50 \\
15\end{array}$ & $\begin{array}{r}2 \\
1 \\
1 \\
40\end{array}$ & $\begin{array}{l}\text { I I } \\
\text { I I } \\
\text { I I } \\
\text { I } \\
\text { I I }\end{array}$ \\
\hline $\begin{array}{l}4786.712 \\
4787.142 \\
4787.374 \\
4787.812 \\
4788.690\end{array}$ & $\begin{array}{r}75 \\
100 \\
18 \\
6 \\
75\end{array}$ & $\begin{array}{l}30 \\
25\end{array}$ & $\begin{array}{l}\text { I } \\
\text { I } \\
\text { I I } \\
\text { I I } \\
\text { I }\end{array}$ & $\begin{array}{l}4811.040 \\
4811.367 \\
4811.877 \\
4812.019 \\
4812.377\end{array}$ & $\begin{array}{r}5 \\
4 \\
18 \\
12 \\
100\end{array}$ & $\begin{array}{l}10 \\
30 \\
10\end{array}$ & $\begin{array}{l}\text { I } \\
\text { II } \\
\text { II } \\
\text { II } \\
\text { I }\end{array}$ & $\begin{array}{l}4830.387 \\
4831.121 \\
4831.603 \\
4832.161 \\
4832.443\end{array}$ & $\begin{array}{r}8 \\
350 \\
200 \\
4 \\
8\end{array}$ & $\begin{array}{l}20 \\
18\end{array}$ & $\begin{array}{l}\text { I } \\
\text { I } \\
\text { I } \\
\text { I } \\
\text { I }\end{array}$ \\
\hline $\begin{array}{l}4789.387 \mathrm{i} \\
4789.907 \\
4790.078 \\
4790.439 \\
4791.343\end{array}$ & $\begin{array}{r}300 \\
3 \\
10 \\
20 \\
12\end{array}$ & $\begin{array}{r}8 \\
8 \\
20 \\
1\end{array}$ & $\begin{array}{l}\text { I } \\
\text { I I } \\
\text { I } \\
\text { I I } \\
\text { I }\end{array}$ & $\begin{array}{l}4812.503 \\
4813.003 \\
4813.727 \\
4813.893 \\
4814.684\end{array}$ & $\begin{array}{r}100 \\
200 \\
300 \\
18\end{array}$ & $\begin{array}{r}100 \\
2 \\
3 \\
5\end{array}$ & $\begin{array}{l}\text { I I I } \\
\text { I } \\
\text { I } \\
\text { I } \\
\text { I }\end{array}$ & $\begin{array}{l}4832.799 \\
4833.157 \\
4833.667 \\
4833.831 \\
4834.087\end{array}$ & $\begin{array}{r}200 \\
150 \\
20 \\
18 \\
35\end{array}$ & $\begin{array}{r}150 \\
3 \\
5\end{array}$ & $\begin{array}{l}\text { I I } \\
\text { I } \\
\text { I } \\
\text { I I } \\
\text { I }\end{array}$ \\
\hline $\begin{array}{l}4791.510 \\
4791.966 \\
4792.895 \\
4793.248 \\
4793.648\end{array}$ & $\begin{array}{r}30 \\
18 \\
6 \\
100 \\
5\end{array}$ & $\begin{array}{r}2 \\
2 \\
20\end{array}$ & $\begin{array}{l}\text { I } \\
\text { I } \\
\text { I } \\
\text { I } \\
\text { I I }\end{array}$ & $\begin{array}{l}4815.050 \\
4815.490 \\
4816.865 \\
4817.018 \\
4817.333\end{array}$ & $\begin{array}{r}50 \\
4 \\
75 \\
75 \\
7\end{array}$ & $\begin{array}{r}3 \\
50 \\
25\end{array}$ & $\begin{array}{l}\text { I } \\
\text { II } \\
\text { II } \\
\text { I } \\
\text { I I }\end{array}$ & $\begin{array}{l}4834.527 \\
4834.651 \\
4834.793 \\
4835.075 \\
4835.310\end{array}$ & $\begin{array}{l}3 \\
2 \\
3 \mathrm{~s} \\
7 \\
2\end{array}$ & 2 & $\begin{array}{l}\text { I } \\
\text { I } \\
\text { I } \\
\text { I } \\
\text { I }\end{array}$ \\
\hline $\begin{array}{l}4793.898 \\
4794.174 \\
4794.466 \\
4795.619 \\
4795.911\end{array}$ & $\begin{array}{r}10 \\
2 \\
3 \\
10 \\
150\end{array}$ & $\begin{array}{l}4 \\
4 \\
5\end{array}$ & $\begin{array}{l}\text { I } \\
\text { I I } \\
\text { I } \\
\text { I I } \\
\text { I }\end{array}$ & $\begin{array}{l}4817.450 \\
4817.844 \\
4818.110 \\
4818.300 \\
4818.650\end{array}$ & $\begin{array}{r}7 \\
8 \\
3 \\
6 \\
300\end{array}$ & 400 & $\begin{array}{l}\text { I } \\
\text { I I } \\
\text { I } \\
\text { I } \\
\text { I I }\end{array}$ & $\begin{array}{l}4835.962 \\
4836.071 \\
4836.191 \\
4836.699 \\
4836.920\end{array}$ & $\begin{array}{l}3 \\
3 \\
5 \\
5 \\
2\end{array}$ & $\begin{array}{l}5 \\
5 \\
1\end{array}$ & $\begin{array}{l}\text { I } \\
\text { I } \\
\text { I I } \\
\text { I I } \\
\text { I I }\end{array}$ \\
\hline $\begin{array}{l}4796.253 \\
4796.499 \\
4796.641 \\
4796.884 \\
4797.497\end{array}$ & $\begin{array}{r}20 \\
1 \\
2 \\
8 \\
125\end{array}$ & 80 & $\begin{array}{l}\text { I } \\
\text { I I } \\
\text { I } \\
\text { I } \\
\text { I }\end{array}$ & $\begin{array}{l}4818.890 \\
4819.021 \\
4819.205 \\
4819.553 \\
4819.928\end{array}$ & $\begin{array}{l}15 \\
10 \\
75 \\
50 \\
20 \mathrm{~h}\end{array}$ & $\begin{array}{l}10 \\
1 \\
1\end{array}$ & $\begin{array}{l}\text { I } \\
\text { II } \\
\text { I } \\
\text { I } \\
\text { I }\end{array}$ & $\begin{array}{l}4837.259 \\
4837.413 \\
4837.699 \\
4837.968 \\
4838.067\end{array}$ & $\begin{array}{r}9 \\
10 \\
10 \\
9 \\
4\end{array}$ & 30 & $\begin{array}{l}\text { I } \\
\text { I } \\
\text { I I } \\
\text { I } \\
\text { I }\end{array}$ \\
\hline $\begin{array}{l}4798.128 \\
4798.603 \\
4798.734 \\
4799.000 \\
4799.130\end{array}$ & $\begin{array}{l}3 \\
8 \\
7 \\
2 \\
1\end{array}$ & $\frac{1}{2}$ & $\begin{array}{l}\text { I } \\
\text { I } \\
\text { I } \\
\text { II } \\
\text { II }\end{array}$ & $\begin{array}{l}4820.477 \\
4820.900 \\
4821.289 \\
4821.597 \\
4821.850\end{array}$ & $\begin{array}{r}75 \\
200 \\
30 \\
150 \\
5\end{array}$ & $\begin{array}{r}1 \\
250 \\
75\end{array}$ & $\begin{array}{l}\text { I } \\
\text { II } \\
\text { II } \\
\text { I } \\
\text { I }\end{array}$ & $\begin{array}{l}4838.362 \\
4838.570 \\
4839.717 \\
4840.481 \\
4840.843 \mathrm{i}\end{array}$ & $\begin{array}{r}25 \\
5 \\
40 \\
125 \\
400\end{array}$ & $\begin{array}{r}125 \\
10 \\
100 \\
10\end{array}$ & $\begin{array}{l}\text { I I } \\
\text { I } \\
\text { I I } \\
\text { I I } \\
\text { I }\end{array}$ \\
\hline
\end{tabular}


Table 1. Wavelengths of Thorium Spectra - Continued

\begin{tabular}{|c|c|c|c|c|c|c|c|c|c|c|c|}
\hline \multirow{2}{*}{$\begin{array}{l}\text { Wave- } \\
\text { length }\end{array}$} & \multicolumn{2}{|c|}{ Intensity } & \multirow{2}{*}{$\begin{array}{l}\text { Spec- } \\
\text { trum }\end{array}$} & \multirow{2}{*}{$\begin{array}{l}\text { Wave- } \\
\text { length }\end{array}$} & \multicolumn{2}{|c|}{ Intensity } & \multirow{2}{*}{$\begin{array}{l}\text { Spec- } \\
\text { trum }\end{array}$} & \multirow{2}{*}{$\begin{array}{l}\text { Wave- } \\
\text { length }\end{array}$} & \multicolumn{2}{|c|}{ Intensity } & \multirow{2}{*}{$\begin{array}{l}\text { Spec- } \\
\text { trum }\end{array}$} \\
\hline & Lamp & Spark & & & Lamp & Spark & & & Lamp & Spark & \\
\hline $\begin{array}{l}4841.362 \\
4842.171 \\
4842.561 \\
4842.769 \\
4843.041\end{array}$ & $\begin{array}{r}60 \\
2 \\
2 \\
20\end{array}$ & $\begin{array}{r}40 \\
5 \\
3\end{array}$ & $\begin{array}{l}\text { I I I } \\
\text { I } \\
\text { I I } \\
\text { I } \\
\text { I }\end{array}$ & $\begin{array}{l}4861.708 \\
4863.1721 \\
4864.150 \\
4864.421 \\
4864.727\end{array}$ & $\begin{array}{c}50 \\
1000 \\
3 \\
15 \mathrm{w} \\
15 \mathrm{w}\end{array}$ & 500 & $\begin{array}{l}\text { I } \\
\text { I I } \\
\text { I } \\
\text { I } \\
\text { I }\end{array}$ & $\begin{array}{l}4884.675 \\
4885.101 \\
4885.286 \\
4885.564 \\
4885.782\end{array}$ & $\begin{array}{l}5 \\
3 \\
4 \\
5 \\
3\end{array}$ & 25 & $\begin{array}{l}\text { II } \\
\text { I } \\
\text { I } \\
\text { I I } \\
\text { I }\end{array}$ \\
\hline $\begin{array}{l}4843.921 \\
4844.171 \\
4844.566 \\
4844.757 \\
4844.923\end{array}$ & $\begin{array}{r}300 \\
200 \\
50 \\
12 \\
12\end{array}$ & $\begin{array}{r}300 \\
75 \\
30\end{array}$ & $\begin{array}{l}\text { I } \\
\text { II } \\
\text { II } \\
\text { II } \\
\text { I }\end{array}$ & $\begin{array}{l}4865.477 i \\
4865.977 \\
4866.594 \\
4867.482 \\
4867.740\end{array}$ & $\begin{array}{r}350 \\
15 \\
18 \\
12 \\
2\end{array}$ & $\begin{array}{r}20 \\
2 \\
8\end{array}$ & $\begin{array}{l}\text { I } \\
\text { I } \\
\text { II } \\
I \\
I\end{array}$ & $\begin{array}{l}4885.992 \\
4886.135 \\
4886.367 \\
4886.870 \\
4887.094\end{array}$ & $\begin{array}{r}4 \\
5 \\
2 \\
10 \\
10\end{array}$ & 10 & $\begin{array}{l}\text { I I } \\
\text { I } \\
\text { I } \\
\text { I } \\
\text { I }\end{array}$ \\
\hline $\begin{array}{l}4845.163 \\
4845.666 \\
4845.929 \\
4846.290 \\
4846.347\end{array}$ & $\begin{array}{c}100 \\
2 \\
25 \\
4 \mathrm{w} \\
20\end{array}$ & $\begin{array}{r}1 \\
25 \\
3\end{array}$ & $\begin{array}{l}\text { I } \\
\text { I } \\
\text { II } \\
\text { II } \\
\text { I }\end{array}$ & $\begin{array}{l}4868.273 \\
4868.533 \\
4868.620 \\
4868.875 \\
4869.673\end{array}$ & $\begin{array}{r}50 \\
30 \\
10 \\
100 \\
3\end{array}$ & 175 & $\begin{array}{l}\text { I I } \\
\text { I } \\
\text { I } \\
\text { I } \\
\text { I }\end{array}$ & $\begin{array}{l}4887.435 \\
4888.150 \\
4888.375 \\
4888.493 \\
4888.731\end{array}$ & $\begin{array}{l}1 \\
1 \\
3 \\
3 \\
3\end{array}$ & $\begin{array}{l}1 \\
4 \\
3\end{array}$ & $\begin{array}{l}\text { I } \\
\text { II } \\
\text { II } \\
\text { II } \\
\text { I }\end{array}$ \\
\hline $\begin{array}{l}4847.327 \\
4847.915 \\
4848.360 \\
4849.043 \\
4849.132\end{array}$ & $\begin{array}{r}80 \\
2 \\
250 \\
75 \\
75\end{array}$ & $\begin{array}{r}2 \\
6 \\
5 \\
400\end{array}$ & $\begin{array}{l}\text { I } \\
\text { II } \\
\text { I } \\
\text { I I } \\
\text { I }\end{array}$ & $\begin{array}{l}4870.133 \\
4870.251 \\
4870.953 \\
4871.289 \\
4871.540\end{array}$ & $\begin{array}{r}2 \\
5 \\
4 \\
25 \\
1\end{array}$ & & $\begin{array}{l}\text { I } \\
\text { I } \\
\text { I } \\
\text { I } \\
\text { I }\end{array}$ & $\begin{array}{l}4888.809 \\
4889.099 \\
4889.496 \\
4889.861 \\
4890.460\end{array}$ & $\begin{array}{l}2 \\
3 \mathrm{w} \\
30 \\
18 \\
30\end{array}$ & $\begin{array}{l}3 \mathrm{~h} h \\
15\end{array}$ & $\begin{array}{l}\text { I } \\
\text { I } \\
\text { I } \\
\text { I I } \\
\text { I }\end{array}$ \\
\hline $\begin{array}{l}4849.424 \\
4849.863 \\
4850.017 \\
4850.441 \\
4851.671\end{array}$ & $\begin{array}{r}10 \\
30 \\
10 \\
600 \\
7\end{array}$ & $\begin{array}{r}40 \\
12 \\
200\end{array}$ & $\begin{array}{l}\text { II } \\
\text { I } \\
\text { II } \\
\text { II } \\
\text { I }\end{array}$ & $\begin{array}{l}4871.685 \\
4872.028 \\
4872.239 \\
4872.913 \\
4873.360\end{array}$ & $\begin{array}{r}4 \\
8 \\
5 \\
300 \\
2\end{array}$ & $\begin{array}{r}2 \\
60 \\
5\end{array}$ & $\begin{array}{l}\text { I } \\
\text { II } \\
\text { I } \\
\text { I } \\
\text { II }\end{array}$ & $\begin{array}{l}4891.035 \\
4891.653 \\
4891.934 \\
4892.030 \\
4892.283\end{array}$ & $\begin{array}{r}30 \\
2 \\
3 \\
4 \\
6\end{array}$ & 8 & $\begin{array}{l}\text { I } \\
\text { I } \\
\text { I I } \\
\text { I } \\
\text { I }\end{array}$ \\
\hline $\begin{array}{l}4851.907 \\
4852.856 \\
4853.608 \\
4853.807 \\
4854.493\end{array}$ & $\begin{array}{r}4 \\
200 \\
12 \\
2 \\
4\end{array}$ & $\begin{array}{r}20 \\
9 \\
2\end{array}$ & $\begin{array}{l}\text { II } \\
\text { I } \\
\text { I } \\
\text { II } \\
\text { I }\end{array}$ & $\begin{array}{l}4874.370 \\
4875.326 \\
4875.678 \\
4876.080 \\
4876.243\end{array}$ & $\begin{array}{r}200 \\
2 \\
2 \\
2 \\
20\end{array}$ & 2 & $\begin{array}{l}\text { I } \\
\text { I } \\
\text { I } \\
\text { II } \\
\text { I }\end{array}$ & $\begin{array}{l}4892.764 \\
4893.451 \\
4893.706 \\
4893.925 \\
4894.374\end{array}$ & $\begin{array}{r}50 \\
80 \\
10 \\
2 \\
3\end{array}$ & 12 & $\begin{array}{l}\text { I } \\
\text { I } \\
\text { I } \\
\text { I } \\
\text { I I }\end{array}$ \\
\hline $\begin{array}{l}4854.636 \\
4854.874 \\
4855.367 \\
4855.524 \\
4855.700\end{array}$ & $\begin{array}{r}2 \\
2 \\
10 \\
3 \\
1\end{array}$ & 3 & $\begin{array}{l}\text { II } \\
\text { I } \\
\text { I } \\
\text { I } \\
\text { I }\end{array}$ & $\begin{array}{l}4876.497 \\
4876.998 \\
4877.250 \\
4877.505 \\
4877.817\end{array}$ & $\begin{array}{r}75 \\
125 \\
5 \\
2 \\
40\end{array}$ & $\begin{array}{r}3 \\
500^{3}\end{array}$ & $\begin{array}{l}\text { I } \\
\text { I I } \\
\text { I } \\
\text { I } \\
\text { II }\end{array}$ & $\begin{array}{l}4894.526 \\
4894.955 \mathrm{i} \\
4895.660 \\
4896.087 \\
4896.258\end{array}$ & $\begin{array}{r}2 \\
350 \\
25 \\
2 \\
2\end{array}$ & $\begin{array}{r}10 \\
50 \\
3\end{array}$ & $\begin{array}{l}\text { I } \\
\text { I } \\
\text { I I } \\
\text { I } \\
\text { I I }\end{array}$ \\
\hline $\begin{array}{l}4856.020 \\
4856.276 \\
4856.557 \\
4856.963 \\
4857.170\end{array}$ & $\begin{array}{r}2 \\
10 \\
3 \\
5 \\
4\end{array}$ & $\begin{array}{r}4 \\
4 \\
10\end{array}$ & $\begin{array}{l}\text { I } \\
\text { I I } \\
\text { I } \\
\text { II } \\
\text { II }\end{array}$ & $\begin{array}{l}4878.012 \\
4878.733 i \\
4879.147 \\
4879.339 \\
4879.500\end{array}$ & $\begin{array}{r}75 \\
200 \\
18 \\
40 \\
5\end{array}$ & $\begin{array}{r}8 \\
20 \\
75\end{array}$ & $\begin{array}{l}\text { I } \\
\text { I } \\
\text { II } \\
\text { II } \\
\text { I }\end{array}$ & $\begin{array}{l}4897.121 \\
4897.591 \\
4897.930 \\
4898.451 \\
4898.797\end{array}$ & $\begin{array}{l}21 \\
15 \\
15 \\
300 \\
100\end{array}$ & $\begin{array}{r}18 \\
300 \\
150\end{array}$ & $\begin{array}{l}\text { I } \\
\text { I } \\
\text { I I } \\
\text { I I } \\
\text { I I }\end{array}$ \\
\hline $\begin{array}{l}4857.534 \\
4857.960 \\
4858.091 \\
4858.328 \\
4860.057\end{array}$ & $\begin{array}{r}50 \\
10 \\
40 \\
300 \\
8\end{array}$ & $\begin{array}{r}2 \\
150 \\
200\end{array}$ & $\begin{array}{l}\text { I } \\
\text { I } \\
\text { II } \\
\text { II } \\
\text { I }\end{array}$ & $\begin{array}{l}4879.703 \\
4880.197 \\
4880.891 \\
4881.210 \\
4881.591\end{array}$ & $\begin{array}{l}6 \\
40 \\
40 d \\
50 \\
7\end{array}$ & $\begin{array}{l}4 \\
60 \\
3 w\end{array}$ & $\begin{array}{l}\text { II } \\
\text { II } \\
\text { I } \\
\text { I }\end{array}$ & $\begin{array}{l}4899.230 \\
4899.325 \\
4899.932 \\
4900.169 \\
4900.740\end{array}$ & $\begin{array}{r}200 \\
75 \\
8 \\
4 \\
3\end{array}$ & 701 & $\begin{array}{l}\text { I I } \\
\text { I } \\
\text { I I } \\
\text { I I } \\
\text { I }\end{array}$ \\
\hline $\begin{array}{l}4860.174 \\
4860.265 \\
4860.423 \\
4860.728 \\
4861.216\end{array}$ & $\begin{array}{r}3 \\
3 \\
10 \\
3 \\
150\end{array}$ & $\begin{array}{r}70 \\
10 \\
7\end{array}$ & $\begin{array}{l}\text { I } \\
\text { III } \\
\text { II } \\
\text { I } \\
\text { I }\end{array}$ & $\begin{array}{l}4881.861 \\
4882.448 \\
4882.891 \\
4883.235 \\
4884.533\end{array}$ & $\begin{array}{c}30 \\
50 \\
3 \\
7 \\
30 \mathrm{~d}\end{array}$ & $\begin{array}{r}100 \\
1\end{array}$ & $\begin{array}{l}\text { I } \\
\text { II } \\
\text { I } \\
\text { I } \\
\text { I }\end{array}$ & $\begin{array}{l}4900.872 \\
4901.344 \\
4901.482 \\
4901.606 \\
4902.030\end{array}$ & $\begin{array}{r}10 \\
2 \\
2 \\
5 \\
250\end{array}$ & 2 & $\begin{array}{l}\text { I } \\
\text { I } \\
\text { I } \\
\text { I } \\
\text { I }\end{array}$ \\
\hline
\end{tabular}


Table 1. Wavelengths of Thorium Spectra - Continued

\begin{tabular}{|c|c|c|c|c|c|c|c|c|c|c|c|}
\hline \multirow{2}{*}{$\begin{array}{l}\text { Wave- } \\
\text { length }\end{array}$} & \multicolumn{2}{|c|}{ Intensity } & \multirow{2}{*}{$\begin{array}{l}\text { Spec- } \\
\text { trum }\end{array}$} & \multirow{2}{*}{$\begin{array}{l}\text { Wave- } \\
\text { length }\end{array}$} & \multicolumn{2}{|c|}{ Intensity } & \multirow{2}{*}{$\begin{array}{l}\text { Spec- } \\
\text { trum }\end{array}$} & \multirow{2}{*}{$\begin{array}{l}\text { Wave- } \\
\text { length }\end{array}$} & \multicolumn{2}{|c|}{ Intensity } & \multirow{2}{*}{$\begin{array}{l}\text { Spec } \\
\text { trum }\end{array}$} \\
\hline & Lamp & Spark & & & Lamp & Spark & & & Lamp & Spark & \\
\hline $\begin{array}{l}4902.340 \\
4902.610 \\
4902.796 \\
4903.063 \\
4903.688\end{array}$ & $\begin{array}{r}5 \\
40 \\
75 \\
20 \\
2\end{array}$ & $\begin{array}{r}2 \\
2 \\
25 \\
3\end{array}$ & $\begin{array}{l}\text { I I } \\
\text { I } \\
\text { I } \\
\text { I } \\
\text { I }\end{array}$ & $\begin{array}{l}4923.700 \\
4924.192 \\
4924.252 \\
4924.421 \\
4924.807\end{array}$ & $\begin{array}{r}5 \\
75 \\
20 \\
100 \\
9\end{array}$ & 75 & $\begin{array}{l}\text { I } \\
\text { I } \\
\text { I } \\
\text { I I } \\
\text { I }\end{array}$ & $\begin{array}{l}4939.272 \\
4939.642 \mathrm{i} \\
4940.661 \\
4940.910 \\
4941.042\end{array}$ & $\begin{array}{r}10 \\
350 \\
1 \\
10 \\
6\end{array}$ & $\begin{array}{r}1 \\
100\end{array}$ & $\begin{array}{l}\text { I } \\
\text { I } \\
\text { I } \\
\text { I } \\
\text { I I }\end{array}$ \\
\hline $\begin{array}{l}4903.812 \\
4904.110 \\
4904.371 \\
4904.692 \\
4905.254\end{array}$ & $\begin{array}{r}2 \\
3 \\
3 \\
50 \\
2\end{array}$ & 1 & $\begin{array}{l}\text { I } \\
\text { I } \\
\text { I } \\
\text { I } \\
\text { I }\end{array}$ & $\begin{array}{l}4924.900 \\
4925.006 \\
4925.426 \\
4925.800 \\
4925.948\end{array}$ & $\begin{array}{r}5 \\
60 \\
75 \\
25 \\
100\end{array}$ & $\begin{array}{r}150 \\
75 \\
25 \\
2\end{array}$ & $\begin{array}{l}\text { I } \\
\text { I I } \\
\text { I I } \\
\text { II } \\
\text { I }\end{array}$ & $\begin{array}{l}4941.420 \\
4941.902 \\
4942.614 \\
4942.839 \\
4943.071\end{array}$ & $\begin{array}{r}12 \\
6 \\
1 \\
9 \\
140\end{array}$ & $\begin{array}{r}2 \\
3 \\
15 \\
5\end{array}$ & $\begin{array}{l}\text { I } \\
\text { I } \\
\text { I } \\
\text { II } \\
\text { I }\end{array}$ \\
\hline $\begin{array}{l}4905.461 \\
4905.600 \\
4906.100 \\
4906.698 \\
4907.044\end{array}$ & $\begin{array}{r}2 \\
4 \\
2 \\
8 \\
40\end{array}$ & $\begin{array}{l}6 \\
4 \\
2\end{array}$ & $\begin{array}{l}\text { I } \\
\text { I I } \\
\text { II } \\
\text { II } \\
\text { I }\end{array}$ & $\begin{array}{l}4926.254 \\
4926.687 \\
4926.946 \\
4927.306 \\
4927.600\end{array}$ & $\begin{array}{r}25 \\
5 \\
5 \\
12\end{array}$ & $\begin{array}{l}20 \\
20\end{array}$ & $\begin{array}{l}\text { I } \\
\text { I } \\
\text { I I } \\
\text { I } \\
\text { I I I }\end{array}$ & $\begin{array}{l}4943.668 \\
4944.150 \\
4944.512 \\
4944.778 \\
4945.210\end{array}$ & $\begin{array}{l}6 \\
1 \\
2 \\
4 \\
3\end{array}$ & 500 & $\begin{array}{l}\text { I I I } \\
\text { I } \\
\text { I } \\
\text { I } \\
\text { I I }\end{array}$ \\
\hline $\begin{array}{l}4907.208 \\
4907.616 \\
4908.017 \\
4908.470 \\
4908.997\end{array}$ & $\begin{array}{c}150 \\
3 \\
4 w \\
50 \\
4\end{array}$ & $\begin{array}{l}4 \\
5 \\
6\end{array}$ & $\begin{array}{l}\text { I } \\
\text { I I } \\
\text { I I } \\
\text { I } \\
\text { I }\end{array}$ & $\begin{array}{l}4927.777 \\
4928.240 \\
4928.457 \\
4928.668 \\
4929.089\end{array}$ & $\begin{array}{r}140 \\
6 \\
3 \\
10 \\
100\end{array}$ & $\begin{array}{r}10 \\
50 \\
2\end{array}$ & $\begin{array}{l}\text { I } \\
\text { I I } \\
\text { I I } \\
\text { I } \\
\text { I }\end{array}$ & $\begin{array}{l}4945.471 \\
4945.856 \\
4946.111 \\
4946.666 \\
4947.022\end{array}$ & $\begin{array}{r}140 \\
5 \\
3 \\
75 \\
2\end{array}$ & $\begin{array}{r}3 \\
20 \\
2 \\
100\end{array}$ & $\begin{array}{l}\text { I } \\
\text { I I } \\
\text { I } \\
\text { I I } \\
\text { I }\end{array}$ \\
\hline $\begin{array}{l}4909.215 \\
4909.694 \\
4909.844 \\
4910.162 \\
4910.323\end{array}$ & $\begin{array}{r}10 \\
7 \\
20 \\
150 \\
2\end{array}$ & $10 \mathrm{w}$ & $\begin{array}{l}\text { I } \\
\text { I } \\
\text { I } \\
\text { I } \\
\text { I I I }\end{array}$ & $\begin{array}{l}4929.371 \\
4929.985 \\
4930.530 \\
4931.059 \\
4931.150\end{array}$ & $\begin{array}{r}5 \\
125 \\
2 \\
5 \\
1\end{array}$ & $\begin{array}{r}12 \\
300\end{array}$ & $\begin{array}{l}\text { I I } \\
\text { I I } \\
\text { I } \\
\text { I } \\
\text { I I }\end{array}$ & $\begin{array}{l}4947.581 \\
4948.107 \\
4948.560 \\
4948.972 \\
4949.082\end{array}$ & $\begin{array}{r}125 \\
4 \\
1 \\
3 \\
2\end{array}$ & 10 & $\begin{array}{l}\text { I I } \\
\text { I } \\
\text { I } \\
\text { I } \\
\text { I }\end{array}$ \\
\hline $\begin{array}{l}4910.800 \\
4911.144 \\
4911.390 \\
4911.940 \\
4912.045\end{array}$ & $\begin{array}{r}175 \\
30 \\
150 \\
6 \\
18\end{array}$ & $\begin{array}{r}2 \\
50 \\
75\end{array}$ & $\begin{array}{l}\text { I } \\
\text { I I } \\
\text { I I } \\
\text { I } \\
\text { I }\end{array}$ & $\begin{array}{l}4931.385 \\
4931.970 \\
4932.260 \\
4932.344 \\
4932.540\end{array}$ & $\begin{array}{r}10 \\
1 \\
1 \\
4 \\
25\end{array}$ & $\begin{array}{r}2 \\
250\end{array}$ & $\begin{array}{l}\text { I } \\
\text { I } \\
\text { I } \\
\text { I I } \\
\text { II }\end{array}$ & $\begin{array}{l}4949.221 \\
4949.991 \\
4950.248 \\
4950.640 \\
4951.397\end{array}$ & $\begin{array}{r}2 \\
5 \\
75 \\
250 \\
5\end{array}$ & $\begin{array}{r}8 \\
200 \\
10\end{array}$ & $\begin{array}{l}\text { I } \\
\text { I I } \\
\text { I } \\
\text { I I } \\
\text { I I }\end{array}$ \\
\hline $\begin{array}{l}4912.524 \\
4913.479 \\
4914.118 \\
4915.418 \\
4915.812\end{array}$ & $\begin{array}{r}300 \\
20 \\
75 \\
50 \\
18\end{array}$ & $\begin{array}{r}200 \\
250 \\
1\end{array}$ & $\begin{array}{l}\text { I I } \\
\text { I } \\
\text { II } \\
\text { I } \\
\text { I }\end{array}$ & $\begin{array}{l}4933.018 \\
4933.486 \\
4933.558 \\
4933.603 \\
4933.850\end{array}$ & $\begin{array}{r}6 \\
7 \\
4 \\
7 \\
100\end{array}$ & $\begin{array}{l}75 \\
80\end{array}$ & $\begin{array}{l}\text { I } \\
\text { I } \\
\text { I I I } \\
\text { I } \\
\text { I I }\end{array}$ & $\begin{array}{l}4951.642 \\
4951.839 \\
4952.008 \\
4952.350 \\
4952.460\end{array}$ & $\begin{array}{l}1 \\
5 \\
1 \\
1 \\
1\end{array}$ & $\begin{array}{c}12 \\
1\end{array}$ & $\begin{array}{l}\text { I } \\
\text { I I } \\
\text { I } \\
\text { I } \\
\text { I }\end{array}$ \\
\hline $\begin{array}{l}4916.132 \\
4916.395 \\
4917.470 \\
4917.824 \\
4917.983\end{array}$ & $\begin{array}{l}8 \\
2 \mathrm{w} \\
10 \\
20 \\
2\end{array}$ & 100 & $\begin{array}{l}\text { I } \\
\text { I } \\
\text { I I I } \\
\text { I } \\
\text { I }\end{array}$ & $\begin{array}{l}4934.328 \\
4934.539 \\
4934.777 \\
4934.963 \\
4935.174\end{array}$ & $\begin{array}{r}5 \\
10 \\
1 \\
1 \\
4\end{array}$ & 40 & $\begin{array}{l}\text { I } \\
\text { I I } \\
\text { I } \\
\text { I } \\
\text { I I }\end{array}$ & $\begin{array}{l}4952.700 \\
4953.542 \\
4953.948 \\
4954.574 \\
4954.669\end{array}$ & $\begin{array}{r}12 \\
25 \\
20 \\
150 \\
150\end{array}$ & $\begin{array}{r}2 \\
7 \\
200 \\
200\end{array}$ & $\begin{array}{l}\text { I I } \\
\text { I } \\
\text { I } \\
\text { II } \\
\text { II }\end{array}$ \\
\hline $\begin{array}{l}4918.146 \\
4918.376 \\
4918.663 \\
4919.760 \\
4919.8161\end{array}$ & $\begin{array}{r}3 \\
3 \\
2 \\
1 \\
600\end{array}$ & 800 & $\begin{array}{l}\text { I I } \\
\text { I } \\
\text { I } \\
\text { I } \\
\text { II }\end{array}$ & $\begin{array}{l}4935.430 \\
4935.630 \\
4935.928 \\
4936.769 \\
4937.363\end{array}$ & $\begin{array}{c}1 \\
1 \\
12 \\
300 \\
4 \mathrm{w}\end{array}$ & $\begin{array}{r}1 \\
200 \\
4\end{array}$ & $\begin{array}{l}\text { I } \\
\text { I } \\
\text { I } \\
\text { I } \\
\text { II }\end{array}$ & $\begin{array}{l}4955.150 \\
4955.320 \\
4955.450 \\
4955.726 \\
4755.958\end{array}$ & $\begin{array}{l}3 \\
4 \\
3 \\
4 \\
3\end{array}$ & 5 & $\begin{array}{l}\text { I } \\
\text { I } \\
\text { I } \\
\text { I } \\
\text { II }\end{array}$ \\
\hline $\begin{array}{l}4920.534 \\
4920.600 \\
4921.615 \\
4922.949 \\
4923.425\end{array}$ & $\begin{array}{r}200 \\
20 \\
400 \\
150 \\
4\end{array}$ & $\begin{array}{r}10 \\
200 \\
150 \\
6\end{array}$ & $\begin{array}{l}\text { II } \\
\text { I } \\
\text { II } \\
\text { II } \\
\text { I I }\end{array}$ & $\begin{array}{l}4937.835 \\
4938.202 \\
4938.495 \\
4938.590 \\
4939.062\end{array}$ & $\begin{array}{r}125 \\
7 \\
12 \\
6 \\
10\end{array}$ & 3 & $\begin{array}{l}\text { I } \\
\text { I } \\
\text { I } \\
\text { I } \\
\text { I }\end{array}$ & $\begin{array}{l}4956.659 \\
4956.833 \\
4957.302 \\
4957.460 \\
4958.102\end{array}$ & $\begin{array}{r}20 \\
8 \\
30 \\
5 \\
75\end{array}$ & 20 & $\begin{array}{l}\text { I } \\
\text { II } \\
\text { I } \\
\text { I } \\
\text { I }\end{array}$ \\
\hline
\end{tabular}


Table 1. Wavelengths of Thorium Spectra - Continued

\begin{tabular}{|c|c|c|c|c|c|c|c|c|c|c|c|}
\hline \multirow{2}{*}{$\begin{array}{l}\text { Wave- } \\
\text { length }\end{array}$} & \multicolumn{2}{|c|}{ Intensity } & \multirow{2}{*}{$\begin{array}{l}\text { Spec- } \\
\text { trum }\end{array}$} & \multirow{2}{*}{$\begin{array}{l}\text { Wave- } \\
\text { length }\end{array}$} & \multicolumn{2}{|c|}{ Intensity } & \multirow{2}{*}{$\begin{array}{l}\text { Spec- } \\
\text { trum }\end{array}$} & \multirow{2}{*}{$\begin{array}{l}\text { Wave- } \\
\text { length }\end{array}$} & \multicolumn{2}{|c|}{ Intensity } & \multirow{2}{*}{$\begin{array}{l}\text { Spec- } \\
\text { trum }\end{array}$} \\
\hline & amp & Spark & & & Lam & Spark & & & & Spark t & \\
\hline $\begin{array}{l}4958.735 \\
4958.820 \\
4958.989 \\
4959.353 \\
4959.440\end{array}$ & $\begin{array}{r}20 \\
2 \\
1 \\
20 \\
1\end{array}$ & $\begin{array}{l}7 \\
2 \\
5\end{array}$ & $\begin{array}{l}\text { II } \\
\text { I } \\
\text { I } \\
\text { I } \\
\text { I }\end{array}$ & $\begin{array}{l}4978.230 \\
4978.706 \\
4978.986 \\
4979.146 \\
4979.558\end{array}$ & $\begin{array}{r}15 \\
5 \\
8 \\
4 \\
15\end{array}$ & $\begin{array}{l}6 \\
2\end{array}$ & $\begin{array}{l}\text { I } \\
\text { I } \\
\text { I } \\
\text { II } \\
\text { I }\end{array}$ & $\begin{array}{l}4999.388 \\
4999.948 \\
5000.249 \\
5000.732 \\
5001.452\end{array}$ & $\begin{array}{r}10 \\
150 \\
100 \\
10 \\
10\end{array}$ & $600 \mathrm{~h}$ & $\begin{array}{l}\text { I } \\
\text { II } \\
\text { I } \\
\text { I } \\
\text { II }\end{array}$ \\
\hline $\begin{array}{l}4959.548 \\
4959.763 \\
4960.461 \\
4961.396 \\
4961.742\end{array}$ & $\begin{array}{r}3 \\
1 \\
200 \\
20 \\
200\end{array}$ & $\begin{array}{r}1 \\
60 \\
5\end{array}$ & $\begin{array}{l}\text { I } \\
\text { I } \\
\text { II } \\
\text { I } \\
\text { I }\end{array}$ & $\begin{array}{l}4980.189 \\
4980.768 \\
4980.957 \\
4981.241 \\
4981.878\end{array}$ & $\begin{array}{r}600 \\
50 \\
160 \\
15 \\
8\end{array}$ & $\begin{array}{r}30 \\
5\end{array}$ & $\begin{array}{l}\text { I } \\
\text { I } \\
\text { II } \\
\text { II } \\
\text { I }\end{array}$ & $\begin{array}{l}5002.097 \mathrm{i} \\
5002.478 \\
5002.578 \\
5002.897 \\
5003.377\end{array}$ & $\begin{array}{r}400 \\
3 \\
5 \\
10 \\
5\end{array}$ & 10 & $\begin{array}{l}\text { I } \\
\text { I } \\
\text { I I } \\
\text { I } \\
\text { I }\end{array}$ \\
\hline $\begin{array}{l}4962.075 \\
4962.478 \\
4962.947 \\
4963.204 \\
4963.560\end{array}$ & $\begin{array}{r}40 \\
3 \\
100 \\
100 \\
1\end{array}$ & $\begin{array}{r}1 \\
20 \\
80 \\
2\end{array}$ & $\begin{array}{l}\text { I } \\
\text { I } \\
\text { II } \\
\text { II } \\
\text { I }\end{array}$ & $\begin{array}{l}4982.480 \\
4983.535 \\
4984.668 \\
4985.385 \\
4985.950\end{array}$ & $\begin{array}{r}300 \\
50 \\
8 \\
300 \\
100\end{array}$ & $\begin{array}{l}4 \\
6 \\
4\end{array}$ & $\begin{array}{l}\text { I } \\
\text { I } \\
\text { I } \\
\text { I } \\
\text { I }\end{array}$ & $\begin{array}{l}5003.496 \\
5003.599 \\
5003.861 \\
5004.018 \\
5004.133\end{array}$ & $\begin{array}{r}75 \\
10 \\
8 \\
200\end{array}$ & $\begin{array}{r}5 \\
10 \\
5 \\
3\end{array}$ & $\begin{array}{l}\text { II } \\
\text { I } \\
\text { I } \\
\text { I } \\
\text { I }\end{array}$ \\
\hline $\begin{array}{l}4963.771 \\
4964.121 \\
4964.250 \\
4964.986 \\
4965.742\end{array}$ & $\begin{array}{l}100 \mathrm{w} \\
175 \\
10 \\
20 \\
250\end{array}$ & $\begin{array}{r}40 \\
300\end{array}$ & $\begin{array}{l}\text { I } \\
\text { II } \\
\text { I } \\
\text { I } \\
\text { I }\end{array}$ & $\begin{array}{l}4986.321 \\
4987.152 \\
4987.556 \\
4988.250 \\
4988.500\end{array}$ & $\begin{array}{r}10 \\
250 \\
100 \\
1 \\
1\end{array}$ & 700 & $\begin{array}{l}\text { I } \\
\text { II } \\
\text { I } \\
\text { I } \\
\text { I }\end{array}$ & $\begin{array}{l}5005.081 \\
5005.353 \\
5005.990 \\
5006.408 \\
5006.820\end{array}$ & $\begin{array}{r}5 \\
12 \\
10 \\
2\end{array}$ & $400 \mathrm{~h}$ & $\begin{array}{l}\text { I I I } \\
\text { I } \\
\text { I } \\
\text { I } \\
\text { II }\end{array}$ \\
\hline $\begin{array}{l}4967.067 \\
4967.341 \\
4967.666 \\
4968.400 \\
4968.757\end{array}$ & $\begin{array}{r}10 \\
9 \\
10 \\
4 \\
150\end{array}$ & $\begin{array}{r}2 \\
20\end{array}$ & $\begin{array}{l}\text { II } \\
\text { I } \\
\text { I } \\
\text { II } \\
\text { II }\end{array}$ & $\begin{array}{l}4988.620 \\
4988.770 \\
4989.310 \\
4990.034 \\
4990.460\end{array}$ & $\begin{array}{r}2 \\
2 \\
200 \\
15 \\
4\end{array}$ & $\begin{array}{r}3 \\
12 \\
3\end{array}$ & $\begin{array}{l}\text { I } \\
\text { I } \\
\text { I } \\
\text { II } \\
\text { II }\end{array}$ & $\begin{array}{l}5006.960 \\
5007.377 \\
5007.858 \\
5008.194 \\
5009.244\end{array}$ & $\begin{array}{r}4 \\
7 \\
5 \\
75 \\
15\end{array}$ & $\begin{array}{r}30 \\
200\end{array}$ & $\begin{array}{l}\text { I } \\
\text { II } \\
\text { I } \\
\text { II } \\
\text { I }\end{array}$ \\
\hline $\begin{array}{l}4969.132 \\
4969.925 \\
4970.060 \\
4970.865 \\
4971.190\end{array}$ & $\begin{array}{r}30 \\
5 \\
300 \\
6 \\
4\end{array}$ & $\begin{array}{r}20 \\
125 \\
5 \\
2\end{array}$ & $\begin{array}{l}\text { II } \\
\text { II } \\
\text { II } \\
\text { I } \\
\text { I }\end{array}$ & $\begin{array}{l}4990.590 \\
4991.200 \\
4991.603 \\
4991.910 \\
4992.129\end{array}$ & $\begin{array}{r}2 \\
10 \\
2 \\
1 \\
15\end{array}$ & $\begin{array}{l}1 \\
3\end{array}$ & $\begin{array}{l}\text { I } \\
\text { I } \\
\text { I } \\
\text { II }\end{array}$ & $\begin{array}{l}5009.453 \\
5009.955 \\
5010.423 \\
5010.952 \\
5011.484\end{array}$ & $\begin{array}{r}10 \\
100 \\
35 \\
75 \\
75\end{array}$ & $\begin{array}{r}8 \\
100\end{array}$ & $\begin{array}{l}\text { I } \\
\text { II } \\
\text { I } \\
\text { I } \\
\text { I }\end{array}$ \\
\hline $\begin{array}{l}4971.558 \\
4972.184 \\
4972.628 \\
4972.763 \\
4973.081\end{array}$ & $\begin{array}{l}300^{3} \mathrm{~d} \\
20 \\
50 \\
10\end{array}$ & $\begin{array}{r}200 \\
30 \\
10\end{array}$ & $\begin{array}{l}\text { I } \\
\text { I } \\
\text { II } \\
\text { I } \\
\text { II }\end{array}$ & $\begin{array}{l}4992.384 \\
4992.646 \\
4993.140 \\
4993.760 \\
4993.908\end{array}$ & $\begin{array}{r}5 \\
15 \\
1 \\
50 \\
12\end{array}$ & $\begin{array}{c}2 \\
200 \mathrm{w}\end{array}$ & $\begin{array}{l}\text { I } \\
\text { I } \\
\text { I } \\
\text { I } \\
\text { II }\end{array}$ & $\begin{array}{l}5011.779 \\
5011.870 \\
5012.269 \\
5012.730 \\
5012.850\end{array}$ & $\begin{array}{r}2 \\
1 \\
10 \\
1 \\
2\end{array}$ & 2 & $\begin{array}{l}\text { I } \\
\text { I } \\
\text { I } \\
\text { II } \\
\text { I }\end{array}$ \\
\hline $\begin{array}{l}4973.401 \\
4973.765 \\
4974.299 \\
4974.440 \\
4974.673\end{array}$ & $\begin{array}{r}125 \\
5 \\
4 \\
5 \\
5\end{array}$ & $\begin{array}{r}200 \\
5\end{array}$ & $\begin{array}{l}\text { II } \\
\text { II } \\
\text { I } \\
\text { I } \\
\text { I }\end{array}$ & $\begin{array}{l}4994.112 \\
4994.568 \\
4994.954 \\
4995.301 \\
4995.638\end{array}$ & $\begin{array}{l}20 \\
10 \\
10 \mathrm{w} \\
10 \\
5\end{array}$ & 3 & $\begin{array}{l}I \\
I \\
I \\
I \\
I\end{array}$ & $\begin{array}{l}5013.170 \\
5013.610 \\
5014.192 \\
5014.463 \\
5014.769\end{array}$ & $\begin{array}{l}12 \\
25 \\
12 \\
10 \\
30\end{array}$ & 40 & $\begin{array}{l}\text { I } \\
\text { II } \\
\text { I } \\
\text { I } \\
\text { II }\end{array}$ \\
\hline $\begin{array}{l}4974.996 \\
4975.100 \\
4975.540 \\
4975.685 \\
4975.955\end{array}$ & $\begin{array}{c}2 \\
30 \\
8 \mathrm{w} \\
10 \\
300\end{array}$ & $\begin{array}{r}6 \\
1 \\
125\end{array}$ & $\begin{array}{l}\text { II } \\
\text { I } \\
\text { I } \\
\text { I } \\
\text { I }\end{array}$ & $\begin{array}{l}4995.799 \\
4995.977 \\
4996.238 \\
4996.540 \\
4996.882\end{array}$ & $\begin{array}{r}6 \\
6 \\
5 \\
18 \\
5\end{array}$ & 4 & $\begin{array}{l}I \\
I \\
I \\
I \\
I\end{array}$ & $\begin{array}{l}5015.286 \\
5015.903 \\
5016.538 \\
5017.255 \mathrm{i} \\
5017.512\end{array}$ & $\begin{array}{r}10 \\
400 \\
75 \\
500 \\
100\end{array}$ & $\begin{array}{l}300 \\
500\end{array}$ & $\begin{array}{l}\text { I } \\
\text { II } \\
\text { I } \\
\text { II } \\
\text { I }\end{array}$ \\
\hline $\begin{array}{l}4976.603 \\
4977.396 \\
4977.531 \\
4977.663 \\
4978.032\end{array}$ & $\begin{array}{r}1.60 \\
30 \\
4 \\
25 \\
10\end{array}$ & $\begin{array}{r}100 \\
1 \\
40\end{array}$ & $\begin{array}{l}\text { I } \\
I \\
I \\
I \\
\text { II }\end{array}$ & $\begin{array}{l}4997.333 \\
4997.674 \\
4997.817 \\
4999.106 \\
4999.250\end{array}$ & $\begin{array}{r}10 \\
100 \\
200 \\
15 \\
10\end{array}$ & $\begin{array}{r}1 \\
75 \\
100 \\
18\end{array}$ & $\begin{array}{l}\text { II } \\
\text { II } \\
\text { II } \\
\text { II } \\
I\end{array}$ & $\begin{array}{l}5017.755 \\
5017.883 \\
5018.055 \\
5018.740 \\
5018.880\end{array}$ & $\begin{array}{r}20 \\
15 \\
100 \\
6 \\
2\end{array}$ & & $\begin{array}{l}\text { I } \\
\text { I } \\
\text { I } \\
\text { I } \\
\text { I }\end{array}$ \\
\hline
\end{tabular}


Table 1. Wavelengths of Thorium Spectra - Continued

\begin{tabular}{|c|c|c|c|c|c|c|c|c|c|c|c|}
\hline \multirow{2}{*}{$\begin{array}{l}\text { Wave- } \\
\text { length }\end{array}$} & \multirow{2}{*}{\multicolumn{2}{|c|}{$\begin{array}{l}\text { Intensity } \\
\text { Lamp Spark }\end{array}$}} & \multirow{2}{*}{$\begin{array}{l}\text { Spec- } \\
\text { trum }\end{array}$} & \multirow{2}{*}{$\begin{array}{l}\text { Wave- } \\
\text { length }\end{array}$} & \multicolumn{2}{|c|}{ Intensity } & \multirow{2}{*}{$\begin{array}{l}\text { Spec- } \\
\text { trum }\end{array}$} & \multirow{2}{*}{$\begin{array}{l}\text { Wave- } \\
\text { length }\end{array}$} & \multicolumn{2}{|c|}{ Intensity } & \multirow{2}{*}{$\begin{array}{l}\text { Spec- } \\
\text { trum }\end{array}$} \\
\hline & & & & & & & & & & & \\
\hline $\begin{array}{l}5019.337 \\
5019.810 \\
5020.550 \\
5021.260 \\
5021.755\end{array}$ & $\begin{array}{r}200 \\
300 \\
100 \\
125 \\
12\end{array}$ & $\begin{array}{r}300 \\
2 \\
200 \\
2 \\
40\end{array}$ & $\begin{array}{l}\text { II } \\
\text { II } \\
\text { II } \\
\text { I } \\
\text { II }\end{array}$ & $\begin{array}{l}5037.578 \\
5037.703 \\
5038.312 \\
5038.420 \\
5039.241\end{array}$ & $\begin{array}{r}9 \\
2 \\
50 \\
12 \\
200\end{array}$ & $\begin{array}{r}3 \\
15\end{array}$ & $\begin{array}{l}\text { I } \\
\text { I } \\
\text { I } \\
\text { I } \\
\text { I }\end{array}$ & $\begin{array}{l}5058.370 \\
5058.567 \\
5058.904 \\
5059.155 \\
5059.400\end{array}$ & $\begin{array}{r}100 \\
200 \\
6 \\
7 \\
2\end{array}$ & 400 & $\begin{array}{l}\text { I } \\
\text { I I } \\
\text { I } \\
\text { I } \\
\text { I }\end{array}$ \\
\hline $\begin{array}{l}5022.016 \\
5022.176 \\
5023.053 \\
5023.130 \\
5023.490\end{array}$ & $\begin{array}{r}250 \\
20 \\
20 \\
15 \\
40\end{array}$ & 100 & $\begin{array}{l}\text { I } \\
\text { I } \\
\text { II } \\
\text { I } \\
\text { I }\end{array}$ & $\begin{array}{l}5039.538 \\
5039.985 \\
5040.569 \\
5040.692 \\
5041.124\end{array}$ & $\begin{array}{r}75 \\
3 \\
20 \\
20 \\
100\end{array}$ & $\begin{array}{l}8 \\
4\end{array}$ & $\begin{array}{l}\text { I } \\
\text { I } \\
\text { II } \\
\text { I } \\
\text { I }\end{array}$ & $\begin{array}{l}5059.865 \\
5061.226 \\
5061.660 \\
5062.501 \\
5062.862\end{array}$ & $\begin{array}{r}200 \\
300 \\
300 \\
15\end{array}$ & $\begin{array}{r}8 \\
400 \\
2 \\
100\end{array}$ & $\begin{array}{l}\text { I } \\
\text { I I } \\
\text { I } \\
\text { I } \\
\text { I II }\end{array}$ \\
\hline $\begin{array}{l}5023.716 \\
5024.113 \\
5024.320 \\
5024.606 \\
5025.135\end{array}$ & $\begin{array}{r}200 \\
20 \\
1 \\
50 \\
3\end{array}$ & $\begin{array}{l}1 \\
1 \\
1 \\
5\end{array}$ & $\begin{array}{l}\text { I } \\
\text { I } \\
\text { I } \\
\text { II }\end{array}$ & $\begin{array}{l}5041.603 \\
5042.194 \\
5042.608 \\
5043.108 \\
5043.522\end{array}$ & $\begin{array}{r}50 \\
10 \\
4 \\
5 \\
100\end{array}$ & $\begin{array}{r}18 \\
4 \\
6 \\
5\end{array}$ & $\begin{array}{l}\text { I } \\
\text { I I } \\
\text { I I } \\
\text { I I } \\
\text { I }\end{array}$ & $\begin{array}{l}5062.939 \\
5063.518 \\
5064.031 \\
5064.346 \\
5064.613\end{array}$ & $\begin{array}{r}250 \\
150 \\
150 \\
75 \\
160\end{array}$ & $\begin{array}{r}2 \\
100 \\
3\end{array}$ & $\begin{array}{l}\text { I } \\
\text { I } \\
\text { I } \\
\text { II } \\
\text { I }\end{array}$ \\
\hline $\begin{array}{l}5025.855 \\
5026.100 \\
5026.164 \\
5026.231 \\
5026.490\end{array}$ & $\begin{array}{c}8 \\
2 \\
20 \mathrm{~d} \\
2 \\
2\end{array}$ & $\begin{array}{l}2 \\
5 \\
5\end{array}$ & $\begin{array}{l}\text { I I } \\
\text { I I } \\
\text { I } \\
\text { II } \\
\text { I }\end{array}$ & $\begin{array}{l}5044.060 \\
5044.728 \\
5044.958 \\
5045.253 \\
5045.528\end{array}$ & $\begin{array}{r}2 \\
400 \\
200 \\
1\end{array}$ & $\begin{array}{c}2 \mathrm{~h} \\
4 \\
300 \\
5 \\
2 \mathrm{~h}\end{array}$ & $\begin{array}{l}\text { I I } \\
\text { I } \\
\text { II I } \\
\text { I } \\
\text { I I }\end{array}$ & $\begin{array}{l}5064.935 \\
5065.194 \\
5066.137 \\
5066.788 \\
5066.841\end{array}$ & $\begin{array}{l}200 \\
200 \\
150 \\
200\end{array}$ & $\begin{array}{r}4 \\
4 \\
5 \\
100\end{array}$ & $\begin{array}{l}\text { I } \\
\text { I } \\
\text { I } \\
\text { I } \\
\text { I I I }\end{array}$ \\
\hline $\begin{array}{l}5026.980 \\
5027.042 \\
5027.120 \\
5027.300 \\
5027.670\end{array}$ & $\begin{array}{r}5 \\
4 \\
2 \\
1 \\
20\end{array}$ & $\begin{array}{l}2 \\
3\end{array}$ & $\begin{array}{l}\text { I } \\
\text { I } \\
\text { I } \\
\text { II } \\
\text { I }\end{array}$ & $\begin{array}{l}5045.780 \\
5046.362 \\
5047.051 \\
5047.431 \\
5048.010\end{array}$ & $\begin{array}{r}2 \\
100 \\
160 \\
100 \\
6\end{array}$ & $\begin{array}{r}2 \\
3 \\
2 \\
100 \\
2\end{array}$ & $\begin{array}{l}\text { II } \\
\text { I } \\
\text { I } \\
\text { I I } \\
\text { II }\end{array}$ & $\begin{array}{l}5067.150 \\
5067.974 \mathrm{i} \\
5068.853 \\
5068.951 \\
5069.085\end{array}$ & $\begin{array}{r}50 \\
900 \\
15 \\
5 \\
10\end{array}$ & $\begin{array}{r}15 \\
2\end{array}$ & $\begin{array}{l}\text { I } \\
\text { I } \\
\text { I } \\
\text { II } \\
\text { I }\end{array}$ \\
\hline $\begin{array}{l}5028.6561 \\
5029.013 \\
5029.634 \\
5029.903 \\
5030.540\end{array}$ & $\begin{array}{r}400 \\
150 \\
75 \\
300 \\
2\end{array}$ & $\begin{array}{r}400 \\
200 \\
2\end{array}$ & $\begin{array}{l}\text { II } \\
\text { I } \\
\text { I I } \\
\text { I } \\
\text { I }\end{array}$ & $\begin{array}{l}5048.946 \\
5049.7961 \\
5050.795 \\
5051.091 \\
5051.356\end{array}$ & $\begin{array}{r}35 \\
400 \\
200 \\
20 \\
12\end{array}$ & $\begin{array}{r}40 \\
5 \\
75\end{array}$ & $\begin{array}{l}\text { I } \\
\text { I } \\
\text { I } \\
\text { I } \\
\text { I I }\end{array}$ & $\begin{array}{l}5069.332 \\
5069.850 \\
5070.020 \\
5070.452 \\
5070.788\end{array}$ & $\begin{array}{r}150 \\
1 \\
2 \\
10 \\
35\end{array}$ & $\begin{array}{l}3 \\
1 \\
1 \\
1\end{array}$ & $\begin{array}{l}\text { I } \\
\text { II } \\
\text { I } \\
\text { I } \\
\text { I }\end{array}$ \\
\hline $\begin{array}{l}5030.630 \\
5031.148 \\
5031.324 \\
5031.815 \\
5031.938\end{array}$ & $\begin{array}{r}3 \\
20 \\
10 \\
15 \\
6\end{array}$ & $\begin{array}{r}10 \\
3\end{array}$ & $\begin{array}{l}\text { I } \\
\text { I } \\
\text { I I } \\
\text { I } \\
\text { II }\end{array}$ & $\begin{array}{l}5051.892 \\
5052.177 \\
5052.588 \\
5052.850 \\
5053.200\end{array}$ & $\begin{array}{c}200 \\
7 \mathrm{w} \\
20 \mathrm{~s} \\
5 \\
2\end{array}$ & $\begin{array}{r}3 \\
18 \\
2\end{array}$ & $\begin{array}{l}\text { I } \\
\text { II } \\
\text { I } \\
\text { II } \\
\text { I }\end{array}$ & $\begin{array}{l}5070.941 \\
5071.752 \\
5071.870 \\
5072.306 \\
5072.634\end{array}$ & $\begin{array}{r}1 \\
20 \\
3 \\
4 \\
30\end{array}$ & $\begin{array}{r}2 \\
4 \\
15 \\
125\end{array}$ & $\begin{array}{l}\text { II } \\
\text { I } \\
\text { I } \\
\text { II } \\
\text { II }\end{array}$ \\
\hline $\begin{array}{l}5032.105 \\
5032.376 \\
5032.790 \\
5033.016 \\
5033.326\end{array}$ & $\begin{array}{r}8 \\
5 \\
2 \\
5 \\
10\end{array}$ & 1 & $\begin{array}{l}\text { I } \\
\text { I } \\
\text { I } \\
\text { I } \\
\text { I }\end{array}$ & $\begin{array}{l}.724 \\
.749 \\
.881 \\
.037 \\
.630\end{array}$ & $\begin{array}{l}3 \\
5 \\
3 \\
5 \\
3\end{array}$ & 5 & $\begin{array}{l}\text { II } \\
\text { I } \\
\text { I } \\
\text { I } \\
\text { I }\end{array}$ & $\begin{array}{l}3.095 \\
3.600 \\
3.987 \\
4.651\end{array}$ & $\begin{array}{r}10 \\
10 \\
8 \\
25 \\
15\end{array}$ & $\begin{array}{r}75 \\
5 \\
12\end{array}$ & $\begin{array}{l}\text { I } \\
\text { I I } \\
\text { I I } \\
\text { I I } \\
\text { I }\end{array}$ \\
\hline $\begin{array}{l}5033.900 \\
5034.297 \\
5034.650 \\
5035.281 \\
5035.327\end{array}$ & $\begin{array}{r}15 \\
35 \\
2 \\
30\end{array}$ & $\begin{array}{r}30 \\
1 \\
18\end{array}$ & $\begin{array}{l}\text { II } \\
\text { I } \\
\text { I } \\
\text { II I } \\
\text { I }\end{array}$ & $\begin{array}{l}5055.358 \\
5055.754 \\
5056.205 \\
5056.324 \\
5558.860\end{array}$ & $\begin{array}{r}400 \\
7 \\
10 \\
5 \\
50\end{array}$ & $\begin{array}{r}10 \\
2\end{array}$ & $\begin{array}{l}\text { II } \\
\text { I } \\
\text { I } \\
\text { II }\end{array}$ & $\begin{array}{l}5075.467 \\
5075.949 \\
5076.144 \\
5076.237 \\
5076.582\end{array}$ & $\begin{array}{r}75 \\
10 \\
4 \\
3 \\
12\end{array}$ & $\begin{array}{r}175 \\
5 \\
50\end{array}$ & $\begin{array}{l}\text { I I } \\
\text { I } \\
\text { I } \\
\text { II } \\
\text { II }\end{array}$ \\
\hline $\begin{array}{l}5035.460 \\
5035.916 \\
5036.214 \\
5036.734 \\
5037.296\end{array}$ & $\begin{array}{r}5 \\
2 \\
3 \\
50 \\
10\end{array}$ & 3 & $\begin{array}{l}I \\
I \\
I \\
I \\
I\end{array}$ & $\begin{array}{l}5057.080 \\
5057.134 \\
5057.604 \\
5057.857 \\
5057.990\end{array}$ & $\begin{array}{l}2 w \\
2 \\
1 \\
1 \\
75\end{array}$ & $\begin{array}{l}2 \\
3\end{array}$ & $\begin{array}{l}\text { I } \\
\text { I } \\
\text { II } \\
\text { II } \\
\text { I }\end{array}$ & $\begin{array}{l}5076.810 \\
5077.195 \\
5077.504 \\
5077.670 \\
5078.856\end{array}$ & $\begin{array}{r}2 \\
2 \\
3 \\
2 \\
10\end{array}$ & & $\begin{array}{l}\text { I } \\
\text { I } \\
\text { I } \\
\text { I } \\
\text { I }\end{array}$ \\
\hline
\end{tabular}


Table 1. Wavelengths of Thorium Spectra - Continued

\begin{tabular}{|c|c|c|c|c|c|c|c|c|c|c|c|}
\hline \multirow{2}{*}{$\begin{array}{l}\text { Wave- } \\
\text { length }\end{array}$} & \multicolumn{2}{|c|}{ Intensity } & \multirow{2}{*}{$\begin{array}{l}\text { Spec- } \\
\text { trum }\end{array}$} & \multirow{2}{*}{$\begin{array}{l}\text { Wave- } \\
\text { length }\end{array}$} & \multicolumn{2}{|c|}{ Intensity } & \multirow{2}{*}{$\begin{array}{l}\text { Spec- } \\
\text { trum }\end{array}$} & \multirow{2}{*}{$\begin{array}{l}\text { Wave- } \\
\text { length }\end{array}$} & \multicolumn{2}{|c|}{ Intensity } & \multirow{2}{*}{$\begin{array}{l}\text { Spec- } \\
\text { trum }\end{array}$} \\
\hline & Lamp & Spark & & & $a m p$ & Spark & & & Lamp & Spark & \\
\hline $\begin{array}{l}5079.139 \\
5079.932 \\
5080.447 \\
5080.770 \\
5081.100\end{array}$ & $\begin{array}{r}25 \\
25 \\
15 \\
1 \\
1\end{array}$ & $\begin{array}{r}30 \\
4 \\
10 \\
2\end{array}$ & $\begin{array}{l}\text { II } \\
\text { I } \\
\text { I I } \\
\text { I } \\
\text { I I }\end{array}$ & $\begin{array}{l}5099.752 \\
5100.197 \\
5100.617 \\
5100.731 \\
5101.120\end{array}$ & $\begin{array}{r}2 \\
3 \\
200 \\
\\
175\end{array}$ & $\begin{array}{r}10 \\
2 \\
10 \\
2\end{array}$ & $\begin{array}{l}\text { I } \\
\text { II } \\
\text { I } \\
\text { I I I } \\
\text { I }\end{array}$ & $\begin{array}{l}5117.091 \\
5117.295 \\
5118.181 \\
5118.284 \\
5119.659\end{array}$ & $\begin{array}{r}100 \\
7 \\
5 \\
3\end{array}$ & $\begin{array}{l}3 \\
8\end{array}$ & $\begin{array}{l}\text { I I I } \\
\text { I } \\
\text { I } \\
\text { I I } \\
\text { I I }\end{array}$ \\
\hline $\begin{array}{l}5081.450 \\
5082.009 \\
5082.032 \\
5082.079 \\
5082.622\end{array}$ & $\begin{array}{r}150 \\
4 \\
2 \\
4 \\
30\end{array}$ & $\begin{array}{l}8 \\
2\end{array}$ & $\begin{array}{l}\text { I } \\
\text { I } \\
\text { II } \\
\text { I } \\
\text { I }\end{array}$ & $\begin{array}{l}5101.480 \\
5101.789 \\
5102.061 \\
5102.402 \\
5102.720\end{array}$ & $\begin{array}{r}4 \\
8 \\
1 \\
5 \\
10\end{array}$ & $\begin{array}{r}1 \\
3 \\
10\end{array}$ & $\begin{array}{l}\text { I } \\
\text { I } \\
\text { II } \\
\text { I } \\
\text { II }\end{array}$ & $\begin{array}{l}5119.860 \\
5120.702 \\
5120.980 \\
5121.323 \\
5121.600\end{array}$ & $\begin{array}{l}10 \\
2 \mathrm{w} \\
1 \\
15 \\
2\end{array}$ & $\begin{array}{l}1 \\
1\end{array}$ & $\begin{array}{l}\text { I } \\
\text { I } \\
\text { I } \\
\text { I } \\
\text { I }\end{array}$ \\
\hline $\begin{array}{l}5083.028 \\
5083.160 \\
5083.579 \\
5084.033 \\
5084.428\end{array}$ & $\begin{array}{l}1 \\
1 \\
2 \\
5 \\
5\end{array}$ & & $\begin{array}{l}\text { I } \\
\text { I } \\
\text { I } \\
\text { I } \\
\text { I }\end{array}$ & $\begin{array}{l}5103.769 \\
5103.900 \\
5104.262 \\
5104.568 \\
5105.127\end{array}$ & $\begin{array}{r}25 \\
4 \\
2 \\
25 \\
2\end{array}$ & 150 & $\begin{array}{l}\text { II } \\
\text { I } \\
\text { I } \\
\text { II } \\
\text { I }\end{array}$ & $\begin{array}{l}5121.920 \\
5122.226 \\
5122.488 \\
5122.893 \\
5123.140\end{array}$ & $\begin{array}{l}1 \mathrm{w} \\
2 \\
50 \\
5 \\
12\end{array}$ & $\begin{array}{l}50 \\
30\end{array}$ & $\begin{array}{l}\text { I } \\
\text { I } \\
\text { I I } \\
\text { I I } \\
\text { I }\end{array}$ \\
\hline $\begin{array}{l}5084.520 \\
5084.799 \\
5084.990 \\
5085.246 \\
5085.844\end{array}$ & $\begin{array}{r}35 \\
150 \\
12 \\
10\end{array}$ & 600 & $\begin{array}{l}\text { I I I } \\
\text { I } \\
\text { I } \\
\text { I II } \\
\text { I }\end{array}$ & $\begin{array}{l}5105.232 \\
5105.544 \\
5105.775 \\
5106.015 \\
5106.317\end{array}$ & $\begin{array}{r}1 \\
5 \\
3 \\
2 \\
10\end{array}$ & $\begin{array}{r}5 \\
5 \\
75\end{array}$ & $\begin{array}{l}\text { I } \\
\text { II } \\
\text { II } \\
\text { I } \\
\text { II }\end{array}$ & $\begin{array}{l}5123.196 \\
5123.241 \\
5124.243 \\
5124.433 \\
5124.677\end{array}$ & $\begin{array}{r}12 \\
15 \\
2 \\
4\end{array}$ & 18 & $\begin{array}{l}\text { I I I } \\
\text { I } \\
\text { I } \\
\text { I I } \\
\text { I }\end{array}$ \\
\hline $\begin{array}{l}5086.174 \\
5086.829 \\
5087.484 \\
5087.722 \\
5087.830\end{array}$ & $\begin{array}{r}50 \\
15 \\
10 \\
1 \\
1\end{array}$ & $\begin{array}{r}2 \\
60\end{array}$ & $\begin{array}{l}\text { I } \\
\text { I I } \\
\text { I I } \\
\text { I } \\
\text { I }\end{array}$ & $\begin{array}{l}5106.668 \\
5107.237 \\
5107.803 \\
5107.960 \\
5108.404\end{array}$ & $\begin{array}{r}18 \\
4 \\
3 \\
1 \\
2\end{array}$ & 800 & $\begin{array}{l}\text { I } \\
\text { I I } \\
\text { I } \\
\text { I } \\
\text { I I I }\end{array}$ & $\begin{array}{l}5125.017 \\
5125.493 \\
5125.820 \\
5125.950 \\
5126.430\end{array}$ & $\begin{array}{c}4 \mathrm{w} \\
125 \\
10 \\
150 \\
2\end{array}$ & $\begin{array}{l}5 \\
2 \\
1\end{array}$ & $\begin{array}{l}\text { I } \\
\text { I } \\
\text { I } \\
\text { I } \\
\text { I I }\end{array}$ \\
\hline $\begin{array}{l}5088.311 \\
5088.705 \\
5089.215 \\
5090.044 \\
5090.554\end{array}$ & $\begin{array}{r}10 \\
5 \\
30 \\
100 \\
50\end{array}$ & $\begin{array}{r}3 \\
1 \\
50 \\
3\end{array}$ & $\begin{array}{l}\text { I I } \\
\text { I } \\
\text { I I } \\
\text { I } \\
\text { I }\end{array}$ & $\begin{array}{l}5108.573 \\
5109.008 \\
5109.470 \\
5109.737 \\
5110.048\end{array}$ & $\begin{array}{r}3 \\
3 \\
1 \\
30 \\
10\end{array}$ & 2 & $\begin{array}{l}\text { I } \\
\text { I } \\
\text { I } \\
\text { I } \\
\text { I }\end{array}$ & $\begin{array}{l}5126.651 \\
5126.764 \\
5126.885 \\
5127.051 \\
5128.080\end{array}$ & $\begin{array}{l}2 \\
1 \\
2 \\
1 \\
2\end{array}$ & $\begin{array}{c}15 \mathrm{w} \\
2 \\
2\end{array}$ & $\begin{array}{l}\text { I } \\
\text { I } \\
\text { II } \\
\text { II } \\
\text { I I }\end{array}$ \\
\hline $\begin{array}{l}5090.753 \\
5091.343 \\
5091.619 \\
5092.253 \\
5093.091\end{array}$ & $\begin{array}{r}75 \\
1 \\
2 \\
1 \\
12\end{array}$ & $\begin{array}{r}1 \\
15\end{array}$ & $\begin{array}{l}\text { I I } \\
\text { I } \\
\text { I } \\
\text { I } \\
\text { I I }\end{array}$ & $\begin{array}{l}5110.222 \\
5110.567 \\
5110.859 \\
5111.063 \\
5111.280\end{array}$ & $\begin{array}{r}6 \\
15 \\
150 \\
40 \\
100\end{array}$ & 250 & $\begin{array}{l}\text { I } \\
\text { I } \\
\text { II } \\
\text { I } \\
\text { I }\end{array}$ & $\begin{array}{l}5128.478 \\
5128.716 \\
5129.193 \\
5129.549 \\
5129.658\end{array}$ & $\begin{array}{r}125 \\
1 \\
10 \\
15 \\
3\end{array}$ & 3 & $\begin{array}{l}\text { I } \\
\text { I } \\
\text { I } \\
\text { I } \\
\text { I }\end{array}$ \\
\hline $\begin{array}{l}5093.283 \\
5094.118 \\
5094.545 \\
5094.687 \\
5095.062\end{array}$ & $\begin{array}{r}5 \\
180 \\
5 \\
5 \\
350\end{array}$ & $\begin{array}{r}100 \\
2 \\
175\end{array}$ & $\begin{array}{l}\text { I } \\
\text { I I } \\
\text { I I } \\
\text { I } \\
\text { I I }\end{array}$ & $\begin{array}{l}5111.568 \\
5111.667 \\
5111.890 \\
5112.753 \\
5113.051\end{array}$ & $\begin{array}{l}5 \\
4 \\
1 \\
4 \\
3\end{array}$ & 2 & $\begin{array}{l}\text { I I } \\
\text { I } \\
\text { I } \\
\text { I } \\
\text { I }\end{array}$ & $\begin{array}{l}5129.843 \\
5129.975 \\
5130.230 \\
5130.481 \\
5130.796\end{array}$ & $\begin{array}{r}15 \\
2 \\
30 \\
2 \\
10\end{array}$ & $\begin{array}{r}40 \\
2\end{array}$ & $\begin{array}{l}\text { I I } \\
\text { I } \\
\text { I } \\
\text { I } \\
\text { I }\end{array}$ \\
\hline $\begin{array}{l}5095.417 \\
5095.628 \\
5096.480 \\
5096.788 \\
5097.638\end{array}$ & $\begin{array}{r}4 \\
2 \\
200 \\
5 \\
2\end{array}$ & $\begin{array}{r}10 \\
8 \\
5\end{array}$ & $\begin{array}{l}\text { I I } \\
\text { I } \\
\text { I } \\
\text { I I } \\
\text { I }\end{array}$ & $\begin{array}{l}5113.293 \\
5113.376 \\
5113.890 \\
5114.066 \\
5115.044 \mathrm{i}\end{array}$ & $\begin{array}{r}25 \\
30 \\
1 \\
3 \\
250\end{array}$ & $\begin{array}{r}50 \\
2\end{array}$ & $\begin{array}{l}\text { I } \\
\text { II } \\
\text { I I } \\
\text { I } \\
\text { I }\end{array}$ & $\begin{array}{l}5131.062 \\
5131.476 \\
5131.701 \\
5131.994 \\
5132.233\end{array}$ & $\begin{array}{r}75 \\
4 \\
4 \\
15 \\
10\end{array}$ & $\begin{array}{r}300 \\
12\end{array}$ & $\begin{array}{l}\text { I I } \\
\text { I I } \\
\text { I } \\
\text { I } \\
\text { I I }\end{array}$ \\
\hline $\begin{array}{l}5098.046 \\
5098.639 \\
5098.916 \\
5099.017 \\
5099.390\end{array}$ & $\begin{array}{r}200 \\
18 \\
40 \\
30 \\
2\end{array}$ & $\begin{array}{r}125 \\
20\end{array}$ & $\begin{array}{l}\text { I I } \\
\text { I I } \\
\text { I } \\
\text { I } \\
\text { I }\end{array}$ & $\begin{array}{l}5115.646 \\
5116.035 \\
5116.292 \\
5116.612 \\
5116.861\end{array}$ & $\begin{array}{l}2 \\
25 \mathrm{~h} \\
50 \\
7 \\
5\end{array}$ & $\begin{array}{c}20 w \\
2\end{array}$ & $\begin{array}{l}\text { I I I } \\
\text { I } \\
\text { I } \\
\text { I } \\
\text { I }\end{array}$ & $\begin{array}{l}5132.488 \\
5132.754 \\
5132.864 \\
5133.120 \\
5133.734\end{array}$ & $\begin{array}{r}2 \\
5 \\
2 \\
50 \\
5\end{array}$ & 2 & $\begin{array}{l}\text { I } \\
\text { I } \\
\text { I } \\
\text { I } \\
\text { I }\end{array}$ \\
\hline
\end{tabular}


Table 1. Wavelengths of Thorium Spectra - Continued

\begin{tabular}{|c|c|c|c|c|c|c|c|c|c|c|c|}
\hline \multirow{2}{*}{$\begin{array}{l}\text { Wave- } \\
\text { length }\end{array}$} & \multicolumn{2}{|c|}{ Intensity } & \multirow{2}{*}{$\begin{array}{l}\text { Spec- } \\
\text { trum }\end{array}$} & \multirow{2}{*}{$\begin{array}{l}\text { Wave- } \\
\text { length }\end{array}$} & \multicolumn{2}{|c|}{ Intensity } & \multirow{2}{*}{$\begin{array}{l}\text { Spec- } \\
\text { trum }\end{array}$} & \multirow{2}{*}{$\begin{array}{l}\text { Wave- } \\
\text { length }\end{array}$} & \multicolumn{2}{|c|}{ Intensity } & \multirow{2}{*}{$\begin{array}{l}\text { Spec- } \\
\text { trum }\end{array}$} \\
\hline & & & & & & & & & & & \\
\hline $\begin{array}{l}5134.026 \\
5134.753 \\
5135.228 \\
5135.420 \\
5135.587\end{array}$ & $\begin{array}{r}2 \\
150 \\
2 \\
2 \\
3\end{array}$ & $\begin{array}{l}1 \\
2 \\
4\end{array}$ & $\begin{array}{l}\text { I I } \\
\text { I } \\
\text { I I } \\
\text { I } \\
\text { I }\end{array}$ & $\begin{array}{l}5159.141 \\
5159.450 \\
5159.625 \\
5159.744 \\
5160.683\end{array}$ & $\begin{array}{r}2 \\
8 \\
30 \\
15 \\
100\end{array}$ & $\begin{array}{r}4 \\
100\end{array}$ & $\begin{array}{l}\text { I I } \\
\text { I } \\
\text { I } \\
\text { I } \\
\text { I I }\end{array}$ & $\begin{array}{l}5177.082 \\
5177.479 \\
5177.627 \\
5178.351 \\
5178.478\end{array}$ & $\begin{array}{r}30 \\
150 \\
30 \\
75\end{array}$ & $\begin{array}{r}15 \\
2 \\
2\end{array}$ & $\begin{array}{l}\text { I I I } \\
\text { I } \\
\text { I } \\
\text { I } \\
\text { I }\end{array}$ \\
\hline $\begin{array}{l}5136.126 \\
5136.390 \\
5137.010 \\
5137.480 \\
5137.714\end{array}$ & $\begin{array}{r}18 \\
2 \\
1 \\
7 \\
5\end{array}$ & $\begin{array}{l}2 \\
1\end{array}$ & $\begin{array}{l}\text { I } \\
\text { I } \\
\text { I } \\
\text { I } \\
\text { I }\end{array}$ & $\begin{array}{l}5160.715 \\
5161.040 \\
5161.282 \\
5161.545 \\
5162.364\end{array}$ & $\begin{array}{r}500 \\
10 \\
8 \\
300 \\
20\end{array}$ & $\begin{array}{l}2 \\
4\end{array}$ & $\begin{array}{l}\text { I } \\
\text { I } \\
\text { I } \\
\text { I } \\
\text { I }\end{array}$ & $\begin{array}{l}5179.137 \\
5179.350 \\
5179.908 \\
5179.992 \\
5180.144\end{array}$ & $\begin{array}{r}8 \\
12 \\
2 \\
2 \\
5\end{array}$ & $\begin{array}{l}1 \\
1\end{array}$ & $\begin{array}{l}\text { I } \\
\text { I } \\
\text { I } \\
\text { I } \\
\text { I }\end{array}$ \\
\hline $\begin{array}{l}5138.090 \\
5138.957 \\
5140.116 \\
5140.766 \\
5141.268\end{array}$ & $\begin{array}{r}2 \\
6 \\
5 \\
200 \\
5\end{array}$ & & $\begin{array}{l}\text { I } \\
\text { I } \\
\text { I } \\
\text { I } \\
\text { I }\end{array}$ & $\begin{array}{l}5162.559 \\
5162.691 \\
5163.412 \\
5163.456 \\
5164.115\end{array}$ & $\begin{array}{r}20 \\
10 \\
300 \\
20\end{array}$ & $\begin{array}{r}2 \\
50\end{array}$ & $\begin{array}{l}\text { I } \\
\text { I } \\
\text { I I I } \\
\text { I } \\
\text { I }\end{array}$ & $\begin{array}{l}5180.732 \\
5181.468 \\
5181.567 \\
5181.681 \\
5182.010\end{array}$ & $\begin{array}{r}75 \\
6 \\
6 \\
6 \\
30\end{array}$ & $\begin{array}{l}2 \\
1\end{array}$ & $\begin{array}{l}\text { I } \\
\text { I } \\
\text { I } \\
\text { I } \\
\text { I I }\end{array}$ \\
\hline $\begin{array}{l}5141.620 \\
5141.960 \\
5142.140 \\
5142.627 \\
5143.276\end{array}$ & $\begin{array}{r}1 \\
1 \\
2 \\
3 \\
150\end{array}$ & 50 & $\begin{array}{l}\text { I } \\
\text { I } \\
\text { I } \\
\text { I } \\
\text { I I }\end{array}$ & $\begin{array}{l}5164.201 \\
5164.405 \\
5164.978 \\
5165.065 \\
5165.607\end{array}$ & $\begin{array}{l}8 \\
30 \mathrm{w} \\
15 \\
20 \\
40\end{array}$ & $\begin{array}{l}1 \\
2\end{array}$ & $\begin{array}{l}\text { I } \\
\text { I } \\
\text { I } \\
\text { I } \\
\text { I }\end{array}$ & $\begin{array}{l}5182.533 \\
5183.103 \\
5183.346 \\
5183.989 \\
5184.444\end{array}$ & $\begin{array}{r}200 \\
20 \\
10 \\
200 \\
100\end{array}$ & $\begin{array}{r}350 \\
5 \\
2 \\
200\end{array}$ & $\begin{array}{l}\text { I I } \\
\text { I } \\
\text { II } \\
\text { II } \\
\text { I }\end{array}$ \\
\hline $\begin{array}{l}5143.918 \\
5145.044 \\
5145.660 \\
5146.064 \\
5147.140\end{array}$ & $\begin{array}{r}200 \\
100 \\
3 \\
50 \\
5\end{array}$ & 2 & $\begin{array}{l}\text { I } \\
\text { I } \\
\text { I } \\
\text { I } \\
\text { I I }\end{array}$ & $\begin{array}{l}5166.249 \\
5166.662 \\
5166.876 \\
5166.910 \\
5167.874\end{array}$ & $\begin{array}{r}18 \\
40 \\
5 \\
30 \\
5\end{array}$ & $\begin{array}{r}75 \\
2\end{array}$ & $\begin{array}{l}\text { I } \\
\text { I } \\
\text { II } \\
\text { I } \\
\text { I I }\end{array}$ & $\begin{array}{l}5184.725 \\
5185.207 \\
5185.895 \\
5186.180 \\
5186.417\end{array}$ & $\begin{array}{c}125 \\
75 \\
12 \\
6 \mathrm{w} \\
75\end{array}$ & $\begin{array}{r}50 \\
300\end{array}$ & $\begin{array}{l}\text { I I } \\
\text { I I I } \\
\text { I } \\
\text { I } \\
\text { I }\end{array}$ \\
\hline $\begin{array}{l}5147.481 \\
5148.221 \\
5148.925 \\
5149.220 \\
5149.813\end{array}$ & $\begin{array}{r}5 \\
75 \\
2 \\
50 \\
25\end{array}$ & $300 \mathrm{~h}$ & $\begin{array}{l}\text { I I } \\
\text { I I } \\
\text { I } \\
\text { I } \\
\text { I }\end{array}$ & $\begin{array}{l}5168.010 \\
5168.170 \\
5168.402 \\
5168.593 \\
5168.920\end{array}$ & $\begin{array}{r}5 \\
1 \\
25 \\
100 \\
75\end{array}$ & 5 & $\begin{array}{l}\text { I } \\
\text { II } \\
\text { I } \\
\text { I } \\
\text { I }\end{array}$ & $\begin{array}{l}5187.117 \\
5187.160 \\
5187.342 \\
5187.455 \\
5187.777\end{array}$ & $\begin{array}{r}75 \\
150 \\
120 \\
4\end{array}$ & $\begin{array}{r}8 \\
10 \\
30\end{array}$ & $\begin{array}{l}\text { I I } \\
\text { I I } \\
\text { I } \\
\text { I } \\
\text { I }\end{array}$ \\
\hline $\begin{array}{l}5150.714 \\
5151.621 \\
5151.871 \\
5151.980 \\
5152.440\end{array}$ & $\begin{array}{r}20 \\
400 \\
10 \\
5 \\
1\end{array}$ & $\begin{array}{c}50 \\
15 \\
1\end{array}$ & $\begin{array}{l}\text { I } \\
\text { I I } \\
\text { I I } \\
\text { I } \\
\text { I I }\end{array}$ & $\begin{array}{l}5169.152 \\
5169.680 \\
5170.232 \\
5170.900 \\
5172.224\end{array}$ & $\begin{array}{r}15 \\
5 \\
200 \\
1 \\
10\end{array}$ & $\begin{array}{r}18 \\
1 \\
10\end{array}$ & $\begin{array}{l}\text { I I } \\
\text { I } \\
\text { II } \\
\text { I } \\
\text { I }\end{array}$ & $\begin{array}{l}5187.948 \\
5188.363 \\
5188.718 \\
5189.017 \\
5189.676\end{array}$ & $\begin{array}{r}8 \\
75 \\
5 \\
20 \\
100\end{array}$ & $\begin{array}{r}75 \\
5 \\
250\end{array}$ & $\begin{array}{l}\text { I } \\
\text { II } \\
\text { I } \\
\text { I } \\
\text { I I }\end{array}$ \\
\hline $\begin{array}{l}5152.700 \\
5152.965 \\
5153.249 \\
5153.529 \\
5154.243 \mathrm{i}\end{array}$ & $\begin{array}{r}2 \\
2 \\
3 \\
15 \\
400\end{array}$ & $\begin{array}{l}30 \\
15\end{array}$ & $\begin{array}{l}\text { I } \\
\text { I } \\
\text { I } \\
\text { I I } \\
\text { I }\end{array}$ & $\begin{array}{l}5172.477 \\
5172.927 \\
5173.260 \\
5173.390 \\
5173.684\end{array}$ & $\begin{array}{r}20 \\
10 \\
5 \\
5 \\
100\end{array}$ & $\begin{array}{r}4 \\
15 \\
2 \\
1 \\
1\end{array}$ & $\begin{array}{l}\text { I } \\
\text { I I } \\
\text { I I } \\
\text { I I } \\
\text { I }\end{array}$ & $\begin{array}{l}5190.220 \\
5190.380 \\
5190.875 \\
5191.719 \\
5191.985\end{array}$ & $\begin{array}{r}2 \\
3 \\
200 \\
2 \\
5\end{array}$ & 200 & $\begin{array}{l}\text { I } \\
\text { I } \\
\text { II } \\
\text { I } \\
\text { I }\end{array}$ \\
\hline $\begin{array}{l}5154.897 \\
5155.076 \\
5155.345 \\
5155.917 \\
5156.268\end{array}$ & $\begin{array}{r}15 \\
8 \\
2 \\
7 \\
7\end{array}$ & $\begin{array}{r}12 \\
3 \\
8 \\
1\end{array}$ & $\begin{array}{l}\text { I I } \\
\text { I } \\
\text { I I } \\
\text { I I } \\
\text { I }\end{array}$ & $\begin{array}{l}5173.847 \\
5174.241 \\
5174.369 \\
5174.582 \\
5174.795\end{array}$ & $\begin{array}{r}50 \\
75 \\
50 \\
1 \\
25\end{array}$ & $\begin{array}{r}75 \\
2\end{array}$ & $\begin{array}{l}\text { I } \\
\text { II } \\
\text { I } \\
\text { I I } \\
\text { I }\end{array}$ & $\begin{array}{l}5192.183 \\
5192.741 \\
5192.977 \\
5193.083 \\
5193.180\end{array}$ & $\begin{array}{r}2 \\
12 \\
2 \\
2 \\
2\end{array}$ & 3 & $\begin{array}{l}\text { I } \\
\text { I } \\
\text { I } \\
\text { I I } \\
\text { I }\end{array}$ \\
\hline $\begin{array}{l}5156.620 \\
5157.006 \\
5157.450 \\
5157.568 \\
5158.604 \mathrm{i}\end{array}$ & $\begin{array}{r}8 \\
2 \\
25 \\
2 \\
700\end{array}$ & $\begin{array}{r}1 \\
2 \\
3 \\
20\end{array}$ & $\begin{array}{l}\text { I } \\
\text { I I } \\
\text { I } \\
\text { I I } \\
\text { I }\end{array}$ & $\begin{array}{l}5175.055 \\
5175.326 \\
5175.914 \\
5176.420 \\
5176.961 \mathrm{i}\end{array}$ & $\begin{array}{r}3 \\
100 \\
100 \\
50 \\
400\end{array}$ & $\begin{array}{r}20 \\
2 \\
3 \\
15\end{array}$ & $\begin{array}{l}\text { I I } \\
\text { I } \\
\text { I } \\
\text { I } \\
\text { I }\end{array}$ & $\begin{array}{l}5193.417 \\
5193.566 \\
5193.640 \\
5193.835 \\
5193.950\end{array}$ & $\begin{array}{r}8 \\
10 \\
4 \\
75 \\
75\end{array}$ & 75 & $\begin{array}{l}\text { I } \\
\text { I } \\
\text { I } \\
\text { II } \\
\text { I }\end{array}$ \\
\hline
\end{tabular}

$5466370-60-5$ 
Table 1. Wavelengths of Thorium Spectra - Continued

\begin{tabular}{|c|c|c|c|c|c|c|c|c|c|c|c|}
\hline \multirow{2}{*}{$\begin{array}{l}\text { Wave- } \\
\text { length }\end{array}$} & \multicolumn{2}{|c|}{ Intensity } & \multirow{2}{*}{$\begin{array}{l}\text { Spec- } \\
\text { trum }\end{array}$} & \multirow{2}{*}{$\begin{array}{l}\text { Wave- } \\
\text { length }\end{array}$} & \multicolumn{2}{|c|}{ Intensity } & \multirow{2}{*}{$\begin{array}{l}\text { Spec- } \\
\text { trum }\end{array}$} & \multirow{2}{*}{$\begin{array}{l}\text { Wave- } \\
\text { length }\end{array}$} & \multicolumn{2}{|c|}{ Intensity } & \multirow{2}{*}{$\begin{array}{l}\text { Spec= } \\
\text { trum }\end{array}$} \\
\hline & $a m p$ & Spark & & & Lamp & Spark & & & Lamp & Spark & \\
\hline $\begin{array}{l}5194.203 \\
5194.457 \\
5195.285 \\
5195.815 \\
5196.017\end{array}$ & $\begin{array}{c}5 \mathrm{w} \\
250 \\
4 \\
400 \\
10\end{array}$ & $\begin{array}{r}5 \\
5 \\
3 \\
10\end{array}$ & $\begin{array}{l}\text { II } \\
\text { I } \\
\text { II } \\
\text { I } \\
\text { II }\end{array}$ & $\begin{array}{l}5212.344 \\
5212.676 \\
5213.349 \\
5214.153 \\
5214.814\end{array}$ & $\begin{array}{r}18 \\
10 \\
300 \\
100\end{array}$ & $\begin{array}{c}3 \\
150 \\
400 \mathrm{w}\end{array}$ & $\begin{array}{l}\text { I } \\
\text { I } \\
\text { I } \\
\text { I I } \\
\text { I I I }\end{array}$ & $\begin{array}{l}5237.042 \\
5237.235 \\
5237.350 \\
5237.621 \\
5237.903\end{array}$ & $\begin{array}{r}5 \\
7 \\
3 \\
70 \\
175\end{array}$ & $\begin{array}{l}300 \\
400\end{array}$ & $\begin{array}{l}\text { I } \\
\text { I } \\
\text { I } \\
\text { II } \\
\text { I I }\end{array}$ \\
\hline $\begin{array}{l}5196.441 \\
5196.558 \\
5196.864 \\
5197.232 \\
5197.580\end{array}$ & $\begin{array}{r}8 \\
3 \\
2 \\
100 \\
4\end{array}$ & 3 & $\begin{array}{l}\text { I } \\
\text { I } \\
\text { I I } \\
\text { I } \\
\text { I }\end{array}$ & $\begin{array}{l}5215.001 \\
5215.518 \\
5215.756 \\
5216.584 \\
5217.276\end{array}$ & $\begin{array}{r}3 \\
2 \\
3 \\
300 \\
10\end{array}$ & 250 & $\begin{array}{l}\text { I } \\
\text { I } \\
\text { I } \\
\text { II } \\
\text { I }\end{array}$ & $\begin{array}{l}5238.808 \\
5239.543 \\
5240.183 \\
5240.760 \\
5241.183\end{array}$ & $\begin{array}{l}300 \\
300 \\
300 \\
10 \\
25 \mathrm{w}\end{array}$ & $\begin{array}{r}3 \\
6 \\
300 \\
1\end{array}$ & $\begin{array}{l}\text { I } \\
\text { I } \\
\text { I I } \\
\text { I } \\
\text { I }\end{array}$ \\
\hline $\begin{array}{l}5197.695 \\
5197.992 \\
5198.066 \\
5198.807 \\
5199.158\end{array}$ & $\begin{array}{r}4 \\
1 \\
3 \\
400 \\
800\end{array}$ & $\begin{array}{r}6 \\
2 \\
200 \\
12\end{array}$ & $\begin{array}{l}\text { II } \\
\text { II } \\
\text { I } \\
\text { I I } \\
\text { I }\end{array}$ & $\begin{array}{l}5218.522 \\
5219.101 \\
5219.960 \\
5220.303 \\
5220.699\end{array}$ & $\begin{array}{r}150 \\
500 \\
12 \\
20 \\
100\end{array}$ & $\begin{array}{r}150 \\
18 \\
10\end{array}$ & $\begin{array}{l}\text { II } \\
\text { I } \\
\text { I I } \\
\text { I } \\
\text { I }\end{array}$ & $\begin{array}{l}5241.857 \\
5242.080 \\
5242.667 \\
5243.104 \\
5243.741\end{array}$ & $\begin{array}{r}50 \\
100 \\
12 \\
3 \\
50\end{array}$ & $\begin{array}{r}15 \\
125 \\
1\end{array}$ & $\begin{array}{l}\text { I I } \\
\text { I I } \\
\text { I } \\
\text { I } \\
\text { I }\end{array}$ \\
\hline $\begin{array}{l}5199.295 \\
5200.028 \\
5200.205 \\
5200.410 \\
5201.325\end{array}$ & $\begin{array}{r}75 \\
7 \\
2 \\
8 \\
10\end{array}$ & 10 & $\begin{array}{l}\text { I } \\
\text { II } \\
\text { I } \\
\text { II } \\
\text { I }\end{array}$ & $\begin{array}{l}5220.924 \\
5221.215 \\
5221.442 \\
5221.617 \\
5222.264\end{array}$ & $\begin{array}{r}150 \\
4 \\
1 \\
3 \\
12\end{array}$ & $\begin{array}{l}1 \\
5 \\
4 \\
1\end{array}$ & $\begin{array}{l}\text { I } \\
\text { II } \\
\text { I I } \\
\text { I } \\
\text { I }\end{array}$ & $\begin{array}{l}5243.835 \\
5244.106 \\
5244.603 \\
5245.341 \\
5245.696\end{array}$ & $\begin{array}{r}30 \\
20 \\
40 \\
4 \\
7\end{array}$ & 40 & $\begin{array}{l}\text { I } \\
\text { I I } \\
\text { I } \\
\text { I } \\
\text { I }\end{array}$ \\
\hline $\begin{array}{l}5201.559 \\
5202.016 \\
5202.730 \\
5203.081 \\
5203.225\end{array}$ & $\begin{array}{r}10 \\
50 \\
3 \\
2 \\
4\end{array}$ & $\begin{array}{l}1 \\
1 \\
6\end{array}$ & $\begin{array}{l}\text { I } \\
\text { I } \\
\text { I } \\
\text { II } \\
\text { I }\end{array}$ & $\begin{array}{l}5222.752 \\
5224.109 \\
5224.714 \\
5225.079 \\
5225.583\end{array}$ & $\begin{array}{r}10 \\
10 \\
5 \\
4 \\
30\end{array}$ & $\begin{array}{r}1 \\
4 \\
\\
4 \\
30\end{array}$ & $\begin{array}{l}\text { I } \\
\text { II } \\
\text { I } \\
\text { II } \\
\text { II }\end{array}$ & $\begin{array}{l}5246.238 \\
5247.193 \\
5247.650 \\
5248.474 \\
5248.864\end{array}$ & $\begin{array}{c}8 \\
300 d \\
400 \\
20 \\
2\end{array}$ & $\begin{array}{r}8 \\
450 \\
5 \\
2\end{array}$ & $\begin{array}{l}\text { I I } \\
\text { I } \\
\text { II } \\
\text { I I } \\
\text { I I }\end{array}$ \\
\hline $\begin{array}{l}5203.844 \\
5204.516 \\
5204.993 \\
5205.152 \\
5205.549\end{array}$ & $\begin{array}{r}150 \\
4 \\
50 \\
200 \\
25\end{array}$ & $\begin{array}{r}4 \\
18\end{array}$ & $\begin{array}{l}\text { I } \\
\text { I } \\
\text { II } \\
\text { I } \\
\text { I }\end{array}$ & $\begin{array}{l}5225.785 \\
5225.961 \\
5226.074 \\
5226.534 \\
5226.706\end{array}$ & $\begin{array}{r}5 \\
3 \\
8 \\
25 \\
2\end{array}$ & 4 & $\begin{array}{l}\text { I } \\
\text { I I } \\
\text { I } \\
\text { I }\end{array}$ & $\begin{array}{l}5248.967 \\
5249.682 \\
5249.880 \\
5250.172 \\
5250.866\end{array}$ & $\begin{array}{r}2 \\
8 \\
2 \\
5 \\
15\end{array}$ & $\begin{array}{l}3 \\
2\end{array}$ & $\begin{array}{l}\text { I } \\
\text { II } \\
\text { II } \\
\text { I } \\
\text { I }\end{array}$ \\
\hline $\begin{array}{l}5205.770 \\
5206.086 \\
5206.228 \\
5206.492 \\
5206.653\end{array}$ & $\begin{array}{r}175 \\
20 \\
10 \\
100 \\
75\end{array}$ & $\begin{array}{r}100 \\
75\end{array}$ & $\begin{array}{l}\text { I I } \\
\text { I } \\
\text { I } \\
\text { II } \\
\text { I I }\end{array}$ & $\begin{array}{l}5227.634 \\
5228.216 \\
5228.987 \\
5229.783 \\
5230.001\end{array}$ & $\begin{array}{r}8 \\
200 \\
60 \\
12 \\
3\end{array}$ & $\begin{array}{r}20 \\
3 \\
2\end{array}$ & $\begin{array}{l}\text { I I } \\
I \\
I \\
I \\
I\end{array}$ & $\begin{array}{l}5251.175 \\
5251.352 \\
5251.779 \\
5251.918 \\
5252.160\end{array}$ & $\begin{array}{r}10 \\
15 \\
5 \\
3 \\
12\end{array}$ & 5 & $\begin{array}{l}\text { I } \\
\text { I } \\
\text { I } \\
\text { I I } \\
\text { I }\end{array}$ \\
\hline $\begin{array}{l}5206.791 \\
5207.067 \\
5207.474 \\
5207.794 \\
5208.090\end{array}$ & $\begin{array}{c}50 \\
12 \\
20 \mathrm{~s} \\
100 \\
4\end{array}$ & $\begin{array}{l}2 \\
1\end{array}$ & $\begin{array}{l}\text { I } \\
\text { I } \\
\text { I } \\
\text { I } \\
\text { I }\end{array}$ & $\begin{array}{l}5230.376 \\
5231.1601 \\
5231.780 \\
5232.015 \\
5232.319\end{array}$ & $\begin{array}{l}5 \\
900 \\
20 \mathrm{w} \\
200 \\
75\end{array}$ & $\begin{array}{r}5 \\
20 \\
2 \\
80\end{array}$ & $\begin{array}{l}\text { II } \\
\text { I } \\
\text { I } \\
\text { II } \\
\text { I }\end{array}$ & $\begin{array}{l}5252.278 \\
5252.571 \\
5252.760 \\
5253.450 \\
5253.682\end{array}$ & $\begin{array}{r}3 \\
3 \\
20 \\
160 \\
10\end{array}$ & $\begin{array}{r}3 \\
100 \\
80\end{array}$ & $\begin{array}{l}\text { II } \\
\text { I } \\
\text { II } \\
\text { I I } \\
\text { I }\end{array}$ \\
\hline $\begin{array}{l}5208.540 \\
5208.754 \\
5209.713 \\
5210.172 \\
5210.238\end{array}$ & $\begin{array}{r}3 \\
2 \\
200 \\
6 \\
8\end{array}$ & $\begin{array}{l}3 \\
3\end{array}$ & $\begin{array}{l}\text { I } \\
\text { I I } \\
\text { I } \\
\text { I } \\
\text { I }\end{array}$ & $\begin{array}{l}5233.209 \\
5233.789 \\
5234.111 \\
5234.241 \\
5234.808\end{array}$ & $\begin{array}{r}350 \\
8 \\
75 \\
50 \\
6\end{array}$ & 400 & $\begin{array}{l}\text { I I } \\
\text { I } \\
\text { I } \\
\text { I } \\
\text { I }\end{array}$ & $\begin{array}{l}5254.254 \\
5254.897 \\
5255.573 \\
5255.958 \\
5256.840\end{array}$ & $\begin{array}{r}150 \\
2 \\
35 \\
30 \\
2\end{array}$ & 125 & $\begin{array}{l}\text { I } \\
\text { I } \\
\text { I } \\
\text { I I } \\
\text { I }\end{array}$ \\
\hline $\begin{array}{l}5210.400 \\
5210.566 \\
5210.848 \\
5211.234 \\
5211.587\end{array}$ & $\begin{array}{r}7 \\
7 \\
10 \\
400 \\
30\end{array}$ & 18 & $\begin{array}{l}\text { I } \\
\text { II } \\
\text { I } \\
\text { I } \\
\text { I I }\end{array}$ & $\begin{array}{l}5235.562 \\
5235.709 \\
5236.428 \\
5236.749 \\
5236.948\end{array}$ & $\begin{array}{r}12 \\
2 \\
18 \\
15 \\
8\end{array}$ & $\begin{array}{l}20 \\
15\end{array}$ & $\begin{array}{l}\text { I } \\
\text { I } \\
\text { II } \\
\text { II } \\
\text { I }\end{array}$ & $\begin{array}{l}5257.046 \\
5258.361 i \\
5259.137 \\
5259.595 \\
5259.788\end{array}$ & $\begin{array}{r}10 \\
300 \\
10 \\
10 \\
70\end{array}$ & $\begin{array}{r}25 \\
1 \\
2 \\
1\end{array}$ & $\begin{array}{l}\text { I } \\
\text { I } \\
\text { I } \\
\text { I I } \\
\text { I }\end{array}$ \\
\hline
\end{tabular}


Table 1. Wavelengths of Thorium Spectra - Continued

\begin{tabular}{|c|c|c|c|c|c|c|c|c|c|c|c|}
\hline \multirow{2}{*}{$\begin{array}{l}\text { Wave- } \\
\text { length }\end{array}$} & \multicolumn{2}{|c|}{ Intensity } & \multirow{2}{*}{$\begin{array}{l}\text { Spec- } \\
\text { trum }\end{array}$} & \multirow{2}{*}{$\begin{array}{l}\text { Wave- } \\
\text { length }\end{array}$} & \multicolumn{2}{|c|}{ Intensity } & \multirow{2}{*}{$\begin{array}{l}\text { Spec- } \\
\text { trum }\end{array}$} & \multirow{2}{*}{$\begin{array}{l}\text { Wave- } \\
\text { length }\end{array}$} & \multicolumn{2}{|c|}{ Intensity } & \multirow{2}{*}{$\begin{array}{l}\text { Spec- } \\
\text { trum }\end{array}$} \\
\hline & Lamp & Spark & & & $\mathrm{amp}$ & Spark & & & amp & Spark & \\
\hline $\begin{array}{l}5260.101 \\
5260.868 \\
5261.184 \\
5261.477 \\
5262.014\end{array}$ & $\begin{array}{r}200 \\
10 \\
10 \\
100 \\
4\end{array}$ & $\begin{array}{r}3 \\
25 \\
2\end{array}$ & $\begin{array}{l}\text { I } \\
\text { II } \\
\text { I } \\
\text { I } \\
\text { I }\end{array}$ & $\begin{array}{l}5282.773 \\
5283.191 \\
5283.326 \\
5283.668 \\
5284.536\end{array}$ & $\begin{array}{r}9 \\
3 \\
3 \\
50 \\
60\end{array}$ & 100 & $\begin{array}{l}\text { I } \\
\text { I } \\
\text { I } \\
\text { I } \\
\text { I I }\end{array}$ & $\begin{array}{l}5306.710 \\
5306.990 \\
5307.459 \\
5308.148 \\
5308.320\end{array}$ & $\begin{array}{l}3 \mathrm{~h} \\
75 \\
300 \\
10 \\
15\end{array}$ & $\begin{array}{r}400 \\
20\end{array}$ & $\begin{array}{l}\text { I } \\
\text { I } \\
\text { II } \\
\text { II } \\
\text { I }\end{array}$ \\
\hline $\begin{array}{l}5262.604 \\
5263.114 \\
5263.472 \\
5263.898 \\
5264.110\end{array}$ & $\begin{array}{r}75 \\
5 \\
12 \\
12 \\
2\end{array}$ & 8 & $\begin{array}{l}\text { I } \\
\text { I I } \\
\text { I } \\
\text { I } \\
\text { I }\end{array}$ & $\begin{array}{l}5285.149 \\
5286.135 \\
5286.502 \\
5287.169 \\
5287.955\end{array}$ & $\begin{array}{c}3 \\
3 \\
10 \\
8 \mathrm{~h} \\
6\end{array}$ & & $\begin{array}{l}\text { I } \\
\text { I } \\
\text { I } \\
\text { I } \\
\text { I }\end{array}$ & $\begin{array}{l}5308.430 \\
5308.750 \\
5309.612 \\
5309.724 \\
5310.254\end{array}$ & $\begin{array}{c}30 \\
4 \mathrm{w} \\
75 \\
50 \\
350\end{array}$ & $\begin{array}{r}30 \\
75 \\
300\end{array}$ & $\begin{array}{l}\text { II } \\
\text { I } \\
\text { II } \\
\text { I } \\
\text { II }\end{array}$ \\
\hline $\begin{array}{l}5264.323 \\
5264.961 \\
5265.742 \\
5266.701 \\
5267.021\end{array}$ & $\begin{array}{r}10 \\
40 \\
10 \\
200 \\
40\end{array}$ & $\begin{array}{r}100 \\
5 \\
4\end{array}$ & $\begin{array}{l}\text { I } \\
\text { I I } \\
\text { I } \\
\text { I } \\
\text { I }\end{array}$ & $\begin{array}{l}5288.960 \\
5289.613 \\
5289.894 \\
5290.475 \\
5290.651\end{array}$ & $\begin{array}{r}3 \\
50 \\
75 \\
10 \\
8\end{array}$ & $\begin{array}{r}50 \\
3\end{array}$ & $\begin{array}{l}\text { I } \\
\text { I } \\
\text { I I } \\
\text { I } \\
\text { I }\end{array}$ & $\begin{array}{l}5310.865 \\
5311.171 \\
5312.001 \\
5312.531 \\
5312.901\end{array}$ & $\begin{array}{r}30 \\
8 \\
400 \\
300 \\
300\end{array}$ & $\begin{array}{r}25 \\
20 \\
10 \\
7\end{array}$ & $\begin{array}{l}\text { I I } \\
\text { I } \\
\text { I } \\
\text { I } \\
\text { I }\end{array}$ \\
\hline $\begin{array}{l}5267.346 \\
5267.690 \\
5268.736 \\
5268.940 \\
5269.364\end{array}$ & $\begin{array}{r}12 \\
4 \\
10 \\
10\end{array}$ & $\begin{array}{l}25 \\
10 \\
12 \\
12\end{array}$ & $\begin{array}{l}\text { I I } \\
\text { I } \\
\text { I I } \\
\text { II } \\
\text { II }\end{array}$ & $\begin{array}{l}5291.124 \\
5291.377 \\
5291.812 \\
5291.895 \\
5292.341\end{array}$ & $\begin{array}{r}2 \\
10 \\
300 \\
10\end{array}$ & 40 & $\begin{array}{l}\text { I I } \\
\text { I } \\
\text { I } \\
\text { I I I } \\
\text { I }\end{array}$ & $\begin{array}{l}5313.604 \\
5314.203 \\
5314.414 \\
5314.607 \\
5315.230\end{array}$ & $\begin{array}{l}20 \\
30 \\
10 \\
10 \mathrm{w} \\
100\end{array}$ & $\begin{array}{r}75 \\
1 \\
10 \\
2\end{array}$ & $\begin{array}{l}\text { I I } \\
\text { I } \\
\text { I I } \\
\text { I } \\
\text { I }\end{array}$ \\
\hline $\begin{array}{l}5269.784 \\
5270.742 \\
5270.823 \\
5271.005 \\
5271.597\end{array}$ & $\begin{array}{r}50 \\
3 \\
8 \\
8 \\
8\end{array}$ & 12 & $\begin{array}{l}\text { I } \\
\text { II } \\
\text { I } \\
\text { I } \\
\text { II }\end{array}$ & $\begin{array}{l}5292.928 \\
5293.122 \\
5293.441 \\
5294.071 \\
5294.391\end{array}$ & $\begin{array}{r}12 \\
15 \\
2 \\
15 \\
200\end{array}$ & 40 & $\begin{array}{l}\text { I I } \\
\text { I } \\
\text { I } \\
\text { I } \\
\text { I }\end{array}$ & $\begin{array}{l}5315.618 \\
5315.754 \\
5315.828 \\
5316.323 \\
5316.793\end{array}$ & $\begin{array}{r}2 \\
4 \\
1 \\
10\end{array}$ & $\begin{array}{l}1 \\
2 \\
1 \\
2 \mathrm{w}\end{array}$ & $\begin{array}{l}\text { I I } \\
\text { I I } \\
\text { I } \\
\text { I } \\
\text { I I }\end{array}$ \\
\hline $\begin{array}{l}5272.641 \\
5272.930 \\
5273.126 \\
5273.517 \\
5274.113\end{array}$ & $\begin{array}{r}50 \\
50 \\
125 \\
5 \\
150\end{array}$ & $\begin{array}{r}100 \\
3 \\
2 \\
3\end{array}$ & $\begin{array}{l}\text { II } \\
\text { I } \\
\text { I } \\
\text { I } \\
\text { I }\end{array}$ & $\begin{array}{l}5294.745 \\
5295.089 \\
5295.605 \\
5295.652 \\
5296.272\end{array}$ & $\begin{array}{l}75 w \\
40 \\
2 \\
125 w \\
200\end{array}$ & $\begin{array}{l}3 \\
4 \\
4\end{array}$ & $\begin{array}{l}\text { I } \\
\text { I } \\
\text { I I } \\
I \\
I\end{array}$ & $\begin{array}{l}5317.494 \\
5318.111 \\
5318.418 \\
5319.514 \\
5319.771\end{array}$ & $\begin{array}{c}300 \\
75 \\
125 \\
7 \\
7 \mathrm{w}\end{array}$ & $\begin{array}{r}6 \\
100\end{array}$ & $\begin{array}{l}\text { I } \\
\text { I } \\
\text { II } \\
\text { I } \\
\text { I }\end{array}$ \\
\hline $\begin{array}{l}5274.384 \\
5274.943 \\
5275.449 \\
5275.778 \\
5276.049\end{array}$ & $\begin{array}{r}75 \\
8 \\
10 \\
7 \\
3\end{array}$ & $\begin{array}{l}2 \\
5 \\
4\end{array}$ & $\begin{array}{l}\text { I } \\
\text { I } \\
\text { II } \\
\text { I } \\
\text { I }\end{array}$ & $\begin{array}{l}5296.683 \\
5296.812 \\
5297.416 \\
5297.743 \\
5298.277\end{array}$ & $\begin{array}{r}5 \\
50 \\
75 \\
250 \\
200\end{array}$ & $\begin{array}{r}15 \\
2 \\
10 \\
2\end{array}$ & $\begin{array}{l}\text { II } \\
\text { I } \\
\text { I } \\
\text { I } \\
\text { I }\end{array}$ & $\begin{array}{l}5320.097 \\
5320.493 \\
5320.768 \\
5320.977 \\
5321.282\end{array}$ & $\begin{array}{c}301 \\
2 \\
50 \\
3 \\
20\end{array}$ & $\begin{array}{r}2 \\
3 \\
25 \\
5 \\
20\end{array}$ & $\begin{array}{l}\text { I } \\
\text { I I } \\
\text { I } \\
\text { II } \\
\text { II }\end{array}$ \\
\hline $\begin{array}{l}5276.404 \\
5277.179 \\
5277.5001 \\
5278.178 \\
5278.368\end{array}$ & $\begin{array}{r}75 \\
100 \\
400 \\
10 \\
8\end{array}$ & $\begin{array}{r}50 \\
200 \\
400 \\
4 \\
3\end{array}$ & $\begin{array}{l}\text { II } \\
\text { II } \\
\text { II } \\
\text { II } \\
\text { II }\end{array}$ & $\begin{array}{l}5298.50 \\
5299.464 \\
5300.519 \\
5301.405 \\
5302.046\end{array}$ & $\begin{array}{r}50 \\
6 \\
250 \\
100 \\
8 d\end{array}$ & $\begin{array}{r}1 \\
2 \\
400\end{array}$ & $\begin{array}{l}\text { I } \\
\text { I } \\
\text { I } \\
\text { I I } \\
\text { I }\end{array}$ & $\begin{array}{l}5321.657 \\
5322.429 \\
5322.866 \\
5322.894 \\
5323.397\end{array}$ & $\begin{array}{r}2 \\
10 \\
150 \\
25\end{array}$ & $\begin{array}{c}30 \mathrm{w} \\
5 \\
1\end{array}$ & $\begin{array}{l}\text { I } \\
\text { I I } \\
\text { I I } \\
\text { I } \\
\text { I }\end{array}$ \\
\hline $\begin{array}{l}5278.706 \\
5279.191 \\
5280.078 \\
5280.333 \\
5280.492\end{array}$ & $\begin{array}{r}8 \\
30 \\
100 \\
75 \\
15\end{array}$ & $\begin{array}{r}1 \\
125 \\
5\end{array}$ & $\begin{array}{l}\text { I } \\
\text { I } \\
\text { I I } \\
\text { I } \\
\text { I }\end{array}$ & $\begin{array}{l}5303.054 \\
5303.171 \\
5303.330 \\
5303.480 \\
5303.873\end{array}$ & $\begin{array}{r}75 \\
50 \\
30 \\
200 \\
5\end{array}$ & 75 & $\begin{array}{l}\text { I I } \\
\text { I } \\
\text { I } \\
\text { I } \\
\text { I }\end{array}$ & $\begin{array}{l}5323.859 \\
5324.057 \\
5324.642 \\
5325.142 \\
5325.426\end{array}$ & $\begin{array}{r}10 \\
8 \\
40 \\
300 \\
125\end{array}$ & $\begin{array}{r}10 \\
600\end{array}$ & $\begin{array}{l}\text { I } \\
\text { II } \\
\text { II } \\
\text { II } \\
\text { I }\end{array}$ \\
\hline $\begin{array}{l}5281.066 \\
5281.940 \\
5282.168 \\
5282.389 \\
5282.538\end{array}$ & $\begin{array}{r}150 \\
10 \\
8 \\
75 \\
6\end{array}$ & $\begin{array}{l}2 \\
1\end{array}$ & $\begin{array}{l}\text { I } \\
\text { I } \\
\text { I } \\
\text { I } \\
\text { I }\end{array}$ & $\begin{array}{l}5304.626 \\
5305.570 \\
5305.735 \\
5306.280 \\
5306.584\end{array}$ & $\begin{array}{l}100 \\
100 \\
30 \\
25 \\
3 \mathrm{~h}\end{array}$ & $\begin{array}{l}200 \\
200\end{array}$ & $\begin{array}{l}\text { I I } \\
\text { I I } \\
\text { I } \\
\text { I } \\
\text { I }\end{array}$ & $\begin{array}{l}5326.274 \\
5326.976 i \\
5328.333 \\
5328.677 \\
5328.826\end{array}$ & $\begin{array}{r}200 \\
400 \\
6 \\
2 \\
2\end{array}$ & $\begin{array}{r}4 \\
30 \\
12\end{array}$ & $\begin{array}{l}\text { I } \\
\text { I } \\
\text { I I } \\
\text { I } \\
\text { I }\end{array}$ \\
\hline
\end{tabular}


Table 1. Wavelengths of Thorium Spectra - Continued

\begin{tabular}{|c|c|c|c|c|c|c|c|c|c|c|c|}
\hline \multirow{2}{*}{$\begin{array}{l}\text { Wave - } \\
\text { length }\end{array}$} & \multicolumn{2}{|c|}{ Intensity } & \multirow{2}{*}{$\begin{array}{l}\text { Spec- } \\
\text { trum }\end{array}$} & \multirow{2}{*}{$\begin{array}{l}\text { Wave- } \\
\text { length }\end{array}$} & \multicolumn{2}{|c|}{ Intensity } & \multirow{2}{*}{$\begin{array}{l}\text { Spec- } \\
\text { trum }\end{array}$} & \multirow{2}{*}{$\begin{array}{l}\text { Wave- } \\
\text { length }\end{array}$} & \multicolumn{2}{|c|}{ Intensity } & \multirow{2}{*}{$\begin{array}{l}\text { Spec } \\
\text { trum }\end{array}$} \\
\hline & & & & & & & & & & & \\
\hline $\begin{array}{l}5329.376 \\
5329.461 \\
5329.747 \\
5330.067 \\
5330.503\end{array}$ & $\begin{array}{r}75 \\
40 \\
75 \\
150 \\
4\end{array}$ & $\begin{array}{c}150 \mathrm{w} \\
40 \\
2\end{array}$ & $\begin{array}{l}\text { II } \\
\text { I } \\
\text { II } \\
\text { I } \\
\text { I }\end{array}$ & $\begin{array}{l}5349.002 \\
5349.698 \\
5350.208 \\
5350.490 \\
5350.536\end{array}$ & $\begin{array}{r}75 \\
3 \\
10 \\
15\end{array}$ & 1 & $\begin{array}{l}\text { I } \\
\text { I } \\
\text { I } \\
\text { I I } \\
\text { I }\end{array}$ & $\begin{array}{l}5371.980 \\
5372.445 \\
5372.696 \\
5372.945 \\
5373.205\end{array}$ & $\begin{array}{r}5 \\
18 \\
200 \\
20 \\
6\end{array}$ & $\begin{array}{r}10 \\
2 \\
20 \\
15\end{array}$ & $\begin{array}{l}\text { I } \\
\text { I } \\
\text { I } \\
\text { I } \\
\text { II }\end{array}$ \\
\hline $\begin{array}{l}5330.855 \\
5331.204 \\
5331.474 \\
5331.743 \\
5332.229\end{array}$ & $\begin{array}{r}25 \\
2 \\
5 \\
30 \\
10\end{array}$ & $\begin{array}{l}2 w \\
1\end{array}$ & $\begin{array}{l}\text { I } \\
\text { I I } \\
\text { I } \\
\text { I } \\
\text { I }\end{array}$ & $\begin{array}{l}5351.133 \\
5351.838 \\
5351.970 \\
5352.309 \\
5353.017\end{array}$ & $\begin{array}{r}20 \\
75 \\
10 \\
12 \\
125\end{array}$ & $\begin{array}{r}6 \\
2 \\
2 \\
18\end{array}$ & $\begin{array}{l}\text { I } \\
\text { I } \\
\text { I } \\
\text { I I } \\
\text { I }\end{array}$ & $\begin{array}{l}5373.396 \\
5374.827 \\
5375.354 \\
5375.773 \\
5376.062\end{array}$ & $\begin{array}{l}10 \\
400 \\
200 \\
300\end{array}$ & $\begin{array}{r}20 \\
200 \\
400 \\
400\end{array}$ & $\begin{array}{l}\text { I } \\
\text { I } \\
\text { I I } \\
\text { I I } \\
\text { I I I }\end{array}$ \\
\hline $\begin{array}{l}5332.590 \\
5333.172 \\
5333.404 \\
5333.652 \\
5333.806\end{array}$ & $\begin{array}{r}1 \\
10 \\
20 \\
10 \\
9\end{array}$ & $\begin{array}{r}10 \\
2\end{array}$ & $\begin{array}{l}\text { I } \\
\text { II } \\
\text { I } \\
\text { II } \\
\text { I }\end{array}$ & $\begin{array}{l}5353.705 \\
5353.985 \\
5354.612 \\
5354.730 \\
5355.643\end{array}$ & $\begin{array}{l}12 \mathrm{~s} \\
15 \\
15 \\
2 \\
125\end{array}$ & $\begin{array}{l}30 \\
3 w \\
2\end{array}$ & $\begin{array}{l}\text { I } \\
\text { I I } \\
\text { I } \\
\text { II } \\
\text { I }\end{array}$ & $\begin{array}{l}5376.776 \\
5377.360 \\
5377.976 \\
5378.170 \\
5378.840\end{array}$ & $\begin{array}{r}200 \\
4 \\
10 \\
30 \\
100\end{array}$ & $\begin{array}{r}1 \\
12\end{array}$ & $\begin{array}{l}\text { I I } \\
\text { I } \\
\text { I } \\
\text { I } \\
\text { I }\end{array}$ \\
\hline $\begin{array}{l}5334.134 \\
5334.293 \\
5334.530 \\
5334.938 \\
5335.373\end{array}$ & $\begin{array}{r}15 \\
4 \\
3 \\
2 \\
10\end{array}$ & 1 & $\begin{array}{l}\text { I } \\
\text { I } \\
\text { I } \\
\text { I } \\
\text { I }\end{array}$ & $\begin{array}{l}5356.153 \\
5356.463 \\
5356.673 \\
5357.684 \\
5357.756\end{array}$ & $\begin{array}{l}8 \\
9 \\
8 \\
6 \\
6\end{array}$ & $\begin{array}{l}4 \\
8\end{array}$ & $\begin{array}{l}\text { I I } \\
\text { I I } \\
\text { I } \\
\text { I } \\
\text { I }\end{array}$ & $\begin{array}{l}5379.121 \\
5379.391 \\
5379.672 \\
5379.878 \\
5380.207\end{array}$ & $\begin{array}{r}200 \\
4 \\
4 \\
10\end{array}$ & $\begin{array}{r}3 \\
2 \\
4 \\
\\
25\end{array}$ & $\begin{array}{l}\text { I } \\
\text { II } \\
\text { II } \\
\text { I } \\
\text { II }\end{array}$ \\
\hline $\begin{array}{l}5335.604 \\
5336.304 \\
5336.790 \\
5337.020 \\
5337.083\end{array}$ & $\begin{array}{l}9 \\
6 \mathrm{w} \\
30 \\
200\end{array}$ & 100 & $\begin{array}{l}\text { I } \\
\text { I } \\
\text { I } \\
\text { I } \\
\text { I I I }\end{array}$ & $\begin{array}{l}5358.101 \\
5358.712 \\
5359.244 \\
5359.436 \\
5359.839\end{array}$ & $\begin{array}{r}2 \\
175 \\
4 \\
2 \\
100\end{array}$ & 10 & $\begin{array}{l}\text { I } \\
\text { I } \\
\text { I } \\
\text { I }\end{array}$ & $\begin{array}{l}5381.347 \\
5381.521 \\
5382.204 \\
5382.930 \\
5384.038\end{array}$ & $\begin{array}{l}40 . \mathrm{w} \\
15 \\
30 \mathrm{w} \\
250 \\
100\end{array}$ & $\begin{array}{r}1 \\
1 \\
150 \\
20\end{array}$ & $\begin{array}{l}\text { I } \\
\text { I } \\
\text { I } \\
\text { II } \\
\text { I }\end{array}$ \\
\hline $\begin{array}{l}5337.714 \\
5338.222 \\
5338.357 \\
5338.632 \\
5339.618\end{array}$ & $\begin{array}{r}2 \\
40 \\
30 \\
15 \\
6\end{array}$ & & $\begin{array}{l}\text { I } \\
\text { I } \\
\text { I } \\
\text { I } \\
\text { I }\end{array}$ & $\begin{array}{l}5360.159 \\
5360.686 \\
5361.014 \\
5361.155 \\
5361.415\end{array}$ & $\begin{array}{r}250 \\
3 \\
10 \\
40 \\
50\end{array}$ & $\begin{array}{r}3 \\
8 \\
200\end{array}$ & $\begin{array}{l}\text { I } \\
\text { I } \\
\text { II } \\
\text { I } \\
\text { I I }\end{array}$ & $\begin{array}{l}5384.302 \\
5385.727 \\
5386.128 \\
5386.432 \\
5386.611 \mathrm{i}\end{array}$ & $\begin{array}{r}75 \\
8 \\
15 \\
\\
300\end{array}$ & $\begin{array}{r}4 \\
10 \\
20 \\
10\end{array}$ & $\begin{array}{l}\text { I } \\
\text { II } \\
\text { I } \\
\text { II } \\
\text { I }\end{array}$ \\
\hline $\begin{array}{l}5339.921 \\
5340.494 \\
5341.198 \\
5341.719 \\
5342.331\end{array}$ & $\begin{array}{c}40 \\
125 \\
25 \\
8 \mathrm{w} \\
5 \mathrm{w}\end{array}$ & $\begin{array}{r}15 \\
7 \\
1 \\
2\end{array}$ & $\begin{array}{l}\text { I I } \\
\text { I } \\
\text { I } \\
\text { I } \\
\text { I I }\end{array}$ & $\begin{array}{l}5361.930 \\
5362.258 \\
5362.572 \\
5363.095 \\
5363.593\end{array}$ & $\begin{array}{r}10 \\
20 \\
125 \\
10 \\
75\end{array}$ & 75 & $\begin{array}{l}\text { I } \\
\text { I I } \\
\text { I } \\
\text { I } \\
\text { I I }\end{array}$ & $\begin{array}{l}5386.778 \\
5387.151 \\
5387.319 \\
5387.815 \\
5388.056\end{array}$ & $\begin{array}{r}15 \\
10 \\
75 \\
150\end{array}$ & $\begin{array}{l}2 \\
4\end{array}$ & $\begin{array}{l}\text { I I } \\
\text { I } \\
\text { I } \\
\text { I } \\
\text { I }\end{array}$ \\
\hline $\begin{array}{l}5342.552 \\
5342.694 \\
5342.798 \\
5343.276 \\
5343.581 \mathrm{i}\end{array}$ & $\begin{array}{r}6 \\
4 \\
3 \\
500\end{array}$ & $\begin{array}{l}30 \\
30\end{array}$ & $\begin{array}{l}\text { I } \\
\text { I } \\
\text { I } \\
\text { I I I } \\
\text { I }\end{array}$ & $\begin{array}{l}5363.898 \\
5364.181 \\
5364.396 \\
5364.635 \\
5365.490\end{array}$ & $\begin{array}{r}6 \\
10 \\
6 \\
15 \\
10\end{array}$ & $\begin{array}{r}50 \\
10 \\
3\end{array}$ & $\begin{array}{l}\text { I } \\
\text { I I } \\
\text { I I } \\
\text { I } \\
\text { I I }\end{array}$ & $\begin{array}{l}5388.909 \\
5389.130 \\
5389.451 \\
5390.174 \\
5390.464\end{array}$ & $\begin{array}{r}15 \\
10 \\
25 \\
12 \\
500\end{array}$ & $\begin{array}{r}18 \\
12 \\
30 \\
20 \\
350\end{array}$ & $\begin{array}{l}\text { I I } \\
\text { I I } \\
\text { I I } \\
\text { I I } \\
\text { I I }\end{array}$ \\
\hline $\begin{array}{l}5344.240 \\
5344.566 \\
5345.307 \\
5346.384 \\
5346.771\end{array}$ & $\begin{array}{r}20 \\
7 \\
300 \\
75 \\
12\end{array}$ & $\begin{array}{r}40 \\
30 \\
350 \\
50\end{array}$ & $\begin{array}{l}\text { II } \\
\text { I I } \\
\text { II } \\
\text { I I } \\
\text { I }\end{array}$ & $\begin{array}{l}5366.712 \\
5367.212 \\
5368.284 \\
5368.468 \\
5369.190\end{array}$ & $\begin{array}{l}20 \\
8 w \\
3 \\
10 \\
50\end{array}$ & 125 & $\begin{array}{l}\text { I } \\
\text { I } \\
\text { I } \\
\text { I } \\
\text { II }\end{array}$ & $\begin{array}{l}5391.083 \\
5391.639 \\
5392.580 \\
5392.874 \\
5393.118\end{array}$ & $\begin{array}{r}40 \\
2 \\
400 \\
3 \\
2\end{array}$ & $\begin{array}{r}20 \\
250 \\
40\end{array}$ & $\begin{array}{l}\text { I } \\
\text { I } \\
\text { I I } \\
\text { I } \\
\text { I I }\end{array}$ \\
\hline $\begin{array}{l}5347.041 \\
5347.428 \\
5347.54 \\
5347.973 \\
5348.309\end{array}$ & $\begin{array}{r}75 \\
12 \\
8 \\
100 \\
15\end{array}$ & 350 & $\begin{array}{l}\text { I I } \\
\text { I } \\
\text { I } \\
\text { I } \\
\text { I }\end{array}$ & $\begin{array}{l}5369.290 \\
5369.450 \\
5369.919 \\
5370.720 \\
5371.133\end{array}$ & $\begin{array}{l}200 \\
100 \\
20 \mathrm{~h} \\
50 \\
201\end{array}$ & & $\begin{array}{l}\text { I } \\
\text { I } \\
\text { I } \\
\text { I } \\
\text { I }\end{array}$ & $\begin{array}{l}5393.967 \\
5394.765 \\
5395.214 \\
5396.103 \\
5396.424\end{array}$ & $\begin{array}{r}300 \\
400 \\
5 \\
50 \\
4\end{array}$ & $\begin{array}{l}3 \\
8\end{array}$ & $\begin{array}{l}\text { I } \\
\text { I } \\
\text { I } \\
\text { I } \\
\text { I }\end{array}$ \\
\hline
\end{tabular}


Table 1. Wavelengths of Thorium Spectra - Continued

\begin{tabular}{|c|c|c|c|c|c|c|c|c|c|c|c|}
\hline \multirow{2}{*}{$\begin{array}{l}\text { Wave- } \\
\text { length }\end{array}$} & \multicolumn{2}{|c|}{ Intensity } & \multirow{2}{*}{$\begin{array}{l}\text { Spec- } \\
\text { trum }\end{array}$} & \multirow{2}{*}{$\begin{array}{l}\text { Wave- } \\
\text { length }\end{array}$} & \multicolumn{2}{|c|}{ Intensity } & \multirow{2}{*}{$\begin{array}{l}\text { Spec- } \\
\text { trum }\end{array}$} & \multirow{2}{*}{$\begin{array}{l}\text { Wave } \\
\text { length }\end{array}$} & \multicolumn{2}{|c|}{ Intensity } & \multirow{2}{*}{$\begin{array}{l}\text { Spec } \\
\text { trum }\end{array}$} \\
\hline & Lamp & Spark & & & Lamp & Spark & & & Lamp & Spark t & \\
\hline $\begin{array}{l}5396.543 \\
5397.454 \\
5397.888 \\
5398.217 \\
5398.710\end{array}$ & $\begin{array}{r}5 \\
5 \\
18 \\
60 \\
125\end{array}$ & 10 & $\begin{array}{l}\text { I I } \\
\text { I } \\
\text { I } \\
\text { I } \\
\text { I }\end{array}$ & $\begin{array}{l}5422.725 \\
5422.901 \\
5423.475 \\
5424.045 \\
5424.118\end{array}$ & $\begin{array}{r}5 \\
5 \\
30 \\
180\end{array}$ & 200 & $\begin{array}{l}\text { I } \\
\text { I } \\
\text { I } \\
\text { I I I }\end{array}$ & $\begin{array}{l}5444.987 \\
5445.162 \\
5445.282 \\
5446.018 \\
5446.329\end{array}$ & $\begin{array}{l}2 \\
1 \\
1 \\
5 \\
2\end{array}$ & 20 & $\begin{array}{l}\text { I } \\
\text { I } \\
\text { I } \\
\text { I I } \\
\text { I }\end{array}$ \\
\hline $\begin{array}{l}5398.913 \\
5399.174 \\
5399.615 \\
5400.144 \\
5400.783\end{array}$ & $\begin{array}{r}200 \\
200 \\
100 \\
125 \\
9\end{array}$ & $\begin{array}{r}20 \\
3 \\
1 \\
3 \\
3\end{array}$ & $\begin{array}{l}\text { I } \\
\text { I } \\
\text { I } \\
\text { I } \\
\text { I }\end{array}$ & $\begin{array}{l}5424.729 \\
5425.6781 \\
5426.165 \\
5426.399 \\
5426.612\end{array}$ & $\begin{array}{r}20 \\
250 \\
6 \\
25 \\
4\end{array}$ & 500 & $\begin{array}{l}\text { I } \\
\text { II } \\
\text { I } \\
\text { I } \\
\text { I }\end{array}$ & $\begin{array}{l}5446.807 \\
5447.003 \\
5447.297 \\
5448.269 \\
5448.958\end{array}$ & $\begin{array}{r}3 \\
15 \\
10 \\
5\end{array}$ & $\begin{array}{c}150 w \\
2\end{array}$ & $\begin{array}{l}\text { I } \\
\text { I I I } \\
\text { I I } \\
\text { I } \\
\text { I }\end{array}$ \\
\hline $\begin{array}{l}5401.552 \\
5402.280 \\
5403.210 \\
5403.660 \\
5404.706\end{array}$ & $\begin{array}{r}15 \\
10 \\
300 \\
4 \\
5\end{array}$ & $\frac{1}{3}$ & $\begin{array}{l}\text { I } \\
\text { I } \\
\text { I } \\
\text { I } \\
\text { I }\end{array}$ & $\begin{array}{l}5427.477 \\
5427.616 \\
5427.758 \\
5427.895 \\
5428.041\end{array}$ & $\begin{array}{r}2 \\
5 \\
1 \\
10 \\
5\end{array}$ & $\begin{array}{r}15 \\
6\end{array}$ & $\begin{array}{l}\text { I } \\
\text { I } \\
\text { I } \\
\text { I I } \\
\text { I I }\end{array}$ & $\begin{array}{l}5449.470 \\
5449.872 \\
5450.136 \\
5450.508 \\
5450.664\end{array}$ & $\begin{array}{r}150 \\
6 \\
2 \\
4 \\
5\end{array}$ & $\begin{array}{r}300 \\
18\end{array}$ & $\begin{array}{l}\text { I I } \\
\text { I I } \\
\text { I } \\
\text { I } \\
\text { I }\end{array}$ \\
\hline $\begin{array}{l}5405.214 \\
5406.331 \\
5406.758 \\
5407.063 \\
5407.654 \mathrm{i}\end{array}$ & $\begin{array}{r}8 \\
10 \\
25 \\
1 \\
200\end{array}$ & $\begin{array}{r}3 \\
20 \\
3 \\
18\end{array}$ & $\begin{array}{l}\text { I } \\
\text { I I } \\
\text { I } \\
\text { I } \\
\text { I }\end{array}$ & $\begin{array}{l}5428.191 \\
5429.106 \\
5430.118 \\
5430.591 \\
5431.112 \mathrm{i}\end{array}$ & $\begin{array}{r}1 \\
40 \\
3 \\
6 \\
300\end{array}$ & $\begin{array}{r}1 \\
1 \\
40\end{array}$ & $\begin{array}{l}\text { I } \\
\text { I } \\
\text { I } \\
\text { I } \\
\text { I }\end{array}$ & $\begin{array}{l}5450.874 \\
5451.075 \\
5452.2191 \\
5452.308 \\
5452.790\end{array}$ & $\begin{array}{r}1 \\
5 \\
250 \\
4\end{array}$ & $\begin{array}{r}10 \\
4\end{array}$ & $\begin{array}{l}\text { I } \\
\text { I } \\
\text { I } \\
\text { I I } \\
\text { I }\end{array}$ \\
\hline $\begin{array}{l}5408.759 \\
5408.858 \\
5409.295 \\
5410.292 \\
5410.761\end{array}$ & $\begin{array}{r}6 \\
5 \\
1 \\
9 \\
180\end{array}$ & 8 & $\begin{array}{l}\text { I } \\
\text { I } \\
\text { I } \\
\text { I } \\
\text { I }\end{array}$ & $\begin{array}{l}5431.593 \\
5431.752 \\
5432.558 \\
5432.763 \\
5433.126\end{array}$ & $\begin{array}{r}5 \\
5 \\
1 \\
18 \\
4\end{array}$ & $\begin{array}{r}12 \\
2 \\
18\end{array}$ & $\begin{array}{l}\text { I } \\
\text { I I } \\
\text { I I } \\
\text { I I } \\
\text { I }\end{array}$ & $\begin{array}{l}5453.068 \\
5453.294 \\
5453.548 \\
5453.808 \\
5453.967\end{array}$ & $\begin{array}{l}2 \\
2 \\
6 \\
1 \\
2\end{array}$ & $\begin{array}{l}2 \\
1\end{array}$ & $\begin{array}{l}\text { I } \\
\text { I I } \\
\text { I } \\
\text { I } \\
\text { I }\end{array}$ \\
\hline $\begin{array}{l}5411.302 \\
5411.610 \\
5411.828 \\
5412.056 \\
5412.690\end{array}$ & $\begin{array}{l}2 \\
1 \\
1 \\
2 \\
2\end{array}$ & $80 w$ & $\begin{array}{l}\text { I } \\
\text { I } \\
\text { I } \\
\text { I I I } \\
\text { I }\end{array}$ & $\begin{array}{l}5433.294 \\
5433.689 \\
5433.870 \\
5434.149 \\
5435.119\end{array}$ & $\begin{array}{r}10 \\
75 \\
5 \\
125 \\
100\end{array}$ & $\begin{array}{r}12 \\
100 \\
200\end{array}$ & $\begin{array}{l}\text { I I } \\
\text { I I } \\
\text { I } \\
\text { I } \\
\text { I I }\end{array}$ & $\begin{array}{l}5454.596 \\
5454.899 \\
5455.141 \\
5456.245 \\
5456.645\end{array}$ & $\begin{array}{r}1 \\
2 \\
40 \\
10 \\
2\end{array}$ & $\begin{array}{l}3 \\
2\end{array}$ & $\begin{array}{l}\text { I } \\
\text { I } \\
\text { I } \\
\text { I I } \\
\text { I }\end{array}$ \\
\hline $\begin{array}{l}5412.812 \\
5413.253 \\
5413.806 \\
5413.935 \\
5414.065\end{array}$ & $\begin{array}{r}10 \\
1 \\
15 \\
10 \\
5\end{array}$ & $\begin{array}{l}25 \\
25\end{array}$ & $\begin{array}{l}\text { I I } \\
\text { I } \\
\text { I I } \\
\text { I } \\
\text { I }\end{array}$ & $\begin{array}{l}5435.881 \\
5436.220 \\
5436.484 \\
5437.380 \\
5438.124\end{array}$ & $\begin{array}{r}400 \\
5 \\
40 \\
200 \\
40\end{array}$ & $\begin{array}{r}500 \\
150 \\
250 \\
1\end{array}$ & $\begin{array}{l}\text { II } \\
\text { I } \\
\text { II } \\
\text { I I } \\
\text { I }\end{array}$ & $\begin{array}{l}5456.965 \\
5457.285 \\
5458.089 \\
5458.244 \\
5458.401\end{array}$ & $\begin{array}{r}1 \\
30 \\
5 \\
4 \\
2\end{array}$ & $\begin{array}{r}2 \\
75\end{array}$ & $\begin{array}{l}\text { I } \\
\text { I } \\
\text { I I } \\
\text { I } \\
\text { I }\end{array}$ \\
\hline $\begin{array}{l}5414.538 \\
5415.462 \\
5417.486 \mathrm{i} \\
5417.818 \\
5418.076\end{array}$ & $\begin{array}{r}15 \\
300 \\
200 \\
50 \\
5\end{array}$ & $\begin{array}{r}20 \\
700 \\
18\end{array}$ & $\begin{array}{l}\text { I I } \\
\text { I I } \\
\text { I } \\
\text { I } \\
\text { I }\end{array}$ & $\begin{array}{l}5438.660 \\
5439.002 \\
5439.848 \\
5440.260 \\
5440.597\end{array}$ & $\begin{array}{r}2 \\
2 \\
50 \\
2 \\
75\end{array}$ & $\begin{array}{l}1 \\
1 \\
3\end{array}$ & $\begin{array}{l}\text { I } \\
\text { I } \\
\text { I } \\
\text { I } \\
\text { I }\end{array}$ & $\begin{array}{l}5458.620 \\
5458.787 \\
5459.174 \\
5460.116 \\
5460.351\end{array}$ & $\begin{array}{l}1 \\
1 \\
5 \\
2 \\
2\end{array}$ & 600 & $\begin{array}{l}\text { I } \\
\text { II I } \\
\text { I } \\
\text { I I }\end{array}$ \\
\hline $\begin{array}{l}5418.210 \\
5419.118 \\
5419.484 \\
5419.653 \\
5420.227\end{array}$ & $\begin{array}{r}6 \\
50 \\
2 \\
2 \\
4\end{array}$ & $\begin{array}{l}1 \\
2 \\
3\end{array}$ & $\begin{array}{l}\text { I } \\
\text { I } \\
\text { I } \\
\text { I I } \\
\text { I }\end{array}$ & $\begin{array}{l}5441.033 \\
5441.208 \\
5441.283 \\
5442.383 \\
5443.113\end{array}$ & $\begin{array}{r}15 \\
75 \\
7 \\
300\end{array}$ & $\begin{array}{l}12 \\
20\end{array}$ & $\begin{array}{l}\text { I } \\
\text { I } \\
\text { II } \\
\text { I } \\
\text { II }\end{array}$ & $\begin{array}{l}5460.744 \\
5461.015 \\
5461.155 \\
5461.737 \\
5462.340\end{array}$ & $\begin{array}{r}6 \\
1 \\
4 \\
75 \\
30\end{array}$ & $250^{3}$ & $\begin{array}{l}\text { I } \\
\text { I } \\
\text { II } \\
\text { I I } \\
\text { I }\end{array}$ \\
\hline $\begin{array}{l}5420.439 \\
5421.221 \\
5421.667 \\
5421.835 \\
5422.089\end{array}$ & $\begin{array}{r}2 \\
6 \\
30 \\
75 \\
5\end{array}$ & $\begin{array}{l}10 \\
80\end{array}$ & $\begin{array}{l}\text { I } \\
\text { I I } \\
\text { I } \\
\text { I I } \\
\text { I }\end{array}$ & $\begin{array}{l}5443.566 \\
5443.839 \\
5444.401 \\
5444.473 \\
5444.790\end{array}$ & $\begin{array}{l}4 \\
6 \\
1 \\
3 \mathrm{~s} \\
3\end{array}$ & 8 & $\begin{array}{l}\text { II } \\
\text { I } \\
\text { I } \\
\text { I } \\
\text { I }\end{array}$ & $\begin{array}{l}5462.610 \\
5463.309 \\
5463.768 \\
5464.210 \\
5464.754\end{array}$ & $\begin{array}{r}100 \\
15 \\
10 \\
75 \\
4\end{array}$ & $\begin{array}{r}250 \\
2 \\
2 \\
3 \\
4\end{array}$ & $\begin{array}{l}\text { I I } \\
\text { I } \\
\text { I I } \\
\text { I } \\
\text { I I }\end{array}$ \\
\hline
\end{tabular}


Table 1. Wavelengths of Thorium Spectra - Continued

\begin{tabular}{|c|c|c|c|c|c|c|c|c|c|c|c|}
\hline \multirow{2}{*}{$\begin{array}{l}\text { Wave- } \\
\text { length }\end{array}$} & \multicolumn{2}{|c|}{ Intensity } & \multirow{2}{*}{$\begin{array}{l}\text { Spec- } \\
\text { trum }\end{array}$} & \multirow{2}{*}{$\begin{array}{l}\text { Wave- } \\
\text { length }\end{array}$} & \multicolumn{2}{|c|}{ Intensity } & \multirow{2}{*}{$\begin{array}{l}\text { Spec- } \\
\text { trum }\end{array}$} & \multirow{2}{*}{$\begin{array}{l}\text { Wave- } \\
\text { length }\end{array}$} & \multicolumn{2}{|c|}{ Intensity } & \multirow{2}{*}{$\begin{array}{l}\text { Spec- } \\
\text { trum }\end{array}$} \\
\hline & Lamp & Spark & & & Lamp & Spark & & & Lamp & Spark & \\
\hline $\begin{array}{l}5465.050 \\
5465.321 \\
5466.012 \\
5466.252 \\
5466.818\end{array}$ & $\begin{array}{l}2 w \\
9 \\
1 \\
5 \\
4\end{array}$ & $\begin{array}{l}2 \\
2\end{array}$ & $\begin{array}{l}\text { I } \\
\text { I } \\
\text { I } \\
\text { I I } \\
\text { II }\end{array}$ & $\begin{array}{l}5488.629 \\
5488.911 \\
5489.069 \\
5489.585 \\
5490.045\end{array}$ & $\begin{array}{r}75 \\
40 \\
40 \\
2 \\
2\end{array}$ & $\begin{array}{l}75 \\
50 \\
15\end{array}$ & $\begin{array}{l}\text { II } \\
\text { II } \\
\text { I } \\
\text { II } \\
\text { I. }\end{array}$ & $\begin{array}{l}5509.081 \\
5509.994 \mathrm{i} \\
5510.366 \\
5510.480 \\
5510.666\end{array}$ & $\begin{array}{r}3 \\
300 \\
5 \\
5 \\
30\end{array}$ & $\begin{array}{r}20 \\
150\end{array}$ & $\begin{array}{l}\text { I } \\
\text { I } \\
\text { I } \\
\text { I } \\
\text { II }\end{array}$ \\
\hline $\begin{array}{l}5467.155 \\
5468.733 \\
5469.190 \\
5469.584 \\
5470.142\end{array}$ & $\begin{array}{r}12 \\
5 \\
5 \\
1 \\
20\end{array}$ & $\begin{array}{r}2 \\
15 \\
1 \\
2\end{array}$ & $\begin{array}{l}\text { II } \\
\text { II } \\
\text { I } \\
\text { I } \\
\text { I }\end{array}$ & $\begin{array}{l}5490.254 \\
5491.276 \\
5491.522 \\
5492.041 \\
5492.071\end{array}$ & $\begin{array}{l}2 \\
4 \\
5 \\
2 \\
5\end{array}$ & $\begin{array}{l}20 \\
10\end{array}$ & $\begin{array}{l}\text { I } \\
\text { I } \\
\text { II } \\
\text { II } \\
\text { I }\end{array}$ & $\begin{array}{l}5511.029 \\
5511.704 \\
5512.152 \\
5512.687 \\
5513.079\end{array}$ & $\begin{array}{r}15 \\
1 \\
12 \\
10 \\
1\end{array}$ & $\begin{array}{l}1 \\
3 \\
4\end{array}$ & $\begin{array}{l}\text { I } \\
\text { I } \\
\text { II } \\
\text { II } \\
\text { I }\end{array}$ \\
\hline $\begin{array}{l}5470.757 \\
5471.381 \\
5471.771 \\
5472.060 \\
5472.534\end{array}$ & $\begin{array}{r}100 \\
10 \\
1 \\
2 \\
5\end{array}$ & $\begin{array}{r}3 \\
100\end{array}$ & $\begin{array}{l}\text { I } \\
\text { I I } \\
\text { I } \\
\text { I } \\
\text { I }\end{array}$ & $\begin{array}{l}5492.322 \\
5492.385 \\
5492.638 \\
5493.190 \\
5493.587\end{array}$ & $\begin{array}{r}75 \\
100 \\
75 \\
3\end{array}$ & $\begin{array}{l}9 \\
8 \\
5 \\
2\end{array}$ & $\begin{array}{l}\text { I I } \\
\text { I } \\
\text { I } \\
\text { I } \\
\text { I }\end{array}$ & $\begin{array}{l}5513.384 \\
5513.858 \\
5514.106 \\
5514.874 \\
5515.824\end{array}$ & $\begin{array}{r}1 \\
3 \\
2 \\
160 \\
2\end{array}$ & $\begin{array}{r}18 \\
5\end{array}$ & $\begin{array}{l}\text { I } \\
\text { II } \\
\text { I } \\
\text { I } \\
\text { I }\end{array}$ \\
\hline $\begin{array}{l}5472.694 \\
5473.405 \\
5473.840 \\
5474.747 \\
5474.865\end{array}$ & $\begin{array}{r}2 \\
10 \\
2 \\
50 \\
75\end{array}$ & 90 & $\begin{array}{l}\text { I } \\
\text { II } \\
\text { I } \\
\text { I } \\
\text { II }\end{array}$ & $\begin{array}{l}5493.925 \\
5494.321 \\
5494.543 \\
5495.815 \\
5496.133\end{array}$ & $\begin{array}{l}75 \\
50 \\
25 \\
80\end{array}$ & $\begin{array}{c}2 w \\
75 \\
75 \\
2\end{array}$ & $\begin{array}{l}\text { II } \\
\text { I } \\
\text { II } \\
\text { II } \\
\text { I }\end{array}$ & $\begin{array}{l}5516.113 \\
5516.494 \\
5516.742 \\
5517.437 \\
5517.602\end{array}$ & $\begin{array}{l}1 \\
9 \\
1 \\
1 \\
1\end{array}$ & & $\begin{array}{l}\text { I } \\
\text { I } \\
\text { I } \\
\text { I } \\
\text { I }\end{array}$ \\
\hline $\begin{array}{l}5475.758 \\
5476.485 \\
5477.047 \\
5477.53 \\
5478.752\end{array}$ & $\begin{array}{r}2 \\
6 \\
12 \\
12 \\
7\end{array}$ & 25 & $\begin{array}{l}\text { I } \\
\text { I } \\
\text { II } \\
\text { I } \\
\text { I }\end{array}$ & $\begin{array}{l}5497.338 \\
5497.749 \\
5497.996 \\
5498.344 \\
5499.255 \mathrm{i}\end{array}$ & $\begin{array}{r}1 \\
5 \\
5 \\
1 \\
250\end{array}$ & $\begin{array}{r}3 \\
15\end{array}$ & $\begin{array}{l}\text { I I } \\
\text { I I } \\
\text { I } \\
\text { I } \\
\text { I }\end{array}$ & $\begin{array}{l}5517.784 \\
5518.156 \\
5518.269 \\
5518.990 \\
5519.222\end{array}$ & $\begin{array}{r}5 \\
1 \\
2 \\
160 \\
30\end{array}$ & $\begin{array}{r}12 \\
3 \\
25\end{array}$ & $\begin{array}{l}\text { II } \\
\text { I } \\
\text { II } \\
\text { II } \\
\text { I }\end{array}$ \\
\hline $\begin{array}{l}5479.074 \\
5479.490 \\
5479.696 \\
5480.141 \\
5480.403\end{array}$ & $\begin{array}{r}75 \\
1 \\
5 \\
1 \\
2\end{array}$ & $\begin{array}{l}5 \\
9\end{array}$ & $\begin{array}{l}\text { I } \\
\text { I } \\
\text { II } \\
\text { I } \\
\text { I }\end{array}$ & $\begin{array}{l}5499.631 \\
5500.021 \\
5500.226 \\
5500.448 \\
5501.279\end{array}$ & $\begin{array}{r}75 \\
5 \\
9 \\
1 \\
75\end{array}$ & $\begin{array}{c}150^{3} \mathrm{w} \\
2\end{array}$ & $\begin{array}{l}\text { I } \\
\text { I I I } \\
\text { I } \\
\text { I } \\
\text { I }\end{array}$ & $\begin{array}{l}5520.240 \\
5520.311 \\
5520.340 \\
5520.725 \\
5520.944\end{array}$ & $\begin{array}{r}2 \\
2 \\
4 \\
10 \\
1\end{array}$ & $\begin{array}{r}18 \\
2\end{array}$ & $\begin{array}{l}\text { I } \\
\text { I I } \\
\text { I } \\
\text { I } \\
\text { I }\end{array}$ \\
\hline $\begin{array}{l}5480.863 \\
5481.463 \\
5481.952 \\
5482.408 \\
5482.490\end{array}$ & $\begin{array}{r}40 \\
20 \\
5 \\
6 \\
6\end{array}$ & $\begin{array}{r}30 \\
3\end{array}$ & $\begin{array}{l}\text { II } \\
\text { I I } \\
\text { I } \\
\text { I } \\
\text { I }\end{array}$ & $\begin{array}{l}5501.521 \\
5501.695 \\
5501.931 \\
5502.434 \\
5502.588\end{array}$ & $\begin{array}{r}3 \\
2 \\
25 \\
2 \\
1\end{array}$ & $\begin{array}{r}15 \\
1 \\
75\end{array}$ & $\begin{array}{l}\text { II } \\
\text { I } \\
\text { II } \\
\text { I } \\
\text { I }\end{array}$ & $\begin{array}{l}5521.747 \\
5522.118 \\
5522.598 \\
5523.159 \\
5523.279\end{array}$ & $\begin{array}{r}100 \\
1 \\
2 \\
5 \\
1\end{array}$ & 5 & $\begin{array}{l}I \\
I \\
I \\
I \\
I\end{array}$ \\
\hline $\begin{array}{l}5482.909 \\
5483.143 \\
5483.609 \\
5484.125 \\
5484.529\end{array}$ & $\begin{array}{r}2 \\
5 \\
1 \\
75 \\
40\end{array}$ & $\begin{array}{r}4 \\
250\end{array}$ & $\begin{array}{l}\text { I } \\
\text { II } \\
\text { I } \\
\text { II } \\
\text { I }\end{array}$ & $\begin{array}{l}5502.704 \\
5502.883 \\
5503.028 \\
5503.372 \\
5503.598\end{array}$ & $\begin{array}{l}2 \\
1 \\
3 \\
2 \\
1\end{array}$ & & $\begin{array}{l}\text { I } \\
\text { I } \\
\text { I } \\
\text { I } \\
\text { I }\end{array}$ & $\begin{array}{l}5523.420 \\
5523.548 \\
5523.673 \\
5524.217 \\
5524.579\end{array}$ & $\begin{array}{r}3 \\
1 \\
2 \\
40 \\
100\end{array}$ & $\begin{array}{r}175 \\
25\end{array}$ & $\begin{array}{l}\text { I I } \\
\text { I } \\
\text { I } \\
\text { I I } \\
\text { I }\end{array}$ \\
\hline $\begin{array}{l}5484.964 \\
5485.422 \\
5485.714 \\
5486.043 \\
5486.156\end{array}$ & $\begin{array}{r}10 \\
2 \\
1 \\
12 \\
10\end{array}$ & $\frac{1}{2}$ & $\begin{array}{l}\text { I } \\
\text { I } \\
\text { I } \\
\text { I } \\
\text { I }\end{array}$ & $\begin{array}{l}5504.297 \\
5504.961 \\
5505.338 \\
5505.930 \\
5506.084\end{array}$ & $\begin{array}{r}160 \\
2 \\
2 \\
2 \\
2\end{array}$ & $\begin{array}{r}30 \\
5\end{array}$ & $\begin{array}{l}\text { I } \\
\text { II } \\
\text { I } \\
\text { I } \\
\text { I }\end{array}$ & $\begin{array}{l}5525.142 \\
5526.087 \\
5526.293 \\
5526.509 \\
5527.281\end{array}$ & $\begin{array}{l}10 \\
1 \\
2 \mathrm{w} \\
25 \\
75\end{array}$ & $\begin{array}{r}15 \\
2\end{array}$ & $\begin{array}{l}\text { I } \\
\text { I } \\
\text { I } \\
\text { I I } \\
\text { I }\end{array}$ \\
\hline $\begin{array}{l}5486.919 \\
5487.493 \\
5487.833 \\
5487.964 \\
5488.328\end{array}$ & $\begin{array}{r}10 \\
10 \\
7 \\
5 \\
1\end{array}$ & 50 & $\begin{array}{l}\text { I I } \\
\text { I } \\
\text { I } \\
\text { I } \\
\text { I }\end{array}$ & $\begin{array}{l}5506.903 \\
5507.531 \\
5507.932 \\
5508.548 \\
5508.886\end{array}$ & $\begin{array}{r}50 \\
50 \\
35 \\
75 \\
2\end{array}$ & $\begin{array}{r}2 \\
1 \\
40 \\
2\end{array}$ & $\begin{array}{l}\text { I } \\
\text { I } \\
\text { II } \\
\text { I } \\
\text { I }\end{array}$ & $\begin{array}{l}5528.003 \\
5528.221 \\
5528.709 \\
5529.085 \\
5529.650\end{array}$ & $\begin{array}{r}10 \\
75 \\
5 \\
30 \\
2\end{array}$ & $\begin{array}{r}75 \\
2 \\
2 \\
10\end{array}$ & $\begin{array}{l}\text { II } \\
\text { I } \\
\text { I } \\
\text { I } \\
\text { I I }\end{array}$ \\
\hline
\end{tabular}


Table 1. Wavelengths of Thorium Spectra - Continued

\begin{tabular}{|c|c|c|c|c|c|c|c|c|c|c|c|}
\hline \multirow{2}{*}{$\begin{array}{l}\text { Wave- } \\
\text { length }\end{array}$} & \multicolumn{2}{|c|}{ Intensity } & \multirow{2}{*}{$\begin{array}{l}\text { Spec- } \\
\text { trum }\end{array}$} & \multirow{2}{*}{$\begin{array}{l}\text { Wave- } \\
\text { length }\end{array}$} & \multicolumn{2}{|c|}{ Intensity } & \multirow{2}{*}{$\begin{array}{l}\text { Spec- } \\
\text { trum }\end{array}$} & \multirow{2}{*}{$\begin{array}{l}\text { Wave- } \\
\text { length }\end{array}$} & \multicolumn{2}{|c|}{ Intensity } & \multirow{2}{*}{$\begin{array}{l}\text { Spec } \\
\text { trum }\end{array}$} \\
\hline & Lamp & Spark & & & Lamp & Spark & & & Lamp & Spark & \\
\hline $\begin{array}{l}5529.770 \\
5530.063 \\
5530.683 \\
5531.565 \\
5532.166\end{array}$ & $\begin{array}{r}4 \\
15 \\
30 \\
7 \\
4\end{array}$ & $\begin{array}{r}15 \\
50 \\
1 \\
20\end{array}$ & $\begin{array}{l}\text { I I } \\
\text { I } \\
\text { I I } \\
\text { I } \\
\text { I I }\end{array}$ & $\begin{array}{l}5551.728 \\
5552.086 \\
5552.608 \\
5553.397 \\
5554.499\end{array}$ & $\begin{array}{r}6 \\
2 \\
50 \\
5 \\
10\end{array}$ & $\begin{array}{l}2 \\
2 \\
1 \\
1\end{array}$ & $\begin{array}{l}\text { I } \\
\text { I } \\
\text { I } \\
\text { I } \\
\text { I }\end{array}$ & $\begin{array}{l}5577.094 \\
5577.228 \\
5577.487 \\
5577.600 \\
5577.889\end{array}$ & $\begin{array}{l}1 \\
1 \\
4 \\
5 \\
7\end{array}$ & $\begin{array}{l}25 \\
60\end{array}$ & $\begin{array}{l}\text { I } \\
\text { I } \\
\text { II } \\
\text { I } \\
\text { II }\end{array}$ \\
\hline $\begin{array}{l}5532.308 \\
5532.748 \\
5533.126 \\
5533.168 \\
5533.584\end{array}$ & $\begin{array}{l}8 \\
1 \mathrm{w} \\
15 \\
10 \\
1\end{array}$ & $\begin{array}{r}25 \\
1\end{array}$ & $\begin{array}{l}\text { I } \\
\text { I } \\
\text { II } \\
\text { I } \\
\text { I }\end{array}$ & $\begin{array}{l}5554.672 \\
5555.092 \\
5555.524 \\
5555.817 \\
5556.142\end{array}$ & $\begin{array}{r}2 \\
1 \\
75 \\
5 \\
25\end{array}$ & $\begin{array}{r}1 \\
2 \\
20\end{array}$ & $\begin{array}{l}\text { I } \\
\text { I } \\
\text { I } \\
\text { I } \\
\text { I I }\end{array}$ & $\begin{array}{l}5578.457 \\
5579.359 \mathrm{i} \\
5580.062 \\
5580.393 \\
5580.747\end{array}$ & $\begin{array}{r}2 \\
300 \\
20 \\
15 \\
75\end{array}$ & $\begin{array}{r}20 \\
2 \\
1 \\
4\end{array}$ & $\begin{array}{l}\text { I } \\
\text { I } \\
\text { I } \\
\text { I } \\
\text { I }\end{array}$ \\
\hline $\begin{array}{l}5533.957 \\
5534.418 \\
5534.564 \\
5535.215 \\
5535.401\end{array}$ & $\begin{array}{l}4 \\
2 \\
3 \\
2 \\
1\end{array}$ & 2 & $\begin{array}{l}\text { I } \\
\text { I } \\
\text { I } \\
\text { I } \\
\text { I }\end{array}$ & $\begin{array}{l}5557.041 \\
5557.296 \\
5557.908 \\
5558.343 \mathrm{i} \\
5558.820\end{array}$ & $\begin{array}{r}200 \\
50 \\
8 \\
400 \\
40\end{array}$ & $\begin{array}{r}12 \\
175 \\
50 \\
2\end{array}$ & $\begin{array}{l}\text { I } \\
\text { II } \\
\text { I } \\
\text { I } \\
\text { I }\end{array}$ & $\begin{array}{l}5581.273 \\
5581.666 \\
5581.849 \\
5581.953 \\
5582.359\end{array}$ & $\begin{array}{r}12 \\
3 \\
2 \\
1 \\
75\end{array}$ & 50 & $\begin{array}{l}\text { II } \\
\text { I } \\
\text { I } \\
\text { I } \\
\text { I }\end{array}$ \\
\hline $\begin{array}{l}5535.680 \\
5535.954 \\
5536.326 \\
5536.967 \\
5537.120\end{array}$ & $\begin{array}{l}1 \\
15 \\
101 \\
15 \\
75\end{array}$ & 150 & $\begin{array}{l}\text { I } \\
\text { I } \\
\text { I } \\
\text { I } \\
\text { II }\end{array}$ & $\begin{array}{l}5559.126 \\
5559.884 \\
5560.038 \\
5560.532 \\
5560.576\end{array}$ & $\begin{array}{r}4 \\
60 \\
10 d\end{array}$ & $\begin{array}{l}3 \\
3 \\
5 \\
8 w\end{array}$ & $\begin{array}{l}\text { II } \\
\text { I } \\
\text { II } \\
\text { II } \\
\text { I }\end{array}$ & $\begin{array}{l}5582.671 \\
5582.849 \\
5583.058 \\
5583.760 \\
5584.150\end{array}$ & $\begin{array}{r}20 \\
40 \\
2 \\
75 \\
10\end{array}$ & $\begin{array}{r}2 \\
4 \\
200 \\
10\end{array}$ & $\begin{array}{l}\text { I } \\
\text { I } \\
\text { II } \\
\text { II } \\
\text { II }\end{array}$ \\
\hline $\begin{array}{l}5537.534 \\
5537.819 \\
5538.490 \\
5538.597 \\
5539.262 \mathrm{i}\end{array}$ & $\begin{array}{r}25 \\
2 \\
20 \\
50 \\
400\end{array}$ & $\begin{array}{l}25 \\
20\end{array}$ & $\begin{array}{l}\text { I } \\
\text { I } \\
\text { II } \\
\text { I } \\
\text { I }\end{array}$ & $\begin{array}{l}5561.177 \\
5561.602 \\
5561.846 \\
5562.161 \\
5562.638\end{array}$ & $\begin{array}{l}2 \\
7 \\
1 \\
5 \\
2\end{array}$ & & $\begin{array}{l}\text { I } \\
\text { I } \\
\text { I } \\
\text { I } \\
\text { I }\end{array}$ & $\begin{array}{l}5584.397 \\
5585.086 \\
5585.594 \\
5585.850 \\
5586.466\end{array}$ & $\begin{array}{c}1 \\
3 \\
2 \\
3 \\
50 \mathrm{w}\end{array}$ & $\begin{array}{r}4 \\
12 \\
20\end{array}$ & $\begin{array}{l}\text { I } \\
\text { II } \\
\text { II } \\
\text { I } \\
\text { II }\end{array}$ \\
\hline $\begin{array}{l}5539.902 \\
5541.125 \\
5541.565 \\
5541.921 \\
5542.743\end{array}$ & $\begin{array}{l}200 \\
401 \\
15 \\
25\end{array}$ & $\begin{array}{r}500 \\
1 \\
2 \\
50\end{array}$ & $\begin{array}{l}\text { I I } \\
\text { I } \\
\text { I } \\
\text { I } \\
\text { I I I }\end{array}$ & $\begin{array}{l}5562.849 \\
5563.359 \\
5564.190 \\
5564.338 \\
5564.783\end{array}$ & $\begin{array}{r}2 \\
5 \\
125 \\
40 \\
6\end{array}$ & $\begin{array}{l}2 \\
400^{-} \\
100\end{array}$ & $\begin{array}{l}\text { I } \\
\text { I I } \\
\text { II } \\
\text { I I } \\
\text { I }\end{array}$ & $\begin{array}{l}5587.027 \mathrm{i} \\
5587.720 \\
5587.847 \\
5588.872 \\
5589.303\end{array}$ & $\begin{array}{r}500 \\
40 \\
15 \\
100 \\
4\end{array}$ & $\begin{array}{r}30 \\
400\end{array}$ & $\begin{array}{l}\text { I } \\
\text { II } \\
\text { I } \\
\text { I } \\
\text { I }\end{array}$ \\
\hline $\begin{array}{l}5542.876 \\
5543.358 \\
5543.458 \\
5544.245 \\
5544.374\end{array}$ & $\begin{array}{r}160 \\
2 \\
2 \\
2 \\
2\end{array}$ & $\begin{array}{l}8 \\
4 \\
4 \\
3\end{array}$ & $\begin{array}{l}\text { I } \\
\text { II } \\
\text { I } \\
\text { II } \\
\text { II }\end{array}$ & $\begin{array}{l}5564.993 \\
5566.144 \\
5566.702 \\
5567.390 \\
5568.001\end{array}$ & $\begin{array}{r}20 \\
2 \\
2 \\
6 \\
300\end{array}$ & $\begin{array}{r}75 \\
3 \\
30 \\
250\end{array}$ & $\begin{array}{l}\text { II } \\
\text { I } \\
\text { II } \\
\text { II } \\
\text { II }\end{array}$ & $\begin{array}{l}5589.654 \\
5590.208 \\
5590.573 \\
5591.325 \\
5591.889\end{array}$ & $\begin{array}{r}5 \\
18 \\
10 \\
7 \\
15\end{array}$ & $\begin{array}{r}30 \\
2\end{array}$ & $\begin{array}{l}\text { II } \\
\text { I } \\
\text { I } \\
\text { I } \\
\text { I }\end{array}$ \\
\hline $\begin{array}{l}5545.041 \\
5545.304 \\
5545.791 \\
5546.104 \\
5546.469\end{array}$ & $\begin{array}{r}3 \\
4 \\
6 \\
80 \\
4\end{array}$ & $\begin{array}{r}4 \\
1 \\
25 \\
10\end{array}$ & $\begin{array}{l}\text { II } \\
\text { I } \\
\text { I } \\
\text { I } \\
\text { I I }\end{array}$ & $\begin{array}{l}5568.519 \\
5568.828 \\
5569.047 \\
5569.241 \\
5571.197\end{array}$ & $\begin{array}{r}10 \\
9 \\
3 \\
1 \\
300\end{array}$ & 18 & $\begin{array}{l}\text { I } \\
\text { I } \\
\text { I } \\
\text { I } \\
\text { I }\end{array}$ & $\begin{array}{l}5592.310 \\
5592.491 \\
5592.794 \\
5593.275 \\
5593.615\end{array}$ & $\begin{array}{r}5 \\
3 \\
5 \\
15 \\
100\end{array}$ & $\begin{array}{r}10 \\
4 \\
3 \\
20 \\
200\end{array}$ & $\begin{array}{l}\text { II } \\
\text { II } \\
\text { II } \\
\text { II } \\
\text { I I }\end{array}$ \\
\hline $\begin{array}{l}5546.601 \\
5547.125 \\
5547.625 \\
5548.1761 \\
5548.519\end{array}$ & $\begin{array}{r}3 \\
25 \\
6 \\
300 \\
5\end{array}$ & $\begin{array}{r}2 \\
1 \\
40 \\
20\end{array}$ & $\begin{array}{l}\text { I } \\
\text { I } \\
\text { II } \\
\text { I } \\
\text { II }\end{array}$ & $\begin{array}{l}5572.097 \\
5572.470 \\
5573.354 \mathrm{i} \\
5573.844 \\
5574.440\end{array}$ & $\begin{array}{r}40 \\
140 \\
350 \\
10 \\
1\end{array}$ & $\begin{array}{l}12 \\
20 \\
40\end{array}$ & $\begin{array}{l}\text { I } \\
\text { I } \\
\text { I } \\
\text { I I } \\
\text { I }\end{array}$ & $\begin{array}{l}5594.157 \\
5594.572 \\
5595.069 \\
5595.607 \\
5595.828\end{array}$ & $\begin{array}{r}7 \\
7 \\
200 \\
30 \\
15\end{array}$ & $\begin{array}{l}50 \\
25\end{array}$ & $\begin{array}{l}\text { I } \\
\text { I I } \\
\text { I } \\
\text { I } \\
\text { I }\end{array}$ \\
\hline $\begin{array}{l}5549.322 \\
5549.948 \\
5550.654 \\
5551.182 \\
5551.357\end{array}$ & $\begin{array}{r}2 \\
5 \\
3 \\
20 \\
125\end{array}$ & 300 & $\begin{array}{l}\text { I } \\
\text { I } \\
\text { I } \\
\text { I } \\
\text { I I }\end{array}$ & $\begin{array}{l}5574.899 \\
5575.303 \\
5575.586 \\
5575.981 \\
5576.207\end{array}$ & $\begin{array}{r}15 \\
2 \\
1 \\
40 \\
100\end{array}$ & $\begin{array}{l}2 \\
5\end{array}$ & $\begin{array}{l}\text { II } \\
\text { I } \\
\text { I } \\
\text { I } \\
\text { I }\end{array}$ & $\begin{array}{l}5595.949 \\
5596.092 \\
5596.812 \\
5597.005 \\
5597.358\end{array}$ & $\begin{array}{c}50 \\
3 \\
4 \\
11 \\
1\end{array}$ & $\begin{array}{r}2 \\
10\end{array}$ & $\begin{array}{l}\text { I } \\
\text { I I } \\
\text { I } \\
\text { I } \\
\text { I }\end{array}$ \\
\hline
\end{tabular}


Table 1. Wavelengths of Thorium Spectra - Continued

\begin{tabular}{|c|c|c|c|c|c|c|c|c|c|c|c|}
\hline \multirow{2}{*}{$\begin{array}{l}\text { Wave- } \\
\text { length }\end{array}$} & \multicolumn{2}{|c|}{ Intensity } & \multirow{2}{*}{$\begin{array}{l}\text { Spec- } \\
\text { trum }\end{array}$} & \multirow{2}{*}{$\begin{array}{l}\text { Wave- } \\
\text { length }\end{array}$} & \multicolumn{2}{|c|}{ Intensity } & \multirow{2}{*}{$\begin{array}{l}\text { Spec- } \\
\text { trum }\end{array}$} & \multirow{2}{*}{$\begin{array}{l}\text { Wave- } \\
\text { length }\end{array}$} & \multicolumn{2}{|c|}{ Intensity } & \multirow{2}{*}{$\begin{array}{l}\text { Spec } \\
\text { trum }\end{array}$} \\
\hline & Lamp & Spark & & & Lamp & Spark & & & Lamp & Spark & \\
\hline $\begin{array}{l}5597.608 \\
5598.070 \\
5598.392 \\
5599.285 \\
5599.640\end{array}$ & $\begin{array}{l}2 \\
2 w \\
1 \\
15 \\
50\end{array}$ & $\begin{array}{r}2 \\
30\end{array}$ & $\begin{array}{l}\text { I } \\
\text { I } \\
\text { I } \\
\text { II } \\
\text { I }\end{array}$ & $\begin{array}{l}5618.595 \\
5618.990 \\
5619.702 \\
5619.980 \\
5620.925\end{array}$ & $\begin{array}{r}5 \\
50 \\
10 \\
125 \\
12\end{array}$ & $\begin{array}{r}2 \\
1 \\
4 \\
30\end{array}$ & $\begin{array}{l}\text { I } \\
\text { I } \\
\text { I } \\
\text { I } \\
\text { I I }\end{array}$ & $\begin{array}{l}5643.337 \\
5643.928 \\
5645.528 \\
5645.670 \\
5645.884\end{array}$ & $\begin{array}{r}5 \\
7 \\
40 \\
75 \\
150\end{array}$ & $\begin{array}{l}100 \\
300\end{array}$ & $\begin{array}{l}\text { I } \\
\text { I } \\
\text { I } \\
\text { I } \\
\text { I I }\end{array}$ \\
\hline $\begin{array}{l}5599.787 \\
5600.251 \\
5600.341 \\
5600.514 \\
5601.125\end{array}$ & $\begin{array}{l}2 \\
1 \\
3 \\
1 \\
2\end{array}$ & 3 & $\begin{array}{l}\text { I I } \\
\text { I } \\
\text { I } \\
\text { I } \\
\text { I I }\end{array}$ & $\begin{array}{l}5621.279 \\
5621.783 \\
5622.072 \\
5623.548 \\
5623.980\end{array}$ & $\begin{array}{l}10 \\
10 \\
12 \\
30 \\
1\end{array}$ & $\begin{array}{r}3 \\
150 \\
1\end{array}$ & $\begin{array}{l}\text { I } \\
\text { II } \\
\text { II } \\
\text { I } \\
\text { I }\end{array}$ & $\begin{array}{l}5646.448 \\
5647.076 \\
5647.697 \\
5648.443 \\
5648.849\end{array}$ & $\begin{array}{r}30 \\
1 \\
12 \\
6 \\
40\end{array}$ & 50 & $\begin{array}{l}\text { I } \\
\text { I } \\
\text { I } \\
\text { I } \\
\text { I I }\end{array}$ \\
\hline $\begin{array}{l}5601.595 \\
5602.241 \\
5602.608 \\
5602.854 \\
5603.520\end{array}$ & $\begin{array}{r}150 \\
7 \\
10 \\
75 \\
1\end{array}$ & 4 & $\begin{array}{l}\text { I } \\
\text { I } \\
\text { I } \\
\text { I } \\
\text { I }\end{array}$ & $\begin{array}{l}5624.145 \\
5624.906 \\
5626.055 \\
5626.246 \\
5626.405\end{array}$ & $\begin{array}{r}3 \\
50 \\
12 \\
5 \\
3\end{array}$ & $\begin{array}{r}5 \\
50 \\
15\end{array}$ & $\begin{array}{l}\text { II } \\
\text { I } \\
\text { II } \\
\text { I I } \\
\text { I }\end{array}$ & $\begin{array}{l}5648.988 \\
5649.261 \\
5649.679 \\
5650.004 \\
5650.340\end{array}$ & $\begin{array}{r}75 \\
15 \\
9 \\
5 \\
75\end{array}$ & $\begin{array}{l}5 \\
8 \\
1 \\
5\end{array}$ & $\begin{array}{l}\text { I I } \\
\text { I I } \\
\text { I } \\
\text { I } \\
\text { I }\end{array}$ \\
\hline $\begin{array}{l}5603.746 \\
5604.512 \\
5605.213 \\
5605.279 \\
5606.379\end{array}$ & $\begin{array}{r}9 \\
400 \\
60 \\
75\end{array}$ & $\begin{array}{r}300 \\
50\end{array}$ & $\begin{array}{l}\text { I } \\
\text { I I } \\
\text { I I I } \\
\text { I } \\
\text { I }\end{array}$ & $\begin{array}{l}5626.732 \\
5627.492 \\
5628.342 \\
5628.594 \\
5629.308\end{array}$ & $\begin{array}{r}40 \\
12 \\
4 \\
4 \\
30\end{array}$ & $\begin{array}{r}125 \\
2 \\
20 \\
2\end{array}$ & $\begin{array}{l}\text { II } \\
\text { I } \\
\text { I } \\
\text { I I } \\
\text { I }\end{array}$ & $\begin{array}{l}5650.800 \\
5651.317 \\
5651.443 \\
5651.876 \\
5652.159\end{array}$ & $\begin{array}{l}1 \\
3 \\
1 \\
2 \\
1\end{array}$ & $\begin{array}{l}1 \\
2 \\
1 \\
5\end{array}$ & $\begin{array}{l}\text { I } \\
\text { I } \\
\text { I I } \\
\text { I I } \\
\text { I I }\end{array}$ \\
\hline $\begin{array}{l}5606.514 \\
5606.795 \\
5607.065 \\
5607.184 \\
5607.622\end{array}$ & $\begin{array}{l}10 \\
75 \\
2 \\
12 \\
1 \mathrm{w}\end{array}$ & $\begin{array}{r}15 \\
2\end{array}$ & $\begin{array}{l}\text { I I } \\
\text { I } \\
\text { I } \\
\text { I } \\
\text { I }\end{array}$ & $\begin{array}{l}5629.848 \\
5630.296 \\
5630.410 \\
5630.912 \\
5631.098\end{array}$ & $\begin{array}{r}20 \\
60 \\
20 \\
5 \\
1\end{array}$ & $\begin{array}{r}3 \\
6 \\
40 \\
3 \\
3\end{array}$ & $\begin{array}{l}\text { I } \\
\text { I } \\
\text { II } \\
\text { I I } \\
\text { I }\end{array}$ & $\begin{array}{l}5652.900 \\
5654.010 \\
5654.512 \\
5655.739 \\
5655.885\end{array}$ & $\begin{array}{r}100 \\
100 \\
50 \\
3 \\
3\end{array}$ & $\begin{array}{r}350 \\
100 \\
3 \\
3 \\
1\end{array}$ & $\begin{array}{l}\text { II } \\
\text { II } \\
\text { I } \\
\text { I } \\
\text { I }\end{array}$ \\
\hline $\begin{array}{l}5608.080 \\
5608.545 \\
5608.748 \\
5609.296 \\
5609.576\end{array}$ & $\begin{array}{r}4 \\
3 \\
6 \\
5 \\
75\end{array}$ & $\begin{array}{r}6 \\
8 \\
20 \\
3\end{array}$ & $\begin{array}{l}\text { II } \\
\text { II } \\
\text { I } \\
\text { II } \\
\text { I }\end{array}$ & $\begin{array}{l}5632.029 \\
5632.121 \\
5632.491 \\
5632.781 \\
5632.876\end{array}$ & $\begin{array}{l}15 \\
20 \\
10 \\
25 \\
50\end{array}$ & 40 & $\begin{array}{l}\text { I I } \\
\text { I } \\
\text { I } \\
\text { I } \\
\text { II }\end{array}$ & $\begin{array}{l}5656.298 \\
5656.724 \\
5657.177 \\
5657.632 \\
5657.926 \mathrm{i}\end{array}$ & $\begin{array}{c}51 \\
10 \\
20 \\
10 \\
100\end{array}$ & $\begin{array}{r}1 \\
40 \\
12\end{array}$ & $\begin{array}{l}\text { I } \\
\text { I } \\
\text { I I } \\
\text { I } \\
\text { I }\end{array}$ \\
\hline $\begin{array}{l}5610.116 \\
5610.249 \\
5610.673 \\
5611.071 \\
5612.073\end{array}$ & $\begin{array}{l}125 \\
100 \\
160 \\
6 \mathrm{w} \\
100\end{array}$ & $\begin{array}{r}3 \\
3 \\
8 \\
1 \\
10\end{array}$ & $\begin{array}{l}\text { I } \\
\text { I } \\
\text { I } \\
\text { I I } \\
\text { I }\end{array}$ & $\begin{array}{l}5633.293 \\
5633.626 \\
5634.081 \\
5634.210 \\
5634.603\end{array}$ & $\begin{array}{r}75 \\
20 \\
5 \\
5 \\
5\end{array}$ & $\begin{array}{r}2 \\
30 \\
10\end{array}$ & $\begin{array}{l}\text { I } \\
\text { I I } \\
\text { I } \\
\text { I I } \\
\text { I }\end{array}$ & $\begin{array}{l}5658.676 \\
5659.194 \\
5659.468 \\
5660.006 \\
5660.652\end{array}$ & $\begin{array}{l}4 \\
4 \\
1 \\
101 \\
18\end{array}$ & $\begin{array}{c}18 \\
1\end{array}$ & $\begin{array}{l}\text { I } \\
\text { I } \\
\text { I } \\
\text { I I } \\
\text { I }\end{array}$ \\
\hline $\begin{array}{l}5612.604 \\
5613.646 \\
5614.416 \\
5614.500 \\
5615.3201\end{array}$ & $\begin{array}{r}40 \\
5 \\
1 \\
2 \\
350\end{array}$ & $\begin{array}{r}1 \\
1 \\
2 \\
12\end{array}$ & $\begin{array}{l}\text { I } \\
\text { I } \\
\text { I } \\
\text { II } \\
\text { I }\end{array}$ & $\begin{array}{l}5634.845 \\
5635.163 \\
5635.877 \\
5637.272 \\
5637.606\end{array}$ & $\begin{array}{r}4 \\
4 \\
75 \\
2 \\
1\end{array}$ & $\begin{array}{l}2 \\
2 \\
1\end{array}$ & $\begin{array}{l}\text { II } \\
\text { I } \\
\text { I } \\
\text { I } \\
\text { I }\end{array}$ & $\begin{array}{l}5661.384 \\
5661.685 \\
5662.018 \\
5662.376 \\
5662.649\end{array}$ & $\begin{array}{l}1 \\
1 \\
4 \\
6 \\
1\end{array}$ & 1 & $\begin{array}{l}\text { I } \\
\text { I } \\
\text { I } \\
\text { I } \\
\text { I }\end{array}$ \\
\hline $\begin{array}{l}5615.684 \\
5616.083 \\
5616.323 \\
5616.493 \\
5616.678\end{array}$ & $\begin{array}{r}75 \\
2 \\
5 \\
4 \\
20\end{array}$ & 200 & $\begin{array}{l}\text { I I } \\
\text { I } \\
\text { I } \\
\text { I } \\
\text { I }\end{array}$ & $\begin{array}{l}5637.946 \\
5638.156 \\
5639.7461 \\
5640.064 \\
5640.365\end{array}$ & $\begin{array}{r}2 \\
1 \\
250 \\
40 \\
15\end{array}$ & $\begin{array}{r}400 \\
50\end{array}$ & $\begin{array}{l}\text { I } \\
\text { I } \\
\text { I I } \\
\text { I I } \\
\text { I }\end{array}$ & $\begin{array}{l}5663.027 \\
5663.360 \\
5663.964 \\
5664.236 \\
5664.619\end{array}$ & $\begin{array}{r}50 \\
5 \\
5 \\
50\end{array}$ & $\begin{array}{r}1 \mathrm{~h} \\
5 \mathrm{w} \\
10\end{array}$ & $\begin{array}{l}\text { I } \\
\text { II } \\
\text { I } \\
\text { I } \\
\text { II }\end{array}$ \\
\hline $\begin{array}{l}5616.934 \\
5617.279 \\
5617.633 \\
5617.966 \\
5618.143\end{array}$ & $\begin{array}{r}1 \\
12 \\
15 \\
1 \\
4\end{array}$ & $\begin{array}{l}2 \\
1 \\
4 w\end{array}$ & $\begin{array}{l}\text { I } \\
\text { II } \\
\text { I } \\
\text { I } \\
\text { II }\end{array}$ & $\begin{array}{l}5640.718 \\
5641.242 \\
5641.560 \\
5641.728 \\
5643.104\end{array}$ & $\begin{array}{r}18 \\
10 \\
30 \\
125 \\
12\end{array}$ & $\begin{array}{r}15 \\
5 \\
1 \\
4 \\
1\end{array}$ & $\begin{array}{l}\text { II } \\
\text { I I } \\
\text { I } \\
\text { I } \\
\text { I }\end{array}$ & $\begin{array}{l}5665.180 \\
5665.619 \\
5666.395 \\
5667.121 \\
5668.021\end{array}$ & $\begin{array}{r}140 \\
125 \\
10 \\
125 \\
1\end{array}$ & $\begin{array}{l}300 \\
300 w\end{array}$ & $\begin{array}{l}\text { I } \\
\text { II } \\
\text { II } \\
\text { I } \\
\text { I }\end{array}$ \\
\hline
\end{tabular}


Table 1. Wavelengths of Thorium Spectra - Continued

\begin{tabular}{|c|c|c|c|c|c|c|c|c|c|c|c|}
\hline \multirow{2}{*}{$\begin{array}{l}\text { Wave- } \\
\text { length }\end{array}$} & \multicolumn{2}{|c|}{ Intensity } & \multirow{2}{*}{$\begin{array}{l}\text { Spec- } \\
\text { trum }\end{array}$} & \multirow{2}{*}{$\begin{array}{l}\text { Wave- } \\
\text { length }\end{array}$} & \multicolumn{2}{|c|}{ Intensity } & \multirow{2}{*}{$\begin{array}{l}\text { Spec- } \\
\text { trum }\end{array}$} & \multirow{2}{*}{$\begin{array}{l}\text { Wave- } \\
\text { length }\end{array}$} & \multicolumn{2}{|c|}{ Intensity } & \multirow{2}{*}{$\begin{array}{l}\text { Spec } \\
\text { trum }\end{array}$} \\
\hline & $\operatorname{amp}$ & Spark & & & Lamp & Spark & & & Lamp & Spark t & \\
\hline $\begin{array}{l}5668.329 \\
5668.897 \\
5669.830 \\
5670.245 \\
5670.658\end{array}$ & $\begin{array}{l}12 \\
3 \mathrm{w} \\
7 \\
2 \\
4\end{array}$ & $\begin{array}{r}18 \\
5\end{array}$ & $\begin{array}{l}\text { II } \\
\text { I I } \\
\text { I } \\
\text { I } \\
\text { I I }\end{array}$ & $\begin{array}{l}5694.040 \\
5694.410 \\
5694.760 \\
5695.112 \\
5695.685\end{array}$ & $\begin{array}{l}6 \\
6 \\
1 \\
7 \\
1\end{array}$ & $\begin{array}{l}1 \\
1 \\
1 \\
2\end{array}$ & $\begin{array}{l}\text { I } \\
\text { I } \\
\text { I I } \\
\text { I } \\
\text { I }\end{array}$ & $\begin{array}{l}5719.631 \\
5720.197 \\
5721.413 \\
5721.688 \\
5722.232\end{array}$ & $\begin{array}{c}200 \\
400 \\
30 \\
2 \\
1 \mathrm{w}\end{array}$ & $\begin{array}{r}20 \\
100 \\
2 \\
3 \\
\end{array}$ & $\begin{array}{l}\text { I } \\
\text { I } \\
\text { I } \\
\text { I I } \\
\text { I }\end{array}$ \\
\hline $\begin{array}{l}5671.054 \\
5671.219 \\
5671.482 \\
5672.725 \\
5672.812\end{array}$ & $\begin{array}{r}15 \\
3 \\
5 \\
2 \\
2\end{array}$ & $\begin{array}{l}1 \\
1 \\
2\end{array}$ & $\begin{array}{l}\text { I } \\
\text { I } \\
\text { I } \\
\text { I I } \\
\text { I }\end{array}$ & $\begin{array}{l}5696.373 \\
5697.072 \\
5697.384 \\
5698.280 \\
5698.530\end{array}$ & $\begin{array}{l}50 \\
6 \\
1 \mathrm{w} \\
50 \\
50\end{array}$ & $\begin{array}{l}3 \\
1 \\
1 \\
2 \\
3\end{array}$ & $\begin{array}{l}\text { I } \\
\text { II } \\
\text { I } \\
\text { I } \\
\text { I I }\end{array}$ & $\begin{array}{l}5722.734 \\
5723.527 \\
5724.235 \\
5724.452 \\
5725.027\end{array}$ & $\begin{array}{r}1 \mathrm{w} \\
9 \\
75 \\
100 \\
50\end{array}$ & $\begin{array}{r}1 \\
4 \\
1 \\
75\end{array}$ & $\begin{array}{l}\text { I } \\
\text { I } \\
\text { I } \\
\text { I } \\
\text { I I }\end{array}$ \\
\hline $\begin{array}{l}5672.974 \\
5673.127 \\
5673.196 \\
5673.828 \\
5674.159\end{array}$ & $\begin{array}{r}2 \\
1 \\
1 \\
50 \\
2\end{array}$ & $\begin{array}{l}1 \\
2\end{array}$ & $\begin{array}{l}\text { I } \\
\text { I } \\
\text { I } \\
\text { I } \\
\text { I I }\end{array}$ & $\begin{array}{l}5699.194 \\
5699.350 \\
5700.453 \\
5700.687 \\
5700.902\end{array}$ & $\begin{array}{r}4 \\
6 \\
200 \\
40 \\
150\end{array}$ & $\begin{array}{r}6 \\
18 \\
75 \\
75 \\
300\end{array}$ & $\begin{array}{l}\text { I I } \\
\text { I I } \\
\text { I I } \\
\text { I I } \\
\text { I I }\end{array}$ & $\begin{array}{l}5725.389 \mathrm{i} \\
5725.603 \\
5727.010 \\
5727.700 \\
5728.454\end{array}$ & $\begin{array}{r}250 \\
30 \\
75 \\
1\end{array}$ & $\begin{array}{r}30 \\
50 \\
2 \\
3 \\
1\end{array}$ & $\begin{array}{l}\text { I } \\
\text { I I I } \\
\text { I } \\
\text { I I } \\
\text { I }\end{array}$ \\
\hline $\begin{array}{l}5674.454 \\
5674.688 \\
5674.980 \\
5675.476 \\
5676.754\end{array}$ & $\begin{array}{r}6 \\
5 \\
120 \\
12 \\
1\end{array}$ & $\begin{array}{r}1 \\
25 \\
2\end{array}$ & $\begin{array}{l}\text { I } \\
\text { I } \\
\text { I } \\
\text { I } \\
\text { I I }\end{array}$ & $\begin{array}{l}5702.006 \\
5702.638 \\
5703.250 \\
5703.916 \\
5704.539\end{array}$ & $\begin{array}{r}5 \\
15 \\
4 \\
15 \\
3\end{array}$ & $\begin{array}{l}1 \mathrm{w} \\
2\end{array}$ & $\begin{array}{l}\text { I } \\
\text { I } \\
\text { I } \\
\text { I I } \\
\text { I }\end{array}$ & $\begin{array}{l}5729.105 \\
5729.391 \\
5729.985 \\
5730.758 \\
5731.464\end{array}$ & $\begin{array}{r}5 \\
1 \\
75 \\
1 \\
4\end{array}$ & $\begin{array}{r}5 \\
1 \\
12\end{array}$ & $\begin{array}{l}\text { I } \\
\text { I } \\
\text { I } \\
\text { I } \\
\text { I I }\end{array}$ \\
\hline $\begin{array}{l}5677.034 \\
5678.324 \\
5678.538 \\
5678.598 \\
5678.972\end{array}$ & $\begin{array}{r}125 \\
2 \\
1 \\
1 \\
75\end{array}$ & $\begin{array}{c}3 \\
2 \\
50 \mathrm{w}\end{array}$ & $\begin{array}{l}\text { I } \\
\text { I } \\
\text { II } \\
\text { I } \\
\text { I I }\end{array}$ & $\begin{array}{l}5705.164 \\
5705.624 \\
5706.128 \\
5707.103 \mathrm{i} \\
5708.650\end{array}$ & $\begin{array}{r}2 \\
15 \\
3 \\
150 \\
15\end{array}$ & 300 & $\begin{array}{l}\text { I } \\
\text { I } \\
\text { I } \\
\text { I I } \\
\text { I }\end{array}$ & $\begin{array}{l}5731.607 \\
5732.037 \\
5732.323 \\
5732.987 \\
5733.885\end{array}$ & $\begin{array}{r}6 \\
5 \\
5 \\
200 \\
7\end{array}$ & $\begin{array}{r}10 \\
500\end{array}$ & $\begin{array}{l}\text { I } \\
\text { I I } \\
\text { I } \\
\text { I I } \\
\text { I }\end{array}$ \\
\hline $\begin{array}{l}5680.187 \\
5680.886 \\
5681.229 \\
5681.760 \\
5682.002\end{array}$ & $\begin{array}{r}4 \\
5 \\
10 \\
1 \\
1\end{array}$ & $\begin{array}{r}20 \\
1 \\
1\end{array}$ & $\begin{array}{l}\text { I } \\
\text { I } \\
\text { II } \\
\text { I } \\
\text { I }\end{array}$ & $\begin{array}{l}5709.080 \\
5709.674 \\
5711.076 \\
5711.989 \\
5712.240\end{array}$ & $\begin{array}{r}1 \\
1 \\
10 \\
15 \\
2\end{array}$ & 2 & $\begin{array}{l}\text { I } \\
\text { I } \\
\text { I } \\
\text { I } \\
\text { I }\end{array}$ & $\begin{array}{l}5734.152 \\
5734.542 \\
5734.872 \\
5735.300 \\
5736.033\end{array}$ & $\begin{array}{r}6 \\
3 \\
1 \\
3 \\
75\end{array}$ & $\begin{array}{l}2 \\
1 \\
1 \\
5 \mathrm{w}\end{array}$ & $\begin{array}{l}\text { I } \\
\text { I I } \\
\text { II } \\
\text { I I } \\
\text { I }\end{array}$ \\
\hline $\begin{array}{l}5682.218 \\
5682.414 \\
5682.822 \\
5683.465 \\
5683.931\end{array}$ & $\begin{array}{l}1 \\
2 \\
2 \\
6 \\
6\end{array}$ & $\begin{array}{l}8 \\
1 \\
1\end{array}$ & $\begin{array}{l}\text { I } \\
\text { I I } \\
\text { I } \\
\text { I I } \\
\text { I I }\end{array}$ & $\begin{array}{l}5712.575 \\
5712.973 \\
5713.141 \\
5713.326 \\
5713.504\end{array}$ & $\begin{array}{r}15 \\
1 \\
3 \\
1 \\
1\end{array}$ & 20 & $\begin{array}{l}\text { I I } \\
\text { I } \\
\text { I } \\
\text { I } \\
\text { I I }\end{array}$ & $\begin{array}{l}5737.360 \\
5737.644 \\
5738.287 \\
5738.960 \\
5739.676\end{array}$ & $\begin{array}{r}2 \\
1 \\
40 \\
15 \\
1\end{array}$ & $\begin{array}{r}2 \\
75\end{array}$ & $\begin{array}{l}\text { I I } \\
\text { I } \\
\text { I I } \\
\text { I } \\
\text { I }\end{array}$ \\
\hline $\begin{array}{l}5684.136 \\
5685.171 \\
5685.810 \\
5686.852 \\
5687.326\end{array}$ & $\begin{array}{r}1 \\
150 \\
1 \\
6 \\
25\end{array}$ & $\begin{array}{l}1 \\
2 \\
1 \\
1\end{array}$ & $\begin{array}{l}\text { I } \\
\text { I } \\
\text { I } \\
\text { I } \\
\text { I }\end{array}$ & $\begin{array}{l}5713.962 \\
5714.883 \\
5714.974 \\
5715.431 \\
5715.719\end{array}$ & $\begin{array}{l}5 \\
1 \\
1 \\
10\end{array}$ & 700 & $\begin{array}{l}\text { I } \\
\text { I } \\
\text { I } \\
\text { I I I } \\
\text { I }\end{array}$ & $\begin{array}{l}5739.824 \\
5740.045 \\
5740.190 \\
5740.628 \\
5740.862\end{array}$ & $\begin{array}{l}1 \\
2 \\
1 \\
1\end{array}$ & $500 \mathrm{w}$ & $\begin{array}{l}\text { I } \\
\text { I } \\
\text { I } \\
\text { I } \\
\text { I I I }\end{array}$ \\
\hline $\begin{array}{l}5687.661 \\
5688.900 \\
5689.624 \\
5690.104 \\
5690.674\end{array}$ & $\begin{array}{r}5 \\
12 \\
5 \\
10 \\
20\end{array}$ & $\begin{array}{r}25 \\
20 \\
5\end{array}$ & $\begin{array}{l}\text { I I } \\
\text { I I } \\
\text { I } \\
\text { I I } \\
\text { I }\end{array}$ & $\begin{array}{l}5715.934 \\
5716.399 \\
5716.800 \\
5717.158 \\
5717.512\end{array}$ & $\begin{array}{r}1 \\
1 \\
6 \\
25 \\
9\end{array}$ & 15 & $\begin{array}{l}\text { I } \\
\text { I } \\
\text { I } \\
\text { I } \\
\text { I }\end{array}$ & $\begin{array}{l}5741.178 \\
5741.837 \\
5742.088 \\
5742.651 \\
5743.054\end{array}$ & $\begin{array}{r}50 \\
50 \\
200 \\
7 \\
9\end{array}$ & $\begin{array}{r}75 \\
2 \\
20 \\
10\end{array}$ & $\begin{array}{l}\text { I I } \\
\text { I } \\
\text { I I } \\
\text { I I } \\
\text { I }\end{array}$ \\
\hline $\begin{array}{l}5691.073 \\
5691.610 \\
5692.131 \\
5692.838 \\
5693.544\end{array}$ & $\begin{array}{r}15 \\
25 \\
7 \\
6 \\
2\end{array}$ & $\begin{array}{r}20 \\
2 \\
12\end{array}$ & $\begin{array}{l}\text { I I } \\
\text { I } \\
\text { I I } \\
\text { I I } \\
\text { I }\end{array}$ & $\begin{array}{l}5718.102 \\
5718.387 \\
5718.516 \\
5718.855 \\
5719.091\end{array}$ & $\begin{array}{l}6 \\
2 \\
2 \\
6 \\
7\end{array}$ & & $\begin{array}{l}\text { I } \\
\text { I } \\
\text { I } \\
\text { I } \\
\text { I }\end{array}$ & $\begin{array}{l}5743.281 \\
5743.578 \\
5744.699 \\
5745.227 \\
5745.678\end{array}$ & $\begin{array}{r}1 \\
2 \\
1 \\
2 \\
40\end{array}$ & 300 & $\begin{array}{l}\text { I I } \\
\text { I } \\
\text { I } \\
\text { I } \\
\text { I I }\end{array}$ \\
\hline
\end{tabular}


Table 1. Wavelengths of Thorium Spectra - Continued

\begin{tabular}{|c|c|c|c|c|c|c|c|c|c|c|c|}
\hline \multirow{2}{*}{$\begin{array}{l}\text { Wave- } \\
\text { length }\end{array}$} & \multicolumn{2}{|c|}{ Intensity } & \multirow{2}{*}{$\begin{array}{l}\text { Spec- } \\
\text { trum }\end{array}$} & \multirow{2}{*}{$\begin{array}{l}\text { Wave- } \\
\text { length }\end{array}$} & \multicolumn{2}{|c|}{ Intensity } & \multirow{2}{*}{$\begin{array}{l}\text { Spec- } \\
\text { trum }\end{array}$} & \multirow{2}{*}{$\begin{array}{l}\text { Wave- } \\
\text { length }\end{array}$} & \multicolumn{2}{|c|}{ Intensity } & \multirow{2}{*}{$\begin{array}{l}\text { Spec- } \\
\text { trum }\end{array}$} \\
\hline & $a m p$ & Spark & & & Lamp & Spark & & & Lamp & Spark & \\
\hline $\begin{array}{l}5746.514 \\
5747.201 \\
5747.391 \\
5747.545 \\
5748.333\end{array}$ & $\begin{array}{l}2 w \\
30 \\
40 \\
7 \\
50\end{array}$ & $\begin{array}{r}1 \\
15 \\
2\end{array}$ & $\begin{array}{l}\text { I } \\
\text { I } \\
\text { I } \\
\text { II } \\
\text { I }\end{array}$ & $\begin{array}{l}5768.626 \\
5769.675 \\
5770.131 \\
5770.468 \\
5770.836\end{array}$ & $\begin{array}{r}5 \\
40 \\
50 \\
7 \\
10\end{array}$ & $\begin{array}{l}200 \\
100\end{array}$ & $\begin{array}{l}\text { I } \\
\text { II } \\
\text { II } \\
\text { I } \\
\text { I }\end{array}$ & $\begin{array}{l}5790.121 \\
5790.591 \\
5791.031 \\
5791.320 \\
5791.706\end{array}$ & $\begin{array}{c}2 \\
31 \\
10 \\
1 \\
50\end{array}$ & 5 & $\begin{array}{l}\text { II } \\
\text { I } \\
\text { I } \\
\text { I } \\
\text { I }\end{array}$ \\
\hline $\begin{array}{l}5748.755 \\
5749.395 \\
5749.616 \\
5749.774 \\
5750.262\end{array}$ & $\begin{array}{r}150 \\
100 \\
\\
75 \\
15\end{array}$ & $\begin{array}{r}4 \\
300 \\
60 \\
15\end{array}$ & $\begin{array}{l}\text { I } \\
\text { II } \\
\text { III } \\
\text { I } \\
\text { II }\end{array}$ & $\begin{array}{l}5771.146 \\
5771.759 \\
5771.941 \\
5772.547 \\
5773.206\end{array}$ & $\begin{array}{r}5 \\
50 \\
15 \\
6 \\
2\end{array}$ & $\begin{array}{r}30 \\
1 \\
5 \\
4\end{array}$ & $\begin{array}{l}\text { II } \\
\text { I } \\
\text { I } \\
\text { I } \\
\text { I }\end{array}$ & $\begin{array}{l}5792.433 \\
5792.475 \\
5792.983 \\
5793.202 \\
5794.377\end{array}$ & $\begin{array}{r}150 \\
10 \\
5 \\
15\end{array}$ & $\begin{array}{l}10 \\
10 \\
75\end{array}$ & $\begin{array}{l}\text { I } \\
\text { II } \\
\text { I } \\
\text { II } \\
\text { II }\end{array}$ \\
\hline $\begin{array}{l}5750.565 \\
5751.168 \\
5751.433 \\
5751.612 \\
5752.309\end{array}$ & $\begin{array}{r}15 \\
2 \\
6 \\
9 \\
3\end{array}$ & $\begin{array}{r}30 \\
5\end{array}$ & $\begin{array}{l}\text { II } \\
\text { II } \\
\text { I } \\
\text { I } \\
\text { II }\end{array}$ & $\begin{array}{l}5773.951 \\
5774.721 \\
5774.976 \\
5775.062 \\
5775.616\end{array}$ & $\begin{array}{r}150 \\
4 \\
2 \\
1 \\
5\end{array}$ & $\begin{array}{l}3 \\
1\end{array}$ & $\begin{array}{l}\text { I } \\
\text { I } \\
\text { I } \\
\text { II } \\
\text { I }\end{array}$ & $\begin{array}{l}5794.870 \\
5795.967 \\
5796.081 \\
5796.424 \\
5797.015\end{array}$ & $\begin{array}{r}12 \\
150 \\
75 \\
3\end{array}$ & $\begin{array}{r}30 \\
5 \\
125\end{array}$ & $\begin{array}{l}\text { I } \\
\text { II } \\
\text { I } \\
\text { II } \\
\text { I }\end{array}$ \\
\hline $\begin{array}{l}5752.581 \\
5753.040 \\
5753.992 \\
5754.555 \\
5754.741\end{array}$ & $\begin{array}{c}2 \mathrm{w} \\
100 \\
1 \\
15 \\
9\end{array}$ & $\begin{array}{r}4 \\
40\end{array}$ & $\begin{array}{l}\text { I } \\
\text { I } \\
\text { I } \\
\text { II } \\
\text { I }\end{array}$ & $\begin{array}{l}5776.167 \\
5776.573 \\
5777.100 \\
5777.397 \\
5777.837\end{array}$ & $\begin{array}{r}40 \\
2 \\
6 \\
75 \\
5\end{array}$ & $\begin{array}{r}2 \\
10 \\
2\end{array}$ & $\begin{array}{l}\text { I } \\
\text { I } \\
\text { II } \\
\text { I } \\
\text { I }\end{array}$ & $\begin{array}{l}5797.319 \\
5798.478 \\
5798.761 \\
5798.891 \\
5799.140\end{array}$ & $\begin{array}{r}50 \\
40 \\
6 \\
7\end{array}$ & $\begin{array}{l}2 \\
1 \\
4 w\end{array}$ & $\begin{array}{l}\text { I } \\
\text { I } \\
\text { I } \\
\text { II } \\
\text { I }\end{array}$ \\
\hline $\begin{array}{l}5754.838 \\
5755.055 \\
5755.717 \\
5755.968 \\
5757.108\end{array}$ & $\begin{array}{r}3 \\
2 \\
10 \\
1 \\
10\end{array}$ & 18 & $\begin{array}{l}\text { I } \\
\text { II } \\
\text { I } \\
\text { I } \\
\text { II }\end{array}$ & $\begin{array}{l}5778.077 \\
5778.470 \\
5778.583 \\
5779.411 \\
5779.825\end{array}$ & $\begin{array}{r}6 \\
4 \\
6 \\
10 \\
50\end{array}$ & $\begin{array}{r}50 \\
2 \\
1\end{array}$ & $\begin{array}{l}\text { I I } \\
\text { I } \\
\text { I } \\
\text { I } \\
\text { I }\end{array}$ & $\begin{array}{l}5799.434 \\
5799.817 \\
5800.125 \\
5800.840 \\
5801.561\end{array}$ & $\begin{array}{c}2 \\
3 w \\
1 \\
175 \\
6\end{array}$ & 15 & $\begin{array}{l}\text { I } \\
\text { I } \\
\text { I } \\
\text { I } \\
\text { I }\end{array}$ \\
\hline $\begin{array}{l}5757.569 \\
5757.654 \\
5758.092 \\
5758.486 \\
5758.728\end{array}$ & $\begin{array}{l}3 \\
5 \\
4 \mathrm{w} \\
2 \\
1\end{array}$ & $\begin{array}{l}2 \\
4\end{array}$ & $\begin{array}{l}\text { II } \\
\text { II } \\
\text { I } \\
\text { I } \\
\text { I }\end{array}$ & $\begin{array}{l}5780.149 \\
5781.173 \\
5781.649 \\
5782.043 \\
5782.284\end{array}$ & $\begin{array}{r}1 \\
4 \\
75 \\
4 \\
125\end{array}$ & $\begin{array}{r}40 \\
2 \\
15 \\
2\end{array}$ & $\begin{array}{l}\text { I } \\
\text { II } \\
\text { I } \\
\text { II } \\
\text { I }\end{array}$ & $\begin{array}{l}5802.231 \\
5802.377 \\
5802.837 \\
5803.201 \\
5804.141 \mathrm{i}\end{array}$ & $\begin{array}{r}10 \\
5 \\
9 \\
4 \\
300\end{array}$ & $\begin{array}{r}30 \\
8 \\
30\end{array}$ & $\begin{array}{l}\text { II } \\
\text { I } \\
\text { I } \\
\text { II } \\
\text { I }\end{array}$ \\
\hline $\begin{array}{l}5758.919 \\
5759.204 \\
5759.601 \\
5760.551 i \\
5761.230\end{array}$ & $\begin{array}{r}4 \\
2 \\
2 \\
600 \\
5\end{array}$ & 100 & $\begin{array}{l}\text { II } \\
\text { I } \\
\text { I } \\
\text { I } \\
\text { I }\end{array}$ & $\begin{array}{l}5783.197 \\
5783.550 \\
5783.837 \\
5784.072 \\
5784.228\end{array}$ & $\begin{array}{r}15 \\
6 \\
9 \\
1 \\
1\end{array}$ & $\begin{array}{r}30 \\
6 \\
2\end{array}$ & $\begin{array}{l}\text { II } \\
\text { I } \\
\text { II } \\
\text { II } \\
\text { I }\end{array}$ & $\begin{array}{l}5804.604 \\
5804.948 \\
5805.475 \\
5805.704 \\
5806.254\end{array}$ & $\begin{array}{r}5 \\
3 \\
2 \\
40 \\
140\end{array}$ & $\begin{array}{r}2 \\
80\end{array}$ & $\begin{array}{l}\text { I } \\
\text { I } \\
\text { I } \\
\text { I } \\
\text { I }\end{array}$ \\
\hline $\begin{array}{l}5761.526 \\
5761.772 \\
5762.068 \\
5762.267 \\
5762.812\end{array}$ & $\begin{array}{l}9 \\
9 w \\
2 w \\
1 \\
75\end{array}$ & $\begin{array}{l}2 \\
2 \\
3 \\
3\end{array}$ & $\begin{array}{l}\text { II } \\
\text { I } \\
\text { I } \\
\text { II } \\
\text { I }\end{array}$ & $\begin{array}{l}5784.394 \\
5784.539 \\
5784.862 \\
5785.116 \\
5785.474\end{array}$ & $\begin{array}{r}1 \\
1 \\
40 \\
3 \\
2\end{array}$ & $\begin{array}{r}2 \\
100 \\
41\end{array}$ & $\begin{array}{l}\text { II } \\
\text { I } \\
\text { I I } \\
\text { I } \\
\text { II }\end{array}$ & $\begin{array}{l}5806.566 \\
5807.682 \\
5808.072 \\
5808.642 \\
5808.962\end{array}$ & $\begin{array}{r}5 \\
40 \\
15 \\
15 \\
2\end{array}$ & $\begin{array}{r}75 \\
5 \\
5\end{array}$ & $\begin{array}{l}\text { I } \\
\text { I } \\
\text { II } \\
\text { I } \\
\text { II }\end{array}$ \\
\hline $\begin{array}{l}5763.540 \\
5765.461 \\
5765.958 \\
5766.111 \\
5766.476\end{array}$ & $\begin{array}{r}80 \\
3 \\
1 \\
6 \\
1\end{array}$ & $\begin{array}{r}30 \\
4 \\
10 \\
2\end{array}$ & $\begin{array}{l}\text { I } \\
\text { I } \\
\text { II } \\
\text { II } \\
\text { II }\end{array}$ & $\begin{array}{l}5785.857 \\
5786.199 \\
5786.676 \\
5787.532 \\
5787.861\end{array}$ & $\begin{array}{l}2 \\
1 \\
5 \\
6 \\
1\end{array}$ & $\begin{array}{l}12 \\
12\end{array}$ & $\begin{array}{l}\text { I } \\
\text { I } \\
\text { II } \\
\text { II } \\
\text { I }\end{array}$ & $\begin{array}{l}5809.401 \\
5809.557 \\
5809.976 \\
5810.236 \\
5810.446\end{array}$ & $\begin{array}{l}5 \\
5 \\
2 \\
2 \\
2\end{array}$ & 5 & $\begin{array}{l}\text { II } \\
\text { I } \\
\text { I } \\
\text { I } \\
\text { I }\end{array}$ \\
\hline $\begin{array}{l}5766.651 \\
5767.134 \\
5767.475 \\
5767.785 \\
5768.191\end{array}$ & $\begin{array}{r}1 \\
1 \\
2 \\
75 \\
150\end{array}$ & $\begin{array}{l}4 \\
4 \\
5\end{array}$ & $\begin{array}{l}\text { I } \\
\text { I } \\
\text { I } \\
\text { I } \\
\text { I }\end{array}$ & $\begin{array}{l}5788.018 \\
5788.188 \\
5788.550 \\
5789.052 \\
5789.644 \mathrm{i}\end{array}$ & $\begin{array}{r}2 \\
4 \\
1 \\
2 \\
200\end{array}$ & 12 & $\begin{array}{l}\text { I } \\
\text { II } \\
\text { I } \\
\text { I } \\
\text { I }\end{array}$ & $\begin{array}{l}5810.964 \\
5811.370 \\
5811.835 \\
5812.094 \\
5812.691\end{array}$ & $\begin{array}{r}5 \\
1 \\
6 \\
1 \\
12\end{array}$ & $\begin{array}{r}12 \\
2 \\
1\end{array}$ & $\begin{array}{l}\text { II } \\
\text { II } \\
\text { I } \\
\text { II } \\
\text { I }\end{array}$ \\
\hline
\end{tabular}


Table 1. Wavelengths of Thorium Spectra - Continued

\begin{tabular}{|c|c|c|c|c|c|c|c|c|c|c|c|}
\hline \multirow{2}{*}{$\begin{array}{l}\text { Wave- } \\
\text { length }\end{array}$} & \multicolumn{2}{|c|}{ Intensity } & \multirow{2}{*}{$\begin{array}{l}\text { Spec- } \\
\text { trum }\end{array}$} & \multirow{2}{*}{$\begin{array}{l}\text { Wave- } \\
\text { length }\end{array}$} & \multicolumn{2}{|c|}{ Intensity } & \multirow{2}{*}{$\begin{array}{l}\text { Spec- } \\
\text { trum }\end{array}$} & \multirow{2}{*}{$\begin{array}{l}\text { Wave- } \\
\text { length }\end{array}$} & \multicolumn{2}{|c|}{ Intensity } & \multirow{2}{*}{$\begin{array}{l}\text { Spec } \\
\text { trum }\end{array}$} \\
\hline & Lamp & Spark & & & Lamp & Spark & & & Lamp & Spark & \\
\hline $\begin{array}{l}5812.972 \\
5813.575 \\
5814.078 \\
5814.354 \\
5814.721\end{array}$ & $\begin{array}{r}150 \\
4 \\
2 \\
2 \\
12\end{array}$ & $\begin{array}{c}3 \\
10 \\
150 \mathrm{w}\end{array}$ & $\begin{array}{l}\text { I } \\
\text { II } \\
\text { III } \\
\text { I } \\
\text { I }\end{array}$ & $\begin{array}{l}5833.546 \\
5833.796 \\
5834.105 \\
5835.141 \\
5835.282\end{array}$ & $\begin{array}{l}2 \\
4 \\
2 \\
2 \\
1\end{array}$ & $\begin{array}{l}3 \\
2\end{array}$ & $\begin{array}{l}\text { I } \\
\text { I } \\
\text { II } \\
\text { I } \\
\text { II }\end{array}$ & $\begin{array}{l}5853.773 \\
5854.119 \\
5855.016 \\
5855.497 \\
5855.898\end{array}$ & $\begin{array}{r}5 \\
100 \\
5 \\
75 \\
1\end{array}$ & $\begin{array}{r}7 \\
3 \\
25 \\
3\end{array}$ & $\begin{array}{l}\text { II } \\
\text { I } \\
\text { II } \\
\text { II } \\
\text { I }\end{array}$ \\
\hline $\begin{array}{l}5815.433 \\
5815.822 \\
5816.168 \\
5816.984 \\
5817.300\end{array}$ & $\begin{array}{r}175 \\
3 \\
5 \\
6 \\
6\end{array}$ & $\begin{array}{r}300 \\
12 \\
5\end{array}$ & $\begin{array}{l}\text { II } \\
\text { I } \\
\text { I } \\
\text { II } \\
\text { II }\end{array}$ & $\begin{array}{l}5835.420 \\
5835.871 \\
5836.238 \\
5836.642 \\
5836.957\end{array}$ & $\begin{array}{r}1 \\
10 \\
10 \\
2 \\
2\end{array}$ & $\begin{array}{r}18 \\
2\end{array}$ & $\begin{array}{l}\text { I } \\
\text { I I } \\
\text { I } \\
\text { II } \\
\text { I }\end{array}$ & $\begin{array}{l}5856.090 \\
5856.209 \\
5856.359 \\
5857.571 \\
5857.690\end{array}$ & $\begin{array}{r}4 \\
4 \\
12 \\
1 \\
1\end{array}$ & $\begin{array}{r}20 \\
1\end{array}$ & $\begin{array}{l}\text { I } \\
\text { I } \\
\text { II } \\
\text { II } \\
\text { I }\end{array}$ \\
\hline $\begin{array}{l}5817.527 \\
5817.733 \\
5818.446 \\
5819.118 \\
5819.312\end{array}$ & $\begin{array}{c}1 \\
151 \\
50 \\
7 \\
9\end{array}$ & $\begin{array}{r}20 \\
2\end{array}$ & $\begin{array}{l}\text { I } \\
\text { II } \\
\text { I } \\
\text { I } \\
\text { I }\end{array}$ & $\begin{array}{l}5837.500 \\
5838.281 \\
5838.545 \\
5838.939 \\
5839.494\end{array}$ & $\begin{array}{r}6 \\
2 \\
1 \\
50 \\
40\end{array}$ & $\begin{array}{r}12 \\
\\
125 \\
2\end{array}$ & $\begin{array}{l}\text { II } \\
\text { I } \\
\text { I } \\
\text { II } \\
\text { I }\end{array}$ & $\begin{array}{l}5858.639 \\
5858.750 \\
5859.544 \\
5859.669 \\
5860.248\end{array}$ & $\begin{array}{r}40 \\
50 \\
30 \\
140 \\
3\end{array}$ & $\begin{array}{r}1 \\
300\end{array}$ & $\begin{array}{l}\text { I } \\
\text { I } \\
\text { I } \\
\text { I I } \\
\text { I }\end{array}$ \\
\hline $\begin{array}{l}5819.601 \\
5819.902 \\
5820.079 \\
5820.167 \\
5820.370\end{array}$ & $\begin{array}{r}30 \\
5 \\
7 \\
7 \\
30\end{array}$ & $\begin{array}{r}18 \\
125\end{array}$ & $\begin{array}{l}\text { I } \\
\text { I } \\
\text { I } \\
\text { II } \\
\text { II }\end{array}$ & $\begin{array}{l}5839.589 \\
5839.844 \\
5840.632 \\
5841.204 \\
5842.043\end{array}$ & $\begin{array}{r}10 \\
30 \\
75 \\
1 \\
40\end{array}$ & 2 & $\begin{array}{l}\text { I } \\
\text { I } \\
\text { I } \\
\text { I } \\
\text { I }\end{array}$ & $\begin{array}{l}5860.468 \\
5860.963 \\
5861.184 \\
5861.292 \\
5861.983\end{array}$ & $\begin{array}{l}5 \\
1 \\
2 \\
4 \\
1 \mathrm{~h}\end{array}$ & & $\begin{array}{l}\text { I } \\
\text { I } \\
\text { I } \\
\text { I } \\
\text { I }\end{array}$ \\
\hline $\begin{array}{l}5820.793 \\
5822.034 \\
5822.514 \\
5822.792 \\
5823.214\end{array}$ & $\begin{array}{r}2 \\
9 \\
6 \\
75 \\
1\end{array}$ & $\begin{array}{r}1 \\
15 \\
2\end{array}$ & $\begin{array}{l}\text { I } \\
\text { I } \\
\text { II } \\
\text { I } \\
\text { I }\end{array}$ & $\begin{array}{l}5842.352 \\
5842.464 \\
5842.836 \\
5843.001 \\
5843.252\end{array}$ & $\begin{array}{l}1 \mathrm{~h} \\
1 \\
2 \\
2 \\
5\end{array}$ & 350 & $\begin{array}{l}\text { I } \\
\text { I } \\
\text { III } \\
\text { I }\end{array}$ & $\begin{array}{l}5862.124 \\
5863.716 \\
5864.122 \\
5864.870 \\
5866.804\end{array}$ & $\begin{array}{r}2 \\
125 \\
1 \\
7 \\
75\end{array}$ & $\begin{array}{r}3 \\
18 \\
1\end{array}$ & $\begin{array}{l}\text { I } \\
\text { I } \\
\text { I } \\
\text { II } \\
\text { I }\end{array}$ \\
\hline $\begin{array}{l}5823.558 \\
5824.521 \\
5824.887 \\
5825.000 \\
5825.341\end{array}$ & $\begin{array}{l}1 \\
1 \mathrm{w} \\
50 \\
30 \\
1\end{array}$ & 3 & $\begin{array}{l}\text { I } \\
\text { II } \\
\text { I } \\
\text { I } \\
\text { I }\end{array}$ & $\begin{array}{l}5843.799 \\
5844.778 \\
5845.021 \\
5845.916 \\
5846.976\end{array}$ & $\begin{array}{r}75 \\
30 \\
1 \\
50 \\
10\end{array}$ & 2 & $\begin{array}{l}\text { I } \\
\text { I } \\
\text { I } \\
\text { I } \\
\text { I }\end{array}$ & $\begin{array}{l}5867.338 \\
5867.608 \\
5867.831 \\
5868.376 \\
5869.153\end{array}$ & $\begin{array}{r}4 \\
5 \\
5 \\
125 \\
6\end{array}$ & $\begin{array}{l}8 \\
8 \\
8\end{array}$ & $\begin{array}{l}\text { II } \\
\text { I I } \\
\text { I } \\
\text { I } \\
\text { I }\end{array}$ \\
\hline $\begin{array}{l}5825.466 \\
5825.667 \\
5826.084 \\
5826.271 \\
5826.706\end{array}$ & $\begin{array}{r}2 \\
3 \\
7 \\
100 \\
3\end{array}$ & 4 & $\begin{array}{l}\text { I } \\
I \\
I \\
I \\
I\end{array}$ & $\begin{array}{l}5847.928 \\
5848.542 \\
5849.064 \\
5849.204 \\
5849.496\end{array}$ & $\begin{array}{r}1 \\
10 \\
6 \\
4 \\
4\end{array}$ & $\begin{array}{r}1 \\
20\end{array}$ & $\begin{array}{l}\text { I } \\
\text { I } \\
\text { II } \\
\text { I } \\
\text { I }\end{array}$ & $\begin{array}{l}5869.847 \\
5870.036 \\
5870.543 \\
5871.178 \\
5871.414\end{array}$ & $\begin{array}{r}100 \\
10 \\
125 \\
100 \\
5\end{array}$ & $\begin{array}{r}2 \\
350 \\
2 \\
7\end{array}$ & $\begin{array}{l}\text { I } \\
\text { I } \\
\text { II } \\
\text { I } \\
\text { I I }\end{array}$ \\
\hline $\begin{array}{l}5827.338 \\
5827.724 \\
5828.071 \\
5828.803 \\
5829.105\end{array}$ & $\begin{array}{r}3 \\
1 \\
2 \\
1 \\
20\end{array}$ & $\begin{array}{l}2 \\
2 w \\
2\end{array}$ & $\begin{array}{l}\text { I } \\
\text { II } \\
\text { I } \\
\text { II } \\
\text { I }\end{array}$ & $\begin{array}{l}5849.750 \\
5849.994 \\
5850.078 \\
5850.254 \\
5850.356\end{array}$ & $\begin{array}{l}3 \\
1 \\
5 \\
1 \\
2\end{array}$ & $\begin{array}{l}8 \\
1 \\
1\end{array}$ & $\begin{array}{l}\text { I } \\
\text { II } \\
\text { I } \\
\text { I } \\
\text { I }\end{array}$ & $\begin{array}{l}5872.299 \\
5872.608 \\
5872.884 \\
5873.316 \\
5873.748\end{array}$ & $\begin{array}{r}5 \\
40 \\
40 \\
3 \\
2\end{array}$ & $\begin{array}{r}8 \\
100\end{array}$ & $\begin{array}{l}\text { II } \\
\text { II } \\
\text { I } \\
\text { I } \\
\text { I }\end{array}$ \\
\hline $\begin{array}{l}5829.846 \\
5830.492 \\
5830.816 \\
5831.114 \\
5831.263\end{array}$ & $\begin{array}{r}40 \\
6 \\
50 \\
1 \\
3\end{array}$ & $\begin{array}{r}2 \\
50 \\
1 \\
12\end{array}$ & $\begin{array}{l}\text { I } \\
\text { II } \\
\text { I } \\
\text { I } \\
\text { II }\end{array}$ & $\begin{array}{l}5850.736 \\
5851.192 \\
5851.383 \\
5851.616 \\
5851.828\end{array}$ & $\begin{array}{l}5 \\
2 \\
1 \\
1 \\
1\end{array}$ & 2 & $\begin{array}{l}\text { I } \\
\text { I } \\
\text { I } \\
\text { I } \\
\text { II }\end{array}$ & $\begin{array}{l}5874.032 \\
5874.336 \\
5874.981 \\
5875.272 \\
5875.497\end{array}$ & $\begin{array}{r}30 \\
30 \\
50 \\
4 \\
10\end{array}$ & $\begin{array}{r}10 \\
3 \\
2 \\
75\end{array}$ & $\begin{array}{l}\text { I } \\
\text { II } \\
\text { II } \\
\text { II } \\
\text { II }\end{array}$ \\
\hline $\begin{array}{l}5831.452 \\
5831.673 \\
5831.911 \\
5832.048 \\
5832.368\end{array}$ & $\begin{array}{c}1 \\
31 \\
1 \\
2 \\
125\end{array}$ & $\begin{array}{r}1 \\
50\end{array}$ & $\begin{array}{l}I \\
I \\
I \\
I \\
I\end{array}$ & $\begin{array}{l}5852.173 \\
5852.484 \\
5852.682 \mathrm{i} \\
5853.056 \\
5853.466\end{array}$ & $\begin{array}{r}9 \\
40 \\
200 \\
20 \\
50\end{array}$ & $\begin{array}{r}18 \\
1 \\
5 \\
50\end{array}$ & $\begin{array}{l}\text { II } \\
\text { I } \\
\text { I } \\
\text { II } \\
\text { I }\end{array}$ & $\begin{array}{l}5876.176 \\
5876.428 \\
5876.655 \\
5876.990 \\
5877.527\end{array}$ & $\begin{array}{l}1 \\
1 \\
1 \\
5 \\
1\end{array}$ & $2 w$ & $\begin{array}{l}\text { II } \\
\text { I } \\
\text { I } \\
\text { I } \\
\text { I }\end{array}$ \\
\hline
\end{tabular}


Table 1. Wavelengths of Thorium Spectra - Continued

\begin{tabular}{|c|c|c|c|c|c|c|c|c|c|c|c|}
\hline \multirow{2}{*}{$\begin{array}{l}\text { Wave- } \\
\text { length }\end{array}$} & \multicolumn{2}{|c|}{ Intensity } & \multirow{2}{*}{$\begin{array}{l}\text { Spec- } \\
\text { trum }\end{array}$} & \multirow{2}{*}{$\begin{array}{l}\text { Wave- } \\
\text { length }\end{array}$} & \multicolumn{2}{|c|}{ Intensity } & \multirow{2}{*}{$\begin{array}{l}\text { Spec- } \\
\text { trum }\end{array}$} & \multirow{2}{*}{$\begin{array}{l}\text { Wave- } \\
\text { length }\end{array}$} & \multicolumn{2}{|c|}{ Intensity } & \multirow{2}{*}{$\begin{array}{l}\text { Spec- } \\
\text { trum }\end{array}$} \\
\hline & $a m p$ & Spark & & & amp & Spark & & & Lamp & Spark & \\
\hline $\begin{array}{l}5877.667 \\
5878.269 \\
5878.922 \\
5879.104 \\
5879.450\end{array}$ & $\begin{array}{r}1 \\
30 \\
12 \\
25 \\
2\end{array}$ & $\begin{array}{r}1 \\
100 \\
70 \\
2\end{array}$ & $\begin{array}{l}\text { II } \\
\text { II } \\
\text { I } \\
\text { II } \\
\text { II }\end{array}$ & $\begin{array}{l}5904.161 \\
5904.709 \\
5905.178 \\
5905.576 \\
5905.746\end{array}$ & $\begin{array}{r}40 \\
2 \\
20 \\
100 \\
30\end{array}$ & 125 & $\begin{array}{l}\text { II } \\
\text { I } \\
\text { I } \\
\text { I } \\
\text { I }\end{array}$ & $\begin{array}{l}5928.862 \\
5929.159 \\
5929.487 \\
5929.942 \\
5930.293\end{array}$ & $\begin{array}{r}10 \\
3 \\
50 \\
60 \\
3\end{array}$ & 50 & $\begin{array}{l}\text { I } \\
\text { I } \\
\text { II } \\
\text { I } \\
\text { I }\end{array}$ \\
\hline $\begin{array}{l}5879.707 \\
5881.409 \\
5881.971 \\
5882.274 \\
5882.440\end{array}$ & $\begin{array}{r}10 \\
5 \\
1 \\
4 \\
1\end{array}$ & $\begin{array}{r}50 \\
8\end{array}$ & $\begin{array}{l}\text { II } \\
\text { I I } \\
\text { I } \\
\text { I } \\
\text { I }\end{array}$ & $\begin{array}{l}5906.323 \\
5906.848 \\
5907.089 \\
5907.344 \\
5907.824\end{array}$ & $\begin{array}{r}20 \\
3 \\
1 \\
1 \\
1\end{array}$ & $\begin{array}{c}10 \\
50 \\
1\end{array}$ & $\begin{array}{l}\text { I I } \\
\text { II } \\
\text { I } \\
\text { I } \\
\text { I }\end{array}$ & $\begin{array}{l}5930.444 \\
5930.963 \\
5931.057 \\
5932.155 \\
5932.424\end{array}$ & $\begin{array}{l}2 \\
3 \\
2 \\
25 d \\
20\end{array}$ & $\begin{array}{r}10 \\
1 \\
15\end{array}$ & $\begin{array}{l}\text { I } \\
\text { II } \\
\text { I I } \\
\text { II } \\
\text { I }\end{array}$ \\
\hline $\begin{array}{l}5882.682 \\
5882.974 \\
5883.520 \\
5883.841 \\
5884.031\end{array}$ & $\begin{array}{r}1 \\
1 \\
2 \\
2 \\
10\end{array}$ & 600 & $\begin{array}{l}\text { I } \\
\text { I } \\
\text { I I I } \\
\text { I } \\
\text { I }\end{array}$ & $\begin{array}{l}5908.397 \\
5908.939 \\
5909.365 \\
5909.592 \\
5910.034\end{array}$ & $\begin{array}{r}25 \\
125 \\
1 \\
1 \\
1 \text { s }\end{array}$ & $\begin{array}{l}50 \\
10\end{array}$ & $\begin{array}{l}\text { I } \\
\text { I } \\
\text { II } \\
\text { I } \\
\text { I }\end{array}$ & $\begin{array}{l}5932.698 \\
5932.907 \\
5933.401 \\
5933.843 \\
5934.429\end{array}$ & $\begin{array}{r}1 \\
3 \\
4 \\
3 \\
50\end{array}$ & $\begin{array}{l}3 \\
3 \\
1 \\
2\end{array}$ & $\begin{array}{l}\text { I I } \\
\text { I } \\
\text { I I } \\
\text { I I } \\
\text { I }\end{array}$ \\
\hline $\begin{array}{l}5884.153 \\
5885.702 \mathrm{i} \\
5886.362 \\
5886.526 \\
5886.716\end{array}$ & $\begin{array}{r}10 \\
120 \\
2 \\
50 \\
30\end{array}$ & $\begin{array}{l}20 \\
25 \\
20 \\
75\end{array}$ & $\begin{array}{l}\text { I I } \\
\text { I } \\
\text { I I } \\
\text { I } \\
\text { I I }\end{array}$ & $\begin{array}{l}5910.227 \\
5910.507 \\
5911.233 \\
5912.095 \\
5912.351\end{array}$ & $\begin{array}{r}5 \\
6 \\
60 \\
10 \\
1\end{array}$ & $\begin{array}{l}100 \\
18 \mathrm{~s}\end{array}$ & $\begin{array}{l}\text { I } \\
\text { II } \\
\text { I } \\
\text { I I } \\
\text { I }\end{array}$ & $\begin{array}{l}5934.708 \\
5935.715 \\
5935.840 \\
5936.175 \\
5936.390\end{array}$ & $\begin{array}{r}6 \\
1 \\
1 \\
3 \\
25\end{array}$ & & $\begin{array}{l}\text { I } \\
\text { I } \\
\text { I } \\
\text { I } \\
\text { I }\end{array}$ \\
\hline $\begin{array}{l}5887.430 \\
5887.850 \\
5888.254 \\
5888.557 \\
5891.453\end{array}$ & $\begin{array}{l}21 \\
1 \\
15 \\
3 \\
70\end{array}$ & $\begin{array}{r}1 \\
3 \\
30 \\
18\end{array}$ & $\begin{array}{l}\text { II } \\
\text { I I } \\
\text { I I } \\
\text { I } \\
\text { I }\end{array}$ & $\begin{array}{l}5913.371 \\
5914.388 \\
5914.681 \\
5916.150 \\
5916.379\end{array}$ & $\begin{array}{r}50 \\
50 \\
140 \\
2 \\
2\end{array}$ & $\begin{array}{r}2 \\
400 \\
100 \\
5\end{array}$ & $\begin{array}{l}\text { I } \\
\text { II } \\
\text { I } \\
\text { I } \\
\text { I I }\end{array}$ & $\begin{array}{l}5936.601 \\
5936.875 \\
5937.169 \\
5937.666 \\
5937.926\end{array}$ & $\begin{array}{l}3 \\
12 \\
40 \\
60 \\
12\end{array}$ & $\begin{array}{r}50 \\
2 \\
2\end{array}$ & $\begin{array}{l}\text { II } \\
\text { I } \\
\text { I } \\
\text { I } \\
\text { I }\end{array}$ \\
\hline $\begin{array}{l}5892.430 \\
5892.787 \\
5893.179 \\
5893.466 \\
5894.700\end{array}$ & $\begin{array}{r}12 \\
1 \\
7 \\
25 \\
40\end{array}$ & $\begin{array}{r}125 \\
40\end{array}$ & $\begin{array}{l}\text { I } \\
\text { I } \\
\text { I } \\
\text { I I } \\
\text { I I }\end{array}$ & $\begin{array}{l}5916.732 \\
5917.270 \\
5917.942 \\
5918.181 \\
5918.944\end{array}$ & $\begin{array}{r}50 \\
4 \\
2 \\
5 \\
75\end{array}$ & $\begin{array}{l}3 \\
2 \mathrm{~h} \\
2\end{array}$ & $\begin{array}{l}\text { I } \\
\text { I } \\
\text { II } \\
\text { I I } \\
\text { I }\end{array}$ & $\begin{array}{l}5938,473 \\
5938.600 \\
5938.826 \mathrm{i} \\
5939.816 \\
5940.572\end{array}$ & $\begin{array}{r}75 \\
140 \\
10 \\
30\end{array}$ & $\begin{array}{l}50 \\
20\end{array}$ & $\begin{array}{l}\text { I } \\
\text { II } \\
\text { I } \\
\text { I } \\
\text { I }\end{array}$ \\
\hline $\begin{array}{l}5895.298 \\
5896.368 \\
5896.634 \\
5896.787 \\
5897.103\end{array}$ & $\begin{array}{r}75 \\
25 \\
8 \\
12 \\
1\end{array}$ & $\begin{array}{l}12 \\
75\end{array}$ & $\begin{array}{l}\text { I } \\
\text { I I } \\
\text { I } \\
\text { I } \\
\text { I }\end{array}$ & $\begin{array}{l}5919.662 \\
5920.363 \\
5920.967 \\
5921.412 \\
5921.842\end{array}$ & $\begin{array}{l}1 \\
2 \\
6 \\
1 w \\
4\end{array}$ & $\begin{array}{l}2 \\
1 \\
3\end{array}$ & $\begin{array}{l}\text { I I } \\
\text { I } \\
\text { I I } \\
\text { I } \\
\text { I I }\end{array}$ & $\begin{array}{l}5940.960 \\
5941.117 \\
5941.808 \\
5942.126 \\
5942.364\end{array}$ & $\begin{array}{r}15 \\
2 \\
10 \\
8 \\
40\end{array}$ & 75 & $\begin{array}{l}\text { I } \\
\text { I } \\
\text { I } \\
\text { I I } \\
\text { I }\end{array}$ \\
\hline $\begin{array}{l}5897.418 \\
5897.934 \\
5898.532 \\
5899.404 \\
5899.520\end{array}$ & $\begin{array}{l}2 \\
1 \mathrm{w} \\
3 \\
30 \\
25\end{array}$ & 75 & $\begin{array}{l}\text { I } \\
\text { I } \\
\text { I } \\
\text { I I } \\
\text { I }\end{array}$ & $\begin{array}{l}5922.825 \\
5923.921 \\
5924.055 \\
5924.392 \\
5924.517\end{array}$ & $\begin{array}{r}50 \\
3 \\
3 \\
30 \\
10\end{array}$ & 400 & $\begin{array}{l}\text { I } \\
\text { I } \\
\text { I } \\
\text { I I } \\
\text { I }\end{array}$ & $\begin{array}{l}5942.793 \\
5943.479 \\
5943.727 \\
5944.069 \\
5944.656\end{array}$ & $\begin{array}{r}30 \\
20 \\
7 \\
10 \\
75\end{array}$ & $\begin{array}{r}75 \\
2 \\
10 \\
50 \\
10\end{array}$ & $\begin{array}{l}\text { I I } \\
\text { I } \\
\text { II } \\
\text { I I } \\
\text { I I }\end{array}$ \\
\hline $\begin{array}{l}5899.843 \\
5900.296 \\
5900.826 \\
5901.620 \\
5901.777\end{array}$ & $\begin{array}{l}75 \\
1 \mathrm{w} \\
4 \\
1 \\
2\end{array}$ & $\begin{array}{l}3 \\
1 \\
2 \mathrm{w} \\
3\end{array}$ & $\begin{array}{l}\text { I } \\
\text { I } \\
\text { I I } \\
\text { I } \\
\text { I I }\end{array}$ & $\begin{array}{l}5924.783 \\
5925.404 \\
5925.894 \\
5926.240 \\
5926.659\end{array}$ & $\begin{array}{r}1 \\
50 \\
100 \\
100 \\
1\end{array}$ & $\begin{array}{r}300 \\
3\end{array}$ & $\begin{array}{l}\text { I } \\
\text { I } \\
\text { II } \\
\text { I } \\
\text { I }\end{array}$ & $\begin{array}{l}5945.471 \\
5945.615 \\
5946.240 \\
5946.839 \\
5947.338\end{array}$ & $\begin{array}{r}2 \\
2 \\
20 \\
2 \\
1\end{array}$ & $\begin{array}{c}10 \\
1\end{array}$ & $\begin{array}{l}\text { I } \\
\text { II } \\
\text { I } \\
\text { II } \\
\text { I }\end{array}$ \\
\hline $\begin{array}{l}5901.968 \\
5902.596 \\
5903.346 \\
5903.493 \\
5903.916\end{array}$ & $\begin{array}{r}15 \\
30 \\
3 \\
5 \\
3\end{array}$ & $\begin{array}{r}50 \\
8 \\
1\end{array}$ & $\begin{array}{l}\text { II } \\
\text { I I } \\
\text { I I } \\
\text { I } \\
\text { I }\end{array}$ & $\begin{array}{l}5926.763 \\
5926.928 \\
5927.944 \\
5928.246 \\
5928.655\end{array}$ & $\begin{array}{l}1 \\
4 \\
3 \\
3 \\
3\end{array}$ & $\begin{array}{r}2 \\
3 \\
25\end{array}$ & $\begin{array}{l}\text { I I } \\
\text { I I } \\
\text { I I } \\
\text { I } \\
\text { I }\end{array}$ & $\begin{array}{l}5947.482 \\
5948.034 \\
5948.395 \\
5948.578 \\
5948.810\end{array}$ & $\begin{array}{r}1 \\
1 \\
3 \\
1 \\
20\end{array}$ & $\begin{array}{l}1 \\
2 \\
3 \\
2\end{array}$ & $\begin{array}{l}\text { I I } \\
\text { I I } \\
\text { I } \\
\text { I I } \\
\text { I }\end{array}$ \\
\hline
\end{tabular}


Table 1. Wavelengths of Thorium Spectra - Continued

\begin{tabular}{|c|c|c|c|c|c|c|c|c|c|c|c|}
\hline \multirow{2}{*}{$\begin{array}{l}\text { Wave- } \\
\text { length }\end{array}$} & \multicolumn{2}{|c|}{ Intensity } & \multirow{2}{*}{$\begin{array}{l}\text { Spec- } \\
\text { trum }\end{array}$} & \multirow{2}{*}{$\begin{array}{l}\text { Wave- } \\
\text { length }\end{array}$} & \multicolumn{2}{|c|}{ Intensity } & \multirow{2}{*}{$\begin{array}{l}\text { Spec- } \\
\text { trum }\end{array}$} & \multirow{2}{*}{$\begin{array}{l}\text { Wave- } \\
\text { length }\end{array}$} & \multicolumn{2}{|c|}{ Intensity } & \multirow{2}{*}{$\begin{array}{l}\text { Spec } \\
\text { trum }\end{array}$} \\
\hline & Lamp & Spark & & & Lamp & Spark & & & Lamp & Spark & \\
\hline $\begin{array}{l}5949.376 \\
5949.679 \\
5950.156 \\
5950.434 \\
5950.642\end{array}$ & $\begin{array}{r}30 \\
1 \\
2 \\
7 \\
3\end{array}$ & $\begin{array}{l}1 \\
1 \mathrm{w} \\
3\end{array}$ & $\begin{array}{l}\text { I } \\
\text { II } \\
\text { I } \\
\text { I } \\
\text { I }\end{array}$ & $\begin{array}{l}5966.209 \\
5967.531 \\
5969.098 \\
5969.754 \\
5970.458\end{array}$ & $\begin{array}{l}2 \\
10 \\
1 \mathrm{w} \\
50 \\
12\end{array}$ & $\begin{array}{l}1 \\
1 \\
3 \\
1\end{array}$ & $\begin{array}{l}\text { I } \\
\text { I } \\
\text { I } \\
\text { I } \\
\text { I }\end{array}$ & $\begin{array}{l}5991.032 \\
5991.717 \\
5992.243 \\
5993.272 \\
5993.516\end{array}$ & $\begin{array}{r}120 \\
2 \\
1 \\
6 \\
10\end{array}$ & $\begin{array}{r}15 \\
3 \\
25\end{array}$ & $\begin{array}{l}\text { I } \\
\text { II } \\
\text { I } \\
\text { II } \\
\text { I }\end{array}$ \\
\hline $\begin{array}{l}5950.778 \\
5951.108 \\
5951.314 \\
5951.508 \\
5951.855\end{array}$ & $\begin{array}{r}20 \\
1 \\
1 \\
1 \\
1\end{array}$ & 3 & $\begin{array}{l}\text { I } \\
\text { I } \\
\text { I } \\
\text { I } \\
\text { I }\end{array}$ & $\begin{array}{l}5970.945 \\
5971.088 \\
5971.370 \\
5971.756 \\
5971.844\end{array}$ & $\begin{array}{l}1 \\
1 \\
1 \\
1 \\
1\end{array}$ & $\begin{array}{l}3 \\
1\end{array}$ & $\begin{array}{l}\text { II } \\
\text { I } \\
\text { II } \\
\text { I } \\
\text { I }\end{array}$ & $\begin{array}{l}5994.156 \\
5995.234 \\
5995.690 \\
5996.100 \\
5996.646\end{array}$ & $\begin{array}{r}200 \\
20 \\
4 \\
5 \\
25\end{array}$ & $\begin{array}{r}20 \\
2 \\
1 \\
1\end{array}$ & $\begin{array}{l}\text { I } \\
\text { I } \\
\text { I } \\
\text { II } \\
\text { I }\end{array}$ \\
\hline $\begin{array}{l}5952.144 \\
5952.342 \\
5952.623 \\
5952.819 \\
5953.262\end{array}$ & $\begin{array}{l}1 \\
3 \\
1 \\
1 \\
6\end{array}$ & $\begin{array}{r}15 \\
2\end{array}$ & $\begin{array}{l}\text { I } \\
\text { II } \\
\text { I } \\
\text { II } \\
\text { I }\end{array}$ & $\begin{array}{l}5972.470 \\
5972.820 \\
5973.665 \mathrm{i} \\
5975.066 \mathrm{i} \\
5975.400\end{array}$ & $\begin{array}{r}10 \\
7 \\
250 \\
250\end{array}$ & $\begin{array}{l}18 \\
75 \\
25 \\
25\end{array}$ & $\begin{array}{l}\text { II } \\
\text { II } \\
\text { I } \\
\text { I } \\
\text { I I I }\end{array}$ & $\begin{array}{l}5997.036 \\
5997.156 \\
5997.349 \\
5997.641 \\
5998.210\end{array}$ & $\begin{array}{l}1 \\
1 \\
1 \\
1 \\
1\end{array}$ & 3 & $\begin{array}{l}\text { I } \\
\text { II } \\
\text { I } \\
\text { I } \\
\text { I }\end{array}$ \\
\hline $\begin{array}{l}5953.594 \\
5954.590 \\
5954.762 \\
5954.889 \\
5955.150\end{array}$ & $\begin{array}{r}25 \\
10 \\
3 \\
4 \\
2\end{array}$ & $\begin{array}{l}2 \\
2 \\
1 \\
1\end{array}$ & $\begin{array}{l}\text { I } \\
\text { I I } \\
\text { I } \\
I \\
\text { I }\end{array}$ & $\begin{array}{l}5976.109 \\
5976.220 \\
5976.445 \\
5976.803 \\
5977.089\end{array}$ & $\begin{array}{r}12 \\
3 \\
1 \\
1 \\
2\end{array}$ & $\begin{array}{l}3 \\
2\end{array}$ & $\begin{array}{l}\text { II } \\
\text { I } \\
\text { II } \\
\text { I } \\
\text { I }\end{array}$ & $\begin{array}{l}5998.672 \\
5999.274 \\
5999.539 \\
5999.915 \\
6000.791\end{array}$ & $\begin{array}{r}6 \\
1 \\
6 \\
2 \\
40\end{array}$ & 12 & $\begin{array}{l}\text { II } \\
\text { I } \\
\text { I } \\
\text { II } \\
\text { I }\end{array}$ \\
\hline $\begin{array}{l}5955.580 \\
5955.960 \\
5956.277 \\
5957.108 \\
5957.493\end{array}$ & $\begin{array}{r}50 \\
6 \\
30 \\
4 \\
6\end{array}$ & $\begin{array}{r}3 \\
30 \\
30\end{array}$ & $\begin{array}{l}\text { I } \\
\text { I I } \\
\text { I } \\
\text { II } \\
\text { I }\end{array}$ & $\begin{array}{l}5977.220 \\
5977.560 \\
5978.095 \\
5978.432 \\
5978.920\end{array}$ & $\begin{array}{l}1 \\
3 \\
7 \\
1 \\
2\end{array}$ & $\begin{array}{l}4 w \\
30\end{array}$ & $\begin{array}{l}\text { I I } \\
\text { I } \\
\text { II } \\
\text { I } \\
\text { I }\end{array}$ & $\begin{array}{l}6000.950 \\
6001.224 \\
6001.658 \\
6001.739 \\
6001.922\end{array}$ & $\begin{array}{r}3 \\
60 \\
3 \\
4 \\
1\end{array}$ & $\begin{array}{r}25 \\
4 \\
1\end{array}$ & $\begin{array}{l}\text { II } \\
\text { I } \\
\text { I } \\
\text { II } \\
\text { I }\end{array}$ \\
\hline $\begin{array}{l}5957.611 \\
5958.084 \\
5958.446 \\
5958.773 \\
5959.712\end{array}$ & $\begin{array}{r}30 \\
4 \\
2 \\
10 \\
40\end{array}$ & $\begin{array}{r}20 \\
1 \\
1 \\
2\end{array}$ & $\begin{array}{l}\text { I } \\
\text { II } \\
\text { I I } \\
\text { I } \\
\text { I }\end{array}$ & $\begin{array}{l}5979.195 \\
5979.326 \\
5979.632 \\
5980.574 \\
5981.048\end{array}$ & $\begin{array}{l}1 \\
1 \\
1 \\
1 \\
1\end{array}$ & $\begin{array}{l}1 \\
1\end{array}$ & $\begin{array}{l}\text { I I } \\
\text { I } \\
\text { I I } \\
\text { I } \\
\text { I }\end{array}$ & $\begin{array}{l}6002.198 \\
6002.374 \\
6002.474 \\
6002.896 \\
6003.166\end{array}$ & $\begin{array}{l}2 \\
3 \\
1 \\
2 \\
3\end{array}$ & 4 & $\begin{array}{l}\text { I } \\
\text { II } \\
\text { I } \\
\text { I } \\
\text { I }\end{array}$ \\
\hline $\begin{array}{l}5960.387 \\
5960.804 \\
5961.229 \\
5961.983 \\
5962.066\end{array}$ & $\begin{array}{r}20 \\
25 \\
1 \\
30 \\
20\end{array}$ & $\begin{array}{r}100 \\
1 \\
100\end{array}$ & $\begin{array}{l}\text { I I } \\
\text { I } \\
\text { I } \\
\text { I I } \\
\text { I }\end{array}$ & $\begin{array}{l}5981.397 \\
5981.908 \\
5982.118 \\
5982.670 \\
5983.169\end{array}$ & $\begin{array}{l}2 \\
20 \\
40 \\
2 w \\
1\end{array}$ & $\begin{array}{r}10 \\
40 \\
2\end{array}$ & $\begin{array}{l}\text { I I } \\
\text { I I } \\
\text { I } \\
\text { II } \\
\text { I }\end{array}$ & $\begin{array}{l}6003.599 \\
6003.937 \\
6004.254 \\
6004.450 \\
6005.184\end{array}$ & $\begin{array}{l}1 \\
1 \\
1 \\
1 \mathrm{~h} \\
50\end{array}$ & $\begin{array}{l}1 \\
3\end{array}$ & $\begin{array}{l}\text { I } \\
\text { I } \\
\text { I } \\
\text { I I } \\
\text { I }\end{array}$ \\
\hline $\begin{array}{l}5962.188 \\
5952.574 \\
5962.764 \\
5962.991 \\
5963.184\end{array}$ & $\begin{array}{r}20 \\
1 \\
1 \\
1 \\
1\end{array}$ & 40 & $\begin{array}{l}\text { I I } \\
\text { I } \\
\text { I } \\
\text { I } \\
\text { I }\end{array}$ & $\begin{array}{l}5983.403 \\
5984.009 \\
5984.996 \\
5985.211 \\
5985.688\end{array}$ & $\begin{array}{l}6 \\
6 \\
2 \\
5 \\
4\end{array}$ & $\begin{array}{l}15 \\
50 \\
12\end{array}$ & $\begin{array}{l}\text { II } \\
\text { II } \\
\text { I } \\
\text { II }\end{array}$ & $\begin{array}{l}6005.604 \\
6005.810 \\
6006.102 \\
6006.296 \\
6007.073 \mathrm{i}\end{array}$ & $\begin{array}{r}2 \\
2 \\
1 \\
1 \\
180\end{array}$ & $\begin{array}{l}2 \\
1\end{array}$ & $\begin{array}{l}\text { I } \\
\text { II } \\
\text { I } \\
\text { II } \\
\text { I }\end{array}$ \\
\hline $\begin{array}{l}5963.322 \\
5963.664 \\
5963.956 \\
5963.999 \\
5964.498\end{array}$ & $\begin{array}{r}2 \\
2 \\
5 \\
5 \\
10\end{array}$ & $\begin{array}{l}1 \\
1\end{array}$ & $\begin{array}{l}\text { I } \\
\text { I } \\
\text { I } \\
I \\
I\end{array}$ & $\begin{array}{l}5986.276 \\
5986.966 \\
5987.474 \\
5988.006 \\
5988.302\end{array}$ & $\begin{array}{r}25 \\
2 \\
3 \\
2 \\
2\end{array}$ & 1 & $\begin{array}{l}\text { I } \\
\text { I } \\
\text { I } \\
\text { I } \\
\text { I }\end{array}$ & $\begin{array}{l}6007.502 \\
6008.049 \\
6008.442 \\
6009.123 \\
6010.176\end{array}$ & $\begin{array}{r}4 \\
1 \\
2 \\
5 \\
90\end{array}$ & $\begin{array}{r}15 \\
3\end{array}$ & $\begin{array}{l}\text { II } \\
\text { I } \\
\text { I } \\
\text { II } \\
\text { I }\end{array}$ \\
\hline $\begin{array}{l}5964.687 \\
5964.957 \\
5965.468 \\
5965.754 \\
5965.838\end{array}$ & $\begin{array}{l}1 \\
1 \\
1 \\
6 \\
8\end{array}$ & 1 & $\begin{array}{l}\text { I } \\
\text { I } \\
\text { I } \\
\text { I } \\
\text { I I }\end{array}$ & $\begin{array}{l}5989.072 \\
5989.754 \\
5989.895 \\
5990.285 \\
5990.801\end{array}$ & $\begin{array}{r}150 \\
10 \\
4 \\
6 \\
2\end{array}$ & $\begin{array}{r}400 \\
18 \\
50 \\
20\end{array}$ & $\begin{array}{l}\text { I I } \\
\text { I } \\
\text { I I } \\
\text { I I } \\
\text { I I }\end{array}$ & $\begin{array}{l}6010.592 \\
6011.215 \\
6011.354 \\
6011.534 \\
6011.991\end{array}$ & $\begin{array}{r}7 \\
7 \\
10 \\
25 \\
1\end{array}$ & 75 & $\begin{array}{l}\text { I } \\
\text { I } \\
\text { II } \\
\text { I } \\
\text { I }\end{array}$ \\
\hline
\end{tabular}


Table 1. Wavelengths of Thorium Spectra - Continued

\begin{tabular}{|c|c|c|c|c|c|c|c|c|c|c|c|}
\hline \multirow{2}{*}{$\begin{array}{l}\text { Wave- } \\
\text { length }\end{array}$} & \multicolumn{2}{|c|}{ Intensity } & \multirow{2}{*}{$\begin{array}{l}\text { Spec- } \\
\text { trum }\end{array}$} & \multirow{2}{*}{$\begin{array}{l}\text { Wave- } \\
\text { length }\end{array}$} & \multicolumn{2}{|c|}{ Intensity } & \multirow{2}{*}{$\begin{array}{l}\text { Spec- } \\
\text { trum }\end{array}$} & \multirow{2}{*}{$\begin{array}{l}\text { Wave- } \\
\text { length }\end{array}$} & \multicolumn{2}{|c|}{ Intensity } & \multirow{2}{*}{$\begin{array}{l}\text { Spec- } \\
\text { trum }\end{array}$} \\
\hline & Lamp & Spark & & & Lamp & Spark & & & Lamp & Spark & \\
\hline $\begin{array}{l}6012.343 \\
6012.749 \\
6013.427 \\
6014.058 \\
6014.304\end{array}$ & $\begin{array}{l}6 \\
1 \\
2 \\
6 \\
3\end{array}$ & $\begin{array}{l}1 \\
1 \mathrm{w} \\
1 \\
1\end{array}$ & $\begin{array}{l}\text { II } \\
\text { II } \\
\text { I } \\
\text { II } \\
\text { II }\end{array}$ & $\begin{array}{l}6036.760 \\
6037.6981 \\
6038.677 \\
6039.214 \\
6039.647\end{array}$ & $\begin{array}{r}8 \\
140 \\
75 \\
6 \\
1\end{array}$ & $\begin{array}{r}10 \\
2 \\
1 \\
1\end{array}$ & $\begin{array}{l}\text { I } \\
\text { I } \\
\text { II } \\
\text { II }\end{array}$ & $\begin{array}{l}6063.048 \\
6065.348 \\
6065.765 \\
6066.487 \\
6067.063\end{array}$ & $\begin{array}{r}3 \\
2 \\
40 \\
30 \\
30\end{array}$ & $\begin{array}{r}12 \\
2 \\
300 \\
125\end{array}$ & $\begin{array}{l}\text { II } \\
\text { I } \\
\text { I } \\
\text { II } \\
\text { II }\end{array}$ \\
\hline $\begin{array}{l}6014.748 \\
6015.432 \\
6016.364 \\
6016.980 \\
6017.262\end{array}$ & $\begin{array}{r}1 \\
75 \\
12 \\
1 \\
2\end{array}$ & 300 & $\begin{array}{l}\text { I } \\
\text { II } \\
\text { I } \\
\text { I } \\
\text { II }\end{array}$ & $\begin{array}{l}6039.911 \\
6040.129 \\
6040.452 \\
6040.865 \\
6041.083\end{array}$ & $\begin{array}{l}1 \\
1 \\
3 \\
2 \\
1\end{array}$ & $10 \mathrm{w}$ & $\begin{array}{l}\text { II } \\
\text { II } \\
\text { I } \\
\text { I } \\
\text { I }\end{array}$ & $\begin{array}{l}6067.505 \\
6068.020 \\
6068.682 \\
6069.015 \\
6069.828\end{array}$ & $\begin{array}{r}1 \\
1 \\
1 \\
40 \\
4\end{array}$ & 2 & $\begin{array}{l}\text { I } \\
\text { II } \\
\text { I } \\
\text { I } \\
\text { I }\end{array}$ \\
\hline $\begin{array}{l}6017.864 \\
6019.005 \\
6019.640 \\
6019.818 \\
6020.266\end{array}$ & $\begin{array}{r}8 \\
30 \\
8 \\
2 \\
4\end{array}$ & $\begin{array}{r}2 \\
175\end{array}$ & $\begin{array}{l}\text { II } \\
\text { II } \\
\text { I } \\
\text { I } \\
\text { I }\end{array}$ & $\begin{array}{l}6041.353 \\
6041.607 \\
6042.583 \\
6042.967 \\
6043.648\end{array}$ & $\begin{array}{l}2 \\
2 \\
40 \\
40 \\
1 \mathrm{w}\end{array}$ & $\begin{array}{r}3 \\
1 \\
125\end{array}$ & $\begin{array}{l}I \\
I I \\
I \\
I I\end{array}$ & $\begin{array}{l}6070.020 \\
6070.315 \\
6070.872 \\
6071.535 \\
6071.968\end{array}$ & $\begin{array}{r}1 \\
10 \\
2 \\
3 \\
6\end{array}$ & $\begin{array}{l}2 \\
3 w \\
1 w\end{array}$ & $\begin{array}{l}\text { II } \\
\text { II } \\
\text { II } \\
\text { II }\end{array}$ \\
\hline $\begin{array}{l}6020.507 \\
6021.0361 \\
6021.415 \\
6022.200 \\
6022.360\end{array}$ & $\begin{array}{c}4 \\
140 \\
30 \\
2 \mathrm{w} \\
3\end{array}$ & $\begin{array}{c}12 \\
150 \\
12 \mathrm{w}\end{array}$ & $\begin{array}{l}\text { I } \\
\text { I } \\
\text { II } \\
\text { II } \\
\text { I }\end{array}$ & $\begin{array}{l}6044.421 \\
6045.274 \\
6045.452 \\
6046.606 \\
6046.991\end{array}$ & $\begin{array}{l}75 \\
1 \\
1 \\
8 \mathrm{w} \\
1\end{array}$ & $\begin{array}{r}200 \\
2\end{array}$ & $\begin{array}{l}\text { II } \\
\text { I } \\
\text { II } \\
\text { I } \\
\text { I }\end{array}$ & $\begin{array}{l}6072.788 \\
6073.093 \\
6073.504 \\
6073.982 \\
6075.081\end{array}$ & $\begin{array}{c}5 \\
50 \\
1 \\
2 \\
1 \mathrm{w}\end{array}$ & 100 & $\begin{array}{l}\text { I } \\
\text { I I } \\
\text { I } \\
\text { I } \\
\text { I }\end{array}$ \\
\hline $\begin{array}{l}6022.681 \\
6023.228 \\
6023.644 \\
6024.669 \\
6024.921\end{array}$ & $\begin{array}{r}2 \\
25 \\
1 \\
2 \\
2\end{array}$ & 2 & $\begin{array}{l}\text { I } \\
\text { I } \\
\text { I } \\
\text { I } \\
\text { I }\end{array}$ & $\begin{array}{l}6047.258 \\
6047.777 \\
6048.165 \\
6049.051 \mathrm{i} \\
6049.766\end{array}$ & $\begin{array}{r}1 \\
3 \\
1 \\
100 \\
8\end{array}$ & $\begin{array}{r}1 \\
8 \\
25\end{array}$ & $\begin{array}{l}\text { I } \\
\text { I } \\
\text { II } \\
\text { I } \\
\text { II }\end{array}$ & $\begin{array}{l}6075.392 \\
6076.188 \\
6077.118 \\
6077.340 \\
6077.532\end{array}$ & $\begin{array}{r}1 \\
2 \\
50 \\
4 \\
2\end{array}$ & $\begin{array}{r}4 \\
2 \\
15\end{array}$ & $\begin{array}{l}\text { I } \\
\text { I I } \\
\text { I } \\
\text { II } \\
\text { I }\end{array}$ \\
\hline $\begin{array}{l}6025.352 \\
6025.794 \\
6026.041 \\
6027.047 \\
6027.217\end{array}$ & $\begin{array}{l}1 \\
30 \\
6 \\
2 \mathrm{w} \\
1\end{array}$ & 1 & $\begin{array}{l}\text { I } \\
\text { I } \\
\text { I } \\
\text { I }\end{array}$ & $\begin{array}{l}6049.910 \\
6050.973 \\
6052.025 \\
6052.428 \\
6053.370\end{array}$ & $\begin{array}{r}3 \\
30 \\
4 \\
4 \\
300\end{array}$ & $\begin{array}{r}10 \\
2\end{array}$ & $\begin{array}{l}I \\
I \\
I \\
I\end{array}$ & $\begin{array}{l}6077.868 \\
6078.418 \\
6078.976 \\
6079.212 \\
6079.446\end{array}$ & $\begin{array}{r}75 \\
75 \\
4 \\
80 \\
20\end{array}$ & $\begin{array}{r}6 \\
3 \\
12 \\
3\end{array}$ & $\begin{array}{l}\text { I } \\
\text { I } \\
\text { I I } \\
\text { I } \\
\text { I }\end{array}$ \\
\hline $\begin{array}{l}6027.461 \\
6027.934 \\
6028.254 \\
6028.618 \\
6028.865\end{array}$ & $\begin{array}{r}1 \\
1 \\
25 \\
1 \\
6\end{array}$ & $\begin{array}{r}2 \\
2 \\
30 \\
15\end{array}$ & $\begin{array}{l}\text { II } \\
\text { II } \\
\text { II } \\
\text { I } \\
\text { II }\end{array}$ & $\begin{array}{l}6053.973 \\
6054.301 \\
6054.543 \\
6055.293 \\
6055.581\end{array}$ & $\begin{array}{r}1 \\
1 \\
1 \\
4 \\
20\end{array}$ & $\begin{array}{l}4 \\
2\end{array}$ & $\begin{array}{l}\text { I } \\
\text { I } \\
\text { I I } \\
\text { I } \\
\text { I }\end{array}$ & $\begin{array}{l}6080.537 \\
6080.885 \\
6081.723 \\
6083.225 \\
6083.512\end{array}$ & $\begin{array}{l}1 \\
2 \\
1 \\
4 \\
1\end{array}$ & 1 & $\begin{array}{l}I \\
I \\
I \\
I \\
I\end{array}$ \\
\hline $\begin{array}{l}6029.224 \\
6029.654 \\
6030.442 \\
6031.907 \\
6032.356\end{array}$ & $\begin{array}{r}30 \\
8 \\
50 \\
6 \\
50\end{array}$ & $\begin{array}{r}2 \\
40 \\
40\end{array}$ & $\begin{array}{l}\text { I } \\
\text { I } \\
\text { I } \\
\text { II } \\
\text { II }\end{array}$ & $\begin{array}{l}6055.922 \\
6056.338 \\
6056.862 \\
6057.153 \\
6057.484\end{array}$ & $\begin{array}{l}2 \\
1 \\
2 \\
2 \\
1\end{array}$ & $\begin{array}{l}1 \\
1\end{array}$ & $\begin{array}{l}\text { I } \\
\text { I } \\
\text { II } \\
\text { I } \\
\text { II }\end{array}$ & $\begin{array}{l}6084.102 \\
6084.486 \\
6085.260 \\
6085.375 \mathrm{i} \\
6085.948\end{array}$ & $\begin{array}{r}3 \\
1 \\
20 \\
100 \\
6\end{array}$ & $\begin{array}{r}75 \\
50 \\
5\end{array}$ & $\begin{array}{l}\text { I } \\
\text { I } \\
\text { II } \\
\text { I } \\
\text { II }\end{array}$ \\
\hline $\begin{array}{l}6032.864 \\
6033.408 \\
6033.941 \\
6034.195 \\
6034.534\end{array}$ & $\begin{array}{r}40 \\
40 \\
8 \\
1 \\
10\end{array}$ & $\begin{array}{r}2 \\
30 \\
25\end{array}$ & $\begin{array}{l}\text { I } \\
\text { I } \\
\text { II } \\
\text { I } \\
\text { II }\end{array}$ & $\begin{array}{l}6057.838 \\
6058.172 \\
6058.597 \\
6059.121 \\
6059.532\end{array}$ & $\begin{array}{r}1 \\
12 \\
7 \\
6 \\
1\end{array}$ & $\begin{array}{r}30 \\
25 \\
2\end{array}$ & $\begin{array}{l}\text { I } \\
\text { II } \\
\text { II } \\
\text { I } \\
\text { II }\end{array}$ & $\begin{array}{l}6086.446 \\
6087.253 \\
6088.031 \mathrm{i} \\
6088.805 \\
6089.082\end{array}$ & $\begin{array}{r}2 \\
75 \\
125 \\
6 \\
2\end{array}$ & $\begin{array}{r}400 \\
7\end{array}$ & $\begin{array}{l}\text { I } \\
\text { II } \\
\text { I } \\
\text { I } \\
\text { I }\end{array}$ \\
\hline $\begin{array}{l}6034.844 \\
6035.188 \\
6035.596 \\
6035.861 \\
6036.079\end{array}$ & $\begin{array}{r}2 \\
50 \\
1 \\
2 \\
1\end{array}$ & 2 & $\begin{array}{l}\text { I } \\
\text { I } \\
\text { I } \\
\text { I } \\
\text { I I }\end{array}$ & $\begin{array}{l}6059.840 \\
6059.941 \\
6060.959 \\
6061.499 \\
6062.592\end{array}$ & $\begin{array}{r}6 \\
1 \\
2 \\
25 \\
6\end{array}$ & $\begin{array}{r}4 \\
75 \\
20\end{array}$ & $\begin{array}{l}\text { I } \\
\text { II } \\
\text { I } \\
\text { I } \\
\text { II }\end{array}$ & $\begin{array}{l}6089.451 \\
6090.101 \\
6090.434 \\
6090.797 \\
6091.228\end{array}$ & $\begin{array}{r}1 \\
10 \\
3 \\
4 \\
1\end{array}$ & $\begin{array}{r}50 \\
8\end{array}$ & $\begin{array}{l}\text { I } \\
\text { I I } \\
\text { I } \\
\text { II } \\
\text { I }\end{array}$ \\
\hline
\end{tabular}


Tabie 1. Wavelengths of Thorium Spectra - Continued

\begin{tabular}{|c|c|c|c|c|c|c|c|c|c|c|c|}
\hline \multirow{2}{*}{$\begin{array}{l}\text { Wave- } \\
\text { length }\end{array}$} & \multicolumn{2}{|c|}{ Intensity } & \multirow{2}{*}{$\begin{array}{l}\text { Spec- } \\
\text { trum }\end{array}$} & \multirow{2}{*}{$\begin{array}{l}\text { Wave- } \\
\text { length }\end{array}$} & \multicolumn{2}{|c|}{ Intensity } & \multirow{2}{*}{$\begin{array}{l}\text { Spec- } \\
\text { trum }\end{array}$} & \multirow{2}{*}{$\begin{array}{l}\text { Wave- } \\
\text { length }\end{array}$} & \multicolumn{2}{|c|}{ Intensity } & \multirow{2}{*}{$\begin{array}{l}\text { Spec } \\
\text { trum }\end{array}$} \\
\hline & Lamp & Spark & & & Lamp & Spark & & & Lamp & Spark & \\
\hline $\begin{array}{l}6091.450 \\
6092.019 \\
6093.018 \\
6093.224 \\
6093.804\end{array}$ & $\begin{array}{l}1 \\
3 \\
3 \\
3 \\
2\end{array}$ & 6 & $\begin{array}{l}\text { I } \\
\text { II } \\
\text { I } \\
\text { I } \\
\text { I }\end{array}$ & $\begin{array}{l}6119.060 \\
6119.690 \\
6120.547 \\
6120.820 \\
6121.397\end{array}$ & $\begin{array}{r}2 \\
40 \\
100 \\
5 \\
75\end{array}$ & $\begin{array}{c}125 \mathrm{w} \\
350 \\
8\end{array}$ & $\begin{array}{l}\text { I I I } \\
\text { I } \\
\text { I I } \\
\text { I } \\
\text { I }\end{array}$ & $\begin{array}{l}6148.358 \\
6149.411 \\
6150.134 \\
6150.334 \\
6150.691\end{array}$ & $\begin{array}{r}1 \\
1 \\
1 \\
3 \\
20\end{array}$ & 2 & $\begin{array}{l}I \\
I I \\
I \\
I \\
I\end{array}$ \\
\hline $\begin{array}{l}6094.042 \\
6094.518 \\
6095.038 \\
6095.204 \\
6095.417\end{array}$ & $\begin{array}{l}3 \\
1 \\
3 \\
1 \\
1\end{array}$ & $\begin{array}{l}3 \\
1\end{array}$ & $\begin{array}{l}\text { I } \\
\text { I } \\
\text { II } \\
\text { I } \\
\text { I }\end{array}$ & $\begin{array}{l}6122.305 \\
6122.693 \\
6123.169 \\
6123.830 \\
6124.063\end{array}$ & $\begin{array}{l}4 \\
1 \mathrm{w} \\
1 \\
25 \\
5\end{array}$ & $\begin{array}{r}10 \\
1 \\
2\end{array}$ & $\begin{array}{l}\text { I I } \\
\text { I I } \\
\text { I I } \\
\text { I } \\
\text { I }\end{array}$ & $\begin{array}{l}6151.9931 \\
6152.930 \\
6153.418 \\
6154.071 \\
6154.510\end{array}$ & $\begin{array}{r}125 \\
1 \\
1 \\
75 \\
50\end{array}$ & $\begin{array}{r}20 \\
12 \\
3\end{array}$ & $\begin{array}{l}\text { I } \\
\text { I } \\
\text { I } \\
\text { II } \\
\text { I }\end{array}$ \\
\hline $\begin{array}{l}6095.968 \\
6096.300 \\
6096.646 \\
6096.871 \\
6097.178\end{array}$ & $\begin{array}{r}1 \\
1 \\
1 \\
1 \\
10\end{array}$ & 20 & $\begin{array}{l}\text { I } \\
\text { I } \\
\text { I } \\
\text { I } \\
\text { I I }\end{array}$ & $\begin{array}{l}6124.473 \\
6125.372 \\
6125.729 \\
6127.390 \\
6127.590\end{array}$ & $\begin{array}{r}75 \\
1 \\
40 \\
5 \\
8\end{array}$ & $\begin{array}{r}2 \\
125\end{array}$ & $\begin{array}{l}\text { I } \\
\text { I } \\
\text { II } \\
\text { I } \\
\text { I }\end{array}$ & $\begin{array}{l}6155.578 \\
6156.087 \\
6156.614 \\
6157.080 \\
6157.494\end{array}$ & $\begin{array}{r}75 \\
5 \\
1 \\
40 \\
1\end{array}$ & $\begin{array}{l}4 \\
1 \\
2 \\
1\end{array}$ & $\begin{array}{l}\text { I } \\
\text { I } \\
\text { I I } \\
\text { I } \\
\text { II }\end{array}$ \\
\hline $\begin{array}{l}6098.111 \\
6099.075 \\
6099.977 \\
6100.391 \\
6100.695\end{array}$ & $\begin{array}{r}75 \\
75 \\
6 \\
1 \\
1\end{array}$ & $\begin{array}{r}10 \\
300\end{array}$ & $\begin{array}{l}\text { II I } \\
\text { I I } \\
\text { I } \\
\text { I } \\
\text { I }\end{array}$ & $\begin{array}{l}6128.121 \\
6128.321 \\
6128.433 \\
6129.528 \\
6130.108\end{array}$ & $\begin{array}{r}2 \\
4 \\
4 \\
25 \\
1\end{array}$ & $\begin{array}{l}1 \\
5\end{array}$ & $\begin{array}{l}\text { I } \\
\text { I } \\
\text { I } \\
\text { I } \\
\text { I I }\end{array}$ & $\begin{array}{l}6159.051 \\
6159.629 \\
6159.843 \\
6160.183 \\
6161.355\end{array}$ & $\begin{array}{r}1 \\
1 \\
1 \\
1 \\
50\end{array}$ & $\begin{array}{l}2 \\
4 \\
4\end{array}$ & $\begin{array}{l}\text { I I } \\
\text { I } \\
\text { I I } \\
\text { I } \\
\text { I }\end{array}$ \\
\hline $\begin{array}{l}6100.966 \\
6101.727 \\
6102.5951 \\
6103.631 \\
6104.567\end{array}$ & $\begin{array}{r}1 \\
75 \\
90 \\
20 \\
100\end{array}$ & $\begin{array}{r}3 \\
10 \\
50 \\
200\end{array}$ & $\begin{array}{l}\text { I } \\
\text { I } \\
\text { I } \\
\text { I I } \\
\text { I I }\end{array}$ & $\begin{array}{l}6130.445 \\
6130.738 \\
6131.382 \\
6131.657 \\
6131.946\end{array}$ & $\begin{array}{l}5 \\
6 \\
1 \\
3 \\
4\end{array}$ & $\begin{array}{r}1 \\
1 \\
10\end{array}$ & $\begin{array}{l}\text { I } \\
\text { I } \\
\text { I } \\
\text { I } \\
\text { I I }\end{array}$ & $\begin{array}{l}6161.764 \\
6162.157 \\
6162.455 \\
6162.965 \\
6163.243\end{array}$ & $\begin{array}{l}3 \\
1 \\
1 \\
8 \\
1\end{array}$ & $\begin{array}{l}1 \\
3 \\
1 \\
5\end{array}$ & $\begin{array}{l}\text { II } \\
\text { I I } \\
\text { I } \\
\text { I I } \\
\text { I I }\end{array}$ \\
\hline $\begin{array}{l}6105.277 \\
6105.948 \\
6106.489 \\
6106.614 \\
6106.840\end{array}$ & $\begin{array}{r}3 \\
1 \\
1 \\
1 \\
12\end{array}$ & 3 & $\begin{array}{l}\text { I } \\
\text { I } \\
\text { I } \\
\text { I } \\
\text { I }\end{array}$ & $\begin{array}{l}6132.215 \\
6132.413 \\
6132.856 \\
6133.053 \\
6133.803\end{array}$ & $\begin{array}{r}3 \\
2 \\
7 \\
30 \\
8\end{array}$ & $\begin{array}{l}7 \\
1\end{array}$ & $\begin{array}{l}\text { I I } \\
\text { I } \\
\text { I I } \\
\text { I } \\
\text { I }\end{array}$ & $\begin{array}{l}6163.717 \\
6163.875 \\
6164.480 \\
6165.249 \\
6165.539\end{array}$ & $\begin{array}{l}6 \\
1 \\
75 \\
1 \mathrm{w} \\
11\end{array}$ & $\begin{array}{l}1 \\
1 \\
3 \\
2 \\
1\end{array}$ & $\begin{array}{l}\text { I I } \\
\text { I I } \\
\text { I } \\
\text { I I } \\
\text { I I }\end{array}$ \\
\hline $\begin{array}{l}6107.524 \\
6108.271 \\
6108.396 \\
6108.891 \\
6109.208\end{array}$ & $\begin{array}{r}50 \\
8 \\
12 \\
1 \\
6\end{array}$ & $\begin{array}{r}4 \\
100 \\
1\end{array}$ & $\begin{array}{l}\text { I } \\
\text { I } \\
\text { II } \\
\text { I I } \\
\text { I }\end{array}$ & $\begin{array}{l}6134.772 \\
6135.590 \\
6135.971 \\
6137.006 \\
6137.923\end{array}$ & $\begin{array}{r}3 \\
4 \\
3 \\
3 \\
25\end{array}$ & 81 & $\begin{array}{l}\text { I I } \\
\text { I } \\
\text { I } \\
\text { I } \\
\text { II }\end{array}$ & $\begin{array}{l}6165.612 \\
6165.826 \\
6166.525 \\
6166.702 \\
6167.198\end{array}$ & $\begin{array}{l}1 \\
2 \\
4 \\
1 \\
1 \mathrm{w}\end{array}$ & & $\begin{array}{l}\text { I } \\
\text { I } \\
\text { I } \\
\text { I } \\
\text { I }\end{array}$ \\
\hline $\begin{array}{l}6109.690 \\
6110.314 \\
6110.654 \\
6111.377 \\
6111.775\end{array}$ & $\begin{array}{l}3 \\
2 \\
1 \\
1 \\
1\end{array}$ & & $\begin{array}{l}\text { I } \\
\text { I } \\
\text { I } \\
\text { I } \\
\text { I }\end{array}$ & $\begin{array}{l}6138.642 \\
6139.320 \\
6140.200 \\
6140.752 \\
6141.626\end{array}$ & $\begin{array}{c}30 \\
1 \\
6 \\
1\end{array}$ & $\begin{array}{c}50 \\
1 \\
10 \mathrm{w}\end{array}$ & $\begin{array}{l}\text { II } \\
\text { I } \\
\text { II I } \\
\text { I } \\
\text { I }\end{array}$ & $\begin{array}{l}6167.906 \\
6168.241 \\
6168.483 \\
6168.580 \\
6168.821\end{array}$ & $\begin{array}{l}2 \\
1 \\
1 \\
1 \\
1\end{array}$ & $\begin{array}{l}5 \\
2\end{array}$ & $\begin{array}{l}\text { II } \\
\text { I } \\
\text { I I } \\
\text { I } \\
\text { I }\end{array}$ \\
\hline $\begin{array}{l}6111.992 \\
6112.827 \\
6113.952 \\
6114.536 \\
6114.909\end{array}$ & $\begin{array}{r}1 \\
125 \\
6 \\
50 \\
3\end{array}$ & $\begin{array}{r}1 \\
250 \\
20 \\
3\end{array}$ & $\begin{array}{l}\text { I I } \\
\text { I I } \\
\text { I I } \\
\text { I } \\
\text { I }\end{array}$ & $\begin{array}{l}6144.282 \\
6144.418 \\
6144.755 \\
6145.278 \\
6145.814\end{array}$ & $\begin{array}{l}1 \mathrm{~s} \\
1 \\
8 \\
25 \\
1\end{array}$ & $\begin{array}{c}3 \mathrm{~s} \\
1 \\
50 \\
1\end{array}$ & $\begin{array}{l}\text { I I } \\
\text { I I } \\
\text { I } \\
\text { II } \\
\text { II }\end{array}$ & $\begin{array}{l}6169.823 \\
6170.676 \\
6170.733 \\
6171.451 \\
6171.892\end{array}$ & $\begin{array}{r}500 \\
20 \\
1 \\
1 \\
1\end{array}$ & 30 & $\begin{array}{l}\text { I } \\
\text { I } \\
\text { I } \\
\text { I } \\
\text { I }\end{array}$ \\
\hline $\begin{array}{l}6116.159 \\
6117.309 \\
6117.726 \\
6118.046 \\
6118.758\end{array}$ & $\begin{array}{r}50 \\
2 \\
1 \\
2 \\
1\end{array}$ & & $\begin{array}{l}\text { I } \\
\text { I } \\
I \\
I \\
I\end{array}$ & $\begin{array}{l}6147.443 \\
6147.625 \\
6147.938 \\
6148.160 \\
6148.202\end{array}$ & $\begin{array}{l}1 \\
1 \\
1 \\
1\end{array}$ & $2 w$ & $\begin{array}{l}\text { I } \\
\text { I } \\
\text { I } \\
\text { II } \\
\text { I }\end{array}$ & $\begin{array}{l}6172.026 \\
6172.563 \\
6173.009 \\
6173.389 \\
6173.868\end{array}$ & $\begin{array}{l}1 \\
1 \\
1 \\
1 d \\
1\end{array}$ & 2 & $\begin{array}{l}\text { II } \\
\text { I } \\
\text { I } \\
\text { I } \\
\text { I }\end{array}$ \\
\hline
\end{tabular}


Table 1. Wavelengths of Thorium Spectra - Continued

\begin{tabular}{|c|c|c|c|c|c|c|c|c|c|c|c|}
\hline \multirow{2}{*}{$\begin{array}{l}\text { Wave- } \\
\text { length }\end{array}$} & \multicolumn{2}{|c|}{ Intensity } & \multirow{2}{*}{$\begin{array}{l}\text { Spec- } \\
\text { trum }\end{array}$} & \multirow{2}{*}{$\begin{array}{l}\text { Wave- } \\
\text { length }\end{array}$} & \multicolumn{2}{|c|}{ Intensity } & \multirow{2}{*}{$\begin{array}{l}\text { Spec- } \\
\text { trum }\end{array}$} & \multirow{2}{*}{$\begin{array}{l}\text { Wave- } \\
\text { length }\end{array}$} & \multicolumn{2}{|c|}{ Intensity } & \multirow{2}{*}{$\begin{array}{l}\text { Spec- } \\
\text { trum }\end{array}$} \\
\hline & Lamp & Spark & & & Lamp & Spark & & & Lamp & Spark & \\
\hline $\begin{array}{l}6174.486 \\
6174.763 \\
6175.379 \\
6175.574 \\
6175.948\end{array}$ & $\begin{array}{l}1 \\
1 \\
1 \\
1 \\
4\end{array}$ & 1 & $\begin{array}{l}\text { II } \\
\text { I } \\
\text { I } \\
\text { I I } \\
\text { I }\end{array}$ & $\begin{array}{l}6208.688 \\
6208.954 \\
6209.947 \\
6210.904 \\
6211.651\end{array}$ & $\begin{array}{r}30 \\
2 \\
6 \\
1 \\
10\end{array}$ & $\begin{array}{r}2 \\
1 \\
1 \\
1000\end{array}$ & $\begin{array}{l}\text { I } \\
\text { I } \\
\text { I } \\
\text { II } \\
\text { II }\end{array}$ & $\begin{array}{l}6234.849 \\
6235.789 \\
6237.090 \\
6237.697 \\
6237.958\end{array}$ & $\begin{array}{r}200 \\
2 \\
3 \\
2 \\
2\end{array}$ & $\begin{array}{l}5 \\
4\end{array}$ & $\begin{array}{l}\text { I } \\
\text { II } \\
\text { I } \\
\text { I } \\
\text { I }\end{array}$ \\
\hline $\begin{array}{l}6176.165 \\
6176.469 \\
6176.678 \\
6178.435 \\
6178.903\end{array}$ & $\begin{array}{r}1 \\
1 \\
1 \\
100 \\
5\end{array}$ & $\begin{array}{l}2 \\
2\end{array}$ & $\begin{array}{l}\text { I } \\
\text { I } \\
\text { I } \\
\text { I } \\
\text { II }\end{array}$ & $\begin{array}{l}6212.720 \\
6213.292 \\
6213.638 \\
6213.934 \\
6214.084\end{array}$ & $\begin{array}{r}30 \\
1 \\
3 \\
2 \\
1\end{array}$ & $\begin{array}{l}1 \\
3 \\
3\end{array}$ & $\begin{array}{l}\text { I } \\
\text { I } \\
\text { II } \\
\text { II } \\
\text { I }\end{array}$ & $\begin{array}{l}6238.080 \\
6239.076 \\
6239.279 \\
6239.528 \\
6239.743\end{array}$ & $\begin{array}{l}6 \\
3 \\
1 \\
1 \\
1\end{array}$ & $\begin{array}{r}20 \\
1\end{array}$ & $\begin{array}{l}\text { I } \\
\text { II } \\
\text { I } \\
\text { II } \\
\text { I }\end{array}$ \\
\hline $\begin{array}{l}6179.152 \\
6179.845 \\
6180.707 \\
6181.529 \\
6182.622 \mathrm{i}\end{array}$ & $\begin{array}{c}3 \\
25 \\
75 \\
5 \mathrm{w} \\
400\end{array}$ & $\begin{array}{r}100 \\
3 \\
50\end{array}$ & $\begin{array}{l}\text { I } \\
\text { II } \\
\text { I } \\
\text { I } \\
\text { I }\end{array}$ & $\begin{array}{l}6214.527 \\
6215.151 \\
6215.687 \\
6215.963 \\
6216.262\end{array}$ & $\begin{array}{l}3 \\
11 \\
1 \\
2 \\
1\end{array}$ & 1 & $\begin{array}{l}\text { I } \\
\text { I } \\
\text { II } \\
\text { I } \\
\text { I }\end{array}$ & $\begin{array}{l}6240.299 \\
6240.597 \\
6240.945 \\
6241.393 \\
6241.668\end{array}$ & $\begin{array}{r}4 \\
2 \\
50 \\
4 \\
2\end{array}$ & $\begin{array}{l}2 \\
8 \\
2\end{array}$ & $\begin{array}{l}\text { I } \\
\text { I } \\
\text { I } \\
\text { I I } \\
\text { I I }\end{array}$ \\
\hline $\begin{array}{l}6184.419 \\
6184.495 \\
6184.773 \\
6185.758 \\
6186.139\end{array}$ & $\begin{array}{r}10 \\
2 \\
100 \\
5 \\
1\end{array}$ & $\begin{array}{c}100 \mathrm{w} \\
50\end{array}$ & $\begin{array}{l}\text { II } \\
\text { I } \\
\text { I } \\
\text { I } \\
\text { I }\end{array}$ & $\begin{array}{l}6216.434 \\
6216.716 \\
6216.853 \\
6217.575 \\
6218.067\end{array}$ & $\begin{array}{l}1 \\
1 \\
1 \\
5 \\
1\end{array}$ & $\begin{array}{l}1 \\
1 \\
1 \\
2\end{array}$ & $\begin{array}{l}\text { II } \\
\text { II } \\
\text { II } \\
\text { I } \\
\text { I }\end{array}$ & $\begin{array}{l}6241.860 \\
6242.690 \\
6243.350 \\
6244.363 \\
6245.033\end{array}$ & $\begin{array}{r}2 \\
12 \\
1 \\
8\end{array}$ & $500 \mathrm{w}$ & $\begin{array}{l}\text { I } \\
\text { I I I } \\
\text { I } \\
\text { I } \\
\text { II }\end{array}$ \\
\hline $\begin{array}{l}6186.360 \\
6186.742 \\
6187.125 \\
6188.122 \\
6189.133\end{array}$ & $\begin{array}{r}3 \\
1 \\
1 \\
160 \\
10\end{array}$ & 2 & $\begin{array}{l}\text { I } \\
\text { I } \\
\text { II } \\
\text { I } \\
\text { I }\end{array}$ & $\begin{array}{l}6218.191 \\
6218.344 \\
6218.570 \\
6219.772 \\
6220.012\end{array}$ & $\begin{array}{r}1 \\
1 \\
1 \\
6 \\
75\end{array}$ & $\begin{array}{l}1 \\
1 \\
2\end{array}$ & $\begin{array}{l}\text { II } \\
\text { I } \\
\text { II } \\
\text { I } \\
\text { I }\end{array}$ & $\begin{array}{l}6245.595 \\
6246.150 \\
6246.913 \\
6247.105 \\
6248.107\end{array}$ & $\begin{array}{r}7 \\
10 \\
4 \\
3 \\
8\end{array}$ & $\begin{array}{r}15 \\
4 \\
2 \\
20\end{array}$ & $\begin{array}{l}\text { II } \\
\text { I } \\
\text { II } \\
\text { II } \\
\text { I I }\end{array}$ \\
\hline $\begin{array}{l}6189.414 \\
6190.742 \\
6191.905 \mathrm{i} \\
6192.578 \\
6193.164\end{array}$ & $\begin{array}{c}10 \\
6 \mathrm{w} \\
100 \\
1 \\
3\end{array}$ & $\begin{array}{c}50 \\
15 \\
1\end{array}$ & $\begin{array}{l}\text { II } \\
\text { I } \\
\text { I } \\
\text { I } \\
\text { II }\end{array}$ & $\begin{array}{l}6221.046 \\
6221.313 \\
6221.565 \\
6222.538 \\
6222.762\end{array}$ & $\begin{array}{r}7 \\
75 \\
6 \\
1 \\
3\end{array}$ & 150 & $\begin{array}{l}\text { I } \\
\text { I } \\
\text { I I } \\
\text { I } \\
\text { I }\end{array}$ & $\begin{array}{l}6249.125 \\
6250.213 \\
6250.478 \\
6250.880 \\
6251.416\end{array}$ & $\begin{array}{c}1 \mathrm{w} \\
1 \\
12 \\
1 \\
2\end{array}$ & $\begin{array}{r}1 \\
15\end{array}$ & $\begin{array}{l}\text { I } \\
\text { I } \\
\text { I } \\
\text { I } \\
\text { II }\end{array}$ \\
\hline $\begin{array}{l}6193.860 \\
6195.313 \\
6196.170 \\
6196.388 \\
6196.959\end{array}$ & $\begin{array}{r}50 \\
10 \\
2 \\
12 \\
1\end{array}$ & $\begin{array}{r}200 \\
1\end{array}$ & $\begin{array}{l}\text { II } \\
\text { I } \\
\text { I } \\
\text { I } \\
\text { I }\end{array}$ & $\begin{array}{l}6223.268 \\
6223.510 \\
6224.528 \mathrm{i} \\
6226.374 \\
6226.623\end{array}$ & $\begin{array}{r}1 \\
10 \\
100 \\
50 \\
3\end{array}$ & $\begin{array}{l}2 \mathrm{~h} \\
20 \\
10 \\
2 \\
5\end{array}$ & $\begin{array}{l}\text { II } \\
\text { I I } \\
\text { I } \\
\text { I } \\
\text { II }\end{array}$ & $\begin{array}{l}6251.630 \\
6251.747 \\
6251.944 \\
6252.298 \\
6252.854\end{array}$ & $\begin{array}{l}1 \\
1 \\
2 \\
2 \\
1\end{array}$ & & $\begin{array}{l}\text { I } \\
\text { I } \\
\text { I } \\
\text { I } \\
\text { I }\end{array}$ \\
\hline $\begin{array}{l}6198.235 \\
6199.042 \\
6199.432 \\
6200.432 \\
6201.506\end{array}$ & $\begin{array}{r}80 \\
2 \\
1 \\
100 \\
1\end{array}$ & $\begin{array}{r}20 \\
3 \\
1 \\
75\end{array}$ & $\begin{array}{l}\text { I } \\
\text { II } \\
\text { II } \\
\text { II } \\
\text { I }\end{array}$ & $\begin{array}{l}6226.930 \\
6227.162 \\
6227.792 \\
6227.935 \\
6228.982\end{array}$ & $\begin{array}{l}1 \\
1 \\
1 \\
1 \\
2\end{array}$ & 4 & $\begin{array}{l}\text { II } \\
\text { I } \\
\text { I } \\
\text { I } \\
\text { I }\end{array}$ & $\begin{array}{l}6253.512 \\
6254.106 \\
6254.763 \\
6255.015 \\
6255.329\end{array}$ & $\begin{array}{l}1 \\
1 \\
3 \\
1 \\
1\end{array}$ & $\begin{array}{l}1 \\
1 \\
1\end{array}$ & $\begin{array}{l}\text { I } \\
\text { II } \\
\text { II } \\
\text { II } \\
\text { I }\end{array}$ \\
\hline $\begin{array}{l}6201.844 \\
6202.066 \\
6203.501 \\
6204.117 \\
6204.761\end{array}$ & $\begin{array}{r}1 \\
1 \\
100 \\
6 \\
3\end{array}$ & $\begin{array}{l}2 \mathrm{~h} \\
15 \\
15 \\
6\end{array}$ & $\begin{array}{l}\text { II } \\
\text { I } \\
\text { I } \\
\text { II } \\
\text { II }\end{array}$ & $\begin{array}{l}6229.599 \\
6230.262 \\
6230.899 \\
6231.122 \\
6231.378\end{array}$ & $\begin{array}{r}2 \\
1 \\
1 \\
4 \\
10\end{array}$ & $\begin{array}{r}3 \\
1 \\
10 \\
50\end{array}$ & $\begin{array}{l}\text { II } \\
\text { I } \\
\text { I I } \\
\text { II } \\
\text { II }\end{array}$ & $\begin{array}{l}6255.514 \\
6255.799 \\
6256.096 \\
6256.329 \\
6256.922\end{array}$ & $\begin{array}{l}1 \\
1 \\
1 \\
1 \\
1\end{array}$ & $\begin{array}{l}3 \\
3\end{array}$ & $\begin{array}{l}\text { I } \\
\text { I } \\
\text { II } \\
\text { I } \\
\text { II }\end{array}$ \\
\hline $\begin{array}{l}6205.011 \\
6205.260 \\
6205.862 \\
6207.2211 \\
6207.729\end{array}$ & $\begin{array}{r}4 \\
4 \\
40 \\
160 \\
30\end{array}$ & $\begin{array}{r}8 \\
1 \\
20 \\
2\end{array}$ & $\begin{array}{l}\text { I } \\
\text { I I } \\
\text { I } \\
\text { I } \\
\text { I }\end{array}$ & $\begin{array}{l}6232.088 \\
6232.976 \\
6233.850 \\
6234.197 \\
6234.294\end{array}$ & $\begin{array}{r}12 \\
20 \\
1 \\
6 \\
3\end{array}$ & $\begin{array}{r}1 \\
40\end{array}$ & $\begin{array}{l}\text { I } \\
\text { II } \\
\text { I } \\
\text { I } \\
\text { II }\end{array}$ & $\begin{array}{l}6257.424 \mathrm{i} \\
6258.597 \\
6259.949 \\
6260.214 \\
6261.060\end{array}$ & $\begin{array}{r}100 \\
30 \\
1 \\
2 \\
40\end{array}$ & $\begin{array}{r}3 \\
75 \\
1 \\
125\end{array}$ & $\begin{array}{l}\text { I } \\
\text { II } \\
\text { II } \\
\text { I } \\
\text { II }\end{array}$ \\
\hline
\end{tabular}


Table 1. Wavelengths of Thorium Spectra - Continued

\begin{tabular}{|c|c|c|c|c|c|c|c|c|c|c|c|}
\hline \multirow{2}{*}{$\begin{array}{l}\text { Wave- } \\
\text { length }\end{array}$} & \multicolumn{2}{|c|}{ Intensity } & \multirow{2}{*}{$\begin{array}{l}\text { Spec- } \\
\text { trum }\end{array}$} & \multirow{2}{*}{$\begin{array}{l}\text { Wave- } \\
\text { length }\end{array}$} & \multicolumn{2}{|c|}{ Intensity } & \multirow{2}{*}{$\begin{array}{l}\text { Spec- } \\
\text { trum }\end{array}$} & \multirow{2}{*}{$\begin{array}{l}\text { Wave- } \\
\text { length }\end{array}$} & \multicolumn{2}{|c|}{ Intensity } & \multirow{2}{*}{$\begin{array}{l}\text { Spec } \\
\text { trum }\end{array}$} \\
\hline & & Spark & & & Lamp & Spark & & & Lamp & Spark & \\
\hline $\begin{array}{l}6261.418 \mathrm{i} \\
6262.869 \\
6263.378 \\
6264.715 \\
6265.586\end{array}$ & $\begin{array}{r}180 \\
1 \\
1 \\
10 \\
6\end{array}$ & 18 & $\begin{array}{l}\text { I } \\
\text { I } \\
\text { I } \\
\text { I } \\
\text { I }\end{array}$ & $\begin{array}{l}6297.461 \\
6297.766 \\
6298.894 \\
6299.421 \\
6300.923\end{array}$ & $\begin{array}{r}1 \\
2 \\
7 \\
1 \\
30\end{array}$ & $\begin{array}{l}1 \\
1 \\
2\end{array}$ & $\begin{array}{l}\text { I } \\
\text { I } \\
\text { I } \\
\text { II } \\
\text { I }\end{array}$ & $\begin{array}{l}6328.778 \\
6329.514 \\
6330.120 \\
6330.804 \\
6331.415\end{array}$ & $\begin{array}{r}6 \\
1 \\
1 \\
1 \\
30\end{array}$ & $\begin{array}{l}6 \\
2\end{array}$ & $\begin{array}{l}\text { I } \\
\text { I } \\
\text { I } \\
\text { II } \\
\text { I }\end{array}$ \\
\hline $\begin{array}{l}6266.170 \\
6266.600 \\
6267.701 \\
6268.188 \\
6268.767\end{array}$ & $\begin{array}{c}100 \\
4 \\
1 \\
3 w \\
2 w\end{array}$ & 50 & $\begin{array}{l}\text { I I } \\
\text { I } \\
\text { I } \\
\text { I } \\
\text { I }\end{array}$ & $\begin{array}{l}6301.408 \\
6302.468 \\
6303.248 \\
6304.252 \\
6305.277\end{array}$ & $\begin{array}{r}90 \\
3 \\
40 \\
30 \\
20\end{array}$ & $\begin{array}{r}3 \\
4 \\
125 \\
2\end{array}$ & $\begin{array}{l}\text { I } \\
\text { I } \\
\text { I } \\
\text { I I } \\
\text { I I }\end{array}$ & $\begin{array}{l}6332.171 \\
6332.505 \\
6333.931 \\
6334.268 \\
6334.688\end{array}$ & $\begin{array}{r}1 \\
10 \\
1 \\
1 \\
1\end{array}$ & 30 & $\begin{array}{l}\text { I } \\
\text { I I } \\
\text { I } \\
\text { I } \\
\text { I }\end{array}$ \\
\hline $\begin{array}{l}6269.166 \\
6269.580 \\
6270.312 \\
6270.574 \\
6271.529\end{array}$ & $\begin{array}{r}1 \\
3 \\
2 \\
1 \\
12\end{array}$ & & $\begin{array}{l}\text { I } \\
\text { I } \\
\text { I } \\
\text { I } \\
\text { I }\end{array}$ & $\begin{array}{l}6306.684 \\
6306.896 \\
6307.214 \\
6307.859 \\
6308.714\end{array}$ & $\begin{array}{l}1 \\
1 \\
1 \\
2 \\
2\end{array}$ & $\begin{array}{l}2 \\
1 \mathrm{~h} \\
1 \\
1\end{array}$ & $\begin{array}{l}\text { I I } \\
\text { I I } \\
\text { I I } \\
\text { I I } \\
\text { I }\end{array}$ & $\begin{array}{l}6335.022 \\
6335.170 \\
6335.713 \\
6336.078 \\
6336.411\end{array}$ & $\begin{array}{l}3 \\
2 \\
3 \\
3\end{array}$ & 600 & $\begin{array}{l}\text { I } \\
\text { I I I } \\
\text { I } \\
\text { I } \\
\text { I }\end{array}$ \\
\hline $\begin{array}{l}6272.071 \\
6274.110 \\
6275.068 \\
6276.152 \\
6277.227\end{array}$ & $\begin{array}{r}25 \\
100 \\
2 \\
20 \\
20\end{array}$ & 150 & $\begin{array}{l}\text { I } \\
\text { II } \\
\text { I } \\
\text { I } \\
\text { II }\end{array}$ & $\begin{array}{l}6309.430 \\
6309.739 \\
6310.817 \\
6311.269 \\
6311.458\end{array}$ & $\begin{array}{r}4 \\
1 \\
30 \\
2 \\
2\end{array}$ & $\begin{array}{r}20 \\
2 \\
6\end{array}$ & $\begin{array}{l}\text { I I } \\
\text { I } \\
\text { I } \\
\text { I I } \\
\text { I }\end{array}$ & $\begin{array}{l}6337.611 \\
6338.372 \\
6338.640 \\
6338.908 \\
6339.328\end{array}$ & $\begin{array}{r}40 \\
1 \\
1 \\
1 \\
3\end{array}$ & $\begin{array}{r}3 \\
2 \\
12\end{array}$ & $\begin{array}{l}\text { I } \\
\text { I } \\
\text { II } \\
\text { II } \\
\text { I }\end{array}$ \\
\hline $\begin{array}{l}6278.102 \\
6279.156 \\
6279.973 \\
6280.880 \\
6281.161\end{array}$ & $\begin{array}{r}1 \\
30 \\
4 \\
1 \\
1\end{array}$ & $\begin{array}{r}125 \\
1 \\
1\end{array}$ & $\begin{array}{l}\text { I } \\
\text { I I } \\
\text { I } \\
\text { I I } \\
\text { I I }\end{array}$ & $\begin{array}{l}6311.834 \\
6311.941 \\
6312.280 \\
6312.640 \\
6314.261\end{array}$ & $\begin{array}{c}1 \\
1 \\
1 \\
15 \mathrm{w} \\
3\end{array}$ & 20 & $\begin{array}{l}\text { I } \\
\text { I } \\
\text { I I } \\
\text { I I } \\
\text { I }\end{array}$ & $\begin{array}{l}6339.450 \\
6339.668 \\
6341.885 \\
6342.860 \mathrm{i} \\
6343.171\end{array}$ & $\begin{array}{c}6 \mathrm{c} \\
40 \\
1 \\
300 \\
2\end{array}$ & $\begin{array}{r}501 \\
30 \\
3\end{array}$ & $\begin{array}{l}\text { II } \\
\text { I } \\
\text { I } \\
\text { I } \\
\text { I I }\end{array}$ \\
\hline $\begin{array}{l}6281.529 \\
6281.944 \\
6283.124 \\
6233.656 \\
6283.783\end{array}$ & $\begin{array}{l}1 \\
1 \\
1 \\
1 \\
1\end{array}$ & $2 w$ & $\begin{array}{l}\text { I } \\
\text { I I } \\
\text { I } \\
\text { I } \\
\text { I }\end{array}$ & $\begin{array}{l}6315.543 \\
6315.776 \\
6315.992 \\
6317.184 \\
6318.302\end{array}$ & $\begin{array}{c}2 \\
10 \\
3 w \\
25 \\
3\end{array}$ & 18 & $\begin{array}{l}\text { I I } \\
\text { I } \\
\text { I } \\
\text { I } \\
\text { I }\end{array}$ & $\begin{array}{l}6344.126 \\
6345.367 \\
6346.116 \\
6346.651 \\
6347.085\end{array}$ & $\begin{array}{r}1 \\
1 \\
20 \\
1 \\
3\end{array}$ & $\begin{array}{l}2 \\
1\end{array}$ & $\begin{array}{l}\text { I } \\
\text { I } \\
\text { I } \\
\text { I } \\
\text { II }\end{array}$ \\
\hline $\begin{array}{l}6285.279 \\
6286.036 \\
6286.706 \\
6286.923 \\
6287.252\end{array}$ & $\begin{array}{r}30 \\
8 \\
1 \\
1 \\
10\end{array}$ & $\begin{array}{r}125 \\
2\end{array}$ & $\begin{array}{l}\text { I I } \\
\text { I } \\
\text { I } \\
\text { I } \\
\text { I }\end{array}$ & $\begin{array}{l}6318.699 \\
6319.158 \\
6319.240 \\
6319.738 \\
6320.096\end{array}$ & $\begin{array}{l}3 \\
5 \\
3 \\
1 \\
1\end{array}$ & 1 & $\begin{array}{l}\text { II } \\
\text { I } \\
\text { I } \\
\text { I } \\
\text { I I }\end{array}$ & $\begin{array}{l}6348.122 \\
6348.567 \\
6348.738 \\
6349.736 \\
6350.432\end{array}$ & $\begin{array}{r}4 \\
6 \\
40 \\
1 \\
1\end{array}$ & $\begin{array}{r}1 \\
175 \\
2\end{array}$ & $\begin{array}{l}\text { I I } \\
\text { I I } \\
\text { I } \\
\text { I I } \\
\text { I }\end{array}$ \\
\hline $\begin{array}{l}6287.562 \\
6288.426 \\
6289.283 \\
6289.486 \\
6290.398\end{array}$ & $\begin{array}{r}2 \\
1 \\
1 \\
10 \\
3\end{array}$ & $\begin{array}{r}1 \\
60\end{array}$ & $\begin{array}{l}\text { I } \\
\text { II } \\
\text { I } \\
\text { II } \\
\text { I }\end{array}$ & $\begin{array}{l}6320.460 \\
6320.793 \\
6321.166 \\
6321.823 \\
6322.191\end{array}$ & $\begin{array}{l}1 \\
1 \\
4 \\
5 \\
1\end{array}$ & $\begin{array}{l}2 \\
5 \\
4 w\end{array}$ & $\begin{array}{l}\text { II } \\
\text { I } \\
\text { II } \\
\text { I } \\
\text { I I }\end{array}$ & $\begin{array}{l}6350.888 \\
6351.390 \\
6351.645 \\
6352.143 \\
6352.505\end{array}$ & $\begin{array}{l}2 \\
1 \\
1 \\
1 \\
2\end{array}$ & & $\begin{array}{l}\text { I } \\
\text { I } \\
\text { I } \\
\text { I } \\
\text { I }\end{array}$ \\
\hline $\begin{array}{l}6291.191 \\
6291.623 \\
6292.056 \\
6292.890 \\
6293.242\end{array}$ & $\begin{array}{r}30 \\
3 \\
6 \\
15 \\
25\end{array}$ & $\begin{array}{l}3 \\
1 \mathrm{~h} \\
1 \\
2\end{array}$ & $\begin{array}{l}\text { I } \\
\text { I I } \\
\text { I } \\
\text { I } \\
\text { I }\end{array}$ & $\begin{array}{l}6322.861 \\
6323.838 \\
6324.388 \\
6324.590 \\
6325.010\end{array}$ & $\begin{array}{l}3 \\
4 \\
6 \\
3 \\
1\end{array}$ & $\begin{array}{l}20 \\
30\end{array}$ & $\begin{array}{l}\text { I } \\
\text { II } \\
\text { I } \\
\text { I I } \\
\text { I }\end{array}$ & $\begin{array}{l}6353.308 \\
6353.620 \\
6354.256 \\
6355.278 \\
6355.626\end{array}$ & $\begin{array}{r}2 \\
1 \\
1 \\
2 \\
25\end{array}$ & 2 & $\begin{array}{l}\text { II } \\
\text { I } \\
\text { I } \\
\text { I } \\
\text { I }\end{array}$ \\
\hline $\begin{array}{l}6295.156 \\
6295.877 \\
6296.537 \\
6296.668 \\
6297.026\end{array}$ & $\begin{array}{l}2 \\
2 \\
1 \\
1 \\
2\end{array}$ & $\begin{array}{l}1 \\
2 \mathrm{~h} \\
3\end{array}$ & $\begin{array}{l}\text { I } \\
\text { I } \\
\text { I I } \\
\text { I I } \\
\text { I I }\end{array}$ & $\begin{array}{l}6325.432 \\
6325.602 \\
6326.372 \\
6327.279 \mathrm{i} \\
6328.254\end{array}$ & $\begin{array}{r}2 \\
3 \\
60 \\
180 \\
1\end{array}$ & $\begin{array}{r}15 \\
4 \\
2\end{array}$ & $\begin{array}{l}\text { II } \\
\text { I } \\
\text { II } \\
\text { I } \\
\text { II }\end{array}$ & $\begin{array}{l}6355.905 \\
6356.214 \\
6356.888 \\
6357.016 \\
6357.321\end{array}$ & $\begin{array}{l}40 \\
3 \\
1 \\
1 \\
1 w\end{array}$ & $\begin{array}{r}3 \\
15\end{array}$ & $\begin{array}{l}\text { I } \\
\text { I I } \\
\text { I } \\
\text { I } \\
\text { I }\end{array}$ \\
\hline
\end{tabular}


Table 1. Wavelengths of Thorium Spectra - Continued

\begin{tabular}{|c|c|c|c|c|c|c|c|c|c|c|c|}
\hline \multirow{2}{*}{$\begin{array}{l}\text { Wave- } \\
\text { length }\end{array}$} & \multicolumn{2}{|c|}{ Intensity } & \multirow{2}{*}{$\begin{array}{l}\text { Spec- } \\
\text { trum }\end{array}$} & \multirow{2}{*}{$\begin{array}{l}\text { Wave- } \\
\text { length }\end{array}$} & \multicolumn{2}{|c|}{ Intensity } & \multirow{2}{*}{$\begin{array}{l}\text { Spec- } \\
\text { trum }\end{array}$} & \multirow{2}{*}{$\begin{array}{l}\text { Wave- } \\
\text { length }\end{array}$} & \multicolumn{2}{|c|}{ Intensity } & \multirow{2}{*}{$\begin{array}{l}\text { Spec. } \\
\text { trum }\end{array}$} \\
\hline & & & & & & & & & & & \\
\hline $\begin{array}{l}6357.683 \\
6357.790 \\
6358.611 \\
6359.126 \\
6359.668\end{array}$ & $\begin{array}{l}1 \\
1 \\
40 \\
30 \mathrm{c} \\
25\end{array}$ & $\begin{array}{r}200 \\
75\end{array}$ & $\begin{array}{l}\text { I } \\
\text { I } \\
\text { II } \\
\text { II } \\
\text { I }\end{array}$ & $\begin{array}{l}6386.381 \\
6386.750 \\
6387.068 \\
6387.389 \\
6387.490\end{array}$ & $\begin{array}{l}3 \\
1 \mathrm{w} \\
4 \\
50\end{array}$ & 20 & $\begin{array}{l}\text { II } \\
I \\
I \\
I \\
\text { III }\end{array}$ & $\begin{array}{l}6414.687 \\
6415.535 \\
6416.103 \\
6416.313 \\
6416.575\end{array}$ & $\begin{array}{r}5 \\
9 \\
15 \\
1 \\
1 \mathrm{w}\end{array}$ & $\begin{array}{r}2 \\
100\end{array}$ & $\begin{array}{l}\text { II } \\
\text { I } \\
\text { II } \\
\text { I } \\
\text { I }\end{array}$ \\
\hline $\begin{array}{l}6360.084 \\
6360.448 \\
6360.778 \\
6360.917 \\
6361.111\end{array}$ & $\begin{array}{l}1 \\
1 \\
1 \\
1 \\
1\end{array}$ & 3 & $\begin{array}{l}\text { I } \\
\text { I } \\
\text { I } \\
\text { II } \\
\text { I }\end{array}$ & $\begin{array}{l}6387.851 \\
6388.154 \\
6388.416 \\
6388.768 \\
6388.812\end{array}$ & $\begin{array}{r}10 \\
1 \\
15 \\
10 \\
40\end{array}$ & $\begin{array}{r}12 \\
3 \\
1 \\
10\end{array}$ & $\begin{array}{l}\text { II } \\
\text { II } \\
\text { I } \\
\text { II } \\
\text { I }\end{array}$ & $\begin{array}{l}6416.913 \\
6417.172 \\
6417.563 \\
6417.891 \\
6418.683\end{array}$ & $\begin{array}{l}2 \\
1 \\
1 \\
3 \\
1\end{array}$ & 1 & $\begin{array}{l}\text { I } \\
\text { II } \\
\text { I } \\
\text { I } \\
\text { I }\end{array}$ \\
\hline $\begin{array}{l}6362.241 \\
6363.012 \\
6363.261 \\
6364.242 \\
6364.409\end{array}$ & $\begin{array}{r}15 \\
1 \\
3 \\
1 \\
2\end{array}$ & $\begin{array}{l}4 \\
6\end{array}$ & $\begin{array}{l}\text { I } \\
\text { I } \\
\text { II } \\
\text { I } \\
\text { I }\end{array}$ & $\begin{array}{l}6389.382 \\
6390.119 \\
6390.546 \\
6390.797 \\
6391.159\end{array}$ & $\begin{array}{r}40 \\
9 \\
1 \\
1 \\
6\end{array}$ & 2 & $\begin{array}{l}\text { I } \\
\text { I } \\
\text { I } \\
\text { I } \\
\text { II }\end{array}$ & $\begin{array}{l}6419.154 \\
6419.518 \\
6419.820 \\
6420.059 \\
6420.930\end{array}$ & $\begin{array}{l}1 \\
1 \\
2 \\
1 \\
2\end{array}$ & 3 & $\begin{array}{l}\text { I } \\
\text { II } \\
\text { I } \\
\text { I } \\
\text { II }\end{array}$ \\
\hline $\begin{array}{l}6364.636 \\
6364.783 \\
6365.429 \\
6365.958 \\
6366.287\end{array}$ & $\begin{array}{l}3 \\
3 \\
1 \\
1 \\
1\end{array}$ & & $\begin{array}{l}\text { I } \\
\text { I } \\
\text { I } \\
\text { I } \\
\text { I }\end{array}$ & $\begin{array}{l}6392.359 \\
6393.181 \\
6394.027 \\
6394.563 \\
6395.604\end{array}$ & $\begin{array}{r}12 \\
1 \\
30 \\
1 \\
1\end{array}$ & $\begin{array}{l}2 \\
1 \\
1\end{array}$ & $\begin{array}{l}\text { II } \\
\text { I } \\
\text { I } \\
\text { I }\end{array}$ & $\begin{array}{l}6421.346 \\
6421.415 \\
6421.703 \\
6422.092 \\
6422.502\end{array}$ & $\begin{array}{r}30 \\
2 \\
1 \\
30 \\
1\end{array}$ & 2 & $\begin{array}{l}\text { I } \\
\text { I } \\
\text { I } \\
\text { I } \\
\text { I }\end{array}$ \\
\hline $\begin{array}{l}6367.633 \\
6368.960 \\
6369.130 \\
6370.785 \\
6371.058\end{array}$ & $\begin{array}{c}1 \mathrm{~s} \\
20 \\
3 \\
1\end{array}$ & $\begin{array}{l}4 \\
4\end{array}$ & $\begin{array}{l}\text { I } \\
\text { II } \\
\text { I } \\
\text { I } \\
\text { I }\end{array}$ & $\begin{array}{l}6395.874 \\
6396.734 \\
6396.949 \\
6397.436 \\
6398.436\end{array}$ & $\begin{array}{r}1 \\
2 \\
15 \\
5 \\
4\end{array}$ & $\begin{array}{l}1 \\
1\end{array}$ & $\begin{array}{l}\text { II } \\
\text { I I } \\
\text { I } \\
\text { I } \\
\text { I }\end{array}$ & $\begin{array}{l}6422.923 \\
6423.393 \\
6424.801 \\
6426.741 \\
6427.006\end{array}$ & $\begin{array}{r}2 \\
1 \\
30 \\
1 \\
1\end{array}$ & $\begin{array}{r}1 \\
300 \\
1 \\
5\end{array}$ & $\begin{array}{l}\text { I } \\
\text { II } \\
\text { II } \\
\text { II } \\
\text { I I }\end{array}$ \\
\hline $\begin{array}{l}6371.929 \\
6372.439 \\
6373.642 \\
6374.246 \\
6374.510\end{array}$ & $\begin{array}{l}30 \\
4 \\
1 \mathrm{~h} \\
1\end{array}$ & $50 \mathrm{w}$ & $\begin{array}{l}\text { I } \\
\text { I } \\
\text { I } \\
\text { I } \\
\text { II I }\end{array}$ & $\begin{array}{l}6399.568 \\
6399.797 \\
6399.963 \\
6400.691 \\
6401.727\end{array}$ & $\begin{array}{r}5 \\
2 \\
5 \\
10 \\
3\end{array}$ & $\begin{array}{r}5 \\
4 \\
\\
30\end{array}$ & $\begin{array}{l}\text { II } \\
\text { I I } \\
\text { I } \\
\text { I } \\
\text { II }\end{array}$ & $\begin{array}{l}6427.485 \\
6428.374 \\
6428.504 \\
6428.761 \\
6429.313\end{array}$ & $\begin{array}{l}4 \\
1 \\
1 \\
4 \\
1\end{array}$ & $\begin{array}{l}1 \mathrm{~h} \\
2 \mathrm{w}\end{array}$ & $\begin{array}{l}\text { II } \\
\text { I } \\
\text { II } \\
\text { I } \\
\text { I }\end{array}$ \\
\hline $\begin{array}{l}6374.874 \\
6376.9311 \\
6377.485 \\
6377.938 \\
6378.482\end{array}$ & $\begin{array}{c}1 \mathrm{w} \\
350 \\
4 \\
3 \\
1\end{array}$ & $\begin{array}{r}30 \\
50 \\
4 \\
1\end{array}$ & $\begin{array}{l}\text { I } \\
\text { I } \\
\text { II } \\
\text { II } \\
\text { II }\end{array}$ & $\begin{array}{l}6402.240 \\
6402.931 \\
6403.850 \\
6404.387 \\
6404.612\end{array}$ & $\begin{array}{l}1 \\
9 \\
2 \\
2 \\
2\end{array}$ & $\begin{array}{r}2 \\
18 \\
15\end{array}$ & $\begin{array}{l}\text { II } \\
\text { II } \\
\text { I } \\
\text { II } \\
\text { I }\end{array}$ & $\begin{array}{l}6429.683 \\
6430.229 \\
6430.478 \\
6430.615 \\
6431.424\end{array}$ & $\begin{array}{l}1 \\
10 \\
1 \\
1 \mathrm{w} \\
1\end{array}$ & $\begin{array}{r}2 \\
15\end{array}$ & $\begin{array}{l}\text { II } \\
\text { II } \\
\text { I } \\
\text { I } \\
\text { I }\end{array}$ \\
\hline $\begin{array}{l}6378.748 \\
6379.157 \\
6379.657 \\
6379.812 \\
6380.280\end{array}$ & $\begin{array}{l}6 \\
5 \\
6 \\
5 \\
1\end{array}$ & $\begin{array}{l}15 \\
12 \\
15\end{array}$ & $\begin{array}{l}\text { II } \\
\text { II } \\
\text { II } \\
\text { II } \\
\text { I }\end{array}$ & $\begin{array}{l}6405.072 \\
6405.326 \\
6405.860 \\
6406.128 \\
6406.441\end{array}$ & $\begin{array}{r}3 \\
1 \\
1 \\
5 \\
75\end{array}$ & $\begin{array}{l}4 \mathrm{w} \\
3\end{array}$ & $\begin{array}{l}\text { I } \\
\text { I } \\
\text { II } \\
\text { I } \\
\text { I }\end{array}$ & $\begin{array}{l}6431.692 \\
6432.175 \\
6432.928 \\
6433.594 \\
6433.956\end{array}$ & $\begin{array}{l}2 \\
1 \\
3 \\
1 \mathrm{~h} \\
1\end{array}$ & $\begin{array}{l}3 \\
1\end{array}$ & $\begin{array}{l}\text { I } \\
\text { I } \\
\text { II } \\
\text { I } \\
\text { II }\end{array}$ \\
\hline $\begin{array}{l}6380.465 \\
6381.358 \\
6381.751 \\
6381.957 \\
6382.790\end{array}$ & $\begin{array}{l}1 \\
6 \\
3 \\
3 \\
1\end{array}$ & 15 & $\begin{array}{l}\text { II } \\
\text { I } \\
\text { I } \\
\text { II }\end{array}$ & $\begin{array}{l}6407.549 \\
6408.595 \\
6409.114 \\
6409.542 \\
6409.847\end{array}$ & $\begin{array}{c}2 \\
15 \\
1 \\
1 \\
1 w\end{array}$ & $\begin{array}{r}20 \\
1\end{array}$ & $\begin{array}{l}\text { I } \\
\text { II } \\
\text { II } \\
\text { I } \\
\text { I }\end{array}$ & $\begin{array}{l}6434.616 \\
6434.819 \\
6435.065 \\
6436.278 \\
6436.437\end{array}$ & $\begin{array}{l}1 \\
1 \\
1 \\
1 \\
1 \\
1\end{array}$ & $\begin{array}{l}1 \\
1\end{array}$ & $\begin{array}{l}\text { I } \\
\text { II } \\
\text { I } \\
\text { II } \\
\text { I }\end{array}$ \\
\hline $\begin{array}{l}6383.220 \\
6383.325 \\
6384.077 \\
6385.046 \\
6385.455\end{array}$ & $\begin{array}{l}5 \\
5 \\
2 \\
3 \\
1\end{array}$ & $\begin{array}{r}20 \\
20 \\
4 \\
3\end{array}$ & $\begin{array}{l}\text { II } \\
\text { II } \\
\text { II } \\
\text { I I } \\
\text { I }\end{array}$ & $\begin{array}{l}6410.969 \\
6411.900 i \\
6412.645 \\
6413.6151 \\
6414.108\end{array}$ & $\begin{array}{r}4 \\
250 \\
1 \\
200 \\
7\end{array}$ & $\begin{array}{r}8 \\
20 \\
1 \\
15 \\
3\end{array}$ & $\begin{array}{l}\text { II } \\
\text { I } \\
\text { II } \\
\text { I } \\
\text { II }\end{array}$ & $\begin{array}{l}6436.634 \\
6437.752 \\
6438.297 \\
6438.378 \\
6438.664\end{array}$ & $\begin{array}{l}2 \\
50 \\
2 \mathrm{w} \\
5 \\
1\end{array}$ & $\begin{array}{l}1 \\
3 \\
1\end{array}$ & $\begin{array}{l}\text { II } \\
\text { I } \\
\text { I } \\
\text { I } \\
\text { I I }\end{array}$ \\
\hline
\end{tabular}


Table 1. Wavelengths of Thorium Spectra - Continued

\begin{tabular}{|c|c|c|c|c|c|c|c|c|c|c|c|}
\hline \multirow{2}{*}{$\begin{array}{l}\text { Wave- } \\
\text { length }\end{array}$} & \multicolumn{2}{|c|}{ Intensity } & \multirow{2}{*}{$\begin{array}{l}\text { Spec- } \\
\text { trum }\end{array}$} & \multirow{2}{*}{$\begin{array}{l}\text { Wave- } \\
\text { length }\end{array}$} & \multicolumn{2}{|c|}{ Intensity } & \multirow{2}{*}{$\begin{array}{l}\text { Spec- } \\
\text { trum }\end{array}$} & \multirow{2}{*}{$\begin{array}{l}\text { Wave- } \\
\text { length }\end{array}$} & \multicolumn{2}{|c|}{ Intensity } & \multirow{2}{*}{$\begin{array}{l}\text { Spec } \\
\text { trum }\end{array}$} \\
\hline & Lamp & Spark & & & Lamp & Spark & & & Lamp & Spark t & \\
\hline $\begin{array}{l}6438.902 \\
6439.026 \\
6439.726 \\
6440.334 \\
6441.262\end{array}$ & $\begin{array}{r}15 \\
20 \\
1 \\
2 \\
5\end{array}$ & $\begin{array}{r}50 \\
2\end{array}$ & $\begin{array}{l}\text { I } \\
\text { I I } \\
\text { I I } \\
\text { I } \\
\text { I }\end{array}$ & $\begin{array}{l}6477.290 \\
6477.694 \\
6478.612 \\
6478.725 \\
6478.945\end{array}$ & $\begin{array}{l}1 \\
1 \\
1 \\
1 \\
2\end{array}$ & 12 & $\begin{array}{l}\text { I } \\
\text { I } \\
\text { I } \\
\text { I } \\
\text { I I }\end{array}$ & $\begin{array}{l}6503.868 \\
6503.972 \\
6504.214 \\
6504.422 \\
6505.040\end{array}$ & $\begin{array}{l}1 \\
1 \\
3 \\
2 \mathrm{w} \\
1\end{array}$ & 1 & $\begin{array}{l}\text { I } \\
\text { I } \\
\text { I } \\
\text { I } \\
\text { I I }\end{array}$ \\
\hline $\begin{array}{l}6442.941 \\
6443.064 \\
6443.812 \\
6444.106 \\
6444.750\end{array}$ & $\begin{array}{l}1 \\
1 \\
9 \\
1 \\
1\end{array}$ & $\begin{array}{l}2 \\
2\end{array}$ & $\begin{array}{l}\text { I I } \\
\text { I } \\
\text { I } \\
\text { I } \\
\text { I }\end{array}$ & $\begin{array}{l}6479.530 \\
6479.950 \\
6480.432 \\
6480.611 \\
6481.293\end{array}$ & $\begin{array}{r}2 \\
2 \\
1 \\
5 \\
12\end{array}$ & $\begin{array}{l}15 \\
10\end{array}$ & $\begin{array}{l}\text { I } \\
\text { I } \\
\text { I } \\
\text { I I } \\
\text { I I }\end{array}$ & $\begin{array}{l}6505.700 \\
6505.930 \\
6506.972 \\
6507.544 \\
6507.929\end{array}$ & $\begin{array}{c}1 w \\
1 \\
50 \\
6 \\
3\end{array}$ & $\begin{array}{l}3 \\
1 w\end{array}$ & $\begin{array}{l}\text { I } \\
\text { I } \\
\text { I } \\
\text { II } \\
\text { I }\end{array}$ \\
\hline $\begin{array}{l}6444.921 \\
6445.625 \\
6446.124 \\
6446.756 \\
6447.961\end{array}$ & $\begin{array}{r}1 \\
4 \\
5 \\
50 \\
1\end{array}$ & 3 & $\begin{array}{l}\text { I I } \\
\text { I } \\
\text { I } \\
\text { I } \\
\text { I }\end{array}$ & $\begin{array}{l}6481.826 \\
6482.492 \\
6482.908 \\
6483.616 \\
6483.740\end{array}$ & $\begin{array}{l}1 \\
1 \\
1 \\
3 \mathrm{w} \\
2\end{array}$ & & $\begin{array}{l}\text { I } \\
\text { I } \\
\text { I } \\
\text { I } \\
\text { I }\end{array}$ & $\begin{array}{l}6508.356 \\
6509.044 \\
6509.848 \\
6510.250 \\
6510.684\end{array}$ & $\begin{array}{r}5 \\
50 \\
1 \\
3 \\
2\end{array}$ & $\begin{array}{l}2 \\
2\end{array}$ & $\begin{array}{l}\text { I I } \\
\text { I } \\
\text { I } \\
\text { I } \\
\text { I }\end{array}$ \\
\hline $\begin{array}{l}6448.294 \\
6448.704 \\
6449.994 \\
6450.338 \\
6450.934\end{array}$ & $\begin{array}{r}1 \\
3 \\
50 \\
15 \\
30\end{array}$ & $\begin{array}{r}15 \\
3 \\
6 \\
3\end{array}$ & $\begin{array}{l}\text { I } \\
\text { II } \\
\text { I } \\
\text { II } \\
\text { I }\end{array}$ & $\begin{array}{l}6484.067 \\
6484.799 \\
6485.119 \\
6485.369 \\
6486.840\end{array}$ & $\begin{array}{l}3 \\
2 \\
1 \\
2 \\
1\end{array}$ & 8 & $\begin{array}{l}\text { I } \\
\text { I } \\
\text { I } \\
\text { I I } \\
\text { I }\end{array}$ & $\begin{array}{l}6510.992 \\
6511.239 \\
6511.348 \\
6512.364 \\
6513.029\end{array}$ & $\begin{array}{r}4 \\
3 \\
5 \\
75 \\
3\end{array}$ & $\begin{array}{r}20 \\
3 \\
25 \\
3\end{array}$ & $\begin{array}{l}\text { II } \\
\text { I I } \\
\text { I I } \\
\text { I } \\
\text { I }\end{array}$ \\
\hline $\begin{array}{l}6452.030 \\
6452.938 \\
6453.327 \\
6453.530 \\
6454.594\end{array}$ & $\begin{array}{l}5 \\
7 \\
2 \\
1 \\
1 \mathrm{~s}\end{array}$ & $\begin{array}{l}2 \\
1\end{array}$ & $\begin{array}{l}\text { I I } \\
\text { I } \\
\text { I } \\
\text { I } \\
\text { I }\end{array}$ & $\begin{array}{l}6486.974 \\
6487.099 \\
6487.477 \\
6488.879 \\
6490.582\end{array}$ & $\begin{array}{l}1 \\
1 \\
9 \\
9\end{array}$ & 10 & $\begin{array}{l}\text { I } \\
\text { I } \\
\text { I } \\
\text { I } \\
\text { I I }\end{array}$ & $\begin{array}{l}6513.452 \\
6514.492 \\
6514.683 \\
6515.130 \\
6515.847\end{array}$ & $\begin{array}{l}1 \\
1 \\
2 \\
1 \\
2\end{array}$ & 1 & $\begin{array}{l}\text { I } \\
\text { I } \\
\text { I } \\
\text { I } \\
\text { I }\end{array}$ \\
\hline $\begin{array}{l}6455.249 \\
6457.283 \mathrm{i} \\
6459.103 \\
6459.509 \\
6460.016\end{array}$ & $\begin{array}{r}7 \\
500 \\
2 \\
1 \\
1\end{array}$ & $\begin{array}{r}1 \\
70\end{array}$ & $\begin{array}{l}\text { I } \\
\text { I } \\
\text { I } \\
\text { I } \\
\text { I }\end{array}$ & $\begin{array}{l}6490.738 \mathrm{i} \\
6491.234 \\
6491.399 \\
6493.192 \\
6494.236\end{array}$ & $\begin{array}{r}120 \\
1 \\
2 \\
75 \\
1\end{array}$ & $\begin{array}{l}8 \\
2 \\
2 \\
1\end{array}$ & $\begin{array}{l}\text { I } \\
\text { I I } \\
\text { I } \\
\text { I } \\
\text { I I }\end{array}$ & $\begin{array}{l}6516.127 \\
6516.824 \\
6517.256 \\
6517.355 \\
6517.921\end{array}$ & $\begin{array}{l}3 \\
1 \\
1 \\
1 \\
1\end{array}$ & 3 & $\begin{array}{l}\text { I } \\
\text { I } \\
\text { I } \\
\text { II } \\
\text { I }\end{array}$ \\
\hline $\begin{array}{l}6460.474 \\
6461.063 \\
6462.620 \\
6464.839 \\
6465.572\end{array}$ & $\begin{array}{r}3 \\
7 \\
400 \\
1 \\
6\end{array}$ & $\begin{array}{l}100 \mathrm{w} \\
125 \\
200\end{array}$ & $\begin{array}{l}\text { I I I } \\
\text { I I } \\
\text { I I } \\
\text { I } \\
\text { I }\end{array}$ & $\begin{array}{l}6495.243 \\
6495.601 \\
6495.902 \\
6496.011 \\
6496.680\end{array}$ & $\begin{array}{l}5 \\
1 \\
1 \\
3 \\
2\end{array}$ & 1 & $\begin{array}{l}\text { I } \\
\text { I } \\
\text { I } \\
\text { I } \\
\text { I I }\end{array}$ & $\begin{array}{l}6518.036 \\
6518.426 \\
6518.945 \\
6520.008 \\
6520.522\end{array}$ & $\begin{array}{l}1 \\
5 \\
2 \\
1 \\
1\end{array}$ & $\begin{array}{l}4 \\
6 \\
3\end{array}$ & $\begin{array}{l}\text { I I } \\
\text { I I } \\
\text { I I } \\
\text { I } \\
\text { I }\end{array}$ \\
\hline $\begin{array}{l}6466.346 \\
6466.708 \\
6467.144 \\
6467.674 \\
6467.915\end{array}$ & $\begin{array}{l}6 \\
7 \\
4 \\
6 \\
6\end{array}$ & $\begin{array}{l}1 \\
1\end{array}$ & $\begin{array}{l}\text { I } \\
\text { I } \\
\text { I } \\
\text { I } \\
\text { I }\end{array}$ & $\begin{array}{l}6496.947 \\
6497.483 \\
6497.765 \\
6497.993 \\
6498.188\end{array}$ & $\begin{array}{l}2 \\
7 \\
1 \\
1 \\
1\end{array}$ & $2 w$ & $\begin{array}{l}\text { I } \\
\text { I } \\
\text { I } \\
\text { I } \\
\text { I I }\end{array}$ & $\begin{array}{l}6520.944 \\
6522.037 \\
6522.492 \\
6523.569 \\
6523.902\end{array}$ & $\begin{array}{r}4 \\
40 \\
9 \\
2 \\
1\end{array}$ & $\begin{array}{r}12 \\
8\end{array}$ & $\begin{array}{l}\text { I I } \\
\text { I I } \\
\text { I } \\
\text { I } \\
\text { II }\end{array}$ \\
\hline $\begin{array}{l}6468.100 \\
6471.216 \\
6471.939 \\
6472.965 \\
6473.412\end{array}$ & $\begin{array}{r}1 \\
20 \\
3 \\
2 \\
2\end{array}$ & $\begin{array}{r}3 \\
150^{3} \\
3 \\
1\end{array}$ & $\begin{array}{l}\text { I I } \\
\text { I I } \\
\text { I } \\
\text { I I } \\
\text { I I }\end{array}$ & $\begin{array}{l}6498.348 \\
6498.762 \\
6499.143 \\
6499.642 \\
6499.846\end{array}$ & $\begin{array}{l}l \\
4 w \\
l w \\
l \\
l w\end{array}$ & $2 w$ & $\begin{array}{l}\text { I } \\
\text { I } \\
\text { I } \\
\text { I } \\
\text { I I }\end{array}$ & $\begin{array}{l}6524.483 \\
6525.053 \\
6525.458 \\
6525.856 \\
6526.571\end{array}$ & $\begin{array}{l}6 \\
1 \\
1 \\
1 \\
5\end{array}$ & 1 & $\begin{array}{l}\text { I } \\
\text { I } \\
\text { I } \\
\text { I } \\
\text { I I }\end{array}$ \\
\hline $\begin{array}{l}6474.793 \\
6475.904 \\
6476.353 \\
6476.657 \\
6476.791\end{array}$ & $\begin{array}{l}40 \\
2 \\
1 \mathrm{w} \\
1 \\
1 \mathrm{w}\end{array}$ & $\begin{array}{l}4 \\
6\end{array}$ & $\begin{array}{l}\text { I I } \\
\text { I I } \\
\text { I } \\
\text { I } \\
\text { I }\end{array}$ & $\begin{array}{l}6500.312 \\
6500.648 \\
6501.404 \\
6501.988 \\
6503.503\end{array}$ & $\begin{array}{l}1 \\
10 \\
1 \mathrm{~W} \\
25 \\
15\end{array}$ & $\begin{array}{r}1 \\
1 \\
2 \\
125\end{array}$ & $\begin{array}{l}\text { I I } \\
\text { I } \\
\text { I } \\
\text { II } \\
\text { I I }\end{array}$ & $\begin{array}{l}6526.898 \\
6527.324 \\
6527.710 \\
6527.936 \\
6528.555\end{array}$ & $\begin{array}{l}6 \\
1 \\
1 \\
1 \\
1\end{array}$ & $\begin{array}{l}1 \\
1 \\
1 \\
2\end{array}$ & $\begin{array}{l}\text { I } \\
\text { I I } \\
\text { I } \\
\text { II } \\
\text { II }\end{array}$ \\
\hline
\end{tabular}


Table 1. Wavelengths of Thorium Spectra - Continued

\begin{tabular}{|c|c|c|c|c|c|c|c|c|c|c|c|}
\hline \multirow{2}{*}{$\begin{array}{l}\text { Wave- } \\
\text { length }\end{array}$} & \multicolumn{2}{|c|}{ Intensity } & \multirow{2}{*}{$\begin{array}{l}\text { Spec- } \\
\text { trum }\end{array}$} & \multirow{2}{*}{$\begin{array}{l}\text { Wave- } \\
\text { length }\end{array}$} & \multicolumn{2}{|c|}{ Intensity } & \multirow{2}{*}{$\begin{array}{l}\text { Spec- } \\
\text { trum }\end{array}$} & \multirow{2}{*}{$\begin{array}{l}\text { Wave- } \\
\text { length }\end{array}$} & \multicolumn{2}{|c|}{ Intensity } & \multirow{2}{*}{$\begin{array}{l}\text { Spec- } \\
\text { trum }\end{array}$} \\
\hline & Lamp & Spark & & & Lamp & Spark & & & Lam & Spark & \\
\hline $\begin{array}{l}6529.089 \\
6529.156 \\
6529.591 \\
6529.764 \\
6530.129\end{array}$ & $\begin{array}{l}1 \\
1 \\
5 \\
4 \\
7\end{array}$ & 40 & $\begin{array}{l}\text { I I } \\
\text { I } \\
\text { I } \\
\text { I I } \\
\text { I }\end{array}$ & $\begin{array}{l}6556.961 \\
6558.564 \\
6558.881 \\
6559.088 \\
6559.205\end{array}$ & $\begin{array}{l}2 w \\
2 \\
10 \\
5 \\
12\end{array}$ & $\begin{array}{r}2 \\
5 \\
10\end{array}$ & $\begin{array}{l}\text { II } \\
\text { II } \\
\text { I } \\
\text { II } \\
\text { I }\end{array}$ & $\begin{array}{l}6583.907 \mathrm{i} \\
6584.226 \\
6584.369 \\
6584.598 \\
6585.676\end{array}$ & $\begin{array}{r}200 \\
5 \\
10 \\
15 \\
10\end{array}$ & $\begin{array}{l}18 \\
2 \mathrm{w} \\
1 \\
1\end{array}$ & $\begin{array}{l}\text { I } \\
\text { I } \\
\text { I I } \\
\text { I } \\
\text { I }\end{array}$ \\
\hline $\begin{array}{l}6531.342 \mathrm{i} \\
6531.758 \\
6532.209 \\
6532.238 \\
6533.401\end{array}$ & $\begin{array}{r}400 \\
2 \\
12 \\
1\end{array}$ & $\begin{array}{l}40 \\
10 \\
40\end{array}$ & $\begin{array}{l}\text { I } \\
\text { I I } \\
\text { I } \\
\text { I I I } \\
\text { I }\end{array}$ & $\begin{array}{l}6559.735 \\
6560.066 \\
6561.223 \\
6561.446 \\
6561.536\end{array}$ & $\begin{array}{l}2 \\
8 \\
1 \\
1 \\
1\end{array}$ & $\begin{array}{r}10 \\
2\end{array}$ & $\begin{array}{l}\text { I } \\
\text { II } \\
\text { II } \\
\text { I } \\
\text { I }\end{array}$ & $\begin{array}{l}6585.992 \\
6586.592 \\
6587.266 \\
6588.5401 \\
6591.176\end{array}$ & $\begin{array}{r}3 \\
2 \\
1 \\
200 \\
40\end{array}$ & $\begin{array}{r}12 \\
1 \\
25 \\
25\end{array}$ & $\begin{array}{l}\text { I I } \\
\text { I } \\
\text { I I } \\
\text { I } \\
\text { I }\end{array}$ \\
\hline $\begin{array}{l}6533.581 \\
6533.774 \\
6534.235 \\
6534.590 \\
6535.484\end{array}$ & $\begin{array}{l}1 \\
1 \\
1 \\
6 \\
1\end{array}$ & $\frac{1}{2}$ & $\begin{array}{l}\text { I } \\
\text { I } \\
\text { I I } \\
\text { I I } \\
\text { I }\end{array}$ & $\begin{array}{l}6562.100 \\
6562.622 \\
6563.103 \\
6563.649 \\
6563.919\end{array}$ & $\begin{array}{l}6 \\
2 \\
2 \\
1 \\
2\end{array}$ & & $\begin{array}{l}\text { I } \\
\text { I } \\
\text { I } \\
\text { I } \\
\text { I }\end{array}$ & $\begin{array}{l}6591.485 i \\
6591.794 \\
6592.214 \\
6593.466 \\
6593.604\end{array}$ & $\begin{array}{r}100 \\
2 \\
1 \\
40 \\
7\end{array}$ & $\begin{array}{l}7 \\
3\end{array}$ & $\begin{array}{l}\text { I } \\
\text { I I } \\
\text { I } \\
\text { I } \\
\text { I I }\end{array}$ \\
\hline $\begin{array}{l}6535.644 \\
6536.130 \\
6536.448 \\
6537.167 \\
6537.599\end{array}$ & $\begin{array}{r}2 \\
7 \\
1 \\
5 \\
10\end{array}$ & $\begin{array}{l}5 \\
1 \\
1 \\
2\end{array}$ & $\begin{array}{l}\text { II } \\
\text { I } \\
\text { I I } \\
\text { I } \\
\text { I }\end{array}$ & $\begin{array}{l}6564.434 \\
6565.053 \\
6566.174 \\
6566.918 \\
6567.142\end{array}$ & $\begin{array}{l}30 \\
10 \\
3 \\
2 \\
1 \mathrm{w}\end{array}$ & & $\begin{array}{l}\text { I } \\
\text { I } \\
\text { I } \\
\text { I } \\
\text { I }\end{array}$ & $\begin{array}{l}6593.940 \mathrm{i} \\
6594.421 \\
6594.869 \\
6595.450 \\
6596.090\end{array}$ & $\begin{array}{r}200 \\
4 \\
2 \\
4 \\
2\end{array}$ & $\begin{array}{r}20 \\
2 \\
2\end{array}$ & $\begin{array}{l}\text { I } \\
\text { I } \\
\text { I I } \\
\text { I } \\
\text { I I }\end{array}$ \\
\hline $\begin{array}{l}6538.290 \\
6539.178 \\
6539.366 \\
6539.607 \\
6539.837\end{array}$ & $\begin{array}{l}7 \\
1 \\
1 \\
1 \\
1\end{array}$ & 1 & $\begin{array}{l}\text { I } \\
\text { I } \\
\text { I } \\
\text { I I } \\
\text { I }\end{array}$ & $\begin{array}{l}6567.584 \\
6567.842 \\
6568.396 \\
6568.666 \\
6569.058\end{array}$ & $\begin{array}{l}1 \\
1 \\
2 \\
1 \\
4\end{array}$ & & $\begin{array}{l}\text { I } \\
\text { I } \\
\text { I } \\
\text { I } \\
\text { I }\end{array}$ & $\begin{array}{l}6596.949 \\
6597.371 \\
6597.999 \\
6598.253 \\
6599.135\end{array}$ & $\begin{array}{l}6 \\
5 \\
1 \\
1 \\
2 \mathrm{w}\end{array}$ & & $\begin{array}{l}\text { I } \\
\text { I } \\
\text { I } \\
\text { I } \\
\text { I }\end{array}$ \\
\hline $\begin{array}{l}6540.112 \\
6540.530 \\
6541.054 \\
6541.764 \\
6542.035\end{array}$ & $\begin{array}{l}3 \\
3 \\
2 \\
2 \\
6\end{array}$ & $\begin{array}{l}1 \\
1 \\
1\end{array}$ & $\begin{array}{l}\text { I I } \\
\text { I } \\
\text { I I } \\
\text { I } \\
\text { I }\end{array}$ & $\begin{array}{l}6569.614 \\
6570.926 \\
6571.327 \\
6572.005 \\
6572.650\end{array}$ & $\begin{array}{l}25 \\
1 \mathrm{w} \\
2 \\
4 \mathrm{~s} \\
1\end{array}$ & $\begin{array}{r}30 \\
3\end{array}$ & $\begin{array}{l}\text { I I } \\
\text { I } \\
\text { I I } \\
\text { I } \\
\text { I }\end{array}$ & $\begin{array}{l}6599.287 \\
6600.086 \\
6600.716 \\
6601.098 \\
6601.421\end{array}$ & $\begin{array}{l}1 \\
5 \\
3 \\
1\end{array}$ & 600 & $\begin{array}{l}\text { I I I } \\
\text { I } \\
\text { I } \\
\text { I I } \\
\text { I }\end{array}$ \\
\hline $\begin{array}{l}6542.499 \\
6543.116 \\
6543.538 \\
6544.054 \\
6544.572\end{array}$ & $\begin{array}{l}5 \\
1 \\
2 \\
1 \\
5\end{array}$ & 10 & $\begin{array}{l}\text { I I } \\
\text { I } \\
\text { I } \\
\text { I } \\
\text { I I }\end{array}$ & $\begin{array}{l}6572.878 \\
6573.457 \\
6573.964 \\
6574.547 \\
6575.118\end{array}$ & $\begin{array}{l}6 \\
5 \\
5 \\
1 w \\
1\end{array}$ & $\begin{array}{r}30 \\
8 \\
9 \\
1\end{array}$ & $\begin{array}{l}\text { I I } \\
\text { I I } \\
\text { I I } \\
\text { I } \\
\text { I I }\end{array}$ & $\begin{array}{l}6601.525 \\
6602.752 \\
6603.026 \\
6603.613 \\
6604.093\end{array}$ & $\begin{array}{l}1 \\
9 \\
2 \\
6 \\
5\end{array}$ & $\begin{array}{r}1 \\
1 \\
20\end{array}$ & $\begin{array}{l}\text { I I } \\
\text { I } \\
\text { I } \\
\text { I } \\
\text { I I }\end{array}$ \\
\hline $\begin{array}{l}6545.706 \\
6546.636 \\
6545.751 \\
6547.516 \\
6548.512\end{array}$ & $\begin{array}{r}20 \\
1 \\
1 \\
1 \\
3\end{array}$ & $\begin{array}{l}2 \\
2 \\
1\end{array}$ & $\begin{array}{l}\text { I } \\
\text { I I } \\
\text { I I } \\
\text { I } \\
\text { I }\end{array}$ & $\begin{array}{l}6576.115 \\
6576.803 \\
6577.210 \\
6577.664 \\
6578.492\end{array}$ & $\begin{array}{r}25 \\
50 \\
75 \\
1\end{array}$ & $\begin{array}{r}4 \\
3 \\
125\end{array}$ & $\begin{array}{l}\text { I } \\
\text { I I } \\
\text { I } \\
\text { II } \\
\text { I }\end{array}$ & $\begin{array}{l}6605.436 \\
6606.071 \\
6606.583 \\
6606.774 \\
6607.113\end{array}$ & $\begin{array}{r}30 \\
2 \\
4 \\
4 \\
1\end{array}$ & 100 & $\begin{array}{l}\text { I I } \\
\text { I } \\
\text { I } \\
\text { I } \\
\text { I }\end{array}$ \\
\hline $\begin{array}{l}6548.932 \\
6549.556 \\
6549.654 \\
6549.800 \\
6550.186\end{array}$ & $\begin{array}{l}2 \mathrm{w} \\
1 \\
1 \\
1 \\
6\end{array}$ & 3 & $\begin{array}{l}\text { I } \\
\text { I I } \\
\text { I } \\
\text { I } \\
\text { I }\end{array}$ & $\begin{array}{l}6578.903 \\
6579.462 \\
6579.863 \\
6580.213 \\
6581.267\end{array}$ & $\begin{array}{l}2 \\
5 \\
2 \\
9 \\
2\end{array}$ & $\begin{array}{l}1 \\
1\end{array}$ & $\begin{array}{l}\text { I } \\
\text { I } \\
\text { I I } \\
\text { I } \\
\text { I }\end{array}$ & $\begin{array}{l}6607.378 \\
6607.926 \\
6608.440 \\
6608.765 \\
6609.021\end{array}$ & $\begin{array}{l}1 \\
1 \\
5 \\
1 \\
2\end{array}$ & $\begin{array}{l}5 \\
1 \\
1 \mathrm{~h}\end{array}$ & $\begin{array}{l}\text { I I } \\
\text { I I } \\
\text { I I } \\
\text { I } \\
\text { I }\end{array}$ \\
\hline $\begin{array}{l}6551.269 \\
6551.707 \\
6552.962 \\
6554.161 \mathrm{i} \\
6555.523\end{array}$ & $\begin{array}{c}1 \mathrm{w} \\
30 \\
1 \mathrm{w} \\
100 \\
1 \mathrm{w}\end{array}$ & $\frac{1}{2}$ & $\begin{array}{l}\text { I I } \\
\text { I } \\
\text { I } \\
\text { I } \\
\text { I I }\end{array}$ & $\begin{array}{l}6582.383 \\
6582.579 \\
6583.180 \\
6583.332 \\
6583.558\end{array}$ & $\begin{array}{r}1 \\
1 \\
2 \\
2 \\
15\end{array}$ & 15 & $\begin{array}{l}\text { I } \\
\text { I } \\
\text { II } \\
\text { I } \\
\text { II }\end{array}$ & $\begin{array}{l}6609.347 \\
6609.659 \\
6610.286 \\
6611.197 \\
6611.816\end{array}$ & $\begin{array}{l}1 \\
1 \\
3 \mathrm{~s} \\
6 \\
2\end{array}$ & $\begin{array}{r}5 \\
20\end{array}$ & $\begin{array}{l}\text { I } \\
\text { I } \\
\text { II } \\
\text { I I } \\
\text { I }\end{array}$ \\
\hline
\end{tabular}


Table 1. Wavelengths of Thorium Spectra - Continued

\begin{tabular}{|c|c|c|c|c|c|c|c|c|c|c|c|}
\hline \multirow{2}{*}{$\begin{array}{l}\text { Wave- } \\
\text { length }\end{array}$} & \multicolumn{2}{|c|}{ Intensity } & \multirow{2}{*}{$\begin{array}{l}\text { Spec } \\
\text { trum }\end{array}$} & \multirow{2}{*}{$\begin{array}{l}\text { Wave- } \\
\text { length }\end{array}$} & \multicolumn{2}{|c|}{ Intensity } & \multirow{2}{*}{$\begin{array}{l}\text { Spec- } \\
\text { trum }\end{array}$} & \multirow{2}{*}{$\begin{array}{l}\text { Wave- } \\
\text { length }\end{array}$} & \multicolumn{2}{|c|}{ Intensity } & \multirow{2}{*}{$\begin{array}{l}\text { Spec } \\
\text { trum }\end{array}$} \\
\hline & Lamp & Spark & & & Lamp & Spark & & & Lamp & Spark & \\
\hline $\begin{array}{l}6612.415 \\
6612.780 \\
6613.376 \\
6615.071 \\
6615.327\end{array}$ & $\begin{array}{r}1 \\
1 \\
30 \\
4 \\
3\end{array}$ & $\begin{array}{l}1 \\
2\end{array}$ & $\begin{array}{l}\text { I } \\
\text { I } \\
\text { I } \\
\text { I } \\
\text { I I }\end{array}$ & $\begin{array}{l}6642.789 \\
6643.099 \\
6643.389 \\
6644.027 \\
6644.676\end{array}$ & $\begin{array}{r}1 \\
2 \\
3 \\
5 \\
30\end{array}$ & $\begin{array}{r}30 \\
150\end{array}$ & $\begin{array}{l}\text { I } \\
\text { I } \\
\text { II } \\
\text { I } \\
\text { II }\end{array}$ & $\begin{array}{l}6672.735 \\
6672.931 \\
6673.559 \\
6674.680 \\
6675.453\end{array}$ & $\begin{array}{r}2 \\
1 \\
20 \\
30 \\
2\end{array}$ & $\begin{array}{l}3 \\
2 \\
5\end{array}$ & $\begin{array}{l}\text { I } \\
\text { I } \\
\text { I } \\
\text { I } \\
\text { I I }\end{array}$ \\
\hline $\begin{array}{l}6615.469 \\
6616.081 \\
6617.081 \\
6617.515 \\
6618.168\end{array}$ & $\begin{array}{r}4 \\
1 \\
10 \\
10 \\
40\end{array}$ & $\begin{array}{r}8 \\
75 \\
2\end{array}$ & $\begin{array}{l}\text { I I } \\
\text { I } \\
\text { II } \\
\text { I } \\
\text { I }\end{array}$ & $\begin{array}{l}6645.731 \\
6646.552 \\
6647.696 \\
6648.496 \\
6648.954\end{array}$ & $\begin{array}{r}2 \\
20 \\
4 \\
25 \\
30\end{array}$ & $\begin{array}{c}2 w \\
100 \\
8 \\
40\end{array}$ & $\begin{array}{l}\text { I I } \\
\text { I I } \\
\text { I I } \\
\text { I I } \\
\text { I }\end{array}$ & $\begin{array}{l}6676.275 \\
6677.051 \\
6677.597 \\
6678.708 \mathrm{i} \\
6679.207\end{array}$ & $\begin{array}{r}1 \\
1 \\
1 \\
30 \\
3\end{array}$ & $\begin{array}{r}4 \\
3 \\
18\end{array}$ & $\begin{array}{l}\text { I } \\
\text { I } \\
\text { I I } \\
\text { I } \\
\text { I I }\end{array}$ \\
\hline $\begin{array}{l}6619.970 \\
6620.397 \\
6620.861 \\
6621.145 \\
6622.106\end{array}$ & $\begin{array}{r}20 \\
4 \\
1 \\
2 \\
1\end{array}$ & $\begin{array}{r}50 \\
1\end{array}$ & $\begin{array}{l}\text { I I } \\
\text { I } \\
\text { I } \\
\text { I } \\
\text { I }\end{array}$ & $\begin{array}{l}6650.551 \\
6651.003 \\
6651.133 \\
6651.329 \\
6652.022\end{array}$ & $\begin{array}{l}5 \\
2 \\
1 \\
1 \\
1\end{array}$ & $\begin{array}{l}2 \\
2 \\
1\end{array}$ & $\begin{array}{l}\text { I } \\
\text { I I } \\
\text { I I } \\
\text { I } \\
\text { I I }\end{array}$ & $\begin{array}{l}6679.607 \\
6680.059 \\
6680.618 \\
6680.918 \\
6681.457\end{array}$ & $\begin{array}{l}1 \mathrm{w} \\
6 \\
1 \\
1 \\
3\end{array}$ & 1 & $\begin{array}{l}\text { I } \\
\text { I } \\
\text { I } \\
\text { I I } \\
\text { I }\end{array}$ \\
\hline $\begin{array}{l}6622.263 \\
6623.227 \\
6623.452 \\
6623.603 \\
6623.735\end{array}$ & $\begin{array}{l}3 \\
2 \\
1 \\
1 \\
1\end{array}$ & 1 & $\begin{array}{l}\text { I I } \\
\text { I } \\
\text { I } \\
\text { I } \\
\text { I I }\end{array}$ & $\begin{array}{l}6652.403 \\
6652.627 \\
6652.966 \\
6653.803 \\
6654.363\end{array}$ & $\begin{array}{r}1 \\
1 \\
1 \\
2 \\
15\end{array}$ & $\begin{array}{c}1 \\
10 w\end{array}$ & $\begin{array}{l}\text { I I } \\
\text { I } \\
\text { I } \\
\text { I I } \\
\text { I }\end{array}$ & $\begin{array}{l}6682.269 \\
6682.549 \\
6682.801 \\
6683.353 \\
6684.027\end{array}$ & $\begin{array}{r}1 \\
4 \\
1 \\
25 \\
15\end{array}$ & & $\begin{array}{l}\text { I } \\
\text { I } \\
\text { I } \\
\text { I } \\
I\end{array}$ \\
\hline $\begin{array}{l}6624.253 \\
6624.789 \\
6626.115 \\
6626.571 \\
6627.509\end{array}$ & $\begin{array}{l}1 \mathrm{w} \\
1 \\
3 \\
1 \mathrm{w} \\
1\end{array}$ & 1 & $\begin{array}{l}\text { I I } \\
\text { I } \\
\text { I } \\
\text { I } \\
\text { I }\end{array}$ & $\begin{array}{l}6655.055 \\
6655.485 \\
6655.913 \\
6656.856 \\
6657.235\end{array}$ & $\begin{array}{l}2 \\
5 \\
5 \\
2 \\
1\end{array}$ & 1 & $\begin{array}{l}\text { I } \\
\text { I } \\
\text { I } \\
\text { I I } \\
\text { I }\end{array}$ & $\begin{array}{l}6684.661 \\
6685.386 \\
6685.657 \\
6685.959 \\
6686.192\end{array}$ & $\begin{array}{l}1 \\
1 \\
1 \\
1 \\
2\end{array}$ & 4 & $\begin{array}{l}\text { I } \\
\text { I } \\
\text { I } \\
\text { I I } \\
\text { I }\end{array}$ \\
\hline $\begin{array}{l}6628.163 \\
6628.671 \\
6629.229 \\
6630.733 \\
6631.137\end{array}$ & $\begin{array}{l}3 \\
2 w \\
2 \\
1 \\
2\end{array}$ & 5 & $\begin{array}{l}\text { I I } \\
\text { I } \\
\text { I } \\
\text { I } \\
\text { I }\end{array}$ & $\begin{array}{l}6657.861 \\
6658.677 \mathrm{i} \\
6659.428 \\
6660.449 \\
6662.269 \mathrm{i}\end{array}$ & $\begin{array}{r}1 \\
50 \\
1 \\
4 \\
250\end{array}$ & $\begin{array}{r}4 \\
2 \\
7 \\
30\end{array}$ & $\begin{array}{l}\text { I } \\
\text { I } \\
\text { I I } \\
\text { I I } \\
\text { I }\end{array}$ & $\begin{array}{l}6686.615 \\
6687.124 \\
6687.503 \\
6689.147 \\
6689.443\end{array}$ & $\begin{array}{l}1 \mathrm{w} \\
3 \\
12 \\
1 \\
1\end{array}$ & $\begin{array}{l}2 \\
2 \\
2\end{array}$ & $\begin{array}{l}\text { I } \\
\text { I I } \\
\text { I I } \\
\text { I I } \\
\text { I }\end{array}$ \\
\hline $\begin{array}{l}6631.545 \\
6631.787 \\
6632.075 \\
6633.450 \\
6634.033\end{array}$ & $\begin{array}{l}2 \\
1 \\
2 \mathrm{w} \\
5 \\
1\end{array}$ & $\begin{array}{r}2 \\
15\end{array}$ & $\begin{array}{l}\text { I } \\
\text { II } \\
\text { I } \\
\text { II } \\
\text { I }\end{array}$ & $\begin{array}{l}6662.601 \\
6663.671 \\
6664.939 \\
6665.606 \\
6666.443\end{array}$ & $\begin{array}{l}7 \\
5 \\
1 \\
1\end{array}$ & $\begin{array}{l}7 \\
8\end{array}$ & $\begin{array}{l}\text { I I } \\
\text { I } \\
\text { I I } \\
\text { I } \\
\text { I }\end{array}$ & $\begin{array}{l}6689.761 \\
6689.899 \\
6690.249 \\
6690.889 \\
6691.347\end{array}$ & $\begin{array}{l}1 \\
1 \\
1 \\
2 \\
1\end{array}$ & $\begin{array}{l}2 \\
l \mathrm{~h}\end{array}$ & $\begin{array}{l}\text { I } \\
\text { I I } \\
\text { I I } \\
\text { I } \\
\text { I }\end{array}$ \\
\hline $\begin{array}{l}6634.220 \\
6634.763 \\
6635.348 \\
6635.814 \\
6636.267\end{array}$ & $\begin{array}{l}1 w \\
2 \\
2 \\
1 \\
3\end{array}$ & 2 & $\begin{array}{l}\text { I } \\
\text { I } \\
\text { I } \\
\text { I I } \\
\text { I }\end{array}$ & $\begin{array}{l}6666.818 \\
6666.956 \\
6667.257 \\
6667.521 \\
6667.783\end{array}$ & $\begin{array}{l}1 \\
1 \\
1 \\
3 \\
2\end{array}$ & $\begin{array}{l}2 \\
2 \\
2\end{array}$ & $\begin{array}{l}\text { I } \\
\text { I } \\
\text { II } \\
\text { II } \\
\text { II }\end{array}$ & $\begin{array}{l}6692.123 \\
6692.707 \\
6693.187 \\
6693.995 \\
6694.473\end{array}$ & $\begin{array}{r}1 \\
10 \\
4 \\
10 \\
7\end{array}$ & $\begin{array}{r}4 \\
20\end{array}$ & $\begin{array}{l}\text { I I } \\
\text { I I } \\
\text { I } \\
\text { I } \\
\text { I }\end{array}$ \\
\hline $\begin{array}{l}6637.325 \\
6638.918 \\
6639.709 \\
6640.119 \\
6640.367\end{array}$ & $\begin{array}{r}10 \\
100 \\
3 \\
1 \\
1\end{array}$ & $\begin{array}{l}1 \\
3 \\
1 \mathrm{~h}\end{array}$ & $\begin{array}{l}\text { I } \\
\text { I } \\
\text { I } \\
\text { II }\end{array}$ & $\begin{array}{l}6668.131 \\
6668.394 \\
6668.812 \\
6668.911 \\
6669.433\end{array}$ & $\begin{array}{l}2 \\
2 \\
9 \\
5 \\
2\end{array}$ & 2 & $\begin{array}{l}\text { I I } \\
\text { I } \\
\text { I } \\
\text { I } \\
\text { I }\end{array}$ & $\begin{array}{l}6694.653 \\
6695.158 \\
6695.759 \\
6696.135 \\
6697.701\end{array}$ & $\begin{array}{r}1 \\
2 \\
1 \\
20 \\
10\end{array}$ & $\begin{array}{l}2 \\
5\end{array}$ & $\begin{array}{l}\text { II } \\
\text { I } \\
\text { I } \\
\text { I } \\
\text { I }\end{array}$ \\
\hline $\begin{array}{l}6640.985 \\
6641.419 \\
6641.645 \\
6641.910 \\
6642.324\end{array}$ & $\begin{array}{l}2 \\
2 \\
2 \\
1 w \\
6\end{array}$ & & $\begin{array}{l}\text { I } \\
\text { I } \\
\text { I } \\
\text { I } \\
\text { I }\end{array}$ & $\begin{array}{l}6669.723 \\
6669.909 \\
6670.400 \\
6671.487 \\
6671.649\end{array}$ & $\begin{array}{l}1 \\
1 \\
1 \\
1 \\
1\end{array}$ & & $\begin{array}{l}\text { I } \\
\text { I } \\
\text { I } \\
\text { I } \\
\text { I }\end{array}$ & $\begin{array}{l}6698.010 \\
6698.585 \\
6698.930 \\
6699.335 \\
6699.643\end{array}$ & $\begin{array}{c}10 \\
2 \\
1 \\
2 \mathrm{~s} \\
1\end{array}$ & 2 & $\begin{array}{l}\text { I } \\
\text { I } \\
\text { I } \\
\text { I I } \\
\text { I }\end{array}$ \\
\hline
\end{tabular}


Table 1. Wavelengths of Thorium Spectra - Continued

\begin{tabular}{|c|c|c|c|c|c|c|c|c|c|c|c|}
\hline \multirow{2}{*}{$\begin{array}{l}\text { Wave- } \\
\text { length }\end{array}$} & \multicolumn{2}{|c|}{ Intensity } & \multirow{2}{*}{$\begin{array}{l}\text { Spec- } \\
\text { trum }\end{array}$} & \multirow{2}{*}{$\begin{array}{l}\text { Wave- } \\
\text { length }\end{array}$} & \multicolumn{2}{|c|}{ Intensity } & \multirow{2}{*}{$\begin{array}{l}\text { Spec- } \\
\text { trum }\end{array}$} & \multirow{2}{*}{$\begin{array}{l}\text { Wave- } \\
\text { length }\end{array}$} & \multicolumn{2}{|c|}{ Intensity } & \multirow{2}{*}{$\begin{array}{l}\text { Spec } \\
\text { trum }\end{array}$} \\
\hline & amp & Spark & & & Lamp & Spark $t$ & & & Lamp & Spark & \\
\hline $\begin{array}{l}6699.937 \\
6700.485 \\
6700.749 \\
6701.345 \\
6701.851\end{array}$ & $\begin{array}{l}1 \\
3 \\
51 \\
2 \\
1\end{array}$ & 18 & $\begin{array}{l}\text { I } \\
\text { II } \\
\text { I } \\
\text { I } \\
\text { I }\end{array}$ & $\begin{array}{l}6727.459 \mathrm{i} \\
6728.121 \\
6728.771 \\
6729.918 \\
6730.500\end{array}$ & $\begin{array}{l}200 \\
20 \mathrm{~h} \\
15 \\
4 \\
4 \mathrm{w}\end{array}$ & 40 & $\begin{array}{l}\text { I } \\
\text { I } \\
\text { I } \\
\text { I } \\
\text { II }\end{array}$ & $\begin{array}{l}6760.888 \\
6761.757 \\
6762.481 \\
6763.313 \\
6763.550\end{array}$ & $\begin{array}{l}8 \\
1 \\
2 \\
2 \\
1\end{array}$ & 20 & $\begin{array}{l}\text { II } \\
\text { I } \\
\text { I } \\
\text { I } \\
\text { I }\end{array}$ \\
\hline $\begin{array}{l}6703.333 \\
6703.888 \\
6704.042 \\
6704.742 \\
6705.129\end{array}$ & $\begin{array}{l}3 \\
4 \\
8 \\
1 \\
2\end{array}$ & $\begin{array}{r}5 \\
8 \\
30\end{array}$ & $\begin{array}{l}\text { II } \\
\text { II } \\
\text { I I } \\
\text { I } \\
\text { I }\end{array}$ & $\begin{array}{l}6731.038 \\
6731.659 \\
6732.204 \\
6732.637 \\
6733.747\end{array}$ & $\begin{array}{r}2 \\
5 \\
1 \\
9 \\
100\end{array}$ & $\begin{array}{r}2 \\
1 \\
2 \\
1 \\
10\end{array}$ & $\begin{array}{l}\text { II } \\
\text { II } \\
\text { II } \\
\text { I } \\
\text { II }\end{array}$ & $\begin{array}{l}6764.353 \\
6765.693 \\
6766.330 \\
6766.831 \\
6767.528\end{array}$ & $\begin{array}{r}1 \\
80 \\
4 \\
1 \\
1\end{array}$ & $\begin{array}{r}40 \\
7\end{array}$ & $\begin{array}{l}\text { I } \\
\text { I I } \\
\text { I } \\
\text { I } \\
\text { I }\end{array}$ \\
\hline $\begin{array}{l}6705.410 \\
6706.051 \\
6706.422 \\
6706.808 \\
6707.735\end{array}$ & $\begin{array}{l}1 \\
2 \\
1 \\
2 \\
1\end{array}$ & 1 & $\begin{array}{l}\text { I } \\
\text { I } \\
\text { I } \\
\text { I } \\
\text { II }\end{array}$ & $\begin{array}{l}6734.652 \\
6735.122 \\
6735.357 \\
6737.226 \\
6737.283\end{array}$ & $\begin{array}{l}2 \\
5 \\
5 \\
1 \\
2\end{array}$ & & $\begin{array}{l}\text { I } \\
\text { I } \\
\text { I } \\
\text { I } \\
\text { I }\end{array}$ & $\begin{array}{l}6767.943 \\
6768.162 \\
6768.530 \\
6769.367 \\
6770.105\end{array}$ & $\begin{array}{l}2 \\
1 \\
1 \\
3 \\
15\end{array}$ & $\begin{array}{r}4 \\
1 \\
15 \\
50\end{array}$ & $\begin{array}{l}\text { II } \\
\text { II } \\
\text { I } \\
\text { II } \\
\text { II }\end{array}$ \\
\hline $\begin{array}{l}6707.883 \\
6708.091 \\
6708.267 \\
6708.372 \\
6708.818\end{array}$ & $\begin{array}{l}1 \\
1 \mathrm{w} \\
1 \\
1 \\
4\end{array}$ & 1 & $\begin{array}{l}\text { I } \\
\text { II } \\
\text { I } \\
\text { I } \\
\text { II }\end{array}$ & $\begin{array}{l}6737.627 \\
6738.170 \\
6739.109 \\
6739.935 \\
6741.900\end{array}$ & $\begin{array}{l}35 \\
25 \\
1 \mathrm{w} \\
2 \\
5\end{array}$ & $5 w$ & $\begin{array}{l}\text { II } \\
\text { I } \\
\text { I } \\
\text { I } \\
\text { I }\end{array}$ & $\begin{array}{l}6771.331 \\
6772.172 \\
6773.087 \\
6773.756 \\
6774.081\end{array}$ & $\begin{array}{r}1 \\
100 \\
20 \\
2 \\
1\end{array}$ & $\begin{array}{l}2 \\
3 \\
2\end{array}$ & $\begin{array}{l}\text { II } \\
\text { I } \\
\text { I } \\
\text { I } \\
\text { I }\end{array}$ \\
\hline $\begin{array}{l}6710.525 \\
6710.861 \\
6711.226 \\
6711.469 \\
6711.811\end{array}$ & $\begin{array}{r}5 \\
2 \\
20 \\
1 \\
2\end{array}$ & $\begin{array}{l}1 \\
2\end{array}$ & $\begin{array}{l}\text { II } \\
\text { I } \\
\text { I } \\
\text { I } \\
\text { I }\end{array}$ & $\begin{array}{l}6742.488 \\
6742.866 \\
6743.356 \\
6744.030 \\
6744.682\end{array}$ & $\begin{array}{l}3 \\
80 \\
1 \\
1 \\
2\end{array}$ & $\begin{array}{l}5 \\
2 \\
1\end{array}$ & $\begin{array}{l}\text { II } \\
\text { I } \\
\text { I } \\
\text { II } \\
\text { I }\end{array}$ & $\begin{array}{l}6774.387 \\
6775.086 \\
6775.381 \\
6775.825 \\
6776.218\end{array}$ & $\begin{array}{l}2 \\
2 \\
1 \\
1 \\
5\end{array}$ & 1 & $\begin{array}{l}\text { I } \\
\text { I } \\
\text { II } \\
\text { I } \\
\text { I }\end{array}$ \\
\hline $\begin{array}{l}6711.975 \\
6712.179 \\
6712.802 \\
6713.585 \\
6713.960\end{array}$ & $\begin{array}{r}1 \\
2 \\
1 \\
5 \\
100\end{array}$ & 2 & $\begin{array}{l}\text { I } \\
\text { I } \\
\text { I } \\
I \\
\text { I }\end{array}$ & $\begin{array}{l}6745.153 \\
6745.521 \\
6746.112 \\
6746.940 \\
6747.164\end{array}$ & $\begin{array}{l}2 \\
1 \\
3 \\
2 \\
1\end{array}$ & 1 & $\begin{array}{l}\text { I } \\
\text { I } \\
\text { II } \\
\text { I } \\
\text { I }\end{array}$ & $\begin{array}{l}6778.312 \\
6778.807 \\
6779.325 \\
6779.461 \\
6780.078\end{array}$ & $\begin{array}{r}80 \\
1 \\
1 \\
1\end{array}$ & 15 & $\begin{array}{l}\text { I } \\
\text { I } \\
\text { I } \\
\text { I } \\
\text { II I }\end{array}$ \\
\hline $\begin{array}{l}6714.469 \\
6714.663 \\
6715.167 \\
6715.493 \\
6716.201\end{array}$ & $\begin{array}{l}1 \\
2 \\
3 \\
5 \\
1\end{array}$ & 18 & $\begin{array}{l}\text { I } \\
\text { I } \\
\text { II } \\
\text { I } \\
\text { I }\end{array}$ & $\begin{array}{l}6747.945 \\
6748.295 \\
6748.571 \\
6749.295 \\
6749.841\end{array}$ & $\begin{array}{l}1 \\
1 \\
1 \\
3 \\
1\end{array}$ & 2 & $\begin{array}{l}\text { I } \\
\text { I } \\
\text { II } \\
\text { I } \\
\text { I }\end{array}$ & $\begin{array}{l}6780.126 \\
6780.408 \\
6780.885 \\
6781.322 \\
6782.041\end{array}$ & $\begin{array}{r}90 \\
140 \\
2 \\
2 \\
1 \mathrm{w}\end{array}$ & $\begin{array}{l}2 \\
4\end{array}$ & $\begin{array}{l}I \\
I \\
I \\
I \\
I\end{array}$ \\
\hline $\begin{array}{l}6716.937 \\
6717.381 \\
6719.181 \\
6720.143 \\
6720.652\end{array}$ & $\begin{array}{c}2 \\
5 \\
100 \\
1 \mathrm{w} \\
1\end{array}$ & $\frac{1}{1}$ & $\begin{array}{l}\text { I } \\
I \\
I \\
I \\
I\end{array}$ & $\begin{array}{l}6751.412 \\
6753.637 \\
6754.243 \\
6754.600 \\
6754.884\end{array}$ & $\begin{array}{r}10 \\
9 \\
7 \\
2 \\
1\end{array}$ & & $\begin{array}{l}\text { I } \\
\text { I } \\
\text { I } \\
\text { I } \\
\text { I }\end{array}$ & $\begin{array}{l}6782.574 \\
6783.088 \\
6783.417 \\
6783.920 \\
6784.191\end{array}$ & $\begin{array}{l}1 \mathrm{w} \\
1 \mathrm{~h} \\
1 \\
1 \mathrm{w} \\
1\end{array}$ & & $\begin{array}{l}\text { I } \\
\text { I } \\
\text { I } \\
\text { I } \\
\text { I }\end{array}$ \\
\hline $\begin{array}{l}6721.067 \\
6721.589 \\
6721.947 \\
6722.125 \\
6722.791\end{array}$ & $\begin{array}{l}2 \\
1 \\
2 \\
2 \\
1\end{array}$ & 5 & $\begin{array}{l}\text { I } \\
\text { I } \\
\text { I } \\
\text { II } \\
\text { I }\end{array}$ & $\begin{array}{l}6755.486 \\
6756.453 \mathrm{i} \\
6757.098 \\
6757.307 \\
6758.197\end{array}$ & $\begin{array}{r}1 \\
250 \\
20 \\
1 \\
25\end{array}$ & $\begin{array}{r}80 \\
3 \\
2\end{array}$ & $\begin{array}{l}\text { I } \\
\text { I } \\
\text { I } \\
\text { II } \\
\text { I }\end{array}$ & $\begin{array}{l}6784.786 \\
6785.590 \\
6786.353 \\
6787.730 \\
6788.835\end{array}$ & $\begin{array}{r}5 \\
1 \\
2 \\
80 \\
80\end{array}$ & $\begin{array}{l}5 \\
2 \\
2\end{array}$ & $\begin{array}{l}\text { I } \\
\text { I } \\
\text { II } \\
\text { I } \\
\text { I }\end{array}$ \\
\hline $\begin{array}{l}6723.095 \\
6724.143 \\
6724.667 \\
6725.349 \\
6726.307\end{array}$ & $\begin{array}{l}1 \\
1 \\
1 \\
1 \\
9\end{array}$ & $\begin{array}{l}3 \\
1 \\
1\end{array}$ & $\begin{array}{l}\text { I } \\
\text { II } \\
\text { II } \\
\text { II } \\
\text { I }\end{array}$ & $\begin{array}{l}6758.703 \\
6759.413 \\
6759.822 \\
6760.312 \\
6760.661\end{array}$ & $\begin{array}{l}1 \\
2 \\
1 \\
1 \\
3\end{array}$ & $\begin{array}{l}3 w \\
1\end{array}$ & $\begin{array}{l}\text { I } \\
\text { I I } \\
\text { I } \\
\text { I } \\
\text { I }\end{array}$ & $\begin{array}{l}6789.693 \\
6791.226 \\
6792.451 \\
6792.886 \\
6793.711\end{array}$ & $\begin{array}{r}1 \\
80 \\
1 \\
2 \\
1\end{array}$ & & $\begin{array}{l}\text { I } \\
\text { I } \\
\text { I } \\
\text { I } \\
\text { I }\end{array}$ \\
\hline
\end{tabular}


Table 1. Wavelengths of Thorium Spectra - Continued

\begin{tabular}{|c|c|c|c|c|c|c|c|c|c|c|c|}
\hline \multirow{2}{*}{$\begin{array}{l}\text { Wave- } \\
\text { length }\end{array}$} & \multicolumn{2}{|c|}{ Intersity } & \multirow{2}{*}{$\begin{array}{l}\text { Spec- } \\
\text { trum }\end{array}$} & \multirow{2}{*}{$\begin{array}{l}\text { Wave- } \\
\text { length }\end{array}$} & \multicolumn{2}{|c|}{ Intensity } & \multirow{2}{*}{$\begin{array}{l}\text { Spec- } \\
\text { trum }\end{array}$} & \multirow{2}{*}{$\begin{array}{l}\text { Wave- } \\
\text { length }\end{array}$} & \multicolumn{2}{|c|}{ Intensity } & \multirow{2}{*}{$\begin{array}{l}\text { Spec. } \\
\text { trum }\end{array}$} \\
\hline & Lamp & Spark & & & amp & Spark & & & Lamp & Spark & \\
\hline $\begin{array}{l}6794.281 \\
6795.026 \\
6795.782 \\
6796.772 \\
6798.481\end{array}$ & $\begin{array}{l}2 w \\
6 \\
5 \\
6 \\
9\end{array}$ & $\begin{array}{r}12 \\
1\end{array}$ & $\begin{array}{l}\text { I } \\
\text { I } \\
\text { I } \\
\text { II } \\
\text { I }\end{array}$ & $\begin{array}{l}6823.505 \\
6823.862 \\
6824.060 \\
6824.678 \\
6825.397\end{array}$ & $\begin{array}{c}20 \\
1 \mathrm{w} \\
1 \\
100 \\
1\end{array}$ & 2 & $\begin{array}{l}\text { I } \\
\text { I } \\
\text { I } \\
\text { I } \\
\text { I }\end{array}$ & $\begin{array}{l}6862.856 \\
6866.146 \\
6866.359 \\
6866.758 \\
6868.449\end{array}$ & $\begin{array}{r}5 \\
3 \\
10 \\
9 \\
30\end{array}$ & & $\begin{array}{l}\text { I } \\
\text { I } \\
\text { I } \\
\text { I } \\
\text { I }\end{array}$ \\
\hline $\begin{array}{l}6798.728 \\
6799.334 \\
6800.452 \\
6801.044 \\
6801.476\end{array}$ & $\begin{array}{l}3 \\
2 \\
5 \\
1 \\
1\end{array}$ & $\frac{1}{10 w}$ & $\begin{array}{l}\text { I } \\
\text { I } \\
\text { I I } \\
\text { I I I } \\
\text { I }\end{array}$ & $\begin{array}{l}6826.298 \\
6827.252 \\
6827.511 \\
6827.744 \\
6828.187\end{array}$ & $\begin{array}{l}2 \\
1 \\
2 \\
1 \\
1\end{array}$ & & $\begin{array}{l}\text { I } \\
\text { I } \\
\text { I } \\
\text { I } \\
\text { I }\end{array}$ & $\begin{array}{l}6868.617 \\
6869.241 \\
6870.100 \\
6870.209 \\
6870.722\end{array}$ & $\begin{array}{l}2 \\
2 \\
2 w \\
2 w \\
5\end{array}$ & $\begin{array}{l}2 \\
4 \\
1\end{array}$ & $\begin{array}{l}\text { I I } \\
\text { I I } \\
\text { I } \\
\text { I I } \\
\text { I }\end{array}$ \\
\hline $\begin{array}{l}6802.106 \\
6802.416 \\
6802.772 \\
6803.287 \\
6804.000\end{array}$ & $\begin{array}{l}1 \\
1 \\
5 \\
2 \\
1\end{array}$ & & $\begin{array}{l}\text { I } \\
\text { I } \\
\text { I } \\
\text { I } \\
\text { I }\end{array}$ & $\begin{array}{l}6828.615 \\
6829.0361 \\
6829.288 \\
6829.926 \\
6831.228\end{array}$ & $\begin{array}{r}1 \\
150 \\
4 \\
2 \\
1\end{array}$ & 10 & $\begin{array}{l}\text { I } \\
\text { I } \\
\text { I } \\
\text { I } \\
\text { I }\end{array}$ & $\begin{array}{l}6870.961 \\
6871.240 \\
6871.464 \\
6872.220 \\
6873.031\end{array}$ & $\begin{array}{l}7 \\
1 \\
1 \\
1 \\
1 w\end{array}$ & & $\begin{array}{l}\text { I } \\
\text { I } \\
\text { I } \\
\text { I } \\
\text { II }\end{array}$ \\
\hline $\begin{array}{l}6804.726 \\
6805.461 \\
6805.735 \\
6806.564 \\
6807.034\end{array}$ & $\begin{array}{l}7 \\
3 \\
3 \\
1 \\
1 w\end{array}$ & 12 & $\begin{array}{l}\text { II } \\
\text { I } \\
\text { I } \\
\text { I } \\
\text { I }\end{array}$ & $\begin{array}{l}6832.211 \\
6832.884 \\
6833.319 \\
6834.281 \\
6834.9251\end{array}$ & $\begin{array}{c}2 \mathrm{w} \\
10 \\
1 \\
1 \\
75\end{array}$ & $\begin{array}{r}-3 \\
1\end{array}$ & $\begin{array}{l}\text { I } \\
\text { II } \\
\text { I } \\
\text { I } \\
\text { I }\end{array}$ & $\begin{array}{l}6874.086 \\
6874.749 \\
6876.288 \\
6877.869 \\
6878.041\end{array}$ & $\begin{array}{r}7 \\
15 \\
4 \\
4 \\
20\end{array}$ & 2 & $\begin{array}{l}\text { I } \\
\text { I } \\
\text { I } \\
\text { II } \\
\text { I }\end{array}$ \\
\hline $\begin{array}{l}6807.305 \\
6807.807 \\
6808.336 \\
6808.532 \\
6809.083\end{array}$ & $\begin{array}{r}5 \\
1 \\
1 \\
1 \\
10\end{array}$ & & $\begin{array}{l}\text { I } \\
\text { I } \\
\text { I } \\
\text { I } \\
\text { I }\end{array}$ & $\begin{array}{l}6835.272 \\
6835.395 \\
6835.616 \\
6835.897 \\
6836.200\end{array}$ & $\begin{array}{l}1 \\
1 \\
1 \\
2 \\
1\end{array}$ & & $\begin{array}{l}\text { I } \\
\text { I } \\
\text { I } \\
\text { I } \\
\text { I }\end{array}$ & $\begin{array}{l}6878.379 \\
6880.190 \\
6882.807 \\
6883.102 \\
6883.332\end{array}$ & $\begin{array}{r}2 \\
50 \\
5 \\
50\end{array}$ & $2 w$ & $\begin{array}{l}\text { I } \\
\text { I I I } \\
\text { I } \\
\text { I } \\
\text { I }\end{array}$ \\
\hline $\begin{array}{l}6809.300 \\
6809.491 \\
6809.655 \\
6810.537 \\
6811.479\end{array}$ & $\begin{array}{r}6 \\
2 \\
7 \\
15 \\
1\end{array}$ & $\begin{array}{l}20 \\
2 w\end{array}$ & $\begin{array}{l}\text { I } \\
\text { I } \\
\text { I } \\
\text { II } \\
\text { II }\end{array}$ & $\begin{array}{l}6836.846 \\
6837.069 \\
6837.482 \\
6838.893 \\
6839.271\end{array}$ & $\begin{array}{l}1 \\
2 \\
2 \\
1 \\
3\end{array}$ & & $\begin{array}{l}\text { I } \\
\text { I } \\
\text { I } \\
\text { I } \\
\text { I }\end{array}$ & $\begin{array}{l}6883.822 \\
6886.399 \\
6888.145 \\
6888.699 \\
6888.829\end{array}$ & $\begin{array}{r}4 \\
50 \\
6 \\
4 \\
16\end{array}$ & & $\begin{array}{l}\text { I } \\
\text { I } \\
\text { I } \\
\text { I } \\
\text { I }\end{array}$ \\
\hline $\begin{array}{l}6811.949 \\
6812.496 \\
6812.767 \\
6813.528 \\
6813.604\end{array}$ & $\begin{array}{r}2 \\
25 \\
15 \\
1 \\
1\end{array}$ & 25 & $\begin{array}{l}\text { I } \\
\text { I } \\
\text { II } \\
\text { I } \\
\text { I }\end{array}$ & $\begin{array}{l}6840.632 \\
6841.681 \\
6842.717 \\
6845.853 \\
6846.449\end{array}$ & $\begin{array}{l}1 \\
1 \\
1 w \\
1 \\
5\end{array}$ & & $\begin{array}{l}I \\
I \\
I \\
I \\
I\end{array}$ & $\begin{array}{l}6889.299 \\
6892.244 \\
6893.614 \\
6894.222 \\
6895.184\end{array}$ & $\begin{array}{c}100 \\
40 \\
2 \\
8 \\
2 w\end{array}$ & 10 & $\begin{array}{l}\text { II } \\
\text { I } \\
\text { I } \\
\text { I } \\
\text { I }\end{array}$ \\
\hline $\begin{array}{l}6814.602 \\
6814.916 \\
6815.594 \\
6816.245 \\
6816.496\end{array}$ & $\begin{array}{l}1 \\
1 \\
5 \\
2 \\
3\end{array}$ & 1 & $\begin{array}{l}\text { I } \\
\text { I } \\
\text { I } \\
\text { I } \\
\text { I }\end{array}$ & $\begin{array}{l}6846.935 \\
6847.400 \\
6849.600 \\
6850.536 \\
6850.683\end{array}$ & $\begin{array}{l}2 \\
2 \\
1 \\
4 \\
2\end{array}$ & 5 & $\begin{array}{l}\text { I } \\
\text { I } \\
\text { I } \\
\text { II } \\
\text { I }\end{array}$ & $\begin{array}{l}6895.717 \\
6897.263 \\
6898.102 \\
6898.981 \\
6900.755\end{array}$ & $\begin{array}{l}2 \\
2 \mathrm{~h} \\
8 \\
2 \\
12\end{array}$ & 1 & $\begin{array}{l}\text { I } \\
\text { I } \\
\text { I } \\
\text { I } \\
\text { I }\end{array}$ \\
\hline $\begin{array}{l}6816.808 \\
6817.383 \\
6817.830 \\
6818.688 \\
6819.539\end{array}$ & $\begin{array}{l}1 \\
1 \\
1 w \\
1 \\
1\end{array}$ & 1 & $\begin{array}{l}\text { I } \\
\text { I } \\
\text { I } \\
\text { II } \\
\text { I }\end{array}$ & $\begin{array}{l}6851.212 \\
6852.150 \\
6852.353 \\
6853.528 \\
6854.114\end{array}$ & $\begin{array}{r}1 \\
2 \\
3 \\
10 \\
30\end{array}$ & $\begin{array}{l}2 \\
1\end{array}$ & $\begin{array}{l}\text { I I } \\
\text { I I } \\
\text { I } \\
\text { I } \\
\text { I }\end{array}$ & $\begin{array}{l}6902.155 \\
6902.230 \\
6902.918 \\
6903.061 \\
6904.548\end{array}$ & $\begin{array}{l}5 \\
5 \\
2 \\
5 \\
6 s\end{array}$ & 3 & $\begin{array}{l}\text { I } \\
\text { I } \\
\text { I } \\
\text { II } \\
\text { I }\end{array}$ \\
\hline $\begin{array}{l}6819.789 \\
6820.428 \\
6821.502 \\
6822.298 \\
6822.808\end{array}$ & $\begin{array}{l}2 \\
1 \\
l w \\
l w \\
l\end{array}$ & & $\begin{array}{l}\text { I } \\
\text { I } \\
\text { I } \\
\text { I } \\
\text { I }\end{array}$ & $\begin{array}{l}6854.497 \\
6855.307 \\
6855.682 \\
6858.438 \\
6861.146\end{array}$ & $\begin{array}{c}41 \\
12 \\
6 \\
1 \mathrm{~h} \\
3\end{array}$ & 1 & $\begin{array}{l}\text { I I } \\
\text { I } \\
\text { I } \\
\text { I } \\
\text { I }\end{array}$ & $\begin{array}{l}6905.822 \\
6906.684 \\
6907.353 \\
6908.971 \\
6909.214\end{array}$ & $\begin{array}{l}2 \\
2 \\
6 \\
4 \\
4\end{array}$ & $1 \mathrm{~h}$ & $\begin{array}{l}\text { I } \\
\text { I } \\
\text { II } \\
\text { I } \\
\text { I }\end{array}$ \\
\hline
\end{tabular}


Table 1. Wavelengths of Thorium Spectra - Continued

\begin{tabular}{|c|c|c|c|c|c|c|c|c|c|c|c|}
\hline \multirow{2}{*}{$\begin{array}{l}\text { Wave- } \\
\text { length }\end{array}$} & \multicolumn{2}{|c|}{ Intensity } & \multirow{2}{*}{$\begin{array}{l}\text { Spec- } \\
\text { trum }\end{array}$} & \multirow{2}{*}{$\begin{array}{l}\text { Wave- } \\
\text { length }\end{array}$} & \multicolumn{2}{|c|}{ Intensity } & \multirow{2}{*}{$\begin{array}{l}\text { Spec- } \\
\text { trum }\end{array}$} & \multirow{2}{*}{$\begin{array}{l}\text { Wave- } \\
\text { length }\end{array}$} & \multicolumn{2}{|c|}{ Intensity } & \multirow{2}{*}{$\begin{array}{l}\text { Spec- } \\
\text { trum }\end{array}$} \\
\hline & Lamp & Spark & & & Lamp & Spark & & & Lamp & Spark & \\
\hline $\begin{array}{l}6909.848 \\
6910.629 \\
6910.825 \\
6911.2261 \\
6911.630\end{array}$ & $\begin{array}{c}100 \\
2 \\
1 \\
400 \\
3 \mathrm{w}\end{array}$ & 15 & $\begin{array}{l}\text { I } \\
\text { I } \\
\text { I } \\
\text { I } \\
\text { I }\end{array}$ & $\begin{array}{l}6963.910 \\
6964.239 \\
6965.941 \\
6969.281 \\
6969.411\end{array}$ & $\begin{array}{r}2 \\
2 \\
40 \\
10 \\
2\end{array}$ & & $\begin{array}{l}\text { I } \\
\text { I } \\
\text { I } \\
I \\
I\end{array}$ & $\begin{array}{l}7020.493 \\
7021.273 \\
7021.874 \\
7022.675 \\
7023.145\end{array}$ & $\begin{array}{r}10 \\
40 \\
2 \\
2 \\
15\end{array}$ & & $\begin{array}{l}\text { I } \\
\text { I } \\
\text { I } \\
\text { I } \\
\text { I }\end{array}$ \\
\hline $\begin{array}{l}6911.705 \\
6913.040 \\
6914.688 \\
6914.972 \\
6916.135\end{array}$ & $\begin{array}{r}2 \\
2 \\
4 \\
2 \\
75\end{array}$ & 1 & $\begin{array}{l}\text { I } \\
\text { I } \\
\text { I } \\
\text { II } \\
\text { I }\end{array}$ & $\begin{array}{l}6972.989 \\
6975.461 \\
6976.035 \\
6977.438 \\
6977.724\end{array}$ & $\begin{array}{l}2 \\
2 \\
2 \\
4 \\
4\end{array}$ & 1 & $\begin{array}{l}\text { I } \\
\text { II } \\
\text { I } \\
\text { I } \\
\text { I }\end{array}$ & $\begin{array}{l}7023.516 \\
7026.454 \\
7028.017 \\
7030.866 \\
7031.194\end{array}$ & $\begin{array}{r}30 \\
4 \\
10 \\
4 \\
2\end{array}$ & & $\begin{array}{l}\text { I } \\
\text { I } \\
\text { I } \\
\text { I } \\
\text { I }\end{array}$ \\
\hline $\begin{array}{l}6916.856 \\
6920.037 \\
6921.424 \\
6923.089 \\
6924.637\end{array}$ & $\begin{array}{r}4 \\
30 \\
10 \\
2 \\
4\end{array}$ & 2 & $\begin{array}{l}\text { I } \\
\text { I } \\
\text { II } \\
\text { I } \\
\text { I }\end{array}$ & $\begin{array}{l}6978.208 \\
6980.161 \\
6981.070 \\
6982.598 \\
6985.461\end{array}$ & $\begin{array}{r}6 \\
2 \\
20 \\
4 \\
25\end{array}$ & $\begin{array}{l}1 \\
1 \\
3\end{array}$ & $\begin{array}{l}\text { I } \\
\text { II } \\
\text { I } \\
\text { I } \\
\text { II }\end{array}$ & $\begin{array}{l}7031.302 \\
7031.500 \\
7032.175 \\
7032.918 \\
7033.046\end{array}$ & $\begin{array}{l}4 \\
2 \\
2 \\
6 \\
4\end{array}$ & & $\begin{array}{l}\text { I } \\
\text { I } \\
\text { I } \\
\text { I } \\
\text { I }\end{array}$ \\
\hline $\begin{array}{l}6925.467 \\
6926.059 \\
6928.951 \\
6932.440 \\
6932.986\end{array}$ & $\begin{array}{c}4 \\
2 \\
15 \\
2 \\
4 \mathrm{w}\end{array}$ & & $\begin{array}{l}\text { I } \\
\text { I } \\
\text { I } \\
\text { I } \\
\text { I }\end{array}$ & $\begin{array}{l}6986.019 \\
6986.937 \\
6987.774 \\
6988.490 \\
6988.816\end{array}$ & $\begin{array}{l}30 \\
20 \\
4 \\
2 \\
2 \mathrm{w}\end{array}$ & 4 & $\begin{array}{l}\text { I } \\
\text { II } \\
\text { I } \\
\text { I } \\
\text { I }\end{array}$ & $\begin{array}{l}7033.348 \\
7033.632 \\
7035.361 \\
7036.281 \\
7037.593\end{array}$ & $\begin{array}{r}12 \\
2 \\
2 \\
75 \\
2\end{array}$ & & $\begin{array}{l}\text { I } \\
\text { I } \\
\text { I } \\
\text { I } \\
\text { I }\end{array}$ \\
\hline $\begin{array}{l}6934.245 \\
6936.647 \\
6937.064 \\
6940.314 \\
6941.557\end{array}$ & $\begin{array}{r}100 \\
20 \\
15 \\
4 \\
15\end{array}$ & 2 & $\begin{array}{l}\text { I } \\
\text { I } \\
\text { I } \\
\text { I } \\
\text { I }\end{array}$ & $\begin{array}{l}6989.072 \\
6989.656 \mathrm{i} \\
6990.103 \\
6990.434 \\
6990.806\end{array}$ & $\begin{array}{l}6 \mathrm{w} \\
900 \\
20 \\
10 \\
40\end{array}$ & $\begin{array}{r}4 \\
20\end{array}$ & $\begin{array}{l}\text { I } \\
\text { I } \\
\text { I } \\
\text { II } \\
\text { I }\end{array}$ & $\begin{array}{l}7037.826 \\
7038.151 \\
7038.706 \\
7044.963 \\
7045.800\end{array}$ & $\begin{array}{r}2 \\
2 \\
4 \\
6 \\
25\end{array}$ & 20 & $\begin{array}{l}\text { I } \\
\text { I } \\
\text { I } \\
\text { I } \\
\text { II }\end{array}$ \\
\hline $\begin{array}{l}6942.530 \\
6943.014 \\
6943.6111 \\
6943.874 \\
6943.966\end{array}$ & $\begin{array}{r}30 \\
4 \\
600 \\
2 \\
2\end{array}$ & 3 & $\begin{array}{l}\text { I } \\
\text { I } \\
\text { I } \\
\text { I } \\
\text { I }\end{array}$ & $\begin{array}{l}6992.691 \\
6993.037 \\
6993.983 \\
6994.731 \\
6995.141\end{array}$ & $\begin{array}{r}30 \\
25 \\
4 \\
6 \\
4\end{array}$ & $\begin{array}{r}2 \\
50\end{array}$ & $\begin{array}{l}\text { I } \\
\text { II } \\
I \\
I \\
I\end{array}$ & $\begin{array}{l}7046.547 \\
7049.814 \\
7051.787 \\
7052.114 \\
7053.620\end{array}$ & $\begin{array}{l}2 \\
2 \\
4 \\
1 \\
5\end{array}$ & 7 & $\begin{array}{l}\text { I } \\
\text { I } \\
\text { I } \\
\text { I } \\
\text { II }\end{array}$ \\
\hline $\begin{array}{l}6944.050 \\
6944.384 \\
6944.573 \\
6944.787 \\
6945.483\end{array}$ & $\begin{array}{l}2 \\
2 w \\
2 \\
2 \\
75\end{array}$ & & $\begin{array}{l}\text { I } \\
\text { I } \\
\text { I } \\
\text { I } \\
\text { I }\end{array}$ & $\begin{array}{l}6995.892 \\
6996.197 \\
6996.766 \\
6997.397 \\
6998.246\end{array}$ & $\begin{array}{l}2 \\
2 \\
2 \\
4 \\
4\end{array}$ & & $\begin{array}{l}\text { I } \\
\text { I } \\
\text { I } \\
\text { I } \\
\text { I }\end{array}$ & $\begin{array}{l}7054.413 \\
7055.288 \\
7055.892 \\
7056.610 \\
7058.489\end{array}$ & $\begin{array}{r}20 \\
8 \\
6 \\
4 \\
30\end{array}$ & 2 & $\begin{array}{l}\text { I } \\
\text { I } \\
\text { I } \\
\text { I }\end{array}$ \\
\hline $\begin{array}{l}6946.198 \\
6946.579 \\
6947.899 \\
6948.203 \\
6950.238\end{array}$ & $\begin{array}{r}10 \\
20 \\
2 \\
75 \\
4\end{array}$ & & $\begin{array}{l}\text { I } \\
\text { I } \\
\text { I } \\
\text { I } \\
\text { I }\end{array}$ & $\begin{array}{l}6998.890 \\
6999.093 \\
6999.621 \\
7000.803 \\
7001.256\end{array}$ & $\begin{array}{r}4 \\
15 \\
300 \\
4\end{array}$ & $3 w$ & $\begin{array}{l}\text { I I I } \\
\text { I } \\
\text { I } \\
\text { I } \\
\text { I }\end{array}$ & $\begin{array}{l}7059.524 \\
7060.042 \\
7060.655 \\
7060.863 \\
7061.386\end{array}$ & $\begin{array}{r}12 \\
40 \\
100 \\
2 \\
10\end{array}$ & 4 & $\begin{array}{l}\text { I } \\
\text { I } \\
\text { I } \\
\text { I } \\
\text { I }\end{array}$ \\
\hline $\begin{array}{l}6951.588 \\
6952.953 \\
6954.656 \\
6955.319 \\
6955.488\end{array}$ & $\begin{array}{r}2 \\
20 \\
40 \\
6 \\
4\end{array}$ & 3 & $\begin{array}{l}\text { I } \\
\text { I } \\
\text { I } \\
\text { I } \\
\text { I I }\end{array}$ & $\begin{array}{l}7002.880 \\
7006.091 \\
7007.092 \\
7008.042 \\
7013.247\end{array}$ & $\begin{array}{r}50 \\
2 \\
20 \\
2 \\
2\end{array}$ & & $\begin{array}{l}\text { I } \\
\text { I } \\
\text { I } \\
\text { I } \\
\text { I }\end{array}$ & $\begin{array}{l}7062.391 \\
7063.830 \\
7064.448 \\
7066.281 \\
7067.507\end{array}$ & $\begin{array}{r}2 \\
6 \\
250 \\
12 \\
10\end{array}$ & & $\begin{array}{l}\text { I } \\
\text { I } \\
\text { I } \\
\text { I } \\
\text { I }\end{array}$ \\
\hline $\begin{array}{l}6956.304 \\
6958.767 \\
6959.461 \\
6962.292 \\
6962.838\end{array}$ & $\begin{array}{r}4 \\
150 \\
4 \\
15 \\
8\end{array}$ & $1 \mathrm{w}$ & $\begin{array}{l}\text { I I } \\
\text { I } \\
\text { I } \\
\text { I } \\
\text { I }\end{array}$ & $\begin{array}{l}7014.506 \\
7014.965 \\
7015.308 \\
7018.568 \\
7019.013\end{array}$ & $\begin{array}{c}2 w \\
40 \\
25 \\
200 \\
2\end{array}$ & & $\begin{array}{l}\text { I } \\
\text { I } \\
\text { I } \\
\text { I } \\
\text { I }\end{array}$ & $\begin{array}{l}7070.200 \\
7070.953 \\
7071.095 \\
7071.476 \\
7072.392\end{array}$ & $\begin{array}{r}1 \\
10 \\
15 \\
15 \\
100\end{array}$ & & $\begin{array}{l}I \\
I \\
I \\
I \\
I\end{array}$ \\
\hline
\end{tabular}


Table 1. Wavelengths of Thorium Spectra - Continued

\begin{tabular}{|c|c|c|c|c|c|c|c|c|c|c|c|}
\hline \multirow{2}{*}{$\begin{array}{l}\text { Wave- } \\
\text { length }\end{array}$} & \multicolumn{2}{|c|}{ Intensity } & \multirow{2}{*}{$\begin{array}{l}\text { Spec- } \\
\text { trum }\end{array}$} & \multirow{2}{*}{$\begin{array}{l}\text { Wave- } \\
\text { length }\end{array}$} & \multicolumn{2}{|c|}{ Intensity } & \multirow{2}{*}{$\begin{array}{l}\text { Spec- } \\
\text { trum }\end{array}$} & \multirow{2}{*}{$\begin{array}{l}\text { Wave- } \\
\text { length }\end{array}$} & \multicolumn{2}{|c|}{ Intensity } & \multirow{2}{*}{$\begin{array}{l}\text { Spec } \\
\text { trum }\end{array}$} \\
\hline & Lamp & Spark & & & Lamp & Spark & & & Lamp & Spark t & \\
\hline $\begin{array}{l}7073.129 \\
7073.615 \\
7074.248 \\
7074.986 \\
7075.333\end{array}$ & $\begin{array}{c}4 w \\
6 \\
6 \\
5 \\
400\end{array}$ & 40 & $\begin{array}{l}\text { I } \\
\text { I } \\
\text { I } \\
\text { I } \\
\text { I I }\end{array}$ & $\begin{array}{l}7128.442 \\
7129.719 \\
7129.886 \\
7130.180 \\
7130.287\end{array}$ & $\begin{array}{l}6 \\
6 s \\
4 \\
25 \\
60\end{array}$ & & $\begin{array}{l}\text { I } \\
\text { I } \\
\text { I } \\
\text { I } \\
\text { I }\end{array}$ & $\begin{array}{l}7166.477 \\
7166.809 \\
7167.199 \\
7167.674 \\
7168.903\end{array}$ & $\begin{array}{c}3 \\
5 \\
10 \\
1 \mathrm{w} \\
500\end{array}$ & $\begin{array}{l}2 \mathrm{w} \\
1 \mathrm{~h}\end{array}$ & $\begin{array}{l}\text { I I } \\
\text { I I } \\
\text { I } \\
\text { I } \\
\text { I }\end{array}$ \\
\hline $\begin{array}{l}7075.766 \\
7075.817 \\
7077.383 \\
7078.236 \\
7078.986\end{array}$ & $\begin{array}{c}6 \\
4 \\
30 \mathrm{w} \\
8 \\
6\end{array}$ & & $\begin{array}{l}\text { I } \\
\text { I } \\
\text { I } \\
\text { I } \\
\text { I }\end{array}$ & $\begin{array}{l}7130.717 \\
7131.357 \\
7132.615 \\
7133.172 \\
7133.899\end{array}$ & $\begin{array}{r}20 \\
90 \\
50 \\
6 \\
4\end{array}$ & 1 & $\begin{array}{l}\text { I } \\
\text { I } \\
I \\
I \\
I\end{array}$ & $\begin{array}{l}7169.284 \\
7169.372 \\
7169.697 \\
7170.350 \\
7172.986\end{array}$ & $\begin{array}{l}2 \\
2 \\
1 \\
7 \\
3\end{array}$ & & $\begin{array}{l}\text { I } \\
\text { I } \\
\text { I } \\
\text { I } \\
\text { I }\end{array}$ \\
\hline $\begin{array}{l}7081.444 \\
7081.816 \\
7082.113 \\
7082.977 \\
7083.294\end{array}$ & $\begin{array}{l}6 \\
4 \\
4 \\
2 \\
2 h\end{array}$ & & $\begin{array}{l}\text { I } \\
\text { I } \\
\text { I } \\
\text { I } \\
\text { I }\end{array}$ & $\begin{array}{l}7134.474 \\
7135.825 \\
7138.120 \\
7138.595 \\
7138.900\end{array}$ & $\begin{array}{l}2 \\
8 \\
3 \\
3 \\
2 w\end{array}$ & · & $\begin{array}{l}\text { I } \\
\text { I } \\
I \\
I \\
I\end{array}$ & $\begin{array}{l}7173.374 \\
7176.188 \\
7176.725 \\
7177.276 \\
7178.546\end{array}$ & $\begin{array}{r}120 \\
8 \\
30 \\
5 \\
3\end{array}$ & $\begin{array}{l}2 \mathrm{~h} \\
6\end{array}$ & $\begin{array}{l}\text { I } \\
\text { I I } \\
\text { I I } \\
\text { I } \\
\text { I }\end{array}$ \\
\hline $\begin{array}{l}7083.568 \\
7084.169 \\
7084.459 \\
7084.585 \\
7084.637\end{array}$ & $\begin{array}{r}6 \\
500 \\
2 \\
8 \\
6\end{array}$ & & $\begin{array}{l}\text { I } \\
\text { I } \\
\text { I } \\
\text { I } \\
\text { I }\end{array}$ & $\begin{array}{l}7139.354 \\
7139.854 \\
7140.447 \\
7141.290 \\
7142.318\end{array}$ & $\begin{array}{r}2 \\
18 \\
20 \\
3 \\
25\end{array}$ & $\begin{array}{l}2 \\
3\end{array}$ & $\begin{array}{l}\text { I } \\
\text { I I } \\
\text { I I } \\
\text { I } \\
\text { I }\end{array}$ & $\begin{array}{l}7179.052 \\
7179.726 \\
7180.314 \\
7180.946 \\
7181.890\end{array}$ & $\begin{array}{r}5 \\
10 \\
2 \\
3 \\
3\end{array}$ & 1 & $\begin{array}{l}\text { I I } \\
\text { I } \\
\text { I } \\
\text { I } \\
\text { I }\end{array}$ \\
\hline $\begin{array}{l}7084.986 \\
7087.279 \\
7088.327 \\
7088.819 \\
7089.337\end{array}$ & $\begin{array}{r}12 \\
350 \\
6 \\
20 \\
250\end{array}$ & 15 & $\begin{array}{l}\text { I } \\
\text { I } \\
\text { I } \\
\text { I } \\
\text { II }\end{array}$ & $\begin{array}{l}7145.372 \\
7145.769 \\
7146.378 \\
7147.130 \\
7147.403\end{array}$ & $\begin{array}{l}1 \\
3 \\
1 \\
1 \\
4\end{array}$ & 1 & $\begin{array}{l}\text { I } \\
\text { I I } \\
\text { I } \\
\text { I } \\
\text { I }\end{array}$ & $\begin{array}{l}7182.183 \\
7185.683 \\
7186.397 \\
7186.630 \\
7187.425\end{array}$ & $\begin{array}{l}4 \\
6 \\
2 \\
1 \\
1\end{array}$ & & $\begin{array}{l}I \\
I \\
I \\
I \\
I\end{array}$ \\
\hline $\begin{array}{l}7090.360 \\
7097.019 \\
7097.150 \\
7100.516 \\
7102.593\end{array}$ & $\begin{array}{r}4 \\
2 \\
1 \\
50 \\
2\end{array}$ & 4 & $\begin{array}{l}\text { I } \\
\text { I } \\
\text { I } \\
\text { I I } \\
\text { I }\end{array}$ & $\begin{array}{l}7148.552 \\
7150.278 \\
7150.680 \\
7151.153 \\
7151.510\end{array}$ & $\begin{array}{l}100 \\
90 \\
5 \\
2 \mathrm{w} \\
3\end{array}$ & & $\begin{array}{l}\text { I } \\
\text { I } \\
\text { I } \\
\text { I } \\
\text { I }\end{array}$ & $\begin{array}{l}7188.545 \\
7189.745 \\
7190.084 \\
7190.513 \\
7191.141\end{array}$ & $\begin{array}{c}10 \\
6 \\
1 \mathrm{w} \\
3 \\
250\end{array}$ & 50 & $\begin{array}{l}\text { I } \\
\text { I } \\
\text { I } \\
\text { I } \\
\text { I I }\end{array}$ \\
\hline $\begin{array}{l}7104.909 \\
7105.635 \\
7109.082 \\
7109.182 \\
7109.346\end{array}$ & $\begin{array}{r}6 \\
2 \\
8 \\
10 \\
8\end{array}$ & & $\begin{array}{l}\text { I } \\
\text { I } \\
\text { I } \\
\text { I } \\
\text { I }\end{array}$ & $\begin{array}{l}7153.583 \\
7154.757 \\
7154.944 \\
7155.381 \\
7156.937\end{array}$ & $\begin{array}{r}25 \\
25 \\
25 \\
3 \\
80\end{array}$ & 1 & $\begin{array}{l}\text { I } \\
\text { I } \\
\text { I } \\
\text { II } \\
\text { I }\end{array}$ & $\begin{array}{l}7192.003 \\
7194.922 \\
7195.948 \\
7196.577 \\
7198.098\end{array}$ & $\begin{array}{c}5 w \\
5 \\
10 \\
2 \\
3\end{array}$ & 1 & $\begin{array}{l}\text { I } \\
\text { I } \\
\text { I } \\
\text { I } \\
\text { I }\end{array}$ \\
\hline $\begin{array}{l}7109.546 \\
7109.847 \\
7111.039 \\
7112.900 \\
7113.979\end{array}$ & $\begin{array}{r}8 \\
5 \\
8 \\
3 \\
12\end{array}$ & & $\begin{array}{l}\text { I } \\
\text { I } \\
\text { I } \\
\text { I }\end{array}$ & $\begin{array}{l}7157.050 \\
7157.690 \\
7157.953 \\
7158.260 \\
7159.932\end{array}$ & $\begin{array}{l}1 \\
12 \\
3 \\
2 \mathrm{w} \\
90^{1}\end{array}$ & $5 w$ & $\begin{array}{l}\text { II } \\
\text { I } \\
\text { I } \\
\text { I } \\
\text { I }\end{array}$ & $\begin{array}{l}7199.527 \\
7200.045 \\
7201.041 \\
7201.805 \\
7202.887\end{array}$ & $\begin{array}{r}3 \\
120 \\
5 \\
12 \\
4\end{array}$ & & $\begin{array}{l}\text { I } \\
\text { I } \\
\text { I } \\
\text { I } \\
\text { I }\end{array}$ \\
\hline $\begin{array}{l}7114.394 \\
7121.691 \\
7124.560 \\
7125.014 \\
7125.821\end{array}$ & $\begin{array}{r}20 \\
6 \\
180 \\
2 \\
20\end{array}$ & & $\begin{array}{l}\text { I } \\
\text { I } \\
\text { I } \\
I \\
I\end{array}$ & $\begin{array}{l}7160.398 \\
7160.753 \\
7161.239 \\
7161.744 \\
7162.602\end{array}$ & $\begin{array}{l}1 w \\
4 \\
5 \\
4 \\
1\end{array}$ & & $\begin{array}{l}\text { I } \\
\text { I } \\
\text { I } \\
\text { I } \\
\text { I }\end{array}$ & $\begin{array}{l}7203.357 \\
7206.475 \\
7206.664 \\
7207.017 \\
7207.381\end{array}$ & $\begin{array}{r}2 \\
80 \\
2 \\
4 \\
6\end{array}$ & & $\begin{array}{l}\text { I } \\
\text { I } \\
\text { I } \\
\text { I } \\
\text { I }\end{array}$ \\
\hline $\begin{array}{l}7126.038 \\
7126.342 \\
7126.818 \\
7127.648 \\
7127.802\end{array}$ & $\begin{array}{c}4 w \\
4 \\
20 \\
4 \\
8\end{array}$ & & $\begin{array}{l}\text { I } \\
\text { I } \\
\text { I } \\
\text { I } \\
\text { I }\end{array}$ & $\begin{array}{l}7163.204 \\
7163.266 \\
7164.442 \\
7165.161 \\
7165.989\end{array}$ & $\begin{array}{l}2 \\
2 \\
2 \\
2 \\
8\end{array}$ & 2 & $\begin{array}{l}\text { I } \\
\text { I } \\
\text { I } \\
\text { I } \\
\text { I I }\end{array}$ & $\begin{array}{l}7208.006 \\
7208.422 \\
7208.507 \\
7208.811 \\
7209.478\end{array}$ & $\begin{array}{r}600 \\
3 \\
3 \\
1 \\
2\end{array}$ & & $\begin{array}{l}\text { I } \\
\text { I } \\
\text { I } \\
\text { I } \\
\text { I }\end{array}$ \\
\hline
\end{tabular}


Table 1. Wavelengths of Thorium Spectra - Continued

\begin{tabular}{|c|c|c|c|c|c|c|c|c|c|c|c|}
\hline \multirow{2}{*}{$\begin{array}{l}\text { Wave- } \\
\text { length }\end{array}$} & \multicolumn{2}{|c|}{ Intensity } & \multirow{2}{*}{$\begin{array}{l}\text { Spec- } \\
\text { trum }\end{array}$} & \multirow{2}{*}{$\begin{array}{l}\text { Wave- } \\
\text { length }\end{array}$} & \multicolumn{2}{|c|}{ Intensity } & \multirow{2}{*}{$\begin{array}{l}\text { Spec- } \\
\text { trum }\end{array}$} & \multirow{2}{*}{$\begin{array}{l}\text { Wave- } \\
\text { length }\end{array}$} & \multicolumn{2}{|c|}{ Intensity } & \multirow{2}{*}{$\begin{array}{l}\text { Spec- } \\
\text { trum }\end{array}$} \\
\hline & Lamp & Spark & & & Lamp & Spark & & & Lamp & Spark & \\
\hline $\begin{array}{l}7209.709 \\
7212.693 \\
7213.192 \\
7214.552 \\
7215.644\end{array}$ & $\begin{array}{c}8 \mathrm{w} \\
180^{\circ} \\
2 \\
2 \\
2\end{array}$ & & $\begin{array}{l}\text { I } \\
\text { I } \\
\text { I } \\
\text { I } \\
\text { I }\end{array}$ & $\begin{array}{l}7250.580 \\
7253.669 \\
7255.352 \\
7256.011 \\
7256.804\end{array}$ & $\begin{array}{r}15 \\
18 \\
20 \\
2 \\
2\end{array}$ & 3 & $\begin{array}{l}\text { II } \\
\text { I } \\
\text { I } \\
\text { I } \\
\text { I }\end{array}$ & $\begin{array}{l}7293.363 \\
7294.300 \\
7296.257 \\
7298.137 \\
7300.037\end{array}$ & $\begin{array}{r}3 \\
4 \\
15 \\
30 \\
2\end{array}$ & & $\begin{array}{l}\text { I } \\
\text { I } \\
\text { I } \\
\text { I } \\
\text { I }\end{array}$ \\
\hline $\begin{array}{l}7215.924 \\
7216.156 \\
7216.657 \\
7217.515 \\
7217.655\end{array}$ & $\begin{array}{l}1 \\
5 \\
4 \\
2 \\
3\end{array}$ & 1 & $\begin{array}{l}\text { I } \\
\text { I I } \\
\text { I } \\
\text { I } \\
\text { I }\end{array}$ & $\begin{array}{l}7256.986 \\
7257.191 \\
7258.173 \\
7258.657 \\
7259.009\end{array}$ & $\begin{array}{r}15 \\
5 \\
30 \\
5 \\
2\end{array}$ & & $\begin{array}{l}\text { I } \\
\text { I } \\
\text { I } \\
\text { I } \\
\text { I }\end{array}$ & $\begin{array}{l}7300.632 \\
7301.330 \\
7302.158 \\
7302.420 \\
7305.188\end{array}$ & $\begin{array}{l}1 \\
3 \\
7 \\
6 \\
2\end{array}$ & & $\begin{array}{l}\text { I } \\
\text { I } \\
\text { I } \\
\text { I } \\
\text { I }\end{array}$ \\
\hline $\begin{array}{l}7217.753 \\
7218.054 \\
7219.153 \\
7220.592 \\
7220.976\end{array}$ & $\begin{array}{r}40 \\
180 \\
70 \\
4 \\
15\end{array}$ & $\begin{array}{l}5 \\
2\end{array}$ & $\begin{array}{l}\text { II } \\
\text { I } \\
\text { I } \\
\text { I } \\
\text { I }\end{array}$ & $\begin{array}{l}7259.488 \\
7260.240 \\
7260.533 \\
7260.859 \\
7261.199\end{array}$ & $\begin{array}{l}2 \\
3 \\
2 \\
2 \\
2\end{array}$ & & $\begin{array}{l}\text { I } \\
\text { I } \\
\text { I } \\
\text { I } \\
\text { I }\end{array}$ & $\begin{array}{l}7305.401 \\
7305.487 \\
7305.617 \\
7308.511 \\
7308.636\end{array}$ & $\begin{array}{r}75 \\
2 \\
2 \\
10 \\
10\end{array}$ & $\begin{array}{l}10 \\
2\end{array}$ & $\begin{array}{l}\text { II } \\
\text { I } \\
\text { I } \\
\text { II } \\
\text { I }\end{array}$ \\
\hline $\begin{array}{l}7222.290 \\
7223.018 \\
7225.105 \\
7225.782 \\
7226.201\end{array}$ & $\begin{array}{r}2 \\
9 \\
12 \\
8 \\
3\end{array}$ & 1 & $\begin{array}{l}\text { II } \\
\text { I } \\
\text { I } \\
\text { I } \\
\text { I }\end{array}$ & $\begin{array}{l}7262.175 \\
7262.728 \\
7264.102 \\
7267.074 \\
7268.822\end{array}$ & $\begin{array}{l}1 \\
2 \\
5 \\
5 \\
5\end{array}$ & & $\begin{array}{l}\text { I } \\
\text { I } \\
\text { I } \\
\text { I } \\
\text { I }\end{array}$ & $\begin{array}{l}7309.244 \\
7310.224 \\
7311.007 \\
7311.514 \\
7312.001\end{array}$ & $\begin{array}{c}8 \\
4 \\
41 \\
10 \\
1\end{array}$ & & $\begin{array}{l}\text { I } \\
\text { I } \\
\text { I } \\
\text { I } \\
\text { I }\end{array}$ \\
\hline $\begin{array}{l}7228.787 \\
7230.863 \\
7231.991 \\
7232.933 \\
7233.388\end{array}$ & $\begin{array}{r}6 \\
80 \\
4 \\
2 \\
15\end{array}$ & 2 & $\begin{array}{l}\text { I } \\
\text { I } \\
\text { I } \\
\text { I } \\
\text { II }\end{array}$ & $\begin{array}{l}7269.040 \\
7270.552 \\
7270.960 \\
7272.522 \\
7272.724\end{array}$ & $\begin{array}{r}2 \\
10 \\
2 \\
8 \\
2\end{array}$ & & $\begin{array}{l}\text { I } \\
\text { I } \\
\text { I } \\
\text { I } \\
\text { I }\end{array}$ & $\begin{array}{l}7312.245 \\
7314.465 \\
7315.067 \\
7315.604 \\
7316.115\end{array}$ & $\begin{array}{r}2 \\
2 \\
15 \\
15 \\
7\end{array}$ & & $\begin{array}{l}\text { I } \\
\text { I } \\
\text { I } \\
\text { I } \\
\text { I }\end{array}$ \\
\hline $\begin{array}{l}7235.539 \\
7235.886 \\
7236.240 \\
7236.359 \\
7237.113\end{array}$ & $\begin{array}{l}3 \\
5 \\
3 \\
2 \\
3\end{array}$ & & $\begin{array}{l}\text { I } \\
\text { I } \\
\text { I } \\
\text { I } \\
\text { I }\end{array}$ & $\begin{array}{l}7274.273 \\
7274.615 \\
7275.824 \\
7276.441 \\
7277.001\end{array}$ & $\begin{array}{l}5 \mathrm{w} \\
1 \\
4 \\
2 \\
5\end{array}$ & & $\begin{array}{l}\text { I } \\
\text { I } \\
\text { I } \\
\text { I } \\
\text { I }\end{array}$ & $\begin{array}{l}7316.371 \\
7320.030 \\
7321.879 \\
7323.206 \\
7323.438\end{array}$ & $\begin{array}{c}2 \\
3 \\
4 \mathrm{~s} \\
12 \\
2\end{array}$ & & $\begin{array}{l}\text { I } \\
\text { I } \\
\text { I } \\
\text { I } \\
\text { I }\end{array}$ \\
\hline $\begin{array}{l}7237.229 \\
7238.362 \\
7239.254 \\
7239.473 \\
7240.177\end{array}$ & $\begin{array}{r}2 \\
1 \\
6 \\
1 \\
12\end{array}$ & $\begin{array}{l}1 \\
3\end{array}$ & $\begin{array}{l}\text { I } \\
\text { I } \\
\text { I I } \\
\text { I } \\
\text { I I }\end{array}$ & $\begin{array}{l}7278.039 \\
7279.130 \\
7280.463 \\
7282.708 \\
7284.158\end{array}$ & $\begin{array}{l}2 \mathrm{w} \\
2 \\
3 \\
8 \\
2\end{array}$ & & $\begin{array}{l}\text { I } \\
\text { I } \\
I \\
I \\
I\end{array}$ & $\begin{array}{l}7323.844 \\
7323.980 \\
7324.810 \\
7324.995 \\
7328.284\end{array}$ & $\begin{array}{r}5 \\
2 \\
120 \\
8 \\
120\end{array}$ & & $\begin{array}{l}\text { I } \\
\text { I } \\
\text { I } \\
\text { I } \\
\text { I }\end{array}$ \\
\hline $\begin{array}{l}7240.853 \\
7240.972 \\
7242.086 \\
7243.337 \\
7243.769\end{array}$ & $\begin{array}{c}3 \\
2 \\
75 \\
12 \mathrm{~s} \\
6\end{array}$ & & $\begin{array}{l}\text { I } \\
\text { I } \\
\text { I } \\
\text { I } \\
\text { I }\end{array}$ & $\begin{array}{l}7284.286 \\
7284.405 \\
7284.901 \\
7285.382 \\
7285.734\end{array}$ & $\begin{array}{c}2 \\
4 \\
120 \\
1 \mathrm{w} \\
2\end{array}$ & & $\begin{array}{l}\text { I } \\
\text { I } \\
\text { I } \\
\text { I } \\
\text { I }\end{array}$ & $\begin{array}{l}7328.714 \\
7329.498 \\
7330.016 \\
7331.106 \\
7332.019\end{array}$ & $\begin{array}{r}1 \\
25 \\
1 \\
2 \\
4\end{array}$ & & $\begin{array}{l}\text { I } \\
\text { I } \\
\text { I } \\
\text { I } \\
\text { I }\end{array}$ \\
\hline $\begin{array}{l}7244.688 \\
7245.324 \\
7245.498 \\
7246.127 \\
7246.332\end{array}$ & $\begin{array}{r}80 \\
7 \\
3 \\
10 \\
2\end{array}$ & & $\begin{array}{l}\text { I } \\
\text { I } \\
\text { I } \\
\text { I } \\
\text { I }\end{array}$ & $\begin{array}{l}7288.591 \\
7288.770 \\
7288.970 \\
7289.185 \\
7289.420\end{array}$ & $\begin{array}{l}3 \\
3 \\
3 \\
2 \\
7\end{array}$ & 1 & $\begin{array}{l}\text { I } \\
\text { I } \\
\text { II } \\
\text { I } \\
\text { I }\end{array}$ & $\begin{array}{l}7335.571 \\
7337.032 \\
7337.552 \\
7337.776 \\
7338.599\end{array}$ & $\begin{array}{l}25 \\
2 \mathrm{w} \\
2 \\
10 \\
3\end{array}$ & $\begin{array}{l}2 \\
1\end{array}$ & $\begin{array}{l}\text { II } \\
\text { I } \\
\text { I } \\
\text { II } \\
\text { II }\end{array}$ \\
\hline $\begin{array}{l}7247.595 \\
7247.974 \\
7248.959 \\
7249.834 \\
7250.090\end{array}$ & $\begin{array}{l}3 \\
7 \\
4 \\
5 \\
3\end{array}$ & & $\begin{array}{l}\text { I } \\
\text { I } \\
\text { I } \\
\text { I } \\
\text { I }\end{array}$ & $\begin{array}{l}7290.998 \\
7291.278 \\
7292.008 \\
7292.411 \\
7292.661\end{array}$ & $\begin{array}{l}4 \\
3 \\
2 \\
2 \\
9\end{array}$ & & $\begin{array}{l}\text { I } \\
\text { I } \\
\text { I } \\
\text { I } \\
\text { I }\end{array}$ & $\begin{array}{l}7338.996 \\
7339.400 \\
7339.599 \\
7341.146 \\
7341.558\end{array}$ & $\begin{array}{r}1 \\
3 \\
10 \\
140 \\
4\end{array}$ & & $\begin{array}{l}\text { I } \\
\text { I } \\
\text { I } \\
\text { I } \\
\text { I }\end{array}$ \\
\hline
\end{tabular}


Table 1. Wavelengths of Thorium Spectra - Continued

\begin{tabular}{|c|c|c|c|c|c|c|c|c|c|c|c|}
\hline \multirow{2}{*}{$\begin{array}{l}\text { Wave- } \\
\text { length }\end{array}$} & \multicolumn{2}{|c|}{ Intensity } & \multirow{2}{*}{$\begin{array}{l}\text { Spec- } \\
\text { trum }\end{array}$} & \multirow{2}{*}{$\begin{array}{l}\text { Wave- } \\
\text { length }\end{array}$} & \multicolumn{2}{|c|}{ Intensity } & \multirow{2}{*}{$\begin{array}{l}\text { Spec- } \\
\text { trum }\end{array}$} & \multirow{2}{*}{$\begin{array}{l}\text { Wave- } \\
\text { length }\end{array}$} & \multicolumn{2}{|c|}{ Intensity } & \multirow{2}{*}{$\begin{array}{l}\text { Spec- } \\
\text { trum }\end{array}$} \\
\hline & Lamp & Spark & & & Lamp & Spark & & & Lamp & Spark t & \\
\hline $\begin{array}{l}7341.906 \\
7342.028 \\
7342.297 \\
7342.576 \\
7343.369\end{array}$ & $\begin{array}{r}2 \\
10 \\
9 \\
80 \\
1\end{array}$ & & $\begin{array}{l}\text { I } \\
\text { I } \\
I \\
I \\
I\end{array}$ & $\begin{array}{l}7388.379 \\
7388.553 \\
7389.246 \\
7390.081 \\
7392.611\end{array}$ & $\begin{array}{l}2 \\
2 \\
9 \\
5 \mathrm{w} \\
2\end{array}$ & $1 \mathrm{~h}$ & $\begin{array}{l}\text { I } \\
\text { I } \\
\text { II } \\
\text { I } \\
\text { I }\end{array}$ & $\begin{array}{l}7435.010 \\
7435.883 \\
7437.693 \\
7438.479 \\
7439.077\end{array}$ & $\begin{array}{l}4 \\
2 \\
5 \\
7 \\
9\end{array}$ & $\begin{array}{l}2 \\
3\end{array}$ & $\begin{array}{l}\text { I } \\
\text { I } \\
\text { II } \\
\text { II } \\
\text { I }\end{array}$ \\
\hline $\begin{array}{l}7344.956 \\
7345.518 \\
7346.337 \\
7347.043 \\
7349.293\end{array}$ & $\begin{array}{r}2 \\
2 \\
20 \\
20 \\
10\end{array}$ & $\begin{array}{l}2 \\
3\end{array}$ & $\begin{array}{l}\text { I } \\
\text { I } \\
\text { I I } \\
\text { II } \\
\text { I }\end{array}$ & $\begin{array}{l}7393.438 \\
7395.998 \\
7396.886 \\
7397.567 \\
7398.559\end{array}$ & $\begin{array}{c}200 \\
18 \\
5 \\
3 \\
6 \mathrm{w}\end{array}$ & $\begin{array}{r}100 \\
4\end{array}$ & $\begin{array}{l}\text { I I } \\
\text { I I } \\
\text { I } \\
\text { I } \\
\text { I }\end{array}$ & $\begin{array}{l}7441.109 \\
7442.032 \\
7443.860 \\
7444.345 \\
7444.751\end{array}$ & $\begin{array}{r}5 \\
4 \\
6 \\
1 \\
75\end{array}$ & & $\begin{array}{l}I \\
I \\
I \\
I \\
I\end{array}$ \\
\hline $\begin{array}{l}7350.442 \\
7351.328 \\
7351.384 \\
7352.569 \\
7353.008\end{array}$ & $\begin{array}{l}6 \\
10 \\
1 \mathrm{w} \\
2 \\
18\end{array}$ & $\frac{1}{5}$ & $\begin{array}{l}\text { I } \\
\text { I } \\
\text { I } \\
\text { I I } \\
\text { I I }\end{array}$ & $\begin{array}{l}7399.238 \\
7399.378 \\
7399.709 \\
7400.051 \\
7402.243\end{array}$ & $\begin{array}{r}2 \\
2 \\
5 \\
4 \\
90\end{array}$ & & $\begin{array}{l}\text { I } \\
\text { I } \\
\text { I } \\
I \\
I\end{array}$ & $\begin{array}{l}7445.735 \\
7447.841 \\
7449.599 \\
7449.806 \\
7450.177\end{array}$ & $\begin{array}{l}10 \\
70 \\
10 \\
12 \\
18\end{array}$ & & $\begin{array}{l}\text { I } \\
\text { I } \\
\text { I } \\
\text { I } \\
\text { I }\end{array}$ \\
\hline $\begin{array}{l}7353.631 \\
7354.594 \\
7355.157 \\
7355.729 \\
7358.355\end{array}$ & $\begin{array}{r}3 \\
2 \\
2 \\
1 \\
30\end{array}$ & 1 & $\begin{array}{l}\text { I I } \\
\text { I } \\
\text { I } \\
\text { I } \\
\text { I I }\end{array}$ & $\begin{array}{l}7403.536 \\
7403.985 \\
7404.245 \\
7404.688 \\
7406.277\end{array}$ & $\begin{array}{r}2 \\
10 \\
7 \\
5 \\
1\end{array}$ & & $\begin{array}{l}\text { I } \\
\text { I } \\
\text { I } \\
\text { I } \\
\text { I }\end{array}$ & $\begin{array}{l}7452.503 \\
7453.176 \\
7453.708 \\
7454.077 \\
7454.383\end{array}$ & $\begin{array}{l}5 \\
3 \\
4 w \\
5 \\
5 w\end{array}$ & & $\begin{array}{l}\text { I } \\
\text { I } \\
\text { I } \\
\text { I } \\
\text { I }\end{array}$ \\
\hline $\begin{array}{l}7359.066 \\
7361.347 \\
7362.190 \\
7363.724 \\
7365.209\end{array}$ & $\begin{array}{r}10 \\
30 \\
10 \\
3 \\
4\end{array}$ & 2 & $\begin{array}{l}\text { I I } \\
\text { I } \\
\text { I } \\
\text { I } \\
\text { I }\end{array}$ & $\begin{array}{l}7409.203 \\
7410.961 \\
7411.731 \\
7411.979 \\
7412.446\end{array}$ & $\begin{array}{r}5 \\
4 \\
20 \\
10 \\
2\end{array}$ & $\begin{array}{l}2 \\
2\end{array}$ & $\begin{array}{l}\text { I } \\
\text { I } \\
\text { II } \\
\text { II } \\
\text { I }\end{array}$ & $\begin{array}{l}7455.200 \\
7455.394 \\
7456.481 \\
7456.811 \\
7457.638\end{array}$ & $\begin{array}{r}75 \\
1 \\
3 \\
7 \\
7\end{array}$ & & $\begin{array}{l}\text { I } \\
\text { I } \\
\text { I } \\
\text { I } \\
\text { I }\end{array}$ \\
\hline $\begin{array}{l}7365.413 \\
7368.583 \\
7369.133 \\
7369.555 \\
7370.342\end{array}$ & $\begin{array}{l}6 \\
2 \\
3 \\
2 \\
4\end{array}$ & & $\begin{array}{l}\text { I } \\
\text { I } \\
\text { I } \\
\text { I } \\
\text { I }\end{array}$ & $\begin{array}{l}7414.035 \\
7415.893 \\
7417.111 \\
7417.778 \\
7418.431\end{array}$ & $\begin{array}{l}10 \\
4 \\
1 \mathrm{w} \\
10 \\
2\end{array}$ & $\begin{array}{l}4 \\
1 \mathrm{~h}\end{array}$ & $\begin{array}{l}\text { I I } \\
\text { I I } \\
\text { I } \\
\text { I } \\
\text { I }\end{array}$ & $\begin{array}{l}7457.747 \\
7460.391 \\
7461.272 \\
7461.481 \\
7461.878\end{array}$ & $\begin{array}{r}10 \\
4 \\
5 \\
2 \\
1\end{array}$ & $300 w$ & $\begin{array}{l}\text { I } \\
\text { I } \\
\text { I } \\
\text { I I I }\end{array}$ \\
\hline $\begin{array}{l}7370.821 \\
7371.075 \\
7372.093 \\
7372.509 \\
7372.603\end{array}$ & $\begin{array}{r}15 \\
3 \\
5 \\
1 \\
1\end{array}$ & & $\begin{array}{l}\text { I } \\
\text { I } \\
\text { I } \\
\text { I } \\
\text { I }\end{array}$ & $\begin{array}{l}7418.532 \\
7419.376 \\
7419.584 \\
7420.512 \\
7421.456\end{array}$ & $\begin{array}{r}50 \\
5 \\
4 \\
2 \\
2\end{array}$ & & $\begin{array}{l}\text { I } \\
\text { I } \\
\text { I } \\
\text { I } \\
\text { I }\end{array}$ & $\begin{array}{l}7463.979 \\
7465.011 \\
7465.227 \\
7465.437 \\
7467.492\end{array}$ & $\begin{array}{r}6 \\
4 \\
4 \\
15 \\
12\end{array}$ & & $\begin{array}{l}\text { I } \\
\text { I } \\
\text { I } \\
I \\
I\end{array}$ \\
\hline $\begin{array}{l}7373.371 \\
7374.467 \\
7376.166 \\
7376.877 \\
7377.877\end{array}$ & $\begin{array}{r}1 \\
1 \\
4 \\
100 \\
6\end{array}$ & & $\begin{array}{l}I \\
I \\
I \\
I \\
I\end{array}$ & $\begin{array}{l}7421.822 \\
7422.007 \\
7422.432 \\
7423.291 \\
7423.797\end{array}$ & $\begin{array}{r}2 \\
12 \\
3 \\
4 \\
12\end{array}$ & & $\begin{array}{l}\mathrm{I} \\
\mathrm{I} \\
\mathrm{I} \\
\mathrm{I} \\
\mathrm{I}\end{array}$ & $\begin{array}{l}7471.323 \\
7471.599 \\
7471.938 \\
7473.419 \\
7474.554\end{array}$ & $\begin{array}{l}2 \\
2 \\
5 \\
5 \\
6\end{array}$ & & $\begin{array}{l}I \\
I \\
I \\
I \\
I\end{array}$ \\
\hline $\begin{array}{l}7379.562 \\
7380.157 \\
7380.685 \\
7381.789 \\
7383.714\end{array}$ & $\begin{array}{r}3 \\
20 \\
1 \\
2 \\
120\end{array}$ & $\begin{array}{l}2 w \\
4 \\
1\end{array}$ & $\begin{array}{l}\text { I I } \\
\text { I I } \\
\text { I I } \\
\text { I } \\
\text { I }\end{array}$ & $\begin{array}{l}7425.238 \\
7428.941 \\
7429.358 \\
7429.455 \\
7429.789\end{array}$ & $\begin{array}{r}10 \\
350 \\
2 \\
2 \\
1\end{array}$ & 3 & $\begin{array}{l}\text { I } \\
\text { I } \\
\text { I } \\
I \\
I\end{array}$ & $\begin{array}{l}7475.661 \\
7477.001 \\
7477.343 \\
7477.984 \\
7479.179\end{array}$ & $\begin{array}{l}3 \\
2 \\
3 \\
2 \\
1\end{array}$ & & $\begin{array}{l}\text { I } \\
\text { I } \\
\text { I } \\
\text { I } \\
\text { I }\end{array}$ \\
\hline $\begin{array}{l}7384.170 \\
7385.501 \\
7386.336 \\
7386.915 \\
7388.119\end{array}$ & $\begin{array}{r}50 \\
300 \\
10 \\
9 \\
2 \mathrm{w}\end{array}$ & & $\begin{array}{l}\text { I } \\
\text { I } \\
I \\
I \\
I\end{array}$ & $\begin{array}{l}7430.252 \\
7431.367 \\
7431.676 \\
7432.182 \\
7433.342\end{array}$ & $\begin{array}{r}160 \\
6 \\
3 \\
1 \\
2\end{array}$ & 1 & $\begin{array}{l}I \\
I \\
I \\
I \\
I\end{array}$ & $\begin{array}{l}7479.803 \\
7480.638 \\
7481.353 \\
7482.618 \\
7483.096\end{array}$ & $\begin{array}{r}12 \\
4 \\
160 \\
5 \\
3\end{array}$ & $\begin{array}{l}5 \\
2\end{array}$ & $\begin{array}{l}\text { II I } \\
\text { I } \\
\text { I } \\
\text { I } \\
\text { I }\end{array}$ \\
\hline
\end{tabular}


Table 1. Wavelengths of Thorium Spectra - Continued

\begin{tabular}{|c|c|c|c|c|c|c|c|c|c|c|c|}
\hline \multirow{2}{*}{$\begin{array}{l}\text { Wave- } \\
\text { length }\end{array}$} & \multicolumn{2}{|c|}{ Intensity } & \multirow{2}{*}{$\begin{array}{l}\text { Spec- } \\
\text { trum }\end{array}$} & \multirow{2}{*}{$\begin{array}{l}\text { Wave- } \\
\text { length }\end{array}$} & \multicolumn{2}{|c|}{ Intensity } & \multirow{2}{*}{$\begin{array}{l}\text { Spec- } \\
\text { trum }\end{array}$} & \multirow{2}{*}{$\begin{array}{l}\text { Wave- } \\
\text { length }\end{array}$} & \multicolumn{2}{|c|}{ Intensity } & \multirow{2}{*}{$\begin{array}{l}\text { Spec- } \\
\text { trum }\end{array}$} \\
\hline & Lamp & Spark & & & Lamp & Spark & & & Lamp & Spark & \\
\hline $\begin{array}{l}7483.363 \\
7483.631 \\
7484.491 \\
7485.103 \\
7485.243\end{array}$ & $\begin{array}{r}1 \\
100 \\
2 \\
1 \\
3\end{array}$ & 3 & $\begin{array}{l}\text { II } \\
\text { I } \\
\text { I } \\
\text { I } \\
\text { I }\end{array}$ & $\begin{array}{l}7520.120 \\
7520.572 \\
7520.686 \\
7521.629 \\
7522.649\end{array}$ & $\begin{array}{r}4 \\
3 \\
7 \\
6 \\
25\end{array}$ & 10 & $\begin{array}{l}\text { I } \\
\text { I } \\
\text { I } \\
\text { I I } \\
\text { I I }\end{array}$ & $\begin{array}{l}7568.587 \\
7569.510 \\
7569.645 \\
7570.520 \\
7571.019\end{array}$ & $\begin{array}{r}1 \\
60 \\
20 \\
15 \\
8\end{array}$ & & $\begin{array}{l}\text { I } \\
\text { I } \\
\text { I } \\
\text { I } \\
\text { I }\end{array}$ \\
\hline $\begin{array}{l}7485.504 \\
7487.714 \\
7487.971 \\
7488.171 \\
7489.611\end{array}$ & $\begin{array}{r}5 \\
8 \\
20 \\
1 \\
8\end{array}$ & 1 & $\begin{array}{l}\text { I } \\
\text { I } \\
\text { I } \\
\text { I } \\
\text { I }\end{array}$ & $\begin{array}{l}7523.133 \\
7525.507 \\
7526.005 \\
7527.342 \\
7527.800\end{array}$ & $\begin{array}{r}50 \\
400 \\
1 \\
7 \\
10\end{array}$ & 100 & $\begin{array}{l}\text { I } \\
\text { I I } \\
\text { I } \\
\text { I } \\
\text { I }\end{array}$ & $\begin{array}{l}7571.916 \\
7572.453 \\
7572.957 \\
7573.328 \\
7574.070\end{array}$ & $\begin{array}{r}2 \mathrm{w} \\
8 \\
3 \\
12 \\
1\end{array}$ & & $\begin{array}{l}\text { I } \\
\text { I } \\
\text { I } \\
\text { I } \\
\text { I }\end{array}$ \\
\hline $\begin{array}{l}7490.104 \\
7489.699 \\
7490.380 \\
7490.608 \\
7492.958\end{array}$ & $\begin{array}{l}6 \\
8 \\
2 \\
2 \mathrm{w} \\
1\end{array}$ & & $\begin{array}{l}\text { I } \\
\text { I } \\
\text { I } \\
\text { I } \\
\text { I }\end{array}$ & $\begin{array}{l}7528.486 \\
7528.466 \\
7528.913 \\
7529.322 \\
7530.346\end{array}$ & $\begin{array}{l}25 \\
30 \\
3 \\
3 \mathrm{w} \\
4 \mathrm{~s}\end{array}$ & 4 & $\begin{array}{l}\text { I } \\
\text { II } \\
\text { I } \\
\text { I } \\
\text { I }\end{array}$ & $\begin{array}{l}7574.445 \\
7574.294 \\
7577.261 \\
7577.539 \\
7578.256\end{array}$ & $\begin{array}{l}5 \\
4 \\
6 \\
6 \\
2\end{array}$ & & $\begin{array}{l}\text { I } \\
\text { I } \\
\text { I } \\
\text { I } \\
\text { I }\end{array}$ \\
\hline $\begin{array}{l}7493.057 \\
7493.426 \\
7495.386 \\
7495.560 \\
7496.971\end{array}$ & $\begin{array}{l}1 \\
5 \\
1 \\
5 \\
2\end{array}$ & & $\begin{array}{l}\text { I } \\
\text { I } \\
\text { I } \\
\text { I } \\
\text { I }\end{array}$ & $\begin{array}{l}7531.123 \\
7534.222 \\
7535.850 \\
7535.976 \\
7536.409\end{array}$ & $\begin{array}{r}30 \\
4 \\
2 \\
12 \\
75\end{array}$ & $\begin{array}{l}3 \\
5\end{array}$ & $\begin{array}{l}\text { I } \\
\text { I } \\
\text { I } \\
\text { II } \\
\text { I I }\end{array}$ & $\begin{array}{l}7578.532 \\
7579.436 \\
7580.326 \\
7580.675 \\
7581.844\end{array}$ & $\begin{array}{r}3 \\
2 \\
10 \\
12 \\
6\end{array}$ & & $\begin{array}{l}\text { I } \\
\text { I } \\
\text { I } \\
\text { I } \\
\text { I }\end{array}$ \\
\hline $\begin{array}{l}7497.523 \\
7497.867 \\
7498.991 \\
7499.606 \\
7500.567\end{array}$ & $\begin{array}{l}2 \\
2 \\
6 \\
4 \\
2\end{array}$ & 1 & $\begin{array}{l}\text { I } \\
\text { II } \\
\text { I } \\
\text { I } \\
\text { I }\end{array}$ & $\begin{array}{l}7537.415 \\
7538.247 \\
7538.399 \\
7540.574 \\
7541.326\end{array}$ & $\begin{array}{l}30 \\
2 \\
4 \\
3 \\
2 w\end{array}$ & & $\begin{array}{l}\text { I } \\
\text { I } \\
\text { I } \\
\text { I } \\
\text { I }\end{array}$ & $\begin{array}{l}7583.407 \\
7585.688 \\
7585.778 \\
7586.209 \\
7586.974\end{array}$ & $\begin{array}{r}12 \\
300 \\
200 \\
12 \\
10\end{array}$ & 3 & $\begin{array}{l}\text { I } \\
\text { I } \\
\text { I } \\
\text { I } \\
\text { I }\end{array}$ \\
\hline $\begin{array}{l}7501.157 \\
7501.913 \\
7503.848 \\
7504.204 \\
7505.288\end{array}$ & $\begin{array}{l}2 \\
1 \mathrm{w} \\
7 \\
2 \\
15\end{array}$ & 5 & $\begin{array}{l}\text { I } \\
\text { I } \\
\text { I I } \\
\text { I } \\
\text { I }\end{array}$ & $\begin{array}{l}7542.597 \\
7544.300 \\
7545.643 \\
7545.814 \\
7546.154\end{array}$ & $\begin{array}{l}3 \\
3 \\
2 \\
2 \\
3\end{array}$ & & $\begin{array}{l}\text { I } \\
\text { I } \\
\text { I } \\
\text { I } \\
\text { I }\end{array}$ & $\begin{array}{l}7591.507 \\
7591.732 \\
7591.979 \\
7593.380 \\
7593.729\end{array}$ & $\begin{array}{l}3 \\
2 \\
5 \\
3 w \\
2\end{array}$ & & $\begin{array}{l}\text { I } \\
\text { I } \\
\text { I } \\
\text { I } \\
\text { I }\end{array}$ \\
\hline $\begin{array}{l}7505.491 \\
7506.275 \\
7507.383 \\
7507.731 \\
7507.917\end{array}$ & $\begin{array}{r}2 \\
4 \\
3 \\
3 \\
20\end{array}$ & & $\begin{array}{l}\text { I } \\
\text { I } \\
\text { I } \\
\text { I } \\
\text { I }\end{array}$ & $\begin{array}{l}7547.272 \\
7549.308 \\
7550.650 \\
7551.448 \\
7551.973\end{array}$ & $\begin{array}{r}12 \\
120 \\
3 \\
10 \\
3\end{array}$ & 4 & $\begin{array}{l}\text { II } \\
\text { I } \\
\text { I } \\
\text { I } \\
\text { I }\end{array}$ & $\begin{array}{l}7596.553 \\
7598.198 \\
7598.742 \\
7599.313 \\
7599.613\end{array}$ & $\begin{array}{r}1 \\
75 \\
10 \\
3 \\
4\end{array}$ & $\begin{array}{l}5 \\
1 \mathrm{~h}\end{array}$ & $\begin{array}{l}\text { I } \\
\text { II } \\
\text { II } \\
\text { I } \\
\text { I }\end{array}$ \\
\hline $\begin{array}{l}7508.475 \\
7508.674 \\
7508.789 \\
7509.693 \\
7511.014\end{array}$ & $\begin{array}{r}30 \\
3 \\
5 \\
2 \\
25\end{array}$ & & $\begin{array}{l}\text { I } \\
\text { I } \\
\text { I } \\
\text { I } \\
\text { I }\end{array}$ & $\begin{array}{l}7553.414 \\
7553.978 \\
7555.320 \\
7555.860 \\
7556.218\end{array}$ & $\begin{array}{r}1 \\
7 \\
12 \\
2 \\
6\end{array}$ & & $\begin{array}{l}\text { I } \\
\text { I } \\
\text { I } \\
\text { I } \\
\text { I }\end{array}$ & $\begin{array}{l}7601.872 \\
7602.774 \\
7603.228 \\
7603.608 \\
7606.300\end{array}$ & $\begin{array}{c}3 \\
8 \\
7 \\
10 \\
3 \mathrm{w}\end{array}$ & & $\begin{array}{l}\text { I } \\
\text { I } \\
\text { I } \\
\text { I } \\
\text { I }\end{array}$ \\
\hline $\begin{array}{l}7511.344 \\
7511.787 \\
7512.311 \\
7513.555 \\
7514.593\end{array}$ & $\begin{array}{r}50 \\
40 \\
5 \\
6 \\
5\end{array}$ & 12 & $\begin{array}{l}\text { I I } \\
\text { I } \\
\text { I } \\
\text { I } \\
\text { I }\end{array}$ & $\begin{array}{l}7556.866 \\
7557.130 \\
7557.727 \\
7560.910 \\
7562.954\end{array}$ & $\begin{array}{r}8 \\
4 \\
12 \\
2 \\
2\end{array}$ & & $\begin{array}{l}\text { I } \\
\text { I } \\
\text { I } \\
\text { I } \\
\text { I }\end{array}$ & $\begin{array}{l}7607.243 \\
7607.388 \\
7607.514 \\
7607.812 \\
7608.636\end{array}$ & $\begin{array}{r}3 \\
10 \\
10 \\
75 \\
6\end{array}$ & & $\begin{array}{l}\text { I } \\
\text { I } \\
\text { I } \\
\text { I } \\
\text { I }\end{array}$ \\
\hline $\begin{array}{l}7514.883 \\
7516.729 \\
7516.993 \\
7517.946 \\
7518.782\end{array}$ & $\begin{array}{r}8 \\
10 \\
4 \\
30 \\
20\end{array}$ & 1 & $\begin{array}{l}\text { I } \\
\text { I } \\
\text { I } \\
\text { I } \\
\text { I }\end{array}$ & $\begin{array}{l}7563.691 \\
7565.308 \\
7565.849 \\
7566.527 \\
7567.741\end{array}$ & $\begin{array}{r}4 \\
1 \\
25 \\
75 \\
300\end{array}$ & 5 & $\begin{array}{l}\text { I } \\
\text { I } \\
\text { I } \\
\text { I I } \\
\text { I }\end{array}$ & $\begin{array}{l}7610.067 \\
7611.251 \\
7612.403 \\
7614.237 \\
7614.773\end{array}$ & $\begin{array}{l}3 \\
4 \\
5 \\
1 \mathrm{w} \\
15\end{array}$ & 3 & $\begin{array}{l}\text { I } \\
\text { I } \\
\text { I } \\
\text { I } \\
\text { I I }\end{array}$ \\
\hline
\end{tabular}


Table 1. Wavelengths of Thorium Spectra - Continued

\begin{tabular}{|c|c|c|c|c|c|c|c|c|c|c|c|}
\hline \multirow{2}{*}{$\begin{array}{l}\text { Wave- } \\
\text { length }\end{array}$} & \multicolumn{2}{|c|}{ Intensity } & \multirow{2}{*}{$\begin{array}{l}\text { Spec- } \\
\text { trum }\end{array}$} & \multirow{2}{*}{$\begin{array}{l}\text { Wave- } \\
\text { length }\end{array}$} & \multicolumn{2}{|c|}{ Intensity } & \multirow{2}{*}{$\begin{array}{l}\text { Spec- } \\
\text { trum }\end{array}$} & \multirow{2}{*}{$\begin{array}{l}\text { Wave- } \\
\text { length }\end{array}$} & \multicolumn{2}{|c|}{ Intensity } & \multirow{2}{*}{$\begin{array}{l}\text { Spec- } \\
\text { trum }\end{array}$} \\
\hline & Lamp & Spark & & & Lamp & Spark & & & Lamp & Spark & \\
\hline $\begin{array}{l}7615.934 \\
7616.669 \\
7616.975 \\
7620.056 \\
7620.573\end{array}$ & $\begin{array}{r}3 \\
12 \\
2 \\
75 \\
7\end{array}$ & I h & $\begin{array}{l}\text { I } \\
\text { I } \\
\text { I } \\
\text { I } \\
\text { II }\end{array}$ & $\begin{array}{l}7664.918 \\
7665.722 \\
7666.566 \\
7668.949 \\
7669.756\end{array}$ & $\begin{array}{c}2 w \\
5 \\
10 \\
5 \\
2\end{array}$ & & $\begin{array}{l}\text { I } \\
\text { I } \\
\text { I } \\
\text { I } \\
\text { I }\end{array}$ & $\begin{array}{l}7718.82 \\
7719.23 \\
7719.56 \\
7720.38 \\
7721.20\end{array}$ & $\begin{array}{r}2 \\
5 \\
2 \\
5 \\
50\end{array}$ & $\begin{array}{l}1 \\
1\end{array}$ & $\begin{array}{l}\text { I } \\
\text { I } \\
\text { I } \\
\text { I } \\
\text { I }\end{array}$ \\
\hline $\begin{array}{l}7620.812 \\
7623.042 \\
7623.552 \\
7624.300 \\
7625.110\end{array}$ & $\begin{array}{r}2 \\
2 \\
10 \\
25 \\
20\end{array}$ & $\begin{array}{l}2 w \\
4\end{array}$ & $\begin{array}{l}\text { I } \\
\text { I } \\
\text { I I } \\
\text { I I } \\
\text { I }\end{array}$ & $\begin{array}{l}7672.253 \\
7674.383 \\
7675.625 \\
7676.217 \\
7676.417\end{array}$ & $\begin{array}{r}10 \\
3 \\
2 \\
50 \\
1\end{array}$ & 8 & $\begin{array}{l}\text { I } \\
\text { I } \\
\text { I } \\
\text { I I } \\
\text { I }\end{array}$ & $\begin{array}{l}7721.78 \\
7722.16 \\
7723.64 \\
7723.94 \\
7724.37\end{array}$ & $\begin{array}{l}1 \\
3 \mathrm{w} \\
2 \\
10 \\
2\end{array}$ & & $\begin{array}{l}\text { I } \\
\text { I } \\
\text { I } \\
\text { I } \\
\text { I }\end{array}$ \\
\hline $\begin{array}{l}7625.700 \\
7625.900 \\
7627.164 \\
7627.386 \\
7627.811\end{array}$ & $\begin{array}{r}120 \\
7 \\
80 \\
2 \\
2\end{array}$ & & $\begin{array}{l}\text { I } \\
\text { I } \\
\text { I } \\
\text { I } \\
\text { I }\end{array}$ & $\begin{array}{l}7678.124 \\
7679.875 \\
7682.044 \\
7683.011 \\
7683.666\end{array}$ & $\begin{array}{r}60 \\
3 \\
5 \\
10 \\
3\end{array}$ & & $\begin{array}{l}\text { I } \\
\text { I } \\
\text { I } \\
\text { I } \\
\text { I }\end{array}$ & $\begin{array}{l}7724.48 \\
7726.15 \\
7728.95 \\
7729.50 \\
7730.11\end{array}$ & $\begin{array}{c}3 \\
1 \\
50 \\
4 \mathrm{~s} \\
3\end{array}$ & 3 & $\begin{array}{l}\text { I } \\
\text { I } \\
\text { II } \\
\text { I } \\
\text { I }\end{array}$ \\
\hline $\begin{array}{l}7629.709 \\
7630.302 \\
7630.519 \\
7632.127 \\
7633.773\end{array}$ & $\begin{array}{r}4 \\
70 \\
20 \\
15 \\
12\end{array}$ & & $\begin{array}{l}\text { I } \\
\text { I } \\
\text { I } \\
\text { I } \\
\text { I }\end{array}$ & $\begin{array}{l}7685.304 \\
7685.493 \\
7686.147 \\
7686.682 \\
7687.070\end{array}$ & $\begin{array}{c}150 \\
2 \\
10 \\
2 \\
1 \mathrm{~h}\end{array}$ & 30 & $\begin{array}{l}\text { I I } \\
\text { I } \\
\text { I } \\
\text { I } \\
\text { I I }\end{array}$ & $\begin{array}{l}7730.46 \\
7730.73 \\
7732.67 \\
7734.62 \\
7737.47\end{array}$ & $\begin{array}{c}4 \mathrm{~s} \\
100 \\
2 \mathrm{w} \\
5 \\
6\end{array}$ & 10 & $\begin{array}{l}\text { I } \\
\text { I I } \\
\text { I } \\
\text { I } \\
\text { I }\end{array}$ \\
\hline $\begin{array}{l}7633.895 \\
7636.166 \\
7637.385 \\
7637.627 \\
7637.759\end{array}$ & $\begin{array}{r}4 \\
50 \\
20 \\
2 \\
5\end{array}$ & $1 \mathrm{~h}$ & $\begin{array}{l}\text { II } \\
\text { I } \\
\text { I } \\
\text { I } \\
\text { I I }\end{array}$ & $\begin{array}{l}7687.791 \\
7690.642 \\
7690.771 \\
7691.167 \\
7691.768\end{array}$ & $\begin{array}{l}4 \mathrm{w} \\
1 \\
2 \\
1 \mathrm{w} \\
20\end{array}$ & & $\begin{array}{l}\text { I } \\
\text { I } \\
\text { I } \\
\text { I } \\
\text { I }\end{array}$ & $\begin{array}{l}7737.79 \\
7738.36 \\
7740.87 \\
7742.56 \\
7743.12\end{array}$ & $\begin{array}{l}2 \\
2 \mathrm{~s} \\
5 \\
60 \\
1\end{array}$ & & $\begin{array}{l}\text { I } \\
\text { I } \\
\text { I } \\
\text { I } \\
\text { I }\end{array}$ \\
\hline $\begin{array}{l}7638.780 \\
7641.193 \\
7641.594 \\
7642.026 \\
7642.868\end{array}$ & $\begin{array}{r}30 \\
3 \\
3 \\
4 \\
4\end{array}$ & & $\begin{array}{l}\text { I } \\
\text { I } \\
\text { I } \\
\text { I } \\
\text { I }\end{array}$ & $\begin{array}{l}7693.802 \\
7697.917 \\
7698.035 \\
7698.916 \\
7699.400\end{array}$ & $\begin{array}{r}25 \\
18 \\
3 \\
5 \\
10\end{array}$ & 3 & $\begin{array}{l}\text { I I } \\
\text { I } \\
\text { I } \\
\text { I } \\
\text { I }\end{array}$ & $\begin{array}{l}7743.23 \\
7743.95 \\
7744.23 \\
7744.78 \\
7745.12\end{array}$ & $\begin{array}{r}2 \\
15 \\
2 \\
3 \\
2\end{array}$ & 2 & $\begin{array}{l}\text { I } \\
\text { I I } \\
\text { I } \\
\text { I } \\
\text { I }\end{array}$ \\
\hline $\begin{array}{l}7644.727 \\
7644.957 \\
7647.377 \\
7648.240 \\
7650.659\end{array}$ & $\begin{array}{c}5 \\
10 \\
500 \\
1 \mathrm{w} \\
4\end{array}$ & $\begin{array}{l}3 \\
4\end{array}$ & $\begin{array}{l}\text { I } \\
\text { II } \\
\text { I } \\
\text { I } \\
\text { I }\end{array}$ & $\begin{array}{l}7699.659 \\
7699.776 \\
7699.868 \\
7699.998 \\
7701.104\end{array}$ & $\begin{array}{r}8 \\
10 \\
5 \\
10 \\
30\end{array}$ & 10 & $\begin{array}{l}\text { I } \\
\text { I I } \\
\text { I } \\
\text { I } \\
\text { I I }\end{array}$ & $\begin{array}{l}7745.25 \\
7745.81 \\
7746.05 \\
7747.41 \\
7748.38\end{array}$ & $\begin{array}{l}2 \\
5 \\
2 \\
4 \\
1\end{array}$ & & $\begin{array}{l}\text { I } \\
\text { I } \\
\text { I } \\
\text { I } \\
\text { I }\end{array}$ \\
\hline $\begin{array}{l}7651.007 \\
7651.726 \\
7652.313 \\
7652.540 \\
7653.827\end{array}$ & $\begin{array}{r}12 \\
30 \\
25 \\
1 \\
120\end{array}$ & & $\begin{array}{l}\text { I } \\
\text { I } \\
\text { I } \\
\text { I } \\
\text { I }\end{array}$ & $\begin{array}{l}7703.680 \\
7705.696 \\
7705.897 \\
7706.45 \\
7709.07\end{array}$ & $\begin{array}{r}30 \\
3 \\
5 \\
2 \\
4\end{array}$ & & $\begin{array}{l}\text { I } \\
\text { I } \\
\text { I } \\
\text { I } \\
\text { I }\end{array}$ & $\begin{array}{l}7748.88 \\
7749.06 \\
7749.30 \\
7749.58 \\
7749.88\end{array}$ & $\begin{array}{l}3 \\
1 \\
6 \\
4 \\
2\end{array}$ & & $\begin{array}{l}\text { I } \\
\text { I } \\
\text { I } \\
\text { I } \\
\text { I }\end{array}$ \\
\hline $\begin{array}{l}7654.256 \\
7654.699 \\
7655.695 \\
7657.064 \\
7657.341\end{array}$ & $\begin{array}{r}4 \\
40 \\
3 \\
2 \\
5\end{array}$ & & $\begin{array}{l}\text { I } \\
\text { I } \\
\text { I } \\
\text { I } \\
\text { I }\end{array}$ & $\begin{array}{l}7709.57 \\
7709.88 \\
7710.27 \\
7711.12 \\
7712.39\end{array}$ & $\begin{array}{r}50 \\
6 \\
60 \\
12 \\
5\end{array}$ & $\begin{array}{l}8 \\
1\end{array}$ & $\begin{array}{l}\text { I I } \\
\text { I } \\
\text { I } \\
\text { I } \\
\text { I }\end{array}$ & $\begin{array}{l}7750.13 \\
7751.43 \\
7752.18 \\
7752.44 \\
7753.72\end{array}$ & $\begin{array}{r}1 \\
4 \\
10 \\
2 \\
2\end{array}$ & 1 & $\begin{array}{l}\text { I } \\
\text { I } \\
\text { I I } \\
\text { I } \\
\text { I }\end{array}$ \\
\hline $\begin{array}{l}7657.913 \\
7658.319 \\
7660.028 \\
7650.890 \\
7661.615\end{array}$ & $\begin{array}{c}3 w \\
60 \\
7 \\
18 \\
4\end{array}$ & & $\begin{array}{l}\text { I } \\
\text { I } \\
\text { I } \\
\text { I } \\
\text { I }\end{array}$ & $\begin{array}{l}7713.28 \\
7713.93 \\
7716.27 \\
7716.95 \\
7718.54\end{array}$ & $\begin{array}{r}2 \\
30 \\
3 \\
3 \\
1\end{array}$ & & $\begin{array}{l}\text { I } \\
\text { I } \\
\text { I } \\
\text { I } \\
\text { I }\end{array}$ & $\begin{array}{l}7754.01 \\
7755.60 \\
7757.49 \\
7758.25 \\
7759.87\end{array}$ & $\begin{array}{l}2 \\
2 \\
1 \\
2 \\
3\end{array}$ & & $\begin{array}{l}\text { I } \\
\text { I } \\
\text { I } \\
\text { I } \\
\text { I }\end{array}$ \\
\hline
\end{tabular}


Table 1. Wavelengths of Thorium Spectra - Continued

\begin{tabular}{|c|c|c|c|c|c|c|c|c|c|c|c|}
\hline \multirow{2}{*}{$\begin{array}{l}\text { Wave- } \\
\text { length }\end{array}$} & \multicolumn{2}{|c|}{ Intensity } & \multirow{2}{*}{$\begin{array}{l}\text { Spec- } \\
\text { trum }\end{array}$} & \multirow{2}{*}{$\begin{array}{l}\text { Wave- } \\
\text { length }\end{array}$} & \multicolumn{2}{|c|}{ Intensity } & \multirow{2}{*}{$\begin{array}{l}\text { Spec- } \\
\text { trum }\end{array}$} & \multirow{2}{*}{$\begin{array}{l}\text { Wave- } \\
\text { length }\end{array}$} & \multicolumn{2}{|c|}{ Intensity } & \multirow{2}{*}{$\begin{array}{l}\text { Spec. } \\
\text { trum }\end{array}$} \\
\hline & Lamp & Spark & & & Lamp & Spark & & & Lamp & Spark & \\
\hline $\begin{array}{l}7759.99 \\
7760.70 \\
7760.87 \\
7761.16 \\
7761.72\end{array}$ & $\begin{array}{r}4 \\
2 \\
2 \\
1 \\
10\end{array}$ & 1 & $\begin{array}{l}\text { II } \\
\text { I } \\
\text { I } \\
\text { I } \\
\text { I }\end{array}$ & $\begin{array}{l}7798.36 \\
7798.94 \\
7799.62 \\
7800.02 \\
7800.46\end{array}$ & $\begin{array}{l}90 \\
6 \mathrm{w} \\
5 \mathrm{w} \\
3 \\
1\end{array}$ & & $\begin{array}{l}\text { I } \\
\text { I } \\
I \\
I \\
I\end{array}$ & $\begin{array}{l}7834.45 \\
7835.26 \\
7835.62 \\
7836.46 \\
7839.28\end{array}$ & $\begin{array}{r}75 \\
7 \\
3 \\
15 \\
4\end{array}$ & $\begin{array}{r}18 \\
2\end{array}$ & $\begin{array}{l}\text { II } \\
\text { I I } \\
\text { I } \\
\text { I } \\
\text { I }\end{array}$ \\
\hline $\begin{array}{l}7762.73 \\
7764.64 \\
7765.63 \\
7766.94 \\
7767.12\end{array}$ & $\begin{array}{r}10 \\
10 \\
10 \\
3 \\
6\end{array}$ & 2 & $\begin{array}{l}\text { I } \\
\text { II } \\
\text { I } \\
\text { I } \\
\text { I }\end{array}$ & $\begin{array}{l}7801.11 \\
7801.74 \\
7801.94 \\
7802.81 \\
7803.16\end{array}$ & $\begin{array}{l}2 \\
3 \\
1 \\
6 \\
1 \mathrm{w}\end{array}$ & & $\begin{array}{l}\text { I } \\
\text { I } \\
\text { I } \\
\text { I } \\
\text { I }\end{array}$ & $\begin{array}{l}7839.53 \\
7840.31 \\
7840.45 \\
7841.01 \\
7841.54\end{array}$ & $\begin{array}{r}1 \\
100 \\
100 \\
2 \\
1\end{array}$ & & $\begin{array}{l}I \\
\text { I } \\
\text { I } \\
\text { I } \\
\text { I }\end{array}$ \\
\hline $\begin{array}{l}7767.31 \\
7767.57 \\
7767.97 \\
7768.46 \\
7770.39\end{array}$ & $\begin{array}{l}3 \\
5 \\
2 \\
2 \\
1\end{array}$ & & $\begin{array}{l}\text { I } \\
\text { I } \\
\text { I } \\
\text { I } \\
\text { I }\end{array}$ & $\begin{array}{l}7803.78 \\
7804.14 \\
7804.53 \\
7806.02 \\
7807.02\end{array}$ & $\begin{array}{l}2 \mathrm{~s} \\
12 \\
18 \\
3 \\
3\end{array}$ & & $\begin{array}{l}\text { I } \\
\text { I } \\
\text { I } \\
\text { I } \\
\text { I }\end{array}$ & $\begin{array}{l}7841.79 \\
7842.03 \\
7842.26 \\
7843.54 \\
7845.35\end{array}$ & $\begin{array}{r}90 \\
4 \\
30 \\
3 \\
3\end{array}$ & & $\begin{array}{l}\text { I } \\
\text { I } \\
\text { I } \\
\text { I } \\
I\end{array}$ \\
\hline $\begin{array}{l}7771.22 \\
7771.95 \\
7773.73 \\
7774.88 \\
7775.17\end{array}$ & $\begin{array}{c}1 \\
30 \\
12 \\
1 \\
5\end{array}$ & 2 & $\begin{array}{l}\text { I } \\
\text { I } \\
\text { I I } \\
\text { I } \\
\text { I }\end{array}$ & $\begin{array}{l}7807.62 \\
7807.87 \\
7808.06 \\
7808.53 \\
7809.23\end{array}$ & $\begin{array}{r}1 \\
10 \\
4 \\
3\end{array}$ & $\begin{array}{l}1 \\
1 \\
2 \\
1\end{array}$ & $\begin{array}{l}\text { I I } \\
\text { I } \\
\text { I I I } \\
\text { I } \\
\text { I }\end{array}$ & $\begin{array}{l}7845.59 \\
7846.86 \\
7846.97 \\
7847.54 \\
7847.80\end{array}$ & $\begin{array}{r}1 \\
1 \\
1 \\
250 \\
50\end{array}$ & 2 & $\begin{array}{l}I \\
I \\
I \\
I \\
I\end{array}$ \\
\hline $\begin{array}{l}7775.44 \\
7775.76 \\
7776.66 \\
7776.82 \\
7777.26\end{array}$ & $\begin{array}{c}10 \\
2 \\
10 \\
2 \mathrm{w} \\
2\end{array}$ & & $\begin{array}{l}\text { I } \\
\text { I } \\
\text { I } \\
\text { I }\end{array}$ & $\begin{array}{l}7809.43 \\
7809.53 \\
7810.62 \\
7810.85 \\
7811.14\end{array}$ & $\begin{array}{r}10 \\
2 \\
12 \\
1 \\
7\end{array}$ & 2 & $\begin{array}{l}\text { I } \\
\text { I } \\
\text { I } \\
\text { I } \\
\text { I I }\end{array}$ & $\begin{array}{l}7848.45 \\
7848.68 \\
7849.64 \\
7849.88 \\
7851.01\end{array}$ & $\begin{array}{c}60 \\
3 \\
70 \\
8 \\
1 \mathrm{w}\end{array}$ & 8 & $\begin{array}{l}\text { I } \\
\text { I } \\
\text { II } \\
I \\
\text { I }\end{array}$ \\
\hline $\begin{array}{l}7778.49 \\
7779.05 \\
7779.74 \\
7780.82 \\
7781.83\end{array}$ & $\begin{array}{r}18 \\
4 \\
4 \\
3 \\
15\end{array}$ & & $\begin{array}{l}\text { I } \\
\text { I } \\
\text { I } \\
\text { I } \\
\text { I }\end{array}$ & $\begin{array}{l}7811.58 \\
7812.12 \\
7813.46 \\
7813.96 \\
7815.01\end{array}$ & $\begin{array}{r}4 \\
2 \\
15 \\
12 \\
1\end{array}$ & & $\begin{array}{l}\text { I } \\
\text { I } \\
\text { I } \\
\text { I } \\
\text { I }\end{array}$ & $\begin{array}{l}7851.62 \\
7853.28 \\
7853.46 \\
7854.92 \\
7855.71\end{array}$ & $\begin{array}{l}1 \\
7 \\
8 \\
6 \\
1\end{array}$ & 1 & $\begin{array}{l}\text { I } \\
\text { I } \\
\text { I } \\
\text { I } \\
\text { I }\end{array}$ \\
\hline $\begin{array}{l}7782.32 \\
7783.41 \\
7784.21 \\
7784.60 \\
7784.78\end{array}$ & $\begin{array}{c}120 \\
4 \\
12 \mathrm{w} \\
5 \\
2\end{array}$ & 2 & $\begin{array}{l}\text { I } \\
\text { I } \\
\text { I } \\
\text { I } \\
\text { I }\end{array}$ & $\begin{array}{l}7816.12 \\
7816.46 \\
7816.80 \\
7817.77 \\
7818.34\end{array}$ & $\begin{array}{r}8 \\
2 \\
1 \\
350 \\
18\end{array}$ & $\begin{array}{l}4 \\
3\end{array}$ & $\begin{array}{l}\text { I } \\
\text { I } \\
\text { I } \\
\text { I } \\
\text { I I }\end{array}$ & $\begin{array}{l}7856.37 \\
7859.20 \\
7860.28 \\
7860.87 \\
7862.34\end{array}$ & $\begin{array}{l}5 \mathrm{w} \\
1 \\
2 \\
1 \\
5\end{array}$ & & $\begin{array}{l}\text { I } \\
\text { I } \\
\text { I } \\
\text { I } \\
\text { I }\end{array}$ \\
\hline $\begin{array}{l}7785.73 \\
7786.42 \\
7787.80 \\
7788.00 \\
7788.35\end{array}$ & $\begin{array}{r}2 \\
6 \\
125 \\
1 \\
1\end{array}$ & $\begin{array}{r}1 \\
20\end{array}$ & $\begin{array}{l}\text { I } \\
\text { I } \\
\text { I I } \\
\text { I } \\
\text { I }\end{array}$ & $\begin{array}{l}7818.65 \\
7820.21 \\
7822.38 \\
7823.02 \\
7823.46\end{array}$ & $\begin{array}{c}2 w \\
1 \\
12 \\
4 \\
8\end{array}$ & & $\begin{array}{l}\text { I } \\
\text { I } \\
\text { I } \\
\text { I } \\
\text { I }\end{array}$ & $\begin{array}{l}7863.41 \\
7864.01 \\
7864.26 \\
7864.38 \\
7864.66\end{array}$ & $\begin{array}{r}25 \\
12 \\
2 \\
3 \\
3\end{array}$ & 2 & $\begin{array}{l}\text { I I } \\
\text { I } \\
\text { I } \\
\text { I } \\
\text { I }\end{array}$ \\
\hline $\begin{array}{l}7788.50 \\
7788.93 \\
7789.91 \\
7790.14 \\
7790.50\end{array}$ & $\begin{array}{r}1 \\
250 \\
4 \\
4 \\
2\end{array}$ & 2 & $\begin{array}{l}\text { I } \\
\text { I } \\
\text { I } \\
\text { I } \\
\text { I }\end{array}$ & $\begin{array}{l}7824.03 \\
7824.81 \\
7828.06 \\
7828.48 \\
7828.81\end{array}$ & $\begin{array}{l}6 \\
2 \\
1 \\
3 \\
2\end{array}$ & & $\begin{array}{l}\text { I } \\
\text { I } \\
\text { I } \\
\text { I } \\
\text { I }\end{array}$ & $\begin{array}{l}7865.08 \\
7865.25 \\
7865.51 \\
7865.97 \\
7866.72\end{array}$ & $\begin{array}{r}4 \\
5 \\
1 \\
200 \\
1\end{array}$ & & $\begin{array}{l}\text { I } \\
\text { I } \\
\text { I } \\
\text { I } \\
\text { I }\end{array}$ \\
\hline $\begin{array}{l}7791.89 \\
7792.79 \\
7793.12 \\
7794.10 \\
7796.23\end{array}$ & $\begin{array}{l}4 \\
1 \\
7 \\
5 \\
1\end{array}$ & & $\begin{array}{l}\text { I } \\
\text { I } \\
\text { I } \\
\text { I } \\
\text { I }\end{array}$ & $\begin{array}{l}7829.26 \\
7829.88 \\
7830.69 \\
7832.89 \\
7833.77\end{array}$ & $\begin{array}{l}3 \\
2 \\
3 \mathrm{w} \\
1 \\
10\end{array}$ & 2 & $\begin{array}{l}\text { I } \\
\text { I } \\
\text { I } \\
\text { I } \\
\text { I }\end{array}$ & $\begin{array}{l}7867.68 \\
7868.40 \\
7868.93 \\
7869.77 \\
7871.27\end{array}$ & $\begin{array}{r}7 \\
75 \\
10 \\
2 \\
4\end{array}$ & 2 & $\begin{array}{l}\text { I } \\
\text { I } \\
\text { I } \\
\text { I } \\
\text { I I }\end{array}$ \\
\hline
\end{tabular}


Table 1. Wavelengths of Thorium Spectra - Continued

\begin{tabular}{|c|c|c|c|c|c|c|c|c|c|c|c|}
\hline \multirow{2}{*}{$\begin{array}{l}\text { Wave- } \\
\text { length }\end{array}$} & \multicolumn{2}{|c|}{ Intensity } & \multirow{2}{*}{$\begin{array}{l}\text { Spec- } \\
\text { trum }\end{array}$} & \multirow{2}{*}{$\begin{array}{l}\text { Wave- } \\
\text { length }\end{array}$} & \multicolumn{2}{|c|}{ Intensity } & \multirow{2}{*}{$\begin{array}{l}\text { Spec- } \\
\text { trum }\end{array}$} & \multirow{2}{*}{$\begin{array}{l}\text { Wave- } \\
\text { length }\end{array}$} & \multicolumn{2}{|c|}{ Intensity } & \multirow{2}{*}{$\begin{array}{l}\text { Spec } \\
\text { trum }\end{array}$} \\
\hline & $\operatorname{Lamp}$ & Spark & & & Lamp & Spark & & & Lamp & Spark & \\
\hline $\begin{array}{l}7872.26 \\
7872.30 \\
7872.60 \\
7872.81 \\
7874.10\end{array}$ & $\begin{array}{l}1 \\
3 \\
1 \\
6\end{array}$ & $\begin{array}{l}4 \\
5\end{array}$ & $\begin{array}{l}\text { I I I } \\
\text { I } \\
\text { II } \\
\text { I } \\
\text { I }\end{array}$ & $\begin{array}{l}7915.15 \\
7916.80 \\
7917.25 \\
7918.28 \\
7918.44\end{array}$ & $\begin{array}{r}5 \\
10 \\
5 \\
1 \\
3\end{array}$ & & $\begin{array}{l}\text { I } \\
\text { I } \\
\text { I } \\
\text { I } \\
\text { I }\end{array}$ & $\begin{array}{l}7951.75 \\
7952.41 \\
7952.76 \\
7953.52 \\
7954.60\end{array}$ & $\begin{array}{r}5 \\
1 \\
2 \\
3 \\
20\end{array}$ & 10 & $\begin{array}{l}\text { I } \\
\text { II } \\
\text { I } \\
\text { I } \\
\text { I }\end{array}$ \\
\hline $\begin{array}{l}7875.46 \\
7877.07 \\
7877.68 \\
7877.87 \\
7878.47\end{array}$ & $\begin{array}{r}40 \\
10 \\
4 \\
1 \\
3\end{array}$ & $\begin{array}{l}4 \\
2\end{array}$ & $\begin{array}{l}\text { I I } \\
\text { I I } \\
\text { I } \\
\text { I } \\
\text { I }\end{array}$ & $\begin{array}{l}7919.45 \\
7920.74 \\
7921.13 \\
7921.78 \\
7922.25\end{array}$ & $\begin{array}{l}1 \\
4 \\
1 \\
1 \\
5\end{array}$ & 1 & $\begin{array}{l}\text { I } \\
\text { I I } \\
\text { I } \\
\text { I } \\
\text { I I }\end{array}$ & $\begin{array}{l}7955.08 \\
7956.69 \\
7958.19 \\
7959.31 \\
7960.34\end{array}$ & $\begin{array}{l}8 \\
5 \\
1 \\
2 \\
2\end{array}$ & & $\begin{array}{l}\text { I } \\
\text { I } \\
\text { I } \\
\text { I } \\
\text { I }\end{array}$ \\
\hline $\begin{array}{l}7878.71 \\
7879.22 \\
7879.42 \\
7880.59 \\
7880.77\end{array}$ & $\begin{array}{l}4 \\
1 \\
2 \\
5 \\
6\end{array}$ & & $\begin{array}{l}\text { I } \\
\text { I } \\
\text { I } \\
\text { I } \\
\text { I }\end{array}$ & $\begin{array}{l}7922.57 \\
7922.81 \\
7922.92 \\
7924.25 \\
7925.00\end{array}$ & $\begin{array}{r}20 \\
2 \\
2 \\
3 \\
10\end{array}$ & 3 & $\begin{array}{l}\text { I } \\
\text { I } \\
\text { I } \\
\text { I I }\end{array}$ & $\begin{array}{l}7961.16 \\
7961.37 \\
7961.98 \\
7962.75 \\
7963.45\end{array}$ & $\begin{array}{r}10 \\
25 \\
25 \\
1 \\
1\end{array}$ & & $\begin{array}{l}\text { I } \\
\text { I } \\
\text { I } \\
\text { I } \\
\text { I }\end{array}$ \\
\hline $\begin{array}{l}7882.10 \\
7882.35 \\
7882.88 \\
7883.61 \\
7883.73\end{array}$ & $\begin{array}{r}75 \\
4 \\
2 \\
2 \\
3\end{array}$ & & $\begin{array}{l}\text { I } \\
\text { I } \\
\text { I } \\
\text { I } \\
\text { I }\end{array}$ & $\begin{array}{l}7925.77 \\
7926.30 \\
7926.73 \\
7927.53 \\
7927.68\end{array}$ & $\begin{array}{r}10 \\
2 \\
1 \\
6 \\
4\end{array}$ & & $\begin{array}{l}\text { I } \\
\text { I } \\
\text { I } \\
\text { I } \\
\text { I }\end{array}$ & $\begin{array}{l}7964.84 \\
7965.71 \\
7966.28 \\
7966.84 \\
7968.41\end{array}$ & $\begin{array}{l}3 \\
3 \mathrm{~s} \\
1 \\
3 \\
1\end{array}$ & & $\begin{array}{l}I \\
I \\
I \\
I \\
I\end{array}$ \\
\hline $\begin{array}{l}7883.96 \\
7886.28 \\
7886.39 \\
7890.94 \\
7891.58\end{array}$ & $\begin{array}{c}2 \\
100 \\
5 \\
2 \mathrm{w} \\
2\end{array}$ & & $\begin{array}{l}\text { I } \\
\text { I } \\
\text { I } \\
\text { I } \\
\text { I }\end{array}$ & $\begin{array}{l}7928.96 \\
7929.38 \\
7929.82 \\
7929.94 \\
7932.27\end{array}$ & $\begin{array}{r}10 \\
2 \\
6 \\
3 \\
1\end{array}$ & 3 & $\begin{array}{l}\text { II } \\
\text { I } \\
\text { I } \\
\text { I } \\
\text { I }\end{array}$ & $\begin{array}{l}7969.78 \\
7970.27 \\
7971.56 \\
7972.59 \\
7972.87\end{array}$ & $\begin{array}{r}1 \\
15 \\
3 \\
120 \\
6\end{array}$ & 4 & $\begin{array}{l}\text { I } \\
\text { I } \\
\text { I } \\
\text { I } \\
\text { I I }\end{array}$ \\
\hline $\begin{array}{l}7891.74 \\
7892.74 \\
7892.86 \\
7893.28 \\
7893.62\end{array}$ & $\begin{array}{r}5 \\
4 \\
2 \\
12 \\
10\end{array}$ & & $\begin{array}{l}\text { I } \\
\text { I } \\
\text { I } \\
\text { I } \\
\text { I }\end{array}$ & $\begin{array}{l}7932.82 \\
7933.25 \\
7933.90 \\
7934.21 \\
7934.88\end{array}$ & $\begin{array}{l}6 \\
2 \\
1 \\
1 \\
1\end{array}$ & & $\begin{array}{l}\text { I } \\
\text { I } \\
\text { I } \\
\text { I } \\
\text { I }\end{array}$ & $\begin{array}{l}7973.13 \\
7973.51 \\
7974.16 \\
7976.18 \\
7976.89\end{array}$ & $\begin{array}{r}1 \\
3 \\
12 \\
5 \\
2\end{array}$ & & $\begin{array}{l}\text { I } \\
\text { I } \\
\text { I } \\
I \\
I\end{array}$ \\
\hline $\begin{array}{l}7894.78 \\
7896.02 \\
7896.43 \\
7896.94 \\
7897.23\end{array}$ & $\begin{array}{r}6 \\
2 \\
18 \\
2 \\
6\end{array}$ & $\begin{array}{l}2 \\
3\end{array}$ & $\begin{array}{l}\text { I I } \\
\text { I } \\
\text { I I } \\
\text { I } \\
\text { I }\end{array}$ & $\begin{array}{l}7936.48 \\
7937.13 \\
7937.73 \\
7937.95 \\
7938.75\end{array}$ & $\begin{array}{r}7 \\
10 \\
75 \\
2 \\
2\end{array}$ & 1 & $\begin{array}{l}\text { II } \\
\text { I } \\
\text { I } \\
\text { I } \\
\text { I }\end{array}$ & $\begin{array}{l}7977.62 \\
7978.00 \\
7978.26 \\
7978.97 \\
7979.52\end{array}$ & $\begin{array}{c}2 \mathrm{w} \\
1 \\
1 \\
400 \\
3\end{array}$ & & $\begin{array}{l}\text { I } \\
\text { I } \\
\text { I } \\
\text { I } \\
\text { I }\end{array}$ \\
\hline $\begin{array}{l}7897.98 \\
7898.44 \\
7899.03 \\
7899.62 \\
7900.32\end{array}$ & $\begin{array}{r}50 \\
1 \\
4 \\
75 \\
180\end{array}$ & $\begin{array}{l}1 \\
1\end{array}$ & $\begin{array}{l}\text { I } \\
\text { I } \\
\text { I } \\
\text { I } \\
\text { I }\end{array}$ & $\begin{array}{l}7939.69 \\
7940.73 \\
7941.27 \\
7941.73 \\
7942.66\end{array}$ & $\begin{array}{r}1 \\
3 \\
2 \\
180 \\
1\end{array}$ & 1 & $\begin{array}{l}\text { I } \\
\text { I } \\
\text { I } \\
\text { I } \\
\text { I }\end{array}$ & $\begin{array}{l}7979.67 \\
7979.89 \\
7980.36 \\
7981.23 \\
7981.68\end{array}$ & $\begin{array}{r}2 \\
2 \\
1 \\
10 \\
10\end{array}$ & $\begin{array}{l}3 \\
3\end{array}$ & $\begin{array}{l}\text { I } \\
\text { I } \\
\text { I } \\
\text { II } \\
\text { II }\end{array}$ \\
\hline $\begin{array}{l}7901.42 \\
7903.34 \\
7904.28 \\
7904.42 \\
7905.70\end{array}$ & $\begin{array}{r}3 \\
4 \\
8 \\
10 \\
2\end{array}$ & 1 & $\begin{array}{l}\text { I I } \\
\text { I } \\
\text { I } \\
\text { I } \\
\text { I }\end{array}$ & $\begin{array}{l}7943.06 \\
7943.38 \\
7945.06 \\
7946.10 \\
7946.66\end{array}$ & $\begin{array}{l}7 \\
2 \mathrm{w} \\
10 \\
4 \\
2\end{array}$ & 6 & $\begin{array}{l}I \\
I \\
I I \\
I \\
I\end{array}$ & $\begin{array}{l}7982.27 \\
7982.51 \\
7983.71 \\
7984.36 \\
7984.84\end{array}$ & $\begin{array}{l}5 \\
6 \\
3 \\
6 \\
3\end{array}$ & 1 & $\begin{array}{l}\text { I } \\
\text { I } \\
\text { I } \\
\text { I I } \\
\text { I }\end{array}$ \\
\hline $\begin{array}{l}7908.14 \\
7911.28 \\
7911.58 \\
7912.40 \\
7914.50\end{array}$ & $\begin{array}{r}10 \\
1 \\
1 \\
1 \\
4\end{array}$ & & $\begin{array}{l}\text { I } \\
\text { I } \\
\text { I } \\
\text { I } \\
\text { I }\end{array}$ & $\begin{array}{l}7946.90 \\
7947.74 \\
7948.58 \\
7949.02 \\
7951.12\end{array}$ & $\begin{array}{l}2 \\
2 \\
2 \\
2\end{array}$ & $20 w$ & $\begin{array}{l}\text { I } \\
\text { I I I } \\
\text { I } \\
\text { I } \\
\text { I I I }\end{array}$ & $\begin{array}{l}7985.39 \\
7986.99 \\
7987.98 \\
7988.52 \\
7989.17\end{array}$ & $\begin{array}{r}2 \\
1 \\
1.80 \\
1 \\
3\end{array}$ & & $\begin{array}{l}\text { I } \\
\text { I } \\
\text { I } \\
\text { I } \\
\text { I }\end{array}$ \\
\hline
\end{tabular}


Table 1. Wavelengths of Thorium Spectra - Continued

\begin{tabular}{|c|c|c|c|c|c|c|c|c|c|c|}
\hline \multirow{2}{*}{$\begin{array}{l}\text { Wave- } \\
\text { length }\end{array}$} & Intensity & \multirow{2}{*}{$\begin{array}{l}\text { Spec- } \\
\text { trum }\end{array}$} & \multirow{2}{*}{$\begin{array}{l}\text { Wave- } \\
\text { length }\end{array}$} & \multicolumn{2}{|c|}{ Intensity } & \multirow{2}{*}{$\begin{array}{l}\text { Spec- } \\
\text { trum }\end{array}$} & \multirow{2}{*}{$\begin{array}{l}\text { Wave- } \\
\text { length }\end{array}$} & \multicolumn{2}{|c|}{ Intensity } & \multirow{2}{*}{$\begin{array}{l}\text { Spec- } \\
\text { trum }\end{array}$} \\
\hline & Lamp Spark & & & Lamp & Spark & & & Lamp & Spark & \\
\hline 7989.64 & 1 & I & 8027.09 & 2 & & I & 8068.74 & 2 & & I \\
\hline 7991.38 & 40 & I & 8027.44 & 1 & & I & 8069.64 & 1 & & I \\
\hline 7992.16 & 15 & I & 8027.73 & 2 & & I & 8070.64 & 2 & & I \\
\hline 7992.93 & 3 & I & 8028.30 & 1 & & I & 8071.84 & 2 & & $\bar{I}$ \\
\hline 7993.27 & 3 & $\bar{I}$ & 8029.39 & 1 & & $\bar{I}$ & 8072.19 & 1 & & I \\
\hline 7993.68 & 40 & I & 8030.20 & 50 & & I & 8073.59 & 4 & & I \\
\hline 7994.14 & 3 & $\bar{I}$ & 8030.42 & 1 & & I & 8073.96 & 2 & & I \\
\hline 7994.75 & 1 & I & 8031.09 & 3 & & I & 8074.53 & $1 \mathrm{w}$ & & I \\
\hline 7995.14 & 2 & I & 8031.98 & 1 & & I & 8075.07 & 1 & & I \\
\hline 7996.20 & 1 & I & 8032.18 & 1 & & $\vec{I}$ & 8075.65 & 90 & & I \\
\hline 7996.98 & 6 & I & 8032.43 & 180 & 15 & I & 8076.25 & 1 & & I \\
\hline 7998.73 & 2 & I & 8032.90 & 2 & & I & 8076.99 & 3 & & I \\
\hline 7998.98 & 2 & I & 8035.62 & 125 & & I & 8078.14 & 2 & & I \\
\hline 8000.04 & 75 & I & 8035.90 & 1 & & I & 8079.64 & 6 & & $\vec{I}$ \\
\hline 8000.27 & 1 & I & 8036.83 & 10 & & $\overline{\mathrm{I}}$ & 8080.32 & 1 & & I \\
\hline 8000.86 & 1 & I & 8037.05 & 2 & & I & 8082.68 & 4 & & I \\
\hline 8002.19 & 15 & $\overrightarrow{\mathrm{I}}$ & 8037.71 & 2 & & $\overrightarrow{\mathrm{I}}$ & 8083.57 & 1 & & I \\
\hline 8003.44 & 1 & I & 8038.65 & 2 & & I & 8084.31 & 1 & & I \\
\hline 8004.16 & $2 \mathrm{w}$ & I & 8044.74 & 2 & & I & 8085.22 & 90 & & I \\
\hline 8004.52 & 1 & I & 8044.96 & 2 & & $\overline{\mathrm{I}}$ & 8086.29 & 2 & & I \\
\hline 8005.21 & 10 & I & 8045.19 & 2 & & I & 8086.57 & 5 & & I \\
\hline 8006.46 & 6 & $\bar{I}$ & 8045.59 & 1 & & $\overline{\mathrm{I}}$ & 8087.50 & 3 & & $\bar{I}$ \\
\hline 8006.63 & 1 & $\overline{\mathrm{I}}$ & 8046.32 & 2 & & $\bar{I}$ & 8088.04 & 1 & & I \\
\hline 8007.36 & 1 & I & 8046.60 & 1 & & I & 8088.72 & 2 & & I \\
\hline 8008.38 & 2 & I & 8046.79 & 1 & & $\bar{I}$ & 8089.48 & 8 & & I \\
\hline 8008.97 & 1 & I & 8047.53 & 1 & & I & 8089.93 & 2 & & I \\
\hline 8009.11 & 4 & I & 8048.18 & 20 & & I & 8092.23 & 20 & & $\bar{I}$ \\
\hline 8009.65 & 3 & I & 8049.74 & 2 & & $\bar{I}$ & 8093.60 & 80 & & $\bar{I}$ \\
\hline 8010.28 & 1 & I & 8050.08 & 1 & & I & 8094.51 & 2 & & I \\
\hline 8010.70 & 15 & I & 8050.64 & 12 & & I & 8095.02 & 1 & & I \\
\hline 8011.54 & 3 & I & 8051.12 & 2 & & I & 8095.32 & 1 & & I \\
\hline 8012.42 & 1 & I & 8051.81 & $2 w$ & & $\bar{I}$ & 8095.80 & 3 & & $\bar{I}$ \\
\hline 8013.50 & 8 & I & 8052.48 & 1 & & $\vec{I}$ & 8096.26 & 10 & & I \\
\hline 8013.99 & $\mathrm{lw}$ & $\overline{\mathrm{I}}$ & 8052.72 & 2 & & I & 8097.18 & 1 & & I \\
\hline 8014.50 & 10 & I & 8053.49 & 2 & & I & 8097.51 & 2 & & I \\
\hline 8015.65 & 4 & I & 8053.62 & 1 & & I & 8097.68 & 1 & & I \\
\hline 8016.37 & 3 & I & 8054.52 & 8 & 3 & I I & 8097.89 & 4 & & I \\
\hline 8017.00 & 1 & I & 8057.11 & 1 & & I & 8100.29 & 4 & & $\bar{I}$ \\
\hline 8017.83 & 1 & $\bar{I}$ & 8057.98 & 18 & & I & 8100.89 & 1 & & $\bar{I}$ \\
\hline 8019.52 & $2 w$ & $\overrightarrow{\mathrm{I}}$ & 8059.01 & 4 & & I & 8101.67 & 1 & & I \\
\hline 8020.31 & 1 & I & 8960.03 & 6 & & I & 8101.90 & 2 & & I \\
\hline 8020.76 & 1 & I & 8060.50 & 1 & & I & 8102.15 & 1 & & I \\
\hline 8022.21 & 50 & I & 8060.75 & 1 & & $\bar{I}$ & 8102.69 & 2 & & I \\
\hline 8022.33 & 50 & I & 8061.13 & 1 & & I & 8104.76 & 2 & & $\bar{I}$ \\
\hline 8023.91 & 1 & I & 8062.63 & 180 & 15 & I & 8105.02 & 2 & & I \\
\hline 8024.25 & 40 & I & 8064.03 & 4 & & $I$ & 8105.16 & & 10 & I I I \\
\hline 8024.99 & 2 & I & 8064.39 & 1 & & I & 8106.30 & 1 & & I \\
\hline 8025.73 & 20 & I & 8065.64 & 2 & & I & 8106.58 & 2 & & I \\
\hline 8026.22 & 50 & $\bar{I}$ & 8066.83 & 100 & & I & 8107.22 & 2 & & $\vec{I}$ \\
\hline 8026.44 & 4 & I & 8068.04 & 2 & & I & 8107.54 & 1 & & I \\
\hline
\end{tabular}


Table 1. Wavelengths of Thorium Spectra - Continued

\begin{tabular}{|c|c|c|c|c|c|c|c|c|c|c|c|}
\hline \multirow{2}{*}{$\begin{array}{l}\text { Wave- } \\
\text { length }\end{array}$} & \multicolumn{2}{|c|}{ Intensity } & \multirow{2}{*}{$\begin{array}{l}\text { Spec- } \\
\text { trum }\end{array}$} & \multirow{2}{*}{$\begin{array}{l}\text { Wave- } \\
\text { length }\end{array}$} & \multicolumn{2}{|c|}{ Intensity } & \multirow{2}{*}{$\begin{array}{l}\text { Spec- } \\
\text { trum }\end{array}$} & \multirow{2}{*}{$\begin{array}{l}\text { Wave- } \\
\text { length }\end{array}$} & \multicolumn{2}{|c|}{ Intensity } & \multirow{2}{*}{$\begin{array}{l}\text { Spec } \\
\text { trum }\end{array}$} \\
\hline & Lamp & Spark & & & Lamp & Spark & & & Lamp & Spark & \\
\hline $\begin{array}{l}8108.94 \\
8109.53 \\
8109.82 \\
8110.80 \\
8111.59\end{array}$ & $\begin{array}{l}2 \\
1 \\
1 \\
2 \\
l w\end{array}$ & & $\begin{array}{l}\text { I } \\
\text { I } \\
\text { I } \\
\text { I } \\
\text { I }\end{array}$ & $\begin{array}{l}8144.91 \\
8145.51 \\
8146.24 \\
8146.76 \\
8148.00\end{array}$ & $\begin{array}{l}1 \\
5 \\
4 \\
4 \\
2\end{array}$ & & $\begin{array}{l}\text { I } \\
\text { I } \\
\text { I } \\
\text { I } \\
\text { I }\end{array}$ & $\begin{array}{l}8183.85 \\
8184.41 \\
8185.60 \\
8186.24 \\
8186.92\end{array}$ & $\begin{array}{c}8 \\
4 \\
2 \mathrm{~s} \\
2 \\
250\end{array}$ & & $\begin{array}{l}\text { I } \\
\text { I } \\
\text { I } \\
\text { I } \\
\text { I }\end{array}$ \\
\hline $\begin{array}{l}8111.80 \\
8112.58 \\
8112.83 \\
8114.56 \\
8115.05\end{array}$ & $\begin{array}{c}1 \\
3 \\
3 \\
20 \\
2 \mathrm{~h}\end{array}$ & & $\begin{array}{l}\text { I } \\
\text { I } \\
\text { I } \\
\text { I } \\
\text { I }\end{array}$ & $\begin{array}{l}8148.30 \\
8148.93 \\
8149.74 \\
8150.36 \\
8150.52\end{array}$ & $\begin{array}{r}1 \\
1 \\
12 \\
18 \\
1\end{array}$ & & $\begin{array}{l}\text { I } \\
\text { I } \\
\text { I } \\
\text { I } \\
\text { I }\end{array}$ & $\begin{array}{l}8187.51 \\
8189.67 \\
8189.98 \\
8190.91 \\
8191.08\end{array}$ & $\begin{array}{r}2 \\
1 \\
2 \\
50 \\
4\end{array}$ & & $\begin{array}{l}\text { I } \\
\text { I } \\
\text { I } \\
\text { I } \\
\text { I }\end{array}$ \\
\hline $\begin{array}{l}8115.29 \\
8115.91 \\
8116.36 \\
8116.47 \\
8117.14\end{array}$ & $\begin{array}{l}1 \\
1 \\
2 \\
2 \\
2\end{array}$ & & $\begin{array}{l}\text { I } \\
\text { I } \\
\text { I } \\
\text { I } \\
\text { I }\end{array}$ & $\begin{array}{l}8150.72 \\
8151.22 \\
8152.40 \\
8152.63 \\
8153.01\end{array}$ & $\begin{array}{r}3 \\
1 \\
20 \\
2 \\
1\end{array}$ & & $\begin{array}{l}\text { I } \\
\text { I } \\
\text { I I } \\
\text { I } \\
\text { I }\end{array}$ & $\begin{array}{l}8192.18 \\
8192.51 \\
8193.25 \\
8194.41 \\
8195.56\end{array}$ & $\begin{array}{r}2 \\
3 \\
4 \\
50 \\
4\end{array}$ & & $\begin{array}{l}\text { I } \\
\text { I } \\
\text { I } \\
\text { I } \\
\text { I }\end{array}$ \\
\hline $\begin{array}{l}8117.58 \\
8118.56 \\
8121.08 \\
8121.65 \\
8122.36\end{array}$ & $\begin{array}{l}3 \\
1 \\
5 \\
2 \mathrm{w} \\
25\end{array}$ & & $\begin{array}{l}\text { I } \\
\text { I } \\
\text { I } \\
\text { I } \\
\text { I }\end{array}$ & $\begin{array}{l}8153.85 \\
8154.20 \\
8154.96 \\
8155.58 \\
8155.97\end{array}$ & $\begin{array}{l}3 \\
5 \\
2 \\
2 \\
3\end{array}$ & & $\begin{array}{l}\text { I } \\
\text { I } \\
\text { I } \\
\text { I } \\
\text { I }\end{array}$ & $\begin{array}{l}8195.95 \\
8196.82 \\
8198.46 \\
8200.01 \\
8200.68\end{array}$ & $\begin{array}{l}4 \\
1 \\
4 \\
2 \mathrm{w} \\
2\end{array}$ & & $\begin{array}{l}\text { I } \\
\text { I } \\
\text { I } \\
\text { I } \\
\text { I }\end{array}$ \\
\hline $\begin{array}{l}8122.74 \\
8123.68 \\
8124.30 \\
8125.16 \\
8125.55\end{array}$ & $\begin{array}{c}120 \\
3 \\
5 \\
1 \mathrm{w} \\
1\end{array}$ & & $\begin{array}{l}\text { I } \\
\text { I } \\
\text { I } \\
\text { I } \\
\text { I }\end{array}$ & $\begin{array}{l}8157.57 \\
8158.66 \\
8159.75 \\
8161.04 \\
8161.54\end{array}$ & $\begin{array}{r}100 \\
4 \\
200 \\
1 \\
1\end{array}$ & & $\begin{array}{l}\text { I } \\
\text { I } \\
\text { I } \\
\text { I } \\
\text { I }\end{array}$ & $\begin{array}{l}8202.17 \\
8203.21 \\
8204.02 \\
8205.13 \\
8205.90\end{array}$ & $\begin{array}{r}10 \\
200 \\
2 \\
10 \\
2\end{array}$ & 2 & $\begin{array}{l}\text { I I } \\
\text { I } \\
\text { I } \\
\text { I } \\
\text { I }\end{array}$ \\
\hline $\begin{array}{l}8125.91 \\
8126.18 \\
8126.48 \\
8127.63 \\
8128.57\end{array}$ & $\begin{array}{l}2 \\
4 \\
3 \\
4 \\
1\end{array}$ & & $\begin{array}{l}\text { I } \\
\text { I } \\
\text { I } \\
\text { I } \\
\text { I }\end{array}$ & $\begin{array}{l}8162.08 \\
8163.15 \\
8164.03 \\
8165.16 \\
8165.78\end{array}$ & $\begin{array}{r}30 \\
50 \\
1 \\
3 \\
5\end{array}$ & 10 & $\begin{array}{l}\text { I } \\
\text { I I } \\
\text { I } \\
\text { I } \\
\text { I }\end{array}$ & $\begin{array}{l}8206.94 \\
8207.48 \\
8208.70 \\
8209.45 \\
8209.75\end{array}$ & $\begin{array}{r}4 \\
10 \\
1 \\
8 \\
2\end{array}$ & & $\begin{array}{l}\text { I } \\
\text { I } \\
\text { I } \\
I \\
I\end{array}$ \\
\hline $\begin{array}{l}8129.41 \\
8129.72 \\
8130.24 \\
8131.52 \\
8132.35\end{array}$ & $\begin{array}{r}50 \\
10 \\
3 \\
2 \\
1\end{array}$ & & $\begin{array}{l}\text { I } \\
\text { I } \\
\text { I } \\
\text { I } \\
\text { I }\end{array}$ & $\begin{array}{l}8166.47 \\
8167.44 \\
8168.00 \\
8169.18 \\
8169.55\end{array}$ & $\begin{array}{r}20 \\
3 \\
2 \\
75 \\
50\end{array}$ & 2 & $\begin{array}{l}\text { I I } \\
\text { I } \\
\text { I } \\
\text { I } \\
\text { I }\end{array}$ & $\begin{array}{l}8210.12 \\
8210.54 \\
8211.60 \\
8212.25 \\
8212.58\end{array}$ & $\begin{array}{r}1 \\
1 \\
1 \\
1 \\
20\end{array}$ & & $\begin{array}{l}\text { I } \\
\text { I } \\
\text { I } \\
\text { I } \\
\text { I }\end{array}$ \\
\hline $\begin{array}{l}8133.53 \\
8134.13 \\
8134.29 \\
8134.67 \\
8134.84\end{array}$ & $\begin{array}{l}1 \\
1 \\
1 \\
2 \\
1\end{array}$ & & $\begin{array}{l}\text { I } \\
\text { I } \\
\text { I } \\
\text { I } \\
\text { I }\end{array}$ & $\begin{array}{l}8169.81 \\
8171.67 \\
8172.14 \\
8173.76 \\
8174.14\end{array}$ & $\begin{array}{r}120 \\
2 \\
40 \\
1 \\
3\end{array}$ & & $\begin{array}{l}\text { I } \\
\text { I } \\
\text { I } \\
\text { I } \\
\text { I }\end{array}$ & $\begin{array}{l}8212.79 \\
8213.46 \\
8214.15 \\
8215.81 \\
8217.23\end{array}$ & $\begin{array}{r}2 \\
10 \\
20 \\
1 \\
40\end{array}$ & & $\begin{array}{l}\text { I } \\
\text { I } \\
\text { I } \\
\text { I } \\
\text { I }\end{array}$ \\
\hline $\begin{array}{l}8135.85 \\
8137.33 \\
8137.96 \\
8138.46 \\
8139.92\end{array}$ & $\begin{array}{r}1 \\
2 \\
20 \\
180 \\
18\end{array}$ & 3 & $\begin{array}{l}\text { I } \\
\text { I } \\
\text { I } \\
\text { I } \\
\text { I I }\end{array}$ & $\begin{array}{l}8174.72 \\
8175.86 \\
8175.94 \\
8176.82 \\
8177.20\end{array}$ & $\begin{array}{l}1 \\
2 \\
2 \\
1 \\
5\end{array}$ & & $\begin{array}{l}\text { I } \\
\text { I } \\
\text { I } \\
\text { I } \\
\text { I }\end{array}$ & $\begin{array}{l}8217.98 \\
8219.26 \\
8219.65 \\
8220.12 \\
8220.39\end{array}$ & $\begin{array}{l}2 \\
1 \\
2 \\
5 \\
2\end{array}$ & & $\begin{array}{l}\text { I } \\
\text { I } \\
\text { I } \\
\text { I } \\
\text { I }\end{array}$ \\
\hline $\begin{array}{l}8140.56 \\
8141.68 \\
8143.16 \\
8143.72 \\
8144.04\end{array}$ & $\begin{array}{r}3 \\
1 \\
300 \\
2 \\
2\end{array}$ & 25 & $\begin{array}{l}\text { I } \\
\text { I } \\
\text { I I } \\
\text { I } \\
\text { I }\end{array}$ & $\begin{array}{l}8178.21 \\
8180.30 \\
8181.23 \\
8181.32 \\
8182.29\end{array}$ & $\begin{array}{l}12 \\
1 \\
2 \mathrm{~h} \\
4 \mathrm{w} \\
5\end{array}$ & & $\begin{array}{l}\text { I } \\
\text { I } \\
\text { I } \\
\text { I } \\
\text { I }\end{array}$ & $\begin{array}{l}8220.50 \\
8220.77 \\
8221.21 \\
8223.43 \\
8224.08\end{array}$ & $\begin{array}{l}3 \\
5 \\
2 \\
2 \\
2\end{array}$ & 1 & $\begin{array}{l}\text { I } \\
\text { I } \\
\text { I } \\
\text { I } \\
\text { I I }\end{array}$ \\
\hline
\end{tabular}


Table 1. Wavelengths of Thorium Spectra - Continued

\begin{tabular}{|c|c|c|c|c|c|c|c|c|c|c|c|}
\hline \multirow{2}{*}{$\begin{array}{l}\text { Wave- } \\
\text { length }\end{array}$} & \multicolumn{2}{|c|}{ Intensity } & \multirow{2}{*}{$\begin{array}{l}\text { Spec- } \\
\text { trum }\end{array}$} & \multirow{2}{*}{$\begin{array}{l}\text { Wave- } \\
\text { length }\end{array}$} & \multicolumn{2}{|c|}{ Intensity } & \multirow{2}{*}{$\begin{array}{l}\text { Spec- } \\
\text { trum }\end{array}$} & \multirow{2}{*}{$\begin{array}{l}\text { Wave- } \\
\text { length }\end{array}$} & \multicolumn{2}{|c|}{ Intensity } & \multirow{2}{*}{$\begin{array}{l}\text { Spec- } \\
\text { trum }\end{array}$} \\
\hline & Lamp & Spark & & & Lamp & Spark & & & Lamp & Spark & \\
\hline $\begin{array}{l}8224.97 \\
8225.92 \\
8226.53 \\
8227.47 \\
8227.68\end{array}$ & $\begin{array}{r}4 \\
1 \\
2 \\
20 \\
10\end{array}$ & & $\begin{array}{l}\text { I } \\
\text { I } \\
\text { I } \\
\text { I } \\
\text { I }\end{array}$ & $\begin{array}{l}8272.43 \\
8273.63 \\
8273.96 \\
8275.63 \\
8276.24\end{array}$ & $\begin{array}{r}1 \\
2 \\
5 \\
300 \\
2\end{array}$ & 20 & $\begin{array}{l}\text { I } \\
\text { I } \\
\text { I } \\
\text { I I } \\
\text { I }\end{array}$ & $\begin{array}{l}8316.61 \\
8317.09 \\
8318.53 \\
8319.39 \\
8320.86\end{array}$ & $\begin{array}{r}4 \\
1 \\
1 \\
1 \\
250\end{array}$ & & $\begin{array}{l}\text { I } \\
\text { I } \\
\text { I } \\
\text { I } \\
\text { I }\end{array}$ \\
\hline $\begin{array}{l}8229.43 \\
8231.41 \\
8232.43 \\
8233.47 \\
8234.36\end{array}$ & $\begin{array}{l}2 \\
20 \\
2 \\
1 \mathrm{w} \\
3\end{array}$ & & $\begin{array}{l}\text { I } \\
\text { I } \\
\text { I } \\
\text { I } \\
\text { I }\end{array}$ & $\begin{array}{l}8276.75 \\
8278.39 \\
8279.52 \\
8280.96 \\
8282.24\end{array}$ & $\begin{array}{l}3 \\
1 \\
1 \\
4 \\
6\end{array}$ & & $\begin{array}{l}\text { I } \\
\text { I } \\
\text { I } \\
\text { I } \\
\text { I }\end{array}$ & $\begin{array}{l}8322.06 \\
8322.91 \\
8323.43 \\
8324.04 \\
8324.60\end{array}$ & $\begin{array}{l}2 \mathrm{~h} \\
3 \\
2 \\
1 \\
2\end{array}$ & & $\begin{array}{l}\text { I } \\
\text { I } \\
\text { I } \\
\text { I } \\
\text { I }\end{array}$ \\
\hline $\begin{array}{l}8234.95 \\
8235.83 \\
8236.33 \\
8236.79 \\
8237.75\end{array}$ & $\begin{array}{l}5 \\
2 \\
3 \\
2 \\
6\end{array}$ & & $\begin{array}{l}\text { I } \\
\text { I } \\
\text { I } \\
\text { I } \\
\text { I }\end{array}$ & $\begin{array}{l}8284.50 \\
8285.56 \\
8286.06 \\
8287.11 \\
8288.42\end{array}$ & $\begin{array}{l}1 \mathrm{w} \\
3 \\
2 \\
8 \\
20\end{array}$ & & $\begin{array}{l}\text { I } \\
\text { I } \\
\text { I } \\
\text { I } \\
\text { I }\end{array}$ & $\begin{array}{l}8325.38 \\
8326.09 \\
8326.98 \\
8327.21 \\
8328.03\end{array}$ & $\begin{array}{l}1 \\
2 \\
3 w \\
4 s \\
2\end{array}$ & & $\begin{array}{l}\text { I } \\
\text { I } \\
\text { I } \\
\text { I } \\
\text { I }\end{array}$ \\
\hline $\begin{array}{l}8241.80 \\
8242.37 \\
8243.50 \\
8243.85 \\
8243.90\end{array}$ & $\begin{array}{l}2 \\
1 \\
2 \\
1 \\
2\end{array}$ & 2 & $\begin{array}{l}\text { I } \\
\text { I } \\
\text { I I } \\
\text { I } \\
\text { I }\end{array}$ & $\begin{array}{l}8290.08 \\
8290.88 \\
8291.57 \\
8292.07 \\
8292.53\end{array}$ & $\begin{array}{r}1 \\
1 \\
1 \\
2 \\
50\end{array}$ & & $\begin{array}{l}\text { I } \\
\text { I } \\
\text { I } \\
\text { I } \\
\text { I }\end{array}$ & $\begin{array}{l}8328.39 \\
8329.01 \\
8329.40 \\
8330.47 \\
8331.95\end{array}$ & $\begin{array}{r}2 \\
4 \\
2 \\
500 \\
2\end{array}$ & & $\begin{array}{l}\text { I } \\
\text { I } \\
\text { I } \\
\text { I } \\
\text { I }\end{array}$ \\
\hline $\begin{array}{l}8245.08 \\
8246.03 \\
8247.83 \\
8248.63 \\
8249.08\end{array}$ & $\begin{array}{l}2 \\
10 \\
5 \mathrm{w} \\
2 \\
2\end{array}$ & & $\begin{array}{l}\text { I } \\
\text { I } \\
\text { I } \\
\text { I } \\
\text { I }\end{array}$ & $\begin{array}{l}8293.50 \\
8294.47 \\
8294.84 \\
8295.03 \\
8295.56\end{array}$ & $\begin{array}{r}5 \\
1 \\
1 \\
4 \\
30\end{array}$ & & $\begin{array}{l}\text { I } \\
\text { I } \\
\text { I } \\
\text { I } \\
\text { I }\end{array}$ & $\begin{array}{l}8332.47 \\
8333.43 \\
8333.79 \\
8334.20 \\
8334.62\end{array}$ & $\begin{array}{r}5 \\
10 \\
2 \\
1 \\
1\end{array}$ & & $\begin{array}{l}\text { I } \\
\text { I } \\
\text { I } \\
\text { I } \\
\text { I }\end{array}$ \\
\hline $\begin{array}{l}8250.03 \\
8251.18 \\
8251.80 \\
8252.39 \\
8252.91\end{array}$ & $\begin{array}{c}4 \\
2 \\
2 w \\
80 \\
2 w\end{array}$ & 5 & $\begin{array}{l}\text { I } \\
\text { I } \\
\text { I } \\
\text { I } \\
\text { I }\end{array}$ & $\begin{array}{l}8297.20 \\
8297.40 \\
8297.88 \\
8298.38 \\
8300.05\end{array}$ & $\begin{array}{l}5 \\
3 \\
2 \mathrm{w} \\
4 \\
3\end{array}$ & & $\begin{array}{l}\text { I } \\
\text { I } \\
\text { I } \\
\text { I } \\
\text { I }\end{array}$ & $\begin{array}{l}8335.16 \\
8335.71 \\
8336.95 \\
8338.46 \\
8339.13\end{array}$ & $\begin{array}{r}2 \\
10 \\
4 \\
2 \\
1\end{array}$ & & $\begin{array}{l}\text { I } \\
\text { I } \\
\text { I } \\
\text { I } \\
\text { I }\end{array}$ \\
\hline $\begin{array}{l}8253.62 \\
8254.12 \\
8254.75 \\
8255.81 \\
8256.83\end{array}$ & $\begin{array}{l}25 \\
2 \mathrm{w} \\
75 \\
2 \\
1\end{array}$ & & $\begin{array}{l}\text { I } \\
\text { I } \\
\text { I } \\
\text { I } \\
\text { I }\end{array}$ & $\begin{array}{l}8300.17 \\
8300.74 \\
8301.43 \\
8302.60 \\
8304.06\end{array}$ & $\begin{array}{l}1 \\
2 \\
2 \\
3 \\
1\end{array}$ & 2 & $\begin{array}{l}\text { I } \\
\text { I } \\
\text { I } \\
\text { I I } \\
\text { I }\end{array}$ & $\begin{array}{l}8339.41 \\
8340.94 \\
8341.49 \\
8342.29 \\
8342.70\end{array}$ & $\begin{array}{r}3 \\
3 \\
30 \\
1 \\
2\end{array}$ & & $\begin{array}{l}\text { I } \\
\text { I } \\
\text { I } \\
\text { I } \\
\text { I }\end{array}$ \\
\hline $\begin{array}{l}8258.35 \\
8258.69 \\
8259.04 \\
8259.51 \\
8261.02\end{array}$ & $\begin{array}{l}5 \mathrm{~s} \\
1 \\
2 \mathrm{w} \\
15 \\
60\end{array}$ & & $\begin{array}{l}\text { I } \\
\text { I } \\
\text { I } \\
\text { I } \\
\text { I }\end{array}$ & $\begin{array}{l}8304.43 \\
8304.87 \\
8307.29 \\
8309.29 \\
8309.61\end{array}$ & $\begin{array}{r}15 \\
2 \\
1 \\
4 \\
3\end{array}$ & 3 & $\begin{array}{l}\text { I I } \\
\text { I } \\
\text { I } \\
\text { I } \\
\text { I }\end{array}$ & $\begin{array}{l}8343.26 \\
8343.58 \\
8344.19 \\
8345.88 \\
8346.54\end{array}$ & $\begin{array}{r}1 \\
1 \\
3 \\
15 \\
15\end{array}$ & & $\begin{array}{l}\text { I } \\
\text { I } \\
\text { I } \\
\text { I } \\
\text { I }\end{array}$ \\
\hline $\begin{array}{l}8261.99 \\
8263.02 \\
8263.93 \\
8265.79 \\
8267.43\end{array}$ & $\begin{array}{r}4 \\
2 \\
25 \\
2 \\
2 \mathrm{~s}\end{array}$ & 5 & $\begin{array}{l}\text { I } \\
\text { I } \\
\text { I I } \\
\text { I } \\
\text { I }\end{array}$ & $\begin{array}{l}8310.10 \\
8310.61 \\
8311.06 \\
8311.64 \\
8312.35\end{array}$ & $\begin{array}{r}3 \\
3 \\
1 \\
20 \\
2\end{array}$ & & $\begin{array}{l}\text { I } \\
\text { I } \\
\text { I } \\
\text { I } \\
\text { I }\end{array}$ & $\begin{array}{l}8347.86 \\
8348.27 \\
8349.24 \\
8349.83 \\
8350.82\end{array}$ & $\begin{array}{l}3 \\
2 \\
1 \\
3 w \\
1\end{array}$ & & $\begin{array}{l}\text { I } \\
\text { I } \\
\text { I } \\
\text { I } \\
\text { I }\end{array}$ \\
\hline $\begin{array}{l}8268.52 \\
8269.24 \\
8269.56 \\
8270.44 \\
8270.96\end{array}$ & $\begin{array}{l}2 \\
2 \\
1 \mathrm{w} \\
2 \\
2 \mathrm{~h}\end{array}$ & & $\begin{array}{l}\text { I } \\
\text { I } \\
\text { I } \\
\text { I } \\
\text { I }\end{array}$ & $\begin{array}{l}8313.29 \\
8313.58 \\
8314.07 \\
8314.29 \\
8315.44\end{array}$ & $\begin{array}{l}1 \\
2 \\
2 \\
1 \\
1\end{array}$ & & $\begin{array}{l}\text { I } \\
\text { I } \\
\text { I } \\
\text { I } \\
\text { I }\end{array}$ & $\begin{array}{l}8351.05 \\
8351.14 \\
8351.38 \\
8351.83 \\
8352.24\end{array}$ & $\begin{array}{l}2 \\
2 \\
3 \\
3 w \\
1\end{array}$ & & $\begin{array}{l}\text { I } \\
\text { I } \\
\text { I } \\
\text { I } \\
\text { I }\end{array}$ \\
\hline
\end{tabular}


Table 1. Wavelengths of Thorium Spectra - Continued

\begin{tabular}{|c|c|c|c|c|c|c|c|c|c|c|c|}
\hline \multirow{2}{*}{$\begin{array}{l}\text { Wave- } \\
\text { length }\end{array}$} & \multicolumn{2}{|c|}{ Intensity } & \multirow{2}{*}{$\begin{array}{l}\text { Spec- } \\
\text { trum }\end{array}$} & \multirow{2}{*}{$\begin{array}{l}\text { Wave- } \\
\text { length }\end{array}$} & \multicolumn{2}{|c|}{ Intensity } & \multirow{2}{*}{$\begin{array}{l}\text { Spec- } \\
\text { trum }\end{array}$} & \multirow{2}{*}{$\begin{array}{l}\text { Wave- } \\
\text { length }\end{array}$} & \multicolumn{2}{|c|}{ Intensity } & \multirow{2}{*}{$\begin{array}{l}\text { Spec- } \\
\text { trum }\end{array}$} \\
\hline & Lamp & Spark & & & Lamp & Spark & & & & Spark & \\
\hline 8353.87 & 1 & & I & 8396.81 & 1 & & I & 8438.49 & 2 & & I \\
\hline 8355.14 & 5 & & I & 8397.05 & 2 & & I & 8438.80 & $4 w$ & & I \\
\hline 8356.07 & 10 & & I & 8398.17 & 25 & & I & 8440.66 & $50 \mathrm{w}$ & & I \\
\hline 8357.53 & 4 & & I & 8399.25 & 25 & & I & 8441.47 & 1 & & I \\
\hline 8357.84 & 4 & & I & 8399.70 & 2 & & I & 8441.81 & 1 & & I \\
\hline 8358.11 & 2 & & I & 8400.12 & 1 & & I & 8442.49 & $2 w$ & & I \\
\hline 8358.72 & 70 & & I & 8401.14 & 1 & & $\bar{I}$ & 8443.21 & $4 \mathrm{w}$ & & I \\
\hline 8359.36 & 1 & & I & 8401.99 & 50 & & I & 8445.49 & 150 & & I \\
\hline 8360.49 & 10 & & I & 8403.79 & 150 & 25 & I I & 8446.51 & 350 & & I \\
\hline 8361.08 & 1 & & I & 8404.45 & 1 & & I & 8447.97 & 4 & & $\bar{I}$ \\
\hline 8362.23 & 1 & & I & 8405.78 & 3 & & I & 8449.27 & $2 w$ & & I \\
\hline 8364.78 & 2 & & I & 8406.31 & 3 & & $\bar{I}$ & 8450.36 & 1 & & I \\
\hline 8365.27 & 2 & & I & 8406.67 & 4 & & I & 8450.67 & 100 & 10 & I I \\
\hline 8366.08 & 75 & & $\bar{I}$ & 8407.01 & 10 & & I & 8452.38 & 2 & & I \\
\hline 8367.40 & 75 & & I & 8407.69 & 3 & & $\bar{I}$ & 8453.40 & 2 & & I \\
\hline 8367.70 & $1 \mathrm{w}$ & & I & 8410.13 & 3 & 2 & I I & 8454.91 & 5 & & I \\
\hline 8368.40 & 5 & & I & 8410.54 & 4 & & I & 8455.37 & 1 & & I \\
\hline 8369.34 & 70 & & I & 8410.78 & 3 & & I & 8455.97 & 1 & & I \\
\hline 8370.71 & 1 & & I & 8411.27 & 2 & & I & 8456.35 & 4 & & I \\
\hline 8371.65 & $2 w$ & & I & 8411.90 & 75 & & I & 8458.12 & 1 & & I \\
\hline 8372.92 & 1 & & I & 8412.96 & 10 & 3 & I I & 8459.77 & 2 & & I \\
\hline 8373.16 & 2 & & I & 8414.76 & 1 & & I & 8460.12 & 3 & & I \\
\hline 8374.20 & 3 & & I & 8414.94 & 2 & & I & 8460.37 & 1 & & I \\
\hline 8374.77 & 5 & & I & 8415.40 & 10 & & I & 8460.48 & $2 w$ & & I \\
\hline 8376.89 & 2 & & I & 8416.72 & 25 & & I & 8461.57 & 1 & & I \\
\hline 8377.32 & 2 & & I & 8418.00 & 90 & & I & 8462.01 & 2 & & I \\
\hline 8377.97 & $\mathrm{l} w$ & & I & 8418.66 & 1 & & I & 8462.66 & 1 & & I \\
\hline 8379.22 & 10 & & $\bar{I}$ & 8419.45 & 1 & & I & 8463.35 & 1 & & I \\
\hline 8379.76 & 12 & & I & 8419.65 & 2 & & I & 8464.23 & 150 & & I \\
\hline 8380.42 & 1 & & I & 8419.92 & 1 & & I & 8464.71 & 4 & & I \\
\hline 8381.07 & $2 w$ & & I & 8421.22 & 300 & & I & 8465.67 & 30 & & I \\
\hline 8382.21 & 1 & & I & 8421.46 & 2 & & I & 8466.36 & 2 & & I \\
\hline 8382.52 & 1 & & I & 8423.54 & 1 & & I & 8467.15 & 2 & & I \\
\hline 8383.07 & 4 & & I & 8424.03 & 75 & 10 & I I & 8467.73 & $2 w$ & & I \\
\hline 8383.75 & 1 & & I & 8426.38 & 25 & & I & 8468.41 & 1 & & I \\
\hline 8384.29 & 1 & & I & 8427.94 & $1 \mathrm{w}$ & & I & 8468.95 & 4 & & I \\
\hline 8384.93 & 4 & & I & 8428.66 & 1 & & I & 8469.45 & 1 & & I \\
\hline 8385.73 & 30 & & I & 8429.14 & 2 & & I & 8469.95 & 2 & & I \\
\hline 8387.10 & 30 & 5 & I I & 8430.61 & 4 & & I & 8470.36 & 3 & & I \\
\hline 8387.76 & 5 & & I & 8432.28 & 2 & & I & 8471.81 & 150 & & I \\
\hline 8388.53 & 12 & & I & 8432.48 & 5 & & I & 8472.59 & 8 & & I \\
\hline 8389.01 & 1 & & I & 8433.26 & 1 & & I & 8473.64 & 1 & & I \\
\hline 8390.36 & 1 & & I & 8433.87 & 3 & & I & 8474.56 & 1 & & I \\
\hline 8390.93 & 4 & & I & 8435.02 & 3 & & I & 8475.40 & 3 & & I \\
\hline 8391.20 & 2 & 5 & I I & 8435.31 & ?. & & I & 8477.86 & 2 & & I \\
\hline 8392.40 & 4 & & I & 8435.74 & 20 & & I & 8478.37 & 300 & & I \\
\hline 8392.55 & 4 & & I & 8436.05 & 2 & & I & 8479.40 & 1 & & I \\
\hline 8393.87 & 5 & & I & 8436.65 & 1 & & I & 8401.74 & 2 & & $\vec{I}$ \\
\hline 8394.76 & 1 & & I & 8437.54 & 1 & & I & 8483.30 & 12 & & I \\
\hline 8395.50 & 2 & & I & 8438.10 & 1 & & I & 8484.68 & 1 & & I \\
\hline
\end{tabular}


Table 1. Wavelengths of Thorium Spectra - Continued

\begin{tabular}{|c|c|c|c|c|c|c|c|c|c|c|c|}
\hline \multirow{2}{*}{$\begin{array}{l}\text { Wave- } \\
\text { length }\end{array}$} & \multicolumn{2}{|c|}{ Intensity } & \multirow{2}{*}{$\begin{array}{l}\text { Spec. } \\
\text { trum }\end{array}$} & \multirow{2}{*}{$\begin{array}{l}\text { Wave- } \\
\text { length }\end{array}$} & \multicolumn{2}{|c|}{ Intensity } & \multirow{2}{*}{$\begin{array}{l}\text { Spec. } \\
\text { trum }\end{array}$} & \multirow{2}{*}{$\begin{array}{l}\text { Wave- } \\
\text { length }\end{array}$} & \multicolumn{2}{|c|}{ Intensity } & \multirow{2}{*}{$\begin{array}{l}\text { Spec- } \\
\text { trum }\end{array}$} \\
\hline & Lamp & Spark & & & Lamp & Spark & & & Lamp & Spark & \\
\hline 8486.56 & 3 & & I & 8526.03 & 1 & & I & 8556.60 & 2 & & I \\
\hline 8487.48 & 8 & & I & 8526.60 & 2 & & I & 8556.83 & 4 & & I \\
\hline 8488.60 & 1 & & I & 8527.89 & 1 & & I & 8557.31 & 2 & & I \\
\hline 8489.63 & 10 & & I & 8528.50 & 1 & & $\bar{I}$ & 8558.46 & 10 & & $\bar{I}$ \\
\hline 8490.46 & 2 & & I & 8528.76 & 3 & & I & 8559.79 & 2 & & I \\
\hline 8491.15 & 3 & & I & 8529.23 & 1 & & I & 8560.44 & 8 & & I \\
\hline 8491.36 & 1 & & I & 8529.54 & 1 & & I & 8561.37 & 2 & & I \\
\hline 8491.80 & 3 & & $\vec{I}$ & 8529.63 & 1 & & I & 8561.75 & 1 & & I \\
\hline 8492.91 & 1 & & I & 8530.14 & 1 & & I & 8562.33 & 3 & & I \\
\hline 8493.47 & 2 & & I & 8530.89 & 8 & & I & 8563.15 & 6 & & I \\
\hline 8494.88 & 15 & & I & 8531.16 & 3 & & I & 8564.14 & 8 & & I \\
\hline 8496.16 & 50 & & I & 8531.45 & 6 & & I & 8564.80 & 50 & & I \\
\hline 8496.34 & 75 & & I & 8531.85 & 2 & 2 & II & 8565.28 & 1 & & I \\
\hline 8496.46 & 2 & & $\vec{I}$ & 8532.07 & 2 & & I & 8565.80 & 3 & & I \\
\hline 8496.73 & 5 & & I & 8532.28 & 4 & & I & 8567.61 & 3 & & I \\
\hline 8497.92 & 1 & & I & 8532.89 & 4 & & I & 8568.22 & 75 & 7 & I I \\
\hline 8498.22 & 3 & & I & 8533.11 & 2 & & I & 8569.93 & 1 & & I \\
\hline 8498.48 & 8 & & $\bar{I}$ & 8534.02 & 3 & & I & 8571.14 & 2 & & I \\
\hline 8500.01 & 8 & & $\vec{I}$ & 8534.67 & 6 & & I & 8571.43 & 1 & & I \\
\hline 8500.67 & 25 & 5 & I I & 8535.12 & 1 & & $\bar{I}$ & 8572.09 & 2 & & I \\
\hline 8501.43 & 12 & & I & 8536.31 & $2 w$ & & I & 8572.49 & 1 & & I \\
\hline 8501.74 & 1 & & I & 8537.37 & 1 & & I & 8573.14 & 180 & & I \\
\hline 8503.29 & 1 & & I & 8537.93 & 1 & & I & 8574.10 & 8 & & I \\
\hline 8503.55 & 1 & & $\vec{I}$ & 8538.80 & 1 & & I & 8574.24 & 1 & & I \\
\hline 8504.32 & 4 & & I & 8539.36 & 2 & & I & 8575.34 & 30 & & I \\
\hline 8505.41 & 2 & & I & 8539.78 & 100 & & I & 8577.29 & 75 & & I \\
\hline 8505.82 & 1 & & I & 8540.23 & 1 & & I & 8579.36 & 2 & & I \\
\hline 8507.79 & 1 & & I & 8541.21 & 1 & & I & 8580.59 & 3 & & $\bar{I}$ \\
\hline 8508.23 & 4 & & I & 8541.76 & 1 & & I & 8581.25 & $1 \mathrm{w}$ & & I \\
\hline 8509.33 & 1 & & I & 8541.87 & 1 & & I & 8582.05 & 3 & & I \\
\hline 8510.62 & 200 & & I & 8542.62 & 1 & & I & 8582.59 & 2 & & I \\
\hline 8511.91 & 15 & & I & 8543.44 & 1 & & I & 8583.24 & 1 & & I \\
\hline 8512.59 & 12 & 10 & I I & 8543.69 & 75 & & I & 8584.01 & 1 & & I \\
\hline 8513.48 & 5 & & I & 8544.58 & 75 & & I & 8584.50 & 2 & & I \\
\hline 8513.63 & 1 & & I & 8546.45 & 1 & & $\bar{I}$ & 8585.21 & 1 & & I \\
\hline 8514.89 & 3 & & I & 8547.42 & 2 & & I & 8586.28 & 5 & & I \\
\hline 8515.61 & 1 & & I & 8548.17 & 2 & & I & 8587.64 & 18 & 50 & I I \\
\hline 8516.55 & 300 & 2 & I & 8548.74 & 2 & & $\bar{I}$ & 8588.23 & 3 & & I \\
\hline 8517.41 & 1 & & $\bar{I}$ & 8549.55 & 1 & & $\bar{I}$ & 8588.44 & 6 & & I \\
\hline 8519.33 & 15 & & I & 8550.20 & 1 & & I & 8589.07 & 1 & & I \\
\hline 8520.10 & $2 w$ & & I & 8550.69 & 2 & & I & 8589.71 & 2 & & I \\
\hline 8520.66 & 1 & & I & 8551.41 & 1 & & I & 8590.76 & 4 & 2 & I I \\
\hline 8521.45 & $6 \mathrm{w}$ & 3 & I I & 8551.76 & 6 & & I & 8591.21 & 6 & & I \\
\hline 8522.19 & 2 & & I & 8552.27 & 1 & & I & 8591.85 & 100 & 50 & I I \\
\hline 8522.60 & 2 & & I & 8553.11 & 2 & & I & 8593.12 & 75 & & I \\
\hline 8523.06 & 4 & & I & 8553.24 & 1 & & I & 8594.12 & 3 & & I \\
\hline 8523.83 & 4 & & I & 8553.81 & 1 & & I & 8595.01 & 2 & & I \\
\hline 8524.80 & 2 & & I & 8553.92 & 2 & & I & 8595.37 & 1 & & I \\
\hline 8525.37 & 2 & & I & 8554.95 & 150 & & I & 8595.98 & 1 & & I \\
\hline 8525.68 & 2 & & I & 8556.32 & 60 & & I & 8596.48 & 4 & & I \\
\hline
\end{tabular}


Table 1. Wavelengths of Thorium Spectra - Continued

\begin{tabular}{|c|c|c|c|c|c|c|c|c|c|c|c|}
\hline \multirow{2}{*}{$\begin{array}{l}\text { Wave- } \\
\text { length }\end{array}$} & \multicolumn{2}{|c|}{ Intensity } & \multirow{2}{*}{$\begin{array}{l}\text { Spec- } \\
\text { trum }\end{array}$} & \multirow{2}{*}{$\begin{array}{l}\text { Wave- } \\
\text { length }\end{array}$} & \multicolumn{2}{|c|}{ Intensity } & \multirow{2}{*}{$\begin{array}{l}\text { Spec- } \\
\text { trum }\end{array}$} & \multirow{2}{*}{$\begin{array}{l}\text { Wave- } \\
\text { length }\end{array}$} & \multicolumn{2}{|c|}{ Intensity } & \multirow{2}{*}{$\begin{array}{l}\text { Spec } \\
\text { trum }\end{array}$} \\
\hline & Lamp & Spark & & & Lamp & Spark & & & Lamp & Spark & \\
\hline $\begin{array}{l}8596.72 \\
8597.05 \\
8597.45 \\
8598.87 \\
8599.48\end{array}$ & $\begin{array}{l}3 \\
3 \\
6 \\
1 \\
1\end{array}$ & & $\begin{array}{l}\text { I } \\
\text { I } \\
\text { I } \\
\text { I } \\
\text { I }\end{array}$ & $\begin{array}{l}8633.93 \\
8634.05 \\
8635.17 \\
8637.27 \\
8638.36\end{array}$ & $\begin{array}{r}2 \\
3 \\
1 \\
3 \\
20\end{array}$ & & $\begin{array}{l}\text { I } \\
\text { I } \\
\text { I } \\
\text { I } \\
\text { I }\end{array}$ & $\begin{array}{l}8680.77 \\
8681.33 \\
8682.23 \\
8682.47 \\
8682.91\end{array}$ & $\begin{array}{r}2 \\
2 \\
10 \\
4 \\
8\end{array}$ & & $\begin{array}{l}\text { I } \\
\text { I } \\
\text { I } \\
\text { I } \\
\text { I }\end{array}$ \\
\hline $\begin{array}{l}8599.79 \\
8600.93 \\
8602.10 \\
8602.42 \\
8603.12\end{array}$ & $\begin{array}{l}5 \\
4 \\
3 \\
2 \\
1\end{array}$ & 30 & $\begin{array}{l}\text { I I } \\
\text { I } \\
\text { I } \\
\text { I } \\
\text { I }\end{array}$ & $\begin{array}{l}8639.44 \\
8640.58 \\
8640.99 \\
8641.31 \\
8641.43\end{array}$ & $\begin{array}{r}60 \\
2 \\
4 \\
1 \\
2\end{array}$ & & $\begin{array}{l}\text { I } \\
\text { I } \\
\text { I } \\
\text { I } \\
\text { I }\end{array}$ & $\begin{array}{l}8683.50 \\
8683.95 \\
8685.74 \\
8686.12 \\
8686.64\end{array}$ & $\begin{array}{r}4 \\
8 \\
12 \\
10 \\
15\end{array}$ & $\begin{array}{l}5 \\
3\end{array}$ & $\begin{array}{l}\text { I } \\
\text { I } \\
\text { I } \\
\text { I I } \\
\text { I I }\end{array}$ \\
\hline $\begin{array}{l}8604.43 \\
8604.53 \\
8604.95 \\
8605.50 \\
8606.16\end{array}$ & $\begin{array}{l}1 \\
1 \\
2 \\
6 \\
8\end{array}$ & & $\begin{array}{l}\text { I } \\
\text { I } \\
\text { I } \\
\text { I } \\
\text { I }\end{array}$ & $\begin{array}{l}8643.31 \\
8643.95 \\
8645.31 \\
8646.25 \\
8647.65\end{array}$ & $\begin{array}{r}1 \\
1 \\
60 \\
1 \\
1\end{array}$ & & $\begin{array}{l}\text { I } \\
\text { I } \\
\text { I } \\
\text { I } \\
\text { I }\end{array}$ & $\begin{array}{l}8687.30 \\
8687.85 \\
8688.65 \\
8689.19 \\
8690.25\end{array}$ & $\begin{array}{r}4 \\
25 \\
4 \\
10 \\
4\end{array}$ & & $\begin{array}{l}\text { I } \\
\text { I } \\
\text { I } \\
\text { I } \\
\text { I }\end{array}$ \\
\hline $\begin{array}{l}8607.73 \\
8608.71 \\
8609.39 \\
8611.45 \\
8611.78\end{array}$ & $\begin{array}{l}5 \\
4 \\
3 \\
1 \\
2\end{array}$ & & $\begin{array}{l}\text { I } \\
\text { I } \\
\text { I } \\
\text { I } \\
\text { I }\end{array}$ & $\begin{array}{l}8648.36 \\
8649.15 \\
8649.86 \\
8650.44 \\
8651.19\end{array}$ & $\begin{array}{r}25 \\
1 \\
1 \\
1 \\
2\end{array}$ & & $\begin{array}{l}\text { I } \\
\text { I } \\
\text { I } \\
\text { I } \\
\text { I }\end{array}$ & $\begin{array}{l}8691.35 \\
8693.70 \\
8694.20 \\
8695.27 \\
8695.95\end{array}$ & $\begin{array}{r}10 \\
4 \\
4 \\
15 \\
4\end{array}$ & & $\begin{array}{l}\text { I } \\
\text { I } \\
\text { I } \\
\text { I } \\
\text { I }\end{array}$ \\
\hline $\begin{array}{l}8612.52 \\
8612.87 \\
8614.69 \\
8616.23 \\
8617.12\end{array}$ & $\begin{array}{r}5 \\
1 \\
5 \\
40 \\
3\end{array}$ & & $\begin{array}{l}\text { I } \\
\text { I } \\
\text { I } \\
\text { I } \\
\text { I }\end{array}$ & $\begin{array}{l}8653.89 \\
8654.73 \\
8655.89 \\
8656.70 \\
8657.53\end{array}$ & $\begin{array}{r}4 \\
4 \\
50 \\
1 \\
4\end{array}$ & & $\begin{array}{l}\text { I } \\
\text { I } \\
\text { I } \\
\text { I } \\
\text { I }\end{array}$ & $\begin{array}{l}8696.57 \\
8698.63 \\
8698.88 \\
8699.23 \\
8702.04\end{array}$ & $\begin{array}{r}1 \\
15 \\
4 \\
4 \\
4\end{array}$ & & $\begin{array}{l}\text { I } \\
\text { I } \\
I \\
I \\
I\end{array}$ \\
\hline $\begin{array}{l}8618.61 \\
8618.98 \\
8620.19 \\
8621.34 \\
8621.84\end{array}$ & $\begin{array}{l}3 \\
2 \\
4 \\
5 \\
1\end{array}$ & & $\begin{array}{l}\text { I } \\
\text { I } \\
\text { I } \\
\text { I } \\
\text { I }\end{array}$ & $\begin{array}{l}8658.45 \\
8659.35 \\
8659.77 \\
8660.67 \\
8661.04\end{array}$ & $\begin{array}{r}1 \\
10 \\
1 \\
1 \\
1\end{array}$ & & $\begin{array}{l}\text { I } \\
\text { I } \\
\text { I } \\
\text { I } \\
\text { I }\end{array}$ & $\begin{array}{l}8703.35 \\
8703.73 \\
8704.88 \\
8707.37 \\
8708.46\end{array}$ & $\begin{array}{r}4 \\
15 \\
8 \\
20 \\
2\end{array}$ & 3 & $\begin{array}{l}\text { I I } \\
\text { I } \\
\text { I } \\
\text { I } \\
\text { I }\end{array}$ \\
\hline $\begin{array}{l}8622.11 \\
8622.87 \\
8623.76 \\
8624.41 \\
8624.87\end{array}$ & $\begin{array}{l}4 w \\
4 \\
3 \\
5 \\
3\end{array}$ & & $\begin{array}{l}\text { I } \\
\text { I } \\
\text { I } \\
\text { I } \\
\text { I }\end{array}$ & $\begin{array}{l}8661.48 \\
8661.92 \\
8662.27 \\
8663.35 \\
8665.50\end{array}$ & $\begin{array}{r}2 \\
4 \\
4 \\
4 \\
160\end{array}$ & & $\begin{array}{l}\text { I } \\
\text { I } \\
\text { I } \\
\text { I } \\
\text { I }\end{array}$ & $\begin{array}{l}8709.24 \\
8710.43 \\
8712.32 \\
8712.82 \\
8713.67\end{array}$ & $\begin{array}{r}90 \\
8 \\
8 \\
4 \\
15\end{array}$ & & $\begin{array}{l}\text { I } \\
\text { I } \\
\text { I } \\
\text { I } \\
\text { I }\end{array}$ \\
\hline $\begin{array}{l}8625.79 \\
8626.22 \\
8626.76 \\
8627.43 \\
8628.06\end{array}$ & $\begin{array}{l}2 \\
1 \\
1 \\
3 \\
4\end{array}$ & & $\begin{array}{l}\text { I } \\
\text { I } \\
\text { I } \\
\text { I } \\
\text { I }\end{array}$ & $\begin{array}{l}8666.97 \\
8667.47 \\
8668.11 \\
8670.08 \\
8672.20\end{array}$ & $\begin{array}{r}4 \\
16 \\
70 \\
4 \\
8\end{array}$ & & $\begin{array}{l}\text { I } \\
\text { I } \\
\text { I } \\
\text { I } \\
\text { I }\end{array}$ & $\begin{array}{l}8714.25 \\
8715.80 \\
8716.22 \\
8717.09 \\
8717.76\end{array}$ & $\begin{array}{r}8 \\
4 \\
2 \\
1 \\
15\end{array}$ & & $\begin{array}{l}\text { I } \\
\text { I } \\
\text { I } \\
\text { I } \\
\text { I }\end{array}$ \\
\hline $\begin{array}{l}8628.65 \\
8629.15 \\
8629.66 \\
8630.08 \\
8630.31\end{array}$ & $\begin{array}{l}1 \\
8 \\
3 \\
1 \\
3\end{array}$ & $10 \mathrm{w}$ & $\begin{array}{l}\text { I } \\
\text { I I } \\
\text { I I I } \\
\text { I } \\
\text { I }\end{array}$ & $\begin{array}{l}8673.20 \\
8674.21 \\
8674.75 \\
8675.40 \\
8676.26\end{array}$ & $\begin{array}{r}8 \\
4 \\
1 \\
10 \\
1\end{array}$ & & $\begin{array}{l}\text { I } \\
\text { I } \\
\text { I } \\
\text { I }\end{array}$ & $\begin{array}{l}8719.63 \\
8721.67 \\
8722.47 \\
8723.72 \\
8724.44\end{array}$ & $\begin{array}{r}80 \\
80 \\
15 \\
8 \\
12\end{array}$ & 5 & $\begin{array}{l}\text { I } \\
\text { I I } \\
\text { I } \\
\text { I } \\
\text { I }\end{array}$ \\
\hline $\begin{array}{l}8630.79 \\
8631.35 \\
8632.17 \\
8633.22 \\
8633.46\end{array}$ & $\begin{array}{r}6 \\
10 \\
5 \\
4 \\
2\end{array}$ & & $\begin{array}{l}\text { I } \\
\text { I } \\
\text { I } \\
\text { I } \\
\text { I }\end{array}$ & $\begin{array}{l}8676.58 \\
8677.27 \\
8678.25 \\
8678.51 \\
8679.46\end{array}$ & $\begin{array}{l}4 w \\
8 \\
4 \\
8 \\
10\end{array}$ & & $\begin{array}{l}\text { I } \\
\text { I } \\
\text { I } \\
\text { I } \\
\text { I }\end{array}$ & $\begin{array}{l}8725.33 \\
8727.44 \\
8727.86 \\
8728.51 \\
8730.83\end{array}$ & $\begin{array}{r}4 \\
4 \\
8 \\
2 \\
12\end{array}$ & & $\begin{array}{l}\text { I } \\
\text { I } \\
\text { I } \\
\text { I } \\
\text { I }\end{array}$ \\
\hline
\end{tabular}


Table 1. Wavelengths of Thorium Spectra - Continued

\begin{tabular}{|c|c|c|c|c|c|c|c|c|c|c|c|}
\hline \multirow{2}{*}{$\begin{array}{l}\text { Wave- } \\
\text { length }\end{array}$} & \multicolumn{2}{|c|}{ Intensity } & \multirow{2}{*}{$\begin{array}{l}\text { Spec- } \\
\text { trum }\end{array}$} & \multirow{2}{*}{$\begin{array}{l}\text { Wave- } \\
\text { length }\end{array}$} & \multicolumn{2}{|c|}{ Intensity } & \multirow{2}{*}{$\begin{array}{l}\text { Spec- } \\
\text { trum }\end{array}$} & \multirow{2}{*}{$\begin{array}{l}\text { Wave- } \\
\text { length }\end{array}$} & \multicolumn{2}{|c|}{ Intensity } & \multirow{2}{*}{$\begin{array}{l}\text { Spec- } \\
\text { trum }\end{array}$} \\
\hline & Lamp & Spark & & & Lamp & Spark & & & Lamp & Spark & \\
\hline $\begin{array}{l}8731.30 \\
8732.42 \\
8733.02 \\
8734.03 \\
8734.53\end{array}$ & $\begin{array}{r}2 \\
140 \\
5 \\
4 \\
8\end{array}$ & 5 & $\begin{array}{l}\text { I } \\
\text { I } \\
\text { I } \\
\text { I } \\
\text { I }\end{array}$ & $\begin{array}{l}8777.35 \\
8778.04 \\
8778.25 \\
8779.94 \\
8781.10\end{array}$ & $\begin{array}{l}8 \\
4 \\
8 \\
8 \\
8\end{array}$ & & $\begin{array}{l}\text { I } \\
\text { I } \\
\text { I } \\
\text { I } \\
\text { I }\end{array}$ & $\begin{array}{l}8831.51 \\
8832.13 \\
8833.67 \\
8834.48 \\
8834.85\end{array}$ & $\begin{array}{l}2 \\
2 \\
8 \\
2 \\
4\end{array}$ & & $\begin{array}{l}\text { I } \\
\text { I } \\
\text { I } \\
\text { I } \\
\text { I }\end{array}$ \\
\hline $\begin{array}{l}8735.20 \\
8735.75 \\
8736.36 \\
8737.14 \\
8737.61\end{array}$ & $\begin{array}{l}8 \\
2 \\
8 \\
4 \\
4\end{array}$ & & $\begin{array}{l}I \\
I \\
I \\
I \\
I\end{array}$ & $\begin{array}{l}8782.47 \\
8782.72 \\
8784.15 \\
8784.57 \\
8785.47\end{array}$ & $\begin{array}{r}4 \\
8 \\
4 \\
20 \\
1\end{array}$ & 20 & $\begin{array}{l}\text { I } \\
\text { I } \\
\text { I } \\
\text { I I } \\
\text { I }\end{array}$ & $\begin{array}{l}8836.12 \\
8837.30 \\
8838.44 \\
8839.11 \\
8841.16\end{array}$ & $\begin{array}{c}25 \\
2 \mathrm{~s} \\
2 \\
1 \\
100\end{array}$ & & $\begin{array}{l}\text { I } \\
\text { I } \\
\text { I } \\
\text { I } \\
\text { I }\end{array}$ \\
\hline $\begin{array}{l}8738.16 \\
8739.80 \\
8743.27 \\
8744.15 \\
8744.60\end{array}$ & $\begin{array}{r}2 \\
50 \\
15 \\
1 \\
8\end{array}$ & & $\begin{array}{l}\text { I } \\
\text { I } \\
\text { I } \\
\text { I } \\
\text { I }\end{array}$ & $\begin{array}{l}8786.05 \\
8788.02 \\
8788.49 \\
8789.59 \\
8790.39\end{array}$ & $\begin{array}{r}2 \\
4 \\
4 \\
4 \\
15\end{array}$ & & $\begin{array}{l}\text { I } \\
\text { I } \\
\text { I } \\
\text { I } \\
\text { I }\end{array}$ & $\begin{array}{l}8841.87 \\
8842.08 \\
8843.53 \\
8844.52 \\
8844.67\end{array}$ & $\begin{array}{r}2 \\
20 \\
5 \\
5 \\
2\end{array}$ & 10 & $\begin{array}{l}\text { I } \\
\text { II } \\
\text { I } \\
\text { I } \\
\text { I }\end{array}$ \\
\hline $\begin{array}{l}8744.99 \\
8745.51 \\
8746.74 \\
8748.03 \\
8749.16\end{array}$ & $\begin{array}{r}4 \\
4 \\
2 \\
300 \\
100\end{array}$ & & $\begin{array}{l}\text { I } \\
\text { I } \\
\text { I } \\
\text { I } \\
\text { I }\end{array}$ & $\begin{array}{l}8792.05 \\
8792.61 \\
8793.33 \\
8794.37 \\
8796.39\end{array}$ & $\begin{array}{c}50 \\
4 \\
1 \\
1 w \\
8\end{array}$ & & $\begin{array}{l}\text { I } \\
\text { I } \\
\text { I } \\
\text { I } \\
\text { I }\end{array}$ & $\begin{array}{l}8847.97 \\
8849.30 \\
8850.30 \\
8852.62 \\
8854.89\end{array}$ & $\begin{array}{r}8 \\
30 \\
15 \\
5 \\
15\end{array}$ & & $\begin{array}{l}\text { I } \\
\text { I } \\
\text { I } \\
\text { I } \\
\text { I }\end{array}$ \\
\hline $\begin{array}{l}8750.21 \\
8751.21 \\
8752.47 \\
8753.72 \\
8754.32\end{array}$ & $\begin{array}{r}1 \\
20 \\
2 \\
2 \\
8\end{array}$ & & $\begin{array}{l}\text { I } \\
\text { I } \\
\text { I } \\
\text { I } \\
\text { I }\end{array}$ & $\begin{array}{l}8798.15 \\
8798.42 \\
8799.00 \\
8799.31 \\
8800.06\end{array}$ & $\begin{array}{r}15 \\
8 \\
1 \\
1 \\
1\end{array}$ & & $\begin{array}{l}\text { I } \\
\text { I } \\
\text { I } \\
\text { I } \\
\text { I }\end{array}$ & $\begin{array}{l}8856.42 \\
8859.00 \\
8859.40 \\
8860.96 \\
8861.57\end{array}$ & $\begin{array}{l}2 w \\
30 \\
15 \\
15 \\
1\end{array}$ & & $\begin{array}{l}\text { I } \\
\text { I } \\
\text { I } \\
\text { I } \\
\text { I }\end{array}$ \\
\hline $\begin{array}{l}8755.03 \\
8755.58 \\
8756.46 \\
8757.22 \\
8758.24\end{array}$ & $\begin{array}{r}4 \\
2 \\
4 \\
1 \\
250\end{array}$ & & $\begin{array}{l}\text { I } \\
\text { I } \\
\text { I } \\
\text { I } \\
\text { I }\end{array}$ & $\begin{array}{l}8800.42 \\
8801.05 \\
8802.45 \\
8803.39 \\
8804.57\end{array}$ & $\begin{array}{r}4 \\
8 \\
1 \\
3 \\
25\end{array}$ & & $\begin{array}{l}\text { I } \\
\text { I } \\
\text { I } \\
\text { I } \\
\text { I }\end{array}$ & $\begin{array}{l}8862.28 \\
8862.73 \\
8864.48 \\
8865.57 \\
8866.71\end{array}$ & $\begin{array}{r}3 \\
8 \\
2 \\
25 \\
15\end{array}$ & & $\begin{array}{l}\text { I } \\
\text { I } \\
\text { I } \\
\text { I } \\
\text { I }\end{array}$ \\
\hline $\begin{array}{l}8759.27 \\
8760.45 \\
8761.67 \\
8762.31 \\
8762.99\end{array}$ & $\begin{array}{r}8 \\
50 \\
8 \\
1 \\
2\end{array}$ & & $\begin{array}{l}\text { I } \\
\text { I } \\
\text { I } \\
\text { I } \\
\text { I }\end{array}$ & $\begin{array}{l}8805.87 \\
8806.23 \\
8807.79 \\
8808.71 \\
8810.23\end{array}$ & $\begin{array}{l}12 \\
4 \\
5 \mathrm{w} \\
5 \mathrm{w} \\
20\end{array}$ & & $\begin{array}{l}\text { I } \\
\text { I } \\
\text { I } \\
\text { I } \\
\text { I }\end{array}$ & $\begin{array}{l}8868.00 \\
8868.81 \\
8870.67 \\
8871.97 \\
8872.22\end{array}$ & $\begin{array}{r}8 \\
250 \\
4 \\
16 \\
1\end{array}$ & & $\begin{array}{l}\text { I } \\
\text { I } \\
\text { I } \\
\text { I } \\
\text { I }\end{array}$ \\
\hline $\begin{array}{l}8763.98 \\
8764.81 \\
8766.74 \\
8768.20 \\
8769.86\end{array}$ & $\begin{array}{r}2 \\
2 \\
50 \\
12 \\
4\end{array}$ & & $\begin{array}{l}\text { I } \\
\text { I } \\
\text { I } \\
\text { I } \\
\text { I }\end{array}$ & $\begin{array}{l}8814.91 \\
8816.16 \\
8817.74 \\
8820.22 \\
8820.38\end{array}$ & $\begin{array}{r}2 \\
15 \\
4 \\
30 \\
30\end{array}$ & 4 & $\begin{array}{l}\text { I } \\
\text { I I } \\
\text { I } \\
\text { I } \\
\text { I }\end{array}$ & $\begin{array}{l}8874.65 \\
8875.23 \\
8876.26 \\
8877.42 \\
8878.35\end{array}$ & $\begin{array}{c}1 \\
30 \\
2 \\
1 \mathrm{~h} \\
4\end{array}$ & & $\begin{array}{l}\text { I } \\
\text { I } \\
\text { I } \\
\text { I } \\
\text { I }\end{array}$ \\
\hline $\begin{array}{l}8771.06 \\
8771.78 \\
8772.39 \\
8772.81 \\
8773.53\end{array}$ & $\begin{array}{r}1 \\
8 \\
8 \\
50 \\
8\end{array}$ & & $\begin{array}{l}\text { I } \\
\text { I } \\
\text { I } \\
\text { I } \\
\text { I }\end{array}$ & $\begin{array}{l}8820.99 \\
8821.73 \\
8822.26 \\
8822.58 \\
8823.17\end{array}$ & $\begin{array}{l}1 \\
8 \\
2 \\
1 \\
1\end{array}$ & & $\begin{array}{l}\text { I } \\
\text { I } \\
\text { I } \\
\text { I } \\
\text { I }\end{array}$ & $\begin{array}{l}8879.10 \\
8880.72 \\
8881.91 \\
8882.50 \\
8883.17\end{array}$ & $\begin{array}{l}4 \\
1 \mathrm{w} \\
4 \\
8 \\
1\end{array}$ & & $\begin{array}{l}\text { I } \\
\text { I } \\
\text { I } \\
\text { I } \\
\text { I }\end{array}$ \\
\hline $\begin{array}{l}8774.06 \\
8774.55 \\
8775.58 \\
8776.15 \\
8777.01\end{array}$ & $\begin{array}{r}4 \\
1 \\
140 \\
1 \\
8\end{array}$ & & $\begin{array}{l}\text { I } \\
\text { I } \\
\text { I } \\
\text { I } \\
\text { I }\end{array}$ & $\begin{array}{l}8824.18 \\
8826.27 \\
8827.88 \\
8829.67 \\
8831.20\end{array}$ & $\begin{array}{r}3 \\
4 \\
4 \\
25 \\
1\end{array}$ & & $\begin{array}{l}\text { I } \\
\text { I } \\
\text { I } \\
\text { I } \\
\text { I }\end{array}$ & $\begin{array}{l}8884.21 \\
8885.04 \\
8886.06 \\
8886.62 \\
8887.70\end{array}$ & $\begin{array}{l}1 \mathrm{~h} \\
2 \\
2 \\
3 \\
3\end{array}$ & & $\begin{array}{l}\text { I } \\
\text { I } \\
\text { I } \\
\text { I } \\
\text { I }\end{array}$ \\
\hline
\end{tabular}


Table 1. Wavelengths of Thorium Spectra - Continued

\begin{tabular}{|c|c|c|c|c|c|c|c|c|c|c|c|}
\hline \multirow{2}{*}{$\begin{array}{l}\text { Wave- } \\
\text { length }\end{array}$} & \multicolumn{2}{|c|}{ Intensity } & \multirow{2}{*}{$\begin{array}{l}\text { Spec- } \\
\text { trum }\end{array}$} & \multirow{2}{*}{$\begin{array}{l}\text { Wave- } \\
\text { length }\end{array}$} & \multicolumn{2}{|c|}{ Intensity } & \multirow{2}{*}{$\begin{array}{l}\text { Spec- } \\
\text { trum }\end{array}$} & \multirow{2}{*}{$\begin{array}{l}\text { Wave- } \\
\text { length }\end{array}$} & \multicolumn{2}{|c|}{ Intensity } & \multirow{2}{*}{$\begin{array}{l}\text { Spec } \\
\text { trum }\end{array}$} \\
\hline & Lamp & Spark & & & Lamp & Spark & & & Lamp & Spark & \\
\hline $\begin{array}{l}8888.23 \\
8889.19 \\
8889.98 \\
8890.26 \\
8892.97\end{array}$ & $\begin{array}{r}1 \\
30 \\
1 \\
8 \\
15\end{array}$ & & $\begin{array}{l}\text { I } \\
\text { I } \\
\text { I } \\
\text { I } \\
\text { I }\end{array}$ & $\begin{array}{l}8962.17 \\
8962.87 \\
8964.00 \\
8966.98 \\
8967.61\end{array}$ & $\begin{array}{r}8 \\
8 \\
4 \\
2 \\
100\end{array}$ & 5 & $\begin{array}{l}\text { I } \\
\text { I } \\
\text { I } \\
\text { I } \\
\text { I }\end{array}$ & $\begin{array}{l}9069.55 \\
9070.38 \\
9072.26 \\
9079.20 \\
9082.50\end{array}$ & $\begin{array}{l}2 \\
10 \mathrm{w} \\
10 \\
20 \\
5\end{array}$ & 5 & $\begin{array}{l}\text { I } \\
\text { I } \\
\text { I I } \\
\text { I } \\
\text { I }\end{array}$ \\
\hline $\begin{array}{l}8893.51 \\
8894.42 \\
8895.52 \\
8897.08 \\
8900.09\end{array}$ & $\begin{array}{r}15 \\
2 \\
2 \\
4 \\
2\end{array}$ & & $\begin{array}{l}\text { I } \\
\text { I } \\
\text { I } \\
\text { I } \\
\text { I }\end{array}$ & $\begin{array}{l}8969.85 \\
8972.85 \\
8973.73 \\
8979.59 \\
8979.69\end{array}$ & $\begin{array}{r}20 \\
1 \\
1 \\
2 \\
1\end{array}$ & & $\begin{array}{l}\text { I } \\
\text { I } \\
\text { I } \\
\text { I } \\
\text { I }\end{array}$ & $\begin{array}{l}9084.62 \\
9089.55 \\
9090.79 \\
9094.70 \\
9094.85\end{array}$ & $\begin{array}{l}1 \\
1 \\
4 \\
5 \\
4\end{array}$ & & $\begin{array}{l}\text { I } \\
\text { I } \\
\text { I } \\
\text { I } \\
\text { I }\end{array}$ \\
\hline $\begin{array}{l}8900.91 \\
8904.14 \\
8904.82 \\
8905.55 \\
8907.00\end{array}$ & $\begin{array}{l}4 \\
2 \mathrm{w} \\
1 \\
2 \\
15\end{array}$ & & $\begin{array}{l}\text { I } \\
\text { I } \\
\text { I } \\
\text { I } \\
\text { I }\end{array}$ & $\begin{array}{l}8980.68 \\
8981.11 \\
8985.28 \\
8985.82 \\
8986.36\end{array}$ & $\begin{array}{l}5 \\
2 \\
2 \\
2 \\
1\end{array}$ & & $\begin{array}{l}\text { I } \\
\text { I } \\
\text { I } \\
\text { I } \\
\text { I }\end{array}$ & $\begin{array}{l}9101.04 \\
9101.76 \\
9119.60 \\
9126.00 \\
9129.10\end{array}$ & $\begin{array}{l}2 \\
5 \\
4 \\
5 \\
1\end{array}$ & 1 & $\begin{array}{l}\text { I } \\
\text { I } \\
\text { I I } \\
\text { I } \\
\text { I }\end{array}$ \\
\hline $\begin{array}{l}8908.65 \\
8910.28 \\
8910.83 \\
8912.77 \\
8913.42\end{array}$ & $\begin{array}{r}8 \\
4 \\
20 \\
15 \\
8\end{array}$ & & $\begin{array}{l}\text { I } \\
\text { I } \\
\text { I } \\
\text { I } \\
\text { I }\end{array}$ & $\begin{array}{l}8987.37 \\
8990.90 \\
8992.04 \\
8995.14 \\
8997.87\end{array}$ & $\begin{array}{r}15 \\
2 \\
1 \\
15 \\
4\end{array}$ & & $\begin{array}{l}\text { I } \\
\text { I } \\
\text { I } \\
\text { I } \\
\text { I }\end{array}$ & $\begin{array}{l}9132.25 \\
9134.68 \\
9140.57 \\
9141.70 \\
9165.01\end{array}$ & $\begin{array}{r}1 \\
5 \\
15 \\
1 \\
1\end{array}$ & & $\begin{array}{l}\text { I } \\
\text { I } \\
\text { I } \\
\text { I } \\
\text { I }\end{array}$ \\
\hline $\begin{array}{l}8914.10 \\
8914.67 \\
8915.32 \\
8915.91 \\
8916.48\end{array}$ & $\begin{array}{l}2 \\
1 \\
4 \\
2 \\
4\end{array}$ & & $\begin{array}{l}\text { I } \\
\text { I } \\
\text { I } \\
I \\
I\end{array}$ & $\begin{array}{l}8999.53 \\
9004.50 \\
9007.78 \\
9009.87 \\
9010.39\end{array}$ & $\begin{array}{l}2 \\
2 w \\
5 \\
8 \\
2\end{array}$ & & $\begin{array}{l}\text { I } \\
\text { I } \\
\text { I } \\
\text { I } \\
\text { I }\end{array}$ & $\begin{array}{l}9165.90 \\
9167.82 \\
9170.79 \\
9178.80 \\
9180.24\end{array}$ & $\begin{array}{r}8 \\
2 \\
2 \\
5 \\
10\end{array}$ & & $\begin{array}{l}\text { I } \\
\text { I } \\
\text { I } \\
\text { I } \\
\text { I }\end{array}$ \\
\hline $\begin{array}{l}8916.98 \\
8917.33 \\
8917.72 \\
8918.66 \\
8919.75\end{array}$ & $\begin{array}{l}1 \\
1 \\
1 \\
1 \\
4\end{array}$ & & $\begin{array}{l}\text { I } \\
\text { I } \\
\text { I } \\
\text { I } \\
\text { I }\end{array}$ & $\begin{array}{l}9011.40 \\
9012.50 \\
9013.97 \\
9016.58 \\
9026.59\end{array}$ & $\begin{array}{r}1 \\
15 \\
1 \\
30 \\
1\end{array}$ & & $\begin{array}{l}\text { I } \\
\text { I } \\
\text { I } \\
\text { I } \\
\text { I }\end{array}$ & $\begin{array}{l}9187.54 \\
9193.60 \\
9199.70 \\
9201.72 \\
9203.93\end{array}$ & $\begin{array}{r}2 \\
2 \\
2 \\
2 \\
30\end{array}$ & 10 & $\begin{array}{l}\text { I } \\
\text { I } \\
\text { I } \\
\text { I } \\
\text { I }\end{array}$ \\
\hline $\begin{array}{l}8922.53 \\
8923.01 \\
8924.22 \\
8925.07 \\
8927.70\end{array}$ & $\begin{array}{l}2 \\
1 \\
4 w \\
4 \\
8\end{array}$ & & $\begin{array}{l}\text { I } \\
\text { I } \\
\text { I } \\
\text { I } \\
\text { I }\end{array}$ & $\begin{array}{l}9029.95 \\
9031.80 \\
9032.60 \\
9034.23 \\
9037.88\end{array}$ & $\begin{array}{l}1 \\
8 \\
2 \mathrm{w} \\
4 \mathrm{w} \\
15\end{array}$ & & $\begin{array}{l}\text { I } \\
\text { I } \\
\text { I } \\
\text { I } \\
\text { I }\end{array}$ & $\begin{array}{l}9208.58 \\
9215.92 \\
9221.44 \\
9222.29 \\
9227.53\end{array}$ & $\begin{array}{r}2 \\
4 \\
2 \\
2 \\
10\end{array}$ & & $\begin{array}{l}\text { I } \\
\text { I } \\
\text { I } \\
\text { I } \\
\text { I }\end{array}$ \\
\hline $\begin{array}{l}8928.08 \\
8930.00 \\
8935.78 \\
8940.31 \\
8941.64\end{array}$ & $\begin{array}{r}15 \\
1 \\
8 \\
1 \\
8\end{array}$ & & $\begin{array}{l}\text { I } \\
\text { I } \\
\text { I } \\
\text { I } \\
\text { I }\end{array}$ & $\begin{array}{l}9038.69 \\
9040.11 \\
9042.37 \\
9045.34 \\
9046.15\end{array}$ & $\begin{array}{l}5 \\
15 \\
1 \\
15 \\
10 \mathrm{w}\end{array}$ & $\begin{array}{r}5 \\
15\end{array}$ & $\begin{array}{l}\text { II } \\
\text { I I } \\
\text { I } \\
\text { I } \\
\text { I }\end{array}$ & $\begin{array}{l}9232.48 \\
9233.24 \\
9233.83 \\
9243.74 \\
9246.48\end{array}$ & $\begin{array}{l}2 \\
2 \\
2 \\
1 \\
1\end{array}$ & 1 & $\begin{array}{l}\text { I } \\
\text { II } \\
\text { I } \\
\text { I } \\
\text { I }\end{array}$ \\
\hline $\begin{array}{l}8942.62 \\
8945.06 \\
8947.99 \\
8948.40 \\
8949.11\end{array}$ & $\begin{array}{r}1 \\
1 \\
1 \\
15 \\
25\end{array}$ & & $\begin{array}{l}I \\
I \\
I \\
I \\
I\end{array}$ & $\begin{array}{l}9046.25 \\
9046.34 \\
9048.24 \\
9050.24 \\
9053.47\end{array}$ & $\begin{array}{c}15 \mathrm{w} \\
5 \\
100 \\
15 \mathrm{w} \\
15\end{array}$ & 15 & $\begin{array}{l}\text { I } \\
\text { I } \\
\text { I } \\
\text { I } \\
\text { I I }\end{array}$ & $\begin{array}{l}9252.84 \\
9260.32 \\
9266.22 \\
9266.98 \\
9267.68\end{array}$ & $\begin{array}{r}1 \\
2 \\
25 \\
15 \\
10\end{array}$ & $\begin{array}{r}2 \\
5 \\
10 \\
8\end{array}$ & $\begin{array}{l}\text { I I } \\
\text { I } \\
\text { I } \\
\text { I I } \\
\text { I I }\end{array}$ \\
\hline $\begin{array}{l}8952.10 \\
8955.82 \\
8956.49 \\
8957.97 \\
8959.22\end{array}$ & $\begin{array}{r}1 \\
15 \\
1 \\
20 \\
1\end{array}$ & 5 & $\begin{array}{l}\text { I } \\
\text { I } \\
\text { I } \\
\text { II } \\
\text { I }\end{array}$ & $\begin{array}{l}9061.80 \\
9062.56 \\
9063.93 \\
9064.48 \\
9066.10\end{array}$ & $\begin{array}{r}1 \\
10 \\
15 \\
1 \\
12\end{array}$ & 10 & $\begin{array}{l}\text { I } \\
\text { I I } \\
\text { I } \\
\text { I } \\
\text { I I }\end{array}$ & $\begin{array}{l}9270.14 \\
9276.28 \\
9276.98 \\
9277.75 \\
9287.58\end{array}$ & $\begin{array}{c}4 \\
10 \\
2 \mathrm{w} \\
1 \\
2\end{array}$ & 1 & $\begin{array}{l}\text { I } \\
\text { I } \\
\text { I } \\
\text { I } \\
\text { I }\end{array}$ \\
\hline
\end{tabular}


Table 1. Wavelengths of Thorium Spectra - Continued

\begin{tabular}{|c|c|c|c|c|c|c|c|c|c|c|c|}
\hline \multirow{2}{*}{$\begin{array}{l}\text { Wave- } \\
\text { length }\end{array}$} & \multicolumn{2}{|c|}{ Intensity } & \multirow{2}{*}{$\begin{array}{l}\text { Spec- } \\
\text { trum }\end{array}$} & \multirow{2}{*}{$\begin{array}{l}\text { Wave- } \\
\text { length }\end{array}$} & \multicolumn{2}{|c|}{ Intensity } & \multirow{2}{*}{$\begin{array}{l}\text { Spec- } \\
\text { trum }\end{array}$} & \multirow{2}{*}{$\begin{array}{l}\text { Wave- } \\
\text { length }\end{array}$} & \multicolumn{2}{|c|}{ Intensity } & \multirow{2}{*}{$\begin{array}{l}\text { Spec. } \\
\text { trum }\end{array}$} \\
\hline & Lamp & Spark & & & $\operatorname{Lamp}$ & Spark & & & Lamp & Spark & \\
\hline 9289.56 & 15 & 1 & I & 9497.20 & 20 & 3 & I & 9665.94 & $5 w$ & & I \\
\hline 9294.97 & 2 & & I & 9500.30 & 2 & & I & 9670.76 & 2 & & I \\
\hline 9300.00 & 2 & & I & 9501.42 & 1 & & I & 9674.74 & 2 & & I \\
\hline 9307.88 & 12 & $i$ & I & 9505.38 & 10 & & I & 9676.09 & 3 & & I \\
\hline 9310.41 & & & I & 9507.63 & 2 & & I & 9676.80 & 3 & & I \\
\hline 9317.72 & 3 & & I & 9509.20 & 1 & & I & 9678.18 & 1 & & I \\
\hline 9319.98 & $1 \mathrm{~h}$ & & I & 9510.96 & 1 & & I & 9678.34 & $1 \mathrm{w}$ & & I \\
\hline 9340.71 & 10 & & I & 9517.18 & 2 & & I & 9679.73 & $2 \mathrm{w}$ & & I \\
\hline 9344.10 & 2 & & I & 9519.65 & $2 w$ & & I & 9695.00 & 1 & & I \\
\hline 9347.42 & 3 & & I & 9528.50 & 2 & & I & 9697.54 & 20 & 3 & I \\
\hline 9355.95 & 2 & & I & 9536.37 & 2 & & $I$ & 9701.44 & 1 & & I \\
\hline 9357.25 & 1 & & I & 9540.35 & 1 & & $\bar{I}$ & 9702.22 & 4 & & I \\
\hline 9360.95 & 10 & 5 & II & 9547.28 & 2 & & I & 9705.69 & 2 & & I \\
\hline 9362.59 & 4 & & II & 9547.99 & 3 & & I & 9705.82 & $2 w$ & & I \\
\hline 9362.73 & $1 \mathrm{~h}$ & 2 & II & 9548.70 & 1 & & I & 9710.64 & $2 w$ & & I \\
\hline 9365.20 & 2 & & I & 9551.67 & 1 & & I & 9715.14 & $1 \mathrm{w}$ & & I \\
\hline 9379.69 & $\mathrm{l} w$ & & I & 9553.96 & 4 & & I & 9716.07 & 2 & & I \\
\hline 9383.23 & 10 & & I & 9555.71 & 1 & & I & 9725.47 & 30 & & I \\
\hline 9388.92 & 8 & & I & 9561.22 & 10 & 2 & I & 9743.55 & 6 & & I \\
\hline 9390.60 & 5 & & I & 9565.56 & 2 & & I & 9744.70 & $10 \mathrm{w}$ & & I \\
\hline 9399.10 & 40 & 20 & I & 9567.28 & 1 & & I & 9746.44 & 25 & 6 & I \\
\hline 9409.34 & 10 & & I & 9567.79 & 1 & & I & 9753.54 & 3 & & I \\
\hline 9413.66 & 3 & & I & 9570.33 & $1 \mathrm{~h}$ & & I & 9758.42 & 1 & 2 & I I \\
\hline 9414.10 & 2 & & I & 9577.31 & 8 & 6 & II & 9769.51 & 3 & & I \\
\hline 9415.76 & 1 & & I & 9578.59 & 1 & & I & 9772.27 & 1 & & I \\
\hline 9417.42 & 1 & & I & 9579.04 & 2 & & I & 9773.54 & 2 & & I \\
\hline 9420.58 & 1 & & $\bar{I}$ & 9579.13 & 2 & & I & 9775.12 & 1 & & I \\
\hline 9431.58 & 10 & & I & 9582.82 & 5 & & I & 9779.41 & 3 & & I \\
\hline 9432.26 & 10 & 6 & I I & 9583.09 & 2 & 2 & II & 9785.32 & 1 & & I \\
\hline 9436.79 & 2 & & I & 9587.00 & 2 & & I & 9789.47 & 3 & & I \\
\hline 9439.30 & $1 \mathrm{~h}$ & & I & 9588.78 & 1 & & I & 9792.86 & 1 & & I \\
\hline 9441.61 & 1 & & I & 9590.32. & 2 & & I & 9794.58 & 1 & & I \\
\hline 9442.00 & 1 & & I & 9595.36 & 4 & & I & 9796.15 & 5 & & I \\
\hline 9446.93 & 1 & & I & 9607.51 & 6 & 5 & I I & 9797.21 & 2 & 7 & I \\
\hline 9450.47 & 1 & & I & 9608.46 & 5 & & I & 9812.67 & 12 & 1 & 1 \\
\hline 9453.58 & 1 & & I & 9613.67 & 15 & 10 & II & 9822.35 & 1 & & I \\
\hline 9455.21 & 3 & & I & 9619.17 & 1 & & I & 9826.45 & 20 & 4 & $I$ \\
\hline 9455.91 & $1 \mathrm{~h}$ & & I & 9620.96 & 4 & & I & 9831.61 & 2 & 2 & $\frac{1}{T}$ \\
\hline $\begin{array}{l}9456.02 \\
9458.57\end{array}$ & $\begin{array}{l}3 \\
1 \mathrm{w}\end{array}$ & & $\begin{array}{l}I \\
I\end{array}$ & $\begin{array}{l}9623.34 \\
9625.15\end{array}$ & $\begin{array}{l}2 \\
4\end{array}$ & & $\begin{array}{l}\text { I } \\
\text { I }\end{array}$ & $\begin{array}{l}9833.41 \\
9833.97\end{array}$ & $\begin{array}{r}20 \\
5\end{array}$ & $\begin{array}{l}2 \\
1\end{array}$ & I \\
\hline 9461.04 & 12 & 2 & I & 9625.76 & 1 & & I & 9845.61 & 1 & & I \\
\hline 9464.99 & 1 & & $\bar{I}$ & 9627.36 & 1 & & I & 9855.68 & 2 & & I \\
\hline 9467.20 & 12 & & I & 9629.54 & 10 & & I & 9863.90 & 1 & & I \\
\hline 9470.68 & 15 & & I & 9632.65 & 15 & 1 & I & 9864.54 & 3 & 1 & II \\
\hline 9474.89 & 25 & 6 & I & 9638.55 & 1 & & I & 9871.99 & 2 & & I \\
\hline 9477.25 & 2 & 3 & I I & 9640.26 & $3 w$ & & I & 9872.58 & 2 & & I \\
\hline 9482.27 & 4 & & I & 9641.82 & 5 & & I & 9873.77 & 10 & & I \\
\hline 9483.80 & 2 & 2 & II & 9642.43 & 3 & 2 & II & 9890.00 & $5 \mathrm{w}$ & & I \\
\hline 9486.91 & 4 & & I & 9643.29 & 1 & & I & 9896.04 & 3 & & I \\
\hline 9495.50 & 15 & 2 & I & 9664.70 & $10 \mathrm{w}$ & 1 & I & 9898.30 & 2 & & 1 \\
\hline
\end{tabular}


Table 1. Wavelengths of Thorium Spectra - Continued

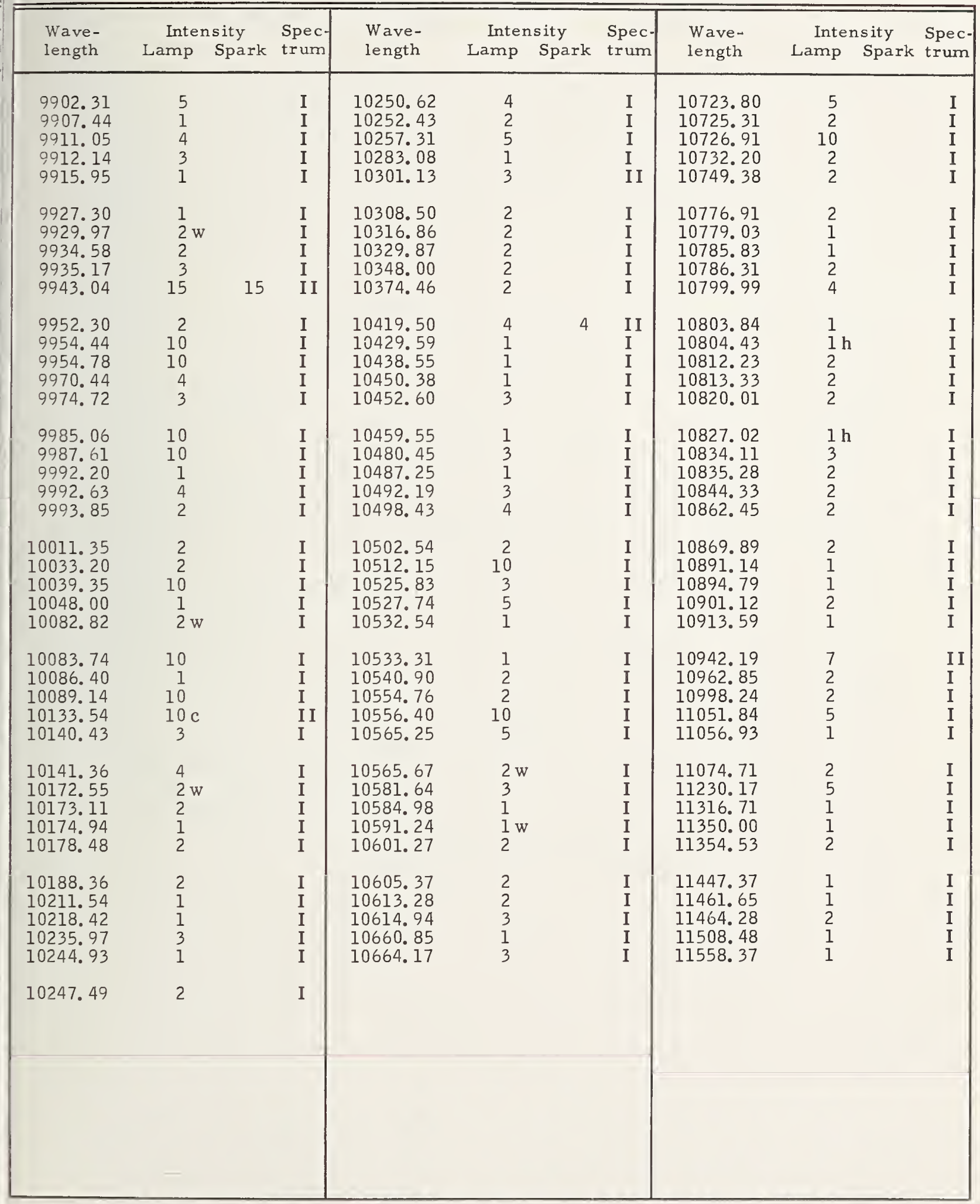





\section{Selected Publications of the National Bureau of Standards}

Table of Wavenumbers, C. D. Coleman, W. R. Bozman, and IT. F. Meggers:

Monograph 3, Volume I. $2000 \mathrm{~A}$ to $7000 \mathrm{~A}$.

Monograph 3, Volume II. 7000 A to $1000 \mu$.

Atomic Energy Levels, C. E. Moore:

Circular 467 , Volume I. H to $\mathrm{V}(\mathrm{Z}=1$ to 23$) 206$ spectra. 309 p. (1949)

Circular 467, Volume II. Cr to $\mathrm{Nb}(\mathrm{Z}=24$ to 41$) 152$ spectra. 227 p. (1952)

Circular 467 , Volume III. $\left\{\begin{array}{l}\text { Mo to La }(\mathrm{Z}=42 \text { to } 57) \\ \text { Hf to Ac }(\mathrm{Z}=72 \text { to } 89)\end{array}\right\} 124$ spectra. 245 p. (1958)

An Ultraviolet Multiplet Table, C. E. Moore:

Circular 488, Section 1. $\mathrm{H}$ to $\mathrm{V}(\mathrm{Z}=1$ to 23$)$; Selected Multiplets of 79 Spectra. 78 p. $(1950)$.

Circular 488, Section 2. Cr to Nb $(Z=24$ to 41$)$; Selected Multiplets of 46 Spectra. 115 p. (1952).

The above publications may be purchased from the Superintendent of Documents, U.S. Government Printing Office, Washington 25 , D.C.

A Multiplet Table of Astrophysical Interest, C. E. Moore. A Reprinting of the 1945 Multiplet Table (Princeton Univ., Obs. Contr. No. 20):

Technical Note 36 (PB151395), Part I. Table of Multiplets and Part II. Finding List, $\lambda \lambda 2951 \AA-13164 \AA .242$ p. (1959).

The above Technical Note may be purchased by the PB number from the Department of Commerce, Office of Technical Services, Washington 25, D.C. 


\section{THE NATIONAL BUREAU OF STANDARDS}

The scope of activities of the National Bureau of Standards at its major laboratories in Washington, D.C., and Boulder, Colorado, is suggested in the following listing of the divisions and sections engaged in technical work. In general, each section carries out specialized research, development, and engineering in the field indicated by its title. A brief description of the activities, and of the resultant publications, appears on the inside of the front cover.

WASHINGTON, D.C.

Electricity and Electronics. Resistance and Reactance. Electron Devices. Electrical Instruments. Magnetic Measurements. Dielectrics. Engineering Electronics. Electronic Instrumentation. Electrochemistry.

Optics and Metrology. Photometry and Colorimetry. Optical Instruments. Photographic Technology. Length. Engineering Metrology.

Heat. Temperature Physics. Thermodynamics. Cryogenic Physics. Rheology. Molecular Kinetics. Free Radicals Research.

Atomic Physics. Spectroscopy. Radiometry. Mass Spectrometry. Solid State Physics. Electron Physics. Atomic Physics.

Radiation Physics. Neutron Physics. Radiation Theory. Radioactivity. X-ray. High Energy Radiation. Nucleonic Instrumentation. Radiological Equipment.

Chemistry. Organic Coatings. Surface Chemistry. Organic Chemistry. Analytical Chemistry. Inorganic Chemistry. Electrodeposition. Molecular Structure and Properties of Gases. Physical Chemistry. Thermochemistry. Spectrochemistry. Pure Substances.

Mechanics. Sound. Mechanical Instruments. Fluid Mechanics. Engineering Mechanics. Mass and Scale, Capacity, Density, and Fluid Meters. Conbustion Controls.

Organic and Fibrous Materials. Rubber. Textiles. Paper. Leather. Testing and Specifications. Polymer Structure. Plastics. Dental Research.

Metallurgy. Thermal Metallurgy. Chemical Metallurgy. Mechanical Metallurgy. Corrosion. Metal Physics.

Mineral Products. Engineering Ceramics. Glass. Refractories. Enameled Metals. Constitution and Microstructure.

Building Technology. Structural Engineering. Fire Protection. Air Conditioning, Heating, and Refrigeration. Floor, Roof, and Wall Coverings. Codes and Safety Standards. Heat Transfer. Concreting Materials.

Applied Mathematics. Numerical Analysis. Computation. Statistical Engineering. Mathematical Physics.

Data Processing Systems. SEAC Engineering Group. Components and Techniques. Digital Circuitry. Digital Systems. Analog Systems. Applications Engineering.

- Office of Basic Instrumentation.

- Office of Weights and Measures

\section{BOULDER, COLORADO}

Cryogenic Engineering. Cryogenic Equipment. Cryogenic Processes. Properties of Materials. Gas Liquefaction.

Radio Propagation Physics. Upper Atmosphere Research. Ionosphere Research. Regular Prediction Services. Sun-Earth Relationships. VIIF Research. Radio Warning Services. Airglow and Aurora. Radio Astronomy and Arctic Propagation.

Radio Propagation Engineering. Data Reduction Instrumentation. Radio Noise. Tropospheric Measurements. Tropospheric Analysis. Propagation-Terrain Effects. Radio Meteorology. Lower Atmosphere Physics.

Radio Standards. High-Frequency Electrical Standards. Radio Broadcast Service. Radio and Microwave Materials.

Atomic Frequencyand Time Standards. Electronic Calibration Center. Microwave Circuit Standards.

Radio Communication and Systems. Low Frequency and Very Low Frequency Research. High Frequency and Very High Frequency Research. Modulation Systems. Antenna Research. Navigation Systems. Systems Analysis. Field Operations. 
